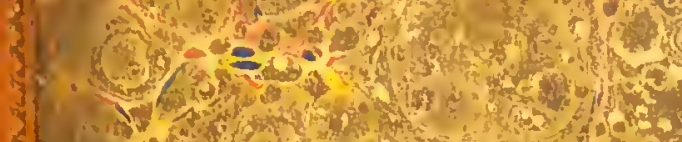

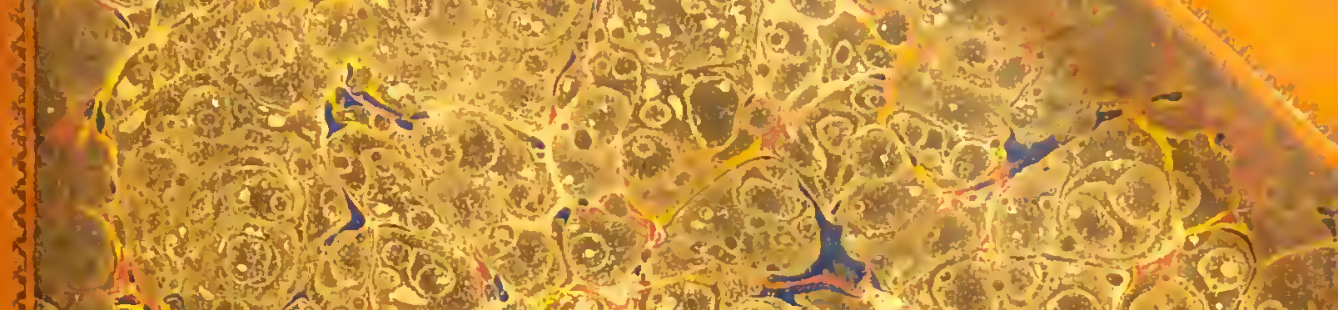

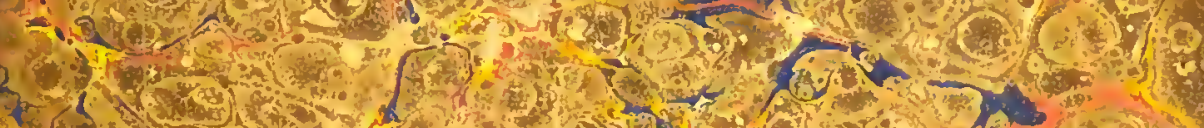

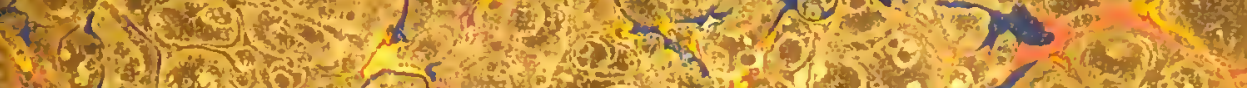

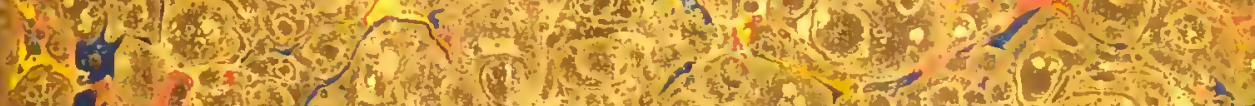

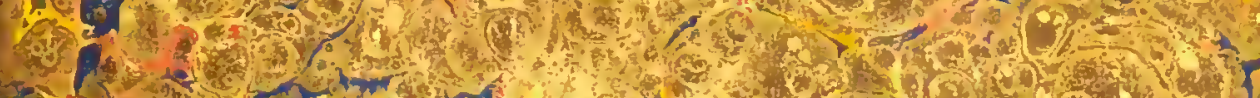

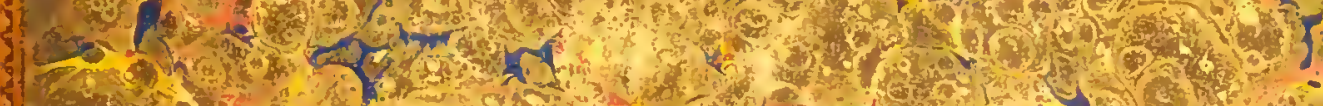

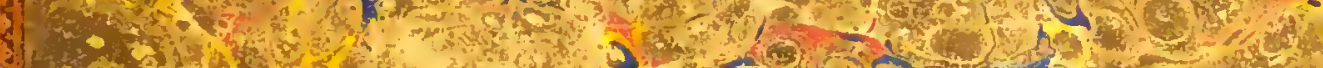

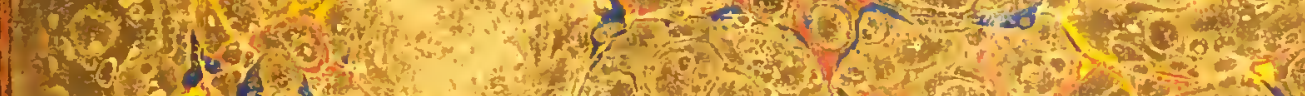

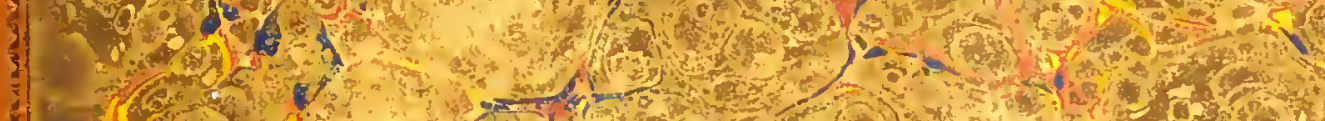
(6)

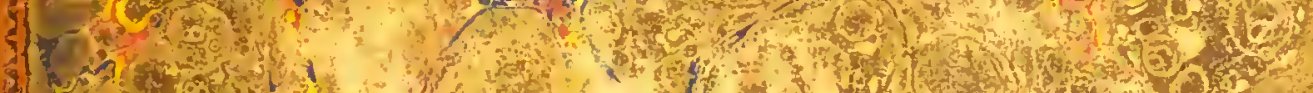

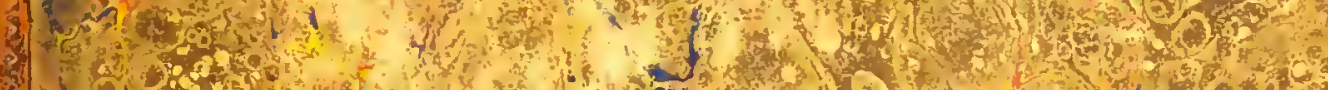

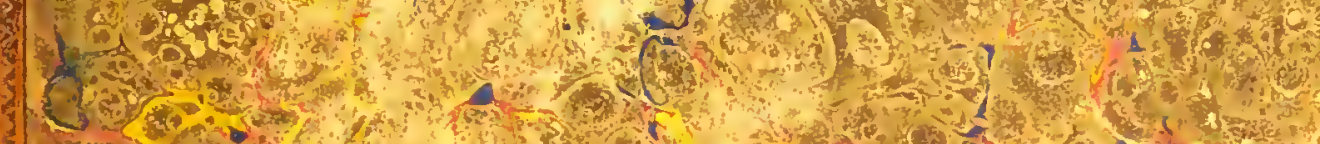

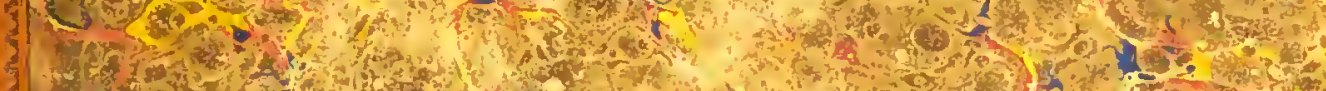

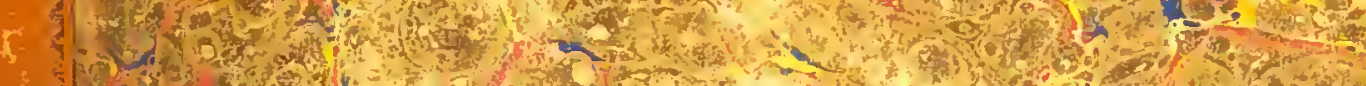

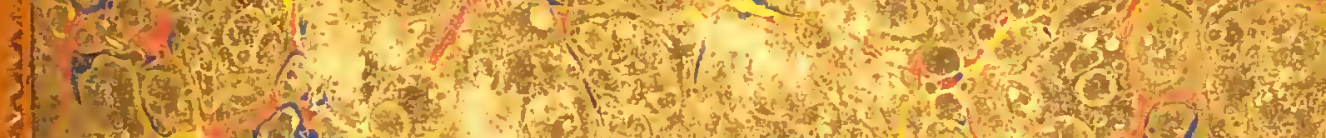

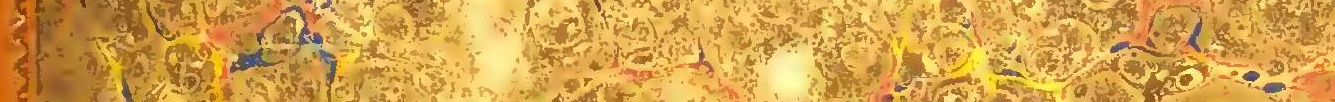

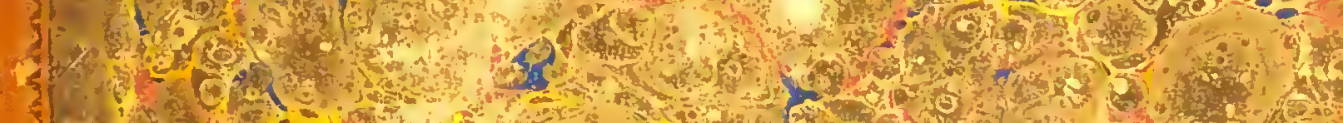

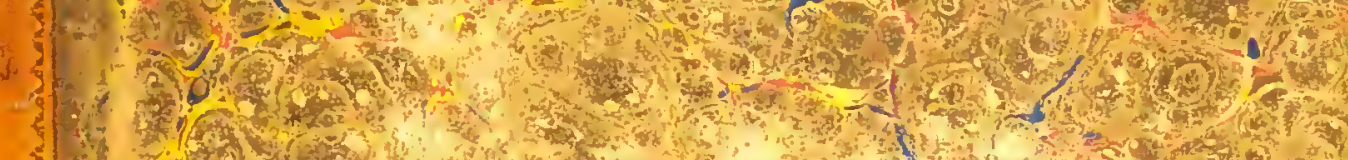
3. 4.

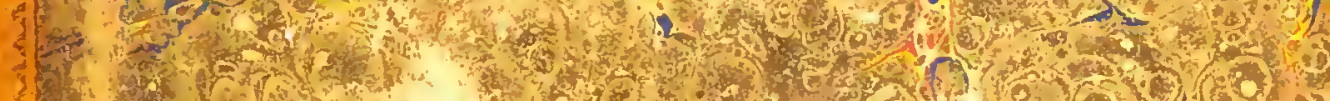

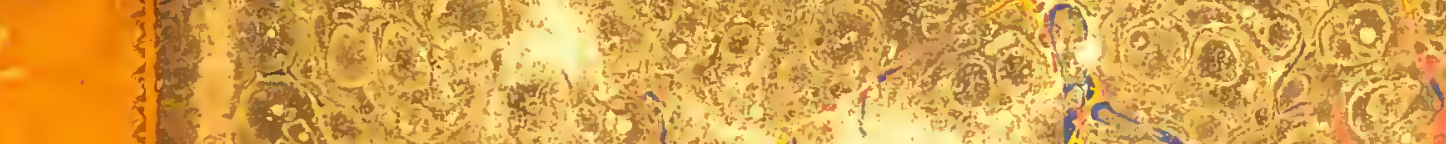

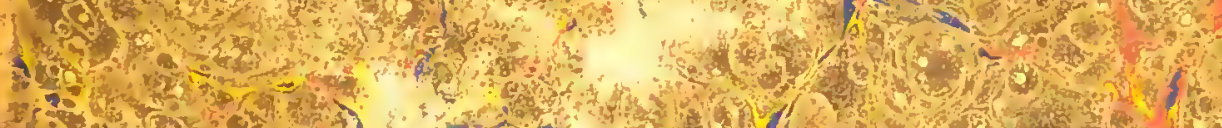

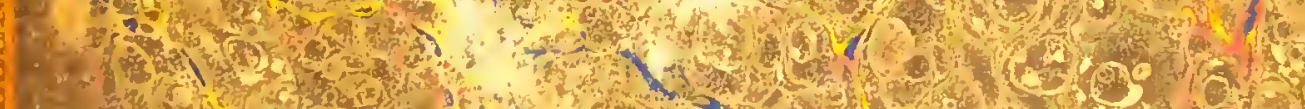

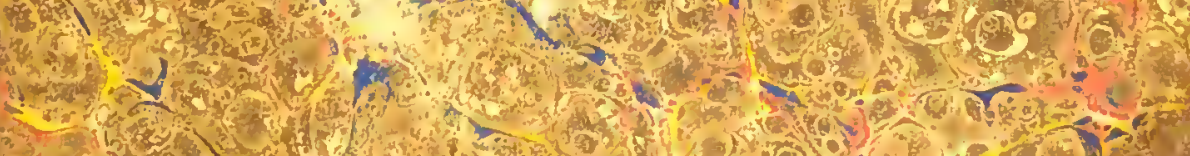

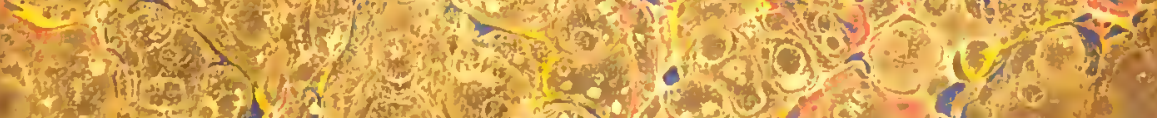

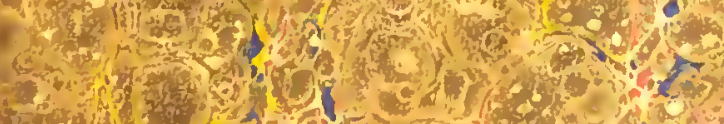

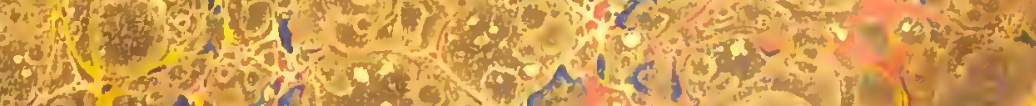

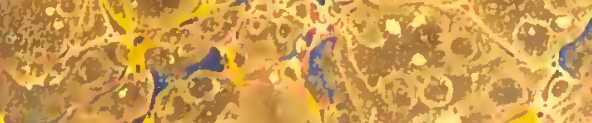

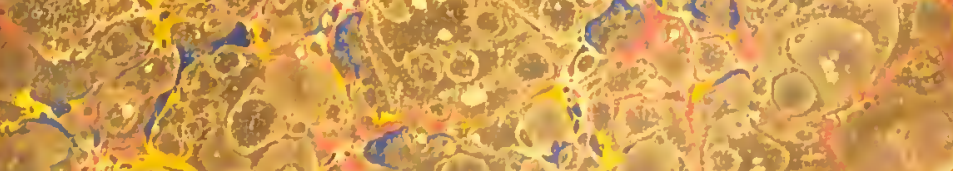

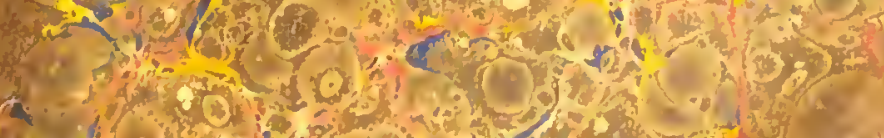


P. 
<smiles>C=C[As](C)C</smiles> 





\section{NOUVEAUX ÉLÉMENS}

\section{DE BOTA NIOUE.}


SE TROUVE AUSSI CHEZ LES LIBRAIRES CI-APRÉS:

\author{
A Bayonne, Bonzom. \\ Bordeaux, LAWAL. \\ Brest, Lefournier et Depériers. \\ Lyon, $\left\{\begin{array}{l}\text { Marre. } \\ \text { Milon. }\end{array}\right.$ \\ Marseille, Camoin. \\ Montpellier, $\left\{\begin{array}{l}\text { Sevaliz. } \\ \text { Pomathio. }\end{array}\right.$ \\ Nantes, Folest. \\ Strasbourg, $\left\{\begin{array}{l}\text { Feviner. } \\ \text { Levraud.T. }\end{array}\right.$ \\ Toulon : Beliue. \\ Toulouse, Senac.
}

PARIS. - IMPRIMERIE DE COSSON,

rue Saint-Germain-des-Prés, $\mathbf{n}^{\circ} \mathrm{g}$. 


\title{
NOUVEAUX ÉLÉMENS
}

\section{DE BOTANIQUE}

ET DE

\section{PHYSIOLOGIE VÉGÉTALE,}

\author{
QUATRIÈME ÉDIṬION, \\ REVUE, CORBTGÉE ET AUGMENTÉE
}

DU CARACTÈRE DES FAMILLES NATURELLES

DU RÈGNE VÉGÉTAL;

\section{PAR AGHILLE RIGAARDจ}

Docteur en Médecine, Agrégé près la Faculté de Médecine de Paris; aide naturaliste au Muséum d'Histoire naturelle; Membrc de l'Académie royale de Médecine; de la société philomatique et de la Société d'Histoire matarelle de Paris, etc.

\section{AVIC HUIT PIANCEIS EN TAIIIE-DOUCE,}

REPRÉSEXTANT LES PRINGIPALES MODIFICATIONS DES ORgANES DES VÉGÉTAUX.

\section{PARIS,}

BECHET JEUNE,

LBRAIRE DE I'ACADÉMIE BOXATE DE MÉDECINE, PLAGR DE L'ÉCOTE DR MÉDECINE, $x^{\circ} 4$.

BRUXELTES,

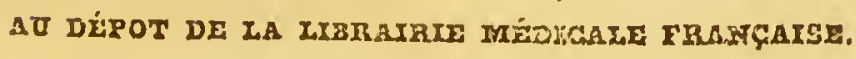




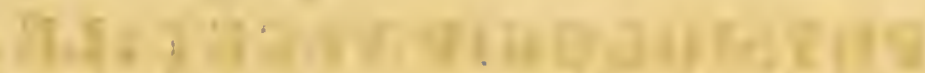

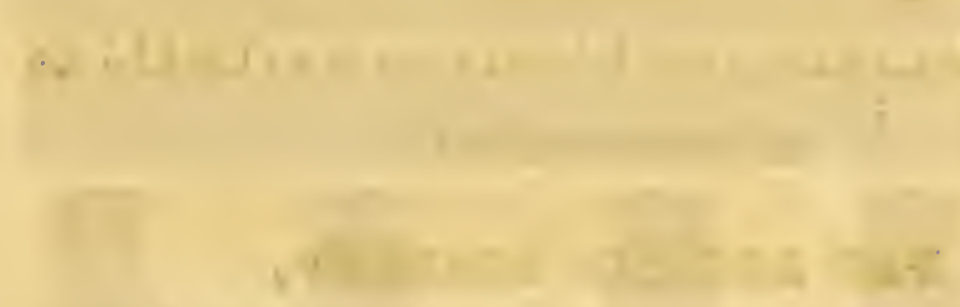

Digitized by the Internet Archive in 2015 


\section{A DI LD BADDN}

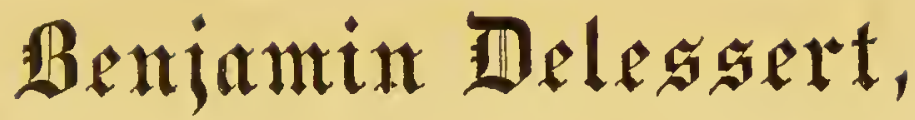

ASรOGré IrBRE

DE L'ACADÉMIE ROYALE DES SCIENCES,

ET DE LA SOCIÉTÉ D'HISTOIRE TATORELLB DE PARIS, ETC.

\section{HOMMAGE}

DU PROFOND RESPECT ET DE IA RECONNAISSANCE DE L'AUTEUR

Stechille Brichard. 



\section{AVERTISSEMENT}

SUR

\section{LA QUATRIÈME EDITION.}

La quatrième édition de nos Élémens de Botaniquf et de Physiologie végétale que nous publions aujourd'hui, a été améliorée autant qu'il a dépendu de nous, afin de tâcher de justifier par nos efforts le succès toujours croissant que cet ouvrage obtient. Indépendamment des changemens et des additions que nous avons faits aux différens chapitres et en particulier à ceux qui traitent de la structure des tiges monocotylédones, de l'organisation générale de la fleur, de celle du pollen et de son action, lors de la fécondation, de celle de l'ovule antérieurement à limprégnation, etc., etc., nous devons signaler surtout l'addition importante des caractères de toutes les familles du règne végétal. Le nombre des familles dont nous présentons ici le tableau s'élè ve à cenc soixante-deux. Il 
eût été encore plus considérable, si nous eussions eu le dessein d'y faire entrer toutes les far milles qui ont été successivement proposées ou établies depuis la publication du Genera plantarum de M. de Jussieu. Mais non-seulement nous avons réuni à d'autres familles dejjà existantes un assez grand nombre de celles qui ont été nouvellement proposées, mais nous avons aussi pensé que, dans un ouvrage élémentaire; nous pouvions, sans inconvénient, omettre quelques familles encore trop imparfaitement connues, soit dans leurs caractères généraux, soit dans les genres qui doivent les composer, soit enfin dans la place qu'elles doivent occuper dans la série des ordres naturels. Nous nous hâtons de faire cette remarque, afin que l'on. ne nous accuse pas de n'avoir pas parlé dans cet ouvrage de plusieurs familles récemment établies.

Nous devóns aussi dire un mot sur la réunion que nous avons cru devoir faire quelquefois de plusieurs familles en une seule. Dans l'état actuel de la science, nous pensons qu'il y a peut-être plus de réductions à faire dans le nombre des genres et des familles, qu'il n'y a lieu à multiplier ce nombre. Un coup d'oil rapide jeté en passant sur les phases de la botanique, depuis l'établissement de la méthode des familles naturelles, démontrera suffisamment cette vérivé. 
Dans les premières années qui suivirent la publication du Genera plantarum de M. de Jussieu, cet ouvrage, qui de nos jours encore est un des plus beaux monumens élevés à la gloire de la botanique, en même temps qu'il est, pour celui qui sait le méditer, une source de connaissances aussi profondes que positives, fut la règle invariable qui servit à caractériser et les genres et les familles résultant du rapprochement de ceux-ci. Mais les progrès que fit faire à la science l'étude plus approfondie de la structure de la graine et du fruit, les avantages qu'elle présenta pour la coordination des genres et des familles, amenèrent de notables changemens dans l'étude de la botanique. On sentil la nécessité de pénétrer encore plus profondément dans l'organisation des diverses parties de la fleur et en particulier de l'ovaire, de la graine et du fruit, qui avaient été reconnus comme fournissant les caracières les plus importans pour y puiser les affinités naturelles des végétaux. On soumit donc à une nouvelle investigation les genres réunis dans chacun des cent ordres naturels préseniés dans le Genera plantarum; et de cette analyse plus précise, dirigée surtont vers les organes les plus essentiels, résulta nécessairement la découverte d'un grand nombre de caractères, d'analogies ou de différences, qui avaient étéjusqu'alors inaperȩus. Cette nouvelle 
marche imprimée à l'étude des végétaux amena la nécessitéd'introduire des modifications et dans la circonscription des genres, dont le nombre fut bientôt plus quedoublé, et dans celle des familles elles-mêmes. Mais dans cette première période de l'ère nouvelle de la science, il était naturel que les observateurs, découvrant chaque jour une foule de modifications nouvelles qui avaient échappé à leurs devanciers, fussent plus frappés des différences qu'ils observaient entre les genres et les familles, que des rapports nouveaux que l'analyse leur dévoilait. En effet, à cette époque, près les principes de la nouvelle école, étaient encore trop peu nombreux, trop isolés pour ne pas présenter en quelque sorte de grandes dissemblances; et comme il n'arrive que trop souvent dans l'étude des sciences, on généralisa trop tòt des faits qui n'étaient encore qu'isolés et spéciaux. De là ce grand nombre de genres et de familles nouvelles qui furent successivement établis, et qui fut bientòt clouble de celui du Genera plantarum. Mais l'impulsion était donnée, la bonne route était ouverte. L'investigation analy tique portée successivement sur un nombre toujour's croissant de végétaux, les découvertes des voyageurs, qui apportent chaque jour de nouveaux types d'organisation, nous paraissent devoir combler successivement un 
grand nombre des intervalles qui séparent les groupesjusqu’à présent établis. Dans la première période, chaque analyse nouvelle amenait la conṇaissance d'une modification nouvelle de l'organisation végétale, et devenait, en quelque sorte, un type isolé. Aujourd'hui que les observations se sont considérablement multipliées, des faits analogues sont venus se grouper autour des premiers, et par les modifications yariées que chacun d'eux présente, des nuances insensiblement graduées les ont en quelque sorte liés les uns aux autres et ont formé cette chaîne, si rarement interrompue, que tous les bons observateurs ont reconnu exister entre toutes les productions de la nature. Dans ce nouvel état de choses, on voit tous les jours disparaitre les caractères tranchés qu'on avait cru d'abord exister, soit entre les espèces qui composent les genres, soit entre les genres réunis en famille. Il en résulte nécessairement que comme les différences disparaissent, on doit anéantir les coupes ou divisions qui avaient été fondées sur elles. Aussi, nous le répétons, les progrès toujours croissans de la botanique nous paraissent devoir présenter pour résultat de diminuer de beaucoup, et le nombre des genres actuellement établis et cclui des groupes ou familles que l'on a formées par leur rapprochement. Mais ce travail est long et demande 
encore de nouvelles observations; si nous nous sommes quelquefois permis de ne pas admettre les idées des autres, nous ne l'avons fait qu'avec une sage réserve, surtout avec bonne foi, et non dans cet esprit étroit et mesquin de substituer nos propres idées à celles de nos devancicrs.

Nous avons suivi dans l'arrangement, ou coordination générale des familles, la série présentée par M. de Jussieu, à laquelle nous avons fait à peine quelques changemens; peu importe d'ailleurs la méthode que l'on suive, pourvu qu'on respecte, autant que possible, les affinités naturelles et évidentes qui existent entre les différentes familles. Car il paraît démontré aujourd'hui, pour tous les bons esprits, qu'il est impossible qu'une série linéaire ne rompe pas fréquemment les rapports naturels; et si l'on adopte, comme servant de base aux divisionś que l'on y établit, soit l'insertion, ainsi que l'avait fait M. de Jussieu, soit l'adhérence ou la non-adhérence de l'ovaire, comme je l'ai essayé dans ma Botanique mídicale, des exceptions nombreuses viennent à chaque instant contrarier la méthode.

Quant à la rédaction des familles elles-mêmes, nous avons en général préféré le nom qui le premier a été imposé, ne croyant pas qu'un simple changement dans la désinence de ce nom dût faire attribuer à un autre l'homneur de l'é- 
tablissement de la famille. Nous avons cité à la suite de ce nom, soit les synonymes de la famille, soit le nom de celles que nous avons cru devoir y être réunies. Tous nos caractères, si l'on en excepte un très-petit nombre dont les matériaux nous ont mancué, ont été faits d'après nature et assez souvent une analyse soignée des genres de chaque famille nous a amené à modifier les caractères qui en avaient été donnés jusqu'alors. Nous n'avons pas cru devoir, dans un ouvrage élémentaire, donner trop d'extensior ${ }_{\perp}$ à ces caractères; mais néanmoins nous $n^{\prime}$ avons rien omis de ce qui pouvait servir à bien distinguer les diverses familles; et comme le fruit et la graine fournissent généralement lés caractères les plus importans, leur description fait toujours partie du caractère général que nous traçons de chaque famille.

A la suite des caractères généraux, nous avons joint quelques observations, soit sur les affinités et les différences de chaque famille avec celles qui l'avoisinent, soit sur les divisions out tribus qui y ont été établies, soit enfin sur les familles qui doivent y être réunies; nous avons également soin d'indiquer les genres principaux qui les composent.

Afin que les personnes qui commencent l'étude de la botanique puissent ne s'occuper que desfamilles les plus distinctes, et surtout de celles 
dont ils peuvent trouver facilement des exemples dans la nature, nous avons marqué d'une astérisque $\left.{ }^{\star}{ }^{\star}\right)$ toutes les familles qui renferment des genres qui font partie de la Flore française.

Quant aux personnes qui, se destinant à l'art de guérir, cherchent dans l'étude de la botanique la connaissance des caractères et des propriétés médicales de tous les végétaux employés en médecine ou de tous les médicamens empruntés au règne végétal, elles trouveront dans notre Botanique médicale (1), tout ce que cette partie de l'histoire naturelle offre d'important à connaître pour le médecin.

En terminant cet Avertissement, nous renouvelons ici l'expression de notre reconnaissance pour ceux de MM. les professeurs qui nous font l'honneur de recommander à leurs élèves la lecture de notre livre, et plus particulièrement à MM. Desfontaines, professeur au Jardin du Roi; Guiart, professeur à l'école de pharmacie; Delile, professeur à la faculté de médecine de Montpellier; Nestler, professeur à la faculté de médecine de Strasbourg, etc.

Paris, rer février I 828 .

(1). Botanique médicane, ou Histoire naturelle et médicale de tous les Médicamens, Alimens ou Poisons tirés du rìgne végétal; 2 rol. in-8. Chez Béchet jeune. 


\section{PRÉFACE}

DE L A

\section{PREMIÈRE ÉDITION.}

L'ouvrage que nous publions aujourd'hui, sous le titre de Nouveadx Élźmens de Botanique appliquée à la médecine ( $\mathrm{r}$ ), était vivement désiré par les, personnes qui se livrent à l'étude de la Botanique, et surtout par les nombreux élèves qui suivent les. cours de la Faculté de médecine de Paris. Depuis long-temps un grand nombre d'entre eux s'étaient adressés à mon père pour l'engager à rédiger et à publier les leçons élémentaires de Botanique que, depuiș vingt-cinq ans, il faisait à la Faculté de mé-

(1) Tel était le titre de la première édition : nous avons cru devoir le modifier pour les suivantes, à cause des changemens et des additions considérables que nous y arons faits. 
decine de Paris. Mais d'autres occupations, et surtout la direction qu'il avait imprimée à ses travaux, dont le but principal était le perfectionnement de la partie philosophique de la science, l'avaient constamment détourné de l'exécution de ce projet. C'est donc d'après ses conseils, et en quelque sorte sous sa direction, que j'ai entrepris le travail que je livre aujourd'hui au public.Je ne me suis point dissimulé ses nombreuses difficultés: la composition d'un livre élémentaire est loin d'être facile. Cependant je ne suis pas très-éloigné de croire que, pour présenter les élémens d'une science avec simplicité, précision et clarté, il ne faut point encore avoir eu le temps d'oublier quels sont les obstacles que l'on a rencontrés soi-même, afin de les aplanir devant ceux que l'on dirige dans la même carrière.

Attaché depuis plusieurs années, en qualité de démonstrateur de Botanique, auprès de la Faculté de médecine de Paris, je me suis principalement occupé des moyens les plus convenables pour simplifier les élémens de cette science. C'est surtout en rédigeant cet ouvrage, que j’ai voulu élaguer de la Botanique les inutiles et vagues hypothèses, les détails fastidieux dont on l'a souvent et inutilement surchargée. Destinant principalement ce livre à l'instruction des jeunes gens qui s'adonnent à l'étude de l'art de guérir, sachant le nombre et l'importance des connaissances qu'ils doivent acquérir, connais- 
sances au nombre desquelles la Botanique occupe un rang distingué, je me suis efforcé de ne leur présenter que les notions en quelque sorte indispensables de cette branche de leurs études. Je n’ai voulu leur offrir de la Botanique que les principes les plus généraux et les mieux établis, que ceux enfin à l'aide desquels ils puissent facilement arriver à la connais: sance exacte des plantes officinales.

Car quel est le but du médecin, en se livrant à l'étude de la Botanique? Il ne veut point embrasser l'immense étendue de cette science :'il cherche simplement à connaître ses principes fondamentaux, et à savoir par quels moyens il peut parvenir à distinguer les différens végétaux utiles à l'homme, pour combattre ses maladies ou satisfaire ses besoins.

En effet la Botanique est une source intarissable de remèdes efficaces pour le médecin qui sait y puiser. Est-il ure autre classe de.corps naturels qui lui offre autant de médicamens utiles que celle des végétaux? Or quel est le médecin instruit, jaloux d'exercer son art avec la noblesse et la supériorité qui l'élèvent au-dessus de tous les autres, quel est le médecin, dis-je, qui peut, sans quelque honte, prescrire chaque jour à ses malades des plantes qu'il connaît à peine de nom, qu'il n'a jamais vues fraîches, et qu'il ne saurait distinguer de celles même avec lesquelles elles n'ont aucun rapport, parce qu'il n'en a point étudié les caractères? C'est le chirur- 
gien qui, pratiquant une opération, ignore les organes que son instrument divise. Le médecin, dans ce cas, se montre non-seulement au-dessous de l'opinion avaritageuse qu'on a $\mathrm{pu}$ concevoir de lui, mais par son inexpérience condamnable, il se met dans le cas d'approuver les erreurs les plus préjudiciables, et de sanctionner les méprises les plus funestes à l'humanité souffrante.

Qui n'a point, en effet, entendu parler de ces empoisonnemens causés par l'ignorance de quelques herboristes qui, au lieu d'une plante salutaire, en avaient donné une autre douée de propriétés vénéneuses? Si le médecin chargé du soin des malades auxquels un pareil accident arrive, eût possédé les connaissances nécessaires de Botanique, il eût reconnu l'erreur grossière de l'herboriste, et en eût prévenu les funestes effets; ou du moins il eût pu, connaissant l'action délétère du végétal employé, administrer à temps les remèdes propres à la neutraliser.

C'est ainsi, pour n'en citer qu'un exemple, que la ciguë a souvent été prise pour une autre ombellifère douée de propriétés bienfaisantes, et avec laquelle elle pouvait avoir quelque ressemblance par les caractères extérieurs, mais dont elle différait essentiellement par les organes de la fructification.

Un avantage non moins inappréciable que le médecin trouve dans l'étude de la Botanique, c'est de 
pouvoir remplacer par d'autres plantes plus communes ou plus à sa portée, les végétaux que l'on emploie habituellement, mais qui ne croissent pas dans le pays qu'il habite, ou qui y sont d'un prix trop élevé. Il pourra, en effet, opérer facilement ces substitutions, quand l'étude des familles naturelles sera venue l'éclairer sur les principes qui doivent le guider dans cette opération. Ainsi il saura que tous les individus d'une même espèce jouissent essentiellement des mêmes propriétés médicales; que les espèces d'un même genre possèdent des vertus analogues, et que souvent tous les genres d'une même famille naturelle de plantes participent aux mêmes propriétés. D'après cette connaissance il substituera indistinctement à tel genre de la famille des Crucifères, tel autre qu'il se procurera plus facilement, parce que tous les genres de cette nombreuse famille ont pour principe une huile essentielle âcre et stimulante qui leur donne ūne propriété tonique et antiscorbutique qu'on retrouve dans presque toutes les espèces. Il en sera de même des familles des Labiées, des Graminées, des Malvacées, et de beaucoup d'autres encore.

Mais il apprendra également qu'il est certaines familles, tout aussi naturelles sous le rapport des caractères botaniques, où ces substitutions ne sont pas praticables, ou du moins ne peuvent être faites qu'avec la plus scrupuleuse attention. Ainsi, dans la famille des Solanées, à côté de la pomme de terre 
on trouve la mandragore; près du bouillon blanc, la jusquiame et la belladone. De même, clans les Euphorbiacées, il trouvera des substances si différentes par leurs propriétés, que les unes sont des alimens, ou des médicamens utiles, les autres de véritables poisons. Par exemple, cette famille nous offre la cascarille, le manioc qui forme la base de la nourriture des Indiens de la Guyane, et à côté le genre Euphorbia, le Hura, et d'autres encore dont le suc laiteux, âcre et brûlant peut devenir un poison violent. Ce que nous venons de dire des Solanées et des Euphorbiacées est encore vrai pour un grand nombre d'autres familles. En résumé, l'étude de la Botanique enseignera au médecin quelles sont les familles naturelles de plantes où tous les genres jouissent des mêmes propriétés, quelles sont celles où l'on retrouve des propriétés analogues dans certains genres; enfin les familles dans lesquelles chaque genre jouit de propriétés différentes, et où toutes les espèces sont souvent délétères.

On exagère en général les difficultés attachées à l'étude de la Botanique. Les jeunes gens surtout qui se destinent à l'art de guérir, se rebutent et se découragent aux premiers obstacles qu'ils rencontrent, sans faire le moindre effort pour les surmonter. Prévenus presque toujours contre cette science, ils ne se donnent pas la peine de l'étudier, ou l'étudient avec tant de légèreté et si peu de méthode, qu'ils emploient, pendant plusieurs années, une partie de 
leur temps pour n'acquérir que des notions vagues et incertaines. Il est facile de démontrer, par l'expérience journalière, que ce peu de réussite dépend évidemment de l'idée fausse qu'ils se sont formée de cette science, et de la mauvaise marche qu'ils ont suivie dans son étude.

Les uns, en effet, croyant que toute la Botanique consiste dans la connaissance pure et simple du nom des plantes, et surtout de celles qui sont empioyées en médecine, ne s'occupent nullement des caractères propres à chacune de ces plantes, c'est-à-dire des signes qui servent à les reconnaître et à les distinguer. Qu'arrive-t-il de là? C'est que bien qu'ils aient un grand nombre de noms dans la tête, ils ne connaissent réellement aucun des végétaux de manière à pouvoir le distinguer de tous les autres : semblables à celui qui, voulant étudier une langue, apprendrait par cour un grand nombre de mots, sans connaître la valeur et le sens attaché à chacun d'eux, et qui cependant voudrait en faire usage.

D'autres, au contraire, n'ayant pas étudié les principes fondamentaux avec soin et attention, veulent sur-le-champ reconnaître et distinguer les différentes plantes, dans les ouvrages où elles se trouvent décrites. Mais à chaque pas ils sont arrêtés par. des difficultés qu'ils ne peuvent vaincre. En effet, d'où sont tirés les caractères au moyen desquels on peut reconnaittre et distinguer un végétal de ceux avec lesquels il a plus ou moins de rapport? Ne 
sont-ce pas les organes des plantes, les nombreuses modifications qu'ils éprouvent, qui servent au botaniste de signes propres à caractériser les différens végétaux? Or il est de toute évidence que pour pouvoir reconnaitre une plante dans une description quelconque, il faut pouvoir apprécier le sens et la valeur des expressions employées pour la décrire. Près de cinquante mille espèces de végétaux sont aujourd'hui connues; trois ou quatre mots bien choisis servent souvent à caractériser une plante, et à la faire distinguer dans un nombre aussi considérable. Le sens attaché à ces mots doit donc être fixe et invariable; et celui qui veut se livrer à l'étude de la Botanique doit, avant tout, s'être familiarisé avec la valeur des mots employés pour dépeindre chaque modification d'organes.

Quelle est donc la meilleure méthode d'étudier la Botanique, surtout pour celui qui, comme le jeune médecin, ne peut y consacrer qu'une partie de son temps? Nous allons indiquer en peu de mots celle que l'expérience nous a démontré étre la plus certaine, et en même temps la plus prompte.

$\mathbf{1}^{0}$ Les organes des végétaux ne sont point en grand nombre, par conséquent les noms substantifs qui les représentent sont peu nombreux, et la mémoire la moins heureuse les retiendra sans efforts. Pénétrez-vous donc bien d'abord du sens attaché aux mots tige, feuille, racine, calice, corolle, etc., avant de chercher à aller plus avant. 


\section{PRÉFACE.}

XXIII

$2^{\circ}$ Ces organes peuvent éprouver diverses modifications que le botaniste exprime par des noms adjectifs, mis à la suite du nom substantif: Ainsi on ajoute au mot TIGE, les adjectifs herbacée, ligneuse, simple, rameuse, dressée, couchée, cylindrique, pentagone, etc., suivant que l'on veut exprimer qu'elle est verte et tendre, ou solide et dure comme du bois; qu'elle est sans rameaux ou divisée en branches, qu'elle est dressée vers le ciel ou étalée sur la terre, etc., etc. La plupart des noms adjectits employés dans le langage botanique sont déjà usités pour désigner d'autres objets, et par conséquent connus de tout le monde. Ainsi il.n'est personne qui ne se figure la forme d'une tige cylindrique, tétragone, pentagone; il en est de même d'un grand nombre d'autres adjectifs. Mais cependant il en existe plusieurs qui, étant particuliers à la langue botanique, ont besoin d'être définis pour être bien compris. C'est donc uniquement ceux-là que l'homme qui veut étudier la Botanique doit chercher à bien connaître et à retenir, puisque sachant déjà la valeur des autres, il n'a besoin que de les voir pour en comprendre aussitôt le sens.

$3^{\circ}$ Celui qui connait les noms des différens organes d'un végétal, le sens attaché aux expressions propres à représenter leurs modifications principales, n'a plus besoin que de faire choix d'un système et de l'étudier, pour être devenu botaniste. Dès lors, en effer, il pourra facilement, au moyen d'un ou- 
vrage où les plantes sont rangées méthodiquement, trouver le nom de la première qui lui sera présentée, lors même qu'il ne l'aurait jamais vue. Or c'est là le but principal de celui qui étudie la Botanique. Cette science, en effet, ne consiste point dans la connaissance purement mécanique du nom des différens végétaux; mais le botaniste est celui qui, au moyen des principes fondámentaux de la science, principes qui reposent uniquement sur la structure, la forme, les usages des différens organes, peut, quand il le désire, trouver le nom d'une plante qu'il ne connaissait pas auparavant.

Telle est la marche que nous avons suivie dans l'exposition des principes fondamentaux de la Botanique, que nous offrons aujourd'hui au public. Notre intention n'a point été de faire un traité complet de Botanique générale ni de physique végétale, car il existe sur ce sujet d'excellens ouvrages qui pourraient être cités comme des modèles; mais nous avons eu pour but principal de présenter à ceux qui se livrent à l'étude de la médecine, des élémens simples et faciles d'une science qui leur est d'une si grande utilité; et qu'ils négligent malheureusement trop souvent. D'après le plan que nous nous étions tracé, nous n'avons pas cru devoir en trer dans les détails les plus minutieux de la science: nous n'avons voulu que faciliter l'étude d'une science si utile pour quelques-uns, siagréable pour tous ceux qui s'y adonnent, et à laquelle nous ayon s youé tous nos momens. 


\title{
NOUVEAUX EETENS
}

\author{
$\mathrm{DE}$ \\ BOTANIOUE.
}

INTRODUCTION.

LA Botantque (I) (Botanica, Res herbaria) est cette partic de l'histoire naturelle qui a pour objet l'étude des végétaux. Elle nous apprend à les connaîlre, à les distinguer et à les elasser.

Cette scienee ne consiste pas, eomme on l'a eru longtemps, dans la eonnaissanee pure et simple du nom donué aux différentes plantes; mais elle s'oceupe aussi des lois qui président à leur organisation générale; de la forme, des fonetions de leurs organes, et des rapports qui les unissent les uns avee les autres.

La Botanique, envisagée par rapport à ses applieations les plus importantes, nous fait également eonnaître les vertus salutaires ou malfaisantes dont sont douées les plantes, et les avanlages que nous pouvons en retirér dans l'éeonomie domestique, les arts ou la thérapeutique:

Une seience aussi vaste a dû́ néeessairement être parta-.

(1) Dérivé de Bossw, Lethe, plante. 
gée en plusieurs branehes distinetes, afin d'en faciliter l'étude; e'est ec qui a eu licu en effet.

I $^{\circ}$ Ainsi l'on nomme Botanreue proprement dite cette partie de la seienee qui eonsidère les végétaux d'une manière générale et eomme des êtres distinets les uns des autres, qu'il faut eomnaître, déerire et elasser. Cette branehe de la seience des végétaux se divise elle-même en :

Glossologie (I), ou eomnaissanee des termes propres à désigner les différens organes des plantes, et leurs nombreuses modifications; eette partie forme la langue de la Botanique, langue dont l'étude est extrềnement importante, et avee laquelle on doit commeneer par se bien familiariser.

Taxonomie (2), ou application des lois générales de la elassifieation au règne végétal. Tei se rapportent les différentes elassifieations proposées pour disposer méthodiquement les plantes.

Phylographic (3); ou art de décrire les plantes.

$2^{\circ}$ La sceoude brauche de la Botanique porte le nom de Pnysique végtrale, ou de Botanique organique. C'est elle qui eonsidère les végétaux eomme des êtres organisés et vivans, qui nous déeèle leur structure intérieure, le mode d'action propre à ehacun de leurs organes, et les altérations qu'ils peuvent éprouver, soit dans leur structure, soit dans leurs fonetions. De là trois divisions sccondaires dans la Physique végélale; savoir :

L'Organograplie (4), ou la description des organes,

(1) Dérivé de $\gamma \gamma_{055} \alpha$, mot, languc ou langage, el de $\lambda 0 \% 0 \leqq$, discours.

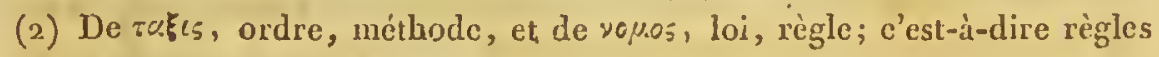
de la classification.

(3) De qurov, plante, et de ypxфw, j'écris on je décris; c'est-à.dire art de décrirc les plantes.

(4) Dérivé de opyxucy, organe, et de rpæzos, je décris; c'est-it-dire deseription des organes. Cette partie est aussi appelée Terminologie, nom inpropre, puisqu'il est composé d'un mot latin et d'un mot grec. 
de leur forme, de leur position, de leur structure et de leurs eonnexions.

La Physiologie végétale, ou l'étude des fonetions propres à chacun des organes.

La Pathologie végétale, qui nous enseigne les diverses altérations ou maladies qui peuvent affeeter les végétaux.

$3^{\circ}$ Enfin oll a donné le nom de Botanique appilquée à eette troisième branehe de la Botariqque générale, qui s'oecupe des rapports existant entre l'homme et les végétaux. Elle se subdivise en Botanique agricole, ou applieation de la eonnaissance des végétaux à la eulture et à l'amélioration dụ sol; en Botanique médicale, ou applieation des eonnaissances botaniques à la détermination des végétaux qui peuvent servir de médicamens, et dont le médecin peut tirer avantage dans le traitement des maladies; en Botanique économique et industrielle, ou celle qui a pour objet de faire connaître l'utilité des plantes dans les arts ou l'économie domestique.

La Botanique étant la seienee qui a pour objet l'étude des végétaux, noús devons nous oceuper d'abord de donner une idée des êtres auxquels on a réservé ce nom.

Les Végétaux (en latin vegetabilia, plantce, et en

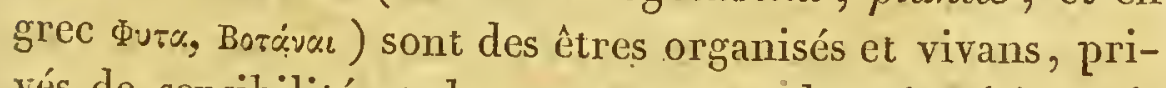
vés de sensibilité et de mouvement volontaire (I); mais jouịssant de l'irritabilité qui fait le caraetère spéeial de tous les êtres organisés. C'est par cette propriété, en vertu de laquelle ils se contractent et se meuvent sous l'influence

(1) Les végétaux sont dépourvus de mouvement volontaire; mais quelques-uns cependant exécutent une sorte de locomotion ou de déplacement bien sensible. Tels sont, par exemple, les orehis, le colehique. En effet, la racine de la plupart des orchis offre deux tubercules charnus, situés l'un à côté de l'autre, à la base de la tige. L'un de ces tnbercules, après avoir donné naissanee à la tigge, dont il contenait le germe dins son intérieur, se fane, se resserre sur lui-mène, et finit par se détruire; mais à mesure qu'il tend is disparaitre, il s'en développe un troisieme auprès de celui quí renferme 
de eertains agens, que les êtres organisés résistent à l'aetion des eauses extéricures qui tendent eontinuellement à les détruire.

Il est extrêmement diffieile de traeer nettement la ligne de démareation qui sépare les végétaux des animaux. Linné, dans son style aphoristique, a dit: Les minéraux croissent; les végétaux croissent et vivent, et les animaux croissent, vivent et sentent. Cette distinetion, qui est en effet bien tranehée, quand on eompare le eristal de roehe à un ehêne, et eelui-ei à un lomme, finit par disparaître insensiblement, lorsque l'on examine eomparativement les êtres qui oeeupent les derniers degrés de ees trois grandes séries. En effet il est bien diffieile de dire en quoi diffèrent essentiellenient eertaines espèees de 'polypes d'avee quelques algues; ear le earaetère essentiel que l'on a attribué aux animaux, la sensibilité, ou la eonscienee de leur existenee et la faeulté de se mouvoir, s'affaiblissent et finissent même par disparaître entièrement dans les dernières classes du règne animal. De plus, un grand nombre d'observateurs s'aceordent à eonsidérer eomme eonstante la transformation de eertaines plantes en animaux, et vice ver'sa. M. $\Lambda$ gardh, eélèbre algologue, et professeur à l'université de Lund en Suède, a publié une dissertation eurieuse sur la métamorphose des algues. Néanmoins cette prétendue transformation paraît être le résultat d'observalions peu exaetes.

Mais, lorsqu'on néglige un instant les faits qui servent ainsi d'intermédiaire et de passage entre les deux grandes

eneore le rudiment de la tige de l'ammée suivante, et remplaee le premier, lorsque celui-ei vient à tomber. Ce développement d'un nouveaù tubercule ayant licu ehaque annéc sur l'un des côtés de ceux qui existent, on eoneoit gue, chaque fois qu'nne nouvelle tige se développe, elle se trouve ćloignée d'un ecrtain espace de terrain de eclle qui l'a précédée. Le même phénomène a lieu à pen près de la méme maniere dans le eolchique, à l'execption que sou bulhe tend continuellenciat ì s'enfonecr de plus en plus. 
divisions des êtres orgat isés, on parvient à trouver des différenees assez marquées entre les animaux et les végétaux. C'est ainsi, par exemple, que chez les premiers, qui sont, doués de la faeulté de se mouvoir, il existe un système de fibres contraetiles, dont l'état de relâehement ou de tẹnsion détermine les mouvemens de l'animal, ee sont les fibres musculaires : dans les végétaux, rien d'analogue; toutes les fibres sont en quelque sorte inertes et impassibles; ehez eux eneore il n'y a rien de semblable au système nerveux, quoiqu'un ingénieux expérimentateur les ait sous ee rapport assimilés aux animaux. Dans eeux-ei, les substanees qui doivent servir à la nutrition sont d'abord absorbées à l'extérieur; elles séjournent pendant un eertain temps dans une eavité partieulière, où elles éprouvent une élaboration convenable avant d'ètre prises par les vaisseaux ehylifères destinés à les répandre dans le torrent de la cireulation; mais dans les végétaux la nutrition se fait d'une manière plus simple; les substanees absorbées sont direetement répandues daus toutes les par-ties du végétal, sans éprouver d'altération préalable, en sorte que chez eux nous ne trouvons ni eanal intestinal, ni estomae, puisqu'il n'y a point de digestion.

Les végétaux diffèrent eneore des animaux par la marehe de leurs fluides. Dans ees derniers, en effet, il y a une véritable cireulation, e'est-à-dire que le sang ou fluide nutritif part d'un point où il reçoit son impulsion, se répand dans toutes les parties du corps, où il dépose, ehemin faisant, les prineipes qui doivent servir à leur mutrition, pour revenir ensuite au point d'où il est parti. Mais dans les végétauxil n'y a point de eireulation à proprement parler; les fluides nourrieiers pareourent le végétal, mais ils manquent deeet agent d'impulsion, du eour, à la fois point de départ et de terninaison du sang dans les animaux.

Les animaux se nourrissent toujours de substanees organisécs végétales et animales; clans les végétaux au con- 
traire la nutrition se fait au moyen de substances inorganiques. Ce sont des gaz, de l'eau, des sels, etc., qui servent áu développement des parties de la plante.

Chez les végétaux il n'y a pas non plus de poumons, et par conséquent de respiration; bien qu'il y ait exhalation des gaz qui ont été absorbés et qui n’ont pas servi à la nutrition, la nature de ces gaz rejetés au-dehors est-elle trèsdifférente dans ces deux grandes classes des êtres organisés. Ainsi dans les animaux c'est de l'acide carbonique, tandis que dans les végétaux c'est de l'oxigène.

La eomposition chimiqne offre encore quelques moyens de distinguer les végétaux des animaux. Ainsi tandis que le carbonne prédomine dans les premiers, c'est l'azote qu'on observe dans les seeonds.

Il nous serait faeile de pousser plus loin eette comparaison entre les végétaux et les animanx; mais nous croyons en avoir dit assez pour faire commaître les différences principales qui existent cntre eux.

L'anatomie nous montre les végétaux composés de parties élémentaires simples et similaires, qui, en se combinant de différentes manières, constituent les organes proprement dits. Nous allons examiner d'abord ces parties élémentaires, dont l'étude constitue l'anatomie végétale. 


\section{PaRTIEs ÉLÍMENTAIRES DES VÍGÉTauX,}

ou

\section{ANATOMIE VÉGETAE.}

LorsQu'on examine l'organisation intérieure d'un végétal à l'oil nu, ou mieux eneore à l'oil aidé d'une forte loupe ou d'un mieroeospe, on voit qu'il se eompose de cellules à parois minees et diaphanes, d'une petitesse extrême, d'une forme variable, tantôt régulières, tantôt irrégulières, et de tubes ou vaisseaux cylindriques, épars ou réunis en faiseeaux. Telles sont les deux formes prineipales sous lesquelles se présentent les parties élémentaires qui entrent dans la eomposition des végétaux, et auxquelles on a donné les noms de tissu cellulaire et de tissu vasculaire. Nous allous les étudier sueeessivement l'un et l'autre.

\section{DU TISSU CELLULAIRE.}

La première modifieation du tissu élémentaire des végétaux est le tissu cellulaire ou aréolaire (voy. pl. I, fig. 7). Il se eompose de eellules contiguës les unes aux autres, et dont la forme dépend en général des résistanees qu'elles éprouvent. Quelques auteurs l'ont comparé à la monsse ou écume légère qui se forme sur l'eau de savon, par l'agitation de ce liquide. On avait généralement pensé que les parois des «ecllules eontiguës les unes aux autres étaient eómmunes aux deux eellules qui se touehaient; mais eependant Malpighi avait déjà émis l'opinion qu'il était composé de vésicules distinetes qu'il nommait utrieules. Le professeur Sprengel de Halle, en r 802 , et une foule d'autres physiologistes distingués, ont fait des observations qui confirment eette opinion. On peut isoler, les unes des autres, les cellules, sans 
déchirement, ec qui prouve que cliaque cellule forme une sorte de petite vésicule qui a ses parois distinetes, et que, là oủ deux ecllules se toneheut, la membrane qui les sépare est forméc de deux feuillets, qui apparticunent à ehacune d'elles. Les reeherehes récentes de M. Du Troehet et du professeur Amici s'accordent avee ecttc opinion. Cette séparation des vésicules formant le tissu cellulaire peut s'opérer soit par la simple coetion dans l'eau, ainsi que l'a fait le professcur Linek, soit par l'ébullition dans l'acide nitriquc. Mais néanmoins, quelquefois les parois des cellules se soudeut si intimement qu'il est presque impossible de les séparer les unes des autres.

Lorsque l'on observe le développement et la formation du tissu ecllulaire dans les végétaux, on aequicrt la eertitude qu'il se compose de eellules d'abord isolées, mais qui, par les progrès de leur dévcloppement, fuissent par se souder plus ou moins entre elles. En effet, dans les vésieules du tissu cellulaire on aperȩoit, au moyen du mieroseope, des corpuseules ovoüdes ou arrondis, généralement de couleur verte, mais néanmoius offrant toutes les teintes possibles, suivant les parois dans lesquelles on les observe. Ce sout ees corpuseules qui colorent le tissu ecllulaire, dont les parois sont toujours diaphancs. M. 'Turpin, qui, dans un excellent mérnoire (Mém. Mus. Vol. xir), a de nouveau appelé l'attention sur' ces corpuscules, lcur a donné le nom générique de globuline. Cliaque grain de globuline est unc petite vésicule, dans laquelle se forment plus tard d'autres petits granules (Globulins, Turp.) qui, s'aceroissant suceessivement, finisscnt par rompre la vésicule qui les renfermait. Alors chaeun d'cux devient à son tour une petite vésicule dáns laquelle se développent de nouvcaux granules qui préscutent les mêmes phénomèues. C'est ainsi que s'aceroît et se développe en tous seus le tissu eellulaire qui forme la masse des végétaux. M. de Candolle, considérant que c'est cette substance granulcuse 
qui colore toutes les parties des vésétaux, a récemment proposé de lui domner le nom de chromule.

Quand elles n'éprouvent que la résistance oecasionée par la présenee des eellules adjacentes, il n'est pas rare de trouver à ees eellules une forme à peu près hexagonale, en sorte qu'elles ressemblent assez bien aux alvéoles eonstruites par les abeilles. Mais elles peuvent être plus ou moins allongéés, arrondies ou comprimées, suivant les obstaeles qui s'opposent à leur libre développement. Il est même fort rare de leur trouver cette forme régulière et hexagonale quenous venons de signaler tout àl'heure. Leurs parois sont minces et transparentes; elles eommuniquent toutes ensemble, soit que leurs eavités s'ourrent mutuellement l'une dans l'autre, soit qu'il existe sur leurs parois des pores, ou même des fentes. Ces pores, qui sont à peine visibles au moyen des instrumens d'optique les plus forts, ont été aperȩus par Leuwenhoek et Hill, et dans ces derniers temps MM. Mirbel et Amiei en ont de nouveau reeonnu l'existence. Selon plusieurs physiologistes, et en partieulier MMI. Rudolphi et Sprengel, les diverses eellules eommuniquent entre elles par un point où leurs parois sont interrompues. Mais Bernhardi le premier démontra que la eommunieation entre les eellules avait uniquement lieu par les pores invisibles de leurs parois. Cette dernière opinion est généralement admise aujourd'bui. Ainsi il paraît très-probable que e'est par exsudation que les fluides passent d'une eellule dans une autre.

Dans les parties ligneuses, les ecllules du tissu aréolaire sont fort allongées, et forment des espèces de petits tubes parallèles entre eux. Leurs parois sont opaques , épaissies, quelquefois même elles finissent par s'oblitérer entièrement. C'est à eette modification que M. Linek a donné le nom de tissu allongé.

Ce tissu allongé existe en abondanee dans les végétaux. Il y est beaucoup plus commun que le tissu cellulaire ré- 
gulier, et se compose de petits tubes étranglés de distance en distance. D'autres fois ils sont fusiformes, c'est-à-dire amincis insensiblement à leurs deux extrémités. C'est à cette modification des eellules du tissu allongé que M. Du Trochet a donné le nom de clostres. Ils sont en général parallèlcs cntre eux, plus ou moins opaques, et très-abondans dans le tissu ligneux. Il arrive parfois quc lcs eellules du tissu allongé ne peuvent se toucher que par les points lcs plus gonflés, d'où il résulte entre cux des intervalles ou vides. Ce sont ees espaees vides qu'Hedwig a nommés vasarevehentia; Tréviranus, meatus intercellulares; et Link, ductus intercellulares. Sclon l'opinion du profcsseur Amici, ces espaees ne eontiennent jamais de liquide, mais seu-lement de l'air' ear les grands pores de l'épiderme, 'qui, ainsi que nous le verrons prochaincment en parlant de eettc membrane, sont des organes qui ne livrent passage qu'à de l'air, sont toujours placés devant un de ces espaces. Quand le tissu est trop compacte et les petits tubes trop serrés pour offrir de ces espaces, on ne trouve pas non plus de pores corticaux.

Il est encore une autre modification du tissu allongé qui mérite d'être mentiomnée ici : ce sont les cellules qui forment les insertions ou rayons médullaires de la tige des végétaux dicotylédons. Elles sont fort petites, allongées et placées horizontalement, au licu d'être vcrticales.

Le tissu cellulaire, dans son état de pureté native, a peu de eonsistanee; il se déchire facilement. Aussi trouvet-on souvent dans certaius végćtaux des espaces vides, remplis seulement par de l'air, et qui résultent de la rupture des parois de plusieurs cellules. Ces espaces, auxqucls on donné le nom de lacunes, sc rencontrent surtout dans les végétaux qui vivent dans l'eau, et dans lesquels ils semblent s'opposer à la macération que ees plantes subiraient infailliblement par leur séjour prolongé dans ce liquide.

M. Amici a une opinion tout-à-fait différente de cclle 
que nous venons d'exposer sur les lacunes. Selon lui, elles ne sont pas, eomme pense M. Mirbel, le résul tat du déehirement des eellules. Ce sont des espaees plus ou moins réguliers, éontenant eonstamment de l'air. Quelquefois elles offrent sur leur paroi interne des poils d'une nature partieulière, en forme de houppe ou de pineeau, qui ont été vus par MM. Mirbel et Amiei. On peut distinguer deux espèees de laeunes : les unes ont pour orifice les pores eortieaux et eommuniquent avee l'air extérieur; les autres n'ont aneune eommunication externe. Ces dernières existent surtout dans les plantes qui manquent de tubes poreux.

En terminant iei ee qui a rapport à l'organisation du tissu vésieulaire, faisons remarquer qu'il jouit de deux propriétés essentielles; l'une qui est sa faeulté d'absorber les liquides, la seconde, sa contraetilité organique. C'est au moyen de ees deux propriétés fondamentales que l'on peut expliquer plusieurs des phénomènes de la vie végétale.

\section{DU TISSU VASCULAIRE.}

Le tissu vasculaire ou tubulaire est la seconde modifieation du tissu élémentaire.

Les vaisseaux sont des lames de tissu élémentaire roulées sur elles-mêmes de manière à former des eanaux ou des eellules plus ou moins allongées, placées bout à bout, et dont les diaphragmes ont souvent disparu. Les parois des vaissenux sont quelquefois assez épaisses, peu transparentes, et pereées d'uin grand nombre d'ouvertures an moyen desquelles ils répandent dans les parties latérales une portion des fluides gazeux ou liquides qu'ils eharrient. Ces vaisseaux ne sont point eontinus depuis la base jusqu'au sommet de la plante, mais ils s'anastomosent fréquemment entre cux, et finissent par se ehanger en tissu aréolaire. 
On comnaît sept espèces principales de vaisscaux, savoir: $I^{\circ}$ Les vaisscaux en ehapelet ou moniliformes; $2^{\circ}$ les vaisseaux poreux; $3^{\text {n }}$ les vaisseaux fendus ou fausses traclées; $4^{\circ}$ les traehées; $5^{\circ}$ les vaisscaux mixtes ; $6^{\circ}$ les vaisseaux propres; $7^{\circ}$ les tubes ou vaisseaux simples.

I $^{\circ}$ aisseaux en chapelet (pl. x, fig. I.). Ce sont des tubes porcux ou ponetués, resserrés de distance en distance, et eoupés de diaphragmes percés de trous à la manic̀re des eribles. Selon la plupart des anatomistes, ces diaphragmes n'existent pas. On les trouve principalement au point de jonetion de la raeine et de la tige, de la tige et des branelies, ete. Ces vaisseaux pourraient bicn, selon nous, être eonsidérćs comme de simples cellules de tissu aréolairc, régulic̀rement disposćcs par séries ou lignes longitudinales.

$2^{\circ} \quad$ aisseaux ponctués (pl. I, fig. 2,3 ). Ils représentent des tubes continus, offrant un grand nombre de points opaques, que d'autres ont considéré comme des pores disposés par lignes transversales. M. Mirbel les nomme vaisseaux poreux. On les trouve dans les eouches ligneuses de la tige, des raeines et des hranches.

$3^{\circ}$ Fausses trachées |( pl. I, fig. 4, 5). Tubes coupés de fentes transversales, suivant l'opinion la plus généralement adoptée. Ce sont, ainsi que les trachées, les principaux conduits de la sève. Ils sont désigués par M. de Candolle sous le nom de vaisseaux fendus ou rayés. IIs sont très-abondans dans les couches ligncuses des végétaux dicotylédons, et dans les faiseeaux ligncux des monoeotylédons.

$4^{\circ}$ Les trachées ( $\mathrm{l}$. x, fig. 6), que Malpighi et Hedwig avaient comparées à l'organe respiratoire des inseetes, sont des vaisseaux formés par une lame argentinc et transparente, roulće sur ellc-même en spirale, ct dont les bords un peu plus épais se touehent de manière à ne laisser aucun espace entre eux, sans ccpendant contracter 
d'adhérence (1). Quelquefois néanmoins les spires des trachées ne se déroulent pas; c'est à eette sorte de tube que Link a donné le nom de vaisseau en spirale soudée. Selon MM. Link et Schrrader la lame roulée en spirale est ereusée en gouttière sur son eôté interne. Dans les dieotylédons, on les observe autour de la moelle; et dans les monocotylédons, e'est ordinaïrement au centre des filets ligneux. L'écoree et les eouches annuelles du bois n'en contiennent jamais. On en trouve quelquefois dans les racines, et il est très-facile de les dérouler encore dans les nervures des feuilles, les pétales, les filets des étamines, ete.

A leurs extrémités, les traehées se terminent en tissu. cellulaire selon M. Mirbel, tandis que d'après M. Du Trochet elles finissent par une sorte de cône plus ou moins aigu.

Hedwig eonsidérait les vaisseaux spiraux ou traehées, que Grew appelait vaisseaux aériens, eomme eomposés de deux parties, savoir d'un tube droit et eentral, rempli d'air, et qu'il nommait pour eette raison vaisseau pneumatophore, et d'un tube roulé en spirale sur le préeédent, rempli de fluide aqueux, et auquel il donnait les noms de vaisseau adducteur, chylifêre, ete. M. Bernhardi a encore émis une autre opinion sur la structure des trachées. II les eonsidère eomme formées d'un tube extérieur trèsminee dans lequel une petite lame argentine est roulée en spirale de manière à en tenir les parois éeartées. Enfin quelques auteurs adméttent que les spires des traehées sont unies entre elles par une membrane très-minee qui se déeliire très-faeilement quand le fil spiral vient à se dérouler. Il résulterait de eette organisation que, dans leur état naturel, les traehées forment un tube eontinu.

Les traehées ne sont pas toujours simples; on trouve

(r) Eilles ont la plus grande resscmblance avec les élastiques en fil de laiton que l'on met daras les bretelles. 
souvent des trachées à double, triple et même à un trèsgrand nombre de spirales parallèles, eomme on l'observe dans beaucoup de plantes monoeotylédones.

$5^{\circ}$ Les vaisseaux mixtes, découverts par M. Mirbel, participent à la fois de la nature de tous les autres, e'est-ìdire qu'ils sont alternativement poreux, fendus ou roulés en spirale dans différeus points de leur étendue. Cependant M. Amiei, qui a fait un grand nombre d'observations mieroseopiques sur l'anatomie végétale, pcnse que jamais les fausses traehées ne deviennent des tráchées. D'ailleurs, ainsi qu'il le fait remarquer, ces deux sortes de vaisseaux oceupent une place tout-à-fait différente.

$6^{\circ}$ Les vaisseaux propres que l'on désigne encore sous le nom de réservoir's des sucs propres, sont des tubes eourts, non porcux, contenant un suc propre, particulier à chaque végétal. Ainsi dans les Conifères ils contiennent de la résine; dans les euphorbes, un sue blane et laiteux, etc.

On les trouve dans les ćcorces, la moelle, les feuilles et les fleurs. Ils sont tantôt solitaires, tantôt réunis en faisceanx.

$7^{\circ}$ Les tubes simples sont des vaisseaux d'un volume variable, souveut ramifiés et anastomosés entre eux, servant au mouvement eirculatoire de la sc̀ve, et clont les parois nimees, ou plus ou moins opaques, ne présentent aueun pore visible.

Ces difiérentes espéces de vaisseaux, auxcuelles on pourrait ajouter un grand nombre d'autres modifications, se réunissent souventplusieurs entre clles, et constituent des faisceaux allongés, soudés enscmble par du tissu cellulaire; clles forment alors les fibres proprement dites. Ce sont ees fibres ou faisecaux de tubes qui constituent la trame et en quelque sortc le squelette de la plupart des organes foliacés des végétaux.

On appelle au contraire parenchyme Ia partie ordinairement molle, composéessentiellement cic lissu cellulaire, 
que l'on observe dans les fruits, dans les fenilles, etc. Cette expression s'emploie par opposition au mot fibre. Toute partie qui n'est point fibreuse cst composée de parenehyme.

C'est en s'unissant et se combinant de diverses manières que les tissus parenchymateux et fibreux eonstituent les différens organes des végétaux. Dans tous, en effet, nous ne trouvons par l'analyse quc ces deux modifications essentielles du tissu fondamental.

Les sept modifieations principales du tissu vaseulaire diffèrent entrc elles non-seulement par leur organisation, leur position respective, mais eneore suivant la nature des fluides qu'ellcs renferment. Sous cc rapport on pcutles distinguer en trois séries, savoir: $\mathrm{I}^{\circ}$ les vaisseaux séveux ou lymphatiques dans lesquels circule la sève; $2^{\circ}$ les vaisseaux des sues propres; $3^{\circ}$ les vaisseaux aériens, dans lesquels on: ne trouve jamais que de l'air ou d'autres fluides élastiques.

Mais les différens auteurs d'anatomie et de physiologie végétales sont loin d'être d'aeeord sur la elasse à laquelle on cloit rapporter les diverses espèees de vaisseaux que nous avons fait connaître. Ainsi, par exemple, Malpighi, Hedwig et plusieurs autres botanistes aneiens eonsidéraient les trachées eomme des vaisseaux destinés à ne eontenir que de l'air. M. Link a soutenu la même opinion, qu'il a étendue aux vaisseaux poreux et aux fausses tranehées. Mais, d'après les observations du professeur Mirbel, l'existenee des vaisseaux aériens avait été révoquée en doute, et mềme niée absolument. Ainsi il eonsidérait tous les tubes des. végétaux comme uniquement destinés à la eireulation de. la sève. Cette opinion, généralement adoptée de nos jours; vient d'être combattue par le professeur Amiei. Cet habile. observatcur dit positivement qu'il s'est assuré par l'observation que les traehées, les fausses traehées, les vaisseaux poreux et en général tous les organes tubuleux ou eellulaires des végétaux qui offrent des trous ou fentes visibles, 
ne conticnnent jamais que de l'air. Quand le diamètre de ces tubes est asscz grand, on peut facilement vérifier cette observation en coupaut ces tubes en travers, on les trouve constamment vides; si l'on fait cette section sous l'eau, on voit que chacun d'eux présente à son orifice une petite bulle d'air.

Les ouvertures ou pores dont sont percés les vaisseaux poreux sont très-fréquemment órganisés comme les porcs de l'épiderme, c'est-à-dire qu'ils offrent à leur contour une sorte de bourrelet circulaire ou de rebord. Cette observation, duc à M. Mirbel, a été confirmée par M. Amici. Ce dernier tire de cette ressemblance une induction de plus en faveur de son opinion sur la nature du fluide contenu dans ces vaisseaux. En effet, ainsi que nous le verrons plus tard, les grands pores de l'épiderme ne livrent jamais passage qu'à des fluides aériformes.

L'air contenu dans les vaisseaux poreux ne communique pas avec l'air extérieur. M. Amici pense qu'il est produit dans l'intérieur même du tissu végétal, inais sa nature n'est pas encore parfaitement comnue.

- Dans les végétaux liğacux, où les vaisseaux aériens finisseut par disparaitre, les rayons médullaires en tienuent lieu et remplissent les mêmes fonctions. Ils sont, en effet, composés de petits tubes placés horizoutalement, ou de cellules poreuses allongées en travers, qui, suivant le professeur de Modène, servent à établir la communication des parties intéricurcs du végétal avec l'extérieur. Ces tubes ou cellules ne contiemuent jamais que de l'air.

D'après ce que nous avons dit précédemment, on voit qu'il existe deux moyens principaux de communication entre les diverses parties du tissu végétal. Dans les cellules ou les tubes aériens, la coinmunication a lieu par le moyen de pores intermoléculaires ou de feutes extrêmement petites, mais dont on peut constater l'existence et reconuaitre l'organisaliou par' le secours du microscope. Ces pores man- 
quent absolument daus le tissu eellulaire proprement dit, et dans les vaisseaux que nous a vons désignés sous le nom. de tubes simples, ou vaisseaux séveux. Dans eette partie du tissu des végétảux, la eommunieation a lieu, soit par une sorte d'imbibition, soit par les espaces intermoléeulaires que les globules qui composent les lames du tissu laissent entre eux.

Quoique les pores que l'on observe sur les parois des cellules allongées des vaisseaux moniliformes et des vaisseaux poreux aient été vus et déerits avec une exaetitude minutieuse par un grand nombre d'auteurs modernes : et spécialement par MM. Mirbel et Amiei, néanmoins M. Du Troehet, dans son Mémoire sur l'anatomie de la sensitive, vient tout réeemment d'en nier l'existence. C'est sur eette assertion qu'il a fondé un système que nous exposerons iei en peu de mots. Cet observateur prétend que les organes déerits par M. Mirbel eomme-des pores entourés d'un bourrelet saillant ne sont rien autre ehose que de petites eellules globuleuses plaeées dans l'épaisseur des parois des aréoles du tissu eellulaire ou des vaisseaux, et remplies d'une matière verte transparente. Ces cellules, dit l'auteur, en. leur qualité de eorps sphériqques transparens, rassemblant les rayons lumineux dans un foyer eentral, doivent paraître opaques dans leur pourtour et transparens à leurr eentre, ee qui les aúra fait croire perforés. Il n'y a done pas de pores. Mais il nous semble évident que M. Du Trochet s'est entièrement mépris. Les eorpuseules qu'il a examinés, et qu'il a eru être les pores déerits par M. Mirbel, sont des organes tout-à-fait différens de ees derniers : il n'est done pas étonnant qu'il ne les ait pas vus perforés. Ce ne sont autre ehose que ees grains de substance amylacće ou ees pelits eorps glanduleux verdâtres, disséminés en abondance dans toutes les parlies du tissu végétal et auxquels M. 'Turpin a récemment donné le nom de grlobuline. La dénégation de M. Du Trochet tombe done 
tout-à-fait d'elle-mêmc, puisque ses observations ont rapport à un organc tout-à-fait différent.

Croyant que les pores du tissu cellulairc étaicnt des ccllules pleines d'une substance verdâtre, l'habile expérimentateur quc nous combattons ici devait faire l'application de cette obscrvation aux vaisseaux sur lesquels on avait décrit des trous ou des fcntes. Aussi a-t-il prétcndu que les vaisseaux porcux ne sont quc des tubes qui offrcnt de ces ccllules globulcuses ct verdâtres disposécs d'une manière plus ou moins symétrique, et que les fausses trachécs ou vaisscaux fendus présentent ces ccllules rangées par lignes transversalcs.

L'autcur a cxaminé cnsuitc quelle est la naturc de cette matière verdàtro , et quels sont ses usages. L'ayant essayée par les réactifs chimiques, il a reconmu qu'elle ćtait concrescible par le moyen de l'acide nitrique, et qu'cnsuite les alcalis la ramenaient à sor état primitif. Or, c'est absolument de eette manière que la substanec cérébralc des animaux se comporte avec les mêmes réactifs. Il arrive done à ectte conséquence, quc ectte matière verdâtre est un véritablc système nerveux, ou plutôt les élémcns épars d'un systèmc ncrveux diffus, qui n'est pas réuni cn masse, mais se préscnte sous l'aspect de petits points épars ou réunis, qu'il nomme corpuscules nerveux. Cette considération, dit-il, appuyée sur l'analogic de la nature chimique des corpuscules globulcux, est encore fortifiéc par l'obscrvation de la strueture intime du système nerveux de certains animaux. Ainsi, daus les mollusques gastéropodes, la substance médullaire du cerveau est composéc de cellulcs globulcuses agglomérées, sur les parois desquelles il existe une grande quantité de corpuseules globuleux ou ovoïdes, qui ne sont que de très-petites cellules remplics de substance médullaire nerveuse. La similitude de cette organisation a vee celle que nous venons d'indiquer dans les végétaux est parfaite, selon M. Du Trochet, et force à con- 
venir que les végétaux sont pourvus d'un système meṛveux.

Nous nous sommes eontenté d'exposer ici les opinions émises réeemment par ee eélèbre physiologiste : nous les cxamiuerons plus en détail en parlant de la motilité des végétaux, après avoir étudié les fonetions des feuilles.

Pour terminer tout ee qui a rapport à l'examen de l'anatomic des différentes parties constituantes et élémentaireș de-l'orgamisation végétale, nous devons nous oceuper des glandes et des poils eonsidérés dans leur structure anatomique.

Les Grandes sont des organes partieuliers qu'on observe sur presque toutes les parties des plantes, et qui sont des tinés à séparer de la masse générale des humeurs un fluide queleonque. Par leurs usages et leur strueture, elles ont la plus grande analogie avee eelles des animaux. Elles paraissent formées par un tissu eellulaire très-fin, dans lequel se ramifient un grand nombre de vaisseaux. Mais on a également étendu ee nom à des corps vésieuleux souvent transparens placés dans la substanee des organes et remplis d'une huile volatile, qui probablement a été sécrétée danș leur intérieur.

Leur forme et leur strueture particulière sont trèsvariées, et les ont fait distinguer en plusieurs espèees. Ainsi il y a des :

$\mathrm{I}^{\circ}$ Glandes miliaires. Elles sont fort petites et superficielles. Elles se présentent sous la forme de petits grains arrondis, disposés par séries régulières, ou dispersés sans ordre dans toutes les parties des plantes exposées à l'air. $2^{\circ}$ Glandes vésiculaires. Ce sont de petits réservoirs remplis d'huile essentielle, logés dans l'enveloppe herbaeéc des végétaux. Elles sont très-apparentes dans les feuilles du myrte et de l'or'anger', et se présentent sous l'aspeet de petits points transparens, lorsqu'on place ces feuilles entre l'oeil et la lumière.

30 Glandes globulaires. Leur forme est sphérique; elles 
n'adhèrent à l'épiderme que par un point. On les observe surtout dans les labiées.

$4^{\circ}$ Glandes utriculaires ou cn ampoules. Elles sont remplies d'un fluide incolore, comme dans la glaciale.

$5^{\circ}$ Glandes papillaires. Elles forment des espèces dis mamelons ou de papilles, qu'on a comparćes à celles de la langue. On les trouve dans plusicurs labiées, par exemple dans la saricttc (satureia hortensis).

Enfin il y en a de lenticulairès, de sessiles, d'autres qui sout portées sur des poils. La tribu des drupacées dans la famille des Rosacées, les familles des Passiflores et beaucoup de Légumineuses, de Malvacées, offrent sur lcur pétiole ou le limbe de leurs feuilles des glandes d'une forme très-variéc, et qui souvent fournissent de bons caractères pour distinguer les espèces.

Les Porls sont des organes filamenteux, plus ou moins déliés, scrvant à l'absorption et à l'exhalation dans les végétaux. Il est peu de plantes qui cn soicnt dépourvues. On les observe prineipalcment sur celles qui vivent dans les licux secs et arides. Dans ce cas, ils ont été regardés par quelques botanistes eomme servant à multiplier et à augmenter l'étcnduc de la surface absorbante des végétaux. Aussi n'en voit-on pas dans les plantes très-succulcntes, comme les plantes grasses, ou celles qui vivent habituellement dans l'cau.

Les poils paraissent être, dans beaucoup de cas, les eanaux excrétcurs des glandes végétales. En effet, ils sont fréquemment implantés sur une glande papillairc. Ne saiton pas que les poils de l'ur'tica urens et de l'urlica dioüca ne détermincnt la formation d'ampoules sur la peau que parec qu'en s'y enfonçant ils y versent en même temps um fluide irritant, sécrété par les glaudes sur lesquelles ils sont implantés, puisque quand, par la dessiccation, ce fluide s'est évaporé, les poils des orlies ne produisent plus le même cffet? On distingue les poils en glandulifères, excréteurs, et 
en lymphatiques. Les premiers sont ou appliqués immédiatement sur une glande, ou surmontés par un petit corps glandulaire partieulier, eomme dans la fraxinelle (dictamnus albus); les seeonds sont plaeés sur des glandes don $\mathrm{t}$ ils paraissent être les canaux exeréteurs, destinés à verser au dehors les fluides sécrétés; enfin les troisièmes ne sont qu'un simple prolongement d'un pore cortieal.

La forme des poils off'c un grand nombre de variétés. Ainsi il y en a de simples, de raneux, de subulés, de capités. D'autres sont creux et eorpés de distanee en distanee par des diaphragmes horizontaux. Dans les Malpighiaeées ils ont la forme et la position horizontale d'une navette.

Ils sont quelquefois solitaires, ou bien rassemblés en faiseeaux, en étoiles, ete.

Quant à Jeur disposition sur une partie (disposition que l'on désigne sous le nom de pubescence), nous en parlerons en traitantsous ee rapport des modifieations dela tige.

Nous venons de eonsidérer la structure anatomique des végétaux, de pénétrer dans l'intérieur de leur tissu, de séparer et d'analyser les rudimens ou parties élémentaires de leur organisation; étudions maintenant le végétal eonsidéré dans sou ensemble : voyons quels sont les organes ou parties qui le eomposent dans son état parfait de développement.

Un végétal, dans son dernier degré de développement et de perfection, offre à considérer les organes suivans :

I La racine, ou cette partie qui, le terminant inférieurement, s'enfonee ordinairement dans la terre, où elle fixe le végétal; flotte dans l'eau, quand celui-ei nage à la surfaee de ee licquide.

$2^{\circ} \mathrm{La}$ tige, qui eroissant en sens inverse de la raeine, se dirige toujours vers le eiel au moment où ellc eornmenee à se développer, se eouvre de feuilles, de fleurs et de fruits, et se divise en branehes et en rameaux.

30 Les feuilles, ou ces espèces d'appendices membra- 
neux, insérés sur la tige et ses divisions, ou bien partant immédiatement du collet de la racine.

$4^{\circ}$ Les fleurs, c'est-à-dire des parties très-complexes, renfermant les organes de la reproduction dans deux enveloppes particulières, destinées à les contenir et à les protéger : ces organes de la reproduction sont le pistil et les étamines. Les enveloppes florales sont la corolle et le calice.

$5^{\circ}$ Le pistil, ou organe sexuel femelle, simple ou multiple, occupant presque toujours le centre de la fleur, se compose d'une partie inférieure creuse, nommée ovaire, propre à contenir les rudimens des graines, ou les oviles, d'une partie glanduleuse, située ordinairement au sommet de l'ovaire, destinée à recevoir l'impression de l'organe mâle, ct que l'on appelle stigmate; quelquefois d'un slyle, sorte de prolongement filiforme du sommet de l'ovaire, qui supporte alors le stigmate.

60 Les étamines, ou organes sexuels mâles, composées essentiellement d'ume anthère, espèce de petite poche membraneuse, le plus souvent à deux loges, renfermant dans sou intéricur la substance propre à déterminerla fécondation oule pollen. Le plus ordinairement l'anthère est portée sur unflet plus ou moins long; dans ce cas l'étamine se trouve formée d'une anthère ou partic essentielle, d'un filet ou partie accessoire.

$7^{\circ}$ La corolle, ou l'enveloppe la plus intérieure de la fleur, souvent peinte des plus riches couleurs, quelquefois formée d'une seule pièce et dite alors corolle monopétale; d'autres fois polypélale, c'est-à-dire composée d'un nombre plus ou moins considérable de pièces distinetes, qui portent chacune le nom de pétale.

$8^{\circ}$ Le calice, ou enveloppe la plus extérieure de la fleur, de nature foliacée, ordinairement vert; composé d'une scule pièce, et dans ce cas nommé monosépale; ou formé de plusicurs pic̀ces distinctes, qui sont nommées sépales, il est appelé alors polysépalc. 
$9^{\circ}$ Le fruit, c'est-à-dire l'ovaire développé et renfermant les graines fécondées, est formé par le péricarpe et les graines.

xo $0^{\circ}$ Le péricarpe, de forme, de eonsistance très-variées, est l'ovaire développé et accru, dans lequel étaient contenus les ovules, qui sont devenus les graines. Il se compose de trois parties, savoir : de l'épicarpe, ou membrane extérieure qui définit la forme du fruit; de l'endocarpe, ou membrane qui revêt sa eavité intérieure simple ou multiple; enfin d'une partie parenchymateuse située et contenue entre ces deux membranes, et qu'on nomme sarcocarpe.

Le sarcocarpe est surtout très-développé dans les fruits charnus.

I I ${ }^{\circ}$ Les graines contenues dans um péricarpe y sont attachées au moyen d'un support partieulier, formé des vaisseaux qui leur apportent la nourriture; ce support est le trophosperme, ou placenta. Le point de la surface de la graine où s'attaehe le trophosperme se nomme liile ou ombilie.

Quelquefois le trophosperme, au lieu de cesser au pourtour du file, se prolonge plus ou moins sur la graine, au point de la recourrir même entièrement. C'est à ce prolongement partieulier qu'on a donné le nom d'arille.

La graine se compose essentiellement de deux parties distinctes, l'épisperme et l'amande.

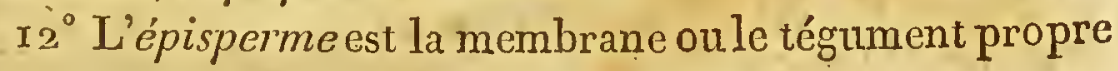
de la graine.

I $3^{\circ}$ L'$^{\prime}$ amande est le eorps contenu dans l'épisperme.

L'amande est composée essentiellement de l'embryon, c'est-à-dire de cette partie qui, mise dans des cireonstanees convenables, tend à se développer et à produire un végétal parfaitement semblable à celui qui lui a donné naissance.

Outre l'embryon, l'amande eontient encore quelquefois 
un corps particulier de nature et de eonsistanee varićes, sur lequel cst appliqué l'embryon, ou dans l'intéricur duquel il esi entièrement eaché; ec corps a reçu les noms d'endosperme, de périsperme el d'albumen.

L'embryon est la partie essentielle du végétal ; e'est pour coneourir à sa formation et à son perfectionnement que tous les autres organes des végétaux paraissent avoir été créćs. Il est formé de trois parties: l'une inféricure ou corps radiculaire; c'est eelle qui, dans la germination, donne naissanec à la racine; l'autre, supérieure, est la gemmule; c'est elle qui, en se développant, produit la tige, les feuilles et les autres parties qui doivent végéter à l'extéricur; enfin une partie intermédiaire et latéralc, qui est le corps cotylédonaire, simple ou divisé en deux parties, nommées colýlédons. De là, la division des végétaux pourvus d'embryon en deux grandes classes : les Monocotylédons, ou ceux dont l'embryon n'a qu'un seul cotylédon ; et les Dicotylédons, ou ecux dont l'cmbryon présente deux cotylédons.

Telle est l'organisation la plus générale et la plus complète des végétaux. Mais on ne doit pas s'attendre à trouver toujours réunies sur la même plante les différentes parties que nous veuons d'énumérer rapidement; plusieurs d'entre elles manquent très-souvent sur le même végétal. C'est ainsi, par exemple, que la tige est quelquefois si peu développée, qu'elle paraît ne point exister, eomme dans le plantain, là primevère; que les feuilles n'existent pas du tout dans la cuscute; qu'on ne trouve pas de corolle dans tous les Monocotylédons, c'est-à-dire qu'il n'existe alors qu'une seule enveloppe autour des organes sexuels; que cette seule enveloppe disparaît quelquefois, comme dans le saule, ete.; que souvent cneore la fleur ne renferme que l'un des deux organes sexuels, eomme dans le coudrier, où les étamines et les pistils sont eontenus dans des fleurs distinetes; ou enfin que les deux organes sexuels disparais- 
sent qquelquefois entièrement, et la fleur alors est dite neutre, comme dans la boule de neige (viburnum opulus), l'hortensia, ete.

Cependant, dans les différens cas que nous venons de citer, eette absenee de eertains organes n'est qu'aeeidentelle, et n'influe pas d'une manière marquée sur le reste de l'organisation; en sorte que eeux de ces végétaux dans lesquels ees organes manquent ne s'éloignent point sensiblement, ni dans leurs earactères extérieurs, ni dans leur mode de végétation et de reproduetion, de eeux qui les possèdent tous.

Mais il est un eertain nombre d'autres végétaux qui, par la privation constante des organes sexuels, par leurs formes extérieures, la manière dont ils végètent et se reproduisent, s'éloignent tellement des autres plantes eonnues, que de tout temps ils en ont été séparés pour former une elasse à part. C'est à ees végétaux que Linné a donné le nom de cryptogames, e'est-à-dire de plantes à organcs sexuels eachés ou invisibles, pour les distinguer des autres végétaux connus, dont les organes sexuels sont apparens, et qui avaient reesu pour eette raison le nom de phanérogames.

Les cryptogames, qui sont mieux nommées agames puisqu'elles sont privées d'organes sexuels, sont fort nombreuses. Elles constituent environ la septième ou huitième partie des einquante mille végétaux connus aujourd'hui.

Comme elles sont dépourvues de graines, et par conséquent d'embryon et.de eotylédons, on les appelle aussi Inembryonées ou Acotylédones. On arrive done ainsi à trouver dans les végétaux trois divisions fondamentales; tirées de l'embryon, savoir :

$I^{\circ}$ Les Inembryonés ou Acolylédons, e'est-ì-dire les plantes dans lesquelles on n'observe ni fleurs proprement dites, ni par eonséquent d'embryon et de cotylédons; telles 
sont les Fougères (I), les Mousses, les Hépatiques, les Lichens, les Champignons, ete.

$2_{2}^{\circ}$ Les Embryonés ou Phanérogames, plantes pourvues de fleurs bien évidentes, de graines et d'embryon. On les distingue en :

Monocorylédones, ou celles dont le eorps cotylédonaire de l'embryon est d'une seule pièce, et développe une seule feuille par la germination; telles sont les Graminées, les Palmier's, les Liliacées, etc.;

Et en Dicotylédones, ou eelles dont l'embryon offrant deux cotylédons développe deux feuilles séminales par la germination; par exemple : les chénes, les orrnes, les Labiées, les Crucifères, ete. Le nombre des végétaux dicotylédons est plus considérable que eelui des acotylédons et des monocotylédons réunis.

Telles sont les grandes divisions fondamentales étałlies dans le règnue végétal. Nous avons cru devoir les exposer iei en abrégé, et en donner une idée sueeincte et générale, paree que, dans le cours de eet ouvrage, nous serons fréquemment obligé d'employer les noms d'acotylédons, de monocolylédons, et de dicotylédons, qui, s'ils n'eussent point été définis d'abord, eussent nécessairement arrêté l'ordre naturel des idées. C'est ici que nous sommes forcés de convenir que la marche des seienees naturelles n'est point aussi rigoureuse que celle des seiences physiques et mathémàtiques. On ne peut pas toujours, dans l'exposition des faits et des notions fondamentales qui appartiennent à l'histoire naturelle, proeéder strictement du. connu

(1) Quelques anteurs ont placé, mais ă tort, selon nous, les Fougères parmi les plantes à embryon monocotylédon. En effet, il est de la dernière évidencc que ces végétaux ne se reproduisent pas au moyen dc véritables graines, mais simplement par des corps particuliers, espèces de bulbiles qu'on observe sur d'autres végétaux, et auxquels on donue le nom de sporules. 
à l'inconuu. Il est souvent impossible d'éviter de passer par eertaines idées intermédiaires, non encore défunies, et de supposer daus ceux pour lesquels on écrit, des connaissances qu'heureusement ils possèdent presque toujours.

Nous avous, autant que possible, eherché à remédier à cet inconvénient dans l'exposition des notions élémentaires de la Botanique que nous venons de présenter. Nous nous sommes efforcé d'exposer ici les faits dans leur dernier degré de simplicité, afin que ecux mème qui n’ont encore aucune connaissance de cette scienee puissent aisément suivre le développement suecessif dans lequel nous allons entrer au sujet des différens organes des végétaux.

Les organes des végétaux sont divisés en deux classes :

$I^{\circ}$ Suivant qu'ils servent à leur nutrition, c'est-à-dire à puiser dans le sein de la terre ou de l'atmosphère les substances nutritives propres à leur développement: on les appelle alors organes de la nutrition ou de la végélation. Tels sont la racine, la tige, les bourgeons et les feuilles, etc.;

$2{ }^{\circ}$ Suivant qu'ils servent à la reproduction de l'espèce: on les nomme organes de la reproduction ou de la fructification. Tels sont la fleur, ses différentes parties, et le fruit qui leur succède.

Nous commencerons d'abord par étudier les organes de la nutrition; et nous ferons suivre eette étude de celle des organe de la fructification.

L'ordre le plus naturel des idées eût été sans doute de commencer par étudier les organes de la plante dans la graine qui les renferme déjà à l'état rudimentaire; d'en suivre ensuite les progrès ultérieurs jusqu'à leur état le plus parfait de développement; mais l'organisation de la graine étant, sans contredit, le point le plus difficile de la Botanique, celui sur lequel il reste encore le plus de doutes et d'obscurité, il nous a semblé qu'il fallait d'a- 
28

INTRODUCTION. ANATOMIE VÉGĹTALF.

bord aeeoutumer en quelque sorte nos leeteurs à des idées et des faits plus simples, afin de les faire arriver ainsi par degrés aux parties les plus eompliquées de l'organisation végétale. 


\section{PREMIÈRE CLASSE.}

\section{ORGANES DEIA NUTEITION OU DI IA TÉGÉTATION.}

Nous avons, dans l'introduction précédente, divisé les organes des végétaux en deux classes, suivant les usages qu'ils remplissent. Dans la première classe nous plaçons les organes de la nutrition ou de la végétation; dans la scconde, ceux de la reproduction ou de la fructification.

Les organes de la nutrition ou dc la végétation sont tous ceux auxquels est confié le soin de la conservation individuelle des végétaux. Ce sont les racines, les tiges, lcs bourgeons, les feuilles, les stipules, et quelques-uns de ces organes dégénérés, tels que les ćpines, les aiguillons, les vrilles. Ces organes ont un but commun, l'entretien de la vie dans le végétal. En effet, la racine, enfouie dans le sein de la terre, absorbe une partie des fluides nutritifs et réparateurs; la tige transmet ces fluides dans tous les points de la plante, tandis que lcs feuilles, étcnducs au milicu de l'atmosphère, y remplissent lcs mêmes fonctions que les racines dans la terre, et servent à la fois d'organes absorbans et cxhalans. On voit, par ce court exposć de leurs fonctions, que ces différens organes tcndent tous à une même fin; qu'ils nourrissent le végétal ct concourent à sa végétation, c'est-à-dire au dévcloppement de toutes ses parlies. 


\section{CHAPITRE PREMIER.}

\section{DE IA RACINE (I):}

ON donne le nom de racinc à cette partic d'un végétal qui, occupant son cxtrémité inféricure, et eachée le plus souvent dans la terre, se dirige et croît constamment en sens inverse de la tige, c'est-à-dire s'enfonce perpendieulairement dans la terre, tandis que eelle-ci s'élève vers le ciel. Un earactère non moins remarquable de la racine est de ne jamais devenir verte ( au moins dans son tissu) quand elle est exposée à l'action de l'air et de la lumière, tandis que toutes les autres parties des végétaux y prennent eette couleur.

A l'exeeption de quelques trémelles et de certaines conferves, qui, plongées dans l'eau ou végétant à sa surface, absorbent les matériaux de leur nutrition par les différens points de leur étendue, tous les autres végétaux sont pourvus de racines, qui servent à les fixer au sol et à y puiscr une partic de leurs prineipes nutritifs.

Les raeines, avons-nous dit, sont le plus souvent implantées dans la terre. C'est ee qui a lieu, en effet, pour le plus grand nombre des végétaux. Mais il cn cst d'autres qui, vivant à la surface de l'eau, préscntent des racines flottantes au milieu de ce liquide; comme on l'observe dans certaines lentilles d'eau. La plupart des plantes aquatiqurs, comme le trèfle d'eau, le nénuphar, l'utriculaire (2), offrent deux espèces de racines. Les unes, enfoncées dans la vase, les fixent au sol; les autres, partant

(1) Radix, lat.; $\rho$ Ł̌ $\alpha$, grec.

(2) Les parties filamenteuses, que la plapart des botanistes ont prises pour des feuilles dans l'utriculaire, ne sont que deș racines flottantes. 
ordinairement de la base des feuilles, sont libres et flottantes au milieu de l'eau.

D'autres plantes végétant sur les roehers, comme les Lichens; sur les murs, comme la giroflée commune, le grand muflier, la valériane rouge; sur le tronc ou la racine des autres arbres, eomme le lierre, certaines Orchidées des tropiques, la plupart des Mousses, l'orobanche et l'hypociste, y implantent leurs raeines, et véritables parasites, en absorbent les matériaux nutritifs, et vivent à leurs dépens.

Le clusia rosea, arbrisseau sarmentenx de l'Amérique méridionale, le sempervivum arboreun, le maïs, le manglier et quelques figuiers. exotiques, outre les raeines qui les terminent inférieurement, en produisent d'autres de différens points de leur tige, qui, d'une hauteur souvent considérable, deseendent et s'enfoneent dans la terre. On a donné à ces racines surnuméraires le nom de racines adventives, et un fait fort remarquable qui les concerne, c'est qu'elles ne'commencent à se développer en diamètre que quand leur extrémité a atteint le sol et y puise les matériaux de son aeeroissement.

Ne confondons pas avee les racines, eomme on l'a fait trèssouvent, certaines tiges souterraines, qui rampent horizontalement sous terre, comme dans l"iris germanica, le seeau de Salomon, etc. Leur direction seule suffirait presque pour les distinguer, si d'autres caractères ne venaient point encorenous éclairer sur leur véritable nature ( $V$ oyez dans le ehapitre suivant ce que nous en disons en parlant de la souche ou tige souterraine.)

Différentes parties dans les végétaux sont suseeptibles de produire des racines; eoupez une branche de saule, de peuplier; enfoncez-la dans la terre, et au bout de quelque temps son extrémité inférieure sera chargée de radicelles. Le même phénomène aura encore lieu lorsqu'on aura implanté les deux extrémités de la branche dans la 
terre: l'une et l'autre s'y fixent, au moyen de raeines qu'elles développent.Dans les Graminées, partieulièrement le maïs ou blé de Turquie, les noends inféricurs de la tige poussent quelquefois des racines qui deseendent s'enfoneer dans la terre. C'est sur cette propriété qu'ont les tiges et même les feuilles dans beaucoup de végétaux de donner naissance à de nouvelles racines, que sont fondées la théorie et la pratique du marcotage et de la bouture, moyens de multiplication très-employés dans l'art de la culture.

Il existe une grande analogic de strueture entre les racines qu'un arbre pousse dans le sein de la terre, et les rameaux qu'il étale au milieu de l'air. Les principales différenees que l'on observe entre ces deux organes dépendent prineipalement déla différence ( I) des milieux daus lesquels ces organes se développent.

Les racines de certains arbres poussent de distance en distance des espéees de cônes ou de bosses d'un bois mou et lâche, entièrement nus et saillans hors de terre, et que l'on a désignés sous le nom d'exostoses. Le cyprès chauve de l'Amérique septentrionale (taxodium distichum. Rieh.) en offre les exemples les plus remarquables.

(r) On a dit que lorsqu'on renversait an jeune arbre de manière que scs branches fussent cnfoncées dans la terre, et scs racines étalées dans l'air, les feuilles se changeaient en racines, et celles-ci en feuilles; ce fait est faux, ou du moins l'explication quc l'on en donne n'est pas cxacte. En cffet, les feuilles ne se changent pas plus en racines que les racines en fevilles. Mais lorsqu'ils sont cachés sous la terre, les bonrgeons situćs à l'aisselle des fcuillcs, au lieu de développer de jeunes xameaux ou scions foliacés, s'allongent, s'ćtiolent, et dcviennent des fibres radicalcs; tandis que les bourgeons latens qui existcut dans les racincs, et qui sont destinés à rcnouveler Ic chcreli chaque année, placés dans un autrc milieu, sc déreloppent en fenilles. On a encore un excmple bien frappant de cette tendance des bourgeons latens de la racine à se chauger en ramcaux foliacés, lorsqu'ils sont exposés au contact de l'air, dans ces rejets qui poussent autour des arbres à ranes rampantes, comme l'acacia, le peuplier, etc. 
La racine, eonsidérée dans son ensemble et d'une manière générale, peut être divisée en trois parties: Io le corps ou pariie moyenne, de forme et de eonsistance variée, quelquefois plus ou moins renflé, eomme dans le navet, la earotte; $2^{\circ}$ le collet ou noend vital : e'est le point ou la ligne de démareation qui sépare la raeine de la tige, et d'où part le bourgeon de la tige ammuelle, dans les raeines vivaees; $3^{\circ}$ les radicelles ou le chevelu : ce sont les fibres plus ou moins délićes qui terminent ordinairement la racine à sa partie inférieure.

$A$. Suivant leur durée, les racines ont été distinguées en annuelles, bisannuelles, vivaces et ligneuses.

Les racines annuelles sont eelles des plantes qui, dans l'espaee d'une année, se développent, fruetifient et meurent : tels sont le blé, le pied-d'alonette (delphinium consolida), le eoquelieot (papaver rhacas), ete.

Les raeines bisannuclles sont eelles des plantes à qui deux années sont néeessaires poir aequérir leur parfait développement. Les plantes bisannuelles ne produisent ordinairement, la première année, que des feuilles; la seeonde année elles meurent après avoir fleuri et fructifié, comme la earotte, ete.

On a donné le nom de racines vivaces à celles qui appartiennent aux plantes ligneuses et à eelles qui, durant un nombre indéterminé d'années, poussent des tiges herbaeées, qui se développent et meurent tous les ans, tandis que leur racine vit pendant un grand nombre d'années; telles sont celles des asperges, des asphodèles, de la luzerne, ete.

Cette division des végétaux en annuels, bisannuels et vivaces, suivant la durée de leurs raeines, est sujette à varier, sous l'influenee de diverses eireonstanees. Le elimat, la température, la situation d'un pays, la eulture même, modifient singulièrement la durée des végétaux. Il n'est pas rare de voir des plantes annnelles végéter 
deux ans, et même davantage, si elles sont mises dans un terrain qui leur soit eonvenable, et abritées contre le froid. Ainsi le réséda odorant, qui ehez nous est une plante annuelle, devient une plante vivaee dans les sables des déserts de l'Egypte. Au eontraire, des plantes vivaees et même ligneuses de l'Afrique et de l'Amérique, transplantées dans les régions septentrionales, y deviennent annuelles. La belle de nuit (nyctago hortensis), le cobcea, sont vivaees au Pérou, et meurent ehaque année dans nos jardins. Le riein, qui, en Afrique, forme des arbres ligneux, est annuel dans notre elimat. Cependant il reprend son earaetère ligneux quand il se retrouve dans une exposition eonvenable. En hierborisant aux environs de Villefranehe, sur les bords de la Méditerranée, au mois de septembre 18 I 8 , j'ai déeouvert sur la montagne qui abrite l'arsenal de cette ville, au couchant, un petit bois formé de rieins en arbre. Leur trone est ligneux, dur. Les plus hauts sont environ vingt-einq pieds d'élévation, et présenteut à peu près le même aspeet que nos platanes. Il est vrai que la situation de Villefranehe, exposée au midi, défendue des vents d'ouest par une ehaîne de eollines assez élevées, la rapproehe singulièrement du elimat de certaines parties de l'Afrique.

En général toutes les plantes exoliques vivaces, dont les graines peuvent former des individus, qui fleurissent dès la première amnée dans nos elimats, y deviennent annuelles. C'est ee qui arrive pour le ricin, le cobcea, la belle de nuit, ete.

Les raeines ligneuses ne diffèrent des racines vivaces que par leur eonsistanee plus solide, et par la persistanee de la tige qu'elles supportent; telles sont eelles des arbres et des arbrisseaux.

$B$. Suivant leur forme et leur strueture, les raeines peuvent se diviser en : $1^{\circ}$ Pivotante (radix perpendicularis), $2^{\circ}$ Fibreuse (radix fibrosa), 30 Tubérifère (radix tuberifera), $4^{\circ}$ Bulbifere (radix bulbifera). 
I $^{0}$ Les racines pirotantes sont celles qui s'enfoncent perpendiculairement dans la terre. Elles sont simples ct sans divisions sensiblcs, comme dans la rave, la carotte; rameuses, dans le frêne et le pcuplier d'Italie, etc. Elles appartiennent exclusivement aux végétaux dicotylédons. (Vorez planche 2, fig. I, 2, 3 et 4.)

$2^{\circ}$ La raeine fïrreuse se compose d'un grand niombre de fibres, quelquefois simples et grêles, d'autres fois épaisses et ramifiées. Telle est celle de la plupart des Palmièr's. Elle ne s'observe que danis les plantics monocotylédones.

$\therefore 3^{\circ}$ J'appelle racines tubérifères celles qui présentent sur différens points de leur étendue, quelquefois à leur partie supérieure, d'autres fois au milieu ou aux extrémités de leurs ramifications, des tubercules plus ou moins nombreux. Ces tubercules ou corps charnus, que l'on a long-temps, et à tort, regardés comme des raeines, ne sont que des amas de fécule amylacée, que la nature a, en quelque sorte, mis en réservc pour servir à la nutrition du végétal. Aussi n’observe-t-on jamais de vćritables tubercules daís les plantes annuclles; ils appartienment exclusivement aux plantcs vivaces; tels sont ceux de la pomme de terre, du topinambour, des Orchidées, des patates, etc. (I) (Voyez pl. 2, fig. 5 et 6.$)$

(r) Le point de vue soüs lequel j'examine ici les tabercules diffère de celni sous lequel on les considère. eommunément. Loin d'être des racines, comme beaucoup d'anteurs l'ont dit, ils ne nous paraissent être, avec M. Sprengel (Linnai Philos. botan.), que des espèces de bourgeons souterrains des plantes vivaces, auxquels la nature a confié le soin et la conservation des rudimens de la tige. La seule différence que présentent les tubereules ainsi considérés, - c'est que la jeune lige, au lieu d'être prolégée par des écailles nombreuses et serrées, se trouve enveloppée par uu corps dense et charnu qui sert non-seulement à l'abriter pendant l'hiver, mais qui lui fournit au printemps les premiers matériaux de son développenent et de sa nutrition.

On pourrait également les considérer comme des tiges souterraines; courtes et charnues, et les yeux qui en maissent, comme des bourgeons. 
$4^{\circ}$ La racine bulbifère est forméc par une espèce de tubercule minee et aplati, qu'on nomme plateau, produisant par sa partie inférieure une raeine fibreuse, ct supportant supérieurement un bulbe ou ognon, qui n'est ricn autre chose qu'un bourgeon d'une nature particulière, formé d'un grand nombre d'écailles. ou de tumiques appliquées les unies sur les autres; par exemple, daus le lis, la jaeinthe, l'ail, et en général les plantes qu'on appelle bulbeuses. (Voyez pl. 2, fig. 8 ct 9.)

Telles sont les modifications prineipales que présente la racine relativement à sa strueture partieulière. Arouons cependant que ces différences ne sont pas toujours aussi tranchées que nous venous de les présenter. Ici, comme dans ses autres ouvrages, la nature ne se prête pas servilement à nos divisions systématiques. Elle fait quelquefois. disparaître par des nuanees insensibles ees différences; que nous avions crues d'abord si constantes et si bien établics.

Toutes les racines qui ue peuvent être rapportées à une des quatre modifications principales que nous venons d'indiquer conservent le nom générique de racines.

Le cherelu des racines, ou cette partie formée de filıres plus ou moims déliées, scra d'autant plus abondant et plus développé, que le végétal vivra dans un terrain plus meuble. Lorsque par liasard l'cxtrémité d'une racine reneontre un filet d'eau, elle s'allonge, se développe en fibrilles capillaires et ramifiées, et eonstitue ec que les jardiuiers désignent sous le nom de queuc de renard. C.c phénomène, que l'on peut produire à volonté, explique pourquoi les plantes aqualiques ont, en génćral, des racines beaucoup plus développées.

Après ees consiclérations gémérales sur la structure des racines, nous devons présenter ici les principales modifications que cet organc peut subir, quant à sa eonsistonce, sa forme el ses autres caractéres extéricurs. 
C. Relativement à sa consistance, la raciné est charnue, lorsque, étant manifcstement plus grossc et plus épaisse que la base de la tige, elle cst cn même temps. plus sueculcntc : tellc cst ccllc de la carotte, du navet, etc. Elle cst ligneuse, air contraire, lorsque son parcnchyme, plus solidc, approche plus ou moins de la durcté du bois. C'cst ee que l'on obscrve dans la plupart des végétaux ligneux.

D. La raeine peut être simple ( simplex), e'est-à-dirc formée par un pivot absolument indivis; commc la bettcrave, lc panais, la rave, ctc. D'autres fois clle est rameuse (ramosa), ou diviséc en ramifications plus ou moins nombrcuses et délićes, toujours de même nature qu'cllc; telle cst ccllc de la plupart des arbres de nos forêts, du chênc, dc l'orme, ctc.

$E$. Considćrée quant à sa direction, la racinc pcut être verticale, comme cellc de la carottc, de la rave; oblique, par cxemplc ccllc des iris ; et cnfin situéc horizontalement sous la terre, eommc dans le Rhus radicans, l'orme, cte. Asscz souvent l'on trouvic ces trois positions réunics dans les différcutes ramifications d'une même racinc.

$F$. Les varićtés de forme les plus remarquables sont les suivantcs :

$I^{\circ}$ Fusiforme, ou cn fuseau (fusiformis), lorsqu'clle cst allongée, plus mince à ses dcux extrémités, plus grosse à sa partie moycnue, commc la rave. (PI. 2, fig. 3.)

$2^{\circ}$ Napiforme, ou cn forme de toupic (napiformis) quand elle cst simple, arrondie, ct renfléc à sa partie supérieure, amincie et tcrminéc brusquement en pointe inférieurcment: lc navet, lc radis, cic. (Pl. 2, fig. 2.)

$3^{\circ}$ Conique (conica), cclic qui préscnite la formc d'un cône renversé : la betterave, le panais, la carotte. (Pl.2, fig. 4.) 
$4^{\circ}$ Arrondie ou presque ronde (subrotunda), comme dans le Bunium bulbocastanum, ete.

$5^{\circ}$ Didyme ou testieulée (x) (didyma, testiculata), lorsqu'elle présente un oudeux tubereules arrondis ou ovoïdes, comme dans l'Orchis militaris, maculata, ete. (Voyez pl. 2, fig. 5 et 6 .)

La raeine didyme est appelée palmée (pailmata), quand les deux tubereules sont divisés jusqu'au milieu environ de leur épaisseur en lobes divergens, eomme les doigts de la main. Ex., Orchis maculata. (P1.2, fig. 6.)

Digitée (digitata), quand les tubereules sont partagés presque jusqu'à leur base, comme dans le Salyrium albidum. C'est d'après cette forme de la raeine didyme que Limué a divisé les espèees du genre Orchis en trois sections.

$6^{\circ}$ Noueuse ou filipendulée (nodosa), lorsque les ramifieations de la racine présentent de distanee en distanee des espèees de renflemens ou de nouds (2) qui lui domnent quelque ressemblanee avee un ehapelet; c'est ee que l'on observe dans la filipendule, l'Avena pracatoria.

$7^{\circ}$ Grenue (granulata). M. de Candolle nomme ainsi celle qui présente un amas de petits tubereules renfermant des yeux propres à reproduire la plante, sans être enveloppé de tissu cellulaire rempli de féeule amylaeée. Par exemple, eelle de la saxifrage grenue.

$8^{\circ}$ Fasciculée (fasciculata), quand elle est formée par la réunion d'un grand nombre de radieelles, épaisses,

(I) Dans la racine testiculée, l'un des tabercules ( $\mathrm{pl} .2$, fig. 5. a.) est ferme, solide, un peu plus gros que l'autre; c'est lui qui renferme le radiment de la tige qui doit se développer l'année suivante; l'autre, au contraire (pl. 2, fig. 5. 6.), mou, ridé, plas petit, contenait le germe de la tige qui vient de se développer, et à l'accroissement de laquelle il a employé la plus grande partie de la fécule amylacée qu'il renfermait.

(2) Ces nocuds ne doivent pas être confondus arec les réritables tubercules, qui renferment toujours les rudimens de nonvclles tiges. 
simples ou peu rameuses, comme celle des asphodèles, des renoneules ( $\mathrm{I}$ ).

$9^{\circ}$ Articulée (articulalia), eelle qui présente, de distance en distanee, des articulations. Par exemple, dans la gratiole.

I $0^{\circ}$ Contournée (contørta), quand elle offre plusicurs courbures en différens sens : eelle de la bistorte.

I I ${ }^{\circ}$ On appelle racine capillaire (capillaris), celle qui est formée de fibres eapillaires très-deliées, comme la plupart des Graminées, le blé, l'orge.

I $2^{\circ}$ Clevelue (comosa), quand les filets eapillaires sont rameux et très-serrés, eomme dans les bruyères.

Quant à la strueture anatomique de la racine, nous n'en ferons l'exposition qu'après eelle de la tige, paree que ees deux organes offrent beaucoup d'analogic sous ec rapport.

\section{Usages des racines.}

Les usages des racines sont relatifs au végétal lui-même ou à ses applications, à l'économie domestique, aux arts, et à la médecine.

Relativement au végétal lui-même, les raeines servent, $x^{0}$ à le fixer à la terre ou au eorps sur lequel il doit vivre; $2^{0}$ à y puiser une partie des matériaux néeessaires à son aceroissement.

Les raeines de beaueoup de plantes ne paraissent remplir que la première de ees fonetions. C'est ee que l'on observe prineipalement dans les plantes grasses et sueeulentes, qui absorbent par tous les points de leur surface les substanees propres à leur nutrition. Dans ee cas, leurs raeines ne servent qu'à les fixer au sol. Tout le monde connaît le magnifique eierge du Pérou ( Caclus pertuvia-

(1) Celles des renoncales, formécs de fibres plus courtes et plus serrées, portent en général le norn de griffes. 
nus) qui existe dans les serres du Muséum d'histoire naturelle. Ce végétal, qui cst d'une hauteur extraordinaire, pousse avec une extrême vigueur des rameaux énormes, et souvent avec une rapidité surprenante; ses racines sont renfermées dans une eaisse, qui contient à peine trois à quatre pieds cubes d'une terre que l'on ne renouvelle et n'arrose jamais.

Les racines des plantes ne sont pas toujours en proportion avec la force et la grandeur des troncs qu'elles supportent. Les Palmiers et les Conifères, dont le tronc acquiert quelquefois une liauteur de plus de cent pieds, ont des racines courtes, s'ćtendant peu profondément dans la terre, et me les y fixant que faiblement. Des plantes herbacées, au contraire, dont la tige, faible et grêle, meurt chaque année, ont quelquefois des racines d'une force et d'une longueur considérables relativement à celles de la tjge, comme on l'observe dans la réglisse, la luzerne, et dans l'Ononis arvensis (qui, à cause de la ténacité et de la profondeur de ses racines, a été appelé arréte-bceuf).

Les racines ont aussi pour usage d'absorber dans le sein de la terre les substances qui doivent servir à l'accroissement du végétal. Mais tous les points de la racine ne concourent pas à celle fonction. Ce n'est que par l'extrémité de leurs fibres les plus délices que s'exerce cette absorption. Les uns ont dit qu'clles étaient terminées par de petites ampoules ou de spongioles plus ou moins renflées, d'autres par des cspèces de bouches aspirantes; quelle que soit leur structure, il cst prouvé que c'est par ces extrémités seules que s'opère cette fonction.

Il n'est point d'expérience plus facile à faire que celle au moyen de laquelle on cémontre d'une manière péremptoire la véritć de ce fait. Si l'on prend un radis ou un navet, qu'on le plonge dans l'eau par l'extrémité de la radicule qui le termine, il poussera des feuilles et végétera. Si, au contraire, on le place daus l'eau de manière à ce que son 
extrémité inférieure soit hors du liquide," il ne donnera aucun sigue dc développernent.

Les racines de.ccrtaines plantes paraissent exeréter une matière partieulière, différente dans les diverses espèees. Duhamel rapporte qu'ayant fait arracher de vieux ormes, il trouva la terre qui environnait les racines d'une eouleur plus foncéc et plus onctucuse. Cette matièrc onctueuse et grasse était le produit d'une sorte d'excrétion faite par les racines. C'est à cettc matière, qui, eomme nous l'avons dit, est différente dans chaque espèec végétale, que l'on a attribué les sympathies et les antipathies que certains végétaux ont les uns pour les autres. On sait, en effet, que certaines plantes se recherehcnt en quelque sortc, et vivent constammént lcs uncs à côté des autres; ee qui forme les plantes sociales; tandis qu'au contrairc d'autrcs semblent ne pouvoir croître dans le même lieu.

On a remarqué quc les raeines ont une tendance marquée à sc dirigcr vers les veines de bonnc terrc, et quc sourcnt elles s'allongent considérablement pour sc portcr vcrs les lieux où la terrc est plus meuble et plus substantielle. Elles s'y développent alors avec plus de forcc et de rapidité. Duhamel rapporte que, voulant garantir un champ de bonne terre des racines d'unc rangée d'ormes qui s'y étendaient et en épuisaient une partie, il fit faire lc long de cette rangée d'arbres une tranchéc profondc qui eoupa toutes les raeines qui s'étendaient dans le ehamp. Mais bientôt les nouvclles racines, arrivécs à l'un des côtés du fossé, se recourbèrent en suivant la pentc dc eeluiei jusqu'à la partic inférieure; là elles se portèrent horizontalcment sous le fossé, sc relevèrcnt ensuite de l'autrc côté, en suivant la pente opposée, et s'étendircnt dc noutveau dans le ehamp.

Les raeines, dans tous les arbres, n'ont pas la même forcc pour pénétrer dans le tuf. Duhamcl a fait l'observation qu'une racine de vigne avait pćnétré profondément 
dans un tuf très-dur, tandis qu'une raeine d'orme avait été arrêtée par sa dureté, et avait en quelque sorte re'broussé chemin.

La raeine, ainsi que nous l'avons dit préeédemment, a une tendanee naturelle et invineible à se diriger vers le centre de la terre. Cette tendanee se remarque surtout dans eet organe, au moment où il eommenee à se prononcer, à l'époque de la germination de l'embryon; plus tard elle est moins manifeste quoiqu'elle existe toujours, surtout dans les raeines qui sont simples, ou dans le pivot des raeines rameuses; ear elle est souvent nulle dans les ramifieations latérales de la raeine.

Quels que soient les obstacles que l'on cherehe à opposer à eette tendanee naturelle de la radieule, elle sait les surmonter. Ainsi plaeez une graine germante de fève ou de pois de manière que les eotylédons soient placés dans la terre et la radieule en l'air, vous verrez bientôt eette radieule se reeourber vers la terre pour aller s'y enfoneer. On a donné beaucoup d'explieations diverses de ee phénomène : les uns ont dit que la racine tendait à deseendre, paree que lè fluides qu'elle eontenait étaient moins élajorés, et par conséquent plus lourds que eeux de la tige. Mais eette explieation est eontredite par les faits. En effet, ne voit-on pas dans eertains végétaux exotiques, tels que le Clusia rosea, ete., des raeines se développer sur la tige à une hauteur très-eonsidérable, et deseendre perpendieulairement pour s'enfoneer dans la terre. Or, dans ee eas, les fluides eontenus dans ees raeines aérienmes sont de la même nature que eeux qui eireulent dans la tige, et néanmoins ees racines, au lieu de s'élever eomme elle, deseendent au eontraire vers la terre. Ce n'est donc pas la différenee de pesanteur des fluides qui leur doune eette tendanee vers le eentre de la terre.

D'autres ont eru trouver eette eause dans l'avidité des raeines pour l'humidité, humidité qui est plus grande' 
dans la terre que dans l'atmosphère. Duhamel, voulant s'assurer de la réalité de eette explieation, fit germer des - graines entre deux éponges humides et suspendues en l'air; les racines, au lieu de se porter vers l'une ou l'autre des deux éponges bien imbibées d'humidité, glissèrent entre elles, et vinrent pendre au-dessous, en tendant ainsi vers la terre. Ce n'est done pas l'humidité qui attire les raeines vers le eentre de la terre.

Serait-ce la terre elle-même par sa nature et par sa masse? L'expérienee eontredit eneore cette explication. M. Dutrochet remplit de terre une eaisse dont le fond était pereé de plusieurs trous; il plaça dans ces trous des graines de haricot germantes, et il suspendit la eaisse en plein air ì une hauteur cle six mètres. De cette manière, dit-il, les graines, plaeées dans les trous pratiqués à la face inférieure de la eaisse, recevaient de bas en haut l'influence de l'atmosphère et de la lumière : la terre humide se trouvait placée au-dessus d'elles. Si la cause de la direetion de cette partie existait dans sa tendanee pour la terre humide, on devait voir la radicule monter dans la terre plaeée au-dessus d'elle, et la tige au eontraire deseendre vers l'atmosphère placée au-dessous d'elle; e'est ce qui n'eut point lieu. Les radieules des graines descendirent dans l'atmosphère, où elles ne tardèrent pas à se dessécher; les plumules au eontraire se dirigèrent en haut dans la terre.

M. Knight, célèbre physieien anglais, a voulu s'assurer par l'expérienee si cette tendanee ne serait pas détruite par le mourement rapide et eireulaire imprimé à des graines germantes. Il fixa des graines de haricots dans les augets d'une roue, mue eontinuellement par un filet d'eau dans 1 plan vertieal, cette roue faisant cent einquante révolutions en une minute. Ces graines placées dans de la mousse sans eesse humeetée, ne tardìrent pas à germer; toutes les radicules se dirigèrent vers la cireonférenee de la roue, et toutes les gemmules vers son eentre. Par chacune de ees 
directions les radicules et les gemmules obćissaient à leurs tendanees naturelles et opposées. Le mème physieien fit une expérience analogue avec une roue mue horizontalement et faisant deux cent cinquante révolutions par minute; les résultats furent scmblables, c'est-à-dire que toutes les radicules se portèrent ver's la circonférence, et les gemmules vers le centre, mais avec une inelinaison de dix degrés des premières vers la terre, et des secondes vers le ciel. Ces expériences, répétées par M. Dutrochet, ont eu les mêmes résultats, excepté que dans la scconde l'inclinaison a été beaucoup plus considérable, et que les radicules et les gemmules sont devenues presque horizontales.

Des diverses expériences rapportées ci-dessus il résulte évidemment que les racines se dirigent vers le ecntre de la terre, non parec qu'elles contiennent un fluide moins élaboré, ni parce qu'elles y. sont attirées par l'humidité ou la nature même de la terre, mais par un mouvement spontanć, une force intéricure, une sorte de soumission aux lois générales de la gravitation.

Mais, quoiqu'on puisse dire que cette loi de la tendance des racines vers le centre de la terre soit générale, néammoins quelques végétaux semblents'y soustrairc; tclles sont en général toutes les plantes parasites, et le gui ( $/$ iscum album) en particulier. Cette plante singulière pousse, en eflet, sa radicule dans quelque position que le hasard la place; ainsi, quand la graine, qui est enveloppée d'une glu épaisse et visqueuse, vient à se coller sur la partie supérieurc d'une branche, sa radicule, qui est ume sorte de tubercule évasé én forme de cor de chasse, se trouve alors perpendiculaire à l'horizon : si, au contraire, la graine est placée à la partie inféricure de la branche, la radicule se dirige ver's le ciel. La graine est-elle située sur les parties latérales de la branche, la radicule se dirige latéralement. En un mot, dans quelque position que la graine 
soit fixée sur la branche, la radieule se dirige toujours perpendieulairement à l'axe de la branelıe.

M. Dutrochet a fait sur la germination dc eette graine un grand nombre d'expérienees pour constater la direetion: de la radicule. Nous rapporterons ici les plus intéressantes. Cette graine, qui trouvc dans la glu qui l'enveloppe les premiers matériaux de son accroissement, germe et se développe non-seulement sur du bois vivant et mort, mais encore sur des pierres, du verre, et même sur du fer. M. Dutrochet en a fait germer sur un boulet de canon. Daus tous ces cas la radicule s'est toujours dirigée vers le centre de ees corps. Ces faits prouvent, ainsi que le remarque cet ingénieux expérimentateur, que ce n'est pas vers un milieu propreà sa nutrition que l'embryon du gui dirige sa radicule, mais que eelle-ei obéit à l'attraction des eorps sur lesquels la graine est fixée, quelle que soit leur nature.

Mais cette attraction n'est qu'une eause éloignée de Ia tendanee de la racine du gui vers les corpss. La véritable cause est un mouvement intérieur et spontané exécuté par l'embryou à l'occasion de l'attraction exercée sur sa radicule. M. Dutroehet colle une graine de gui germée à l'une. des extrémités d'une aiguille de cuivre, semblable à, une aiguille de boussole, et plaeée de mème sur un pivot; une petite boule de eire mise à l'autre extrémité forme le. contre-poids de la graine. Les ehoses ainsi disposées, M. Dutroehet approehe latéralement de la radicule une petite planche de bois, à euviron un millimètre de distanee. Cet appareil est ensuitc recouvert d'un récipient de verre, afin de le garantir de l'action des agens extérieurs. Au bout de cinq jours la tige de l'embryon s'est fléehie et a dirigé la radieule vers la petite planche qui l'avoisinait, sans que l'aiguille eût cliangé de position, malgré son extrême mobilité sur le pivot. Deux jours aprè̀s, la radieule était dirigée perpendiculairement yers la planche avec la- 
quelle elle s'était mise en eontaet, sans que l'aiguille, qui portait la graine, eût éprouvé le moindre dérangement.

La radieule du gui présente eneorc unc autre tendanee constante; c'est eelle de fuir la lumière. Faites germer des graines de gui sur la face interne des vitres d'une croisée d'appartement, et vous verrez toutes les radicules se diriger vers l'intćrieur de l'appartement pour y chcrcher l'obscuritć. Prenez une de ces graines germées, appliquezla sur la vitre en dehors de l'appartement, et sa radicule. s'appliquera contre la vitre, comme si elle tendait vers i'intérieur de l'appartement pour fuir la lumière.

Dans l'économie domestique, beaucoup de racines sont utilement employées comme alimens. Ainsi lcs carottes, les navcts "les panais, les salsifis, et bcaucoup d'autres racines sont trop universcllement usitées pour que nous soyons obligés d'eutrer dans des détails à cet égard.

C'est avec les tubcrculcs d'un grand nombre d'orehis eonvenablement préparés que se fait le salep.

On extrait de la betterave, par des procédés quc la ehimic a singulièrement perfeetionnés, un sucre qui peut avantageuscment remplaccr eelui que nous tirons à grands frais des eolonies.

Certaines plantes, ayant la faeulté de pousser des racines qui se ramifient ct s'étendent à de grandes distances, on s'en cst servi pour ramifier et consolider les terrains mouvans. C'est ainsi qu'en Hollande, et aux environs de Bordeaux, on plante lc Carex arenaria sur les dunes et' les bords des eanaux, afin de consolider et fixer les teries. Dans plusieurs autres pays on plante, pour remplir le même objet, l'Hippophae rhaminoüdes ou argousier, le genêt d'Espagne, etc.

Plusieurs racines sont employées avee avantage daus la. teinture. Telles srnt celles de garanee, d'oreanette, de eurcuma, cte.

Quant aux usages médicinaux des raeines, on sait que 
la thérapeutique leur emprunte des médieamens préeieux. Relativement aux prineipes qui y prédominent, les raeines offieinales ont été divisées en :

§. I. Raeines fades : principe muqueux ou amylaeé.

Guimauve offieinale (Althoea officinalis. L.)

Grande Consoude (Symplyytum officinale. L.)

Chiendent (Triticum repens. L.) ete., ete.

§. 2. Racines douees et suerées.

Réglisse (Glycyrrhiza glabra. L, )

Polypode (Polypodium commune. L.), ete., ete.

§.3. Racines peu sapides, ou légèrement amères.

Salsepareille (Smilax Salsaparilla. L.)

Squine (Smilax China. L.)

Bardane (Arctium Lappa. L.)

Patience (Rimex Patientia. L.)

$\$$ 4. Raeines aromatiques et odorantes.

Valériane (Valeriana officinalis. L.)

Serpentaire de Virginie (Aristolochia serpentaria. L.)

Angélique (Angelica Archangelica. L.)

Aunée (Inula Helenium. L.)

Benoite (Greum urbanum. L.)

Raifort (Coclulearia armoracia. L.)

Ginseng (Panax quinquefolium. Lamk.)

\$.5. Raeines amères.

Grande Gentiane (Gentiana lutea. L.)

Rhubarbe (Rheum palmatum et $R$. undulatum. L.)

Columbo (Cocculus palmatus. DC.)

Polygala amer (Polygala amara. L.)

Chicorée sauvage (Cichorium In'ybus. L.)

\$.6. Raeines acerbes.

Bistorte (Polygonum Bistorta. L.)

Tormentille (T'ormentilla erecta. L.)

§. \%. Racines âeres et nauséabondes. 
Ipécacuanha annclé (I) (Cephaelis Ipecacuanha. Rich.

Ipćcacuanha simple ou strié (Psycholria emetica. L.)

Cabaret (Asarum europaum. L.)

Hellébore noir (Helleborus.niger.)

Hellébore blanc (Veratrum album.)

Jalap (Convolvulus Jalappa. L.), ctc., etc.

\section{CIIAPITRE II. \\ DE LA TIGE (Caulis, L.)}

Nous vehons de voir la racine tcndre généralement à s'enfoncer vers le centre de la terre. La tige, au contraire, est cette partic de la plante qui, croissant en scns inverse dc la racine, cherclic l'air ct la Iumière, ct sert dc support aux feuilles, aux flcurs ct aux fruits, lorsque la plante en est pourvue.

Tous les végétaux Phanérogames ont une tige proprement ditc. Mais quelquefois cctte tigc est si peu développée, clle cst tcllenient cour'tc, qu'clle parait ne pas exister. Lcs plantcs qui offrent ccttc disposition ont été dites sans tigc ou acaules; telles sont la primcvère, la jacinthe, et beaucoup d'autrcs.

Ne confondons pas avec la véritable tige la Hampe et le Pédoncule radical. La Hampe (Scapus) est un pédoncule floral nu, c'est-à-dire ne portant pas de fcuilles, qui part

(1) Voyez mon Mémoire sur les deux espèces d'ipécacuanha tirées de la famille des Rubiacées, inséré dans les bulletins de la Soeiété de la Faculté, pour l'aunée 1818 , et mon Histoire maturelle et médicale des différentes espèees d'ipécacuanla du commerce, Paris, 1820 . Un vol. in-4, fig. C̣hez Béchet jeune. 
du eollet de la racine, et qui se termine par une ou plusicurs fleurs, eomme dans la jaeinthe.

Le Pédoncule radical (pedunculus radicalis) diffère de la Hampe en ee qu'au lieu de naître du eentre d'un assemblage de feuilles radieales, il sort de l'aisselle d'une de ees feuilles; par exemple, dans les plantains (Plantago media, P. lanceolata, ete.)

On distingue einq espèces prineipales de tiges, fondées sur leur organisation et leur mode partieulier de dévelóppement. Ces espèees sont : $r^{\circ}$ le Tronc, $2^{\circ}$ le Stipe; $3^{\circ}$ le Chaume, $4^{\circ}$ la Souche, $5^{\circ}$. la Tige proprement dite.

${ }^{\circ}$ On appelle Tronc (Truncus), la tige des arbres de nos forêts, du ehêne, du sapin, du frêné, etc. İl a pour earactères d'ètre eonique, allongé, e'est-à-dire d'offrir sa plus grande épaisseur à sa base. Il est nu inférieurement, terminé à son sommet pär des divisions suceessivement plus petites, anxquelles on a douné le nom de branehes, de rameaux et de ramilles ou ramuseules, et qui portent ordinairement les feuilles et les organes de la reproduetion. Le trone est propre auxi arbres dieotylédonés; eomposé intérieurement de eouehes eoneentriques. superposées, il eroît en longueur et en épaisseur, par l'addition de nouvelles eouehes à śa cireonférenee.

$2^{\circ}$ Le Stipe (Frons, Stipes) est une sorte de tige qu'on n'observe que dans les arbres monoeotylédonés, tels que' les Palmiers, les Dracœna, les Yucca, et dans eertains Dieotylédons, savoir, le Cycas et le Zamia. Il est formé par une espèee de eolomne (r) eylindrique, c'est-à-dire aussi grosse à son sommet qu'à sa base (ee qui est le eontraire dans le trone), souvent mêtme plus renflée à sa partie moyenne qu’à ses deux extrémités, rärement raünifiće, eouronnée à son sommel par un boưquet de feuilles cntremèlées de fleurs. Son écoree, lorsqu'il cu a une, est or-

(I) On le désigne souvent par lc nom de trone on tige à colonne. 
dinairement peu distinete du reste de la tige. Son aecroissement en hauteur se fait par le développement du bouton qui le termine supéricurement; il s'aceroît en épaisseur par la multiplication des filets de sa circonférenee.

Nous ferons voir bientôt, en traitant de la strueture anatomique des tiges, que le stipe ne diffère pas moins du trone par son organisation intérieure que par les caraetères physiques que nous venons d'indiquer.

30. Le chaume (culnuls) esi propre aux Graminćes, e’est¿̀-dire au blé, à l'orge, à l'avoine, etc.; aux Cypéracées et aux jones, etc. C'est une tige simple, rarement ramifiée, le plus souvent fisiuleuse (I) (e'est-à dire ereuse dans son intéricur) el séparée de disiance en distance par des espèces de nouds ou cloisons desquels partent des feuillẹs alternes et engainantes.

$4^{\circ}$ La souche ou rhizoma (2). On a donné ec nom aux tiges souterraines et horizontales des plantes vivaees, eachées cntièrement ou en partic sous la terre, poussant de leur extrémité an térieure de nouvelles tiges, à mesure que leur extrémité postérieure se détruit. Ciest à cette tige souterraine que l'on donne, en général, le nom impropre de racine progressive, de racine succise. Exemple: l'iris, la scabjeuse suecise, le sceau de Salomon (3). Outre sa direction à peu près horizontale sous la terre, un des earactères prineipaux de la souche, caractère qui la distingue de la

(1) Quelquefois cependant ello est pleine intéricurement, comme dans Ia canre à sucre, le mais.

(2) Rhizoma, dérivẹ de pǐ̌s, racine, et ossus, corps.

(3) Le nombre des plantes pourvues de souehc ou de tige sontcriaine est beaucoup plus considćrable qu'on ne l'imagine communément. Un grand nowbie de plantes dites sans tigc ou acaules, et des plantes vivaces, sont pourvies d'unc souehe plus ou moins développée. C'cst ce que l'on observe par exemple, dans la sylvie (anemone nemorosa), la mosehatelline (adoxa moschacellina), Ic Paris quadrifolia, etc. La partie de ecs plantes, qui a ćté décrite comme une racine tubéreuse, cst une véritable souchc. 
racine, e'est d'offrir toujours, sur quelques points de son étendue, lcs traces des feuilles des années précédentes; ou des écailles qui en tiennent lieu, et de s'aeeroître par sa base ou point le plus rapproehé des feuilles, ee qui est le contraire pour la véritable raeine. ( $V_{\text {oy }} \cdot \mathrm{pl} .2$, fig. 7. )

$5^{\circ}$ Enfin l'on donne le nom commun et général de liģës à celles qui, différentes des quatre espèees précédentes, ne peuvent être rapportées à aueune d'elles. Le nombre des végétaux pourvus d'une tige proprement dite est beaucoup phis considérable que eelui des végétaux qui ont un stipe, un chaume ou un tronc.

Nous allons maintenant étudier la tige en général, quant aux modifications qu'elle peut offrir.

$A$. Sous le rapport de la consistance, on distingue la tige :

I Herbacée (herbaceus), celle qui est tendre, verte, et périt chaque année. Telles sont eelles des plantes amnuelles, bisannuelles et vivaees, le mouron des ehamps, la bourrache, la consoude, ete. Toutes ees plantes prement le nom général d'herbes (herbce).

$2^{0}$ Demi-ligneuse ou sous-ligneuse (suffruticosus), quand la base est dure et persiste hors de terre un grand nombre d'aunées, tandis que les rameaux et les extrémités des branches périssent et se renouvellent tous les ans. Tels sont la rue odorante (ruta graveolens), le thym des jardins (thrmus vulgaris), la sauge offieinale (salvia officinalis). iLes végétaux qui offrent une semblable tige portent le nom de sous-arbrisseaux (suffrutices). Ils sont dépourvus de bourgeons écailleux.

3o Ligneuse (lignosus), quand la tige est persistante,' ct que sa dureté est semblable à celle que l'on eonnaît au bois en général. Ees végétaux à tige ligneuse se divisent en : Arbustes (frutices) quand ils se ramificnt dès leur base ct ne portent pas de bourgeons; par exemple, les bruyires. Arbrisseaux (arbusculce), s'ils sont ramifiés à leur base 
et portent des bourgeons, eomme le noisettier et le lilas, etc.

Enfin ils retiemnent le nom d'arbres proprement dits, lorsqu'ils présentent un trone d'abord simple et nu dans sa partie inférieure, ramifié seulement vers sa partie supéricure : le chêne, l'orme, le pin, ete.

Cette division est tout-à-fait arbitraire, et n'existe point dans la nature. En effet, un arbre de la même espèce peut offrir ees trois modifieations de grandeur suivant les expositious auxquelles il est soumis, ou par l'art du cultivateur. Ainsi l'ormille, le petit buis, dont on fait des bordures de plate-bandes dans nos jardins, en ayant soin de les tailler fréquemment, sont absolument de la même espèce que l'orme et le buis ordinaires, dont les tiges, surtout celle du premier, s'élèvent ordinairement à une grande hauteur, lorsque ees végétaux sont abandonnés à eux-mêmes.

$4^{\circ}$ Solide ou pleine (solidus), quand elle n'offre aneme cavité intérieure. Par exemple, la eanne à suere, le tronc de la plupart des arbres. Cette épithète s'emploic toujours par opposition à la suivante.

$5^{\circ}$ Fistuleuse ( fistulosus), offrant une cavité intérieure, continue ou séparée par des eloisons horizontales : l'arundo donax, l'angélique, l'cnanthe fistulosa, le bambou, le cecropia peltata, grand arbre de l'Amérique méridionale, dont le trone toujours ereux est pour cette raison nommé bois-canon par les liabitans.

$6^{0}$ Médulleuse (medullosus); remplie de moelle : l'hyè̀ble, le sureau, le figuier.

$7^{0}$ Spongieuse (spongiosus), formée intérieurement d'un tissu élastique, spongieux, compressible, retenant l'humidité à la manière des éponges; ex. : typla lalifolia, scirpus lacustris, ete.

$8^{\circ}$ Molle (mollis, flaccidus), quand elle ne peut se soutenir d'elle-mème et qu'elle tombe sur la terre : par exemple, le mouron des champs (anagallis arvensis). 
$9^{\circ}$ Ferme ou roide (rigidus), lorsqu'elle s'élève directement, se soutient droite; ex. : la bistorte (polygonum bislorta).

I $0^{\circ}$ Flexible (flexibilis), quand on peut la plier ou la fléehir aisément saus qu'elle se rompe : l'osier.

I I $^{\mathbf{0}}$ Cassante ( fragilis), quand elle est raide, et se easse aisément : celle de l'herbe à Robert (geranium robertianum), les différentes espèees de elaragnes, ete.

${ }_{12}{ }^{\circ}$ Charnue (succulentus), eelle qui renferme une grande quantité de sue ou de substanee aqueuse: par exemple, la bourrache, le pourpier.

Les tiges charnues peuvent être laiteuses, c'est-à-dire renfermer un suc blanehâtre et laetiforme ou jaunâtre, comme les euphorbes, la grande éclaire (chelidonium majus), le pavot, ete.

$B$. Quant à sa forme, la tige peut offrir un grand nombre de modifieations; ainsi on l'appelle:

I Cylindrique(I) (cylindricus), quand sa forme générale approche de eelle d'un eylindre, e'est-à-dire que sa seetion transversale offre un eerele dont les différens diamètres sont à peu près égaux. Cette forme se trouve daus le tronc de la plupart des arbres de nos forêts, et dans eertaines plantes herbacées, eommme la stramoine (dalura stramonium), le lin, eto.

$2^{\circ}$ Effilée (virgatus), ou en baguette, eelle qui est grêle, longue, droite, et s'allonge eonsidérablement en diminuant de la base vers le sommet. Telle est eelle de la guimauve (althrea officinalis), de la gaude (reseda luteola), de la salieaire (lythrum salicaria).

(I) Remarquons ici que clans le règne organique les formes géométriques ne sont jamais aussi régulières, aussi rigoureusement déterminées que dans les minéraux. Ainsi, quand on dit d'une tige qu'elle est cylindrique, on exprime seulement par ce mot que c'est du cylindre que sa forrao se rapproche davantage. 
$3^{\circ}$ Comprimée (compressus), lorsqu'clle est légèrcment aplatie sur deux eôtés opposés (le poa compressa).

$4^{\circ}$ Ancipitée (anceps), quand la eompression est portée jusqu'au point de formẹ deux tranehans scmblables à ceux d'un glaive.

$5^{\circ}$ Angule (angulatus), lorsqu'clle est marquée d'angles ou de lignes saillantes longitudinales, dont le nombre est détcrminé.

Selon que ecs angles sont aigus ou obtus, on la dit:

\section{Aculangulée, \\ Obtusangulée.}

Suivant le nombre des angles, et par conséquent des faces distinetes qu'clle présente, on la nomme:

Triangulairc, trigone ou triquètre (triangularis, trigonus, triqueter), quand elle offre trois augles. Tels sont beaueoup de carex, le scirpus sylvalicus, etc.

Quadrangulaire, tétragone (quadrangularis, tetragonus), quand clle a quatre augles ct quatre faees. Si les angles sont égaux ainsi que les faces, clle est carréc; telles sont la plupart des Labiées, eomme la menthe, la sauge, le marrubc, cte.

Pentagone(pentagonus), lorsqu'clle présente einq faces. Hexagone (hexagonus), quand elle en offre six.

$6^{\circ}$ On dit de la tige qu'clle est anguleuse (angulosus), lorsque le nombre des angles est très-considérable, on que l'on ne veut pas le déterminer avee préeision.

$7^{\circ}$ Noucuse (nodosus), offrant des noeuds ou renflemens de distance en distanec : les Graminées, le geranium Robertianum.

$8^{\circ}$ Articulée (ariculatus), forméc d'artieulations superposées et réunies bout à bout : le gui, beaneonp de Caryophyllées, ete.

$9^{\circ}$ Gériculée (geniculatus), quand les artienlations sont fléehies angulairement: exemple, l'alsine media, le geranium sanguinoum. 
io Sarmenteuse (sarmentosus), une tige frutiqueuse trop faible pour pouvoir se soutenir elle-mème, ct s'élcvant sur les eorps voisins, soit au moyen d'appendiees partieuliers, nommés vrilles, soit par sa simple torsion autour de ees eorps : par exemple, la vigne, le ehèvrefeuille.

I I Grimpante (scandens, radicans), celle qui s'élève sur les corps enviromnans et s'y attaehe au moyen de racines, comme le lierre (hedera helix), le bignonia radicans, cte.

I $2^{\circ}$ Volubile (volubitis), la tige qui s'entortille en forme de spirale autour des eorps voisins. Une chiose bien digne de remarque, e'est que les mêmes plantes ne commencent point leur spirale indistinctement à droite ou à gauche. Elles se dirigent constamment du mème côté dans une même espèee. Ainsi, quand la spirale a lieu de droite à gauche, la tige est dite dextrorsìm volubilis, comme dans le haricot, le doliehos, le lizerou. On dit au contraire qu'elle est sinistror'sum volubilis, quand elle commence sa spirale de gauehe à droite : par exemple, le houblon, le chèvrefeuille.

I $3^{\circ}$ Gréle (gracilis), quand elle est très-longue en comparaison de sa grosseur : par exemple, la stellaria holostea, l'orchis conopsea, etc.

$14^{\circ}$ Filiforme (filiformis), quand clle est fort grêle et couchée à terre, eomme dans la eanneberge (vaccinium oxycoccos).

C. D'après sa composition, on distingue la tige en :

I Simple ( simplex), lorsqu' elle est sans ramifieations marquées : cxemple, le bouillon blane (verbascum thap-. sus ), la digitale pourprée (digitalis purpurea).

$2^{\circ}$ Rameuse (ramosus), divisée en branches et en rameaux. La tigc peut être rameuse dòs sa base (basi ramosus), comme l'ajone ou landier (ulex europaus), ou seulement vers son sommet (apice ramosus).

$3^{\circ}$ Dichotome (dichotomus), lorsqu'olle sc divise par 
bifurcations successives; telle est eelle de la màchc ( $v a$ lerianella locusta.), de la stramoine (datur'a stramonium).

$4^{\circ}$ Tricholome (tricholomus), se divisant par trifureations, eornme dans la belle-de-nuit (nyctago hortensis).

Quant à la disposition des rameaux, relativentent à la tige, eomme leurs diverses modifieations sont parfaitement analogues à eelles que nous observerons dans les feuilles, nous croyons inutile d'en parler iei, ee que nous dirons bientồt de la position des feuilles sur la tige pouvant s'appliquer également à eelle des branches et des rameaux.

$D$. Suivant sa direction, on dit que la tige est :

I" Verlicale ou dressée (I) (2'erticalis, erectus), quand elle est dans une direetion verticale rela tivement à l'horizon; par exemple, eelle de la raiponee ('campanula rapunculus), de la linaire (antirrhinum linaria).

$2{ }^{\circ}$ Couchée (prostratus, procumbens (2) Tumifusus) (3); lorsqu'elle ne s'élève point, mais se eonelıe sur la terre sans s'y enraeiner; par exemple, la mauve ( malva rotundifolia), le serpolet (thymus serpyllum), ete.

$3^{\circ}$ Rampante (repens), quand clle est eouchée sur la terre et qu'ellc s'y enracine par tons les points de sou étendue; exemple : la nummulaire (lysimachia nummularia).

$4^{\circ}$ Traçante ou stolonifere (replans s. stoloniferus), poussant du pied prineipal de petites tiges latérales grêles, nommées stolons ou coulans, suseeptibles de s'enraeiner et de reproduire de nouveaux pieds; par exemple, le fraisier ( fragaria vesca).

(I) Il ne fant pas confondre la tige droite (rectus) aree la tige dressée (crectus). La première s'ćlève direetement sans former aucune courbure, aucune déviation latérale, comme daus le bouillon blane, par exemple : la seconde, au contraire, n'exprime que l'opposition à tige couchce (prostratus). Une tige dressce peut donc ne point être droite; de mêne une tige droite n'est pas nécessairement dressée.

(2) Prostratus, couchée d'un scul côté.

(3) Humifusus, étalée en tous sens. 
$5^{\circ}$ Oblique (obliquus), s'élevant obliquement à l'horizon.

$6^{\circ}$ Ascendante (ascendens), formant à sa base une courbe dont la convexité regarde à terre, et redressée dans sa partie supérieure; par cxemple, le trèfle des prés (trifolium pratense), la véronique en épi (veronica spicata).

$7^{\circ}$ Réclinée (reclinatus), dressée, mais réfléehie brusquement à son sommet, comme, par exemple, quelques cspèces de groseillers.

$8^{\circ}$ Tortuetuse (tortuosils), formant plusieurs courbures en différens sens, le bunias cakile, par exemple.

$9^{\circ}$ Spiralée (spiralis), formant des courbures en forme de spirale : par exemple, la plupart des costus.

$E$. D'après sa vestiture et ses appendices, la tige est :

I Feuillée (foliatus), portant les ferilles; telles sont en général la plupart des tiges.

On dit, dans un autre sens, d'une tige, qu'clle cst feuillue (caulis foliosus), quand elle est couverte d'un nombre trèsconsidérable de feuilles.

$2^{\circ}$ Aphylle ou sans feuilles (aplyllius), dépourvue de feuilles (la cuscute).

$3^{\circ}$ Ecailleuse (squamosus), portant des feuilles cn forme d'écailles; telles sont les orobanches.

$4^{\circ}$ Ailée (alatus), garnie longitudinalement d'appendices membraneux ou foliacés, venant le plus souvent des feuilles, comme dans la grande eonsoude (symphytum officincle), le bouillon blanc (verbascum thapsus).

$F$. En considérant la superfieie de la tige, celle-ci est :

$1^{\circ}$ Unie (lacvis), dont la surface n'a aucune sorte d'aspérité ni d'éminences (tamus communis).

$2^{\circ}$ Glabre (glaber), dépourvue de poils : la pervenche (vinca major).

$3^{\circ}$ Lisse (lavigatus), glabre et unie.

${ }_{4}^{\circ}$ Pulvérulente (pulverulenlus), couverte d'une sorte de poussière produite par le végétal (primula farinosa). 
$5^{\circ}$ Glauque (glaucus), quand eette poussière forme une couche cxcessivement mince, qu'on cnlève facilement, ct qui est de eouleur,vert de mer (1); cxcmple : lc cucubalus behen, la chlora perfoliata, etc.

$6^{\circ}$ Ponctuée (punctalus), offrant des points plus ou moins saillans et nombreux, commc dans la ruc (ruta graveolens). Ces points sont ordinairement de petites glandes vésiculeuses, remplies d'huile cssenticlle.

$7^{\circ}$ Maculée (maculatus), marquée de taehes de couleur varićc; par cxcmplc, le gouet (arum maculatum), la grande ciguë. (conium maculalum), l'orchis maculata, etc.

$S^{\circ}$ Rude (scaber, asper), dont la surfaec offre au doigt unc aspérité inscusible à la vue, et qui paraît due à de très-petits poils, rudes ct extrêmemcnt eourts, commc dans l'hcrbc aux perles (lithospernum arvense).

$9^{\circ}$ Terruqueuse (verrucosus), offrant de petites cxcroissances callcuses (appclées galcs ou vcrrues); tellocst la tigc du fusain galcux (Evonymus verrucosus).

$10^{\circ}$ Suber'euse (suberosus), cellc dont l'écorce cst de la nature du liége, eommc lc liége proprement dit (Quercus suber).

I I Crevassée ou fendillée (rimosus), offrant des fentes inégalcs et profondes, comme l'orme, le cliêne, ct un grand nombre d'autres arbres.

I $2{ }^{\circ}$ Striée (striatus), offrant de petitcs lignes longitudinales saillantes, nommées strics, eomme l'oscillé (rumex acetosa).

I $3^{\circ}$ Sillonnée (sulcatus), présentant des sillons longitudinaux, plus ou moins profonds : la ciguë, lc panais.

$G$. La pubescence de la tige lui a fait imposer les dénominations suivantes :

(I) C'est cette poussière que l'on désigne vulgairement sous lo nom de jour dans ocrtains fruits, les prunes, lo raisin, ctc. 
$I^{\circ}$ Pubescente (pulens) (I), garnie de poils mous, trèsfins et rapprochés, mais distincts; par exemple: la digitale pourprée (digitalis purpurea), la saxifrage grenue (saxifraga granulata).

$2^{\circ}$ Poilue (pilosus), eouverte de poils longs, mous et peu nombreux; exemple : l'aigremoine (agrimonia eupatorium), la renoneule âcre (ranunculus acris).

$3^{\circ}$ Velue (villosus), quand les poils sont mous, longs, très-riapproehés.

$4_{4}^{\circ}$ Laineuse (lanatus), eouverte de poils longs, un peu crépus et rudes, semblables à de la laine; par ex. : la Ballota lanata.

$5^{\circ}$ Cotonneuse, quand les poils sont blancs, longs et doux au toueher comme du coton; ex. : le Stachys germanica, l'Hieracium eriophorum.

$6^{\circ}$ Soyeuse (sericeus), quand les poils sont longs, doux au toueher, luisans et non entremêlés, comme sont des fils de soie (Protea argentea).

$7^{\circ}$ Tomenteuse (tomentosus), quand les poils sont eourts, entremêlés, et semblent ètre tissus comme un drap; exemple: le Bouillon blane.

$8^{\circ}$ Ciliée (ciliatus)', quand les poils sont disposés par rangées ou lignes plus ou mòins régulières; exemple : la $V$ eronica chamodrys, qui offre deux rangées opposées; le mouron des oiseaux, qui en présente une seule.

$9^{\circ}$ Hispide (hispidus), garnie de poils longs', roides et à

(I) C'est à tort que l'on se sert du mot pubescens pour signifier une partie couverte de poils. Les Latins, que nous devons imiter servilement quand nous cmployons leur langae, se servaient $\mathrm{du}$ verbe pubescere, en parlant des végétaux, pour exprimer leur accroissement. C'est ainsi que Pline dit: Jam pubescit arbor, déjà l'arbré commence à croitre. Tandis qu'il dit dans un autre lieu: Folia quercís pubentia, pour exprimer la pubesccnce des feuilles du chêne. Il me semble, d'après ccla, que nous n'avons rien de mieux à faire dans ce cas que de copicr les Latins; car, à coup sûr, ils devaient mieux oañ naltro que nous la valeur et la proprióté des mots de leur langne. 
base tuberculée; comme le Galeopsis tetrahit, le Sinapis arvensis.

IT. L'Armure, dont la tige est quelquefois revêtue, l'a fait nommer :

$I^{\circ}$ Eppineuse (spinosus), armée d'épines (I): Genista anglica, Gleditsclia ferox, etc.

$2^{\circ}$ Aiguillonneuse (aculeatus), offrant des aiguillons (les Rosiers).

$3^{\circ}$ Inernne (inermis), se dit par opposition aux deux expressions prćcćdentes, c’est-à-dire sans épines ni ai guillons.

\section{Structure anatomique des tiges.}

En parlant précćdemment de la distinćtion du tronc et du stipe, nous avons dit que ces deux espèees de tiges, dont l'une appartient à la grande classe des Dicotylédons, et l'autre aux Monocotylédons, différaient autant par leur structure intérieure, et la disposition respective des parties élémentaires qui les eomposent, que par leurs earactères extéricurs. C'est, eomme nous l'exposerons bicntôt, à M. Desfontaines que la seienee doit ectte importante découverte. Ce savant botaniste est le premicr qui ait fait connaître avec cxactitude et précision l'organisation intérieure ou strueture anatomique de la tige des végćtaux, ct prineipalement des Monoeotylédons. Aussi les notions que nous allons exposer sur ee sujet sont-elles dues en grande partic à ce eélèbre naturaliste. Mais il convient d'examiner' sćparćment l'organisation des tiges des Dicotylédons, ct ensuite eelle des Monocotylćdons.

(1) Voyez plus loin la description des épines et des aiguillons. 


\section{SECTION PREMIÌRE.}

ORGANISATION DE LA TIGE DES DICOTYLÉDONS.

Le trone des arbres dicotylédonés est formé de coulẹ es coneentriques superposées, de sorte qu'il représente en quelque manière une suite d'étuis emboités les uns dans les autres, et augmentant d'étendue du eentre à la eireonférence. Coupé transversalement, il offre à considérer les objets suivans: $\mathrm{I}^{\circ}$ au centre; le Cancul médullaire, formé de l'Étui médullaire, qui constitue les parois de ee canal, et de la Moelle, qui en occupe la eavité; $2^{\circ}$ tout-à-fait à sa circonférence, on voit l'Écorce, qui se compose de l'Épiderme, ou de cette pellicule extérieure reeouvrant toutes les parties du végétal; de l'Enveloppe herbacée, des Couchos corticales et du Liber; $3^{\circ}$ enfin, entre l'étui médullaire et l'éeorce, se trouvent les Couches ligneuses, formées extérieurement par l'Aubier ou faux bois , intérieurement par le Bois proprement dit. Nous allons étudier suceessivement ces différentes parties en proeédant de l'extérieur vers l'intérieur.

\section{ऽ. De l'Épiderme.}

L'Epiderme (Epidermis, cuticula) est une lame minee, presque diaphane, formée d'un tissu uniforme, qui paraît composé de eellules d'une forme exeessivement variable; et qui présente un grand nombre de petites ouvertures ou pores, que quelques auteurs regardent eomme des espèces de bouches aspirantes. Il enveloppe toutes les parties du végétal; mais il est surtout apparent sur les jeunes tiges ; dont on peut facilement l'isoler avec quelque précaution. Comme il ne jouit que d'mn eertain degré d'extensibilité au-delà duquel il ne pent plus s'étendre, il se déchire et se fendille quand le tronc a acquis un certain volume, 
ainsi qu'on l'observe dans lc ehêne, l'orme; d'autres fois il sc détaelıe par lambeaux ou par plaques, comme dans lc boulcau, le platanc. Lorsqu'on l'enlève sur unc jeunc tige, il se régénèrc avec assez de facilité. C'cst la partic du végétal qui résiste le plus long-temps à la décomposition; la putréfaction n'excrec sur lui aucunc action sensible. La coulcur qu'il présente n'est point inhérente à sa naturé; clle est duc à la coloration particulićre du tissu. sur lequel il est appliqué.

La nature et l'originc de l'épidcrme sont deux points assez obscurs de l'anatomie végétalc. Quelques autcurs discnt, avec Malpighi, que l'épiderme n'est pas une membrane distincte du reste du tissu végétal. Ils le considèrent comme formé par la paroi cxtcrne des cellules sous-jacentes, qui appartiennent à l'cnveloppe herbacée, laquclle paroi a été endureic par l'action prolongée de l'air et de la lumière. D'autres, au contraire, ct c'csı Grew qui peut être considéré comme l'anteur de ectle opinion, le r'cgardent comme une membrane tout-à-fait distinetc, simplement appliquéc sur le tissu eellulairc sons-jacent. On doit à M. le professeur Amici des obscrvations microscopiques de la plus haute importance, qui jettent le plus grand jour sur cette question, et semblent confirmer la scconde de ces deux opinions. Sclon ce savant, l'épiderme est une membranc tout-à-fait distincte du tissú ccllulaire sur lcquel elle est appliquée. Examinée au microscope, clle se compose d'unc couche simple de cellules dont la forme est exeessivement variable, suivant les diverses espèces. C'est eetle structure cellulcusc qui en a imposé aux auteurs qui ont eru l'épiderme formé par la paroi cxterne du tissu cellulaire. Mais, s'il en était ainsi, les cellules qui constituent l'épiderme devraient avoir constamment la mêmc formc que le tissu sous-jacent, ce qui n'a pas lieu. Ainsi, dans l'oeillet, les cellules de l'épiderme ont unc forme quadrilatère, tandis que la eouehe, plaeće immé- 
diatement dessous, eonsiste en une multitude de petits tubes perpendiculaires à l'épiderme. Il en est de même dans un grand nombre d'autres végétaux; d'où l'on peut -conclure que l'épiderme est une mẹmbrane eelluleusce, entièrement distincte du tissu sous-jaeent sur lequel on la trouve simplement appliquée.

L'épiderme, ainsi que nous l'avons dit prćeédemment; offre un grand nombre de petites ouvertures nommées pores corticaux, glandes corticales, glandes epidermödales, et enfin stomates. Plusieurs auteurs en avaient nić l'existence; mais les observations mieroseopiques du professeur Amiei ne laissent plus aucun doute à eet égard. Il les a vues dans un grand nombre de vćgćtaux, et en a donné une description et dẹs figures extrêmement exactes. Ce sont des espèces de petites poches placées danś l'épaisseur de l'épiderme, s'ouvrant à l'extérieur par une fente ou ouverture ovalaire allongée, bordée d'une sorte de bourrclct formé pär des eellules partieulières de l'épiderme. Ce bourrelet, qui manque très-rarement, joue l'offiee d'une sorte de sphincter qui resserre ou dilate l'ouverture suivant diverscs cireonstanees. Ainsi l'humiditć ou l'eau ferme les pores, tandis que la sécheresse et l'action des rayons solaires les tiennent ouvertes et leurs bords éeartés. Les mouvemens de dilatation et de res'serrement s'exécutent non-seulement sur la plante vivante, mais également sur des fragmens d'épiderme détachés d'un végétal. Par leur fond, ces pores ou petites poehettes correspondent toujours à des espaees vides, remplis d'air, et qui résultent de l'arrangement des ccllules ou des tubes entre eux. Ces espaces intercellulaires communiquent presque toujours les uns avec les autres', et servent ainsi de moyen de eommunication aux fluides aćriformes qui se trouvent dans l'intéricur des végétaux. Quelques parties eependant paraissent dépourvues de $s t o-$ mates; telles sont les racines, les pélioles non foliacés, 
les pétales en général, l'épiderme des vicilles tiges, eclui des fruits charnus, des graines, etc. Certaines feuilles n'en présentent qu'à l'une de leurs faces; d'autres au contraires à toutes les deux.

Quel est l'usage de ces pores corticaux? Sont-ils, dit M. Amici, destinés à l'absorption de l'humidité? Non : nous avons déjà vu qu'ils correspondent à des vides intérieurs privés de suc, que l'eau les fait fermer, que la lumière et la sécheresse les font ouvrir; en outre, ilsmanquent daus toutes les racines, ainsi que dans les plantes qui vivent constamment sous l'cau; ils ne servent donc pas à l'absorption de l'eau. Servent-ils à l'évapor'ation? Pas davantage : sinous laissons sécher une plante détachée de sa racine, quoique les pores se ferment au bout de quelque temps, l'évaporation n'en continue pas moins, tant qu'il reste des fluides dans son intérieur; on a observé en outre que les corolles et les fruits, qui n'ont pas de pores corticaux, produisent eependant une évaporation abondante. Ils ne peuvente ètre mis, ainsi que M. Linek l'avait pensé, au nombre des organes excrétoires, puisqu'ils correspondent toujours à des espaces vides. La véritable fonetion des pores corticaux consiste à donner passage à l'air. Mais il ri'est pas facile de déterminer avec certitude s'ils servent à l'inspiration plutôt qu'à l'expiration, ou à ces deux fonctions également. Si nous considérons que pendant la nuit, lorsque les grands pores de l'épiderme sont fermés, les feuilles absorbent le gaz acide carbonique dissous dans la rosée, lequel pénèire indubitablement daus les cellules en traversant leur membrane, et si nous réfléchissons en outre que ees feuilles décomposent le gaz acide carbonique, lorscue ces pores sout ouverts, c'est-à-dire pendant le jour, nous pouvons conjecturer qu'ils sont uniquement destinés à l'exhalation de l'oxigène. Cet usage devient encore plus probable, si nous ajoutons que les corolles qui, d'après les obscrvatious de M. De Candolle, 
manquent de pores, sơnt également privées de la propriété de dégager de l'oxigène.

La surface de l'épiderme présente quelquefois certains organes qui s'offrent sous la forme de pctites taehes allongées dans le sens longitudinal sur les jeunes branches, et dans le scns transversal sur les branches plus anciennes, que Guettard a le prcmier désignées sous lc nom de glandes lenticulaires ct que M. De Candollc a plus récemment nommées lenticelles. On n'en a cneore trouvé aucune trace ni dans les plantes monocotylédonées, ni dans les acotylédonécs. Elles manquent également dans les herbes dieotylćdones. Elles sont très-apparentes sur l'ćpiderme du boulcau et surtout du fusain galeux ( $E v-$ nymus verrucosus $L$.) où clles sont très-proéminentes et très-rapproehées. C'est des lentieelles que sortent les racines que certains arbres dévcloppent sur leur tige, comme quelques figuiers, par cxemplc, ou eelles qui sc forment lorsqu'on enfonee une branche cn terre, comme dans l'opération du marcottage. On peut donc en quelcque sorte les considérer comme les bourgeons des racines.

C'est encore sur la surfacc de la cuticule que naissent les poils de différente nature, que l'on remarque sur un grand nombre de végétaux.

\section{§2. De l'enveloppe herbacée.}

Au-dessous de l'épiderme, on voit une lame de tissu cellulairc, qui l'unit aux couehes corticales, et à laquelle M. Mirbel domne le nom d'enveloppe herbacée. Sa coulcur est le plus souvcut vertc dans les jeunes tigcs. Ellc recouvre le trone, les branehes et leurs divisions, et remplit les espaees qui existent entre lcs ramifieations des nervures des feuilles. M. Dutrochet la nomme médulle externe, par opposition au nom de médulle interne qu'il domuc à la moclle. Sa conleur n'est pas propre au 
tissu cellulaire qui la eompose; elle est due aux petits grains de globuline, placés dans les parois des cellules, et que M. Dutrochet considère comme des corpuscules nerveux.

L'enveloppe herbacée, ou médulle externe, renferme souvent les sucs propres des végétaux, qui sont contenus dans des eanaux ou réservoirs partieuliers. Elle se répare faeilement sur la tige des végétaux ligneux; mais ce phénomène n'a pas lieu dans les plantes annuelles. Elle paraît avoir une organisation et des usages analogues à eeux de la moelle renfermée dans l'étui médullaire. C'est 'cette enveloppe herbaeće qui, ayant aequis une épaisseur eonsidérable et des qualités physiques particulières, constitue la partie connue sous le nom de liége dans le Quercus Suber et daus quelques autres végétaux. L'enveloppe herbacée est le siége d'un des phénomènes chimiques les plus remarquables que présente la vie du végétal. Fn effet, c'est dans son intérieur que, par une eause difficile à appréeier, s'opère la décomposition de l'acide carbonique absorbé dans l'air par la plante. Le carbonne reste dans l'intérieur du végétal, l'oxigène mis à nu est rejeté à l'extérieur. Remarquons cependant que cette décomposition n'a lieu que lorsque la plante est exposée aux rayons du soleil, tandis que l'acide carbonique est rejeté indéeomposé, quand le végétal ne se trouve plus sous l'influenee de eet astre. Cet organe se renouvelle en partie ehaque année. Il joue encore un rôle très-important dans les phénomènes de la végétation; c'est lui, èn efíet, qui, au retour de la belle saison, sollicite la sève à monter jusque vers les bourgeons, et devient ainsi un des mobiles les plus puissans de leur élongation aérienne.

Il est trés-facile de déeouvrir l'enveloppe herbaeée sur les jeunes bramehes d'un arbre; ear e'est elle que l'on, aperȩoit lorsque l'on a enlevé l'épiderme. 


\section{§3. Des conches corticales.}

Les couches corticales n'existent pas toujours, on du moins elles sont parfois si peu développées, si peu distinctes du liber, qu'il devient fort difficile de les reconnaître. Situées au-dessous de l'enveloppe herbacée, elles sont appliquées sur les couches les plus extérieures du liber, dont on les distingue avec peine. Nul végétal nè les offre plus apparentes et plus remarquables, par la disposition singulière du tissu qui les compose, que le bois dentelle (Lagetto). Ici, en effet, elles forment plusieurs couches superposées qui, lorsqu'elles viemnent à être étendues, ressemblent parfäitement à une toile tissue, ou plutôt à une sorte de dentelle assez régulièré. Mais dans le plus grand nombre des plantes, il est difficile de distinguer cette partie d'avec le liber.

\section{\$. Du Liber.}

Entre les couches corticales, qui sont à l'extérieur, et le corps ligneux, qui est plus intérieurement, se troúve le liber. Cet organe est composé d'un réseau vasculaire, dont les aréoles allongées sont remplies par du tissiu cellulaire. Il est rare que, comme l'indique son nom, on puisse le séparer facilement en feuillets distincts, que l'on a comparés à ceux d'un livre (I). Mais, par la macération, on parvient presque toujours à obtenir cet effet.

Les différentes lames qui forment le liber, et qui ont été créées successivement, sont séparées les unnes des autres par une couche mince de tissu cellulaire. Lorsqu'on fait macérer le liber, c'est ce tissu cellulaire qui se détruit, et qui permet la séparation des feuilles du liber.

De même que toutes les autres parties de l'écorce, le

(1) On l'appelle indifféremmenț liber ou livret. 
liber peut se réparer lorsqu'il a été enlevé. Cependant il faut, pour que sa régénération ait lieu, que la place dont on l'a détaché soit garantic du eontact de l'air. C'est à Duhamel que l'on doit eette importante déeouverte. Cet habile naturaliste, à qui la physiologie végétale doit un si grand nombre de résultats heureux, enleva une portion d'éeoree sur un arbre vigoureux et en pleine végétation; il garantit la plaie du contact de l'air, et vit bientôt suinter, de la superficie du corps ligneux et des bords de l'écoree, une substanee visqueuse qui, s'étendant sur la plaie, prit de la eonsistanee, devint verte, eelluleuse, et reproduisit la partic du liber qui avait été enlevée.

C'est à cette substance visqueuse qui s'épanehe des parties dénudées pour reformer le liber que Grew, et après lui Duhamel, ont donné le nom de cambium. Plusieurs auteurs pensent avee quelque raison que le Cambium n'est autre ehose que la sève deseendante et élaborée. Je suis d'autant plus porté à admettre eette opinion, que ce fluide visqueux remplit absolument dans l'économie végétale les mêmes fonetions que eelles que l'on attribue généralement à la sève deseendante, et qu'il est eharrié par les mêmes parties.

Quelle que soit l'origine du eambium, il n'en joue pas moins un rôle extrêmement important dans l'accroissement des tiges. En effet, dans toutes les hypothèses émises pour expliquer ee phénomène, sa présenee n'est pasmoins indispensable, eomme nous le démontrerons prochainement en traitant de l'aeeroissement des tiges dicotylédones.

Un grand nombre de phénomènes prouvent la néeessité indispensable du liber pour la végétation. Une greffe ne reprendra qu'autant que son İiber se trouvera en contaet avee eelui de l'arbre sur lequel on l'implante. Une marcotte dont la partie inférieure est privéc de liber ne s'enracinera pas. Si l'on enlève sur le trone d'un arbre une bande eireulaire de liber, de manic̀re à laisser le eorps 
ligneux à nu, non-seulement toute la partie supérieure de l'arbre ne se développera pas l'année suivante, mais l'arbre entier finira même par périr.

Chaque année le liber s'endureit; il se forme à son intérieur de nouvelles contehes par le moyen du eambium.

\section{§5. De l'Aubier, ou faux bois.}

Les eouches ligneuses les plus extérieures, celles qui touelient le liber, eonstituent l'aubier. Cette partie n'est point un organe distinet du bois proprement dit, dont les couehes sont situées au-dessous; e'est du bc is, mais eneore jeune, et qui n'a point eneore aequis toute la dureté ni toute la ténaeité qu'il doit présenter plus tard. Aussi l'aubier.offre-t-il absolument la même strueture que le bois, en observant toutefois que son tissu est formé de fibres plus faibles, plus éeartées les unes des autres, et en général d'une teinte plus elaire.

La différenee de eoloration entre le bois et l'aubier est très-remarquable dans les arbres dont le bois est très-dur, très-eompaete, et partieulièrement dans ceux où il offre une teinte plus ou moins foneéc : ainsi, dans les bois d'ébène et de Campèelıe, le bois proprement dit est noir ou rouge foneé, tándis que les eouehes d'aubier présentent une teinte grisâtre, très-elaire; mais dans les arbres à bois blane et à gros grains, la différenee entre les eouehes ligneuses et l'aubier est peu sensible.

Nous présenterons, en parlant de l'aeeroissement des tiges en diamètre; les opinions très-diverses des auteurs sur l'origine de l'aubier.

\section{§6. Du Bois proprement dit.}

Le bois tire son origine des eonehes les plus intérieures de l'aubier, qui aequièrent sueeessivement une dureté plus eonsidérable, et finissent par se eonvertir en véritable bois. 
Celui-ciest done eomposé de toutes les eouehes eirculaires situées entre l'aubier et l'étui médullaire. A une eertaine époque de la vie du végétal il se forme, elaqque année, une couehe de bois et une eouehe d'aubier, c'est-à-dire que la eouehela plus intérieure de l'aubier se eonvertit en bois à mesure qu'il se régénère à l'extérieur une nouvelle eouche d'aubier, en sorte qu'il s'ajoute tous les ans une nouvelle zone eoneentrique à eelles qui existaient déjà.

Le bois est, en général, la partie la plus dure du trone; mais sa dureté n'est point la même dans toutes les zones qui le constituent. Dans les arbres dieotýlédonés, les eouches les plus intérieures, qui sont en même temps les plus aneiennes, -ont une solidité et une eompaeité plus grandes que les extérieures, qui se rapproehent en général, à eet égard, de l'aubier. Ordinairement le passage du bois à l'aubier est presque insensible, parce que le plus souvent leur eouleur est la nuême. Mais quelquefois la différenee est des plus tranehées, eomme nous l'avons fait remarquer pour l'ébène et le bois de Campêehe.

Une différenee non moins remarquable entrelc bois et l'aubier, e'est que ce dernier est totalement privé de vaisseaux, tandis qu'on en aperȩoit manifestement dans le bois. Les vaisseaux du bois sont des fausses traehcées, des vaisseaux poreux, mais jamais de véritables traehées. C'est au moyen de ees tubes, tantôt dispersés sans ordre dans la substance du bois, tantôt réunis en faiseeaux, que la sève est portée dans l'épaisseur du tronc. Mais il arrive une époque où, par les progrès de l'âge, les parois de ees vaisseaux s'épaississent, leur eavité diminue, finit même par disparaître, et le eours des liquides est pour toujours interrompu dans la substanee ligneuse.

Duhamel a démontré d'une manière péremptoire la transformation de l'aubier en bois. Il fit passer un fil d'argent dans les eouehes de l'aubier, il en ramena les deux bouts au dehors et les noua. Ayant coupé la branche quel- 
ques années après, et exarniné les fils qu'il avait passés dans l'aubier, il les trouva engagés dans le bois; par eonséquent l'aubier était devenu bois.

\section{§. De l'Etui médullaire.}

L'étui médullaire, eomme nous l'avons déjà dit, oecupe le eentre de la tige; il tapisse la eouehe la plus intérieure du bois, et a pour usage de eontenir la moelle. Ses parois sont formées de vaisseaux très-longs, parallèles et disposés longitudinalement. Ces vaisseaux sont des traehées, de fausses traehées et des vaisseaux poreux. C'est dans l'étui médullaire seulement qu'on a jusqu'à ee jour observé les traehées. La forme de l'étui médullaire n'est pas la même dans tous les végétaux. Assez souvent elle est arrondie; quel'quefois eependant l'aire de l'étui médullaire est elliptique, émprimée, à trois, à quatre, à einq ou à un grand nombre d'angles. Cette forme, ainsi que l'a prouvé Palisot de Beauvois, paraît déterminée par la position des feuilles sur les branehes. Ainsi, quand les feuilles sont opposées, la eoupe du eaual médullaire est elliptique, eomme dans le frêne, par exemple; si les feuilles sont vertieillées par trois, le eanal médullaire sera triangulaire, ainsi qu'on l'observe dansle laurier-rose, et áiusi de suite. Néanmoins cette loi est loin d'être générale, et l'on y trouve d'assez nombreuses exeeptions. Ainsi, par exemple, l'Hortensia, qui a les feuilles opposées, offre un eanal médullaire régulièrement hexagone.

L'étui médullaire une fois formé, sa forme et ses dimensions ne ehangent plus, et restent eonstamment les mêmes pendant toute la vie du végétal. C'est done à tort que l'on dit généralement que le eanal médullaire se resserre petit à petit sur lui-même, et qu'il finit par disparaître par les progrès de l'àge. C'est M. Du Pelit-Thouars qui a le premier prouvél'invariabilité du canal médullaire, 
§. De la Moelle.

La moelle ou médulle interne est ectte substance spongieuse, lâche, diaphane et légère, formée, presque en totalité, de tissu eellulaire à son état de simplieité, qui remplit l'étui médullaire. Quelques vaisseaux semblent la pareourir longitudinalement. Les eellules du tissu eellulaire qui eonstituent la moclle ont en général une grande régularité; comme eelles du tissu cellulaire des autres parties, elles communiquent toutes les unes avee les autres. Quelquefois, et surtont dans les jeunes branehes et les plantes herbaećes, le tissu cellulaire de la moelle est abreuvé de fluides et rempli de granulations vertes. C'est ee que l'on voit, par exemple, en cassant une jeune branehe de sureau d'une année. La moelle paraît être une substanee charnue, verte et très-humide. Mais, par les progrès de la végétation, toutes ees substanees, en quelque sorte étrangères à la nature propre de la moelle, disparaissent, et il ne reste plus dans l'étui médullaire qu'un tissu diaphane.

Dans quelques végétaux, à mesure que la tige s'accroît, le eanal médullaire se vide en partie et quelquefois en totalité, toute la moelle finit par disparaître, et la tige devient ereuse ou fistuleuse; c'est ee que l'on observe, par exemple, dans un grand nombre de plantes de la famille des Ombellifères.'

La moclle eommunique avee la eouehe celluleuse et herbacée de l'éeorce au moyen de prolongemens partieuliers, qu'elle envoic à travers le corps ligneux. C'est à ees prolongemens, disposés sur une eoupe transversale du trone, comme des rayons partant en divergeant du eentre à la eirconférenee, que l'on a donné le nom d'insertions ou de prolongemens médullaires. Ils servent à établir une eommunieation directe entre la moelle et le tissu eellulaire extéricur de la tige. 
Les rayous médullaires cxistent également dans la plus grande partic de l'épaisseur de l'écor'ce, puisqu'ils servent à établir la communication entre la médulle interne et la médulle extcrne; mais ceux de l'écoree n'ont point une communication directe avec ceux des couches ligneuses.

II. le professeur Amici a reconnu qu'ils sont formés de petits tubes poreux, placés en travers, qui ne contiennent jamais que de l'air, et qui établissent la communication entre les parties internes et externes de la plante.

Si maintenant nous cherchons à savoir quels sont les usages de la moelle, nous verrons que les. opinions ont beaucoup varié à cet égard. Ainsi, selon le célèbre Hales, elle est l'agent essentiel de la végétation. Étant élastique et dilatable, elle agit, à la manière d'un ressort, sur les autres parties, qu'elle sollioite ainsi à se développer. D'autres, au contraire, la considèrent comme un corps tout-àfait incrte. M. Dutrochet a, dans ces derniers temps, reproduit l'opinion de Hales, en faisant.jouer à la moelle un rôle extrêmement important dans les phénomènes de l'accroissement des végétaux. Nous reviendrons prochainement sur cette opinion.

Tels sont les différens organes que l'on trouve en analysant la tige des végétaux dicotylédons. Cependant toutes ces parties sont loin d'être toujour's réunies et visibles sur la même plante. Quelquefois elles se confondent tellement les unes avec les autres, qu'il est presque impossible de les distinguer et de les isoler. Mais, lorsqu'on comnaît bien la structure la plus compliquée d'une partie, il devient facile de se représenter, dans certains cas, ceux de sés organes qui peuvent y manquer accidentellement.

Il nous reste maintenant à étudier comparativement la structure de la tige des monocotylédóns, afin d'exposer ensuite lc modé particulier de développement et d'aecroissement propre à ehacuue de ces deux grandes divisions du règne végétal. 


\section{SECTION II.}

ORGANISATION DE LA TIGE DES MONOCOTYLÉDONS.

M. Desfontaines a le premicr eonfirmé la grande division des végćtaux phanćrogames en monoeotylédons ct en dicotylćdons, par la strueture anatomique de leur tige, si différente dans l'unc et l'autre de ccs deux classes. Il est en effet le premier qui, dans un exeellent mémoire inséré dans le premier volume des Mémoires de l'Institut, ait fait connaître la vćritable organisation des tiges monocotylédones, et lcs différences qui la distinguent de eelle des dicotylćdones.

En génćral, la tige des monocotylćdons 'est plus ćlaneée, plus simple que celle des arbres à deux eotylćdons. Trèsrarement elle se divise en ramcaux, eomme eelle que nous venons d'ćtudier préećdemment.

Le stipe d'un arbre monocotylćdoné, d'un palmier, par exemple, eoupé en travers, ne présente pas, eomme le tronc d'un chênc, d'un orme ou de tout autre arbre de nos forĉts, un aspect régulier et symćtrique; des zones cireulaires de bois, d'aubier, de liber et d'écorce, toujours disposćes dans le même ordre; un canal médullaire oecupant constamment la partie eentrale de la tigc. Ici, toutes ees parties semblent réunies, ou plutôt confonducs les unes avee les autres. La moelle remplit toute l'ćpaisseur de la tige; le bois, disposć par faisecaux longitudinaux, se trouve en quelque sorte perdu, et eomme dispersé sans ordre au milicu de la substance médullaire. L'ćcorec n'existe pas toujours, et quand elle ne manque pas, elle est si peu distincte des autres parties de la tigc, qu'on pourrait eroire également qu'elles n'en sont pas rceouvertes. Dans les arbres dieotylédonés, la partic la plus dure est cclle qui se rapproehe le plus du eentre de la tige, paree qu'elle est forméc des couches ligneuses les plus anciennes. 
Le eontraire a lieu dans lcs arbres monoeotylédonés, où la partic la plus voisine de la circonfćrenee sc trouve avoir la solidité la plus grandc. Dans les prcmiers, cn cfict, les couches les plus anciennes sont au centre; elles occupent au contrairc la circonférenee dans les seconds. C'est ce que l'on concevra facilement tout à l'heurc, quand nous aurons cxposé le mode particulier suivant lequel se formc et s'accroît la tige des arbres monocotylédons. Les faisccaux ligneux de la tige, qui se réunissent fréquemment enscmblc par lcurs parties latérales, de manière à former un réscau plus ou moins régulier, sont, comme dans les dicotylédons, accompagnés de vaisseaúx poreux, de trachécs et de fausșcs trachées, destinés à charrier la sève et lcs autres fluides nutritifs dans tous les points de la tige.

Ainsi donc les arbres monocotylédons se dịstinguent des arbrcs dicotylédons, non-seulemcnt par la structure de leur embryon, mais encore par cclle de leur tigc. En cffet, leur stipe, qui cst en général simple ct cylindriquc, n'offre point commc le tronc des chênes et des ormes, des eouches de bois emboîtées les unes dans lés autres, et disposées rćgulièrement autour d'un eanal eentral rcnfcrmant la moelle; mais la moelle forme, en quelque sorte, toute l'épaișseur de leur trone, et lcs fibres ligneuses, au lieu d'être réunies et rapprochées lcs unes contre lcs autres, sont écartées, isolées, ct leurs faisceaux épars au milieu de la substance spongieusc de la moelle. En traitant, dans la quatrième section, de l'aecroissement et du développepement des tiges, nous espérons prouver que l'organe ainsi nommć dans les monocotylćdons ligneux, et spécialement dans les palmiers, les Dracuena, Yucca, ete., n'est. pas une véritable tige, mais un organe tout-à-fait différent. 


\section{SECTION III.}

\section{DE L'ORGANISATION DE LA RACINE.}

Maintenant que la strueture intéricure des diverses espèecs de tiges nous est eonnuc, il nous scra plus facile d'étudier comparativement eclle que présentent les racines.

Toutes les racines sont généralement organisées comme les tiges. Ainsi, dans les arbres dicotylédons, la coupe transversalc de la racine offre des zones coneentriques de bois disposées eirculaircment et emboîtées les unes dans les autres. On a dit que le caraetèrc vraiment distinctif entre la tige ct la raeine, c'cst que cette dernière cst dépourvue de canal médullaire, ct par conséquent de moclle, tandis qu'au eontrairc nous savons que ect organc cxistc constamment dans les arbres dieotylédons. Il suit de là néccssairement que les insertions médullaires manquent aussi dans lcs racines.

Cependant cettc différcnec nous paraît de peu d'importance, ct même tout-à-fait contraire aux faits. En effet, nous avons trouvé dans un grand nombre de végétaux que le canal médullaire de la tige se prolonge sans aucune intcrruption dans le corps de la racine. Si, par exemple, on fend longitudinalement la tige ct la raeine d'un jeune maromnier d'Inde d'un à deux ans, on verra lc canál médullaire de la tige s'étcndre jusqu'à la partic la plus inféricure de la racine. Il en sera de même si l'on cxamine une jeune plantulc de syeomore ou d'érable planc. Mais, très-fréquemment, ce canal qui était très-manifeste dans la plante peu de temps après sa germination, finit par diminuer, et même disparaître insensiblement par les progrès de la végétation, en sortc qu'on ne le retrouve plus dans les plantes adultes chez lcsquclles il a d'abord cxisté. Il résulte de là qu'on ne peut donner commc un caractère anatomique distinctif entre la tige et la racine le manque de 
canal médullaire dans eette dernière; puisqu'il existe presque eonstamment dans la radieule de la graine germante, et souvent dans la raeine d'un grand nombre de végétaux, long-temps après cette première époque de leur vie. Cependant les raeines pivotantes ne l'offrent jamais dans leurs ramifieations, même dans celles qui sont les plus grosses.

Jusqu'en ces derniers temps, on avait donné comme caractère distinetif entre la strueture anatomique de la raeine et eelle de la tige le manque de vaisseaux trachées dans ce premier organe; eependant deux des savans qui en Allemagne' se' sont oceupés de l'anatomie végétale avee le plus de sueeès, MM. Link et Tréviranus, sont parvenus à trouver ces vaisseaux dans la raeine de quelques plantes. Plus récemment eneore M. Amici a déroulé des trachées dans les racines de plusieurs plantes, et entre autres de l'Agapanthus umbellatus et du Crinum erubescens.

La différenee que nous avons vu exister dans l'organisation du trone des dieotylédons et du stipe des monoeotylédons, se remarque également dans leurs racines. En effet, jamais dans les plantes monocotylédones on ne trouve de pivot faisant suite à la tige. Cette disposition est une conséquenee du mode de développement de la graine à l'époque de la germination, puisque, comme nous le verrons plus en détail en traitanț de eette fonetion, la radieule centrale et principale se détruit toujours peu de temps après la germination.

Il existe eneore une autre différenee très-remarquable entre les raeines et les tiges. Ces dernières, en général, s'aeeroissent en hauteur par tous les points de leur étendue, tandis que les racines ne s'allongent que par leur extrémité seulement. C'est ce qui a été prouvépar les expéricnces de Duhamel. Que l'on fasse à une jeune tige, au moment de son déreloppement, de petites marques élojguées 
les unes des autres, d'un pouce, par excmple, et l'on verra, lorsque l'accroissement sera terminć, que les cspácés situés entre ces marquies sć sonit considérablement augmentés. Que l'on répète la même expéricrice sur des raciines, et l'on se convaincra que, ces cspaccs rcstant les mêmés, tandis quie la racine s'cst allongéc, l'augmentation en longucur a eu lieu par son extrémité sculement.

\section{SECTION IV.}

CONSLDÉRATIONS GÉNÉRALES SUR L'ACCROISSEMENT DES VÉGÉTAUX, ET EN PARTICULIER SUR LE DÉVELOPPEMENT DE LA TIGE.

Tous les corps de la naturc tendent à s'accroître. Cette loi est commune aux corps inorganiqucs aussi bicn qu'aux ćtres organisés. Mais l'accroisscincnt présente des différenccs très-marquées, suivant qu'on l'étudie dans ces dcux groupes primitifs des corps de la nature. Dans les minćraux, en effct, il n'offrc point de limites détermiriécs; ces corps s'accroisscnt continucllemcnt, jusqu'à ce 'qu'une cause fortuitc vicnne mettre un tcrmc à leur dévcloppement. Lcs animaux et les végétaux ayant en général une existence dont la durće est détcrminée, chcz eux l'accroissement est toujoui's en rapport avec la durćc dc leur existence. Daus lcs minćraux cc sont de nouvclles moléculcs qui s'ajoutent extéricurement à cellcs qui existaicnt dćjà et qui en constituaient lc noyau primitif; cn sortc quc la superficic de ccs corps se renouvellc à chaque ínstant ct à mcsurc que leur volumc augmentc. De là la déńomination de juxta-position domée au mode particulier de l'accroisscmcnt dans les corps bruts. Si au contraire vous étudicz l'accroisscment dans les êtres doués d'organisation, vous verrcz qu'il a lieu de l'intéricur vers l'cxtérieur, que ce sont ou des parties primilivement existantes 
qui s'allongent, ou des organes nouveaux qui se forment dans l'intérieur des premières et se développent en tous sens", pour augmenter la masse et le volume du eorps. Aussi a-t-on nommé intus-susception eétte manière de s'aecroître, partieulière aux animaux et aux végétaux.

L'aeeroissement ne présente pas des différcnees moins frappantes lorsque l'on eompare entre eúx sous cé rapport les végétaux et les an̈imaux. Dans les premiers, en éffet, l'aeeroissement n'est pas renfermé dans des limites aussi rigoureusement déterninées que dans les seeonds. Le volüme du eorps, aussi-bien que le nombre de ses partics constituants, ne sont point fixes. L'art et la eulture peuvent exereer sur le développement des végétaux linflúenee la plusmarquée. Il suffit, pour s'en eonvainere, de èomparè entre eux deux arbres d'une même espèee, dont l'un vit abandonné dans un terrain see et roeailleux, tandis que l'autre est eultivé dans un terrain substantiel et profond. Le premier est petit, ses rameaux eourts, et ses feuilles étroites; le seeond, au eontraire, élève majèstueusement son trone eouromné de brainehes longues et vigotireuses; et ornées d'un feuillage épais. Dans les animaux, le volume et la forme générale du eorps, le nombre des päties qui doivent le eonstituer, sont plus fixes, et sujets à moins de variations; tandis que dans les végétaux, il est en quelque sorte impossible de trouver deux individus de la même espèee qui offrent un nombré égál de párties.

Si maintenant nous eherehons à étudier les phénomènes de l'aeeroissement dans les végétaux en partieulier, nous verrons que cés êtres se développent en deux sens, e'està-dire qu'à mesure que leur hauteur augmente, leur diamètre devient plus eonsidérable. Nous avons vu, en traitant del'organisation de la tige, que les arbres dieotylédons et les arbres monoeotylédons étaient loin d'avoir la même structure intérieure, et qu'il existait entre cux des différenees extrêmement tranchées. Ces différences dépendent 
évidemment du mode particulier suivant lequel les végétaux de ces deux grandes séries se développent. Aussi traiterons-nous séparément de l'accroissement des arbres monocotylédons et des dicotylédons.

Cette partie de la physiologic végétale est sans contredit une des plus intéressantes, et cepcndant c'est une de celles qui offrent encore le plus d'obscurité et d'incertitude. En cffet, tous les auteurs, surtout depuis un certain nombrc d'années, sont loin d'être d'accord, d'avoir une seule et même opinion sur la manière d'expliqucr les phénomènes de l'aceroissement de la tige, particulièrement dans leš arbres dicotylédons. Il existe même sur ce point des opinions tellement différentes, que nous croyons nécessaire de les faire connaître séparément.

§. Accroissement de la tige des arbres dicotylédons.

\section{A. Accroissement en diamètre.}

Tous les végétaux s'accroissent en diamètre. Il suffit de jeter les yeux sur les arbres qui végètent autour de nous, pour nous convaincre de cette vérité; aussi personne ne l'a-t-il contesté. Mais par quel mécanisme cet accroissement a-t-il lieu? C'est ici que l'on est loin de s'accordcr: Parmi les opinions diverses qui ont été émises par les physiologistes, nous distinguerons particulièrement les trois suivantes : $I^{\circ} l^{\prime}$ accroissement a lieu par la transformation annuelle du libcr en aubier ; $2^{\circ}$ par le développement des bourgeons ; $3^{\circ}$ par le eambium, qui forme chaque année une couche distincte de liber et d'aubier. Nous allons les exposcr ici avec quelques détails.

I L'accroissement en dianètre a lieu dans les arbres dicotylédons par la transformalion annuelle du liber en aubier, de l'aubier en bois, et par le renouvellement successif diu liver.

Tel est le foudement de la théoric de Duhamel, de celle 
que cet auteur célèbre a développée dans sa $\mathrm{P} / \mathrm{h}$ sique des arbres. Nous allons la faire connaître dans tous ses développemens, paree que c'est elle qui est la plus généralement adoptée, et presque la seule qui pendant long-temps ait été publiquement professée, du moins en France.

Nous prendrons la tige à l'époque de son premier développement, e'est-à-dire lorsque, par l'effet de la germination, elle sort de la graine qui la eontenait, et eommence à se montrer à l'extérieur.

Toutes les parties du végétal renfermées dans la graine, avant la germination, ne sont formées cue par un tissu cellulaire dense et régulier. La tige se trouve, comme les autres organes, entièrement privée de vaisseaux. On n'apcrçoit, à proprement parler, aueune traec d'écoree, de moelle, de liber, etc. Miais à peine la germination est-elle commeneée, à peine la tige a-t-elle aequis quelque développement, qu'on voit des trachées, de fausses traehées et des vaisseaux poreux se former, pour eonstituer, en se réunissant, les parois de l'étui médullaire. C'est eette partie intérieure de la tige qui la première est apparente et s'organise. La moelle se trouve eontenue dans son intérieur; mais elle est eneore verte et abreuvée d'une grande quantité de fluides aqueux. Bientôt on voit la surfaee externe de l'étui médullaire se recouvrir d'un tissu cellulaire fluide; c'est la première eouehe de eambium, qui d'un côté va former le premier liber, et de l'autre constituer les couches corticalcs. Cc liber se convertira bientôt en aubier, à mesure qu'une nouvelle eouehe s'organisera pour remplacer la premic̀re. L'année suivante, le nouveau liber formera une seeonde zone d'aubier, et suecessivement ainsi, tous les ans, une eouche d'aubier se conver'ira en véritable bois, tandis que le liber aura lui-mêrne aequis les propriétés et Ja nature de l'aubier. Ce développement régulier de la tige explique la formation des eouehes ou zoues concentriques que l'on observe sur la eoupe trans- 
versale de la tige d'un arbre dịeotylédon. Nais ees eouehes n'ont pas toutes la même épaịsseụr, et cette épaisseur n'est souvent pas égale dans toute leur eireonférenee. Une obscrvation attentive.explique facilement ecte disposition singulière. On a remarqué, en effet, que la plus grande épaisseur des eouches ligneuses eorrespondait eonsțamment au côté où se trouvaient les raeines les plus eonsidérables, qui, par eonséquent, avaient puisé dans la terre une nourriture plus abondante. C'est ainsi, par exemple, que les arbres sịtués sur la lisièrc d'une forêt présentent toujours des conehes ligneuses plus épaisses du eôté extéricur, paree qu'en effet leurs racincs, n'y éprouvant pas d'obstaeles, s'y étendent et y aequièrent un développement plus eonsidérable.

Dans eette théoric de Duhamel, on voit que e'est le liber qui joue le rôle le plus important dans la formation des eouehes ligneuses, puisque e'est lui qui chaque année se eonvertit en une nouvclle zone d'aubier qui s'ajoute à eelles qui existaient déjì.

Le liber étant l'organe essentiel de la végétation, et ehangeant chaque annce de forme cı de consistanee, la natụre a dû pourvoir aux moyens de le reproduire aussi chaque année. C'est ee qui à lieu en effet. Si nous étudions avee attention le développement suecessif des divers organes qui eomposent la tige des dieotylédons, nous verrons que, la premic̀re annće, entre les eouelies eortieales et l'étui médullaire, se troure un liquide gélatineux, auquel Grew et Duhamel ont domné le nom de cambium. C'est ce fluide particulier qui eontient les premiers rudimens de l'organisation. A mesure que la jeune tige se développe, l a eouche la plus intérieure de ee liquide prend de la consistance, s'organise, se dürcit, se ehange en liber, qui, à la fin de la prenière année; se troure converti en une substance lignense encorc molle ct mal formće. L'automne arrive, el la végétations'arrête en cel état. La conehe exté- 
ricure du cambium, qui n'a point encore entièrement changé de nature, reste stationnaire et comme engourdie. Cependant, au retour du printemps, quand la chaleur douce du saleil vient tirer les végétaux de leur sommeil. hivernal, le cambium reprend sa force végétative; il développe les bourgeons et les nouvelles racines; et, lorsqu'il a produit toutes les parties qui doiỵent servir à l'entretien de la vie du végétal, ils se durcit peu à peu, devient compacte, en un mot, suit et éproụve les mêmeș changemens que celui quị l'a précédé. Maị, à mesure que ces chạngemens s'opèrent, que le liber sc durcịt et change de nature, que la couche qu'il a remplacée ạequiert une so= lidité plus grande, il se développe un nouveau liber. De tous les points de la surface cxtéricure de celui qụi est prèt à se convertir en bois, suinte me humeur visqueuse, sous forme de petites gouttelettes qui s'étendent et se réunissent : c'est un nouveau cambium, un nouveau liḅer qui va s'organiser, se développer, eț suivre les différentes époques d'accroissement parcourues par ceux qui l'ont précédé, et dont il a tiré son orịigine.

Tels sont les mojens que la nature met en usage pour renouveler chaque année la partic végétante de la tigc. C'est ici que se présente la grande différence des tiges ligneuses, et des tiges herbacées. Daụs les tiges ligṇcuses, cn effet, c'est au développement successif d'une nouvelle couche de liber qụe l'arbre doit sa durée et la persịstance de sa végétation. Dans les íges herbacées, au contraire, tout le cambium sc consume à produirc les différens organes de la plante, et à la fin de l'année se trouve entièrement converti en une sorte de substance ligniforme, sèche et aride. Il ne reste donc point, comme dans la tige ligneuse, une certaine quantité de natière gélatineuse, chargée de conserver d'une annéc à l'autre les germes d'une nouvelle végétation, et la plante ineurt nécessairement, faute d'une substance propre à renouveler son développement. 
Après avoir développé avec quelques détails la théorie de la formation des couehes ligneuses au moyen de la transformation annuelle du liber en aubier, nous devons faire comnaître eelle qui a été émise par MI. Du Petit-Thouars, et qui a fait, entre plusieurs physiologistes, le sujet de tant de contestations.

$2^{\circ}$ La formation successive des couches ligneuses, c'est$\grave{a}$-dire l'accroissement en diamètre, est produic par le développement des bourgeons.

Dans la théorie préeédente, e'est au liber que l'on attribue la plus grande part dans les phénomènes de l'aeeroissemcnt en diamètrc; iei, au eontrairc, se sont les bourgcons qui jouent le rôle le plus important dans eette opération. M. Du Petit-Thouars ayant remarqué que les bourgeons sont assis sur le parenehyme extérieur, et que leurs fibres communiquent avee eelles des seions ou jeunes xameaux qui les supportent, cn a tiré les eonséquenees saivantes, qui forment la base de sa théoric de l'organisation végétale.

$I^{\circ}$ Les beurgeons sont les premiers phénomènes sensibles de la végétation. En cffet, toutes les parties qui, dans les végétaux, doivent se développer à l'extérieur, sont d'abord renfcrmées dans des bourgeons.

Il en existe un à l'aisselle de toutes les fenilles; mais ec bourgeon n'est apparent que dans les plantes dieotylédones, et parmi les monoeotylédones, dans la famille des Graminées seulement. Dans les autres monoeotylédones, ce bourgeon est latent, et ne consiste que dans un point vital, suseeptible, dans eertaines cireonstances, de se développer à la manière des bourgeons des dieotylédons.

2 . Par leur développement, les liourgeons donnent naissanee à des scions ou jeunes branehes chargées de feuilles, et le plus souvent de fleurs. Chaque bourgeon a une existenee en quelque sorte indépendante de celle des autres. MI. Du Petit-Thourars les regarde comme analogues dans 
leur développement et lcur strueture aux embryons renfermés dans l'intérieur des graines, qui, par l'aete de la germination, développent une jeune tige que l'on peut comparer, avee juste raison, au seion produit par l'érolution d'un bourgeon. Aussi donne-t-il à ees derniers le nom d'embryons fixes ou adhérens, par opposition à eelui d'embryons libres, conservé pour eeux renfermés dans l'intérieur de la graine.

$3^{\circ} \mathrm{Si}$ l'on examine l'intérieur de ces bourgeons sur un seion ou jeune branehe de l'année, on voit qu'ils eommuniquent directement avee le parenehyme intérieur ou la moelle. Or eette moelle, eomme nous l'avons dit, est d'a. bord verte, et scs eellules sont remplies de fluides aqueux très-abondans. C'est dans ees fluides aqueux que les bour geons puisent les premiers matériaux de leur développement. Ils se mourrissent done aux dépens du parenehyme intérieur; et en absorbant les fluides qu'il eontient, ils le dessèchent, et le font passer à l'état de moelle proprement dite, plus ou moins opaque ou diaphane.

$4^{\circ}$ Dès que ees bourgeons se manifestent, ils obéissent à deux mouvemens généraux, l'uu montant ou aérien, l'autre deseendant ou terrestre. C'eșt ici que M. Du Petit-Thouars rapproche la strueture et les usages des bourgeons de eeux des embryons-graines. Il considère en quelque sorte les bourgeons eomme des embryons germans. La couche de eambium située entre l'éeoree et le bois est, pour le bourgeon, anloggue au sol sur lequel la graine eommenee à germer. Son évolution aérienne donne naissanee à un seion, ou jeune branehe; tandis que dé sa base, e'est-à-dire du point par lequel il adhère à la plante-mère, partent des fibres (qué l'auteur compare à la radieule de l'embryon), et qui, -glissant dans la eouehe humide du eambium, entre le liber et l'aubier, deseendent jusqu'à la partie inférieure du végétal. Or, ehemin faisant; ees fibres reneontrent eelles qui deseendent des 
autres bourgeons; clles s'y r'ćunissent, s'anastomosent entre elles, et forment ainsi une couche plus ou moins épaisse, qui prend de la consistanée, de la solidité, et constitue eháque année une liourelle eouche ligneúse. Quant au liber, une fois formé, il re eliangè plus de riature, et n'éprouve aucune transformation.

Cette théorie est cxtrêmement ingériieuse, et M. Dú Petit-Thouars s'appuie sur plusieurs faits pour en prouver l'exaetitude. Ainsi, dit-il, lorsque l'on fait au tronc d'un arbre dieotylédon une forte ligature eireulaire, il se forme au-dessus de l'obstaele un bourrelet, et l'aecroissement en diamètre eesse d'avoir lieu au-dessous de lá ligature. Ce bourrelet est formé par les fibres ligneuses quii deseendent de la base des bourgeons en glissant dans le eambium situé entre le liber et l'aubier. Ces fibres ligineuses reneontrent un obstacle qu'elles ne peuvent surmonter, s'y aeeumulent et s'y arrêtent. Dès-lors il ne peut plus se former de nouvelles couehes ligneuses audessous de la ligature, puisque les fibres qui doivent les constituer éessent d'y arriver. Telle est l'explication donnée par M. Du Petit-Thouars du fait de la ligature et du bourrelet eireulaires, que la plupart des auteurs expliquent d'une manière tout-à-fait différente.

M. Du Petit-Tliourars s'autorise eneore des phénomènes de la greffe pour étayer sa théorie. Lorsque l'on greffe en écusson, on prend ordinairement un bourgeon eneore stationnaire, on appliquesa base sur lacouehe du eambium que l'on a mise à nu; dès-lors les radieelles ou filires qui partent de la base du bourgeon glissent entre l'éeorce et l'aubier, et le nouveau sujet s'est ainsi identifié à eelui sur lequel oul l'a greffé.

J'ai vu ehez M. Du Petit-Thouars une pièee bien précieuse, qui semble un argument bien fort en faveur de sa thćorie, dont il a domné une très-bonne figure dans un recueil de mémoires, imprimé mais resté 
inédit, je crois. C'est une branche de robinia pseudoacacia, sur laquelle avait été greffé un jeune scion de robinia hispida. Le sujet est mort, mais la greffe ayant continué de végéter, on voit partir de sa base une sorte d'empâtement formé de fibres très-distincles, qui em-. brassent l'extrémité de la branche dans unie assez girande étendue, et lui forment une sorte d'étui. Dans cet exemple, on reconhaît avec la dernière évidence que les fibres deseendent de la base de la grefie pour sc répandre sur le sujet.

Malgré toutes les raisons alléguées par l'autcur, cn faveur de sa théorie; aucùn physiologiste ne l'a encore entièrement adoptée. Au contraire, présque tous eeux qui s'occupent de la physique des végétaux l'ont plus ou moins eombattue. Les prineiparix argumens que l'on a cherché à opposcr à la théorie de M. Du Petit-Thouadrs, sont: $\mathrm{I}^{\circ}$ que rien ne prouve d'une maniêre irréfragable que les fibres qui établissent la communiealion entre les bourgeons et les tiges qui les supportent descendent ainsi dè ees bourgeons jusque dans les racines : mais à eela M. Du Petit-Thouars répond que les bourgeons sont bien la souree, l'origine première des fibres ligneuses, mais que ee ne sont pas les bourgeons qui fournisssent tous les matériaux de leur élongation; une fois sorties de la base des bourgeons, les fibres se trouvent plongées dans le eambium? où elles ałsorbent tout cc qui est nécessaire à leur aceroissement; $2^{\circ}$ que les phénomèncs du bourrelet cireulaire, formé à la suite de la ligature du trone, pẹvent s'expliquer par. l'interception et la stase de la sève deseendante : mais, objeete M. Du Petit-Thouars, l'expérience de Hales, constatée par Duhamel, répond à cettc objeetion : ayant isolé complétemient deux cylindres d'écorec par trois enlèvemens d'anneaux eireulaires, dont l'un était pourvu d'un bourgeon et l'autre n'en avait pas, il en résulta que ce fut sur le premier seulement gu'il se 
fit un bourrelet inférieur, preuve évidente que ee sont les bourgeons qui donnent naissance aux fibres ligucuses; $3^{\circ}$ qu'il est impossible de eoneeroir comment des fibres aussi grêles que eclies qui unissent les bourgeons aux tiges peuvent, dans un espace de temps aussi court que eclui durant lequel la tige s'accroît en diamètre, descendre, de leur propre poids, du sommet d'un arbire de 60 à 80 pieds, jusqu'à sa base : comme l'opinion du savant académicien n'est pas que les fibres sortent et descendent toutes formées de la base des bourgeons, mais qu'au eontraire elles se forment ch traversant les eouches de cambium, eetle oljection tombe d'clle-même; $4^{\circ}$ que, puisque ee sont les fibres qui descendent de la base des beurgeons qui constituent les couches ligneuses, si dans la greffe en ćeusson on greffe un bourgeon d'un arbre à bois eoloré sur un indiridu à bois blane, les fibres qui partent de ees bourgeons devraient conscrver leur couleur, et les nouvelles couehes ligneuses.qu'elles forment en préscnter une semblable, ce qui u'a pas lieu: cette objection, use de eclles dont on a fait le plus de bruit, est aussi une de celles que l'auteur réfute avec le plus de facilité; car e'est paree qu'on n'a pas bicn eompris son opinion, qu'on lui a opposé cette objection; en effet, comme M. Du Petit-Thouars n’a cessé de le répéter, les fibres sorties de la base du bourgeon se nourrissent du cambium de la branche à la surface de laquelle •lles se forment; or, dans le eas de la greffe de deux sujets dont le bois est d'une eoulcur différente, tant que les fibres nouvelles sont plongées dans le eambium du sujet à bois eoloré, elles prennent et conservent la teinte qui leur est naturelle; lorsqu'au contraire elles se forment aux dépens du cambium du sujet à bois clair, clles prennent la teintc particulière à ee nouveau bois; $5^{\circ}$ enfin, si e'est le développement des bourgeons qui donne lieu à la formation du bois, eomment la première couehe ligneuse a-t-elle pu se former sur le jeune 
scion de l'amnée, puiscque aucun des bourgeons qu'il supporte nc s'est encorc dévcloppé? Sclon lc célèbre académicien dont nous exposons ici la théorie, au moment où un bourgeon se développe pour former un scion, les feuillcs qui le composcnt s'ćloignent les uncs des autres, et laissent entre ellcs des espaces que l'on a nommés mérithalles. Si l'on examine à cette époquc la structure intérieure du jeune scion, on verra que de la base de chaque feuille il part un faisceau de fibres dont la rćunion constitue l'étui médullaire; mais à mesure que ces feuilles se déreloppent, il sc manifestc à l'aisselle de chacune d'elles un bourgeon qui tend de suitc à établir sa communication radicale, en détcrminant la formation de fibres ligneuses. Ce sont donc celles-ci qui recouvrent graduellement l'étui médullaire, ct en composent une couchc continue.

Les dcux théories dont nous venons de faire l'exposition nc peuvcnt donc pas être adoptécs danslcur entier, comme donnant unc cxplication rigoureuse de tous les phénomènes dc l'accroissemcnt en diamètrc dans les végétaux dicotylédons. En effet, cclle dc Duhamel est cssentiellement fondée sur la transformation annuclle du liber en aubier, ct sa régénération au moyen de la couchc dc cambium. L'expériencc par laqucllc ce célćbrc physicien dit qu'ayant fait passcr un fil d'argent dans le liber, il l'a retrouvé l'année suivante dans l'aubier, cst tout-à-fait inexacte. En effet, tous ceux qui après Duhamel ont cherché à la répéter n'ont pu obtenir le mêmc résultat, et lorsque le fil d'argent avait été réellcment passé à travers lc liber, on l'a toujours retrouvé dans cet organc, et non dans l'aubicr. Cette thćoric dòit donc néccssairement s'écrouler, si nous sapons la base sur laquclle l'auteur l'avait élevée. Voyons maintenant l'explication qui nous paraît s'oecorder le mieux avec les faits.

$3^{\circ}$ La formation annuelle des' couches ligneuses est 
due au cambium, qui chaque année forme à la fois une nouvelle couche d'aubier et une nouvelle couche de liber.

Cette opinion est eelle qu'en dernier licu avait professée M. Mirbel, et qui nous paraît réunir en sa faveur le plus de probabilités.

Lé liber, quel'on avait jusqu'à présent eonsidéré eomme l'organe le plus essentiel de la végétation, comme eelui qui opérait ehaque année l'augmentation en diamètre du trone des arbres dieotylédons, étant au contraire neutre et passif dans eette opération, on doit ehereher une autre explieation des phénomênes de l'aecroisssement en diamètre. Or voiei celle qui nous paraîtla plus probable et lá plús en rapport avec l'observation rigoureuse des faits. Si l'on examihe une jeune branehe à l'époque de la végétation, c'est-à-dire quand la sève eireule abondamment dans toutes les parties du vésétal, voiei ee que l'on observe : entre le liber et l'aubier, on trouve une couche d'un fluide d'abord élaiir et linpide, qui peu à peu s'épaissit, et prend de la eonsistance ; ee fluide, ou lé cambium, est formé par la sève deseendante, mélangée à une partie des sues propres des végétaux. A mesure que le eambium s'épaissit, on voit des filamens se former dans son intérieur, et bientôt il s'organise et prend l'aspeet d'un tissu végétal. Cette transformation est graduelle, et continue pendant tout le temps du développement des bourgeons, en sorte que la formation dè la eouehe annuelle a lieu d'une manièré lenté et progressive. C'est pour éette raison que les eouehes nouvellés d'aubier présentent très-souvent plusieurs zones eoncéntriques qui annoneent que toute leur épaisseur n'a pas été formée d'une seulc fois.

Ainsi done l'aubier n'est pas formé par le liber, qui s'épaissit et prend plus de eonsistanee, mais par le eambium, qui s'organise, et devient ainsi l'agent de l'accroissement en diamètre, donnant lieu ehaque année à la formation d'un eouche d'aubier et d'une eouehe de liber, 
toutes deux distinetes l'une de l'autre, quoique tirant leur origine du mềme organe: Lorsque Duhamel a retrouvé dans l'aubier le fil d'argent qu'il avait eru avoir engagé dans le liber, e'est quie ee fil avait été passé à travers la couehe organique du eambium.

Il suit également de là que ehaque année le liber s'äeeroît en épaisseur pàr sa faee interne: En effet; la éouehe de cambium qui baigne sa surfaee intérieure s'organise; et s'ajoute à eet organe, en sorte qu'il prend graduellement plus de dév́eloppement. C'est pour eette raison que le liber se trouve formé de plusieurs lanies ou feuillets; réunis les uns aux autres par un eouehe exeessivement minee de tissu eellulairc.

Ainsi done, pour nous résumer, il se forme ehaque année dans le trone des arbres dieótylédons une nouvelle eouehe ligneuse. Cette nouvelle eouehe est produite par une partie du eambium, qui s'organise et se solidifie: L'aubier formé l'année préeédente aequiert plus de densité, et se ehange en bois. Mais le liber n'éprouve aueune transformation; seulement il se répare et s'aeeroît par sả faee interne au moyen d'une partie du eambium, qui forme suecessivement de nouveaux feuillets.

C'est par ee méeanisme qu'a lieu; selon nous, l'àeeroissement en épaisseur des tiges des dieotylédons; éxpliquons de même leur développeinent en hautetir.

\section{$B$. Aecroissement en hauteur.}

A l'époque de la germination, lä rádiculé s'enfonêể dans la terre, tandis que le eaiudex ascendant s'élèvè vers lẻ eiel. La première eouehe du cámibium s'organisé; èt obéit à eette impulsion. Vêrs l'automine; quand élle est organisée en aubier et en liber, son aeeroissement s'arrête. Quand, au retour du printemps, la végétation recommenee, le tissu végétal est gorgé de fluides nourrieiers qui vivifjent les bourgeons; de la partie supérieure de la tige 
part un nouveau eentre de végétation d'où s'élève une jeume pousse qui éprouve dans son-développement les mêmes phénomènes que la première; à cette seeonde en suecède une troisième, qui l'année d'ensuite est surmontée d'une quatrième, ete.

Le trone se trouve donc formé par une suite de eônes très-allongés, dont le sommet est en haut, et qui sont superposés les uns aux autres, Mais le sommet du cône le plus intérieur s'arrête à la base de la sceonde pousse, et ainsi sueeessivement, en sorte que ce n'est qu'à la base du tronc que le nombre des couches ligneuses eorrespond au nombre des années de la plante. Ainsi, par exemple, une tige de dix ans offrira à sa base dix couehes ligneuses. Elle n'en présentera que neuf, si on la eoupe à la hauteur de la seconde pousse, que huit à la troisième, et enfin qu'une seule vers son sommet. C'est pour cette raison que le trone des arbres dicotylédons est plus ou moins conique, le nombre de ses eouehes ligneuses étant graduellement moins considérable, à mesure que l'on deseend du sommet vers la basc.

Il est des arbres sur lesquels ee développement en hauteur est des plus manifestes : dans les pins et les sapins, pair exemple. Au bout de la première année, on voit au sommet de la tige un bourgeon eonique, d'où part un vertieile de jeunes rameaux, au centre desquels en est un qui s'élève verticalement; e'est lui qui est destiné à eontinuer la tige. A la fin de la seconde année, de son sommet part égalenient un semblable bourgeon qui présentera les mêmes phénomènes dans son développement. Ainsi l'on peut connaître dans ees arbres le nombre de leurs années par le nombre de verticilles de rameaux qu'ils présentent sur leur tige. 
\$2. Accroissement de la tige des arbres monocotylédons.

Si nous examinons l'accroissement du stipe d'un palmier, nous voyons qu'il se développe de la manière suivante :

Après la germination, les feuilles, ordinairement plissées sur elles-mêmes, se déroulent et se déploient en formant un faisceau circulaire, qui naît du collet de la racine. Du centre de ce faisceau part, la seconde année, un autre bouquet de feuilles, qui rejettent en dehors celles qui existaient déjà. Alors les plus anciennes se fancnt, se dessèehcnt et tombent. Mais leurs bases étant intimement adhérentes au sommet de la racine, restent, persistent, ct constituent, cn se soudant, un anneau solide qui devient la base du stipe. Chaque année un nouveau bourgeon central venant à se développer, les feuilles les plus extérieures de celui qui l'a prćcédé tombent, et leur base qui persiste forme un nouvel anneau qui s'ajoute au-dessus de ceux qui existaient déjà.

Tel est le développement de la tige des Monocotylédons. Leur stipe, au lieu d'être formé, comme le tronc des Dicotylédons, de couches concentriques, est composé d'anneaux superposés. D'après cela, on voit que le tronc des Monocotylédons ne doit eroître que très-peu cn épaisseur. En effet, son développement latéral ne peut avoir lieu qu'autant que la base persistante des feuilles ne s'est point cncore assez solidifiée et cndureie pour résister à la pression exeentrique que le bourgeon tend à opérer sur ellc. Aussi voyons-nous que les palmiers, qui ont quelqucfois jusqu'à cent vingt et ccnt quarante pieds de hauteur, ont unc tige qui a sourent à peine un pied de diamètre.

Dans les arbres dieotylédonés c'est lc cambium qui est l'agent essentiel de l'augmentation de la tige, puisque c'est 
lui qui, chaque annéc, s'organise et forme une nouvelle couche ligneuse. Iei, au contraire, e'est le bourgeon terminal couronnant le stipequi remplit le même usagé. Aussi l'arbre périrait-il infailliblement, si l'on retranchait ee centre de végétation.

Si nous comparons d'unc manière générale l'aceroissement en diamètre de la tiģe des arbres dieotylédons et eelui des monocotylédons, nous verrons qu'il ne diffère pas moins que leur structure anatomique. Fn effet, dans les dieotylédons il y a deux systèmes distinets, le système central, formé de l'étui médullaire et des couches ligneuses, et le système cortical, qui se compose de l'écoree. Ces deux systèmes s'accroissent séparément, en sorte qu'il y a deux surfaces d'aceroissement dans ectte classe de végétaux. Le système central s'aceroît par les nouvelles couehes qui s'ajoutent à sa surface externe, et le système cortical s'aceroît au contraire par sa face interne.

Dans les végétaux monocotylédonés, au contraire, il n'y a qu'une seule surface d'aecroissement, et par conséquent qu'un seul système. M. Thém. Lestiboudois, professeur à Lille, remarquant, et avec juste raison, que dans ee système unique qui forme la tige des monocotylédons, l'aceroissement se fait par la face interne, en tire eette eonclusion que ee système est le cortical, et que le eentral manque. D'ou il suit que le stipe des palmiers est organisé comme l'écorce des dicotylédons.

De ces diverses eonsidérations ondoit tirer eette première observation, e'est que le stipe des pálmiers et des autres arbres monocctylédonés ligneux diffère essenticllement, et par son organisation et par son mode de développement, du urone des végétaux dicotylédonés. Si mêune on pousse plus loin cette observation, on verra que le stipe diffère tellement du trone par son origine première et son mode de développement, qu'il n'est point ćionnant que sou organisation intérieure, qui n’est que le résultat de ec mode 
de développemens, oflre avec la tige ligneuse des arbres à deux cotylédoụs des différences aussi tranchées. Rappelonsnous en effet comment se forme et s'aceroît la tige d'un chêne ou de tout autre végétal dieotylédané : la graine germe, la radieule s'enfonce dans la terre, la tigelle ou l'organe qui la représente, e'est-à-dire qui serț de support à la gemmule et l'élève au-desșus de la base de la radicule, se redreșe; en un mot, dès ces premiers temps de la vie de la plante, l'organe qui doit eonstịtuer la tige existe déjà sous la forme d'un cylindre plus ou moins allongé, com posé intérịeurement d'un tișsu cellulaire qui représente la moẹlle, et extérieurement de tupes ou de fibres, premiers rudimens du bois, de l'éeoree et en général de toutes les partịes filamenteuses de la tige. Examinons comparativement une graine de palmier, au moment où elle germe : son extrémité radiculaire ș'allonge plus ou moịns, se rompt à son sommet pour laisser sop̣tir la radicule, emprisonnée d'abord dans une sorte de bourse close, nommée coléorhize, qu'elle déchire pour pouvoir s'enfoneer dans la terre et devenir lą racine. L'extrémité opposée à la radicule, c’est-à-dire le cotylédon, prend un léger développement, mais bientôt on le voit se fendre sur l'un de ses côtés, au-deśsous de son sommet, et, par cette fente ou rupture, sortent un nombre plus ou moins considérable de feuilles d'abord emboîtées les unes clans les autres. Mais dans eet embryon de palmier nous n'apercevons pas, comme dans celui du chêne, du tilleul, dụ pin, ete., une tigelle ou rudiment de tige. L'organe auquel on domnera plus tard ce nom va se former suecessivement aux dépens d'un autre organe. En effet, ainsị que n̦ous l'avons exposé précédemment, ce sont les bases des feuilles suecessivement développées qui, en se rapprochant les mes des autres par. suite du refoulement que les plus extérieures éprouvent, à mesure que de nouvelles se développent à l'intérieur, se soudent ensemble ct finissent par former une sorte 
de plateau charnu, formé de tissu cellulaire et pareouru de fibres éparses. Ce que l'on appelle stipe ou tronc dans un palmier est done un organe composé d'un grand nombre d'écailles qui ne sont que des bases de feuilles plus ou moins soudées entre elles, et offrant à leur intérieur un bourgeon central et terminal qui en est l'organe essenticllement végétant. Ainsi done le stipe d'un palmier n'est véritablement pas une tige, ni par son origine, ni par son développement, ni par son organisation. Tojons s'il n'offre pas quelque aualogue dans la série des autres végétaux. Et d'abord, qu'est-ee que la prétendue tige souterraine, vulgairement nommée raeine dans la plupart des espèees du genre iris? C'est un eorps charnu, ofrrant quelques fibres longitudinales intérieurement, et présentant à sa surface externe les eieatrices des écailles qui la composent. Or, si nous en suivons le développement, nous verrons qu'elle doit sa formation aux bases des feuilles qui ont persisté, tandis que leur partic supéricure s'est détruite, qui se sont soudées et ont formé le eorps eharnu que l'on désigne communément sous les noms de raeine, de rhizome, de souclie ou de tige souterraine dans les iris. Par conséquent cet organe n'est, en réalité, comme le stipe des palmiers, ni une racine, ni une tige, mais une réunion de bases de feuilles tontes soudées cn une seule masse. Une espèce d'ail (allium senescens) nous offre in organe entiérement senublable, e'est-à-dire une souclıe plus ou moins charnue et rameuse. Or, de ectte souche de l'allium senescens et des iris aux bulbes solides ou éeaillcux des Liliaeées, la transition me parait insensible. Un bulbe, en effet, n'est qu'un organe composé d'écailles variables dans leur forme et leur disposition, mais toujours assises sur un plateau charuu et reeouvrant un bourgycon eentral et terminal ; toujours ces ćçailles ne sont que des feuitles ou dont la base seules'est développée ou dont la base seule a résisté, tandis que la parlie supérieure s'est détruite. Si, 
comme noüs croyons l'avoi- prouvé, la souche souterraine des iris a la même origine, le même mode de développement et la mêméorganisation que le stipe des Palmiers, si d'un autrc côté nous avons démontré quc sous ces divers rapports il n'existe aucunc différence sensiblc entre cette prétendue souelic des iris et le bulbc de la plupart des Li-, liacées, il nous paraît impossiblc de ne pas tirer cctte conclusion, que le stipe des Palmiers, au lieu d'être une tige, n'est véritablemcnt qu'un bulbe. Cette opinion pourra paraître paradoxalc à celui qui ne fera pas abstraction de la formc générale, de la grandeur et de la dureté du stipe. des Palmiers, comparćcs avec le bulbe des autrcs monocotylédones. Mais si l'on réfléchit attentivement queccs divers attributs ne sont pas essentiels à la nature de cet organe, qu'ils manquent fréquemment dans un grand nombre d'cs: pèces, qu'ainsi dans quelques-unes le stipc, au lieu d'ètre long et cylindrique, est court, à peine sensible, ct consiste quelquefois seulcment en une sorte de renflcment bulbiforme, que dans d'autres espèccs ce stipe, loin d'êtrc dur et ligneux, est mou, charnu, et se laisse aisément entamerpar les instrumens tranchans, ces différcnccs, d'abord si frappantcs, disparaîtront à l'instant. Si d'un autre côté on examine l'origine, lc mode de formation et de développemcnt du stipe comparés à ceux du bulbe, on devra conclure que ces deux organes sont essentiellement les mêmcs.

Dans cette manière d'envisagcr le stipe on peut très bien expliquer pourquoi eet organe se ramifie si raremcnt En effet on sait qu'un rameau n'est jamais que le résul tat de l'élongation d'un bourgeon plaeé en général à l'aissclle d'une feuille: or dans lcs monocotylédones ces bourgcons axillaires avortent presque constamment, ou restent à l'état rudimentaire, comme dans la plupart des Graminées par exemple : il en est de nuêmc dans les Palmiers, leurs bourgeons axillaires restcnt cn général à l'état rudimentaire, et alors le stipe est parfaitement simple ; mais 
dans certaines eirconstanees quelques-uns de ees bourġeons recevant plus de nóturriture que les autres se développent, c'est-à-dire que les feuilles qui les composent è se soudant pár leur base, finissent à la longue par forinèr un nouveau stipe partant du premier; c'est ee qu'on observve, par exemple, dans ecrtaines espèécs d'yueca, dans le Doom de la Thébaïde, ete.

Poür terminer ici tout ee qui a rapport à l'aceroisseinent de la tige clans les végétaux, il nous reste à faire connaître le résultat des observations publiées récemment par M. Dutroeliet. (Mém. du Muséum, vol. vir et vir.) Jusqu'à présent on n'avait généralement admis l'aceroissement en diamètre que eomme le résultat des nouvelles eouches qui s'ajoutcut chaque année entre l'aubier et l'écorce. M. Dutrochict a prouvé que les végétaux s'aceroissent en diamètre en deux sens, savoir ${ }^{\circ}$ en épaisseur, par la formation des houvelles couches entre l'écorce et l'aubier, $2^{\circ}$ en largeur, par le développement látéral de la nouvelle couehe et la formation de nouveaux faisccaux de fibres. Cet accroissement, dans le sens de l'épaisseur et de la largeur, a lieu également dans les racines et dans les tiges. Mais nous 'devons faire remarquer que c'est le professeur Linek, qui dans son Anatomie des plantes et plus tard dans sa Plitosophie'botanique a le premier établi que la tige s'aceroissait non-seulement vers'son eentre et sa periphérie, mais encore latéralcment par la multiplication des faisecaux vasculaires. (Voyez Linek, Grundl: d. Anat.f. d. Pfl. p. $1 / 46$, f. $58-60$.

C'est d'abord sur la tige de la clématite que M. Dutrochet a fait ses premicrs essais. Lorsque l'on coupe trans versalement l'extrémité d'une jeunc brauche de clématite, on trouve qu'clle se compose de six faisceaux de fibres longitudinales, séparés les uns des autres par des rayons ou espaces inédullaires assez larges. Peu à peu, et par les progrès de la végétation, il se forme au eentre de eliaque 
espaee mćdullaire un nouveau faisceau de fibres longitudinales qui acquiert bientôt le même volume que les six faisceaux primitifs, en sorte qu'à la fin de la premic̀re année la tige se trouve composée de douze faisceaux de fibrcs, sćparés par autant de rayons médullaires.

Pendant la seconde annéc chaeun des six faisceaux primitifs sc divise cn trois par la production médiane d'un uouveau faisceau de fibres longitudinales sćparé des deux autres, au milicu desquels il s'est développé, par deux rayons médullaires incomplets, qui n’atteignent pas jusqu'à la moellc ccntrale; d'um autre côté, les six autres faisceaux secondaires de la premic̀re année se diviscnt chacun en deux par la formation médiane d'un nouveau rayon médullaire incomplet : d'où il résultc qu'à la fin de la seconde année il y a trente faisceaux de fibres distingués les uns des autres par autant de rayons ou espaccś médullaires, dont douze seulemcnt, savóir ceux qui existaient à la fin de la premic̀re annće, sont seuls complets et établissent une communication directe entre la mćdulle externe et l'internc.

Pour pcu qu'on réfléchissc avec quelque attention à la manicre dont les faisceaux de fibres longitudinalcs se sont multipliés, on verra que l'accroissement s'est fait latéralcment. En effet la production médiane de nouveaux faisceaux de fibres au céntre des rayons médullaỉres, ou celle de nouvcaux rayons médullaircs au centrc des faisceaux de fibres, a dû nécessairemcnt dilater latćralcment, et par conséquent augmenter la largeur de la couche circulaire dans laquellc ee développement s'est općrć. Or c'est cctte dilatation latérale qui n'avait point encore été aperçue avant l'habile expérimentateur dont nous faisons connaître ici les observations.

L'accroissement en largeur s'arrête dans les parties dès l'instant qu'elles se sont solidifiées. Ainsi il n'a plus lieu. dans les couches tigneuses; mais il se eontinuc dans l'é- 
corcc, et c'est ainsi qu'elle permet l'aecroissement en épaisseur des couehes ligncuses.

L'aeeroissement en largeur a égalcment licu dans les racines, ainsi que nous l'avons déjà annoneé. Mais dans eet organe il commence toujours par la production médiane de nouveaux rayons mćdullaires au ccntre des faisecaux de fibres. Plus tard ces nouveaux cspaces médullaires domnent eux-mèmes naissance à d'autres agglomérations des fibres.

D'après ec qui préeède, on voit que les ćlémiens organiqques des végétaux ont une tendance naturelle à la production médiane. Ainsi les faisceaux de fibres tendent à produire dans leur partic mojenne de nouvcaux rajons médullaires; d'un autre côté les rayons médullaires tendent à produirc de nouveaux faisceaux de fibres longitudinales.

Nous venons de fairc eonnaîtl'e l'opinion de l'auteur relativement à l'accroissement en largeur; exposons aussi scs idćes sur le développement en épaisseur. Les couches ligncuses de nouvelle formation qui sc développent ehaque annéc sont séparćes des anciennes par unc couche mince de médulle centrale. Ces couelıcs de médulles, qui isolent les eouches ligneuses les unes des autres, ne sont pas toujours faciles à apercevoir; mais elles sont très-visibles dans quelques arbres, par excmple, dans le Rhus typhinum, où leur conlcur plus foncéc les fait distinguer au premicr coup d'œil des couches de bois, qui sont plus claires. $\Lambda u$ printemps l'accroisscment en épaisseur commence toujours par la formation de cette couehe mincc de tissu ccllulairc, ou de médullc. Bientôt, par sa proprićté de domner naissanee à des fibres longitudinales, cettc couche de moelle produit des vaisseaux qui l'environnent, ct constitucnt ainsi une sorte de canal médullairc, destiné à devenir plus tard la nouvelle couche ligneuse.

Dans cette tliéorie on voit le rôlc important que l'auteur' fait jouer à la moelle. C'est elle cn effet qui devient l'agent cssenticl de l'accroissement cu diamètre, puisque 
e'cst elle qui donne naissanee aux vaisseaux qui doivent eonstituer plus tard la nouvelle eouẹe de bois.

Les mêmes phénomènes ont lieu dans le liber. Chaeun de ses feuillets est séparé par une eouche minee dé tissu cellulaire, qui appartient à la méculle corticale, et qui est l'agent de son aceroissement annuel.

Théorie de quelques procédés pour la multiplication artificielle des végétaux expliquée par les lois de la physiologie végétale.

Le moyen de multiplication le plus naturcl et le plus facile dans les végétaux est sans contredit celui qui a lieu au, moyen des graines et de leur développement; e'est celui par lequiel. les végétaux dispersés sur la surfaee du globe se renouvellent naturellement; mais il en est eneore d'autres que l'art de la eulture met fréquemment à contribution pour perpétuer et multiplier eertaines raees ou variétés d'arbres que l'on ne pourrait reproduire par le mojen des graines. Ces proeédés sont la mareotte, la bouture et la greffe. Nous allons en peu de mots exposer la théorie de ees trois opérations, eonsidérées d'unc manière générale, et quant à leurs rapports avee la physique végétale.

I ${ }^{\circ}$ La mancotte est une opération par laquelle on entoure de terre la base d'une jeune branche, et on lui fait pousser des racines avant de la détaeher du sujet. Tantôt eette opération se pratique sur les branehes inférieures d'un jeune arbuste; on les ineline et on les eouche légèrement: tantôt e'est sur les branehes supérieures, que l'on fait passer à travers un pot ou une cage de verre remplic de tcrre de bruyère.

f Pour faeiliter le marcottage on pratique ordinairement à la base de la jeune branche une incision ou uns: forte ligature, afin de déterminer la formation des racines. 
Ces racines sont des bourgeons qui, plongés dans la terre, s'allongent en fibres grêles et radicellaires, tandis qu'exposés à l'air ils se seraient développés en jeunes scions. On emploic la marcotte pour multiplier un grand nombre de végétaux, tels que les oeillets, les hortensia, les bruyères, les groseillers, etc.

$2^{\circ}$ La bouture diffère de la marcotte en ec que l'on sépare la jeune branche du sujet avant de la fixer en terre. Il $\mathrm{y}$ a des arbres chez, lesquels les boutures reprennent avee une graude facilité. En général, ceux dont le bois est blanc et léger se prêtent plus facilement à ectte opération: ainsi une branche de saule, de peuplicr, de tilleul, cnfoneće en terre, s'y cnracine au bout de quelque temps, et ne tarde pas à pousser avee vigueur.

Une bouture réussira d'autant plus sûrement que le cultivateur aura eu le soin de laisser deux ou trois jeunes bourgeons au-dessous de la terrẹ, e'est-ì-dire sur la partic inférieure de la jeune branche. Ces boutons s'allongent en racines, et aident singulièrement la suceion qui doit amener le développement des jeunes scious.

Assez, souvent on pratique à la base des houtures des incisions ou des ligatures, afin d'en assurer la réussite. Quelquefois même on les fend longitudinalement à leur base, et l'on y introduit une petite éponge imbibée d'eau.

Il est des espèces ligneuses qui repremnent très-difficilement de bouture; tels sont les pins, les sapins, les chênes, les bruyèrés, et eu général les arbres à bois trèsdense ou résineux.

$3^{\circ}$ La GREFFE est une opération par laquelle on ente sur un individu un bourgén ou un jeune scion, qui s'y développe et s'identifie avec le sujet sur lequel il a été greffé.

La grefíc ne peut réussir qu'autant qu'elle a licu entre des parties végétantes : e'est ainsi, par exemple, que l'on ne peut greffer le bois ni même l'aubier. C'est dans l'opération et les phénomènes de la grefle que l'on peut re- 
marquer la grande analogie qui existe entre les gemmes on bourgeons et les graines, surtout sous le rapport de leur développement. Ces deux organes, en effet, sont destimés à donner naissance à de nouveaux individus, dont les uns vivent aux dépens du sujet sur lequel ils se déve: loppent, tandis que les autres subsistent par eux-mêmes, et sans avoir besoin de seeours étrangers.

Remarquons que la greffe ou soudure des parties ne peut avoir lieu qu'entre des végétaux de la même espèce, des espèces du même genre, ou enfin des genres d'une même famille; mais jamais entre des individus appartenant à des ordres naturels différens ; c'est ainsi, par exemple, que l'on peut greffer le pêeher sur l'amandier, l'abricotier sur le prunier, les pavia sur le marronnier d'Inde; mais eette opération ne pourrait pas réussir entré ce dernier arbre, par exemple, et l'amandier; il faut qu'il y ait une sorte de convenance, d'analogie entre la sève des deux individus pour que la sondure d'une greffe puisse s'effectuer.

C'est au moyen du eambium, ó sue propre dẹs végétaux, que s'opère la soudure des greffes. Cette matière fluide sert de móyen d'union entre l'individu et la greffe, comme dans les animaux la lymphe coagulable s'interpose entre les deux lèvres d'une plaie réeente qu'elle réunit et rapproehe. Lorsque l'on examine la plaie d'une greffe, environ quinze jours après l'opération, on voit entre les deux parties rapproehées une eouehe minee de petites granulations verdâtres dispersées dans un flụide viscụeux. Ces petites granulations, rudimens de l'organisation végétale, sont produites par le eambium, qui se solidifie et s'organise, phénomène qui se répète toutes les fois que l'on fait une plaie superfieielle à un arbre, et qu'on la garantit du eontaet de l'air.

Ce moyen de multiplication procure plusieurs avantages dans l'art de la culture : $x^{\circ}$ il sert à conserver et à multi- 
plier des variétés ou monstruosités remarquables, qui ne pourraient se reproduire au mojen des graines; $2^{\circ}$ à prócurer promptement un grand nombre d'arbres intéressans, qui se multiplient difficilement par tout autre mojen; 3० d'aeeélérer de plusieurs années la fructification de certains végétaux; $4^{\circ}$ de bonifier et de propager les variétés d'arbres à fruits, etc.

Le professeur Thouin, dont les sciences déplorent la perte récente, a publié une excellente Monographie des greffes, dans'laquelle il rapporte tous les proeédés connus aux quatre sections suivantes : $\mathbf{I}^{\circ}$ greffes par approche; $2^{\circ}$ greffes par scions; $3^{\circ}$ greffes par gemmes ou bourgeons; $4^{\circ}$ enfin greffes des végétaux herbaeés. Nous allons faire comnaître rapidement les procédés mis en usage pour opérer ces différentes greffes.

\section{$\oint I^{e_{r}}$ \\ Greffes par approche.}

Elles s'exécutent entre deux individus enracinés que l'on veut réunir et souder ensemble par un ou plusieurs points de leur longueur. Pour eela on fait aux parties que l'on veut grefler des plaies qui se correspondent cxaetement; et, en enlevant des plaques d'éeorce d'égale grandeur, on réunit ees plaies, on les tient rapprochées, et on les garantit du eontaet de l'air.

On peut grefler par ce procédé des tiges, des branehes, des raeines entre elles, des fruits et même des fleurs avec des feuilles.

\section{\$II.}

\section{Gieffes par'scions.}

On pratique les greffes par scions avee de jemnes rameaux, ou mìme avec des racines que l'on sćpare de leur individu pour les placer sur un autre, afin quils y vivent 
et s'y dévcloppent à ses dépens. Ordinaircment on sćpare les ramilles que l'on veut greffer, que]ques jours, quelquefois même plusieurs' mois avant dc pratiqucr cctte opération, afin qu'ils soient moins en sève que les sujcts sur lesquels ils doivent être placés. On a soin, dans ce cas, de les conserver, en plongeant leur cxtrćmité inférieure dans l'eau ou dans la terre.

Avant d'opérer eette cspèce de greffe, on coupe ordinairement la tête du sujet sur lequel on veut la pratiquer; quelqucfois même cettc résection se fait à fleur de terre, surtout pour les arbres dont la greffe doit être enterrée, comme la vigne, etc.

Remarquons qu'une condition indispensable pour la réussitc de cette espècć de greffc, c'est qu'il faut que le liber du rameau eoïneide, dans la plus grande partie de son étendue, avee celui du sujet sur lequel on l'a implanté.

La greffe par scions se fait de plusieurs manières : tantôt on fend la tête du sujet en deux, et l'on implante dans cette fente le ramille que l'on veut greffer; cettc espèee est eonnuc sons le nom de greffe en fente : tantôt on écarte l'écorce dẻs couehcs ligneuscs sous-jaeentcs, ct l'on insinuc cntre elles plusieurs petits ramcaux que l'on dispose eireulaircment; c'est la gr'effe en couroine: d'autres fois on perfore le tronc de l'arbre, et l'on y adapte une jcune branehe quc l'on y maintient fixéc; eette greffe, aujourd'hui peu employéc, porte.lc nom de greffe en vilebrequin: quelquefois on pratique la greffc par scions avec de jeunes rameaux chargés de feuillcs, de fleurs, et même de jeuncs fruits; clle s'cffeetuc alors dans le plcin de la premièrc sève. Par ee procédé, il n'est pas rare, dit M. Thouin, d'obtcnir des fruits d'un arbre quinze à vingt ans plus tôt qu'il n'en eût donné sans son seeours; on est mêmc parvenu, en semant un pépin à une époque détcrminéc, à en recueillir avant la fin de l'anuée des fruits parfaitement mûrs. 
La greffe par scions se pratique encore sans couper la tête du sujet; on entaille seulement un de ses côtés, et l'on y applique la greffe. Cette espèce, qui a pour but principal de regarnir la tête d'un arlore qui a perdu quelqu'une de ses branches, porte le nom de greffe de côté.

Enfin on doit rapporter à cette section les greffes que l'on općre avec un scion sur une racine laissée en place, ou avee une raeine súr la raeine d'un autre sujet.

\section{§ III.}

\section{Greffes par gemmes ou boutons.}

Ces greffes consistent à transporter sur un autre individu une plaque d'éeoree à laquelle adhèrent un ou plusieurs bourgeons ou gemmes. A cette seetion se rapportent les greffes en ćeusson, en flîte, en sifflet, en chalumeau, etc. Cette espèee de greffe est la plus employée, surtout pour la multiplication en grand des arbres fruitiers.

En effet, elle est d'une exécution facile et expéditive. Elle se pratique, soit au printemps, lors de l'ascension de la sève, soit à la sève d'août. La forme à domer à la greffe, et celle de l'incision, varient singulièrement, suivant le procédé d'après lequel on opère.

\section{§IV.}

Greffe des parties herbacées des végétaux, ou greffe Tschoudy.

La découverte de eette espèce de greffe date d'une époque assez récente. Il y a peu d'années qu'elle fut pratiquée pour la première fois par, son inventeur M. le baron 'Tschoudy. Elle peut s'effeetuer avec les jeunes pousses herbacées des arbres, dans le fort de la sève ou avee des plantes annuelles.

Pour que eette greffe puisse réussir, il faut l'insérer 
dans l'aisselle ou dans le voisinage d'une feuille vivante du sujet. Cette feuille sert à appeler la sève dans la greffe, et en facilite la reprise et le dévcloppement.

Les procédés mis en usage sont à peu près les mêmes que ceux employés pour exćeuter les autres espèees de greffes.

Telles sont les différentes espèees de greffes employées pour la multiplication des végétaux. Il n'entre point dans notre sujet de déerire les procédés nombreux et variés mis en usage pour les pratiquer; nous renvoyons pour cct objet aux traitćs d'agrieulture, et particulièrement à la Monographie que le professeur André Thouin a publiée en 1822 .

\section{De la Hauteur des arbres.}

Les arbres sont, en général, d'autant plus forts et plus élevés que le sol, le elimat et la situation dans lesquels ils se trouvent sont plus convenables à leur nature et plus favorables à leur accroissement. Une certaine humidité , jointe à un degré de chaleur assez considérable, paraît "êtrc la eireonstance la plus propre au développement des arbres : aussi cst-ee dans les régions qui présentent ces conditions atmosphériques qu'ils acquièrent la hauteur la plus grande. Les forêts de l'Amérique méridionale sont peuplées en général d'arbres qui, par leur port, leur taille élevée, la beauté de leur feuillage ct de leurs fleurs, l'emportent de beaueoup sur ceux de nos elimats tempérés.

Il est eertains arbres qui n'acquièrent que par une longue suite d'années une hauteur ct un diamc̀tre considérables; tels sont, par exemple, le chệne, l'orme, le cèdrc. D'autres, au contraire, prennent un accroissement plus rapide dans un temps beaueoup plus court; ee sont principalement ceux dont le bois est tendre et léger, 
comme les peupliers, les sapins, les aeaeias, etc., etc. Enfin il est eertaines plantes qui se développent avee tant de rapidité, qu'on peut, en quelque sorte, suivre de l'œil les progrès de leur développement : l'agave arrojicana est de ee nombre. Cette plante, que j'ai vue tapissant les roehers qui bordent la Méditerranée dans le golfe de Gènes, lorsqu'elle fleurit, développe, dans l'espaee de trente à quarante jours, souvent plus rapidement, une hampe qui aequiert quelquefois trente pieds de hauteur. Croissant ainsi de près d'un pied par jour, on eonçoit qu'il serait en quelque faeon possible que son développement sueeessif fût pereeptible aux yeux de l'observateur.

En général, le plus grand aeeroissement en hauteur que puissent aequérir les arbres de nos forêts est de eent vingt à eent trente pieds. En Amérique les Palmiers et beaucoup d'autres arbres dépassent souvent eent einquante pieds.

\section{De la Grosseur des arbres.}

La grosseur des arbres n'est pas moins variée que leur hauteur. Il en est qui acquièrent quelquefois des dimensions monstrueuses. Nous ne parlerons pas iei de ee ehâtaignier si renommé du mont Etna, qui, au rapport de quelques voyageurs, avait eent soixante pieds de eirconférenee, paree qu'on s'aeeorde à le eonsidérer eomme composé de plusieurs trones soudés en un seul ; mais nous pouvons eiter eomme exemples bien avérés d'une grosseur énorme, les baobabs observés par Adanson aux îles du Cap-Vert, et dont quelques-uns présentaient quatre-vingt-dix pieds de eireonférenee.

Dans nos elimats on voit des ehênes, des ormes, des tilleuls, des poiriers et des pommiers aequérir jusqu'ì vingt-cinq et trente pieds de eireonférenee. 


\section{De la Durée des arbres.}

Les arbres placés dans des terrains qui leur conviennent, dans une situation appropriée à leur nature, sont susceptibles de vivre pendant des siècles. Ainsi l'olivier peut exister pendant trois cents ans; le chêne environ six cents. Les cèdres du Liban paraissent en quelquic sorte indestructibles. D'après des calculs fort ingénieux, Adanson estime quc les baobabs, dont nous venons de parler tout à l'heure, pouvaient avoir environ six mille ans.

Dans les arbres dicotylédons on peut connaître l'àge d'un arbre par le nombre des couches ligneuses qu'il présente sur la coupe transversale de son tronc. En effct, comme chaque année il se forme une nouvelle couche de bois, on conçoit qu'un arbre de vingt ans, par exemple, doit offrir, mais à sa base seulement, vingt zones concentriques de bois, ct ainsi successivement.

\section{Usages des tiges.}

Le bois est employé à tant d'usages variés dans l'écono=' mie domestique et les arts, il est tellement indispensable à la construction de nos bàtimens de terire et de mer, de la plupart de nos machines et de nos instrumens, qu'il n'est aucune partie des végétaux qui puisse lui disputer à cet égard la'supériorité.

Beaucoup de tiges herbacées sont usitées pour la nourriture de l'homme et des animaux.

La tìge du Saccharum officinarum fournit la plus grande partie du sucre répandu dans le commeree, et qu'on nomme sucre de cannes.

Bcaucoup de bois sont employés dans la teinture : tels sont le santal, le bois de Campêche, le bois de Brésil, etc.

C'est arec les écorces du cliêne, et en général arce 
toutes celles qui renferment une grande quantité de tannin et d'acide gallique, que l'on tanne les cuirs.

- Sous le rapport des propriétés médieales, les tiges, le bois et les écorces occupent un des premicrs rangs dans la thérapeutique. Qui 'ne sait, en effet, qu'à cette classe $d^{2}$ organies se rapportent les quinquinas, la cannelle, l'écorce de Winter, le sassafras, le gayae, et tant d'autres médicamens qui jouissent d'une réputation si bien méritéc? Suivant leurs propriétés ehimiques les plus remarquables, on peut diviser ainsi les principales écorces et les bois employés en médecine :

\section{$x^{\circ}$ Écorces et bois anuers.}

Le Simarouba (Simarouba Guyanensis).

Le Quassia (Quiassia amara).

$2^{\circ}$.Amers, astringens et légèrement aromatiques.

L'Angusture (Cusparia febrifuga).

Le Quinquina gris (Cinchona Condaminea. Humb. et Bonpl. Pl. équinox.).

Le Quinquina rouge (Cinchona oblongifolia. Mutis).

Le Quinquina jaune (Cinchona coidifolia. Mutis).

Le Quinquina orangé (Cinchoná lancifolia. Mutis).

Le Quinquina blanc (Cinchona ovalifolia. Mutis).

La Cascarille (Croton Cascarilla).

$3^{\circ}$ Astringens.

L'écorce de Chêne (Quercus robur').

Le Vinaigrier (Rhus coriaria).

Le Marromier d'Inde (Esculus hippocastanuim).

$4^{\circ}$ Aromatiques.

La Cannelle (Laums Cinnamomum).

L'écorce de Winter (D'ymis Winteri). 
La Cannelle blanehe (Cannella alba).

Le Sassafras (Laurus Sassafras).

$5^{\circ}$ Aeres.

Le Garou (Daphne Mezereum). Bois et écorce de Gayac (Ǵuaiacum officinale).

\section{CH $\triangle$ PITRE III:}

DES BOURGEONS.

Sous le nom général de bourgeons nous comprenons $\mathrm{r}^{\circ}$ les Bourgeons proprement dits, $2^{\circ}$, le Turion, $3^{\circ}$ le Bulbe, $4^{\circ}$ le Tubercule, $5^{\circ}$ les Bulbilles, dont nous al-... lons traiter successivement.

\section{§. Des Bourgeons proprement dits.}

Les bourgeons proprement dits (gemma) sont des corps de forme, de nature et d'aspect variés, généralement formés d'éeailles étroitement imbriquées les unès sur les autres, et reifermant dans leur intérieur les rudimens des tigeș; des branches, des feuilles et des organes de la fruetifieation. Ils se développent toujours sur les branehes; dan's' l'aisselle des feuilles, où à l'extrémité des rameaux. Ils' sont ovoïdes, coniques ou arrondis, eomposés d'écailleś superposées les unes sur les autres, et imbriquées, couverts à l'extérieur, dans les arbres de nos climats, d'un cinduit visqueux et résineux', et garnis à l'intéricur d'un tissu tomenteux et d'une sorte de bourre destinés à garantir les organcs qu'ils renferment des rigueurs de la froide saison : aussi n'observe-t-on point d'enveloppes de' eette sorte sur les arbres de la zone torride, ni sur ceux qu’on abrite dans nos serres: mais les végétaux qui en 
sont dépourvus ne peuvent résister aux froids de nos hivers, et périraient immanquablement si on les y laissait exposés.

Les bourgeons eommeneent à paraître en été, c'est-àdire à l'époque où la végétation est dans son plus grand état de vigueur et d'aetivité; ils portent alors le nom d'yeux. Ils s'aeeroissent un peu en automne, eonstituent les boulons, et restent stationnaires pendant l'hiver. Mais au retour du printemps, ils suivent l'impulsion générale communiquée aux autres parties de la plante; ils se dilatent, se gonflent; leurs éeailles s'éeartent et laissent sortir les organes qu'ils protégeaient. C'est alors qu'on les appelle proprement des bourgeons.

Les éeailles, qui eonstituent la partie la plus extérieure des bourgeons, n'ont pas toutes une même nature, une même origine. Le seul point commun de ressemblanee qu'elles aient entre elles, e'est de n'ètre jamais que des organes avortés et imparfaits. Ainsi quelquefois ce sont des feuilles, des pétioles, des stipules, qui n'ont point acquis leur entier développement, et qui eependant, dans certaines eireonstanees, s'aeeroissent, se déploient et décèlent ainsi leur véritable nature.

Les bourgeons sont divisés en zus et écailleux. Les premiers sont eeux qui n'offrent point d'éeailles à l'extérieur, c'est-à-dire que toutes les parties qui les composent poussent et se développent. Tels sont eeux de la plupart des plantes herbaećes.

On appelle, au eontraire, bourgeons éccilleux eeux dont la partie externe est formée d'écailles plus ou moins nombreuses, eomme on l'observe dans les arbres de mos climats.

Suivant les organes dont leurs éeailles sont formées; on distingue les bourgeons écailleux en :

I $^{\circ}$ Foliacés (semma foliacece), ceux dont les écailles ne sont que des feuilles avortées, sourent susceptibles de 
se développer, comme dans le bois-gentil (daphne mezereum).

$2^{\circ}$ Pétiolacés (gemmee petiolacece), quand lcurs écailles sont constituécs par la base persistante des pétioles, eomme dans le noyer (juglans regia).

$3^{\circ}$ Stipulacés (gemmee stipulacea), lorsque ce sont lcs stipules qui, 'en se réunissant, enveloppcnt la jcunc pousse, comme on l'obscrve dans le charme (carpinus sylvestris), le tulipicr (briodendrum tulipifera), et surtout certaincs espèces dc figuiers, par cremple, dans le ficus elastica, et d'autres encorc.

$4^{\circ}$ Fulcracés (gemma fulcracea), quand clles sont formées par des pétioles garnis de stipules, comme dans le prunier.

Les bourgeous sont le plus souvcnt visibles à l'extérieur, long-temps avant lcur épanouissement. Il est ccrtains arbres, au contraire, dans lesqucls ils sont eommc engagés dans la substance même du bois, et ne se montrcnt qu'au moment où ils commenccnt à se développer; tels sont les acacias (robinia pseudo-acacia. L.) et beaucoup d'autres Légumineuses.

Les bourgeons peurent être simples, c'est-à-dire ne donner naissance qu'à un seul scion, comme dans lc lilas, le chêne; ou bien composés, c'est-à-dire rcufcrmant plusieurs tiges ou ramcaux, comme ceux des pins.

Selon lcs parties qu'ils rcnferment, on a encore distimgué les bourgeons en : florifêres, foliifềes et mixtes.

$\mathrm{I}^{\circ}$ Le bourgcon florifère ou fructifère (gemma florifera seu fructifera) est colui qui renferme une ou plusieurs fleurs sans fcuilles. Il est en général assez gros, ovoïde et arrondi, eomme dans les poiricrs, les pommicrs, ctc.

$2^{\circ}$ Lc bourgcon foliifère (gemma foliifera) nc rcnferme que des feuilles; tel cst eclui qui terminc la tige du boisgeniil (daplune mezereum).

$3^{\circ}$ Enfin on appelle bourgcon mixte (gemma foliiflori- 
fera) eclui qui contient à la fois des fleurs et des feuilles; eomme dans le lilas.

Les eultivateurs ne se trompent jamais sur la nature d'un bourgeon, qu'ils reconnaissent en général dans les arbres fruitiers, d'après sa forme : ainsi, eclui qui porte fleurs est conique, gonflé; celui qui ne porte que des feuilles, au eontraire, est effilé, allongé, pointu.

\section{§2. Du Turion.}

On donne le nom de turion (turio) au bourgeon souterrain des plantes vivaces; c'est lui qui, en se développant, produit ehaque année les nouvelles tiges. Ainsi la partie de l'asperge que nous mangeons est le turion de la plante de ee nom. La différenee entre le bourgeon proprement dit et le turion, c'est que ee dernier naît constamment d'une raeine vivaee, ou d'un rhizome, e'est-ìdire que son origine est sonterraine, tandis que l'autre naît toujours sur une partie exposée à l'air et à la lumière.

\section{§3. Du Bulbe (I).}

Le bulbe (bulbus) est une sorte de bourgeon appartenant à certaines plantes vivaees, et partieulièrement aux monoeotylédons. Nous avons déjà vu, en parlant des racines bulbifères, qu'il était supporté par une espèce de plateau solide, horizontal, intermédiaire à lui et à la véritable raeine. C'est à ce tubercule aplati que sont fixées par leur base les éeailles charnues qui forment le bulbe à l'extérieur. L'intérieur renferme les rudimens de la liampe et des feuilles. Ces éeailles sont d'autant plus épaisses,

(I) Bulbus, $i$, étant masculin en latin, et tiré d'un mot grec ( $\beta 0 \lambda 60 s$ ) également masculin, nous avons cru devoir lui conserver le même genre cn français. 
cliarnues et suceulentes, qu'on les observe plus à l'intérieur du bulbe; les plus extérieures, au eontraire, sont sèches, minees eł eomme papyraeées.

Tantôt ees écailles sont d'une seule pièee, ct s'emboîtent les unes dans les autres, e'est-à-dire qu'une seule embrasse toute la eireonférenee du bulbe, comme dans l'ognon ordinaire (allium cepa), la jaeinthe (hyacintluzs orientalis). On les nomme alors bulbes en tuniques (bulbi tunicati). ( $V o y \cdot$ pl. 2, fig. 7,7 a.)

D'autres fois ces éeailles sont plus petites, libres par leurs eôtés, et ne se recouvrent qu'à la manière des tuiles d'un toit. Par exemplc, dans le lis (litiun candidum). Ils constituent dans ee eas les bulbes écailleux (bulbi squamosi, imbricati). (Voy.pl. 2, fig. II.)

Enfin quelquefois les tuniques qui eonstituent le bulbe sont tellement serrées et confondues, qu'on ne peut les distinguer, et qu'il paraît formé d'une substance solide et homogène. Ce bulbe porte alors le nom de solide (bulbus solidus). Par exemple dans lc safran (crocus sativas), le colehique (colchicum autumnale), le glaïeul (gladiolus communis ).

C'est iei que nous ferons remarquer le passage insensible du bulbe proprement dit au véritable tubercule. C'est iei que nous trouverons en même temps la preuve et la eonfirmation du principe que nous avons préeédemment ćnoncé, savoir : que les tubercules, regardés pendant si long-temps comme des racines, ne sont que de véritables bourgeons. En effet, personne ne eontestc que l'on ne doive regarder eomme des bourgeons les bulbes à tuniques et les bulbes écailleax, même les bulbes solides du. colehique, du safran, ete. Or, nous le demandons, quelle différenee y a-t-il entre ees bourgeons solides et les deux tubercules des Orchidées, ou ceux de la pomme de terre? Si, darus un cas, l'on a appliqué un nom à l'un de ces organes, 
pourcquoi en domnerail - on un autre à une partie absolument analogue par sa strueture et ses usages ( 1 )?

Les bulbes ont en général une forme ovoïde ou globuleuse; quelquefois eependant ils sont plus ou moins allongés et comme eylindraeés, ainsi qu'on l'observe dans quelques espèees d'ail. Dans les bananiers le bulbe est très-allongé, cylindrique et en forme de tige. Nous avons déjà di tet prouvé précédeniment quc le stipe des Palmiers, des draccena, yucca, ete., était un véritable bulbe.

Le bulbe est tantòt simple, c'cst-à-dire formé d'un seul corps, comme eelui de la tulipe, de la seille.

Ou bien il est mulliple, e'est-à-dire que sous une même enveloppe on trouve plusieurs petits bulbes réunis, auxquels on donne le nom de caïeux. Par exemple dans l'ail (allium salivım).

Les bulbes, étant les bourgeons de eertaines plantes vivaees, doivent se régénérer elıqque année. Mais eette régénération n'a pas lieu de la même manière dans toutes les espèees. Quelquefois les nouveaux bulbes naissent au centre mème des aneiens, comme dans l'ognon ordinaire (allium cepa); d'autres fois, de la partie latérale de leur' substanee, eomme dans le colchique, l'ornithogalum minimum, ete.; ou bien les nouveaux se développent à côté des aneiens, comme dans la tulipe, la jaeinthe; ou audessus d'cux, dans le glaïeul; ou au-dessous, dans un grand nombre d'ixia, ete.

A mesure qu'un bulbe pousse la tige qu'il renferme, les éeailles extérieures diminuent d'épaisseur, se fanent et finissent par se desséeher entièrement. Elles paraisseut

(1) Dans les bulbes solides, le plateau n'est plus distinct. Ne pourrait-on pas admettre, dans ce cas, que c'est la substance dú plateau qu'on regarde comme un véritable tubercule, qui a pris un accroisscuent extraordinaire, et a recouvert tout le bourgcon? 
donc fournir ì la jeune tige une partie des matériaux néeessaires à son développement.

\section{§4. Des Tubercules.}

Les tubercules (tubercula) sont de véritables bourgeons souterrains, appartenant à eertaines plantes vivaees. Nous ne reviendrons point iei sur ee que nous avons déjà dit touehant la nature des tubereules; -nous ne rapporterons point de nouveau les faits et les raisons qui nous ont déterminé à regarder ees excroissanees charnues comme de véritables bourgeons.

Ils sont tantôt simples, et ne développent qu'une seule tige, comme dans les orehis;

Tantôt multiples, e'est-à-dire plusieurs réunis ensemble et eomme agglomérés, dont ehaeun pousse une tige partieulière, comme dans la saxifrage grenue (saxifraga sranulata);

Tantôt composés, e'est-à-dire que d'un tubereule simple il sort plusieurs tiges, eomme dans la pomme de terre.

\section{Des Bulbilles.}

On nomme bulbilles (bulbilli) des espèees de petits bourgeons solides ou écailleux, naissant sur différentes parties de la plante, et qui peuvent avoir une végétation à part, e'est-à-dire que, détaehés de la plante-mère, ils se développent et produisent un végétal parfaitement analogue à eelui dont ils tirent leur origine. Les plantes qui offrent de semblables bourgeons portent le nom de vivipares (plantce viviparce).

Ils existent, ou bien dans l'aisselle des feuilles, eomme , ceux du lis bulbifère (hilium, bulbiferum). Dans ce eas, ils sont dits axillaires.

D'autres fois ils se développent à la place des fleurs, 
comme dans l'ornithogalum viviparum, l'allium carinatum, ete.

On a dit aussi que les bulbilles pouvaient quelquefois se développer dans lintérieur du périearpe et oeeuper la place des graines. Mais nous avons fait voir ( $A$ nnı. des Sciences nat., i 824 ) que ees prétendus bulbilles ne sont antre ehose que les véritables graines, qui ont acquis, souvent aux dépens du périearpe lui-même, un développement extraordinaire. Mais leur organisation intérieure reste absolument la même.

La nature des bulbilles est semblable à eelle des bulbes proprement dits. Tantôt ils sont écailleux, eomme dans le lilium bulbiferum, tantôt solides et eompaetes.

On doit regarder eomme de véritables bulbilles les petits eorps qui se développent dans différentes parties des plantes agames, telles que les Fougères, les Lyeopodiacées, les Mousses, les Liehens, ete., et que l'on a fort improprement nommés des graines. Quoique ces eorps, que nous nommons sporules, soient suseeptibles de reproduire une plante analogue à eelle dont ils se sont détaehés, on ne peut les eonfondre avee les véritables gräines. En effet, le earaetère essentiel de la graine est de renfermer un cmbryon, e'est-à-dire un corps eomplexe de sa nature, composé d'une radieule ou rudiment des raeines, d'une gemmule ou germe de la tige, et d'un corps eotylédonaire. Par l'aete de la germination, l'embryon proprement dit ne fait que développer les parties qui existaient déjà err lui toutes formées. Ce n'est pas la germination qui leur donne naissanee; elle ne fait que les mettre dans une cireonstanee propre à leur aeeroissement. Dans les bulbilles, au eontraire, et surtout dans les sporules des agames, il n'y a pas d'embryon. Il n'y existe nulle traee de radieule, de eotylédons et de gemmule. C'est la germination qui crée ees parties. Ce ne sont donc pas de véritables graines. 
Usuges des Bourgeons, des Bulbes, eic.

Plusieurs bourgeons sont employés dans l'éeonomie domestique eomme alimens : tels sont, par exemple, les turions de l'asperge et de plusieurs antres plantes de la même famille. Tout le monde connait l'emploi journalier que l'on fait des différentes espèees du genre allium, telles que l'ognon commun (allium cepa), l'ail (allium sativum), le poireau (allium porrum), l'éehalotte (allium ascalonicum), etc.

La thérapeutique emploie aussi les bourgeons ou bulbes de quelques végétaux. Ainsi c'est avee les bourgeons de la sapinette (pinus picea), infușés dans la bịère, que se prépare 'la bière-sapinette. Les squames du bulbe de la scille (scilla maritima) sont un puissant diurétique. On l'emploie également comme excitant de l'organe pulmonaire. L'ail, comme on sait, est un excellent anthelmintique, etc.

\section{CHAPITRE IV.}

DES FEUILLES (I).

Avant leur entier développement, les feuilles sont toujours renfermées dans des bourgeons. Elles y sont diversement arrangées les unes'à l'égard des autres, mais toujours de la même manière, dans toutes les plantes de la même espèce, souvent du même genre, quelquefois même de toute une famille naturelle.

Cette disposition des feuilles dans le bourgeon a reçu

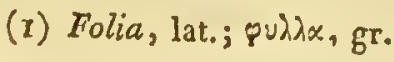


le nom de préfoliation. On peut souvent en tirer de fort bons earactères pour la eoordination des genres en familles naturelles.

Les modifieations prineipales des feuilles ainsi disposées sont les suivantes :

$1^{\circ}$ Elles peuvent être pliées en longueur, moitié sur moitié, c'est-à-dire que leur partie latérale gauelie est appliquée sur la droite, de manièrc que leurs bords se correspondent parfaitement de chaque eôté, comme daus le syringa (Philadelphus coronarius).

$2^{\circ}$ Elles peuvent être pliées de haut en bas, plusieurs fois sur elles-mêmes, comme dans l'aconit (A conitum napellus).

$3^{\circ}$ Elles peuvent être plissées, suivant leur longueur, de manière à imiter les plis d'un éventail, comme celle des groseillers, de la vigne, etc.

$4^{\circ}$ Les feuilles peuvent être roulées sur elles-mêmes en. forme de spirale, comme dans eertains figuiers, dans l'abricoticr, ete.

$5^{\circ}$ Leurs bords peuvent être roulés en delıors ou en dessous : telles sont eelles du romarin.

$6^{\circ}$ D'autres fois ils sont roulés en dedans ou en dessus, comme eelles du peuplier, du poirier, ete.

$7^{\circ}$ Enfin les feuilles peuvent être roulées en crosse ou cn volute; e'est ee qui a licu, par exemple, dans toutes les plantes de la famille des Fougères.

Étudions maintenant les feuilles quand clles se sont développées.

Les FEurLess sont des organes ordinairement membraneux, planes, verdàtres, horizontaux, naissant sur la tige et les rameaux, ou partant inmédiatement du eollet de la racine. Par les pores nombreux qu'elles présentent à leurs surfaees, les feuilles servent à l'absorption et à l'exhalation des gaz propres ou devenus inuliles à la nutrition du végétal. 
Les feuilles semblent formées par l'épanouissement d'un faiseeau de fibres provenant, de la tige. Ces fibres, qui sont des raisseaux, en se ramifiant diversement, constituent une sorte de rćseau, qui représente en quelque manière le squelette de la feuille, et dont les mailles sont remplies par un tissu cellulaire, plus ou mnins abondant, qui tire son origine de l'enveloppe herbaeée de la tige.

Lorsque le faisčeau de fibres caulinaires, qui, par son épanouissement, doit constituer la feuille', se divise et se ramifie aussitôt qu'il se sépare de la tige, la feuille lui est alors attachée sans le secours d'aucun support particulier, et 'est désignée sous le nom de feuille sessile (folium sessile), comme dans le pavot.

$\mathrm{Si}$, au contraire, ce faisceau se prolonge avant de s'étendre en membrane, il forme alor's une espèee de pédicelle, nommé communément queue de la feuilile, et auquel on domne, en botanique, le nom de pétiole (petiolus). Dans ce cas, la feuille est dite pétiolée (folium petiolatum ); par exemple, dans le tilleul, le tulipier, le marronier d'Inde, etc.

Cette disposition étant la plus générale, on peut eonsidérer la feuille comme formée de deux parties; savoir, le pétiole et le disque ou limbe, c'est-à-dire eette partie le plus souvent plane et verdâtre, qui constitue la feuille proprement dite.

De même que le pétiole manque dans un grand nombre de feuilles, de même aussi le limbe lui-même avorte et la feuille ne se eompose alors que du pétiole, qui souvent se dilate et prend la forme et les caraetères d'une feuilie sessile. C'est ce que l'on observe, par exemple, dans toutes les espèces d'aeacia à feuilles simples de la NouvelleHollande; il cst même probable que, dans les buplevrum, les feuilles ne sont que des pétioles. On leur a donné le nom de phyllodes. 
On distingue à la feuille une faee supérieure ordinairement plus lisse, plus verte, eouverte d'un épiderme plus adhérent et offrant moins de pores eorticaux; une faee inférieure, d'une eouleur moins foneée, souvent eouverte de poils ou de duvet, dont l'épiderme est plus lâehement uni à la eouehe lierbaeée, présentant un grand nombre de petits pertuis, qui sont les orifiees des vaisseaux intérieuis ciu végétal. Aussi est-ee surtout par leur face inférieure queles feuilles absorbent les fluides qui s'exhalent de la terre, ou qui sont répandus et mêlés dans l'atmosphère.

On distingue aussi dans la feuille : sa base, ou la partie par laquelle elle s'attaehe à la tige; son sommet, ou le point opposé à la base; sa circonférence, ou la ligne qui détermine extérieurement sa surface.

La face inférieure de la feuille est eneore remarquable par un grand nombre de prolongemens saillans disposés en divers sens, qui ne sont que des divisions du pétiole, et qu'on appelle nervures (nervi).

Parmi les nervures, il en est une qui offre une disposition presque eonstante. Elle fait suite au pétiole, offre ordinairement une direetion longitudinale, et divise la feuille en deux parties latérales assez souvent égales entre elles. Elle a reẹu le nom de cốte ou nervure médiane. C'est de sa base et de ses partics latérales que partent en différens sens, et en s'anastom sant fréquemment entre elles, les autres nervures.

Suivant leur épaisseù et la saillie qu'elles forment à la face inférieure de la feuille, les nervures prennent différens noms. Elles eonservent eelui de nervures proprement dites (nervi) quand elles sont saillantes et très-prononcées: on les appelle veines (vence), lorsqu'elles le sont moins; enfin les dernières ramifieations des veines, qui s'anastomosent fréquemment, et constituent, à proprement parler, le squelette de la feuille, sont appelées veinules (venuloe). 
Les nervures, malgré la ressemblance de leur nom, n'ont aucune analogie de structure ou d'usage avec lesnerfs des animaux. Ce sont des faisceaux de vaisseaux poreux, de trachées et de fausses trachées, enveloppés d'une certaine quantité de tissu cellulaire.

Quelquefois les nervurres se prolongent au-delà de la circonférence du disque de la feuille, et forment àlors, quand elles ont une certaine rigidité, des épines plus ou moins acérées, comme on le voit, par exemple, dans le houx (Ilex aquifolium).

La disposition des nervures sur les feuilles mérite la plus grande attention. En effet, elle peut servir à caractériser certaines divisions des végétaux. Ainsi, par exemple, dans la plupart des Monocotylédons, les nervures sont presque toujours simples, peu ramifiées, et souvent parallèles entre elles (I). Dans les Dicotylédons, elles peuvent offrir cette disposition; mais elles sont le plus fréquemment très-ramifiées et anastomosées entre elles.

On peut rapporter aux suivantes les variétés les plus remarquables de la disposition des nervures :

$I^{\circ}$ Les nervures peuvent partir toutes de la base de la feuille, et se diriger vers son sommet, sans éprouver de division sensible : par exemple, dans un grand nombre de plantes monocotylédonées.

Les feuilles qui présentent une semblable disposition sont appelées feuilles basinerves ou digitinerves (folia basinervia, digitinervia).

$2^{\circ}$ Quand, au contraire, les nervures naissent des côtés de la nervure médiane, et se dirigent, soit horizontalement, comme dans le bananier (Musa paradisiaca), soit obliquement vers son sommet, comme dans l'Amomum Zerumbet, les feuilles prennent le nom de laté-

(I) Les Aroidées et certaines Asparaginées font exception à cette règle presque constante. 
rinerves ou penninerves (folia lalerinervia, penninervia).

$3^{\circ}$ Enfin, si les nervures naissent à la fois de la base et des partics latérales de la nervure médiane, les feuilles sont dites alors mixtinerves (folia mixtinervia), comme on l'observe dans beaneoup de Nerpruns.

Toutes les autres dispositions que les nervures des feuilles sont suseeptibles d'offrir peuvent se rapporter à quelqu'un des trois types prineipaux que nous venons d'établir, ou n'en sont que de légères modifieations.

Une feuille, sessile ou pétiolée, peut être fixée de différentes manières à la tige ou aux branehes qui la supportent. Quelquefois elle y est simplement articulée, e'est-àdire qu'elle ne fait pas immédiatement eorps avee elles par toute sa base, mais y est simplement fixée par une sorte de rétréeissement ou d'artieulation, eomme dans le platane, le marronier d'Inde. Ces feuilles sont alors caduques, ct tombent de très-bonne heure.

D'autres fois la feuille est tellement unie à la tige, qu'elle ne peut s'en séparer sans déchirure. Dans ee cas ees feuilles persistent aussi long-temps que les branehes qui les supportent, comme dans le lierre, ete.

La manière dont les feuilles sessiles sont attaehées à la tige mérite également d'être étudiée.

Ainsi, quelrquefois la nervure médiane s'élargit, et embrasse la tige, dans environ la moitić de sa eirconférenee. Les feuilles sont alors appelées semi-amplexicanles (folic semi-amplexicaulia).

On dit au eontraire de la feuille qu'elle est amplexicaule (folium amplexicanle) quand elle embrasse la tige dans toute sa eireonférenee; par exemple, dans le salsifis sauvage (Tragopogon pratense), le pavot blane (Papaver somnifertum), cte.

Souvent eneore la base de la feuille se prolonge en formant une gaine qui circonscrit entièrement la tige et l'en- 
FEUILLES.

veloppe dans une eertaine longueur. Dans ee cas, ees feuilles sont nommées engainnantes (fol:a vaginantia), comme dans les Graminées, les Cypéraeées, ete. Cette gaine peut être regardée eomme un péliole très-ćlargi, dont les deux bords se sont quelquefois soudés pour former une espèee de tube. Le point de réunion du limbe de la feuille et de la gaîne a reȩu le nom de collet. Tantôt il est nu, tantôt garni de poils, eomme dans le Poa pilosa, ou d'un petit appendiee membraneux nommé ligule ou collure; e'est ce que l'on observe prineipalement dans les Graminées. La forme de la ligule est très-variée dans les différentes espèees, et fort souvent elle est employée comme un bon earaetère spéeifique.

La gaîne est ordinairement entière; d'autres fois elle est fendue longitudinalement; ee caraetère distinguie, à très-peu d'exceptions près, la famille des Graminées de eelle,des Cypéracées; les premières ayant, en général, la gaìne fendue, tandis qu'elle est entière dans les Cypéraeées.

Quelcquefois le limbe de la feuille, au lieu de se terminer à son point d'origine sur la tige, se prolonge plus ou moins bas sur eet organe, où il forme des espèees d'ailes membraneuses. Dans ee eas, les feuilles sont dites décurrentes (folia decurrentia) et la tige est appelée ailée (caulis alatus), eomme dans le bouillon blane (Verbascum Thapsus), la grande consoude (Symphytum officicinale), etc.

On nomme feuille perfoliée (folium perfoliatum) eelle dont le disque est en quelque sorte traversé par la tige, comme dans le Bupleurum rotundifolium, ete. (Voyez pl. 3 , fig. Ir.)

On a donné le nom de feuilles connées ou conjointes (folia connata, coadnata) anx feuilles opposćes qui se réunisscnt ensemble par lcur base de manière que la tige passe au milieu de leurs limjes soudés. Telles sont les 
feuilles supérieures du elièvrefeuille (Lonicera caprifolium), eelles du ehardon à foulon (Dipsacus fullonum), de la saponaire (Saponaria officinalis). (Voyez pl. 3, fig. Iо.)

On appelle feuille simple (fo?ium simplex) eelle dont le pétiole n'offre aueune division sensible, et dont le limbe est formé d'une seule et même pièee; par exemple, le lilas, le tilleul, l'orme, etc. (Voy. toutes les fig. de la pl. 3.)

La feuille composée, au eon traire (folium compositum), résulte de l'assemblage d'un nombre plus ou moins eonsidérable de petites feuilles isolées et distinetes les unes des autres, qu'on appelle folioles, toutes fixées ou rémies sur les parties latérales, ou au sommet d'un péliole commun, qui, dans le premier eas, porte le nom de rachis. Chaque foliole peut être sessile sur le rachis, e'est-à-dire attaehée par la base seulement de sa nervure moyenne; ou bien elle peut être portée sur un petit pétiole particulier, qui prend le nom de pétiolule. Telles sont les feuilles de l'aeaeia, du marronier d'Inde, ete. ( $V_{\text {oy }}$. les fig. de la pl. 4.).

On distingue les feuilles composées', en articulées, et en non articulées. Les premières sont eelles dont les folioles sont fixées au péliole commun, au moyen d'une sorte d'artieulation, suseeptible de mobilité, eomme on l'observe dans l'aeaeia, les easses, et en général dans la plupart des plantes de la famille de Légumineuses. Cesont les seules dans lesquelles ait lieu le phénomène que LinNaUs désigne sous le nom de sommeil des feuilles, les autres, qui sont privées d'artieulations, ne le présentant pas.

Entre la feuille simple et la feuille composée il existe une série de modifieations qui servent en quelque sorte à établir le passage insensible de l'une à l'autre. Ainsi il y a d'abord dès feuilles dentées; d'autres qui sont divisées 
jusqu'à la moitié de leur profondeur en lobes distinets; d'antres enfin dont les ineisions parviennent presque jusqu'à la nervure médiane, et simulent ainsi une feuille eomposée. Mais il sera toujours faeile de-les bien distinguer de la feuille vraiment composée, en remarquant que dans eelle-ei on pourra détacher ehaeune des pièees dont elle est formée sans endommáger aueunement les autres; tandis que dans une feuille simple, quelque profondément divisée qu'elle soit, la partie foliaeée, oul le limbe de chaque division, se eontinue à sa base avee les divisions voisines, en sorte qu'on ne peut en séparer une sans déchirer les deux autres, entre lesquelles elle se trouve plaeée (I).

Toutes les feuilles d'une plante ne présentent pas toujours une forme parfaitement semblable. Il y a même à eet égard, dans eertains végétaux, une différenee des plus marquées. Ainsi tout le monde a dû observer que le lierre (hedera helix) offre des feuilles entières, et d'autres qui sont profondément lobées. En général, les plantes qui ont des feuilles partant immédiatement de la raeine, et d'autres naissant des différens points de la tige, les ont rarement semblables. La valériane phu a les feuilles radicales déeoupées latéralement, tandis que les feuilles de sa tige sont entières.

Les feuilles varient eneore suivant le milieu dans lequel elles végètent. Les plantes aquatiques ont ordinairement deux espèces de feuilles; les unes nageant à la surface de l'eau, ou un peu élevées au-dessus de son niveau; les autres, au eontraire, eonstamment plongées dans ee liquide. Ainsi, par exemple, la renoneule aquatique (ranunculus

(1) On peat encore reconnaître unc fenille composée cn ce que chacunc de ses folioles a une base rétrécic, ct ne, s'attache au rachis que-par sa nervure moyenne ou le pétiolc qui le continue; tandis qu'une feuille simple, même profondément divisée, s'y attache toujours par une portion plus ou. moins large de sa partic foliacée. 
aquatilis) a des feuilles lobées qui surnagent, et des feuilles divisées en lanières extrêmement étroites et très-nombrenses, plongées dans l'eau. Il en est de même d'un grand nombre d'autres plantes analogues.

Nous allons eonsidérer nuaintenant les nombreuses modifications de forme, de direction, de nature, ete., que peuvent présenter la feuille simple et la feuille composéc.

\section{\. De la feuille simple.}

\section{A. Relativement au lieu d'où elles naissent, les feuilles sont :}

I Séminales (folia seminalia), quand elles sont formées par le développement du eorps eotylédonaire. D'après cela, on voit qu'il peut en exister une ou deux,

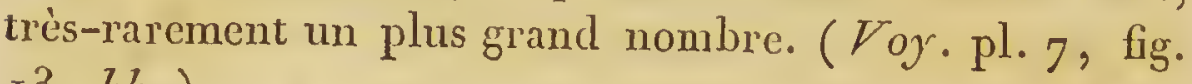
$x 3,66$.

$2^{\circ}$ Primordiales (fol. primordialia); ee sont les premières qui se développent après les feuilles séminales. Elles sont formées par les deux folioles extérieures de la gemmule. (Voy. pl. 7 , fig. $\mathrm{x} 3, d d$. )

$3^{\circ}$ Radicales (fol. radicalia), celles qui naissent immédiatement du eollet de la racine, comme dans le plantain (plantago major), le pissenlit (taraxacum dens leoris), etc.

$4^{\circ}$ Caulinaires ( $f o l$. caulinaria), celles qui sont fixées sur la tige.

$5^{\circ}$ Ramaires (fol. rancalia, ramea), quand elles naissent sur les rameaux.

$6^{\circ}$ Florales (fol. floralia), eelles qui aceompagnent les fleur's et sont placées à leur base, mais qui n'ont pas changé de forme ni de nature, eomme dans le elièrefeuille. Quand la forme des feuilles florales difiere beaucoup de celles des autres feuilles, elles portent alors le 
nom de bractées. Nous parlerons bientôt des bractées, en traitant des organes floraux.

B. Suivant leur disposition sur la tige ou les rameaux, elles sont :

I Opposées (fol. opposita), disposées une à une à la même hauteur sur deux points diamétralement opposés de la tige; comme dans la sauge (Salvia officinalis) et toutes les Labiées, la véronique (Veronica officinalis), etc.

On dit des feuilles qu'elles sont opposées en croix (cruciatim opposila, s. deczssata), quand les paires de feuilles superposées se croisent de manière à former des angles droits, comme dans l'épurge (Euphorbia lathyris).

$2^{\circ}$ Alternes (fol. alterna), naissant, seule à seule, en échelons et à des distanees à peu près égales, sur différens points de la tige, comme dans le tilleul (Tilia enropara).

3० Eparses (fol. sparsa), quand elles n'affectent aucune disposition régulière, et qu'elles sont en quelque sorte dispersées sans ordre sur la tige, comme dans la linaire (Linaria vulgaris), etc.

$4^{\circ}$ Verticillées (fol. verticillata), lorsqu'elles naissent pł̣us de deux à la même hauteur, autour de la tige, ou sur les rameaux, comme dans le laurier-rose (Nerium oleander), la garance (Rubia tinctorum), ete.

Suivant le nombre des feuilles qui forment chaque verticille, on dit qu'elles sont:

Ternées ( $f o l . t e r n a)$, quand le vertieille est formé de trois feuilles, eomme dans la verveine à odeur de citron (Verbena triphylla), le laurier-rose, etc.

Qua!ernées (fol. quaterna), quand le vertieille est composé de quatre feuilles; par exemple, dans la eroisette (Valantia cruciatci).

Quinées (fol. quina), vertieille de einq feuilles : plusieurs caille-laits, le Myriopiryllum verlicillatum.

Senées ( $\int u l$ senci), verticille de six feuilles, comme dans le Galium uliginosum. 
Octonées (fol. octona), verticille de huit feuilles : par exemple, celle de l'aspérule odorante (Asperula odorata).

$5^{\circ}$ Géminées (fol. gemina), naissant deux à deux, l'une à eôté de l'autre, du même point de la tige. Les feuilles supérieures de la belladone (Atropa Belladona), de l'alkekenge (Plyssalis Alkekengi).

$6^{\circ}$ Distiques (fol. disticha), disposées sur deux rangs opposés l'un à l'autre, comme dans l'orme (Ulmus campestris), le laurier-eerise (Cerasus lauro-cerasus).

$7^{\circ}$ Unilatérales ( $f o l$. unilateralia), quand elles sont tournćes toutes d'un seul et même côté; par exemple, le Convallaria multiflora, ete.

$8^{\circ}$ Ecarlées (fol. remota), quand elles sont très-éloignées les unes des autres.

$9^{\circ}$ Rapprochées (fol. approximata, conferta), naissaut à une très-petite distance les unes des autres.

( Ces deux expressions ne s'emploient jamais isolément; elles servent toujours à exprimer une eomparaison avec d'autres espèces connues.)

${ }^{0}$ Imbriquées (fol. imbricata), quand elles se recouvrent en partie, à la manière des tuiles d'un toit, comme dans certaines espèees d'aloës, les ThuyYa, etc.

On dit des feuilles imbriquées qu'elles sont bisériées, quand elles sont disposées sur deux lignes longitudinales.

Trisériées (fol. triseriata), disposées sur trois rangées longitudinales.

Quadrisérices (fol. quadriseriata), formant quatrè séries.longitudinales; telles sont eelles du thuya.

Enfin on dit qu'elles sont imbriquées de tous côtés, quand elles n'offrent aueun ordre régulier.

I ${ }^{\circ}$ Fasciculées (fol. fasciculata), naissant plus de deux ensemble du mème point de la tige, eomme dans le ce-isier (Cerasus communis), le mélèse (Larix vulgaris), l'épine vinette (Berberis vulgaris), etc.

I $2^{\circ}$ Couroniantes (fol. coronantia, terminantia), réul- 
nies en forme de bouquet, au sommet de la tige; eomme dans les Palmiers, le papayer (Carica Papaya).

I $3^{\circ}$ Roselées ou en rosette (fol. rosulata), alternes et rapprochées en forme de rosaee, comme dans la joubarbe (Sempervivum tectorum), le pissenlit, etc.

C. Quant à leur direetion relativement à la tige, les feuilles sont :

$x^{\circ}$ Dressées ( fol. erecta), formant un angle très-aigu avee la partie supérieure de la tige, comme dans la massette (Typha latifolia).

$2^{\circ}$ Apprinées (fol. adpressa), quand le limbe de la feuille est appliqué sur la tige.

3० Etalées ou ouvertes (patentia), quand elles forment avec la tige un angle presque droit, comme dans le lierre terrestre (Glechoma hederacea), l'androsème (Hypericumn androscemum), etc.

$4^{\circ}$ Infléchies (fol. inflexa), quand elles sont fléehies en dedans, eomme eelles de plusieurs Malvacées.

$5^{\circ}$ Involutées (fol. involuta), lorsqu'elles sont roulées en dedans; telles sont celles des Fougères.

$6^{\circ}$ Réfléchies (fol. reflexa), celles qui sont rabattues brusquement en dehors, eomme dans l'Inula pulicaria, le Dracana reflexa, etc.

$7^{\circ}$ Révolutées (fol. revoluta), roulées en dehors.

$8^{\circ}$ Pendantes (fol. pendentia), celles qui s'abaissent presque perpendiculairement vers la terre, comme dans le liseron des haies (Convolvnlus sepium), le daphné lauréole (Daphne laureola).

$9^{\circ}$ Inverses (fol. inversa), quand le pétiole se tord de 1 manière que la faee inférieure devicnt supérieure, eomme (dans le Pharus.

10 ${ }^{\circ}$ Thumifuses (fol. humifusa), quaid elles sont radicales, molles et étalées sur la terre, commc dans la paquereite (Bellis perennis). 
I I $^{\circ}$ Nageantes (fol.natanlia), se soutenant sur l'eau, le némuphar (Nymphaca alba).

$12^{\circ}$ Submergées (fol. submersa, demersa), cacliées sous l'eau; celles de l'Hottonia palustris.

I $3^{\circ}$ Emergées (fol. emer'sa), quand leur point d'attache est sous l'eau, et que leur pétiole les élève au-dessus du liquide, comme celles du plantain d'eau (Alisma Plantago), de la sagittaire (Sagittaria sagittcefolia).

\section{Circonscription ou figure.}

I Orbiculées (fol. orbiculata) celles dont la circonférence approche de la figure d'un cercle, comme l'écuelle d'eau (Hydrocotyle vulgaris). (Voy• pl. 3, fig.9.)

$2^{\circ}$ Ovales (1) ( fol. ovalia), allongées, arrondies aux dcux extrémités, l'extrémité inféricure étant plus large. Exemples: l'aunée (Inula helenium), le mouron des oiseaux (Alsine media), la grande pervenche'( Vinca major ). ( Voy. pl. 3, fig. I.)

$3^{\circ}$ Obovales (2) (fol. obovalia), la précédente renversée, c'est-à-dire que la grosse extrémité est tournée en haut, comme dans la busserole (Arbutus uva ursi), le Samolus valerandi, etc.

$4^{\circ}$ Elliptiques (3) (fol. elliptica), allongées, les deux bouts arrondis et égaux entre eux, comme dans le muguet (Convallaria maialis). (Voy. pl. 3, fig. 2.)

$5^{\circ}$ Oblongues (oblonga), elliptiques très-allongées et étroites.

$6^{\circ}$ Lancéolées (fol. lanceolata), oblongues et finissant insensiblement en pointe vers le sommet : Plantago lan-

(I) La figure ovale est celle qu'on obtient par la section oblique d'un còne.

(2) Obovalia, par abréviation de obversè ovalia.

(3) I a figure elliptique est celle que l'on obtient par la section oblique d'un cylindre. 
ceolata, le laurier-rose (Nerium oleander), le pêeluer (Amygdalus persica).

$7^{\circ}$ Linéaires (fol.linearia), lancéolécs, mais étroites; la plupart des Graminées.

$8^{\circ}$ Rubanaires ou en ruban (fol. fasciaria, graminea), un peu plus larges que les précédentes, mais bien plus allongéés : la Valisneria spiralis, le Typha latifolia.

$9^{\circ}$ Subulées ou eñ alène (fol. subulata), très-étroites à leur base, et rétrécies insensiblement en une pointe aiguë au sommet : le genévrier (Juniperus communis).

ro ${ }^{\circ}$ Aciculées et sétacées ( fol. acicularia, setacea), allongées, roides et aiguës, ayant quelque ressemblance avec des aiguilles ou des soies de cochon; par exemple, celles de l'Asparagus acutifolius, etc.

I ${ }^{\circ}$ Capillaires ( $f o l$. capillaria); déliées et flexibles comme des cheveux: celles de l'asperge (Asparagus officinalis), etc.

I $2^{\circ}$ Filiformes (fol. filiformia), minces, grêles, trèsdéliées comme un fil. Exemple, la renoncule aquatique (Ranunculus aquatilis).

I $3^{\circ}$ Spatulées ou en forme de spatule (fol. spatulata), minees, étroites à la base, larges et arrondies à leur sommet : la paquerette (Bellis perennis). (Voy. pl. 3, fig. 3.)

I $4^{\circ}$ Cunéaires, ayant la figure d'un coin (fol. cuneata), très-étroites à la base, s'élargissant jusqu'au sommet, qui est comme tronqué. Exemple : le Saxifraga tridentata, elc. (Voy. pl. 3, fig. I 2.)

I $5^{\circ}$ Paraboliques (fol. parabolica), oblongues, arrondies du haut, et comme tronquées du bas.

I6 Falquées (fol.falcata), ou enfer de faux: Bupleurum falcatum, etc.

i $7^{\circ}$ Inéquilatères ( fol. incequilatera), quand la nervure médiane partage la feuille en cleux moitiés inéigales : par exemple, dans le tilleul, le Begonia obliqua, etc. 
$E$. Les feuilles peuvent être diversement échanerées à Jeur base, ec qui leur domne des figures variées. Ainsi on dit qu'clles sont :

$I^{\circ}$ Cordées, ou en cour, ou cordiformes (fol. cordata, cordiformia), quand elles sont écliancrécs à leur basc de manière à représenter deux lobes arrondis; et qu'elles se terminent supérieurcment en s'amincissant, éomme dans le Tamus communis, lc nénuphar.(Nymphoea alba), ete. ( Voyez pl. 3, fig. 4, 5.)

Les feuilles cordiformes peuvent être en même temps obliques ou inéquilatères (obliquè cordata), comme dans lc tilleul, etc.

$2^{\circ}$ Rénaires ou réniformes, en forme de rein (reniformia), quand elles sont beaueoup plus larges que hautes, et sont arrondics au sommet, et éehancrées en eocur à la base; par excmple: l'asaret ( $A$ sarzm enropceum), le lierre terrestre (Glechoma hederacea). (Voy. pl. 3, fig. 6.)

$3^{\circ}$ Lunulées ou en croissant ( fol. lunata), arrondies et divisées à leur base en deux lobes étroits.

$4^{\circ}$ Sagittées ou en fer de fièche (fol. sagiltata), quand elles sont aiguës, et quc leur base est prolongéc en deux lobes pointus, peu divergens. Exemple: la sagittaire ( $\mathrm{Sa}$ gittaria sagittafolia). (Voy. pl. 3, fig. 7.)

5. Hastées (fol. hastata), à base prolongée cn deux lobes aigus, très-ćeartés et rejetés en dehors, eomme dans l'A rum maculatum, cte. (Voy. pl. 3, fig. 8.)

F. Les feuilles peurent être terminées de diverses manières à leur sommet. De là elles prennent les noms de:

I $^{\circ}$ Aiguës ( fol. acula), quand clles s'amincissent insensiblement en pointe à leur sommet, eomme eclles du laurier-rose. ( Voy. pl. 3, fig. 4, 7.)

$2^{\circ}$ Piquantes (fol. pungentia), terminées par unc 
pointe roide, comme dans le landier (Ulex eliropceus), lo petit houx (Ruscus aculeatus).

$3^{\circ}$ Acuminées (fol. acuminata), quand, vers le sommet, lcurs deux bords changent de direction, ct se prolongent en se rappoehant, comme dans le eoudrier ( $C_{O}$ rylus Avellana), le eornouiller (Cormus mascula).

$4^{\circ}$ Mucronées (fol. mucronata), surmontécs d'une petite pointe, grêle ct isolée, qui ne paraît pas faire suite au sommet de la feuille : dans la joubarbe des toits (Sempervivun tectorum).

$5^{\circ}$ Uncinées ( fol. uncinata), terminées par une pointe recourbée en eroehet.

$6^{\circ}$ Obtuses ( fol. obtusa), terme général mis en opposition à celui de fcuilles aiguës : comme eelles du Nymphaea alba, ete. (Voy.pl. 3, fig. I, 2, 5.)

$7^{\circ}$ Echancrées (fol. emarginata), offrant à leur sommet un sinus.rentrant en forme de crénelure, comme le Buis (Buxus sempervirens), l'asaret (Ásarumsuropaun). (Voy.pl. 3, fig.6.)

$8^{\circ}$ Rétuses ( fol. reíusa), offrant un sinus peu profond, comme la busserole ( $V$ accinium vilis idcea).

$9^{\circ}$ Obcordées ( $f \circ$. obcordaia) (1), en eour renversé. Les folioles de l'alléluia (Oxalis acetosella).

Io ${ }^{\circ}$ Bifides ( fol. apice bifida), fendues au sommet en deux lanières aiguës, peu profondes.

I I Bi,lobćes (fol. apice biloba), quand les deux divisions sont séparées par un sinus obtus.

I $2^{\circ}$ Bipartites (fol. apice bipartita), quand les deux divisions sont très-profondes et aiguës.

$G$. Les feuilles peuvent offrir, dans leur contour, des angles plus ou moins nombreux, plus ou moins maŕqués,

(I) Obcordata. Ce mot est employé par abréviation pour obversè gordata. 
ee qui leur donne des figures particulières; ainsi on les appelle :

I Rhomboïdales (fol. rhomboïlea), quand elles présentent quatre angles, dont deux opposés plus aigus. Exemple : Campanula rhomboüdalis, ete.

$2^{\circ}$ Deltoïdes ( fol. deltö̈dea), quand elles ont la figure d'un rhomboïde, dont l'angle inférieur est très-eourt, en sorte qu'elles paraissent eomme triangulaires, ou approchant de la forme du delta des Grecs $(\Delta)$. Exemple : le Mesembryanıtiemum delloüdes.

30 Trapézoüdes (fol. trapezoïdea), ayant la figure d'un trapèze, e'est-à-dire d'un quadrilatère dont les quatre côtés sont inégaux. Par exemple, plusieurs Fougères.

$4^{\circ}$ Triangulées (fol. triangulata), oflrant trois angles saillans.

$5^{\circ}$ Quadrangulées (fol. quadrangulata).

H. Les feuilles simples, comme nous l'avons dit préeédemment, peuvent offrir des incisions' plus on moins profondes, sans pour eela devoir être considéréés comme composées. Ainsi elles peuvent être :

- Trifides (fol. trifida),

$2^{\circ}$ Quadrifides (fol. quadrifida),

$3^{\circ}$ Quinquéfides (fol. quinquefida),

$4^{\circ}$ Sexfides (fol. sexfida),

$5^{\circ}$ Multifides (fol. mullifida),

quand elles présentent trois, quatre, einq, six ou un plus grand nombre de divisions étroites et peu profondes.

$6^{\circ}$ Trilobées (fol. trilobata),

$7^{\circ}$ Quadrilobées (fol. quadrilobata),

$8^{\circ}$ Quinquélobées (fol. quinquelobata),

$9^{\circ}$ Mrultilobées (fol. multilobata),

lorsque les divisions sont plus larges, et séparées par des sinus obtus. 
Io $0^{\circ}$ Tripartites (fol. tripartita), (Voy. pl. 3, fig. I5.)

$\mathrm{II}^{\circ}$ Quadripartites (fol. quadripartita),

I $2^{\circ}$ Quinquépartites (fol. quinquepartita), (Voy.pl. 3, fig. I6.)

I $3^{\circ}$ Multipartites (fol. multipartita),

si les incisions sont assez profondes pour arriver jusqu'aux deux tiers au moins du limbe de la feuille.

I $4^{\circ}$ Laciniées ( fol. laciniata), celles dont les divisions sont profondes et manifestement inégales, comme dans bęaucoup de Synanthérées. (Voy. pl. 3, fig. I4.)

I $5^{\circ}$ Palmées (fol. palmata), quand toutes les nervures, partant en rayonnant du sommet du pétiole, se dirigent chacune vers le milieu des divisions, comme dans le ricin (Ricinus communis). (Voyez pl. 3, fig. i6.)

$16^{\circ}$ Auriculées (fol: auriculata), offrant à leur base deux petits appendices qu'on nomme oreilletles, comme dans la sauge officinale (Salvia officinalis), la scrofulaire aquatique (Scrophnlaria aqnatica), etc.

I $7^{\circ}$ Pandurées ou Panduriformes (fol. pandurata, panduriformia), approchant de la figure d'un violon, c'est-à-dire allongées, arrondies aux deux extrémités et présentant deux sinus latéraux rentrans; par exemple, dans le Convolvulus panduratus, le Rumex pulcher, etc:

I $8^{\circ}$ Sinuées ( fol. sinuata), quand elles présentent une ou plusieurs échancrures arrondies, ou sinus en nombre déterminé.

r $0^{\circ}$ Sinueuses ( fol. sinuosa), présentant des sinus arrondis et des saillies également arrondies et convexes, en nombre indéterminé : dans le chêne ( Quercus robur ).

$20^{\circ}$ Pinnatifides (fol. pinnatifida), diviséeslatéralement en lobes plus ou moins profonds, comme dans le Polypodium vulgare, le Coronopus Ruellii.

$2 \mathrm{r}^{\circ}$ Interrompues ( fol. interruptè-pinnatifida); ce sont celles dont les divisions supérieures sont confluentes par leur base, tandis que les inférieures sont entièrement li- 
bres; en sorte que ees feuilles représentent supéricurement une feuille pinnatifide, et inférieurement une feuille pinnée. Mais on ne peut les eonfondre avec les feuilles vraiment eomposées.

I $2^{\circ}$ Pectinées ou en forme de peigne (fol. pectinata), feuilles pimnatifides, dont les divisions sont étroites, rapproehées 'et presque parallèles. Par exemple, dans l' $\boldsymbol{A}$ chillaca pectinata.

$23^{\circ}$ Lyrées (fol. Lyrata), feuilles pinnatifides, terminées par un lobe arrondi, beaucoup plus eonsidérable que les autres, eomme dans la benoite (Geum urbanum); le radis sauvage (Raphanus Raphanistrum), etc. (Voyez pl. 3, fig. x/.)

$24^{\circ}$ Roncinées (fol. runcinata), feuilles pinnatifides, dont les lobes latéraux sont aigus et reeourbés en bas. Par excmple, celles du pissenlit (Taraxacum dens leonis ), du Prenanthes muralis, etc. (Voy. pl. 3, fig. x3.)

I. Quant à leur contour, ou aux modifieations que présente leur bord même, les feuilles sont :

$I^{\circ}$ Entiëres (integra), quand leur bord se continue sans présenter ni dents, ni ineisions, ni sinus. Exemple: la pervenehe (Vinca major), le lilas, ete. (Voy.pl. 3, lig. $2,3,4,5$.)

$2^{\circ}$ Erodées (fol. erosa), présentant de petites dentelures inégales, en sorte que le bord de la feuille semble avoir été rongé par un inscete, eomme celles du Sinapis alba, etc.

$3^{\circ}$ Crénelées (fol. crenata), dont le bord offre des crénelures ou petites parties saillantes, arrondies, séparées par des angles rentrans. Par exemple, dans le lierre terrestre (Glecoma hederacea), le marrube blane (Marnibium vulgare), la betoine (Benotica officinalis ).

$4^{\circ}$ Doublement crénelées (fol. duplicato-crenata), quand chaque crénelure principale en offre de plus petites, 
comme dans le Chrysosplenium alternifernium, et l'Hydrocotyle vulgaris. (Voy. pl. 3, fig. 9.)

$5^{\circ}$ Dentées ( fol. dentata), dont le bord est découpé en petites dents aiguës, ne sinelinant ni vers le sommet, ni vers la base de la feuille. Exemple : l'alliaire ( $E_{\text {ry }}$ simım Alliaria) le seneçon (Senecio vulgaris), etc.

$6^{\circ}$ Serrées ou Dentées en scie (fol. serrata), quand les dents sont inclincées vers le sommet de la feuille, eomme dans la violette (Viola odorata); la viorne ( iburnum Lantana), etc. (Voy. pl. 3, fig. I.)

$7^{\circ}$ Doublement servées (fol. duplicato-serrata), dont chaque deutelure est elle-même serrée, eomme dans le coudrier ( Corylus Avellana), l'orme (Ulmus campestris).

8o Epinenses ( $f \circ l$. margine spinosa), bordées de dents raides, aiguës et picquantes, comme dans le houx (Ilex aquifolizm), beaucoup de ehardons.

$9^{\circ}$ Cilicées ( $f o l$. ciluluta), ayant le bord garni de poils disposés en série eomme les cils des paupières; par exemple dans l'Erica tetralix, la Luzzula vernalis, ete.

$K$. Expansion.

Les feuilles peuvent être :

I $^{\circ}$ Planes ( fol. plana), quand leur surface n'est ni eoncave ni convexe : celles de la plupart des plantes.

$2^{\circ}$ Convexes ( $f \circ$. convexa), quand elles sont bombées par leur faee supérieure.

$3^{\circ}$ Concaves ( fol. concava), bombées par leur faee inférieure, de manière à ee que la supérieure présente une cavité.

$4^{\circ}$ Gladićes ou Ensiformes ( fol. ensiformia), comprimées fortement sur leurs parties latérales, en sorte que leurs faces sont devenucs latérales, et leurs bords postérieur et antérieur, eomme dans l'Iris germani$\mathrm{ca}$, etc. 

sens.

$5^{\circ}$ Striées ( fol. striata), offrant des stries en différens

$6^{\circ}$ Onduleuses ( $f o l$.ondulosa), offrant des saillies et des enfoncemens irrégulicrs, qu'on a comparés aux ondulations de l'eau agitée. La rhubarbe ondulée ( Rheum undulalum).

L. Superficic.

I $^{\circ}$ Luisantes (fol. lucida), ayant leur surface unic et réflechissant la lumière : le laurier-eérise, le lierre.

$2^{\circ}$ Unies (fol. lavia), n'offrant aucunc saillie ni aspérité : le Nymphaea, etc.

$3^{\circ}$ Glabres (fol. glabra), déponrvucs de toutu espèce de poils : la petite ccntauréc (Erythrcea Centaurium), le laurier-rose.

$4^{\circ}$ Pertuses ( fol. pertusa), percées de trous très-sensibles : Dracontium pertusum.

$5^{\circ}$ Cancellées ( fol. cancellata), quand le parenchyme n'existe pas, et qu'elles sont simplement formées par les ramifications des nervures fréquemment anastomosées, et représentant une sorte de treillage, comme celle de l'Hy drogeton fenestralis.

$6^{\circ}$ Gladuleuses ( fol. glandulosa), offrant à lcur surface de petites glandes.

$7^{\circ}$ Scabres ( fol. scabra), rudes au toucher. L'orme (Ulmus campestris), le grémil (Lithospermum officincale), etc.

$8^{\circ}$ Glutineuses (fol. glutinosa); oflrant, quand on les touche, une viscosité plus ou moins grande: Inula viscosa.

M. Pubescence. (Voyez ce que nous avons dit précédemment en parlant de la tige).

$N$. Consistance et tissu.

I $^{\circ}$ Membraneuses ( fol. membranacea), n'ayaut pas 
d'épaisseur sensible, molles et souples, eomme celles de la grande aristoloche (Aristolochia Sypho ).

$2^{0}$ Scarieuses (fol. scariosa), minces, sèches, demitransparentes.

$3^{\circ}$ Coriaces (fol. coriacea), quand elles sont épaisses et qu'elles ont une certaine eonsistanee : eelles du gui (Viscum album), du laurier-cerise, etc.

$4^{\circ}$ Molles ( $f o l$. mollia ), ayant peu de solidité, et douces an toueher : l'épinard (Spinacia oleracea), la guimauve (Althcea officinalis).

$5^{\circ}$ Raides ( fol. rigida), coriaees et résistant à la flexion : le petit houx (Ruscus aculeatus).

$6^{\circ}$ Charnues ( fol. carnosa) : la joubarbe des toits (Sempervivum tectorum), et en général toutes les plantes grasses.

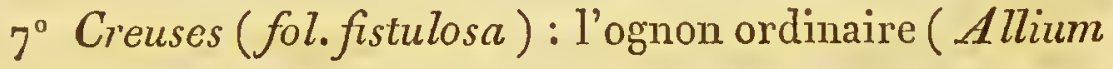
Cepa).

O. Forme (I) (épaisseur ou solidité notable).

$I^{0}$ Ovées (fol. ovala), ayant la forme d'un ouf.

$2^{\circ}$ Obovées ( $f o l$ obovata), ayant la forme d'un ouf renversé.

$3^{\circ}$ Conoïdales ( fol. conoïdea), ayant la forme d'un cône.

$4^{\circ}$ Cylindriques ( fol. cylindrica, teretia), ayant la forme d'un cylindre allongé : le Sedum album, l'ognon.

(1) Il ne faut pas confondre, comme on le fait très-souvent, la forne et la figure d'un corps. La forme ne s'entend que des corps solides, c'est-à-dire de ceux qui présentent l'étendue, la largeur et l'épaisseur. La partic de la géométrie qui s'en occape porte le nom de stéréométric. Lc terme de figure n'est applicable qu'aux corps plans, c'est-à-dire aux surfaces qui n'offrent que deux dimensions, la largeur ct la longuear. On donne le nom de planimétrie à la partie de la géométrie qui traite de la figure des corps plans. Ainsi un œuf a unc forme ovée : une fcuille plane, reprćsentant la section longitudinale d'un cuf, a une figure,ovale. On voit donc la nécessité de distinguer les expressions fornaires des expressions figuraires. 
$5^{\circ}$ Linguiformes (fol. linguifornia), ayant l'épaisscur et la forme d'une langue : la joubarbe des toits (Sempervivum tectorum).

$6^{\circ}$ Triquètres ( fol. triquetra), allongécs en prisme à trois faccs : le jone flcuri (Butomuls umbellatus).

$7^{\circ}$ Tétragonées ( fol. tetragona) allongées en prisme à quatrc faccs : Gladiolus tristis.

$8^{\circ}$ Comprimées ( fol. compressa), épaisscs, charnucs, aplatics latéralement, ayant plus d'épaisscur que de 'largeur.

P. Coloration.

$I^{\circ}$ Vertes (fol. viridia) : la plupart des fcuilles. lc vert.

$2^{\circ}$ Colorées ( $f o l$. colorata), d'une autre coulcur que

3o Glanques ( fol. glanca) : Magnolia glanca, le chou (Brassica oleracea). Celles qui sont d'unc couleur vert de mer. Cette coloration est due à une couche légère d'unc matièrc résincuse, semblable à celle qui recouvre ccrtains fruits, et cn partieulicr les pruncs ct les raisins. Un fait remarquable c'est que les feuilles glauques nc sont pas susceptibles d'ètrc mouillées quand on les trempc dans l'eau, cc qui démontre bicn la nature de l'cnduit qui leur domne la coulcur glauque.

$4^{\circ}$ Discolores ( fol. discolora), quand les deux faccs ne sont pas de la même coulcur. Ainsi dans la cymbalaire (Antirrhinum cymbalaria), lc cyelamen (Cyclanzen Europoum), la facc supéricurc est verte, l'inféricurc est pourpréc.

50 Tachetées ( fol. maculata), offrant des taches pliss ou moins considérables, d'unc coulcur différentc de eclle de la feuillc : Arum maculatum.

6o Incanes ('fol. incana), d'un blanc pur' Achillaea incana. 
Q. Pétiolation.

I $^{0}$ Sessiles (fol. sessilia) : le buis ( Buxus sempervivens), ctc.

$2^{\circ}$ Pétiolées ( fol. petiolata) : le platane, le poiricr, l'abrieotier.

30 Peltées ( fol. peltata), quand lc pétiolc s'insèrc au centre de la face inféricure des feuilles, et que les nervures partent de ce point, cn rayonnant vers la eirconférence : eomme dans la eapucine (Tropoolum majus), l'écuelle d'eau (Hydrocotylevulgaris). (Voyez pl.3, fig.9.)

Quand les fcuilles sont pourvucs d'un pétiole, il ne faut pas négliger les earaetc̀res qu'on peut tirer de ses différcntes modifications.

Ainsi, il pcut être cylindrique, comprimé, triquètre, filiforme, court, long, etc. Nous n'avons pas bcsoin de donner ici l'explication dc ces expressions, que nous avons déjà définies, pour la plupart, dans un autre lieu.

Le pétiole peut être tordu sur 'lui-même, comme dans plusieurs Cucurbitacées, ctc.

Claviforme, en forme de massue ( $p$. claviformis), quand il cst renflé d'unc manière manifeste à sa partie supérieure, comme dans la châtaigne d'eau (Trapa natans).

Canaliculé, ou creusé en gonttière ( $p$. canaliculatus), 'quand il cst convexe à sa facc cxterne, coneave du eôté (dc la tige; par cxemplc, dans beaueoup d'Ombellifères.

Ailé ( $p$. alatus), quand le limbe de la fcuille sc proIlonge sur lui de manière à formér de eliaquc côté un :appendice membraneux. Par cxemple, dans l'oranger (Citrus Aurantium).

Dans les fcuilles eomposées, le pétiolc commun cst quel'qucfois formé d'autant de pièccs articulécs ct mcmbraneuses qu'il y a de paires de folioles; e'est ce qu'on obsserve dans le Quassia anara par exemple, et un' granid rnombre d'cspèces d'Inga. 
Foliiforme, ou en forme de feuille (foliiformis), quand il est large, minee, et a l'aspeet d'uue feuille. Dans ee cas, il remplaee fort souvent les véritables feuilles, qui n'existent que dans les individus encore jeunes, et qui tombent à une eertaine époque. Ainsi les prétendues feuilles simples des Mimosa de la Nouvelle-Hollande ne sont que des pétioles élargis et foliiformes, etc. On leur a donné le nom de Phyllodes.

Le pétiole est quelquefois aeeompagné d'une gaîne membraneuse à laquelle on a donné le nom d'Ochrea et qui embrasse la tige dans toute sa eireonférenee. La présenee de eelte Ochrea est un des meilleurs earaetères pour distinguer les plantes qui appartiennent à la famille des Polygonées, qui en sont toutes pourvues.

$R$. Suivant leur durée sur la tige, on distingue les feuilles en :

$x^{\circ}$ Caduques ( $f o l$. caduca), lorsqu'elles tombent peu de temps après leur,apparition, eomme eelles de beaucoup de cactus.

$2^{\circ}$ Décidues (fol. decidua), quand elles tombent avant unenouvelle foliation : eelles du marromnier, du tilleul, ete.

30 Marcescentes ( fol. marcescentia), lorsqu'elles se dessèehent sur la plante avant de tomber, comme eelles du eliêne.

$4^{\circ}$ Persistentes (fol. persistentia), eelles qui restent. sur le végétal plus d'une année. Par exemple, dans les pins, les buis, le laurier-ecrise, ete. Ces arbres portent le nom général d'arbres tonjours verts.

\section{§2. Des Feuilles Composées.}

La feuille vraiment composée, avons-nous dit, est celle qui, sur un pétiole commun, porte plusieurs folioles qu'on peut isoler, les unes des autres. Ces folioles sont, ou artieulées sur le rachis, c'est-à-dire attachées par un point très-rétréci de la base de leur petit pétiole, 
ou continues avec lui par toute la base de leur pétiole.

Il y a différens degrés de eomposition dans les feuilles. Ainsi le pétiole eommun peut être simple, ou bien il peut se ramifier.

Quand le pétiole commun ne se ramifie pas, la feuille est dite simplemént composée. On l'appelle feuille décomposée quand il se ramifie.

Nous allons étudier les modifications qu’elle présente dans ces deux cas.

Les feuilles simplement eomposées offrent deux modifications principales, suivant la position qu'affeetent les folioles qui les eomposent. Aiusi tantôt toutes les folioles partent du sommet même du pétiole eommun, comme dans le marronnier d'Inde, le trèfle, ete.; tantôt au eontraire ees folioles naissent sur les parties latérales du pétiole commun ou raehis, eomme daus le frêne, le bagnenaudier, l'aeacia, ete. On a donné le nom de feuilles digitées à la première de ces deux modifieations; et celui de pennées à la seeonde.

Les feuilles digitées ( fol. digitata) sont done eelles dont toutes les folioles partent en divergeant du sommet du pétiole eommun, à la manière des doigts de la main. lorsqu'ils sont éeartés.

Le nombre des folioles qui eonstituent les feuilles digitées est très-variable, eomme on peut le voir en eomparant ensemble les feuilles du trèfle, qui en offrent trois, avee eelles des Pavia, qui én ont einq; cellés du marronnier d'Inde, qui en présente sept; eelles des lupins, qui en offrent un grand nombre, ete. Aussi est-ee d'après ec nombre que l'on a divisé les feuilles digitées en :

Io Unifoliolées ( fol. unifoliolata), quand elles n'offrent qu'une seule foliole, mais qui est articulée au sommet du pétiole. Dans ee eas, des raisons d'analogie eı la présenee d'une artieulation font ranger eette feuille parmi les composécs. Telles sont celles de l'oranger ( Citzus Au- 
rantium ), du Rosa simplicifolia, etc. (Voy.pl.4., fig. x.) $2^{\circ}$ Trifoliolées (fol. trifololiolata), quand elles ont trois folioles comme dans le trèfle d'eau (Menyanthes trifoliata), l'alleluia (Oxalis acetosella). (Voy. pl.4, fig. 5.)

$3^{\circ}$ Quadrifoliolées (fol.quadrifoliolala), composées de quatre folioles (Marsilea quadrifolia).

$4^{\circ}$ Quinquéfoliolées (fol. quinquefoliolata) : Cissus quinquefolia, Potentilla reptans, etc.

50 Septemfoliolées ( $f o l$. septemfoliolata), le marronnier d'Inde, etc. (Voy. pl. 4, fig. 6.)

$6^{\circ}$ Multifoliolées ( fol. multifoliolata), composées d'un grand nombre de folioles, comme le Lupinus varius.

Les feuilles pennées ( fol. pennala), comme nous l'avons dit, sont celles qui, sur un pétiole commun, portent un nombre plus ou moins considérable de folioles, disposées sur les parties latérales à la manière des barbes d'une plume sur leur tige commune; telles sont celles de l'acacia (Robinia pseudo-acacia), du frêne (Fraxinus excelsior ). (Voyez pl. 4, fig. 3.)

Les folioles d'une feuille pennée peuvent être opposées l'ume à l'autre et disposées par paire; dans ce cas, on dit qu'elles sont oppositi-pennées; ou bien ses folioles sont alternes, et les feuilles sont dites alternati-pennées.

Les feuilles oppositi-pentrées sont également appelées conjuguées. On dit qu'elles sont:

I Unijuguées ( fol. unijugata) quand le pétiole commun porte une seule paire de folioles, comme dans le Lailyyrus latifolius, le Lathyrus sylvestris, etc. (Voy.pl. 4, fig. 4.)

$2^{9}$ Bijuguées (fol.bijugata), composées de deux paires de folioles, comme dans ccrtains Mimosa. (Voy. pl. 4, fig. 2.)

$3^{\circ}$ Trijuguées (fol. trijugata), composées de trois paires de folioles, comme celle de l'Orobus tuberosus. $4^{\circ}$ Quadrijuguées ( fol. quadrijugata). 
$5^{\circ}$ Quinquéjuguées (fol.quinquejugata), comme celles de la casse (Cassia fistula).

$6^{\circ}$ Multijuguées ( $\left.f o l . m u l t i j u g a t a\right)$, quand les paires de folioles sont en nombre indéterminé, comme celles de la fausse réglisse : Astragalus glycyphyllos, la Vicia crac$c a$, etc.

Les feuilles oppositi-pennées sont dites pari-pennées ou pennées sans impaire, quand les folioles sont attachées par paires, et que le sommet du pétiole commun ne présente pas de foliole solitaire nil de vrille qui en tienne lieu, comme dans le caroubier (Ceratonia siliqua), l'Orobus tuberosus, etc. (Voy.pl. 4, fig. 2.)

Elles sont dites au contraire impari-pennées, ou pennées avec impaire (impari-pennata), quand le pétiole commun est terminé par une foliole solitaire, comme dans l'acaeia (Robinia pseudo-acacia), le frêne (Fraxinus excelsior ). ( Voy. pl.4, fig. 3.)

Les feuilles impari-pennées sont appelées trifoliolées ( fol. impari-pennata trifoliolata), quand au-dessus de l'unique paire de folioles dont elles sont formées, se trouve une foliole solitaire pétiolée, comme dans les espèees de Dolichos, de Glycine, de Phaseolus, etc.

On appelle feuilles interrupté-pennées (fol. interruptepennata) celles dont les folioles sont alternativement grandes et petites, comme dans l'aigremoine ( $A$ grimonia Eupatoria).

Quant aux feuilles decursivé-pennées, c'est-à-dire celles dont Ie pétiole commun est ailé par le prolongement de la base des folioles, nous ne les rangeons pas parmi les feuilles composées, puisque aucune foliole ne peut être enlevée sans en déehirer la partic foliaeée. Ce ne sont que des feuilles plus ou moins profondément pinnatifides.

Les feuilles décomposées ( fol. decomposita) sont le deuxième degré de composition des feuilles; le péiole 
commun est divisé en pétioles sccondaires, qui portent les folioles. On les appelle :

I Digitées-pennées ( digitato-pennala) quand les pétioles secondaires représentent des feuilles pennées partant toutes du sommet du pétiole commun. Exemple: certains Mimosa.

$2^{\circ}$ Bigéminées (fol. decomposito-bigeminatä), quand chacun des pétioles secondaires porte une seule paire de folioles. Exemple : Mimosa unguiscati.

30. Bipennées ( fol. bipennata, duplicato-pennata), quand les pétioles secondaires sont autant de feuilles pennées, partant du pétiolc eommun, comme dans le Mimosa Julibrizin, ete. (Voy.pl.4, fig. 7.)

On nomme feuilles surdécomposées le troisième et dernier degré de composition que présentent les feuilles. Dans ee eas les pélioles seeondaires se divisent en pétioles tertiaires, portant les folioles. Ainsi on appelle feuille surdécomposée-triternée celle dont le pétiole commun se divise en trois pétioles sceondaires, divisé chaeun en trois pétioles tertiaires, portaut aussi eliaeun trois folioles, comme daus l'Actcea spicala, l'Epimedium alpinum. (Voy.pl.4, fig. 8.)

Nous yenons d'exposer avec quelques détails les nombreuses variétés de forme, de figure, de eonsistance, de simplieité et de eomposition, que présentent les feuilles. Nous avons eru devoir domer quelque développement à cet article, paree 'que beaucoup d'autres organes, que nous étudierons suecessivement, telles que les stipules, les sépales, les pétales, ete., nous ofrirons des modifieations analogues dans leur figure, leur forme, leur strueture, ete., qui, une fois déerites et défnies, n'anront plus besoin que d'ètre cilées pour être parfaitement comprises. 
Structure, usages ei fonctions des Feuilles.

Les feuilles, comme nous l'avons dit préeédemment, sont formées par trois organes prineipaux, savoir par un faisseau vasculaire provenant de la tige, par du parenchyme, prolongement de l'enveloppe herbacée de l'éeoree, et enfin par une portion d'épiderme qui les reeouvre dans toute leur étendue.

Le faisceau vaseulaire constitue le pétiole quand celuici existe. Ces vaisseaux sont des traelıées, des fausses trachées et des vaisseaux poreux; ils sont, dans le pétiole, enveloppés à l'extérieur par une eouche de la substance herbaece, qui se prolonge sur eux au moment où ils sortent de la tige. C'est par leur épanouissement et leurs ramifieations suceessives qu'ils eonstituent le réseau de la feuille. Les mailles ou espaees vides qu'ils laissent entre eux sont remplis par le tissu parenehymateux venant de l'écorce. Ce parenehyme manque quelquefois, eomme dans l'Hydrogeton; et alors la feuille, qui n'est eomposée que par son réseau vaseulaire, offre l'aspeet d'une sorte de treillage ou de dentelle.

L'épiderme qui reeouvre les surfaces de la feuille est en général mince et très-poreux, surtout à lạ surface inférieure.

Ces deux lames d'épiderme reeouvrent la partie formée par les fibres vasculaires et le parencluyme, et que le professeur De Candolle propose de nommer mésophylle. Cet organe est quelquefois très-mince, ainsi qu'on l'observe pour les feuilles qui sont planes et membraneuses; inais dans toutes les feuilles épaisses et eharnues, dans les plantes grasses par exemple, le mésopliylle est très-développé, et domne la forme à la feuille.

Les stomates ou pores qu'on observe sur les feuilles ne sont, suivant plusieurs auteurs, que l'orifice supérieur des 
vaisseaux séveux; il résulte de là qu'ils sont d'autant plus abondans que la feuille est plus fibreuse.

Les feuilles sont, avec les raeines, les organes priucipaux de l'absorption et de la nutrition dans les végétaux. En effet elles absorbent dans l'atmosphèrc les substances nutritives qui peuvent servir à l'accroisscment. Aussi quelques autcurs les ont-ils désignées sous le nom de racines aériennes. Elles remplissent encore d'autres usages d'une haute importance dans l'économic végétale. Elles servent à la transpiration et à l'exhalation des fluides devenus inutiles à la végétation, et e'est par elles que la sève se dépouille des sucs aqueux qu'elle contient, ct qu'clle aequiert toutes ses qualités nutritives.

C'est principalement par les portes situés à la face inférieure de la feuille des plantes ligneuses que les fluides vaporcux et les gaz répandus dans l'atmosphère sont absorbés. Cette face inférieure, cn cffet, est plus mollc, moins lisse, et présente presque toujours un duvet léger qui favorise cetteabsorption; leur face supérieure, au eontrairc, plus lisse, plus souvent glabre, sert à l'cxcrétion des fluides inutiles à la nutrition du végétal; c'est ec qui constituc la transpiration dans les végétaux.

Les feuilles des plantes herbácées, plus rapprochées du sol, plongées cn quclque sorte dans unc atmosphère continucllement humide, absorbent égalcment par leur face supérieure et lcur faee inférieure. C'est au célèbre Bonnet que l'on doit ees eonnaissances. Ce pliysicicn posa des feuilles d'arbre sur l'eau par leur face inférieure; clles se conservèrent fraîches et vertes pendant plusieurs mois. Il cn posa d'autres par lcur faee supérieure, qui, en peu de jours, ne tardèrent point à se faner. Des feuilles de plantes herbacées sc conservèrent saines pendant fort longtemps dans les deux positions.

C'est dans le parenelıme des feuilles, de même que dans toutes les autres partics vertes et herbacées du végétal, que 
s'opère la décomposition de l'aeide carbonique absorbé dans l'air. Lorsqu'elles sont exposées à l'aetion du soleil, elles déeomposent ce gaz, retiennent le earbone et dégagent l'oxygène. Le eontraire a lieu quand elles sont soustraites à l'ácion de la lumière; ear alors elles preunent dans l'air une portion de son oxygèné, qu'elles remplacent en dégageant une égale quantité de gaz aeide earbonique. On sait que les végétaux privés de l'influenee du soleil s'étiolent, c'est-ì-dire qu'ils perdent leur eouleur verte, deviennent mous, aqueur, et contiennent une plus grande proportion de prineipe sueré:

Mais nous reviendrons tout à l'heure avee plus de détails sur les phénomènes de l'absorption et de la transpiration, en traitant de la nutrition dans les plantes.

Les feuilles sont suseeptibles de eertains mouvemens qui dépendent évidemment de l'irritabilité dont elles sont douées. Des faits nombréux et bien eonstatés mettent hors de doute l'existence de eette propriété dans les végétaux.

Si l'on place une branche tenant encore à sa tige, de manière que la faee inférieure des feuilles regarde vers le ciel, on verra les feuilles se retourner, peu à pet, et reprendre leur position maturelle. Ce fait peut s'observer chaque jour lorsqu'on taille et que l'on palissade les arbres tenus en espalier, eomme le pêeher, la vigne, ete.

Ce sont surtout les feuilles composées et artieulées, c'est-à-dire eelles dont les fólioles sont attaehées par artieulation au pétiole eommun, qui présentent les mouvemensles plus remarquables. Ainsi, pendant la nuit, les folioles d'un grand nombre de Légumineuses, dont les feuilles sont toutes articulées, ont une position différente de eelle qu'elles oecupent pendant le jour. Linnæus a domné le nom de sommeil des plantes à ee phénomène singulier. Par exemple, les folioles de l'Acacia, au lever du soleil, sont étendues presque horizontalement. Mais à mesure 
que eet astre s'élève au-dessus de l'horizon; ses folioles se redressent, de plus en plus, et devieunent presque vertieales; clles eommeneent au contraire à baisser à mesure que le jour déeline.

D'autres plantes présentent eneore des phénomènes analogues qui nous paraissent dépendre de l'influenee de la lumière. C'est en effet ee que l'on peut eonelure des ex périenees ingénieuses de M. De Candolle. Cet habile botaniste, ayant placé dans un eaveau, à l'abri de la lumière, des plantes à feuilles eomposées, est parvenu, en les privant pendant le jour de la lumière, et les éelairant au eontraire fortement la nuit, à ehanger dans quelquesunes les heures de Jeur veille et de leur sommeil.

Mais les feuilles de eertains végétaux exéeutent aussi des mouvemcns d'irritabilité que. l'on ne peut pas attribuer uniquement à l'influenee de la lumière. La sensilive ( $M_{i-}$ mosa prudica) esı de ce nombre. La seeousse la plus légère, l'air faiblement agité par le vent, l'ombre d'un nuage ou d'un eorps queleonque, l'aetion du fluide électrique, la chalcur, le froid, les vapeurs irritantes, telles que celles du ehlore, du gaz nitreux, suffisent pour faire éprouver à ses folioles les mouvemens les plus singuliers. Si l'on en touehe une seule, elle se redresse eontre eelle qui lui est opposée, et bientôt toutes les autres de la même feuille suivent et exécutent le même mouvement, et se eouehent lẹs unes sur les autres, en se reeouvrant ì la manière des tuiles d'un toit. La feuille elle-mềme tout entière ne tarde pas à se fléchir vers la terre. Mais peu de temps après, si la eause a eessé d'exereer son aetion, toutes ces parties qui semblaient s'être fanées, repremment leur aspeet et leur position naturelle.

L'Hedysarum gyrans, plante singulière, originaire du Bengale, offre des mouvemens très-remarquables. Ses feuilles sont unifoliées, aeeompagnées latćralement de deux stipules plus petites. Les deux stipules sont animées 
d'un double mouvement de flexion et de torsion sur ellesmêmes, qui paraît indépendant dans chacune d'elles. En effet, l'une se meut quelquefois rapidement, tandis que l'autre reste en repos. Ce mouvement s'exéeute sans l'intcrvention d'aueun stimulant extérieur. La nuit ne le suspend pas. Celui de la foliole médiane, au eontraire, paraît dépendre de l'aetion de la lumière, et cesse quand la plante n'y est plus exposée.

Les folioles du Porlier'a se rapprochent et s'accolent aussitôt que le ciel se eouvre de nuages.

Le Dioncea muscipula, plante originaire de l'Amérique septentrionale, présente, à l'extrémité de ses feuilles, deux lobes réunis par une charnière médiane. Quand un inseete, ou un corps queleonque, touehe et irrite l'un des petits corps glanduleux que l'on remarque sur leur faee supérieure, ces deux lobes, se redressant vivement, se rapproehent, et saisissent l'inseete qui les irritait. Aussi eette plante porte-t-clle le nom vulgaire d'attrapemouche. Mais il cst à remarquer qu'il n'y a d'irritable dans eette feuille que les deux ou. trois petit points glanduleux, qu'on observe sur la faee supérieure.

M. Dutroehet, que nous avons déjà eité avantageusement dans le eours de eet ouvrage, s'est beaueoup oceupé des mouvemens des feuilles dans les végétaux, et plus partieulièrement dans la sensitive. Nous exposerons ici brièvement le résultat de ses opinions.

A la base du pétiole des feuilles dites articulées, qui sont les seules dans lesquelles se manifestent les mouvemens d'irritabilité, on aperçoit un renflement ou bourrelet qui se termine ensuite par un rétrécissement manifeste. Jusqu'à. présent on avait pensé que les mouvemens se passaient daus ee point rétréci, que l'on regardait comme semblable à l'articulation des membres chez les animaux. Les expériences de MI. Dutrochet tendent à prouver que tous les nouvemens ont lieu dans le bourrelet lui-même, 
et qu'ils se réduisent à la flexion et au redressement. Dañs le premier eas il forme une courbe dont la eonvexité est tournée vers le eiel; dans le second cas il est presque droit. Ce bourrelet est essentiellement composé d'un tissu eellulaire fin et délieat, garni d'une très-grande quantité de petits grains verts, qui sont pour M. Dutrochet autant de eorpuscules nerveux. Au eentre se trouve un faiseeau de vaisseaux nourrieiers. C'est ee tissu eellulaire du bourrelet, qui est le siége des mouvemens du pétiole, que l'on peut à volonté anéantir en enlevant ee tissu eellulaire. Ainsi, quand on enlève le tissu eellulaire du eôté inférieur du bourrelet, la feuille reste fléehie et ne peut se redresser; si au eontraire on ôte la partie supéricure, la feuille eonserve la faculté de se redresser, mais elle ne peut plus se fléehir. Il résulte évidemment de cette expérienee que la flexion de la feuille est produite par l'aetion du bourrelet supérieur, et que son redressement est dû̀ à eelle du bourrelet inférieur. Ce sont en quelque sorte deux ressorts antagonistes, dont l'un devient alternativement plus fort que l'autre.

En voulant étudier avec plus de soin l'organisation intime du bourrelet, l'habile expérimentateur dont nous exposons iei les idées est arrivé à ume autre découverte. Si l'on coupe une tranche très-mince du tissu eellulaire du bourrelet sur le eôté supérieur, on la voit sur-lechamp se ployer en eerele, dont la coneavité est constamment tournée vers l'axe du bourrelet. Si l'on répète la même opération sur le côté inférieur, la coneavité du cerele regarde également vers le ecntre; en sorte que le bourrelet est eomposé de deux ressorts antagonistes, qui tendent à se eourber en sens inverse: le ressorı inférieur redresse le pétiole, tandis que le supérieur le fléchit. M. Dutroehet donne le nom d'incurvation à cette propriété que possèdent les lames du bourrelet de se rouler dans un sens ou dans un autre. 
La cause immédiate de ces mouremens d'incurvation réside, sclou notrc autenr, dans l'action nerveuse: mise en jeupar les agens du dehors. Il était naturel quie M. Dutrochet, ayant attribué aux plantes un système nerveux, analogue à celui des animaux, lui fít jouer dans les phénomènes dc la végétation le rôle important que ce système remplit dansles actions dela vieanimale. Ainsi donc l'action du système nerveux est la cause des mouvemens visibles ḋes végétaux comme dans les animaux. Mais, s'il en est ainsi ce système nervcux doit, comme dans ces derniers, êtrè l'organe de transmission de ces mouvemens, ou, en d'autres termes, la partie qui transmet le stimulus qui met eu jeu l'action de ce système. Or, c'est ce qui n'a päs lieu, du propre aveu de M. Dutrochet; car, d'après des expériences extrêmement délicates, il est parvenu à recounaîtrc que l'action nerveuse qui détermine les mouveméns des feuilles se transmet uniquement par les vaisseaux qui forment l'étui médullaire, vaisseaux entièrement privés de tubercules nerveux. Ainsi donc le système nerveux des végétaux serait l'agent de la puissance nerveuse, sans être l'organe de la transmission de cette puissance.

D'après ce court exposé, il nous semble que l'importante question de la cause des mouvemens des feuilles n'est point encore complètement résolue, et que de nouvelles expériences sont encore nécessaires pour arriver à une solution satisfaisante.

\section{Défoliation ou chute des Feuilles.}

Il arrive chaque année une époque où la plupart des végétaux se dépouillent de leurs feuilles. C'est ordinairemcnt à la fun de l'été ou au commencement de l'automne quc les arbres perdent leur feuillagé.

Ccpcudant ce phénomène n'a pas liè à la mếme épóque pour toutes les plantes. On remarque en général quíe les 
arbres dont les feuilles se développent de bomne heure sont aussi ceux qui les perdent les premiers, comme on l'observe pour le tilleul, le marronnier d'Inde, ete. Le sureau fait exception à eette règle; ses feuilles paraissent de bonne heure, et ne tombent que très-tard. Le frêne ordinaire présente une autre particularité; ses feuilles se montrent très-tard, et tombent dès la fin de l'été.

Les feuilles pétiolées, surtout eelles qui sont articulées avec la tige, s'en détachent plutôt que celles qui sont sessiles, et à plus forte raison que eelles qui sont amplexicaules. En général, dans les plantes herbacées, annuelles ou vivaces, les feuilles meurent avee la tige, sans s'en détaeher.

Mais il est des arbres et des arbrisseaux qui restent en tout temps ornés de leur feuillage. Ce sont en général les espèces résineuses, telles que les pins, les sapins, ou certains végétaux dont les feuilles sont raides et eoriaces, comme les myrtes, les alaternes, les lauriers-roses, etc. On leur donne le nom d'arbres verts.

Quoique la chute des feuilles ait généralement lieu aux approches de l'Jiver, on ne doit cependant pas regarder le froid comme la principale eause de ee phénomène. Elle doit être bien plus naturellement attribuée à la cessation de la végétation, au manque de nourriture que les feuilles éprouvent à cette époque de l'année, où le cours de la sève est interrompu. Les vaisseaux de la feuille se resserrent, se déssèehent, et bientôt eet organe se détache du rameau sur lequel il s'était développé.

Usages économiques et médicinaux des Feuilles.

Un grand nombre de végétaux sont cultivés dans nos potagers à eause de leurs feuilles, qui sont d'exeellens alimens. C'est ainsi qu'on emploie fréquemment les choux, 
les épinards, l'oseille, le céleri', les cardons et beaucoup d'autres espèces. Remarquons ici que les cultivateurs se servent souvent de la propriété que possèdent les végétaux privés de l'action de la lumière, de devenir tendres et sucrés, pour les rendre plus propres à la nourriture de l'homme.

La médecine trouve aussi dans les feuilles un grand nombre de médicamens utiles, que l'on peut ranger de la manière suivante :

$\$$ x. Feuilles émollientes;

De guimauve (Althcea officinalis).'

De mauve (Malva rotundifolia).

De poirée (Beta vulgaris).

$\$ 2$. Tuilles amères ou toniques.

Trèfle d'eau ( Menyanthes trifoliata).

Véronique officinale ( Veronica officinalis).

Beccabunga (Veronica Beccabunga).

Petite centaurée (Erythrcea Centaurium).

$\$ 3$. Feuilles excitantes.

Oranger (Citrus Aurantium).

Menthe poivrée (Mentka piperita).

Mcnthe crépue (Mentha crispa).

Sauge ( Salvia officinalis).

Cresson de fontaine ( Sisymbrium Nasturtium ).'

Cochléaria (Cochlearia officinalis).

Cresson alenois (Lepidium sativum).

$\oint$ 4: Feuilles vireuses.

Ciguë ( Conium maculatum).

Stramoine (Dalura Stramonium).

Tabac (Nicotiana Tabacum).

Belladone (Atropa Belladona).

Digitale pourprée (Digitalis purpurea), ètc. 


\section{\$5. Feuilles purgatives.}

Séné d'Italie (Cassia Senna).

Séné d'Alexandrie (Cassia lanceolata).

Gratiole (Gratiola officinalis).

Baguenaudier (Colutea arborescens).

\section{CHAPITRE V.}

DES STIPULES (I).

Les siipules sont des organes aeeessoires des feuilles. Elles n'existent point dans les végétaux monoeotylédonés, mais seulement dans les dicotylédonés, qui n'en sont pas tous pourvus. Ce sont de petits appendices squamifores ou foliacés, qu'on reneontre au point d'origine des feuilles sur la tige. Elles sont ordinairement au nombre de deux, une de ehaque côté du pétiole, eomme dans le charme, le tilleul; le plus souvent elles sont libres, e'est-à-dire qu'elles ne sont pas fixées aut pétiole; d'autres fois elles font corps avee la base de eet organe, comme dans le rosier.

Les stipules fournissent d'excellens caraetères pour la coordination des plantes. Quand un végétal d'une famille naturelle en présente, il est extrêmement rare que tous les autres n'en soient pas également pourvus. Ainsi elles existent dans toutes les plantes de la famille des Légumineuses, des Rosacées, des Tiliacées, etc.

Comme elles tombent très-facilement quand elles sont libres, on pourrait quelquefois s'en laisser imposer par leur absenee, et eroire que la plante en est dépourvue; mais on pourra éviter facilement cette erreur en obser-

(I) Stipula, Fulera. 
vant qu'elles laissent toujours sur la tige, au licu qu'elles oceupaient, une petite cicatrice qui atteste ainsi qu'elles ont existé.

Dans les Rubiacées exotiques, à feuilles opposées, tel que le Coffoea, le Psychotria, le Cinchona, les stipules sont situées entre les feuilles, et paraissent être de véritables feuilles avortées. En effet, dans les Rubiacées de nos elimats, telles que les Galium, les Rubia, les Asperula, elles sont remplaeées par de véritables feuilles, qui alors forment un verticile autour de la tige.

Quelques plantes ne présentent qu'une seule stipule, eomme le vinettier (Berberis vulgaris).

Quand il en existe deux, elles sont presque toujours distinctes l'une de l'autre; mais quelquefois elles se soudent et sont conjointes (stipulce connatce), eomme dans le houblon (Humulus Lupulus). Les stipules peuvent se souder ensemble en dedans de l'aisselle de la feuille, la tige restant en dehors; dans ee eas les stipules sont axillaires, eomme on le remarque dans le Meliantus major. Il est très-probable que la gaine membraneuse des Polygonées, à laquelle on a donné le nom d'ochrea, est formée de la soudure de deux stipules.

Leur nature et leur consistance sont très-sujettes à varier. Ainsi elles peuvent être foliacées, c'est-à-dire semblables à des feuilles, comme dans l'aigremoine ( $A \mathrm{gri}$ monia eupatoria); membraneuses, eomme dans le figuier, les Magnolia; spinescentes, eomme dans le jujubier (Zizyphus vulgaris), le groseiller à maquereau (Ribes grossularia).

Leur figure ne varie pas moins que celle des feuilles. Ainsi il y en a d'orbieulaires, d'ovales, de sagittées, de réniformes, etc. Elles peuvent encore être entières, dentées ou laciniées.

Quant à leur durée, les unes sont fugaces, e'est-à-dire tombant avant les feuilles; par exemple celles du figuier 
(Ficus Carica), du tilleul (Tilica europaca). Lès autres sont simplement caduques, quand elles tombent en même temps que les feuilles. C'est ce qui a licu pour le plus grand nombre. Enfin, il en est d'autres qui persistent sur la tige plus ou moins long-tcmps après la chute des feuilles; telles sont eelles du jujubier, du groseiller à maquereau, ete.

L'usage des stipules parait être de protéger les feuilles avant leur développement, ainsi que le montre évidemment leur disposition respeetive dans les bourgeous des Amentaeées, des Rosacées, ete.

\section{CHAPITRE VT.}

DES VRILLES, CIRRHES OU MATNS.

On désigne sous ces noms des appendiees ordiuairement filamenteux, d'origines diverses, simples ou rameux, se roulant en spirale autour des eorps voisins, et servant ainsi ì soutenir la tige des plantes faibles et grimpantes.

Les vrilles ne sont jamais que des organes avortés. Tantôt, en effet, ee sont des pédoneules floraux qui se sont allongés considérablement, eomme dans la vigne : aussi les voit-on quelquefois porter des fleurs et des fruits. Tantôt ec sont des pétioles, comme dans beaueoup de Lathyrus, de Vicia, ete. D'autres fois enfin ce sont des stipules, ou même des rameaux avortés. $\Lambda$ ssez souvent ce sont les feuilles elles-mêmes dont l'extrémité se roule ainsi et constitue des espèees de vrilles, eomme dans l'oillet.

La position relative des vrilles mérite beaucoup d'être obscrvéc; ear elle indique l'organe dont elles tiennent la place. Ainsi dans la vigne elles sont, comme les grappes de fleurs, opposées aux feuilles, ce qui fait voir que ee 
sont des grappes avortées; elles sont axillair'es dans les passiflores; pétioléentnes dans le Latlyrus lalifolius, la Fumaria vesicaria; pédonculéennes dans la vigne; stipuléennzes daus ecrtains Smilax; cnfin elles peuvent ètre simples, comme dans la bryonc (Bryonia alba), ou rameuses, commc dans le Cobaer scandens.

On donne le nom particulier de griffes aux racines que les plantes sarmentcuses ct grimpantes enfoncent dans les corps sur lesquels elles s'élèvent, comme celles du lierre, du Bignonia radicans. On appclle suçoirs les filamens très-déliés que l'on rencontre sur la surface des griffes, et qui paraissent destinés à absorber les partics nutritives eontenues dans le eorps où ellcs sont implantées.

\section{CHAPITRE VII.}

DES ÉPINES ET DES AIGUIILONS.

Les épines (spina) sont des piquans formés par le pro: longement du tissu interne du végétal, tandis que les aiguillons (aculei) ne proviennent que de la partic la plus cxtérieure des végétaux, e'cst-à-dire de l'épiderme, dont on peut les détacher avec la plus grandc facilité.

L'origine et la nature des épines ne sont pas moiṇs variées que leur siége. Ellcs, remplaeent les feuilles dans eertaines espèces d'asperges de l'Áfrique, les stipules dans le jujubier, le groseiller à maquereau. Très-souvent elles ne sont que des rameaux̃ avortés; par exemple, dans le prunier sauvage. Aussi eet arbre, transplanté dans un bon terrain, ehange-t-il.ses épines cn rameaux. Le trone de quel(ques arbres est hérissé d'épincs qui les rèndent inabordables; telies sont les diverses espèces de Gleditscia. 
Les pétioles persistans de l'Astragalus tragacanthos se converlissent en épines.

Suivant leur situation et leur origine, elles sont caulinaires, quand elles naissent sur la tige, comme dans les cierges ( Cactus), les Gleditscia.

Elles sont terminales quand elles se développent à l'extrémité des branches et des rameaux, comme dans le prunier sauvage (Prunus spinosa).

Axillaires, quand elles sont situćes dans l'aisselle des feuilles, eomme dans le citronnier (Citrus medica).

Infra-axillaires, lorsqu'elles maissent au-dessous des feuilles et des rameaux, conmme dans le grosciller à maquereau.

Infin elles peuvent être simples, rameuses, solitaires ou fasciculées. Ces expressions s'entendent d'elles-mêmes et n'ont pas besoin d'être définies.

Les aiguillons ont été regardés par quelques physiologyistes comme des poils endurcis. Ils sont très-peu adhérens aux parties sur lesquelles on les observe, et peuvent s'en détacher facilement, comme on le voit dans les Rosiers.

Les modifications qu'ils présentent quant à leur siluation, leur forme, ete., sont les mèmes que celles des épines. 


\section{DE LA NUTRITION}

DANS LES VÉGÉTAUX.

Nous venons d'étudier tous les organes de la végétation, c'est-à-dire tous eeux qui servent au développement et à la formation du végétal ; voyons maintenant comment s'opère la nutrition; quelle part y prend ehaeun de ces organes en particulier, et quelles sont les eonditions nécessaires pour qu'elle ait lieu.

La mutrition est une fonetion par laquelle les végétaux s'assimilent une partie des substances solides, liquides ou gazeuses répandues dans le sein de la térre ou au milieu de l'atmosphère, et qu'ils y absorbent, soit par l'extrémité la pís déliée de leurs radicules, soit au moyen des parties vertes qu'ils développent dans l'atmosphère.

C'est en vertu d'une force partieulière de suceion, dont ces diverses parties sont douées, que l'on voit s'effeetuer l'absorption de ees matières et leur introduetion dans le tissu végétal. Nous ferons d'abord connaitre la sueeion ou. l'absorption exercée par les raeines dans le sein de la terre, par les feuilles et les autres parties vertes au milieu de l'atmosphère, puis nous décrirons la marehe des sues nourriciers, ou de la sève des raeines vers les feuilles. Alors nous étudierons les phćnomènes de la transpiration, de l'expiration et de l'exerétion, et nous suivrons eusuite la sève dans son eour's rétrograde des feuilles vers les raeines. 
Nous avons déjà dit que e'cst par les extrémités de leurs fibrilles les plus délicées que les racines absorbent dans l'intérieur de la terre les fluides et les gaz qui s'y trourent répandus. Mais toutes les parties vertes des végétaux, telles que les feuilles, les jcunes branches, ete., sont égalẹment douées d'une foree de succion fort remarquable, et coneourent aussi à cette fonction importante.

Plongées dans le sein de la terre, les radicules capillaires y pompent, par les espèces de spongioles ou de bouches aspirantes qui les terminent, l'humidité dont elle est imprégnée. L'eau est le vélieule nécessaire des substanees nutritives des végétaux. Ce n'est point elle qui forme la base de l'alimentation du végétal, comme le croyaient les aneiens' physiciens; mais elle sert de dissolvant et de menstrue aux corps qu'il doit s'assimiler. Fin effet, si l'on fait végéter une plante dans l'eau distillée, à l'abri de toute influence étrangère, elle ne tardera pas à périr. L'eau seule ne sert done pas à sa nutrition. Il faut qu'elle contienne d'autres principes qui lui soient étrangers. D'ailleurs les végétaux ne renferment-ils point du carbone, des gaz, des substances terreuses, des sels, et mềne des métaux à l'état d'oxides ou en combinaison avec les acides? Or, l'eau aurait - elle pu domner naissance à ces différentes substanees? Voyons donc par quel moyen elles se sont introduites dans l'iutérieur de la plante, dont elles sont devenucs parties constituantes.

Comment le carbone s'est.il introduit dans les végétaux? Ce ne peut être à l'état de pur'eté et d'isolement, puisqu'alors il est fort rare dans la nature, et n'est pas soluble dans l'eau. Mais tout le monde ccinnaît la grande affinité du carbone pour l'oxygèue; on sait que l'acide carbonique, qu'ils forment en se combinant, est très- 
abondanment répandu dans la nature; qu'il se trouve dans lc sein de la terre, dans les cngrais, le fumier qu'on y mêle; que, très-soluble dans l'eau, cc liquide cn conticnt toujours unc certainc quantité. C'cst donc à l'ćtat d'acide que le carbone est porté dans le tissu des végétaux. Or, nous avons dit précćdemment qu'exposées à l'action des rayons du soleil, lcs plantcs décomposent l'acide carbonique, rctiennent et s'assimilent le carbonc, tandis qu'elles rejcttent la plus grande partie dc l'oxyg̣ène au dehors. L'eau ne pcut done scrvir quc de véhiculc à cette substance alimentaire de la végétation.

L'oxygène fait égalcment partic de la substance des végćtaux. Il nous sera faeile d'y expliquer la présencc de ce fluide. En effet, commc le prouvent les expćriences dc Théodore dc Saussure, les plantes nc rcjettcnt point tout l'oxygène qui acidifiait lc carbone; elles cn rctiennent une certaine quantité. L'air atmosphériquc qui circulc dans les végćtaux leur cèdc égalcmcnt unc portion de l'oxygène qu'il contient; mais c'cst prineipalcment l'eau qui, par la déeomposition qu'clle éprouve dans lc tissu végétal, décomposition dont les lois ordinaircs de la chimie ne peuvent pas plus nous donner unc, explication satisfaisante que de cclle de l'acidc carbonique, lui fournit à la fois la majcure partic de son oxygènc, ct l'hỹdrogène, qu'il renfcrme aussi cn si grandc proportion.

L'azote, quc l'on trouvc également dans lcs substances végćtalcs, tire évidcmment son origine de la dćcomposition de l'air atmosphérique dans l'intéricur de la plantc.

Tclles sont lcs différcntes substances inorganiquca qui entrent csscnticllement dans la composition du tissu végétal; cc sont elles qui cn forment la basc. Mạis il cn est d'autres encorc qui, sans faire partic néccssairc de leur organisation, s'y retrouvent toujours dans des quantités plus ou moins eonsidérables; tcls sont la chaux, la silice, le carbonate, le phosphate et le malate de chaux' les 
carbonates de soude'et de potasse, le nitrate de potasse, le fer, etc. Or, il est prouvé, d'après lès cxpériences de M. Théodore de Saussure, que ees substances arrivent toutes formées dans l'intérieur du végétal. Déposées dans le sein de' la terre ou dans l'atmosphère, elles sont dissoutes ou entraînées par l'eau qui les eharrie et les transporte dans l'intéricur du tissu végétal.

Ce n'est point l'aete de la végétation qui forme ees substances, ainsi que quelques botanistes et physieiens l'avaient avaneć. C'est la terre ou le milicu dans lesquels les végétaux se développent qui leur eèdent les alcalis, les terres et les substanees métalliques que l'aualyse chimique y fait découvrir. Ce fait, déjà prouvé par les nombreux essais de M. Théodore de Saussure, vient d'être mis dans son dernier degré d'évidenee par les expérienees réecntes de M. Lassaigne. Ce jeune et habile ehimiste répéta de la manière suivante les expérienees de M. Théodore de Saussure :

"Au 2 avril dernier, je plaçai, dit-il, dix grammes de graines de sarrasin (Polygonum Fagopyrum) dans une capsule de platine eontenant de la fleur de soufre lavée et que j’avais humectée aree de l'cau distillée, réeemment préparéc; je la posai sur unc assictte de porcelaine qui contenait un demi-centimètre d'eau distillée, et je recouvris le tout avee une cloehe de verre, à la partic supérieure de laquelle il y avait un robinet, qui, au moyen d'un tube de verre recourbé en siplion et terminé par un entonnoir, me permettait de verser de l'eau de temps en temps sur le soufre.

"Au bout de deux ou trois jours les graines avaient germé pour la plus grande partie; on eontinua de les arroser tous les jours, et dans l'espace d'une quinzaine elles avaicnt poussé des tiges de six centimètres de hauteur, surmontées de plusieurs feuilles.

"On les rassembla avec soin, ainsi que plusieurs grai- 
nes qui n'avaient point levé, et on les ineinéra dans un creuset de platine; la cendre qu'on en obtint pesait 0,220 grammes; soumise à l'analyse, elle a donné rgo de phosphate de ehaux; 25 de earbonate de ehaux, et 5 dé silicé.

j) Dix grammés de ces nêmes semènees ineinéréés fournirent la mềie quantité de éeridre, formée exáetement dès mênies prineipes. )

Il résul té évidémment de cettè expérienee, qui 'fut répétée unie seconde fois et qui donina le même réstiltat, qu'après leứ dévéloppenenent dans'l'eáu distillée, les jeunès

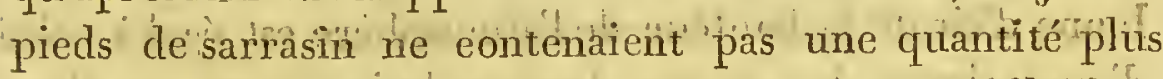
considérable dé sels alealins 'que les graines dont ils piovenaient. D'óu 1 'on peut ēonclure avec $M$. Théodóre de Saussure que les alcalis et les terres que l'on trouvedans les planies ont été absorbés èt tirés diu sól.

Mais quelle est la puissanee qui détermine la suecion dés räeines?'Les lois de lá phýsique et de la mécanique sont insuffisantés pour expliquér un semblable phénomène. La foree extraordinai ave laquelle s'opère celte absorption ne peut être encue d'une manièré sàtisfaisante qu'en ádmettant une puissance, une énergie vitale inhérentè au 'îssú même dès végétaux, et déterminant par son influenee, dont la näứ nous est inconnue, les phénomènes sensibles de la végétation.

C'est au eélèbre physieien Hales que l'on doit les expérienees les plus préeisè et les plúsingénieuses au moyen desquelles on démontre la forée prodigieúse de sueeion dont sont dóuées les racinés et les branehes. Il déeouvrit une des raeines d'un poirier, en eoupa la pointe, 'y adapta T'une des extrémités d'un tube rempli d'eau, dont l'autre extrémité était plongée dans une euve à mercure; et én six minutes le mereure s'éleva de huit pouees dans le tube.

Hales, pour mesurer la force avee laquelle la vigne absorbe l'humidité dans le sein de la terre, fit une expérience dont les résultats paraîtraient inexaets et exagérés, 
s'ils n'eussent été vérifiés dans ees derniers temps par M. Mirbel, qui répéta l'expérience. Le physicicn angiais coupa, le 6 avril, un eep de vigne sans ramcaux, d'environ sept à liuit lignes de diamètre, à trente-trois poutces au-dessus de la terre. Il y adapta un tube à double courbure, qu’il remplit de mereure jusqu'aup̣ès de la courbure qui surmontait la section transversalc de la tige. La sève qui en sortit eut assez de foree pour élever en quelques jours la eolonne de mercureà trente-deux pouees et demi au-dessus de son niveau. Or', le poids d'une eolonne d'aịr de la hauteur de l'atmosphère est égal à celui d'une colonne de mereure de vingt-huit pouces, ou d'une colonne d'cau d'cuviron trente-trois pieds. Dans ce eas, la force avec laquelle. la sève s'élevait des racines dans la tige était done beaucoup plus considérable que la pression "de l'atmosphèrc.

Un grand nombre de faits ct d'expéricnces démontrent la part que les feuilles prennent au phénomène de la succion ct de l'absorption. Ainsi, une branehe détachéc de l'arbre dont elle faisait partie absorbe encore avee une grande force le liquide dans lequel on plonge son extrémité. Il ẹn est de même si on la retourne et que son sommet trempe dans l'eau; sa puissanec absorbante n'en sera pas diminuée.

Pendant l'été nious vojons la ehaleur du soleil flétrir et faire faner les plantes qui ornent nos parterres; mais qu'ou les examine pendant la nuit ou dans la matinée, la rosće que les feuilles ont absorbée lcur a rendu leur force et leur firaîeheur.

Si l'on dépouille cntièrement un vegétal de ses feuilles, il ne tárdera pas à périr, parce que la succion exercéc par ses racines sera insuffisante pour fournir tous les matériaux de sa nutrition.

Dans bcaucoup de plantes, particulièrement dans les Cactus et autres plantes grasses, dont les raciues sont très- 
petites, et qui végètent d'ordinaire sur les roehers ou dans les sables mouvans des déserts, il est évident que l'absorption des fluides nutritifs a lieu presque exelusivement par les feuilles et les autres parties plongées dans I'atmosphère; ear la petitesse de leurs raeines, l'extrême aridité du sol dans lequel elles eroissent, ne suffiraient point pour les faire végéter.

D'après ee qui vient d'ètre dit, on voit eombien dans les végétaux la surfaee absorbante est grande, lorsqu'on la eomparc à leur volume général. Elle est ineomparablement plus eonsidérable que eelle des animaux.

\section{De la Marche de la Sève.}

La sève est ce liquide incolore, essentiellement aqueux, que.les raeines puisent et absorbent đans le sein de la terre, les fcuilles dans l'atmosphère, pour le faire servir à la nutrition du végétal. C'est elle qui, eontenant en dissolution les véritables prineipes nutritifs, les dépose dans l'intérieur de la plante à mesure qu'elle traverse leur tissu.

Les aneiens se sont disputés long-temps pour savoir par quelle partie de la tige l'aseension de la sève avait lieu. Les uns eroyaient que e'était par la moelle; les autres, au eontraire, pensaient que l'éeorce était le siége de ec singuliẹ phénomène. Mais, quand on a eu recours à des expérienees positives, il a été prouvé que ees deux opinions étaient également erronées. En effet, la marche dęla sève se fait à travers les eouehes ligneuses. Ce sont les vaisseaux lymphatiques répandus dans le bois et l'aubier qui servent de eanaux pour eharrier ee fluidè nutritif. Mais e'est la partic la plus voisine de l'étui médullaire qui paraît être le siége prineipal de eette asccnsion. En effet, si l'on fait tremper une branehe ou un jeune végétal dans une liqueur eolorée, on pourra suivre, surtout dans les vaisseaux qui avoisinent l'étui médullaire, les traces du fluide absorbé : 
or, ce fluide ne se verra ni dans la moelle ni dans l'éeorce. Le hasard dévoila eette vérité à Coulon. Ce physiejen faisait ajoattre une allée de grands peupliers en pleine végétation. Il vit sur un pied seié eireulairement à sa base, qui avait été renversé et qui néanmoins tenait encore à sa souehe par. șon eentre, il vit, dis-je, des bulles de liquide et d'air s'élever des fibres intérieures rompues en faisant entendre un bruissement très-manifeste. Il tenta dès lors quelques expérienees sur les arbres qui lui restaient à abattre. Ajnsi, en les faisant pereer avee une large tarière, il vit que les fragmens que l'on retirait des eouches extérieures du bois étaient presque sèehes, et qu'elles devenaient de plus en plus lumides à mesure que la tarière s'enfonęait plus profondement, ęt qu'arrivée vers le centre de la tige, la sève eommenȩait à s'éeouler à l'extérieur. Ces expérienees furent présentées à l'aeadémie des seienees, et MM. Desfontaines et Thouin, qui les répétèrent, en eonstatèrent l'éxaetitude. Ainsi donc ee fait prouve évidemment que l'aseension du fluide séveux se fait par les eonehes ligneuses, et en partieulier par eelles qui sont les plus voisines du eanal médullaire. L'expérienee a eneore démontré que la marehe de la sève ne s'est point arrêtée dans des arbres privés de leur éeoree, et dans lesquels la moelle était plus ou moins obstruée, tandis quiè, si l'on enlève sur un arbre toutes les eouehes ligneuses, l'asecnsion de la sève n'a plus lieu. Cependant elle pourrait eneore se faire s'il restait un petit eylindre de eouches ligneuses; tels sont les arbres ereux, et prineipalement les saules, dont le trone est le plus souvent earié à l'intérieur.

En traversant ainsi les eouches du bois dans sa marehe ascendante, la sève communique avee les parties et branches latérales de la tige, soit direetement par l'anastomose de leurs vaisseaux, soit en se répandant de proche en proche, par les pores intermoléculaires dont sont percés les 
eanaux qui la eharrient. L'eau qui en forme la base essentielle, ehargée des prineipes nourrieiers et réparateurs, s'cn dépouille elnemin faisant, et les dépose dans le tissu végétal.

En parlant précédemment de la suceion des raeines, nous a vons rapporté les expérienees de Hales qui prouvènt la force avee laquelle a lieu la marche de la sève dans une tige, même d'un petit diamètre, puisque eette foree agit avee plus de puissanee sur le mereure qu'une colonne d'air égale à la hauteur de l'atmosphère. Boninet a également expérimenté pour eonnaître la rapidité avec laquelle la sève peut s'élever. Ainsi, en plongeant des jeunes pieds 'dé harieot dans des fluides eolorés, il a vu ees dérniers s'y élever, tantôt d'un demi-pouee dans une demi-heure; tantôt de trois pouees en une heure, tantôt enfin de quatre pouees en trois heures.

Il résulte des observations et des expérienees du professeur Amiei de Modène (I) que les fluides renfermés dans les vaisseaux ou dans les aréoles du tissu eellulaire des plantes se meuvent et cireulent d'une manièré tout-àfait indépendante dans èhaeune de ees eellìles oú de ees vaisseaux. Chaque eavité, dit-il, eonstitue un organe distinet, et e'est dans son intéricur que le fluide se meut en tournoyant, indépendamment de la cireulation particulière qui a lieu dans ehaeune dés eavités adjaeentes. C'est prineipalement sur les Chara vulgaris et flexilis et sur le Caulinia fragilis, plantes aquatiques dont l'organisation se laisse plus faeilement apereevoir, à eausé de la transparenee de leurs parties élémentaires, qué le professeur de Modène a fait ses observations. Pendant son séjoư à Paris, dans l'été de 1827 , j'ai vu ehez M. 'le professeur Amiei, au moyen de son admirable mieroscope; un grand

(r) Atti della Societ. italiana, t. xvifr et xrx; et Annales des Sciences naturelles, $t$. Ir. 
nombre des faits qui ont scrvi de base à scs observations. Ce mouvement du fluide dans chaque eavité du tissu eellulairc ou daus chaquc vaisscau peut être apcrçu, à eause des particules solides qui nagent dans ee fluide. On voit ces particules qui sont des globules d'ume tćnuité cxtrêmc, et quelquefois d'une tcinte verte très-prononcéc, rcmontcr le long d'unc des parois de la eavité; arrivécs vers le diaphragme horizontal qui sépare cette cellule de cclle qui lui est supcrposéc, elles changent de direetion, suivent un 'eours horizontal jusqu'à ce qu'atteignant la paroi opposéc, elles descendeui en la suivant jusqu’à la partie inférieurc, où leur cours rcdevient liorizontal, pour recommencer cnsuitc la mêmc marehe. Il résulte de là que dans un mêne vaisscau il $\mathrm{y}$ a eonstamment quatre couraus différcns, savoir un ascendant, et deux horizontaux cn scns opposés.

Une chosc bicn remarquable, c'est que la direction du mouvement dans chaquc vaisscau nc semble avoir aueun rapport avee celle qui s'cxécute dans les tubes eirconvoisins. Aiusi; quelquefois deux vaisscaux qui sc touehent offriront le mème mourement; tandis quc ceux qui les environnent auront daus le mouvement de lcurs fluides unc dircction tout-à-fait opposée.

Le même obscrvateur fait également remarquer qu'on ne voit aucun globule mobile passer d'unc cavité dans une autre. "Cepcndant, dit-il, je nc prétends pas établir que le sue renfermé dans un vaisseau ne pénètrc pas, quand les eirconstances l'cxigent, dans les vaisscaux voisins. Je me suis même persuadć quc cettc transfusiou est nécessairc pour lc dévcloppement de la plante; mais la partie la plus fluide et la plus subtilc du sue cst la seule qui puissc pénétrer invisiblement à travers la mcmbrane, en traversant des trous quc l'ocil armć du mieroscope ne saurait apcrcevoir. "

Quclle est la causc de ce mouvement indépendant du 
fluide dans chaque partie organique du végétal? Quelques-uns l'out attribué à l'irritabilité dont est douée la membrane qui forme ces tubes. Le piofesseur Amici ne partage pas cette opinion. Il croit reconnaitre la force motrice du fluide dans les espèces de pctits grains verts ou transparens tapissant les parois des tubes où ils sont disposés par rangées ou chapelets, et qui, par unc action analogue à celle des piles voltaïques, impriment au fluịde son mouvement. Ces grains verts sont évidemment les mêmcs que ceux quc M. Dutrochet considère comme le système nerveux des végétaux, et qui, ainsi que nous l'avons dit, ne sont quc des globules remplis de matière verte.

Mais quelle est la cause de cette ascension dc la sève? Comment ce fluide peut-il s'élever des racincs vers la partie supérieure des tiges? On pense bien quc dans les temps anciens chaque auteur a dû avoir une opinion différente pour expliquer cct étonnant phénomènc.

Grew en trouvait la cause dans le jeu des utricules. Cet auteur, qui considérait le tissu végétal comme formé de petitcs utricules juxtaposées les unes au-dcssus des autres, ct communiquant toutcs eutre elles, pcnsait que la sèvc, une fois entrée dans les utriculcs inférieures, celles-ci sc contractaient sur elles-mêmcs, la poussaient dans cellcs qui leur était immédiatement supérieures; et que par ce mécanisme la sève parvenait ainsi' jusqu'au sommet du végétal.

Malpighi, au contrairc, l'attribuait à la raréfaction et à la condensation alternatives de la sève par la chalcur.

De La Hire, qui croyait les vaisseaux séveux garnis de valvulcs comme les veincs des animaux, pensait qu'elle dépendait de cette disposition.?

Pérault la croyait produite par une sorte de fcrmentation.

D'autres cnfin, ct ccux-la sont en grand nombre, ont 
comparć l' narehe de la sève, dans le tissu végétal, à l'aseension des liquides dans les tubes capillaires. Mais on scnt combicn de semblables hypothèses sont insuffisantes pour cxpliquer les phénomèncs dont il s'agit. Si, cn effet, ils étaicnt dus à la capillarité des vaisscaux sévcux, leur action devrait être indépendante des cireonstances extériẹres, et même de la vie du végétal. Or, e’est ce qui n'a pas lieu. Personne n'ignore que la sève ne eireule plus dans un végétal privé de la vic. La vie a done une action direetc et puissante sur l'cxercicc dc eette fonetion. De même que pour la suecion opérée par les racincs dans le sein de la tcrre, nous avons admis une forcc vitale particulière d'où dépendent tous les phénonıènes de la végétation, force qui fait le caraetèrc distinctif des êtres vivans, qui les soustrait à l'empire des eauses plissiques ct ehimiques; dc mênc aussi nous sommes foreés de reeourir encore à elle pour expliquer la marehe de la sève. En effet, si tous les phénomèncs de la végétation n'étaient produits que par l'aetion des agcos méeaniques ou èhimiqucs, par quels earactères distiuguerions-nous les végétaux des êtres inorganiques? Nous devons done admcttre dans les végétaux eomme daus les animaux une force vitale qui présidc à toutes leurs fonctions.

Mais quoique cette forcc vitalc soit lc véritable agent de la marche ascensionelle de la sève, cependant ecrtaincs causes interncs et extcrncs peuvcnt faeilitcr. l'excrciee do ce phénomènc.

Parmi les causes extcrues on doit placcr la températurc, l'influcnce de la lumièrc et du fluide élcetriquc.

On sait généralcment qu'une température ehaudc favorise singulièrcment le cours dc la sève. En cffet, pondant l'hiver, l'arbre cn est gorgé, mais clle est épaisse et stagnante; lc printemps, en ramenant la ehalcur, détcrmine aussi l'ascension des sucs, dans les vaisseaux de la tige qui semblaient en ètre obstrués. 
DES VEGETAUX.

La lumière et le fluide éleetrique ont aussi une influence marquée sur les phénomènes de la marche de la sève. On. sait que, quand l'atmosphère reste long-temps ehargée d'éleetricité, les végétaux aequièrent un développement considérable, ce qui annonee néeessairement que la sèvo a un cours plus rapide et plus puissant.

Certaines eauses internes, e'est-à-dire inhérentes au végétal lui-méme, paraissent agir aussi sur l'aseension de la sève. Telle est la quantité plus ou moins grande de pores eortieaux que présente le végétal, et l'étendue plus eonsidérable de sa surface. Ces deux circonstances favorisent évidemment la rapidité et la foree de la marehe du. fluide séveux.

Nous venons de voir par quelle foree et par quels organes la sève s'élève des raeines jusque vers l'extrémité de torites les branches du végétal. Iei s'opèrent de nouvẹux phénomènes, ici va commencer une nouvelle cireulation.

Fín effet, lorsque la sève est parvenue vers les extrémités des branches, elle se répand dans leurs feuilles. Là elle perd une partie des principes qu'elle contenait, et en aequiert de noureaux. Les feuilles et les parties vertes sont le siége de la transpiration, de l'expiration et de l'excrétion végétales. La sève s'y dépouille de l'air atmosphérique qu'elle eontient eneore, de sa quantité surabondante de principes aqueux, et des substances qui sont devenues étrangères ou inutiles à sa nutrition. Mais en même temps qu'elle perd ainsi tme partie des prineipes qui la eonstituaient auparavant, elle éprouve une élaboration partieulière; elle aequiert des qualités nouvelles, et, suivant tme route inverse de eelle qu'elle vient de parcourir, elle redeseend des feuilles vers les racines, à travers le liber ou la partie végétante des eouches eorticales.

Examinons en particulier tous les phénomènes qui s'opèrentdans les feuilles par l'effet de l'ascension de la sève. 


\section{\$3. De la Transpiralion.}

La transpiration ou émanation aqueuse des végétaux est cette fonction par laquelle la sève, parvenue dans les organes foliacés, perd et laisse ćchapper la quantité surabondante d'eau qu'elle contenait.

C'est en général sous forme de vapcur que ectte eau s'exhale dans l'atmosphère. Quand la transpiration est pen considérable, cette vapcur est absorbéc par l'air à mesure qu'elle se forme. Mais si la quantité augmente, et si la tcmpérature de l'atmosphère est peu élcvée, on voit alors ce liquide transpircr sous forme de gouttclettes extrêmement petites, qui souvent se réunissent plusieurs ensemble et deviennent alors d'un volume remarquable. Ainsi on trouve fréquemment, au lever du soleil, des gouttelettes limpides qui pendent de la pointe des,feuilles d'un grand nombre de Graminées. Les feuilles du choux en présentent aussi de très-apparentes. On avait cru long-temps qu'clles étaicnt produites par la rosée; mais Musschenbrock prouva le premier, par des expériences concluantes, qu'elles provenaicnt de la transpiration végétale condensée par la fraîcheur de la nuit. En effet, il intercepta toutc communication à une tige de pavot avec l'air anbiant, en la recouvrant d'une cloche, et avec la surface de la terre, en recouvrant le vase dans lequel était la plante d'unc plaque de plomb, et le lendemain matin les gouttclettes s'y trouvèrent comme auparavant.

Hales fit égalcment des expériences pour évaluer le rapport cxistant entre la quantité des fluides absorbés par les racines, ct celui que ces feuilles exhalent. Il mit dans um vase vernissé un pied de l'Helianthus annutus (grand soleil), recouvrit le vase d'une lame de plomb percée de deux ouvertures, l'une par laquelle passait la tige, l'autre destinéc à pouvoir l'arroser. Il pesa exactement cet appa- 
reil pendant quinze jours de suite, et vit que pour terme moyeu la quantité d'eau expirée pendant les douze henres de jour était de viugt onees environ. Un temps see et ehaud favorisait singulièrement eette transpiration, qui s'éleva à trente onees dans une eireonstance semblable. Une atmosphère ehargée d'humidité diminuait au eontraire sensiblement. eette quantité; aussi la trauspiration n'étaitelle au plus que de trois onees pendant la nuit, et même quelquefois la quantité de liquide expirée devenoit insensible quand la nuit était fraîehe et humide.

Ces expériencès ont été depuis répétées par MM. Desfontaines et Mirbel, qui ont eneore eu oceasion d'admirer l'exaetitude et la sagaeité du physieien anglais.

Sénebier a prouvé par des expériences multipliées que la quantité d'eau expirée était à eelle absorbée par le végétal dans le rapport de $2: 3$; ee qui démontre, eneore qu'une partie de ee liquide est fixée ou déeomposée dans. l'intérieur du végétal.

Ces faits prouvent d'une manière ineontestable, I que les végétaux transpirent par leurs feuilles, e'est-à-dire qu'ils rejettent une eertaine quantité de fluides aqueux;

$2^{\circ}$ Que eette transpiration est d'autant plus grande que l'atmosphère est plus ehaude et plus sèehe; tandis que, quand le temps est humide, et surtout pendant la nuit, la transpiration est presque nulle;

$3^{\circ}$ Que eette fonetion s'exéeute avee d'autant plus d'àetivité que la plante est plus jeune et plus vigoureuse ;

$4^{\circ}$ Que la nutrition se fait d'autant mieux que la transpiration est en rapport avee l'absorption: Car, lorsque l'une de ees deux fonctions se fait avee une foree supérieure à eelle de l'autre, le végétal languit. C'est ee que I'on observe, par exemple, pour les plantes qui, exposées aux ardeurs du soleil, se fauent et perdent leur vigueur, parèe que la transpiration n'est plus en équilibre ayce la succion excrcée par les racines. 


\section{\$4. De l'Expiration.}

Nous avoins dit et prouvé préećdemment que les végétaux absorbent ou inspirent une ecrtaine quantité d'air ou d'autres fluides aériformes, soit directement, soit mélangés avee la sève, e'est-à-dire tout à la fois par le moyen de leurs raeines et de leurs feuilles : or, e'est la portion de ees flujđes qui n'a point été dćeomposée pour servir à l'alimentation qui forme la matière de l'expiration. Les plantes sont done, eomme les animaux, douées d'une sortc de respiration, qui sc eompose également des deux phénomènes,' de l'iuspiration et de l'expiration, toutefois aree cette différence très-notable qu'il n'y a point iei développement de ealorique. Cette fonetion devient très-manifeste si l'on' plonge une branche d'arbre ou une jeunc plante dans une cloche de verre remplic d'eau, et qu'clle soit cxposée à l'action de la lumière; en effet, on verra s'élcver de sa surface un grand nombre de petites bulles qui sont formécs par un air très-pur et presque cntièrement eomposé de gaz oxygène. Si, au contraire, cette expérienee était faite dans un lieu obseur, les feuilles expireraicnt de l'acide eaibonique et du gaz azote, et non du gaz oxygène. 11 faut noter jei soigneusement que toutes les autres parties du végétal qui n'offrent pas la couleur verte, telles que les raciues, l'éeorec, les fleur's, les fruits, soumises aux mềmes expériences, rejetteront toujours au dehor's de l'acide earbonique, ct jamais de l'oxygène. Par eonséquent l'expiration du gaz oxygène dépend non-seulement de l'influenec dircete des rayons lumineux, mais eneore de la coloration verte des parlies.

Noits savons que les végétaux absorbent une grande quantité d'aeide earbonique qu'ils déeomposent dans l'intérieur de leur tissu, quand ils sont exposés à l'action du soleil, et rejettent à l'extérieur la plus grande partie de l'oxygène 
qui était eombiné avee le carbone. Or, ee phénomène est encore une véritable expiration.

Lorsqu'une plante esit morte ou languissante, ou bien l'expiration eesse entièrement, ou bien le fluide expiré est eonstamment du gaz azote. Il est eertains végétaux qui, mêmé exposés à l'influcucé des rayons du soleil, n'expirent que de l'azote; tels sont la sensitive, le houx, le lauriercerise, et quelques autres. Il nous paraît difficile d'indiquer la véritable eause d'une pareille anomalie.

\section{\$5. De l'Excrétion.}

Les déjeetions végétales sont des fluides plus on moins épais, susceptibles quelquefois de se eondenser et de se solidifier. Leur nature est très-variéc. Ce sont tantôt des résines, de la eire, des huiles volatiles; tantôt des matières sucrées, de la manne, des huiles fixes, ete. Toutes ces substanees sont rejetées à l'extérjeur par la foree de la végétation. Ainsi le Fraxinus Ornus, et quelques autres espèces de frênes, laissent sujnter, en Calabre, un liquide épais et sucré, qui, par l'action de l'air, se concrète et forme la manne. Les pins, les sapins, et en général tous les arbres de la famille des Conifères, fournissent des quantités eonsidérables de matières résineuses. Beaucoup de végétaux, tels que le Ceroxylon andicola, superbe espèce de palmier, déerite par MM. de Humboldt et Bonpland, le $M$ Yyriça cerifer a de l'Amérique septentrionale, domnent une grande quantité de eịre utilement employée dans la patrie de ces végélaux.

Les racines excrètent aussi par leurs extrémités les plus délićes eertains fluides qui nuisent ou sont utiles aux plantes qui végètent dans leur vioisinage. C'est de eette manjère que l'on peut expliquer les eonvenances ou les antipathies de eertains végétaux. Ainsi l'on sait que le chardon hémorrhoïdal nuit à l'avoine, I'Erigeron acre au froment, la scabieuse au lin, ete. 
Tels sont les différens phénomènes qui dépendent de la présence de la sève, quand elle est parvenne it la partic supérienre des régétaux. Suivons-la maintenant daus son cours rétrograde, des feuilles vers les racines.

\section{\$6. De la Sève descendunte.}

Ce point a été l'objet d'un grand nombre de discussions parmi les physiologistes. Plusicurs, en effet, ont longtemps nié l'existenee d'une sère desecndante. Mais les phénomènes sensibles de la végétation, et les cxpérienecs les plus précises ont démontré qu'il existc une sceonde sève, qui suit une marche inverse de celle que nous avons précédemment examinée. En effet, si l'on fait au tronc d'un arbre dicotylédon une forte ligature, il se formera au-dessus ä'elle un bourrelet circulaire qui deviendra de plus en pluss saillant. Or, ce bourrelct pourrait-il c̀tre formé par la sève qui des racines monte vers les feuilles? On conçoit qu'alors il devrait se présenter au-dessous de la ligatnre, et non an-dessus. Mais le contrairc a licu; ce bourrelet ne peut done dépendre que de l'obstacle éprouré par les sucs qui desecndent de la partic supéricure vers linféricure, à travers les couches corticales. Done il existe une sève descendante.

La sève desendante, dépouilléc de la plus grande partic de ses principes aqueux, beaneoup plus élaborée, contenant plus de principes nutritifs que la première, concourt essenticllement à la nutrition du végétal. Circulant dans la partie végétante de la tige, dans celle qui est sculc susceptible d'accroisscment, ses usages ne peurcut paraitre équivocques.

En effet, examinons cncore de plus près les phénoniènes qui résultent de la ligature circulaire faite an trone d'mu arbre dicotylédon, et nous verrous que non-seulement il se forme un bourrelet au-clessus de ectte ligature, mais que la partie du tronc siluce au-dessous d'eile cesse de 
s'aeeroître, et qu'aueune couehe cireulaire nouvelle ne s'ajoute à celles qui existaient déjà. Or, ne voyons-nous point ici, de la manière la plus éviden te, l'usage de la sìre descendante? C'est elle qui renouvelle et cutreticnt contiuucllement le cambium : e'est donc cllc qui concourt essentiellement à l'aecroissement et au développement des arbres dicotylédonés.

Mais cette seconde sève n'est point de la inême nature dans tous les végétaux. Il cn est dans leque!s clle formc un suc blanc et laiteux, comme dans les Euphorbes; dans d'autres (les Papavéracées), c'est un suc jaunàtre ou brunâtre; dans les Conifères, elle est plus ou noins zésincuse, cte. Néanmoins nous devons fairc remarquer que, suivant un grand nombre de physiologistes, les sucs propres des végétaux ne sont pas la sève descendante ellc-même, mais des fluides que l'acte de la végétation cn séparc. La diversité de naturc que préscntent ces sucs, leur préscucc dans quelques végétaux sculement, leur situation daus cies vaisscaux détcrminés ct cu petit nombre, nous paraissent aulant de preuves qui ćtayent cette dernic̀re opinion:

Nous venons de passcr successirement en revue les différens pliénomèncs qui ont r'apport ou concourent à la nutrition des végétaux. Nous a vons vu les sućs puisés par les racines dans le sein de la terre portés par ıne force particulièrc, dépcndante de la vic du végétal, jusqu'aux parlies les plus élevécs des dermières ramifications de la tige; là, en se mêlant avec les flêtides absorłés, en se déponillant des principes aqueux et aériformes inutiles à la nutrition, acquérir des proprićtés notuvelles; ct, suivant une marclic rétrograde, devenir les véritables alimens du végétal.

On voit par-lì que la nuḷilion dans les plantes, quoique 
ayant de grands rapports avee la même fonetion dans les animaux, en diffère essenticllement.

En effet, e'est par leur bouehe que les animaux introduisent dans leur intérieur les diverses substances qui doivent servir à leur nutrition. C'est au moyen des bouehes aspirantes qui terminent leurs raeines que les végétaux absorbent, dans l'intérieur de la terre, l'eau mélangée des matières néeessaires ou inutiles à leur développement.

Dans les animaux, les matières absorbées suivent un seul et même eanal, depuis la bouehe jusqu'à l'endroit où la substanee vraiment nutritive (le chyle), doit être séparće des matières inutiles ou exerémentitielles.

Dans les végétaux, le même phénomène a lieu: les fluides absorbés pareourent un eertain trajet avant d'arriver jusqu'aux feuilles, où s'opère la séparation des partics néeessaires ou inutiles à la nutrition.

Les animaux et les végétaux rejettent au dehors les substanees impropres à leur développement.

Une des différenees les plus tranehées qui existent entre les végétaux et les animaux, c'est que les premiers se nourrissent esscntiellement de matières inorganiques, telles que d'eau, de 'earbone, d'hydrogène, d'oxygène, ete., tandis que dans les animaux les matières qui servent à la nutrition sont uniquement des substanees organiques, tirées des règnes animal et végétal.

Le ehyle, ou la partic nutritive des animaux se mêle au sang, qu'il entretient et répare eontinuellement, pareourt toutes les parties du corps, et sert au développement et à la nutrition des organes.

La sève des végétaux, après avoir éprouvé l'influenee de l'atmosphère dans les feuilles, après avoir aequis une nature et des propriétés nouvelles, redeseend dans toutes les parties du végétal pour y porter les matériaux de leur aeeroissement et scrvir au développement de toutes leurs parties. 


\section{DEUXIEME CLASSE.}

\section{ORGANES DE LA REPRODUGTION.}

$\mathbf{L}_{\mathrm{ES}}$ organes de la reproduetion, que nous désignons encore sous le nom d'organes de la fruetifieation, sont ceux qui servent à la eonservation de l'espèce et à la propagation des raees. Leur rôle n'est pas moins important que celui des organes dont nous venons d'étudier la strueture et les usages. En effet, si les premiers sont nécessaires à l'existence de l'individu, au développement de toutes ses parties, les seconds sont indispensables pour que cet individu puisse devenir apte à procréer d'autres êtres semblables à lui, qui puissent renouveler et perpétuer'son espèee.

Dans les plantes, ee sont la fleur, le fruit et les différentes parties dont ils sont formés, qui composent les 'organes de la reproduetion. Aussi les avons-nous distingués en deux seetions, savoir : les organes de la floraison et les organes de la fruetifieation. 


\section{SECTION PREMIÈRE.}

DES ORGANES DE LA FLORAISON.

\section{Considérations générales sur la Fleur.}

Nous connaissons déjà les parties qui servent à fixcer la plante au sol, à absorber dans le sein de la terre, ou-au milicu de l'atmosphère, les fluides aqueux et aériformes nécessaires à la nutrition ct au développcment du végétal; nous venons d'étudier la série d'organes qui concourent à l'entretien de la vie individuelle : occupons-nous maintenant des organes, non moins essenticls, dont l'action tend à renotzveler et à perpétuer l'espèec.

Ici se présente une grande ressemblanee entre les végétaux et les animaux. Les uns et les autres, en effet, sont pourvus d'organes partieuliers, qui, par leur influence réciproque, concourent à la fonction la plus importante de leur vie. La génération est le but final pour lcquel la nature a créé les différens organes des végétaux ct des animaux. L'analogic la plus parfaite existc entre eux danseette grandc fonction. C'est de l'action que l'organe mâle exeree sur l'organc femelle que résulte la fécondation, ou ce phénomènc par lequel l'cmbryon, encore à l'état rudimentaire, reçoit et conservele principe animatcur de la vie. Cependant remarquons ici lesmodifications que la nature a imprimées à ecs dcux grandcs elasses d'êtres organisés. La plupart des animaux apportent cn naissant les organes qui doivent servir un jour à les reproduire; ces organes restent engourdis jisqu'à l'époque où la nature, dirigeant sur cux une nouvelle énergic, les rend eapables de remplir les usages pour lesquels elle les a eréés. Les végétaux, 
au contraire, sont à leur naissance dépourvus d'organes scxuels. La nature ne les y développe qu'au moment où ils doivcnt-scrvil à la féeondatioì. Une autr'c grande disscmblance entrc les animaux et les" végétaux, c'est que, dans les premicrs, les organes; scxucls peuvent scrvir plusieurs fois ì la mếme fonetion, naissent ct meurent avee l'ètre qui les portc; tandis que dans les végétaux, dont le tissu est mol et délieat, ecs organes n'ont qu'une existcnce passagère : ils paraissent pour aecomplir le voeu de la nature, se fanent et se détruisent aussitôt qu'ils l'ont rempli.

Admirons la prévoyance de la nature dans la distribution des sexes parmi les êtres organisés. Les végétaux fixés invariablement au lieu qui les a vus naître, privés de la faeulté locomotivc, portent, lc plus souvent, sur le mêmc individu, les deux organes dont l'action mutuelle doit produirc la fécondation. Les animaux, au eontraire, qui, donćs de la volonté et de la faculté de se mouvoir, peuvent se diriger dans tous les sens, ont cn général les sexes séparés sur des individus distincts. C'cst pour cette raison que l'hermaphroditisme est aussi eommun chez lcs végétaux qu'il est rare parmi les animaux.

La fleur est esscnticllement constituée par la préscnca d'un des deux organes sexucls, ou des deux réunis sur un support commun, avec ou sans enveloppes cxtéricures destinées à les protéger.

Réduitc à son dernier degré de simplicité, la flcur peut donc n'être formée que par un scul organc sexuel, mảlc ou femclle, c'est-à-dirc par unc étcmine ou un pistil.

Ainsi, dans les saules, dont les fleurs sont unisexuées, lcs flcurs mäles eonsistent simplement en une, deux ou trois étrmines, attachćes sur une petite éeaillc. I_cs fleurs fenclles sont formées par un pistil, également aecompaguć d'une ćcaille, sans aụtres organes aeccssoires. Daus 
ce eas, comme dans un grand nombre d'autres, la fleur est aussi simple que possible. Elle prend alors le nom de fleur mäle ou de fleur femelle, suivant les organes qui la composent.

La fleur hermaphrodite est cellc, au contraire, qui présente réunis les deux organes sexuels, mâle et femelle.

Mais les différentes fleurs que nouis venons d'examiner ne sout pas complètes. En effet, quoique l'essence de la fleur consiste dans les organes scxuels, pour être parfaite, il faut encore qu'clle présente d'autres organes qui, bicn qu'accessoires, ne lui appartiennent pas moins, et servent à favoriser ses fonctions. Ces organes sont les cnveloppes florales ou périanthe, c'est-à-dire le calice et la corolle. La fleur complète sera donc celle qui présentera les deux organes sexuels entourés d'une corolle et d'un calice.

Sous le point de vue de son organisation primitive, on peut dire que la fleur, eomplète se compose de quatre vertieilles de feuilles diversement modifiées, très-rapprochés les uns des autres. Nous développerons plus tard cette idéc quand nous aurons fait connaître les diverses partics constituantes de la fleur et leur position respeetive.

Il est important d'cxaminer ici dans quel ordre symétrique sont disposés entre eux les différens organes coustituant une fleur complète.

En allant du ccntre à la circonférenec, nous verrons : le pistil, ou organe scxucl femelle, oceuper toujours la partie eentrale de la fleur. Il se compose de l'ovaire, du style et du sligmate. Plus en dehors, sont les organes scxuels mâles, ou les étamines, ordinairement en nombre plus considérable que les pistils, et composées d'un filet et d'une anthère.

A l'extéricur des étamines se trouve la plus intérieure dẹs deux enveloppes florales, ou la corolle : on l'appelle monopétale, quand elle est forméc d'unc sculc pièce; 
polypétale, quand elle est formée de plusieurs pièces, nommées pélales: cnfin la plus extéricure des deux cnveloppes florales est le calice, qui est monosépale ou polysépale, suivant qu'il est eomposé d'une ou de plusieurs pièccs nommées sépales. Tout eequi est en dehors du ealiee n'appartient plus en propre à la flcur; telles sont les fenilles florales ou les bractées qui les aceompagnent fort souvent, et qui doivent en être considérées comme des parties accessoires.

Prenons dans la nature quelques exemples de fleurs daus lesquelles nous ehercherons à reconnaître ct à dénommer les diflérentes parties que nous venons d'énumérer. La giroflée jaune (Cheiranthus Cheiri) va nous servir d'exemple.

Tous verrons le eentre de la fleur occupé par un petit corps allongé, un peu comprimé d'avant en arrière, présentant, lorsquion lc fend longitudinalement dans scs deux tiers inférieurs, dcux eavités dans lesquelles sont renfermés les ovules : ce eorps est le pistil. Il se compose d'un ovaire ou partic inférieure, d'un style, prolongement filiforme du sommct de l'ovairc, terminé par un petit eorps visqueux, glandulaire et bilobé : c'est le stigmate. En dchors du pistil nous trouvons six organes de même forme, de même strueture, disposés eirculairement autour de l'organe femelle, composés chacun d'une partie inférieure filamentiforme, que surmonte une espèce de petit sae ovoïde, à dcux loges, remplis d'une poussière jaunâtre. A leur posilion ct à leur structure, nous reeonnaîtrons ces corps pour les étamines, ou organes sexuels mâles. Lcur partic inférieure filamentiforme est le filet; leur partie supéricure est l'anthère; la poussière qu'ils renferment est 1c pollen. En examinant ce qui reste au dehors des organes sexuels, nous apercevons huit appcndices membraneux, disposés par deux sérics, quatre plus intérieurs, et quatre occupant la partic externe de lá fleur. 
Les quatre intéricurs, plus grands, d'une coulcur jaune, parfaitement scmblables entre etx, eonstituent un seul et même organe; e'est la corolle, qui dans ee cas est eomposée de quatre pièees distinetes ou de quatre pétales. II nous sera très-facilc maintenant de dénommer les quatre pic̀ees verdàtres, plus petites, situées en dehors de la corolle. En effet, nous savons decja que la plus externe des deux enveloppes florales est le calice. Ici le calice est done formé de quatre pièces ou sépales.

Telle est la strueture et la position respeetive des différens organes qui eonslituent une fleur eomplète. Examinons maintenant quelques fleurs dans lesquelles tous les organes que nous venons d'énumérer ne se rencontrent pas. Dans la tulipe, par exemple, nous trouvons au centre de la fleur le pisiil, eomposé d'un ovaire prismatique et à trois faecs, dont le sommet est couromné par un eorps glandulaire, qui est le sligmale : il n'y a point de style. En dehors nous vojons six étamines, dont la strueture n'a. ricn de remarquable. Voili donc les deux organes sexucls; mais à leur extérieur nous trouvons six pic̀ees, ou segmens membrancux, parfaitement scmblables entre eux, ne formant évidenment qu'un scul et même organe. Dans cette fleur il manque donc une des deux enveloppes tlorales; mais quclle est celle qui manque? Cette question a bcaucoup occupé les botanistes, qui tous ne sont pas encore d'accord à ee sujct. Les uns, en eflet, aree Linnæus, veulent que, lorsqu'il n'existe qu'ume scule enveloppe florale autour des organes sexucls, on la nomme corolle, quand elle offre des couleurs vives; calice, quand elle est verte. On voit eombien ectle distinction est fondée sur des caractères peu fixes. Les autres, au contraire, avec M. de Jussieu, eonciuits par les lois de l'analogic, la regardent eomme un calice, quelles que soicnt sa couleur et sa eonsistance. Nous partageons eette opinion, et nous appellerons calice l'enveloppe florale smique qui se trouve 
autour des organes sexuels. D'autres autchrs, voulant remédier ì cette dircrsité d'opinions, et concilier en quelque sorte les deux partis, doment lc nom de périgone à l'envcloppe florale unique qui entoure les organes sexucls. La tulipe, que nous examinons, a donc un calice formé de six sépales, ou un périgone composé de six pièces distinctes.

Eufin, comme nous l'avons vu précédemment, il est des fleurs dans lesquelles les deux enveloppes florales unauquent en mème temps. On les a appelćes fleurs nues, pour les distinguer de celles qui sont munies d'enveloppes, floralcs.

\section{CHAPITRE PREMIER.}

DU PÉDONGULE ET DES BRACTÉES.

LA fleur peut être fixéc de diverses manières atix branclics ou aux rameaux qui la supportcnt. Ainsi tantôt elle y cst immédiatement attachée par sa base, sans le secours d'aucune partie accessoire ou intermédiaire; dans ce cas clle est dite sessile (flos sessilis). On la nomme au contìaire Rleur pédonculée ( Ros pedunculatus ), quand elle y est fixée au moyen d'un prolongement particulier, nommé vulgaircment queue de la fleur, et.désignć en botanique sous le nom de pédoncule. Le pédoncule de la fleur, de mème que le pétiole de la feuille, peut être simple ou ramifié. Quand il est ramifié, chacune de ses divisions, portant une seule fleur, prend le nom de pédicelle, et les flcurs sont dites pédicellées (flores pedicellati). Ainsi la fleur de l'oillet ordinaire est pédonculéc, et chacune des fleurs qui composent la grappe du lilas ou de la vigne est pédicelléc. 
Le Pédoncule, ou support des fleurs, affeete différentes modifications qu'il est utile de faire eonnaître.

Ainsi, suivant sa situation, il est radical quand il part de l'aissclle d'une feuille radicale, comme dans le pissenlit (Taraxacum dens leonis), la primevère (Primula veris).

On lui donne le nom spéeial de hampe (scapus) quiand il part immédiatement d'un assemblage de feuilles radi cales, eomme dans la jaciuthe, les nareisses, ete.

Il est caulinaire ou ramaire, suivant qu'il naît de la tige ou des rameaux; ce qui est la disposition la plus ordinaire.

Il est pétiolaire quand il fait corps, dans une partic de sa longucur, avec le pétiole.

Epiphylle, lorsqu'au lieu de naître sur la tige ou les rameaux, il prend origine sur la surface même des feuilles; tel est eelui du petit houx (Ruscus aculeatus).

Axillaire, lorsqu'il naît sur la tige ou les rameaux dans l'aisselle des feuilles.

Extraxillaire ou latéral, quand il prend naissance sur les partics latérales du point d'insertion de la feuille, comme dans les Solanées.

Terminal, quand il termine le sommet de la tige, dont il ne paraît être que la continuation.

Le Pédoncule est uniflore, biflore, triflore, multiflore, suivant le nombre des fleurs qu'il supporte.

Il est quelquefois roulć en spirale ou cn tirc-bouchon, eomme dans le Vallisneria spiralis; le pain de poureeau (Cyclamen europaum) offre aussi cette singulière disposition, lorsque son fruit approche de la maturité.

Il arrive fréquemment qu'autour d'une ou de plusieurs fleurs réunies on trouve un certain nombre de petites feuilles tout-à-fait différentes des autres par leur couleur, leur forme, leur consistanee, etc. On leur a donnéle nom de bractées (bractece). Ne confondez pas les braetées avec les feuilles florales proprement dites. Celles-ci, en ellet, 
ne différent point notablement des autres feuilles de la même plante; mais elles sont seulement plus petites et plus rapproekées des fleurs. Ainsi, dans le Salvia hormirum et le Salvia sclarcea, les bractées-sont très-apparentes, et fort distinetes des feuilles; elles sont eolorées en bleu.

Quand les bractées ou les feuilles florales sont disposées symétriquement autour d'une ou de plusieurs fleurs, de nunière à leur former une sorte d'enveloppe aeeessoire, on donne à leur réunion le nom d'iıvolucre. Ainsi, dans les anémones, on trouve au-dessous de la:fleur trois feuilles florales disposées symétriquement, qui eonstituent un involucre triphylle. L'involucre est dit tétra-, phylle, pentaplyylle, hexaphylle, polyphylle,' suivant qu'il est formé de quatre, cinq, six, ou d'un grand: nombre de braetées. Quand le pédoncule: est divisé, et qu'à.la base de ehaque pédicelle se trouve un petitinvoluere, on nomme eelui-ci involucelle : par. exemple, dans la carotte, à la base des pédoncules, on "observe un involucre polyplyylle, et à la base des pédicelles, un involucelle également polyphylle.

Les bractées sont le plus souvent libres de toute adhérenee; d'autres fois elles adhèrent avee lè pédoneule de la fleur, comme dans le tilleul (Tilia europaea).

Elles ont ordinairement une strueture et une eonsistanee foliacées; quelquefois cependant ce sont de petites éeailles, plus ou moins nombreuses et serrées autour de la fleur. Dans ce cas, si elles sont persistantes, et qu'elles entourent la base du fiuit ou l'enveloppent entièrement, à l'époque de sa maturité, elles forment ce que les botanistes nomment une cupule (cupula), comme dans les ehênes, etc.

La cupule peut être squanacée, e'est-à-dire formée de petites éeailles très-serrées, eomme dans le chêne (Quercus Robur). 
Elle peut ĉtre foliacée, c'est-ì-dire formće par de petites folioles, plus ou moins libres et distinctes, eomme dans le noisetier (Corylus Avellana).

Enfun elle est quelquefois pericarpoïde, e'est-à-dire formée d'une seule pièee, recouvrant et eachant entièrement les fruits, s'ouvrant quelquefois régulièrement, pour les laisser éehapper à l'époque de leur maturité, eomme dans le châtaignjer, le hêtre, ete.

Quand l'involucre entoure une seule fleur, qu'il en est très-rapproehé, et semblable au ealice, on l'appelle calicule ou ealice extérjeur, eomme dans la mauve, la gujmauve; les fleurs qui ont un caljcule sont dites caliculées (Aores caliculati).

La spathe (spattua). est un involuere membraneux, renfermant une ou plusieurs fleurs, qu'il recouvre entièrement avant leur épanouissement, et qui ne se montrent à l'extérieur qu'après son déroulement ou son déehirement. Par exemple, dans les Palmiers, les nareisses, les différentes espèees d'Allium, telles que l'ognon commun, ete. La spathe est monophylle, e'est-à-dire eomposée d'une seule pièce, eomme dans le gouct (Arum maculatum); composée de deux pièees, ou diphylle, dans l'ail, l'ognon, ete.

Elle est cuculliforme (s. cucullata), ou roulée en cornet, dans l'Arum.

Ruptile, c'est-à-dire se déchirant irrégulièrement pour laisser sortir les fleurs, eomme dans les narejsses.

Uniflore, biflore ou multiflore, suivant qu'elle renferme une, deux ou un grand nombre de fleurs.

Membraneuse, quand elle est minee et demi-transparente, eomme dans les narcisses, les Allium.

Lignense, quand elle offre la consistanee et le tissu du bois, eomme dans plusieurs Palmiers. Par exemple le dattier (Phonix dactylifera), ete.

Pétaloïde, quand elle est molle ct eolorée comme la eorolle. Fxemple : le Richardia athiopica, etc. 
Quelquefois les fleurs eontenues dans une spathe sont enveloppées ehaeune dans une petite spathe partieulière, qui porte le nom de spathille, eomme la plupart des Iridées.

Les Graminées et les Cypéraećes, qui s'ćloignent tant des autres fanilles de plantes par leur aspeet général et la structure de leurs organes, n'ont ni ealiee ni eorolle proprement dits. Les parties auxquelles on avait donné ee nom diffèrent essentiellement de ces mêmes organes dans les autres végétaux phanérogames. Ce ne sont que de véritables involueres, mais qui affeetent une disposition particulière, qu'on ne retrouve pas dans les autres vẹ́gélaux; aussi leur a-t-on donné des noms partieuliers:

Ainsi on appelle glume (gluma), dans les Graminées; les deux éeailles, de forme très-variée, qui sont les plus voisines des organes sexuels. (Voy. pl. 6, fig. 15, 6 b.) Quelquefois ees deux paillettes sont soudées en une seule, qui alors est bifide, eomme dans l'A lopecur'us, le Cornucopice. Toutes les autres paillettes qui sont en dehors de la glume eonstituent la lépicène (lepićena): Leur nombre est très-variable. Ainsi il y en a une dans l'Agrostis canina, L. ; deux dans le plus grand nombre des autres Agrostis, le Cynodon, ete. (Voy.pl. 6, fig. i 4, a a.) Souvent, en dehors des organes sexuels, on trouve un ou deux petits eorps de forme très-variable; ils portent le nom de paléoles, et leur ensemble eonstitue la glumelle (glumella). (Voy.pl. 6, fig. r5, a a.)

Lorsque dans les Graminées, deux ou un plus grand nombre de fleurs sont réunies de manière à former une sorte de petit épi nommé épiet (spicula) ou lodicule, leur enveloppe eommune reẹoit également le nom de lépicène; elle peut ĉtre unipaléacée, eomme dans le Lolium, ou bipaléacée, eommedansle Poa; ou multipaléacée, eomme dans quelques espèces d'Uniola. Il résulıe de là que ehaque petite fleur en partiçulier est dépởurvue de lepicènę 
propre, et n'est entourée que d'une glume, qui, dans ce cas, est toujours bipaléacée. On dit alors que l'épiet ou la lépicène est biflore, triflore, etc., suivant le nombre des fleurs qu'ils renferment.

\section{CHAPITRE II.}

DE L'INFLORESCENCE (I).

On donne le nom d'inflorescence à la disposition générale ou à l'arrangement que les fleurs affectent sur la tige ou les autres organes qui les supportent.

Les fleurs sont dites solitaires, toutes les fois qu'clles naissent seule à seule de différens points de la tige, à des distanees plus ou moins grandes les unes des autres; par exemple, dans la tulipe, le rosier ì eent feuilles.

Elles sont terminales, quaud elles sont situées au sommet de la tige, eomine dans la tulipe.

Latérales, quand elles se développent sur les côtés des tiges ou des rameaux.

Axillaires, quand elles naissent à l'aisselle des feuilles, comme dans la pervenche ( $V$ inca major), la Véronique à feuilles de lierre ( $V$ eronica hederafolia), etc.

On appelle fleurs géminées (flores gemini) celles qui naissent deux à deux d'un même point de la tige, comme dans le Viola biflora.

Ternées ( flores ternati), celles qui naissent trois à trois d'un même point de la tige; par excmple, eclles du Teucrium flavun.

Fasciculéés ou en faisceau (flores fasciculali), quand

(I) M. Roper, professeur de botanique à Bâle, a récemment publié ua mémoire très-intéresşant sur l'inlloreşcence. 
clles sortent plus de trois ensemblc d'un même point dc la tigc ou des rameaux, eomme dans le ecrisicr (Cerasus communis ).

Examinons les espèees d'infloreseence qui ont reçu des noms particuliers.

$I^{\circ}$ Lorsque les fleurs sont disposécs sur un axe eommun, simple et non ramifié, qu'elles soient sessiles ou pédonculées, quc le pédoncule soit droit ou penehé, elles forment un épi (spica, flores spicáti); exemple : le blé, l'orge, le seigle, le plantain lancéolé, le eassis (Ribes nigrum), l'épine-vinette (Berberis unlgaris); les orehis, ete.

La base de ehaque fleur est souvent aeeompagnće d'une écaille ou braetée, l'épi alors est dit squammifère ou braetéolé, par exemple, dans l'Orchis militaris.

Quclquefois les fleurs sont disposées en spirale autour du rachis, [comme dans l'Ophrys cestivalis et l'O. autumnalis (Spiranthes, Rich. )

D'autrefois lcs fleurs sont très-serrées, l'épi est court et globuleux ( spica globosa) eomme dans l'Orchis globosa, plusieurs espèces dc scille, etc.

$2^{\circ}$ Si le pédoncule commun se ramifie plusieurs fois et d'une manière irrégulière, cettc disposition prond le nom de grappe (racemus, flores racemosi) comme dans la vigne.

Les caraetères qui ont été donnés par la plupart des auteurs pour distingucr l'épi dc la grappe sont tcllement incertains, qu'il est presque impossible de distinguer ces deux modes d'inflorescence. En eff'et, les uns ont dit que dans l'épi lcs fleurs étaient sessiles, et pćdonculées dans la grappe; d'autres ont dit que la grappe était toujours pendante et l'épi dressé. Nous croyons inutile d'insistcr sur le peu de valcur de ees caractères. Cclui quc nous prenons nous paraît plus fixe et surtout d'une applieation plus facile dans la pratique : l'axe d'un épi est toujours simple; eclui d'ume grappe est constamment ramifié. 
$3^{\circ}$ Quand l'axe commun est dressé, les pédoncules irrégulièrement divisés en pédicelles portant les fleurs, si cet assemblage a une forme à peu près pyramidale, on lui dome le nom de thyrse (thyrsus, flores thyrsoïdei ). Tcls sont le lilas (Syringa vulgaris), le troëne (Ligustrum vulgare), le marronier d'Inde (Assulus Hippocastanum). Cette espèee d'infloresecnee sc distingue à peine de la grappe.

$4^{\circ}$ On dit que les flcurs sont disposées en panicule ( lores paniculati), quand l'axe eommm se ramifie, et que ses divisions secondaires sont très-allongées et éeartées les unes des autres، Cette espèec d'infloreseenee appartient presque exelusivement aux Graminées; telles sont, par cxemple, les fleurs mâles du blé de Turquie (Zea Mays), l'Agrostis spica venti, la canne (Arundo Donax), ete.

$5^{\circ}$ Les fleurs sont disposées cn corymbe (flores corymbosi), quand les pédoneules et les pédicelles partent de points différens de la partie supérieure de la tige, mais arrivent tous à peu près à la même hauteur, eomme on le remarque dans la millcfcuille (Achillcea Millefolium ).

$6^{\circ}$ La disposition en cyme ( Jlores cymosi) est celle dans laquelle les pédoneules partent d'un mème point, les pédieelles étant inégaux, ct partant de points différens, mais élevant toutes les fleur's à la même hauteur, eomme on le remarque dans le surean noir (Sambucus nigra), le eornouiller (Cornus sanguinea), cte.

$7^{\circ}$ Les fleurs sont dites en ombelle (Roresumbellati), quand tous les pédoneules, égaux entre eux, partent d'un même point de la tige, divergent, se ramifient en pédieclles, qui partent également tous de la même hauteur, en sorte que l'ensemble des fleurs représente une surfaee bombée, comme un parasol étendu( umbella). Cette disposition se reucontrc dans toute une famille très-naturelle de plantes, les Ombellifères; tclles sont la carottc ( Daucus $\mathrm{Ca}$ - 
rotta), la ciguë ( Conium maculatum), l'opoponax (Pastinaca Opoponax), etc.

L'enscmble des pédoncules réunis forme une ombcllc; cliaque groupe de pédicclles constitue une ombcllulc.

Très-souvent, à la base de l'ombelle, on trouve un involucrc, et à la base dc chaquc ombellule un involucelle, comme dans la carotte. D'autres fois l'involucre manque, ct il cxiste des involucellcs, comme dans le cerfeuil ( Cllacrophyllum sativum ). Enfin l'involucre et les involucellcs pcuvcnt ne pas exister du tout, comme dans le Pimpinella saxifraga, Pimpinella magna, etc.

$8^{\circ}$ Les fleurs sont disposées en sertule (fores sertulati), quand les pédoncules sont simples, partant tous du même point, ct arrivant à peu près à la même hauteur, eomme dans le jonc fleuri (Butomus umbellaius), la plupart des espèces du genre Allium, les primevères, ete.

Cettc espèce d'inflorescence avait été réunie à l'ombellc; mais elle en est trop différente pour ne pas mériter un nom particulier.

$9^{\circ}$ Les fleurs sont en verticille ou verticillées ( Rores verticillati), quand elles forment un anneau autour d'un même point de la tige. Exemplc : le genre Myriophyllum, l'Hippuris vulgaris, etc.

On dit en général que les Labiées 'ont leurs fleurs verticillées, mais dans ce cas on s'en laisse imposer par l'apparcnee. En cffet, dans toutes les plantes de cette famille les fleurs sont placées à l'aiselle des deux feuilles opposées et portécs sur des pédoncules divisés. Ainsi donc elles ne naisscnt que de deux points opposés, et non de toute la eirconférence de la tige.

$10^{\circ}$ On nomme spadice (spadix, flores spadicei ) une espèce d'infloresccncc dans laquelle lc pédoneule commun cst eouvcrt dc fleur's unisexuées nues, c'est-à-dire sans ealiee propre, ordinairement distinctes et séparées les unés des autres, comme dans l'Arum maculatum, le Calla pa- 
lustris, etc. Quclquefois cependant on trouve des écailles qui entrecoupent les différentes flcurs; mais elles ne peuvent être regardćes comme des calices, puisqu'clles naisscnt de la substance même du pédonculc, dont clles paraissent être des appendices, , et sont toujours situćes au-dessous du point qui donne attache aux fleurs, comme dans certaines espèces de poivrier.

Le spadice est propre aux plantes monocotylédonées et aux diverses espèces de poivricrs. Quclquefois il est nu, c'est-à-dire sans enveloppe destinée à le recouvrir, comme dansles poivricrs.D'autres fois il est enveloppé d'unc spathe, comme dans les Aroïdes ct ecrtaines espèces de Palmicrs.

i I ${ }^{\circ}$ Le chaton (amentum, flores amentacei) est une disposition dans laquelle des flcurs uniscxućcs sont insérćes sur des ćcailles qui lcur servent en quelque sorte de pédoncule; telles sont les fleurs mâles du noyer (Juglans regia), du noiscticr (Corylus avellana), les fieurs mîles ct femelles des saulcs, etc. Cette cspèce d'inflorescencc sc rencontre dans toutc unc famille de végćtaux, composée d'arbres plus ou moins élevés, et que l'on a nommée Amentacées (I). Tels sont les saulcs, les pcuplicrs, les auncs, le boulcau, le charmc, le chêne, le hêtre, ctc.

I $2^{\circ}$ On donne les noms de capitule (capitu? $u$ ), de $c a$ lathide ou d'anthodium à la disposition des fleurs que les ancicns nommaicnt improprement fleurs composées. C'est ce que l'on remarque dans les chardons, l'artichaut, la scorzonère, et cn génćral dans toutcs lcs plantes de la famille des Synantherćes. Le capitule cst formé par un nombre plus ou moins considérable de petites fleurs, réunics sur un

(I) La famille des Amentacées de Jussieu a été partagée, d'après les observations réeentes de quelques botanistes, en plusieurs groupes ou familles très-distinctes par la structure des différentes parties de leurs fleurs et de lears fruits ; tclles sont les Cupulifères, les Bétulinées, les Salicinécs \& lcs Dlraacées, etc. 
réceptaele commun, manifestement plus renflé et plus large que le sonmet du pédoncule, dont il est néanmoins la terminaison, et que l'on nomme phoranthe; et entourées d'un involucre partieulier, qu'on désignait autrefois sous le nom de ealice eommun. Ainsi, par exemple, dans l'artichaut (Cinara Scolymus), les feuilles vertes dont on mange la base appartiemnent à l'involuere : la partie inférieure, large et eharnue, est le phoranthe ou clinanthe. Les fleurs sont au eentre des folioles de l'involucre. Elles sont très-petites et entremêlées de soies raides et dressées.

Le phoranthe n'a pas toujours la même disposition. Quelquefois il est légèrement concave, comme dans l'artiehaut; d'autres fois très-eonvexe, proéminent et eomme cylindrique, dans quelques Anthemis, le Rudbeckia, ete.

Il est plus souvent lisse; d'autres fois eependant il offre des espèces d'alvéoles dans lesquelles la base des petites fleurs est eontenue, comme dans l'Onopordurm. Tantôt il est nu, e'est-à-dire qu'il ne porte que les fleurs; d'autres fois les fleurs sont aceompagnées d'éeailles ou de poils pius ou moins raides et aeérés.

L'involuere ne varie pas moins. Tantôt, en effet, il est formé d'un seul rang de folioles, eomme dans le salsifis (Tragopogon); quelquefois ees éeailles sont très-nombreuses, imbriquées, et formant plusieurs rangées, comme dans les eentaurées, les ehardons, etc.

\section{CHAPITPE III.}

DE LA PREFLEURAISON.

Os entend par le mot de préfleuraison (prafloratio, cestivatio) la manière d'être des différentes parties d'une fleur avant son épanouissement. On voit, d'après eelte définition, que nous eomprenons ici les positions variées 
que les diverses parlies d'une fleur affectent dans le bouton.

Cette eonsidération a étélong-temps négligée, et mérite eependant la plus grande attention de la part des botanistes; car la préfleuraison est en général la même dans toutes les plantes d'une mème famille naturelle. Jusqu'iei on n'a étudié que la préfleuraison de la eorolle; mais eelle du ealiec et des organes sexuels n'est pas moins importante à connaître :

$I^{\circ}$ Les pétales ou les divisions de la eorollc peuvent être imbriqués (petala imbricata, prafloratio imbricativa), quand ils se reeouvrent latéralement les.uns les autres par une petite portion de leur largeur, eomme dans le genre Rosa; les pommiers, les eerisicrs, le lin, ete.

$2^{\circ} \mathrm{La}$ eorolle monopétale peut être pliée sur elle-méme à la manière des filtres de papier (corolla plicata, procfloratio plicativa), eomme dans les Convolvulacées, plusieurs Solanées.

$3^{\circ}$ Les pétales, ou les divisions de la eorolle monopétale, sont quelquefois rapprochés et ronlés en spirale (petala spiraliter contorta, prafloratio torsiva), eomme dans les Oxalis, les $A$ poeinées, ete.

$4{ }^{\circ}$ Les pétales sont souvent eliffonnés (petala corrugala, prafloratio corrugativa), e'est-à-dire pliés en tous sens, eomme dans les pavots, lc grenadier, les eistes, etc..

5 5 Les pétales peuvent ètre rapproehés bords à bords,' eomme les valves d'une eapsule (prafloratio valvaris); dans les Araliaećes, par exemple.

$6^{\circ}$ Quand les pétales sont au nombre de cinq, qu'il y cn a deux extérieurs ct deux intérieurs, et un qui recouvre les intérieurs par un de ses eôtés, et est reeouvert 'de l'autre par les extérieurs, M. De Candolle nomme ectte disposition praforaison quinconciale; par exemple daus l'oeillet.

Il existe encore plusicurs autres modes de préfleurai- 
son; mais moins importans à commaître, parce qu'ils se rencontrent moins fréquemment.

Ces différentes modifications sont également applieables au calice.

Dans les Ombellifères, les Urticées, les étamines sont infléchies vers le centre de la flcur; elles se redressent, quelquefois même se rabattcnt en dehors, lors de son épanouissement.

\section{CHAPITRE IV.}

DES ENVELOPPES FLORALES EN GÉNÉRAI.

Nous avons déjà vu précédemment que les enveloppes florales n'étaient point des organes essentiels de la fleur, puisque beaucoup de plantes en étaient entièrement dépourvues. Ainsi donc nous ne serons point étonnés quaud nous verrons des flcurs dans lesquelles le calice et la corolle manquent, et qui cependant sont remplacés par des fruits parfaits.

Linnæus donnait le nom général de périanthe (perianthiuin) à l'ensemble des enveloppes florales qui entourent les organes sexuels.

Le périanthe est simple ou double.

Quand il est simple, on lui donne le nom de calice, quelles que soient sa couleur, sa consistance, sa forme, comme dans la tulipe, le lis, les Thymélées, etc.

Toutes les plantes monocotylédonées n'ont jamais de corolle; leur périanthe est toujours simple; elles n'ont qu'un calice.

Quand le périanthe est double, l'enveloppe la plus intérieure, c'est-à-dire celle qui est la plus voisinc des organes sexucls, prend le nom de corolle. On nomme calice 
l'enveloppe la plus extérieure. On a dit eneore que le caliee faisait suite à l'éeorce du pédoneule, la eorolle au corps ligneux, ou à la partie située entre la moelle et l'ćeoree, dans les plantes annuelles. Mais eette assertion est peu fondée.

'Telle est l'opinion généralement admise par les auteurs qui s'oeeupent des rapports naturels des plantes. Et $t_{y}$ en effet, elle paraît, dans le plus grand nombre des eas, eonforme à la nature. Mais remarquons eependant iei, à l'égard des Monoeotylédons, que dans beaucoup de eirconstanees, surtout quand le périanthe se eompose de segmens séparés, on pourrait croire à l'existenee de deux enveloppes autour des organes sexuels. En effet, les six pic̀ees qui forment le périanthe simple d'un grand nombre de Monocotylédons sont le plus souvent disposées comme sur deux rangs; en sorte que trois paraissent plus intérieures, et trois plus extérieures. Si nous ajoutons à cela que les trois intérieures sont souvent eolorées et pétaloïdes, tandis que les trois externes sont vertes et semblables au calice, nous pourrons eoneevoir eomment on a pu admettre dans ees plantes un périanthe double, e'està-dire une eorolle et un ealiee. Cette disposition est surtout remarquable dans l'éphémère de Virginie ( Tradescantia virginica) : son périanthe simple est à six divisions, trois intérieures plus grandes, minees, délieates, d'une belle eouleur bleue; trois extérieures plus petites, vertes, et tout-à-fait différentes des premières. Il en est de même dans l'Alisma Plantago, le sagittaire, ete. qui ont toujours les trois divisions intérieures de leur périanthe colorées et pétaloïdes, tandis que les trois extérieures sont vertes et ealieiformes.

Mais ees exeeptions n'existent qu'en apparenee : elles s'évanouissent devant une observation plus exaete. Car, bien que les six segmens du périanthe d'un grand nombre de Monocotylédoins soient disposés sur deux rangs, ce- 
pendant ils ne forment sur le sommet du pédoncule qui les supporte, qu'un seul et mème eerele, e'est-à-dire qu'ils n'ont qu'um point d'origime eommum, et se eontimuent manifestement tous les six avee la partie la plus extérieure du pédoneule. Ils ne forment done qu'un seul et même organe, e'est-à-dire un ealiee. En effet, s'ils eonstituaient deux euveloppes distinetes, un caliee et une corolle, le point d'insertion de la eorolle serait plus intérieur que eelui du ealiee, puisqu'elle se continue avee la substanee ligneuse de la tige ou la partie qui la représente, tandis que le calice est une suite de l'épiderme ou de la partie la plus extérieure du pédoneule. De tout eeei nous pouvons conelure que dans les Monoeotylédons, il n'y a jamais de corolles, mais seulement un ealiee, quelles que soient la eoloration et la disposition des parties qui le eonstituent.

La vaste et intéressante famille des Orchidées, qui s'éloigne autant des autres plantes monoeotylédonées par la forme et l'apparenee extérieure de ses fleurs que par leur organisation intérieure, nous présente également un périanthe simple à six divisions, mais qui subit des modifications partieulières qu'il est important de noter ici. De ees divisions, trois sont plus intérieures, trois plus extérieures que les préeédentes. Les trois externes sont fort souvent réunies ensemble, avee deux des intérieures, à la partie supérieure de la fleur, et constituent, en se rapproehant intiniement les unes eontre les autres, une espèee de voûte ou de èasque qui recouvre et protége les organes sexuels. De là le caliee est dit en casque (calyx galeatus). Des trois divisions intérieures, l'une est moyenne et inférieure, d'une forme et d'une eouleur ordinairement différentes de celles des deux autres. Elle a reçu le nom partieulier de labelle (labellum). C'est eette troisième partie qui, dans un grand nombre d'espèees, offre des formes s' variées et si extraordinaires. Tautôt, en effet, on croirait apereevoir une abeille-bourdon se reposant sur la 
plante (Ophryss apifera), tantôt une araignée (Ophrys aranifera); d'autres fois un singe dont les parties inférieurcs sont écartées (Orchis zoophora, Oplirys anthropophora). Dans plusieurs genres de cettc famille, le labelle présente à sa partie inférieure un prolongcment creux, en forme de eornet, auquel on a donné le nom d'éperon (calcar). Dans ee cas il est dit éperonné ( labellum calcaratum ). La présenee, l'absence ou la longueur respective de l'éperon servent de earactére distinetif à ccrtains genres d'Orchidées.

Les enveloppes florales, malgré la délicatesse de leur tissu et les eouleurs variées dont clles sont fort souvent cmbellies, ne sont en général que des feuilles légèrement modifiécs. C'est surtout pour le caliee que cette analogie, eette identité même de structure est plus frappante. En effet, il cst des fleurs dans lesquelles les sépales ou folioles du ealiee ont tant de ressemblanee avec les feuilles, qu'il est diffieile de ne pas les eonsidérer eomme un seul et même organe. Cependaut, pour faeiliter l'établissement des earaetères génériques des plantes, les botanistes sont convenus de regarder comme tout-à-fait distincts des organes dont la strueture est identiquement la même.

Nous allons maintenant étudier séparément les deux enveloppes florales qui eomposent le périanthe double, c'est-à-dire le caliec et la corollc.

\section{CHAPITRE V.}

\section{DU CALICE.}

LE calice est l'cureloppe la plus extéricure du périanthe double, ou ee péricanthe lui-même, quand il est simple.

Il se compose d'un nombre variable de feuilles formant le vcrticille le plus extérieur de la fleur, ct tantòt parfai- 
tement distinctes les unes des autres, tantôt plus ou moins soudécs entre elles.

Il est facile de prouver par l'analogic que le périanthe simple est un calicc, et non point une corolle, comme Linnæus le nommait souvcnt.

En effet, un principe général, sanctionné par tous les botanistes, e'est que l'ovaire est appclé infêre (ovarium inferum) toutes les fois qu'il fait eorps, ou qu'il est soudé avee le tube du ealice par tous les points de sa pćriphérie. Or, l'ovaire est infère dans un grand nombre de Monocotylédons qui n'ont qu'un périanthe simple, tels que dans les Iridées, les Narcisses, les Orelidées, ete. On doit done conelure de là que cetté enveloppe unique, entic̀rement sondée par sa base avec l'ovaire, est-un véritable calice.

Le calice est monosépale (caly $x$ 'monosepalus) toutes les fois qu'il est d'une-seule pièce, ou pour parler plus exactement toutes les fois.que les feuilles calycinales sont toutes soudées 'ensemble, comme dans la stramoine et toutes les autres Solanées, dans la sauge et toutes les autres Labiées. (Voy. pl. 5, fig. 1, 2, 3.)

M. De Candolle propose de substituer le nom de calice gamosépale à celui de monosépale; le premier de ccs. noms signifiant que le calice dans ce cas est composé de plusieurs sépales soudés, et non d'un seul sćpalè, comme l'indique eelui de calice monosépale.

Il est poly sépale (caly $x$ polysepalus ) quand il est formé d'un nombre plus ou moins considérable de pièees distinctes, qu'on peut isolcr les unes des autres sans aucune dćchirure de leur substance, et auxquelles on donne le nom de sépales, commc dans la giroflée, le crcsson, etc.

Toutes les fois que le ealice fait corps avec l'ovaire, ou, ce qui est la mème clıose, toutes les fois quc l'ovaire est infère, lè ealice est naturcllemcrit monosépale.

Le caliec monosépale persiste presque toujours après la 
féeondation. T'rès-souvent il aceompagne le fruit jusqu'à l'ćpoque de sa maturité. Quelquefois même il prend de l'aceroissement à mesure que le fruit approehe de la maturité, eomme on le remarque dans l'Alkékenge (Physalis Alkekengi), etc.

Le ealiee polysépale est généralement eadue; il tombe le plus souvent à l'époque de la fécondation, quelquefois même aussitôt que la fleur s'épanouit, conme dans les pavots.

On distingue dans le ealiee monosépale $x^{\circ}$ le $t u b e$, ou la partic inféricure, ordinairement allongée et rétréeie; $2^{\circ}$ le limbe ou la partie supéricure, plus ou moins ouverte et étalée; $3^{c}$ la gorge $(f a u x)$, ou la ligne qui séparc le tube du limbe.

Le limbe du ealiee monosépale peut être plus ou moins profondément divisé. Ainsi il est simplement:

$x^{\circ}$ Denté (calyx dentatus), quand il offre des dentelures aiguës. Il peut êtretridenté, (c. tridentatus), eomme dans la cameléc (Cneorum tricoccum); quadridenté (c. quadridentatus), eomme dans le troëne, le lilas (voy. pl. 5, fig. I); quinquédenté (c. quinquedentatus), dans un grand nombre de Labiées et de Caryophyllées ; ete. suivant qu'il présente trois, quatre ou einq dents. Ces dents elles-mêmes peuvent offrir différentes dispositions. Ainsi elles sont égales ou inégales, dressées, étalées ou réfléehies. Ces diverses cxpressions s'entendent d'elles-mêmes, ctn'ont pas besoin d'être définies plus longuement.

$2^{\circ}$ Le ealiee monosépale peut être fendu (c. fissus), quand les ineisions attcignent environ la moitié de la liaulteur totale du ealiee. De là on dit qu'il est :

Bifide (c. bifidus), eomme dans la pédieulaire des marais (Pedicularis palustris);

Trifide (c. trifidus);

Quadrifide (c. quadrifidus), comme dans le Rilinanthus crisia galli, etc. ; 
Quinquéfide(e.quinquefidus), dans la jusquiame (Hyosciamus niger), le tabac (voy. pl. 5, fig. 2);

Mullifide (multifidus), etc.;

$3^{\circ}$ Quand les divisions sont très-profondes, et parviennent presque jusqu'à sa base, on dit alors du calice qu'il est :

Biparti (c. bipartitus), 'comme dans le genre Orobanche;

Triparti (c. tripartitus), comme dans l'Anona triloba;

Quadriparti (c. quadripartitus), dans la vćronique officinale (Veronica officinalis);

Quinquéparti (c. quinquepartitus), dans.la bourrache (Borrago oficinalis), la digitale pourprée (digitalis purpurea), etc.;

Multiparti (c. multipartitus), etc.

Enfin, par opposition à toutes ces expressions, on dit du calice qu'il est entier (caly $x$ integer), quand son limbe, ne présente ni dentelures ni incisions; par exemple, dans beaucoup de genres d'Ombellifères.

Le calice monsépale peut ĉtre régulier ou irrégulier. ?

Il est régulier (c. regularis) quand toutes ses incisions sont parfaitement égales entre elles, quelles que soient d'ailleurs leur figure ou leur forme; par exemplé, celui de: la bourrache, de l'oeillet, etc.

Il est irrégulier, au contraire (c. irregularis), quand les parties correspondantes n'ont point une même figure ni une grandcur ćgale, comme dans la capucine (Tropcoolum majus).

Quant à sa forme, le calice est tubuleux (c. tubulosus), quand il est étroit, trc̀s-allongé, et que son limbc n'est. point ćtalé, comme dans la primevère (Primula veris) l'oiillet, etc. (voy. pl. 5, fig. ro);

Turbiné (c. turbinatus), ayant la formc d'une poire ou d'une toupie; par exenple, dans la bourgène;

Urcéolé (c. urceolatus, ventricosus), renflé à sa bạse, 
resserré à la gorge, le limbe étant dilaté, comme dans le genre Rosa, la jusquiame;

Enflé ou vésiculeux (inflatus, vesiculosus), quand il est mince, membraneux, dilaté, comme une vessie, beaucoup plus large que la base de la corolle qu'il entoure, comme dans le Cucubalus Behen, le Rhinanthus crista galli, etc.;

Campanulé ou en clocke (c. campanulatus), dilaté de la!lbase vers l'orifice, qui est très-ouvert, comme dans la fausse mélisse (Melittis melissophyllum), la, molucellé, etc. ;

Cupulé (c. cupuliformis), aplati ou légèrement concave, comme daus le citronnier (Citrus medica);

Cylindrique (c. cylindricus), lorsque, de sa base jusqu'à sa partic supérieure, il forme un tube dont tous les diamètres sont à peu près égaux, comme dans l'ocillet (voy. pl. 5, fig. 10);

Claviforme ou en massue (c. clavalus, claviformis), quand le tube est légèrement renflé à son sommet, eonmme dans le Silene armeria;

Comprimé (c. compressus), large et aplaii latéralement, comme dans la pédiculaire des marais (Pedicularis palustris);

Prismatique (c. prismaticus), ayant des angles et des faces bien marqués, comme dans la pulmonaire (Pulnonaria officinalis);

Anguleux (c. angulosus), offrant un grand nombre d'angles saillans et longitudinaux;

Sillonié (c. sulcatus), offrant des lignes rentrantes longitudinales;

Bilabié (c. bilabiatus), ayant ses divisions disposées de manière à oflrir une lèvre supérieure et une inférieure, éeartées l'une de l'autre; par exemple, dans la sauge (Salvia officinalis) et un grand nombre d'autres Labiées;

Eperoniné (c. calcaralus), présentant un prolonges- 
ment creux à sa base, comme daus la capucine (Tropcec: Tum majus);

Diptère (c. dipterus), présentant deux appendices latéraux et membraneux, en forme d'ailes;

Triptère (c. tripterus), offrant trois appeńtices latéraux, membraneux, en forme d'ailes.

Le calice est souvent colorć assez vivement, surtont quand il n'existe pas de corolle; dans ce cas il est dit pétaloüde ou corolliforme (c. petaluïdeus, corolliformis), comme dans le bois gentil (Daplune Mezereum), les narcisses, les Orchidées, etc.

Il est important de mentionner les proportions relatives du calice et de la corolle. Ainsi, ordinairement, le calice est plus court que la corolle (caly $x$ corolla brevior). D'autres fois il est plus long (calyx corolla longior'), comme dans la nielle des blés (Agrostemnna Githago). Enfin il peut être égal à la corolle (caly’x corolla a qualis).

Le calice peut être libre de toute adhérence, ou bien il peut être soudé el faire corps, en tout ou cn partic, avec l'ovaire: dans ce cas, le calice est dit adlérent (calyx ovario adliceriens), et l'ovaire est nécessairement infère.

Le calice polysépale peut ètre composé d'un nombre plus ou moins considérable de sépales ou pic̀ces distinctes; ainsi il est :

Disépale (c. disepalus), quand il est formé de deux sépales, comme dans le pavou (Papaver somniferum), la fumeterre (Fumaria officinalis);

Trisépale ( c. trisepalus), formé de trois sépales, comme dans la ficaire (Ficaria ranunculoüdes);

Tétrasépale (c. Tétrasépalus), offrant quatre sépalcs, comme dans le cbou, la rave, le cresson, et les autres Crucifẹ̀res (voy.pl. 5, fig. 9 );

Pentasépale (c. pentasepalus), quand il est composé de ciuq sépales, comme celui du lin ( Linum usilatissimuml), ctc. 
Quant aux sépales, leur figure ou leur forme doit être étudiée ct considérée comme celle des feuilles ou des divisions du ealice monosépale; ainsi ils pcuvent être lancéolés, aigus, obtus, cordiformes, ctc.

Un calice polysépale peut aussi présenter différentes formes par l'arrangement que les sćpales prennent cutre eux; ainsi il est tubulaire (c. tubularis), quand les sćpales sont longs, dressés, rapprochés de manière à former un tube. Beaucoup de Crucifères sont dans ce cas. (Voy. pl. 5, fig. 9. )

Il peut être campanulaire (c. campanularis);

En étoile ( c. stellaris ), quand il est formé de cinq sépales étalés et égaux, comme dans plusicurs Caryophyllées.

\section{CHAPITRE VI.}

DE IA COROLLE.

LA cor:olle n'existe jamais que lorsqu'il y a un périanthe double; c'en est l'cuvcloppe la plus intérieure. Elle entoure immédiatement les organes de la reproduction; quoique faisant suite à la partie ligneuse de la tige, son tissu est mou et délieat. Souvent peinte des plus riches coulcurs, elle attire principalement les regards du vulgaire, qui ne voit de fleurs que là où il y a de grandes et brillantes corolles, ou des périanthes colorés. Le botaniste, au eontraire, ne considère eet organe que comme accessoire à l'essence de la fleur; tandis qu'un pistil ou une étamine quelquefois à peine visibles constituent pour lui une véritable fleur.

La corolle peut êtremonopétale ou gamopétale (corolla monopetala, c. gamopetala), c'est-à-dire dout les diverses. 
pièces sont réunies en un seul tout, comme dans la digitale pourprée (Digitalis purpurea), le liseron (Convolvulus arvensis), la belladone (Atropa belladona). (Voyez pl. 5, fig. I, 2, 3, 4.)

Elle peut être eomposée d'un nombre plus ou moins considérable de segmens isolés, qu'on nomme pétales. (petala) : dans ee eas elle est appelée polypétale (cor. polypetala), eomme dans la rose, l'oeillet, le ehou, la giroflée. (Voyez pl. 5, fig. 9, Io, I I.)

Tout pétale offre à eonsidérer, I l'onglet (unguis), ou la partie inférieure rétréeie, plus ou moins allongée, par laquelle il est attaché; $2^{\circ}$ la lame (lamina) ou la partie élargie, de forme variée, qui surmonte l'onglet.

La figure des pétales varie singulièrement, et peut être, en général, rapportée aux différentes modifications que nous avons indiquées pour les feuilles; ainsi il y en a qui sont arrondis, d'autres allongés, aigus, obtus, dentés, entiers, etc., ete.

De même que le calice, la eorolle peut être régulière ou irrégulière.

Elle est régulière toutes les fois que ses incisions et ses divisions sont égales entre elles, ou que ses parties paraissent être disposéés régulièrement autour d'un axe commun. Par exemple, celle de la campanule raiponce (Campanula Rapunculus), de la giroflée jaune ( Cleeiranthus Cheiri). (Voyez pl. 5, fig. 1, 2, 3, 9.)

Elle est irrégulière, au contraire, quand ses ineisions sont inégales, ou que les différentes parties qui la composent ne paraissent pas disposées symétriquement autour d'un axe commun fictif, comme dans le muflier (Antirrhinum majus), l'utriculaire (Utricularia vulgaris), la eapucine (Tropceolum majus), ete.(Voy.pl. 5, fig. 7, 8, 1.2.)

La eorolle monopétale tombe d'une seule pièce en se fanant. Quclquefois sa base persiste, comme dans la bellede-nuit. ( Nyctago horlensis). 
Dans la corolle polypélale, au contraire, eliacun des pétales tombe isolément. Cependant il péut arriver que, dans une corolle polypétale, les segmens ou pétales tombent tous ensemble et réunis par leur base, comme dans la mauve (Malva rotundifolia), la guimauve (Althaea officinalis), etc. Dans ee cas, la eorolle n'en est pas moins polypétale; mais les pétales sont réunis aceidentellement à lcur base par un prolongement de la substance des filets des étamines. On pourrait eiter cneore plusieurs autres exemples analogues.

On dit d'une corolle monopétale qu'elle est éperonnée (c.calcarala), quand elle offre à sa base un prolongement creux, cn forme de cornet, comme dans la linaire ( Linaria vulgaris ). ( Voy. pl. 5, fig. 7.)

La corolle monopétale ofire à considérer trois partics, $\mathrm{I}^{\circ}$ une inféricure, ordinairement eylindrique et tubuliforme, plus ou moins allongée, qu'on appelle tube (tubus); $2^{\circ}$ une partie supérieure au tube, plus ou moins évasée, quelquefois étaléc et même réfléchie : on la nomme limbe (limbus). Enfin la ligne cireulaire qui sépare le tube da limbe prend le nom de gorge ( faux, palatum ). Ces trois parties sont essenticlles à considérer; en effet, leurs formes, variées, leurs proportions relatives fournissent au botaniste des caractères propres à distinguer certains genres de plantes. (Voyez pl. 5, fig. 1, 2, ete.)

En général la corolle monopétale donne attache aux étamines.

Nous allons maintenant passer en revue les différentes modifieations que présente la corolle monopétale et la corolle polypétale, quand elles sout régulières ou irrégulières. 
§. Corolle monopétale régulière.

La corolle monopétale régulière offue des formes trèsvariées :

$r^{\circ}$ Ainsi elle est tubulée (tubulata) quand son tube est très-allongé, comme dans la belle-de-nuit ( $N$ jctago hortensis), le lilas (Syringa vulgaris). (Voy. pl. 5, fig. I, 2.)

Le tube est quelquefois capillaire ou filiforme, comme dans certaines Synanthérées.

$2^{\circ} \mathrm{La}$ corolle est en cloche ou campanulée (cor. campanulata), lorsqu'elle ne présente pas de tube manifeste, mais qu'elle va en s'évasant de la base vers la partie supérieure, commé dans la raiponce (Campanula Rapunculus), le liseron des haies (Convolvulus sepium), le jalap (Convolvullus Jalappa), etc. (Voyez pl.5, fig. 3')

$3^{\circ}$ Elle est infundibuliforme ou en entonnoir (cor. innfundibuliformis), quand le tube est d'abord étroit à sà partie inférieure, puis se dilate insensiblement, de manière que le limbe est campanulé. Par exemple, le tabac (Nicotiana Tabacum), ete. (Voy. pl.5, fig.2.)

$4^{\circ} \mathrm{On}$ la dit hypocratériforme (cor. hypocrateriformis), quand son tube est long, étroit, non dilaté à sa partie supérieure, que le limbe est étalé à plat, de sorte qu'ellé représente la forme d'une coupe antique, comme le lilas (Syringavulgaris), le jasmin (Jasminum officinale), etc. (Voyez pl. 5, fig. r.)

$5^{\circ} \mathrm{La}$ corolle est rotacée ou en roue (corolla rotata), quand le tube est très-court et le limbe étalé et presque plane comme dans la bourrache (Borrago officinalis') et la plupart des Solanum.

On dit que la corolle est étoilée (cor. stellata), quand elle est très-petite; son tube fort eourt, et les divisions de son limbe aiguës et allongées : par exemple, dans les caillelaits (Galium), les aspérules (Asperúla), ete.

$6^{\circ}$ Elle est urcéolée (cor. urceola! a), renflée comme une 


\section{4}

ORGANES DE LA REPRODUCTION.

petite outre à sa base, rétrécie vers l'orifiee, comme dans beaucoup de bruyères (Erica), de Vaccinium, ete. (Voy. pl.5, fig. 4.)

$7^{\circ}$ On l'appelle scutcllée (cor. scutellata, scutelliformis), quand elle a la forme d'une écuelle, e'est-à-dire qu'elle est étalée et légèrement concave.

\section{\$2. Corolle monopétale irrégulière.}

I La eorolle monopétale irrégulière est dite bilabiée (corbilabiata), quand le tube est plus ou moins allongé, la gorge ouverte et dilatée, le limbe partagé transversalement en deux divisions, l'une supérieure, l'autre inférieure, qu'on a comparées à deux lèvres éeartées. Cette forme de la corolle earaetérise spéeialement toute unc famille de plantes, l'une des plus naturelles du règne végétal : ee sont les Labiées (voy.pl. 5, fig. 8); par exemple, le thym (Thymus vulgaris), la mélisse (Melissa officinalis), la sauge (Salvia officinalis), le romarin (Rosmarinus officinalis), ete.

Ces deux lèvres peuvent offrir une foulc de modifications, sur lesquelles reposent les caraetères propres à distinguer les genres nombreux de eette famillc. Ainsi la lèvre supérieure est tantôt plane, tantôt redressée, ou en voúte, ou en fer de faux. Ellc peut être entière et sans ineisions; échancrée, bidentée, bilobée, bifide, ete.

La lèvru inférieure est ordinairement réfléchie; quelquefois elle est concave et plissée sur les bords, eomme dans le genre Nepeta. Elle peut également être trifide, trilobée ou tripartie.

Quelquefois la lèvre supéricure semble ne pas exister, ou du moins est si peu développée, qu'on la distingue diffieilement, eomme dans les genres Teugrium et Ajuga.

$2^{\circ}$ On appelle corolle personnée (1) (corolla perso-

(r) Des nuances insensibles rapprochent les corolles labiées des personnées. Aussi cst-il très-difficile de les bien caractériser. On cst obligé d'em- 
nata) celle dont le tube est plus ou moins allongé, la gorge très-dilatée et elose supérieurement par le rapprochement du limbe, qui esti, à deux lèvìes inégales, de manière à représenter grossierement le muffle d'un animal, ou eertains masques antiques. Telles sont célles de l'A $l^{\prime}$ tirrhinum majus, de la linaire (Linaria vulgaris), etc. (Voy. pl. 5, fig. 7.)

Enfin'on a réuni sous le nom de corolles monopétales irrégulières anomales toutes eelles qui, par leur forme extraordinaire, l'impossibilité où l'on est de les émparer à aucune autre forme eonnue, s'éloignent des différens types que nous venons d'établir, et ne peuvent être rapportées à aucun d'eux. Ainsi la eorolle de la digitale pourprée (Digitalis purpurea) qui offre à peu près la forme d'un doigt de gant (I); les eorolles de l'utrieulaire ( Utricularia), de la grassette ( $P$ inguicula), ete. sont également des eorolles irrégulières et anomales.

Dans les diverses formes de eorolles monopétales régulière et irrégulière que nous venons d'examiner., les' trois parties qui eomposent ces eorolles, c'est-à-dire le tube, le limbe et la gorge, présentent des modifieations qu'il est utile d'indiquer.

Aiusi le tube peut être :

Cylindrique (.cylindricus), comme dans le lilas (Syringa vulgaris), la belle-de-nuit (Nyctago hortensis), etc. (Voy.pl. 5, fig. ז.)

Il peut être long ou court, relativement au ealice ou. au limbe.

$V$ entru ou onflé( ventricosus aut inflatus), soit dans

ployer un caractère anxiliaire tiré dc la forme et de la structure de l'ovaire. Dans les Lahiées, en effet, l'ovaire est profondément quadrilobé; il est simple au contraire dans toutes les véritables Pcrsonnćcs.

(I) Aussi cette plante porte-elle le nom valgaire de gantelée: 
sa partie inféricure, soit ver's son sommet; dans ce cas il est dit :

Claviforme ou en massue (claviformis), comme dans le Spigclia marylandica.

Enfin il peut être lisse, strié, anguleux, prismatique, etc. Nous avons déjà plusieurs fois donné la valeur de ces expressions.

La gorge (faux) peut être :

Close (clausa), quand elle est entièrement fermée, comme dans le grand muflier (Antirrhinum majus).

Ouverle et dilatée (aperta, patens), comme dans la digitale pourprée, certaines Labiées, ete.

Elle peut être garnie de poils, comme dans le thym, l'origan, ete.

Ciliée (ciliata), garnie de eils, eomme dans la Gentiana amarella, ete.

Couronnée par des appendices saillans de forme variée, eomme dans la bourrache (Borrago officinatis), la eonsoude (Symplytum consolida), la buglosse (Anchusa italica), et beaucoup d'autres Borraginées.

Enfin on dit, par opposition aux expressions précédentes, qu'elle est nue, quand clle n'offre ni poils, ni bosses, ni appendices.

Le limbe, ou la partie de la corolle qui surmonte la gorge, peut être :

Dressé (erectus), eomme dans la cynoglosse (Cynoglossum officinale).

Etalé, ouvert (patens), lorsqu'il forme un angle droit avee le tube, comme dans le laurier-rose ( $N$ erium Oleander).

Réfléchi ou renversé en dehors (reflexus), comme celui de la donce-amère (Solanum dulcamara), de la eanneberge (Vaccinium oxycoccos), etc.

Le limbe peut être aussi plus ou noins profondément incisé. Ainsi il est quelquefois simplement denté sur son bord. 
Il est également trifide', quadrifıde, quinquéfide, ou quadriparti, quinquéparti, ete.', suivant la profondeur de ses ineisions.

La forme des différentes divisions d'un limbe ineisé offre un grand nombre de variétés qui peuvent être rapportées à eelles des pétales et des feuilles.

Remarquons ici, en terminant ee qui a rapport à la corolle monopétale, que sa forme n'est point un earaetère essenticl dans la eoordination des genres en familles naturelles. En effet, on trouve souvent plusieurs formes réunies ensemble, dans des groupes essentiellement naturels. Ainsi, dans les Solauées, on voit réunies des eorolles

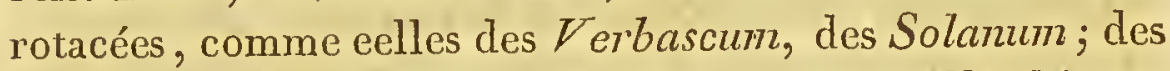
eorolles infundibuliformes (le tabae); des corolles hippoeratériformes, eomme eertains Cestrum, et des corolles campanulées, comme dans la jusquiame, la belladone. Nous pourrions encore faire un rapproehement semblable dans beaucoup d'autres familles tout aussi naturelles.

\section{Corolle polypétale.}

Le nombre des pétales varie singulièrement dans les différentes corolles polypétales. Ainsi, il y a des corolles formées de deux pétales, "comme daus la cireée (Circcea lutetiana). Dans ee eas elle est dite dipétale (corolla dipetala).

Tripétale (cor. tripetala), eomposée de trois pétales, eornme celle de la eamélée (Cneorumn tricocum), ete:

Tétrapétale (cor' tetrapetala); eomposée de quatre pétales. Par exemple, toutes les Crueifères, telles que le cresson de fontaine (Sisymbrium Nasturtium), le raifort (Cochlearia Armoracia), la passeragi' (Lepidium latifolium); ete. (Voyez pl. 5, fig. 9.)

Pentapétale (cor, pentapetala), formée de einq pétales, eomme toutes les Ombellifères, les Rosaeées. Par 
exemple, le panais (Pastinaca sativa), le persil (Apium Petroselinum), la ejguë (Conium maculatum), le fraisier. (Voyez pl. 5, fig. ro, гx.)

Hexapétale (cor. hexapetala), ayant six pétales, comme l'épine-vinette (Berberis vulgaris), etc.

Les pétales ou segmens d'une eorolle polypétale peuvent ètre onguiculés, e'est-à-dire munis d'un onglet trìsapparent, eomme dans l'œillet, la giroflće jaune. (Voyez pl. 5, fig. 9, a.) Ou bien ils peuvent être sessiles, c'està-dire sans onglet ou inonguieulés, comme dans la vigne (Vilis vinifera), la gypsophile (Gypsophilamuratis), ete.

La longueur et la proportion de l'onglet, relativement au calice, mérite aussi d'être notće. En effet, l'onglet est souvent plus court que le ealiee (umguis calice brevior); d'autres fois, au contraire, il est plus long que lui et le dépasse (unguis calycelongior).

Les pétales sont souvent dressés (petala erecta), e'està-dire qu'ils suivent une direction parallèle à l'axe de la fleur, eomme dans le Geum rivale.

Ils sont quelquefois infléchis (petala inflexa), eourbés vers le centre de la fleur, eomme ùus beaucoup d'Ombellifères.

Etalés (petala patentia), eomme dans le fraisier ( $F r a-$ garia vesca), la benoite (Geum urbanum), ete. (Voyez pl. 5, fig. II.)

Réfléchis (pet. reflexa), se renversant en dehors.

La figure des pétales est extrĉmement variable; ses principales modifications peuvent être rapportées à celles déjà établies préeédemment pour les feuilles ou les sépales. Cependant ils offrent quelquefois des formes singulières que nous allons faire connaître.

Les pétales sont concaves (pet. concava), dans le tilleul (Tilia enropcea), la rue (Rula graveolens), etc.

Galéiformes ou en casque (pet. galeiformia), lorsqu'ils 
sont voùtés, ereux, et qu'ils ressemblent à un casque, eormme dans l'aeonit (A conitum Napellus), etc.

Cuculliformes (pet. cuculliformia), ayant la forme d'un capuchon ou d'un cornet de papier, comme dans l'aneolie (Aquilegia vulgaris), le pied d'alouette (Delphinium consolida).

Eperonnés (pet.calcarata), munis à leur base d'un éperon, comme dans la violette, le pied d'alouette, ete.

La corolle polypétale peut être régulière ou irrégulière, suivant que les parties qui la composent sont disposées ou non avec symétrie autour de l'axe de la fleur. Dans l'un et l'autre eas, les pétales, par leur forme, leur nombre et leur disposition respective, donnent à la corolle un aspect, une forme partieulière, qui ont servi à la diviser en plusieurs groupes.

\section{$\$ \mathrm{I}$. Corolle polypétale régulière.}

La corolle polypétale régulière peut offrir trois modifieations principales. Elle peut être :

I ${ }^{\circ}$ Cruciforme (cor. cruciformis), eomposée de quatie pétales onguiculés, disposés en eroix. Les plantes dont la eorolle présente une semblable disposition eonstituent un des groupes les plus naturels du règne végétal. Elles ont reçu le nom de Crucifères. Tels sont le ehou, la giroflée, le cresson, ete. (Voy. pl. 5, fig. 9.)

Les quatre pétales d'une eorolle erueiforme ne sont pas toujours égaux et semblables entre eux; il y en a souvent plusieurs qui sont ou plus petits ou plus grands. Ainsi, dans le genre des Iberis, deux pétales sont eonstamment plus grands.

$2^{\circ}$ Rosacée ou roselée (cor. rosacea), eelle qui est eomposée de trois à einq pétales, rarement d'un plus grand. nombre, dont l'onglet est très-eourt, et qui sont étalés et disposés en rosaee. Telles sont toutes les Rosaeées, 
comme la rose simple, l'amandier, l'abricotier, le prunier, ete., la ehélidoine, et des plantes d'autres familles. (Voy.pl. 5, fig. I I.)

$3^{\circ}$ Caryophyllée (cor. caryophyllata), corolle formée de einq pétales dont les onglets sont fort allongés, et eachés par le ealiee, qui est très-long et dressé, eomme dans l'œillet, les Silene, les Cucubalus, ete. (Voy. pl. 5 , fig. Io.)

\section{\$2. Corolle polypétale irrégulière.}

$I^{\circ}$ Papilionacée (cor. papilionacea). Cette eorolle est eomposée de cinq pétales très-irréguliers, qui ont chacun une forme partieulière; ee qui leur a fait donner des noms propres. De ees einq pétales, l'un est supérieur, deux latéraux, et deux inférieurs. Le supérieur porte le nom d'étendard ou de pavillon (vexillum) (voy. pl.5, fig. I 2,a); il est ordinairement redressé, d'une figure très-variée, et reeouvre les quatre autres avant l'épanouissement de la fleur. Les deux inférieurs, le plus souvent réunis et soudés l'un à l'autre par leur bord inférieur, forment la carène (carina) (fig. I2, c). Les deux latéraux eonstituent les ailes (alce) (fig. 12, $b$ b).

C'est par la ressemblanee que l'on a eru trouver à ectte fleur avee un papillon dont les ailes sont étalées, qu'on lui a douné le nom de papilionacée.

La eorolle vraiment papilionacće appartient exelusivement à la vaste famille des Légumineuses : tels sont les pois (Pisum), les haricots (Phaseolus), l'aeaeia (Robinia psendo-(acacia), les astragales, ete.

$2^{\circ}$ On nomme eorolle anomale (cor. anomala) eelle qui est formée de pétales irréguliers, qu'on ne peut rapporter à la corolle papilionaeée. Telles sont eelles des aeonits, des pieds d'alouette, de la violette, de la balsamine, de la capucine, etc. 
La position des pétales ou des divisions de la corolle monopétale, relativement aux sépales ou aux divisions du caliee monosépale, présente les deux modifieations suivantes :

Les pétales peuvent être opposés aux divisions du ealice, e'cst-à-dire placés de manière à se eorrespondre par leur's faces, comme dans l'épine-vinette (Berberis vulgaris), l'Epinedium alpinum, ete.

Ils peuvent être alternes avee les divisions du ealice, e'est-à-dire qu'ils eorrespondent aux ineisions du ealiee, et non à ses divisions. Cette disposition est bien plus fréquente que la préeédente, qui est très-rare. Les pétales sont alternes aux sépales dans les Crucifèrẹs, ete., ete.

La grandeur relative de la eorolle et du calice mérite également d'être bien observée; ear on peut souvent én tirer de fort bons caractères distinctifs.

Suivant sa durée, la corolle est fugaee ou caduque (caduca, fugax), quand elle tombe aussitôt qu'elle s'épanouit, eomme dans le Papaver Argemone, plusieurs eistes, etc.

Décidue (c. decidua), tombant après la fécondation. La plupart des eorolles sont dans ee cas.

Marcescente (c. marcescens), persistant après la féeondation, et se fanant dans la fleur avant de s'en détaeher, eomme dans les Bruyères et certaines Cucurbitacées.

La corolle cst ordinairement la partie la plus brillante de la fleur. La délicatesse de son ìssu, l'éclat et la frầeheur de ses couleurs, le parfum suave qu'elle exhale souvent, en font une des plus agréables produetions de la nature. Ses usages, de mème que ceux du caliee, paraissent être de protéger les organes sexuels avant leur parfait développement, ei de favoriser, à l'époque de la fécondation, l'aetion mutuelle que ces deux organes excrcent l'un sur l'autre. 


\section{CHAPITRE VII.}

\section{DES ORGANES SEXUEIS.}

LA découverte des organes sexuels dans les plantes ne remonte point à une époque très-éloignée. Jusqu'au seizième sièele, on n'avait vu dans les fleurs qui couvrent les végétaux qu'un simple ornement dont la nature s'était plue à les parer. Camérarius et Grew, à eette époque, démontrèrent par l'expérienee l'utilité des différentes parties de la fleur dans la produetion de la graine, l'entretien et la sueeession des espèees. Ils firent voir que le pistil, qui oecupe le eentre de la fleur, devait être comparé, pour sa structure et surtout ses usages, aux organes générateurs de la femelle dans les animaux. En effet, nous y trouvons également les rudimens imparfaits de l'embryon (ovules); une cavité destinée à les contenir et à les protéger pendant leur développement (ovaire); un organe particulicr propre à reevoir l'impression féeondante du mâle (stigmate); un autre organe encore par lequel cette impression est transmise jusqu'aux embryons (style). Ils prouvèrent également que l'étamine devait être assimilée aux organes qui sont l'apanage du mâle dans les animaux. Car elle contient dans une eavité spéciale (anthère), une substance partieulière dont les usages sont de féconder les ovules (pollen).

Dès lors il fut prouvé que les plantes, de même que les animaux, sont pourvues d'organes sexuels, destinés à leur reproduction. L'organe sexuel mâle est eonstitué par l'étamine; le pistil forme l'organe sexuel femelle.

Presque toujours dans les végétaux les deux organes de la reproduetion sont réunis dans une même fleur, ee qui constitue l'hermaphroditisme, et la fleur est dite herma- 
phrodite. D'autres fois, au eontraire, on n'y rencontre qu'un seul des deux organes sexuels, et la fleur est dite unisexuée.

La fleur unisexuée pcut ètre mâle ou femelle', suivant qu'elle renferme des étamines ou un pistil.

Les fleurs males et les fleurs femelles sont quelquefois réunies sur la même plante ; e'est ee qui eonstitue les végétaux monoïqnes. Le châtaignier (Castanea vulgaris), le eoudrier (Corylus Avellana), sont de ee nombre.

D'autres fois, au contraire, les fleurs mâles et les fleur's femelles se trouvent séparées les unes des autres sur des pieds différens; les plantes qui présentent une semblable disposition sont appelées dioüques. Telles sont la mereuriale (Mercurialis annua), le mûrier à papier (Broussonetia papyrifer'a), le dattier ( Phoenix dactylifera).

Enfinqquelquefois on trouve mêlées ensemble sur le même pied, ou sur des pieds différens, des fleurs mâles, des fleurs femelles, et des fleurs hermaphrodites; e'est aux végétaux qui offrent ee mélange irrégulier des trois sortes de fleurs qu'on a donné le nom de polygames. Telles sont la pariétaire ( Parietaria officinalis), la croisette (Valantia cruciata), ete.

Ces trois divisions fondées sur la séparation, la réunion ou le mélange des sexes, ont servi de base à Linnæus pour établir les trois dernières classes des plantes phanérogames de s on système, savoir : la Monoecie, la Dïoecie et la Polygamie. 


\section{CHAPITRE VIII.}

DE L'ETAMINE OU ORGATE SEXUEL MALE.

L'ÉtAMrne dans les végétaux remplit absolument les mêmes usages que les organes mâles daus les animaux, c'est-à-dire qu'elle renferme la substanee qui opère la fécondation des germes.

L'étamine est ordinairement eomposée de trois parties; savoir: I I'anthère (anthera), espèee de petit sae membraneux dont la eavité intérieure est double, c'est-à-dire formée de deux loges soudées ensemble; $2^{\circ}$ du pollen ( $p o l-$ len ), substanee ordinairement formée de petits grains vésieuleux, qui eontiennent les parties néeessaires à la féeondation; $3^{\circ}$ l'anthère est souvent portée sur un appendiee filiforme auquel on donne le nom de filet (filamentumn).

Telles sont les trois parties qui eomposent ordinairement l'élamine. Mais remarquons iei que deux seulement lui sont ıéeessaires; ce sont l'anthère et le pollen. Le filet, en effet, n'est qu'une partie aceessoire de l'étamine; aussi manque-t-il souvent, e'eșt-à-dire que l'anthère est immédiatement attachée au eorps sur lequel elle est insérée, sans le seeours d'un filet. Dans ee eas l'étamine est appelée sessile (stamen sessile), comme dans beaucoup de Thynúlées.

L'essenee et la perfeetion de l'étamine résident doue dans la présenee de l'anthère. Mais une eondition indispensable pour que cet organe soit apte à remplir les fonetions que la nature lui a eonfićes, e'est qu'il faut que non-seulement il eontienne du pollen, mais eneore qu'il s'ouvre, pour que eette substanee soit mise en eontact avec le stignate; ear, sans cette circonstancc, la féeondation ne pourrait pas avoir lieu. 
Le nombre des étamines varie singulièrement dans les différentes plantes. C'est même d'après cette eonsidération du nombre des organes sexuels màles contenus dans chaque fleur, que Linnæus a établi les premières classes de son système.

Ainsi il y a des fleurs qui ne renferment qu'une seule étamine; on leur donne le nom de fleurs monandres (flores monandri). Tels sont l'Hippuris vulgaris, la valériane rouge (Centranthus ruber), le Blitum virgatum, etc.

On les appelle fleurs diandres ( flores diandri), quand elles eontiennent deux étamines. Par exemple, le lilas (Syringa vulgaris), le troëne (Ligustrum vulgare), la véronique offieinale ( $V$ eronicà officinalis), la sauge (Salvia officinalis), ete.

Fleurs triandres (flores triandri) : la plupart des Graminées, des Cypéraeées, des Iridées, ete.

Fleurs tétrandres ( flores tetrandri) : le caille-lait (Galium verum), la garance ( Rubia tinciorum), la plnpart des Labiées, des Antirrhinées, des Dipsacées, ete.

Fleurs pentandres (flores pentandri): le bouillon blanc (Verbaseum Thapsus ), et la plupart des Solanées; la cynoglosse (Cynoglossum officinale), et la plupart des Borraginées; la carotte (Dancus Carotta), et toutes les Ombellifìres, etc.

Fleurs hexandres (flores hexandri); le lis (Lilium candidum ), la tulippe (Tulipa gesneriana), et la plupart des Liliacées, des Asphodèles, le riz (Oryza sativa).

Fleurs heptandres (flores heptandri) : le marronier d'Inde ( Esculus Hippocastanum ).

Fleurs'octandres ( flores octandri) : celles des bruyères, des Vaccinium, des Daplune, des Polygonum, ete.

Fleurs ennéandres (flores enneandri ), comme celles du jonc fleuri (Butomus umbellatus). 
Fleurs décandres (flores decandri), comme dans l'ocillet, la saponaire ( Saponaria officinalis ), et la plus grande partie des Caryophyllées; la ruë (Ruta graveolens), la pyrole ( Pyrola rotundifolia), Ies saxifrages, ete.

Passé dix, le nombre des étamines n'est plus fixe dans les fleurs; ainsi, on dit qu'elles sont :

Dodécandres (flores dodecandri), quand elles eontiennent de douze à vingt étamines, comme dans la gaude (Reseda luteola), l'aigremoine (Agrimonia eupatoria).

Polyandres (flores polyandri), quand elles eontiennent plus de vingt étamines, comme le pavot ( Papaver somniferum ), les renoneules, ete.

Les étamines peuvent être toutes égales entre elles, comme dans le lis, la tulipe, ete.

Elles peuvent être inégales, e'est-à-dire les unes plus grandes, les autres plus petites dans la même fleur.

'Tantôt cette disproportion se fait avee symétrie, tantôt elle a lieu sans aueune espèce d'ordre. Dans les Greranium, les Oxalis, il y a dix étamines, einq grandes et einq plus petites, disposées alternativement, en sorte qu'une grande se trouve entre deux petites, et réeiproquement.

Quand une fleur renferme quatre étamines, dont deux sont eonstamment plus eourtes, ees étamines premnent lo nom de didynames ( stamina didynama) : la plupart des Labićes, le marrube (Marrubium vulgare), le thym, ete.; la plupart des Antirrhinées, eomme la linaire (Linaria vu/garis), le grand mufle de veau (Antirrhinum majus), ont les étamines didynames.

Lorsqu'au contraire elles sont au nombre de six dans une fleur, et que quatre d'entre elles sont plus grandes que les deux autres, elles sont appelées tétradynames (sta- 
mina tétradynama). Cette disposition existe dans toute la famille des Crucifères, comme dans le cochléaria ( $\mathrm{Co}$ chiearia officinalis), le radis (Brassica N'apus), etc.

La situation des étamines, relativement aux divisions de la corolle et du calice, mérite aussi d'être soigneusement observée. Ordinairement chaque étamine répond aux incisions de la corolle, c'est-à-dire que les étamines sont alternes avec les divisions de la corolle ou les pétales, lorsqu'elles sont en nombre égal à ces divisions, comme dans la bourrache et les autres Borraginées.

Quelquefois cependant chaque étamine, au lieu de correspondre aux incisions, est située vis-à-vis chaque lobe ou chaque pétale; dans ce cas, les étamines sont dites opposées aux pétales, comme on l'observe dans la primevère, la vigne, etc.

Quand le nombre des étamines est double de eelui des divisions de la eorolle, la moitié de.ces étamines sont alternes, l'autre moitié opposées aux divisions de la corolle.

Les étamines sont, dans le plus grand nombre des cas, opposées aux sépales ou aux divisions du calice, excepté dans les eas rares où elles sont opposées aux pétales.

Dans le lis, la tulipe, les six étamines sont opposées aux six segmens du périanthe simple.

Quelquefois les étamines sont plus courtes que la corolle ou le calice; dc manière qu'elles me sont pas saillantes ạ̀ l'extérieur; on les nomme alors incluses (stamina inclu$s(\iota)$, comme dans la primevère, les narcisses, les daphnés, etc.

On lesnomme, au contraire, exertes (stamina exerta), lorsqu'elles dépassent la hauteur de la corolle ou du calice, comme dans le jasminoïde (Lycium europccum), les menthes, le plantain, ctc.

Suivant leur direction, les étawines sont : 
Dressées ( stam. erecta), comme dans la tulipe, le lis, le tabae (Nicotiana Tabacum), ete.

Infléchies ( stam. inflexa), quand elles sont plićes en arc, et que leur sommet se eourbe vers le centre de la fleur, comme dans les sauges, la fraxinelle (Dictamnus Fraxinella).

Réfléchies ( stam. reflexa), quand elles sont reeourbées en dehors, comme dans la pariétaire (Parietaria officinalis), le mûrier à papier (Broussonetia papyrifera), etc.

Etalées (slam. patentia), lorsqu'elles s'étendent horizontalement, comme dans le lierre (Hedera Helix).

Pendantes ( stam. pendentia), quand leur filet est très-grêle et trop faible pour soutenir l'anthère, comme dans la plupart des Gramincées.

Ascendantes ( stam. ascendentia), quand elles se portent toutes ver's la partie supérieure de la fleur, comme dans la sauge.

Déclinées ou décombantes ( stam. declinata, decumbentia), quand elles se portent toutes vers la partie inférieure de la fleur, comme dans le marronier d'Inde (AEsculus Hippocastanum), la fraxinelle.

Les étamines sont quelquefois réunies par leurs filet: ou par leurs anthères; d'autres fois elles sont réunies e comme confondues avec le pistil : nous parlerons de ce diverses modifications en traitant du filet et de l'anthèr considérés en partieulier.

Dans certaines fleurs on voit un nombre détermin d'étamines avorter constamment. Le plus souvent, les éta mines qui manquent sont remplacées par des appendice de forme très-variée, auxquels on donne le nom de sta minodes ( staminodia), comme dans l'éphémère de Vir ginic (Tradescantia virginica), la plupart des Or chidées, etc.

Une seule étamine avorte constamment dans l'Antir 
rhinum, et beaucoup de Personnćes; deux dans la sauge, le Lycopus, le romarin, ctc., et dans toutcs les Labiées diandres, ainsi que dans toutes les Orchidées, à l'exception du Cypripedium; trois dans le Bignonia, la gratiole; cinq dans l'Erodium, etc.

\section{§. Du Filet.}

Lefilet, comme nous l'avons déjà vu, n'est point une partie cssentielle et indispensable dc l'étamine, puisque assez souvent il manque entièrcmcnt.

Le plus généralemcnt sa formc correspond à son nom, c'est-à-dire qu'il est allongé, étroit et filiforme.

Il est aplati (fil: planum, compressumi) dans l'Allium fragrans, la pervenche, etc.

Cunéaire (fil. cuneiforme), ayant la forme d'un coin, dans le Thalictrum petaloïdeum.

Subulé (fil. subulatum), ou en forme d'alène, quand il est allongé et va en s'amincissant vers le sommet, comme dans la tulipe, etc.

Capillaire ( fll. capillare), quand il est grêle comme un chevcu; par excmple, dans lc blé, l'orge et la plupart des Graminées.

Il est pétaloïde ( fil.petaloïdeum), quand il est large; mince ct coloré à la manière des pétales, comme dans le Nymphaca alba, lcs Amomées; etc.

Quelquefois il cst dilatérà sa base, comme dans l'Ornithogaluim pyrenaïcum.

D'autres fois il est comme voúté ( fil.basifornicatum ), comme dans l'asphodèle, les campanules, etc.

Le sommet du filet est ordinairemcnt aigu : comme dans la tulipc, le lis, ctc.

D'autres fois il est obtus, et même renflć en tête ou cápitulé, commc dans le Cephalotus, etc.

C'est, dans lc plus grand nombre des cas, au sommęt 
du filet que s'attaehe l'anthère. Cependant il arrive quelquefois qu'il se prolonge au-dessus du point d'insertion de eet organe; dans ee eas il est dit proéminent ( fil.prominens ), comme dans le Paris quadrifolia, ete.

Les étamines sont, le plus souvent, libres de toute adhérenee, et isolées les unes des autres. Mais il arrive quelquefois qu'elles sont réunies par leurs filets en un ou plusieurs eorps, que nous désignerons, avee M. Mirbel, sous le nom d'androphore (androphorum).

Quand tous les filets sont réunis ensemble en un seul androphore, les étamines prennent le nom de monadelphes ( stamina monadelpha), comme dans la mauve, la guimauve, ete. (Voy. pl.6, fig. ıо.)

Dans ee cas, l'androphore forme un tube plus ou moins complet. Quelquefois eependant l'union des filets n'a lieu que par leur base, en sorte qu'ils sont libres dans la plus grande partic de leur étendue, comme dans le Geranium, l'Erodium.

D'autres fois ils sont soudés jusqu'à la moitié de leur hauteur, eomme dans plusicurs Oxalis.(Voy•pl.6, fig. 1o.)

Enfin ils sont soudés en tube à peu près eomplet, dans la plupart des Malvacées. A sa partie supérieure, l'androphore tubuleux se divise en autant de petits filets eourts et distinets qu'il y a d'anthères.

Lorsque toutes les étamines sont réunies en deux àndrophores, e'est-à-dire que leurs filets se soudent en deux corps distinets, on les nomme diadelphes ( stamina diadelpha). Par exemple, le fumeterre (Fumaria officina7is ); les harieots, les aeaeias, ete., et la plus grande partie des Légumineuses. (Voyez pl.6, fig. I1.)

Quand les filets sont réunis en trois ou en un nombre plus eonsidérable d'androphores, les étamines sont dites alors polyadclphes (stamina polyadelpha). Il y a trois androphores dans l'Hypericum agyptiaocum, einq et un plus grand nombre dans les Melaleuca. 
La nature et la structure organique du filet des étamines paraissent être entièrement analogues à celles de la eorolle. En effet, l'on voit très-souvent ees deux organes se changer l'un dans l'autre. Ainsi, par exemple dans le nénuphar ( $N$ ymphoea alba ), on aperçoit suceessivement les filets staminaux, à partir du eentre vers la eirconférenee de la fleur, devenir de plus en plus larges et s'amineir; l'anthère, au contraire, diminuer et finir par dispa-, raitre entièrement quand les filets se sont tout-à-fait changés en pétales. C'est cette dégradation insensible des filets des étaninines en pétales qui a fait penser à quelques botanistes que la corolle et les segmens qui la composent n'étaient que des étamines avortées, dont les filets avaient acquis un développement extraordinaire.

Cette opinion; que nous ne voulons ni admettre ni rejeter entièrement, semble encore trouver un appui dans la formation des fleurs nommées doubles et pleines. La rose, en effet, dans son élat primitif et sauvage, n'a que cinq pétales, mais un nombre très-eonsidérable d'étamines. Dans nos jardins, par les soins du eultivateur, nous voyons les étamines de la rose se changer en pétales, et la fleur devenir stérile. Iei la transformation des étamines en pétales est manifeste, et paraît confirmer l'opinion des botanistes qui regardent la corolle eomme de véritables étamines avortées.

\section{\2. De l'Anthère.}

Lianthère (anthera) est cette partie essenticlle de l'étamine qui renferme le pollen ou poussière féeondante avant l'aete de la féeondation. Le plus généralement elle est formée par deux petites poehes membraneuses, adossées immédiatement l'une à l'autre par un de leurs eồtés (voy. pl. 6 , fig. $6,7,8)$, ou réunies par un eorps intermédiaire particulier, auquel on a donné le nom de connectif. (Pl. 6, fig. 9...a.). 
Chacun de ces petits saes membraneux, nommés loges de l'anthère, est partagé intérieurement en deùx parties par une eloison longitudinale, et s'ouvrent à l'époque de la féeondation, pour laisser sortir le pollen.

Les anthères sont done le plus communément biloculaires (antherce biloculares), e'est-à-dire formées de deux loges, comme dans le lis, la jaein the, ete.

Quelquefois elles ne sont formées que d'une seule loge; dans ee eas elles sont dites uniloculaires (antherœ uniloculares), eomme dans les Conifères, les Epacridées, les Malvaećes, le coudrier, ete.

Plus raremcut encore l'anthère est composée de quatre loges, et on la nomme quadriloculaire (anthera quadrilocularis), eomme daus le Butomus umbellatus, ete.

Chaque loge d'une anthère offre ordinairement sur l'une de ses faees un sillon longitudinal, par lequel elle s'ouvre dans le plus grand nombre des eas. La partie de l'anthère du côté de laquelle sont les sillons porte le nom de face proprement dite; la partie opposée à celle-ci, et par laquelle l'anthère s'attaehe au filet, est nommée le dos de l'anthère.

L'anthère est eommunément fixée au sommet du filet staminal. Cette insertion, qui fouruit de très-bons earactères, peut se faire de trois manières différentes :

$x^{\circ} L^{2}$ 'anthère peut ĉtre attachée au sommet du filet par sa base même, eomme dans l'iris, le glaïeul, ete. Elle porte le nom de basifixe (anthera basifixa).

$2^{\circ}$ Elle peut être fixée par la partie moyenne de son dos, comme dans le lis. Dans ee eas elle a été appelée médiifixe (anthera mediifixa);

$3^{\circ}$ Assez souvent elle est attaehée par son sommet; dans ce cas elle es mobile et vacillante. On l'appelle alors apicifixè (anthera apicifixa);

Lorsque la faee des anthères est toumée vers le centre de la fleur, elles sont dites introrses (antherce intror'sa), eomme cela a lieu dans la plupart des plantes. 
On les appellc, au eontrairc, extrorses (antherce extror$s(e)$, quand leur face regarde la circonfércnce de la fleur, comme, par exemplc, dans les Iridées, le concombre; ctc. Cette disposition est plus rare quc la précédcnte.

La forme dcs anthères présente un grand nombre devariétés. Ainsi on dit qu'clles sont :

Sphéroiddales (anth. spheroïdales, subglobosa), quand elles se rapprochent de la forme ronde, comme celles dc la mercuriale (Mercurialis annula).

Didymes (anth. didymee), offrant deux lobes sphćroïdaux, rénnis par un point de leur circonfércnce, comme dans l'épinard (Spinacia oleracea), les Euphorbes, etc.

Ovoüdales (anth. ovoüdea). ,Cette forme est une des plus fréquentes.

Oblongues (anth. oblonga), comme dans le lis (Lilium. candidım), cıc.

Linéaires (anth. lineares), quand elles sont très-alongées et très-étroites, comme celles des campanules, des Magnolic, etc.

Sagittées (anth. agittatce), ou en fer de flèche : par excmple, cellcs du laurier-rosc (Nerium oleander), di safran (Crocus sativuls), ctc.

Cordiformes (anth. cordiformes), comme dans lc basilic (Ocymuin basilicum), ctc.

Reniformes (anth. réniformes), ou en forme de rein; dans la digitale pourprée (Digilalis purpurea), un grand nombre de Mimosa, ẹtc.

Tétragones (anth. tetragona), ayant la forme d'un prisme à quatre faces, comme celles de la tulipc (Tulipa gessneriana).

A son sommet, l'anthère peut être terminée de différentes manières; ainsi ellc cst :

Aiguë (anth. apice acuta), dans la bourrache (Borrago officinalis).

Bifide (anth. bifida), fendue à son sommet (ou à sabase) 
en deux lobes étroits et écartés, comme dans un grand nombre de Graminées.

Bicorne (anth. bicornis), terminće à son sommet par deux eornes allongées, eomme dans l'airelle myrtille (Vaccinium myrtillus), la pyrole (Pyrola rotundifolia).

Appendiculée (anth. appendiculata), eouronnée d'appendices, dont la forme est très-variable, comme daus l'aunée (Inula lelenium), le laurier-rose (Nerium oleander).

Les deux loges qui composent une anthère biloculaire peuvent être soudées l'ume à l'autre de différentes manières.

Io Elles peuvent être réumies immédiatement l'une à l'autre sans le seeours d'aucum autre eorps intermédiaire, comme dans les Graminées. (Voy.pl. 6, fig. 6, 7,8.)

Quand les deux loges sont réunies immédiatement, elles peuvent offrir deux modifications différentes. En effet, tantôt leur union a lieu par l'un de leurs côtés, de manière que les deux sillons se trouvent encore sur la mème faee et eomme parallèles; les loges sont dites alors apposées (loculis apposiils), eomme dans le lis, ete.

D'autres fois, au eontraire, elles sont soudées par la faee opposće à leur sillon, en sorte que les deux sillons se trouvent situés de ehaque côté de l'anthère; les deux loges sont alors appelées opposées (loculis oppositis). Mais cette disposition est moins fréquente que la première.

$2^{\circ}$ Elles peuvent être réunies immédiatement par la partie supérieure du filet qui se prolonge entre elles, comme dans un grand nombre de renoneules.

3० Enfin elles peuvent être éloignées plus ou moins l'une de l'autre par un eorps intermédiaire, manifestement distinet du sommet du filet; c'est à ce eorps qu'on a dommé le nom de connectif (connectivnum), paree qu'il sert de moyen d'union entre les deux'loges. (Voy.pl. 6, fig. 9, a.)

Le connectif n'est quelquefois apparent qu'au dos de 
l'anthère; alors il est appelé dorsal, comme on l'observe dans le lis, etc.

D'autres fois il est apparent sur les deux faces de l'anthère, dont il écarte assez manifestement les deux loges, comme dans le Melissa grandiflora, l'éphémère de Virginie, etc. (Voy. pl. 6, fig. 9.)

Enfin quelquefois le eonneetif est tellement grand, tellement développé, que ce n'est que par analogie qu'on le reeonnaît; dans ce cas il a reẹu le nom de connectif distractilde. Ainsi, par exemple, dans la sauge, ce connectif est sous forme d'un long filament recourbé, posé transversalement sur le sommet du filet; à l'une de ses extrémités, on voit une des loges de l'anthère remplie de pollen; à l'autre extrémité se trouve la seconde loge, mais presque eonstamment avortée et à l'état rudimentaire.

Cette singulière conformation se trouve également dans les Mélastomes, et plusieurs espèces de Labiées et de Scrophularinées.

Chaeune des loges d'une anthère peut s'ouvrir de différentes manières, dans les divers genres de plantes, et les caractères tirés de cette déhiscence servent, dans quëlques eas, à distinguer certainś genres.

Le plus souvent cette déhiseence a lieu par la suture du sillon longitudinal qui règne sur la surfaee de ehaque loge; dans ee cas, on dit que les loges sont: longitudinäaliter déhiscentes, eomme dans le lis, la tulipe et un grand nombre d'autres plantes.

La déhiscence peut avoir lieu par des pores ou des fentes situées dans 'différens points.'

Ainsi, dans les Erica, les Solanum, etc., chaque loge s'ouvre par un petit trou placé à son sommet (locul. apice dehiscentes.) (Voy. pl. 6, fig. 7, $a$ a.)

Dans la pyrole, ce trou est placé à la partie infériéurc ('locul. basi dehiscentes).

D'autres fois ce sont des espèces de petites valvules, 
qui se soulèvent de la partie inféricure vers la supérieure; comme dans les lauriers, l'épinevinette, l'Epimedium alpinum, etc. (Voy.pl. 6, fig. 8.)

Nous venons d'examiner jusqu'ici les anthères, libres de toute adhérence; mais, aussi bien que les filets staminaux, elles peuvent se rapprocher et se souder entre clles de manière à former une sorte de tube. Cette disposition remarquable se reneontre dans toute la vaste famille des Synanthérées, auxquelles on donnait autrefois le nom de plantes à fleurs composées; tels sout les ehardons, les artichauts, les soueis, etc. Linnæus a donné le nom de syngénésie à la elasse de son système dans laquelle sont réunies toutes les plantes à anthères soudées latéralement, qu'il désignait aussi sous le nom de syngénèses. (Voy. pl. 6, fig. i3.)

Il existe un grand nombre de plantes dans lesquelles les étamines, au lieu d'ètre libres, ou simplement réunies ensemble par leurs filcts ou leurs anthères, font corps avec le pistil, e'est-à-dire qu'elles sont intimement soudées avee le style et le stigmate. C'est à ees plantes qu'on a donné le nom de gynandres. (V.pl. 6, fig. 14.)

La coaleseenee des étamines n'a jamais lieu avee l'ovaire. Ce ne sont que les filets et le style qui s'unissent, en sorte que les anthères et le stigmate sont portés par un support commun, avee lequel ils se confondent. C'est ee que l'on observe dans les Aristoloches, les Orchidées, Zingibéracées, etc.

Daus les Orehidées, on donne le nom de gynostème ( gynostemium) au support commun du stigmate et des anthères.

\section{§. Du Pollen.}

Le pollen, ou la substanee contenue dans les loges de l'anthère, et qui sert à la féeondation, se préscnte ordinairement sous l'apparence d'une poussière composée de 
petits grains d'une extrême ténuité; quelquacfois il est en masses solides plus ou moins considérables. Cette dernière forme, étant restreinte à un petit nombre de végétaux, ne fixera notre attention qu'après qque nous aurons examiné avec détail la structure du pollen sous forme pulvérulente.

Avant le perfectionuement des instrumens d'optique, les renseignemens que l'on possédait sur les formes variées des grains polliniques et surtout sur lcur structure intime, étaient extrêmement vagues. On avait bien aperçu une grande diversité dans ceux que l'on avait examinés avec de fortes loupes; mais ces différences avaient été indiquécs, sans en tirer d'utiles conséquences pour l'avancement de la science. La structure du grain pollinique avait aussi été un objet de rccherches de la plupart des anciens bota-nistes qui, faute de moyens rigoureux d'obscrvation, s'étaicnt long-temps disputés, mais sans tombcr d'accord; sur la composition intérieure de corps aussi élémentaires. L'étude microscopique du pollen était donc un sujet digne de révision, et ne pouvait manquer de fixer l'attention des observateurs modernes. M. Amici que nous avons eu occasion de citer si avantageusement dans cet ouyrage, a publié dans les Actes de la société italienne, vol. xvir, un chapitre sur le pollen, où il a fait connaître des circonstances très-intéressantes et que nous mentionnerous plus bas. A l'aide du microscope acromatique de M. Sclligue( I), notre ami M. Guillemiu a fait, dans le cours de l'été de i 824 , des observations nombrcuses sur le pollen, observations dont il nous a communiqué les priucipaux résultats.

Les grains polliniques sont des utriculcs de formes variées, sans adhérence dans l'anthèrè à l'époque de la maturité, et renfermant une multitude de granules d'une extrême ténuilé.

(r) Voyez la description et la figure de cet instrument dans les Annales des Sciences naturelles. Nop. 1824, t. 3, p. 345, et tab. I 8 . 
I a membrane utrieulairc est tantôt lisse, tantôt marquée d'éminences ou d'aspérités; quelquefois elle offre de simples faeettes ou des bosses disposées entre elles symétriquement. Lorsque le pollen est parfaitement lisse dans sa superficie, il n'est en mćmc temps recouver t d'aucun enduit visqueux, tandis que les moindres éminences sont des indices de viscosité. Les papilles, les éminences mamelonnées, etc., qui reeouvrent certains grains polliniques, sont de véritables organes séeréteur's, et l'enduit visqueux ordinairement eoloré qui les recouvre en est le produit. Parmi les pollens pulvérulens, on peut done établir deux ordres principaux, savoir, les pollens visqueux et les pollens non visqueux. Les considérations tirécs de la forme générale sont moins importantes, c'est-à-dire quc la différenee est moins grande entre les pollens sphériques, elliptiques, cycloïdes, polyédriques, etc.

M. Guillemin s'est eonvaineu par un grand nombre d'observations que la nature des grains polliniques était la même dans chaque famille naturcllc de plantes, ou, en d'autres tcrmes, que dans une de ecs familles on ne rencontrait point en même temps des pollcns visqueux et des pollens non visqueux. Il a vu de plus que tous les genres d'une même famille n'offrent que des modifications dans les formes de leurs grains polliniques; mais que des familles très-éloignées par d'autres caractères se rapproehaient nćanmoins par une identité dans leurs pollens. Nous nous contenterons d'énumércr la naturc et la formc de cet organe dans quelques familles remarquables.

Le polleu des Malvacées et des Convolvulacées est formé de grains sphériques papillaires et d'un blanc argentin. Dans les Cucurbitacées, ils sont sphériques, papillaires et d'un beau jaune dorć. Ceux de la tribu des Mélicunthées, dc la famille des Synanthérées, sont égalcmcnt sphériques, papillaires, et d'un beau janne orangé. La tribu ou plutòt l'ordre des Chicoracées nous présente des grains spliéri- 
ques visqueux, mais dont la superficie est taillée à facettes. Un pollen à grains eouverts d'éminenees mamelounées, surmontés ehaeun d'un point brillant, s'observe dans le Cobrea scandens. Celui des Phlox est très-analogue à ee dernier, eireonstanee qui appuie l'opinion de eeux qui considèrent ees deux genres eomme étant de la même famille. Enfin, pour ne pas pousser trop loin eette énumération des pollens visqueux, les grains dans les Onagraires ont une forme trigone très-manifeste, avee une dépression considérable dans leur eentre.

Les familles où l'on trouve des grains non visqueux, sont en très-grand nombre. Il nous suffira de eiter les Solanées, Scrophularinées, Gentianées, Caryophyllées, Graminées, Euphorbiacées, etc. Ces grains ont toujours une forme elliptique, et sont marqués d'une rainure longitudinale; le plus souvent ils sont eolorés en jaune, quelquefois en rouge, eomme dans les V erbascum. Dans les Légumineuses papilionacées, le pollen est bien d'une nature non visqueuse, mais il a une forme eylindroïde très-prononcée.

Lorsqu'on soumet les grains non visqueux à l'aetion de l'eau, ils ehangent de forme à l'instant même; d'elliptiques qu'ils étaient, ils deviennent parfaitement sphériques. Les grains visqueux se dépouillent d'abord de leur enduit; puis ils éelatent plus ou moins promptement, et laneent au dehors un liquide plus dense que l'eau, et dans lequel se meuvent des myriades de petits grains que leur eouleur verdàtre rend pereeptibles à la vue, par un grossissement de plusieurs eentaines de fois leur diamètre. M. Amiei a vu un grain pollinique de Portulaca oleracea en eontaet avee un poil du stigmate, se rompre, lancer au dehors une sorte de boyau dans lequel les granules ont cireulé pendant plus de quatre heures. Gleichen, qui avait déjà observé les granules eontenus dans les grains polliniques, les avait considérés comme jouant le principal rôle dans 
l'acte de la féeondation, et M. Guillemin, raisonnant d'après l'analogie de ces organes avee les animalcules spermatiques des animaux, n'est pas éloigné d'adopter eette opinious.

Telles étaient nos eomnaissanees sur la nature et l'organisation des grains polliniques, lorsque notre ami, M. Adolphe Brongniart, entreprit son beau travail sur la génération daus les végétaux. Nous allons faire eonnaître iei son opinion sur la nature et l'organisation des grains de pollen. Lorsque l'on examine l'intérieur des loges d'une jeune anthère dans un bouton de fleur, long-temps avant son épanouissement, on voit que l'intérieur de eette loge est rempli d'une masse eelluleuse distinete de ses parois. Petit à petit ehacune des eellules, en général fort petites, dont se eompose la masse celluleuse, s'isolent les unes des autres et finissent par former les granules que l'on a nommés pollen. Quelquefois ces ecllules partieulières ou grains polliniques sont renfermés dans d'autres vésieules plus grandes qui se dćehirent, et dont on peut eneore reeonnaître les traees.

Chaque grain de pollen, dont la forme est très-variable, ainsi que nous l'avons dit précédemment, offre une organisation uniforme. Il se eompose de deux membranes, l'une externe plus épaisse, munie de pores et quelquefois d'appendiees plus ou moins saillans; l'autre interne, minee, transparente et sans adhérenee avee la préeédente. Soumis à l'aetion de l'eau, la membrane interne se gonfle, l'externe se rompt dans un point de son étendue, et à travers eette ouverture sort un prolongement tubuleux, qui forme une sorte de hemie, et qui a été observé pour première fois par Needharn. M. Amiei l'a également vu sur les grains polliniques du Portulacı pilosa. Quelquefois il en sort deux, par deux points opposés, comme dans l'OEnolhera biennis. La eavité de la membrane interne est remplie de granules sphériques, d'une excessive ténuité, 
qui paraissent jouer le rôle le plus important dans l'acte de la féeondation.

Nous parlerons maintenant du pollen des Asclépiadées et des Orchidées, qui présente des modifications très-remarquables. Dans plusieurs genres de ces deux familles, tout le pollen contenu dans une loge est réuni en un corps qui a la même forme que la loge dans l'intéricur de laquelle. il est contenu. On donne à ce pollen ainsi réuni le nom de massepollinique (massa pollinica). Quand ces masses sont partagées en plusieurs autres masses plus petites, on appelle ces dernières des massettes (massulae). Les masses polliniques des Orehidées sont tantôt formées de grains solides réunis ensemble par une sorte de réseau élastique; on les appelle alors masses sectiles (massce sectiles), comme dans les genres Orchis, Ophrrss. D'autres fois elles sont tout-ì-fait granuleuses ou farinacées (massa granulosa) ; telles sont eelles des gemres Epipactis, Loroglossum, ete. Enfin elles sont quelquefois d'une substance solide et eompacte (massce solidce), comme dans les genres Corallorliza, Malaxis. Ces trois formes ne se trouvent jamais réunies ni eonfondues dans ùn même genre.

Le pollen, projetésur des eharbons ardens, brûle ets'enflamme avee rapidité. Dans beaucoup de plantes, il répand une odeur qui a l'analogie la plus frappante avec la substance à laquelle on le compare dans les animaux, eomme on l'observe très-bien dans le châtaignier, l'épine-vinette, etc.

Le polleu, lorsqu'il eommence à se développer, e'està-dire long-temps a vant l'épanouissement de la fleur, se présente sous la forme d'une masse celluleuse, quelquefois recouverte d'une membrane, propre extrèmenent mince, mais sans aucune adhérenee avec la cavité qui la renferme. Les utrieules qui composent cette masse, sỏat d'abord très-intimement unis les uns avec les autres; on aperçoit dans leur intérieur quelques granules épars. Petit 
à petit lès utricules se séparent, les granules qu'ils contiennent sc.réunissent, et bientòt ils déehirent, par leur développement sueeessif, les utrieules qui les contenaient, prennent la forme qu'ils doivent conserver, et deviennent cnfin les grains de pollen. On voit que ee mode de développement est entièrement analogue à eelui du tissu eellulaire, et dont nous avons parlé en traitant des parties élémentaires des végétaux.

\section{CH $\triangle$ PITRE IX.}

DU PISTIL OU ORGANE SEXUEL FEMELIE.

LE pistil, comme nous l'avons déjà vu précédemment, est l'organe sexuel femelle dans les végétaux. Il oecupe presque coustamment le centre de la fleur, et se compose de trois parties, savoir : $1^{\circ}$ de l'ovaire, $2^{\circ} \mathrm{du}$ style, $3^{\circ} \mathrm{du}$ sligmate.

Ordinairement on ne rencontre qu'un seul pistil dans une fleur, comme dans le lis, la jaeinthe, le pavot, ete.

D'autres fois il $\mathrm{y}$ en a plusieurs dans la même fleur, comme dans la rose, les renoneules, ete.

Le pistil ou les pistils, lorsqu'il y en a plusicurs, sont souvent attaehés à un prolongement partieulier du réecptacle, auquel on donne le nom de gynophore.

Il ne faut pas eonfondre le gynophor'e avee le podogyne, amincissement de la base de l'ovairc qui élève un peu le pistil au-dessus du fond de la fleur. Le gYnophore, en efiet, n'appartient pas essentiellement au pistil; il reste au fond de la fleur, quand eelui-ei vient à s'cn détacher. Le podogyne au contraire, qui fait partic du pistil, l'aeeompagne dans toutes les époques de son développement. 11 y a un gynophore dans le fraisicr, le framboisicr, et un podogyne dans le caprier, lc pavot, etc. 
Lorsqu'ily a plusieurs pistils dans une fleur, il n'est pas s rare de voir le gynophore devenir épais et eharnu : e'est ee ' qu'on observe d'une manière très-manifeste dans le framI boisier, et surtout le fraisier. La partie de la fraise qui 'est pulpeuse, sucrée, ct que nous mangeons, n'est qu'un gynophore trc̀s-développé : les petits grains brillans qui lla recouvrent sont autant de pistils. Il est faeile de reconinaitre la nature de ees différentes parties, et d'en suivie 1 les dćveloppemens sueeessifs dans la fleur.

La base du pistil est toujours représentée par le point au moyen duquel il s'attache au réeeptaele. Le sommet, au contraire, eorrespond toujours au point où les styles ou tbien le stigmate sont insérés sur l'ovaire. Comme quelqueifois cette insertion a lieu latćralement, on conçoit que le sommet organique de l'ovaire ne rćpond pas toujours à sson sominet géométrique. Cc dernier, en cffet, est le point de plus élevé par lequel passe une ligne qui traverse l'ovaire dans sa partie eentrale.

\section{ऽ. De l'ovaire.}

L'ovaire (ovarium) occupe toujours la partie inférieure du pistil. Son earaetère essenticl est dc prćsenter, quand on le coupe longitudinalement ou cn travers, une ou plusieurs eavitćs, nommćcs loges, dans lesquelles ont contcnus les rudimens des graines ou les ovules. C'est lans l'intćrieur dc l'ovaire que les ovules acquièrent tout eur dévcloppement et se changent en gr'aines. Cet organe icut done être considéré, sous le rappori de ses fonctions, omme l'analogue de lovaire et de l'utérus dans les nimaux.

La formc la plus générale et la plus habituelle de l'o vaire, st d'ètre ovoïde; eependant il est plus ou moins eomprimé $t$ allongé dans eertaines familles de plantes, comme daus is Crucifères, les Légumineuses, etc. 
L'ovaire est le plus souvent libre au fond de la fleur; c'est-à-dire que sa base eorrespond au point du réeeptaele, où s'insèrent également les étamines et les enveloppes florales, sans qu'il contraete d'adhérenee avee le ealiee, comme on le voit dans le jaeintle, le lis, la tulipe, ete. (Voy.pl. 6, fig. r, 3.)

Mais quelquefois on ne reneontre pas l'ovaire dans le fond de la fleur; il scmble plaeé entièrement au-dessous du point d'insertion des autres parties; e'est-à-dire que, faisant eorps par tous les points de sa périphérie avee le tube du ealiee, son sommet seul se trouve libre au fond de la fleur. Dans ee eas, l'ovaire a été appelé adliérent ou infère (ovarium inferum), pour le distinguer de eelui où, étant libre, il porte le nom d'ovaire supère (ovarium superum ); les Iris, les Nareisses, les Myrtes, les Groseiller's ont un ovaire infère. (Voy. pl. 6, fig. 4.)

Lor's done qu'au fond d'une fleur on ne trouvera pas l'ovaire, mais que le eentre en sera oceupé par un style et un stigmate, on devra examiner si au-dessous du fond de eette fleur on ne voit pas un renflement partieulier, distinet du sommet du pédoneule. Si ee renflement, eoupé en travers, offre une ou plusieurs eavités, eontenant des ovules, on sera dans la eertitude qu'il existe un ovaire infère.

La position de l'ovaire infére ou supère fournit les earaetères les plus précicux pour le groupement des genres en familles naturelles.

Toutes les fois que l'ovaire est infère, le ealiee est nécessairement monosépale, puisque son tube est intimement uni avee la périphérie de l'ovaire.

Quelquefois l'ovaire n'est pas entièrement infère, e'està-dire qu'il est libre par son tiers, sa moitié ou ses deux tiers supérieurs. Le genre saxifrage oftre ees différentes nuanees.

Mais il est une position de l'ovaire qui, presque toujours eonfondue avee l'ovaire infère, mérite cependant d'en être distinguée. C'est le cas où plusieurs pistils réunis dans une 
fleur sont attachés à la paroi interne d'un caliee très-resserré à sa partie supérieure, en sorte qu'au premier eoup d'oeil il représente un ovaire infère. Ces ovaires reçoivent alors Je nom de pariétaux (ovaria parietalia), eomme dans la rose, et un grand nombre d'autres Rosaeées. (Voyez pl.6, fig. 2.)

Nous devons également faire eonnaître une modifieation de l'ovaire à laquelle on a donné le nom d'ovaire gynobasique. Un grand nombre de familles en présentent des exemples; telles sont entre autres les Labiées, les Borraginées, les Oehnaeées, les Simaroubées, ete. L'ovaire appliqué sur un disque hypogyne, qui dans ee cas a reẹu le nom partieulier de gynobase, est plus ou moins profondément partagé en un eertain nombre de lobes eorrespondans à eelui des loges, et son axe eentral est tellement déprimé qu'il paraît en quelque sorte nul et que le style semble naître immédiatement du disque; en sorte qu'à l'époque de la maturité ehaeune des parties ou eoques dont se eompose l'ovaire se sépare, et semble en quelque sorte eonstituer un fruit partieulier.

L'ovaire infère étant eelui qui fait eorps par tous les points de sa périphérie avee le tube du ealiee, il déeoule de là une loi générale à laquelle on n'a point fait attention : e'est que la position infère de l'ovaire exelut néeessairement la multiplieité des pistils dans la même fleur. En effet, dans le eas d'ovaires pariétaux, on voit qu'ils ne touehent au ealiee que par un seul point : il est de toute impossibilité que eet organe en enveloppe plusieurs par toute leur périphérie. Il suit done de là que ees ovaires ne sont pas infères, mais seulement pariétaux, puisqu'ils ne font pas eorps par tous les points de leur périphérie avec le tube du ealiee. Cette modifieation mérite d'être signalée.

L'ovaire est sessile au fond de la fleur (ovarium sessile), quand il n'est élevé sur aucun support particulier, comme dans le lis, la jacinthe, etc. (Voy. pl. 6, fig. I et 3.) 
Il peut être stipité (ovarium stipitatum), quand il est porté sur un podogyne très-allongé, eomme dans le eâprier (Capparis spinosa).

Coupé transversalement, l'ovaire offre souvent une - seule eavité intérieure ou loge, eontenant les ovules. Il est dit alors uniloculaire (ovarium uniloculare), eomme eelui de l'amandier, du eerisier, de l'oillet, ete.

On l'appelle biloculaire (ovarium biloculare), quand il est composé de deux loges; par exemple dans le lilas, la linaire, la digitale, ete.

Triloculaire (ovarium triloculare); tel est eelui du lis, de l'iris, de la tulipe, ete. (Voy·pl. 6, fig. 5.)

Quadriloculaire (ovarium quadriloculare), eomme dans le Sagina procumbens.

Quinquéloculaire (ovarium quinqueloculare), eomme dans le lierre (Hedera Helix).

Multiloculaire (ovarium multiloculare), quand il présente un grand nombre de loges: Ex. le nénuphar.

Mais chaque loge peut eontenir un nombre d'ovules plus ou moins eonsidérable. Ainsi il y a des loges qui ne renferment jamais qu'un seul ovule : on les appelle uniovulées (locula uniovulala), eomme dans les Graminées, les Synanthérées, les Labiées, les Ombcllifères, ete.

D'autres fois chaque loge eontient deux ovules, e'està-dire qu'elle est biovulée (locula biovulata). Dans les eas où chaque loge d'un ovaire renferme deux ovules seulement, il est très-important d'étudier leur position respective. Tantôt, en effet, les deux ovules naissent d'un même point et à la même hauteur; dans ce eas ils sont dits apposés (ovulis appositis), comme dans les Euphorbiaeées. D'autres fois, au eontraire, ils naissent l'un au-dessus de l'autre; on les appelle alors superposés (ovulis superpositis), eomme dans le Tamus communis.

On dit au eontraire qu'ils sont alternes (ovulis alternis), lorsque les points d'attache des ovules ne sont pas 
sur le même plan, quoique les ovules se touchent latéralement, par exemple, dans le pommier, le poirier, ete.

Nous reviendrons plus en détail sur les différentes positious des ovules entre eux, et relativement à l'ovaire, en pärlant de la graine.

Quelquefois enfin eliaque loge d'un ovaire renferme un nombre très-considérable d'ovules, eomme dans le tabae, le pavot, ete.; mais ees ovules peuvent être disposés de diverses manières. Ils sont assez souvent superposés régulièrement les uns au-dessus des autres, sụr une ligne longitudinale, eomme dans l'aristoloche (Aristolochica Sypho). On les appelle alors unisériés (ovulis uniseriatis). D'autres fois ils sont disposés sur deux lignes longitudinales : ils sont bisériés, eomme dans les iris, le lis, la tulipe, etc.

Quelquefois ils sout épars et sans ordre, comme dans la pomme épineuse. D'autres fois ils sont conglobés, ou réunis et serrés les uns eontre les autres, de manière à former un globe, comme dans un grand nombre de $\mathrm{Ca}-$ ryophyllées.

Les ovules féeondés deviennent des graines; mais il arrive fréquemment qu'un certain nombre d'ovules avortent eonstamment dans le fruit. Quelquefois même plusieurs eloisons se détruisent et disparaissent. Ils est done essentiel de reehereher dans l'ovaire la véritable structure du fruit. C'est par ee moyen seul qu'on peut rapprocher.les uns des autres, dans la série des ordres naturels, eertains genres qui, au premier eoup-d'oeil, s'éloignent beaucoup par la structure de leurs fruits et la disposition de leurs graines.

\section{§2. DuS Siyle.}

Le style (stylus) est ce prolongement filiforme du sommet de l'ovaire qui supporte le stigmate. (Voyez pl. 6, fig. I, 3). Quelquefois il manque entièrement; et alors le stigmate est sessile, comme dans le parot, la tụlipe, etce. 
L'ovaire peut être surmonté d'un seul style, eomme dans le lis, les Légumineuses; de deux styles, comme dans les Ombellifìres; de trois styles, eomme dansla viorne (Viburnum lantana), ete. Il y a quatre styles surl'ovaire, dans le Parnassia; einq daus le Statice, le lin, ete.

Dans d'autres eas, au eontraire, il n'y a qu'un seul style pour plusieurs ovaires, eomme dans les Apocynées, ete.

Presque toujours le style oceupe la partie la plus élevée, e'est-à-dire le sommet géométrique de l'ovaire, eomme dans les Crueifères, les Liliaeées, ete. On l'appelle alors style terminal (stylus terminalis).

On le nomme latéral (stylus lateralis) quand il naît des parties latérales de l'ovaire, comme dans la plupart des Rosacées, le Daphné, etc. Il indique alors le sommet organique de l'ovaire, qui, dans ee eas, est différent du sommet géométrique.

Dans quelques eireonstanees beaucoup plus rares, le style paraît naître de la base de l'ovaire. On lui a domné le nom de style basilaire (stylus basilaris), eomme dans l'alchimille (Alchimilla vulgaris), l'arbre à pain (Artocarpus incisa).

Quelquefois eneore le style, au lieu de naître sur l'ovaire, semble partir du réeeptacle, comme dans les Labiées, eertaines Borraginées, ete. Cette eireonstanee se reneontre toutes les fois qu'il y a un gynobase. ( $V \cdot$ p. 224.)

Le style peut être inclus (styluns inclusus), e'est-à-dire renfermé dans la fleur, de manière à n'être pas visible à l'extérieur, eomme dans le lilas (Syringa vulgaris), le jasmin (Jasminum officinale), ete.

Il peut être saillant (sylus exsertus), eomme dans la valériane rouge (Centranthus ruber).

Les formes du style ne sont pas moins nombreuses que celles des autres organes que nous avons étudiés jusqu'iei. En effet, quoique le plus généralement il soit grêle et filiforme, eependant il offre, dans eertains végétaux, une 
apparence tout-à-fait différente. Ainsi il est trigone (stylus trigonus) dans l'Ornithogalum luteum, le Lilium bulbifernum, etc.

Il est claviforme; ou en massue (stylus claviformis) dans le Leucoium astivum.

Il est creux (stylus fistulosus) dans le lis (Lilium candidum).

Pétaloüde (stylus petaloïdeus), large, mince, membraneux, coloré à la manière des pétales, dans les Iris, etc. , etc.

Suivant sa direction, relativement à l'ovaire, il est vertical, dans le lis;

Asccndant (stylus ascendens), formant un arc dont la convexité est tournée vers le haut de la fleur, 'comme dans la sauge et plusieurs autres Labiées;

Décliné (stylus declinatus) (r), lorsqu'il s'abaisse vers la partie inférieure de la fleur, comme dans le dictame blanc (Dictamnus albus), certaines Labiées et Légumineuses.

Le style peut être simple (stylus simplex), et sans aucune-division, comme dans la pervenche, le lis.

Il est bifide dans le groseiller rouge (Ribes rubrum), trifide dans le glaïeul (Gludiolus communis); quinquéfide, dans l'Hibiscus; multifide, dans la mauve, suivant qu'il est fendu en deux, trois, cinq, ou un grand nombre dé divisions peu profondes.

Si, au contraire, ces divisions sont très-profondes, et atteignent jusqu'au-dessous de son milieu, il est dit alors biparti, comme dans le groseiller à maquereau (Ribes grossularia) ( voy.pl. 6, fig.4); triparti, quinquéparti, multiparti, etc., suivant le nombre de ses divisions.

(I) Assez souvent les étamines et le pistil sont déclinés dans la même fleur : on dit alors que les organes sexuels sont déclinés (genitalia declinata), comme dans la fraxinelle. 
Le style est quelquefois comme artieulé avec le sommet de l'ovaire, en sorte qu'il tombe après la féeondation; on lui donne le nom de caduc ( stylus caducus) : dansce cas, il n'en reste aueune trace sur l'ovaire, comme dans la cerise, la prune, ete. D'autres fois, au contraire, il est persistant ( slylus persistens) quandil survit à la féconda tion: ainsi, dans les Crucifères, le buis, les Anémones, les Clématites, le style persiste et fait partie du fruit.

Enfin, quelquefois non-seulement il persiste, mais il prend eneore de l'aeroissement après la fécondation, comme dans les Pulsatilles, les Clématites, la benoite, etc.

\section{§3. Du stigmate.}

Le stigmate ( stigma) est eette partie du pistil ordinairement glaudulaire, placée au sommet de l'ovaire ou du style et qui est destinée à recevoir l'impression de la substance fécondante. Sa surfaee est en général inégale et plus ou moins visqueuse.

Le stigmate considéré sous le rapport anatomique se compose d'utrieules allongés, convergens de la surfaee du stigmate vers le style, lâchement unis les uns aux autres par une matière mucilagineuse. Ces utricules sont généralement nus, rarement ils sont recouverts par une membrane fort minee et transparente.

Le nombre des stigmates est déterminé par celui des styles et des divisions du style. En effet, il y a toujours autant de stigmates que de styles distinets ou de divisions manifestes dans le style.

Le stigmate est sessile, c'est-à-dire inmédiatement attaehé au sommet de l'ovaire, quand le style manque, comme dans le pavot, la tulipe.

Il n'y a qu'un seul stigmate dans les Crueifères, les Légumineuses, les Primulaeées, ete.

Il y en a deux dans les Ombellifẹres et un grand nombre de Graminées. 
On cn trouve trois dans les Iridées, les Silene, la rhubarbe, les Rumex, ete.

Il y en a cinq dans le lin; six et'même un nombre plus eonsidérable dans beauecup d'autres plantes, telle que la mauve.

Le stigmate est le plus souvent terminal (stigma terminale), e'est-à-dire situé au sommet du style ou de l'ovaire, eomme dans le lis, le pavot, ete. (Voyez pl. 6, fig. I, 3. )

Il est latéral ( stigma laterale), quand il oceupe les eôtés du style ou de l'ovaire, lorsque le style n'existe pas, eomme dans les Renoneulaeées, le platane, etc.

Selon la substance qui le eonstitue, il est charnu ( stigma carnosum), quand il est épais, ferme et sueeulent, eomme eelui du lis.

Glandulaire (stigma glandulare), quand il est évidemment formé de petites glandes plus ou moins rapprochées.

Membraneux (stigma membranaceum)', quand il est aplati et minee.

Pétaloïde, quand il est minee, membraneux et eoloré à la manière des pétales, eomme dans les iris, ete.

Suivant sa forme, le stigmatc peut être globuleux ou capité ( globulosum, capitatum), arrondi en forme de petite tête : la primevère (Primula veris), la belladone (Atropa belladona.), la belle-de-nuit (Nyctago hortensis).

Hémisphérique (stigma hemisphericum), présentant la forme d'une demi-sphère, eomme dans la jusquiame jaune (Hyosciamus aureus).

Discoïde ( stigma discoïdeum), aplati, large et en forme de bouelier, eomme dans le pavot, le coquelieot, ete.

Claviforme ou en massue (stigma clavatum), dans le Jasione montana, ete.

Gapillaire ou fliforme ( stigma capilla re, filiforme) ? 
grêle ct très-allongé, comme dans le maïs ou blé de Turquie.

Linéaire ( stigma lineare), étroit et allongé, comme dans les campanules et beaueoup de Caryophyllées.

Trigone (stigma trigonum), ayant la for.ne d'un prisme à trois faees, comme dans la tulipe sauvage ( $\boldsymbol{T} u$ lipa sylvestris).

Trilobé (stigma trilobum), formé de trois lobes arrondis, eomme dans le lis. (Voy.pl. 6, fig. I.)

Etoilé (stigma stellatum), plane et déeoupé en lobes à la manière d'une étoile, eomme dans les Érieinées, la pyrole, ete.

Ombiliqué ( stigma umbilicatum), offrant dans son centre une dépression plus ou moins profonde, comme dans le lis, la Viola rothomagensis, ete.

Sémilunéou en croissant (stigma semilunatum), comme dans la fumeterre jaune (Corydalis lutea.).

De même que le style, le stigmate peut être simple êt indivis, eomme dans la bourraehe (Borrago officinalis), la primevère, etc.

Bifide ( stigma bifidum), partagé en deux divisions étroites, eomme dans la sauge, et le plus grand nombre des Labiées, des Synanthérées, ete.

Trifide (stigma trifidum), dans la camélée (Cneorum tricoccum), les nareisses, ete.

Quadrifide ( stigma quadrifidum) dans la dentelaire (Plumbago europara), ete.

Multifide (stigma multifidum), quand le nombre de ses divisions est plus considérable.

Il est bilamellé ( stigna bilanellatum), formé de deux lames mobiles l'une sur l'autre, dans le Mimulus. (Voy. pl. 6, fig. 3. )

Suivant sa direction, on dit du stigmate qu'il est:

Dressé ( stignıa erectum), lorsqu'il est allongé et dirigé suivant l'axe de la fleur. 
Oblique (stigma obliquum), quand il se dirige obliquement par rapport à l'axe de la fleur.

Tors, roulé en tire-boure, comme dans la Nigella hispanica, etc.

La superfieie du stigmate est tautôt glabre, țantôt veloutée, comme dans le Chelidonium Glaucium, le Mimulus aurcantiacus, etc. Elle est pubescente dans le platane.

Le stigmate est plumeux (stigma plumosum), quand il est filiforme, et que de chaque eôté il offre une rangée de poils disposés comme les barbes d'une plume; exemple, beaueoup de Graminées.

Pénicelliforme (stigma penicelliforme), ou en forme de pinceau, quand les poils sont rassemblés par petites touffes ou bouquets, et eonstituent des espèces de houppes ou de pinceaux, comme dans le Triglochinmaritimum, etc.

Nous venons d'examiner et de faire comnaître les organes de la floraison, savoir : le pistil, les étamines, et les enveloppes florales. Nous avons remarqué que l'essence de la fleur réside uniquement dans la présenee des organes sexuels, et que le calice et la eorolle ne doivent être eonsidérés que eomme purement accessoires, e'est-à-dire servant seulement à favoriser l'exereice des fonctions que la nature a eonfiées à la fleur, mais n'y concourant qu'indirectement. Aussi les voit-on manquer assez fréquemment, sans que leur absence paraisse avoir aucune influenee sur les phénomènes et l'action réeiproque des organes sexuels.

Les enveloppes florales semblent done avoir pour principal usage de protéger les organcs de la génération jusqu'à leur parfait accroissement, c'est-à-dire jusqu'à l'époque où ils sont propres à la fécondation. 
Avant d'exposer les phénomènes de eette importante fonetion, revenons encore à quelques eonsidérations générales sur la fleur.

On a donné le nom d'anthèse à l'ensemble ides phénomènes qui se manifestent au moment où toutes les parties d'une fleur, ayant aequis leur entier développement, s'ouvrent, s'éeartent et s'épanouissent.

Toutes les plantes ne fleurissent pas à la même époque de l'année. Il existe à eet égard des différenees extrêmement remarquables, qui tiennent à la nature même de la plante, à l'influenee plus ou moins vive du ealorique et de la lumière, et enfin à la position géographique du végétal.

Les fleurs sont un des plus beaux ornemens de la nature. Si elles s'étaient montrées toutes dans la mêmesaison et à la même époque, elles eussent disparu trop tôt, et les végétaux seraient restés trop long-temps sans parure.

L'hiver même, malgré ses frimas, voit éelore des fleurs. Les Galanthus nivalis, les Leucoium, les hellébores, les Dapline, poussent et développent leurs fleurs quand la terre est eneore couverte de neige. Mais ees exemples ne sont en quelque sorte que des exeeptions. Le froid, en effet, paraît s'opposer au développement et à l'épanouissement des fleurs, tandis qu'une ehaleur douee et modérée les favorise et les entretient. Aussi voyons-nous régner en quelque sorte un printemps perpétuel, ct la terre se couvrir toujours de fleurs nouvelles, dans les pays où la température se maintient toute l'année dans un terme moyen.

Danis nos elimats tempérés, c'est atr printemps, quand une ehaleur donee et vivifiante a remplaeć les rigueurs de l'hiver, qu'éeartant insensiblement leurs enveloppes, les fleurs se montrent et s'épanouissent à nos yeux. Les mois de mai et de juin, dans nos elimats, sont ceux qui voient éelore le plus de fleurs.

Suivant la saison durant laquelle elles développent leurs 
fleurs, les plantes ont été distinguées en quatre elasses, savoir en :

I'Printanières (plantce vernales, verna), cclles qui fleurissent pendant les mois de mars, avril et mai : telles sont les violettes, les primevères, etc.

$2^{\circ}$ Estivales (plantce cestivales), celles qui fleurissent depuis le mois de juin jusqu'à la fin d'août : la plupart des plantes sont dans ce cas.

$3^{\circ}$ Automnales (plantce autumnales), celles qui poussent et développent leurs fleurs depuis le mois de septembre jusqu'en décembre. 'Tels sont beaueoup d'Aster, le colehique (Colchicum autumnale), le Chrysanthemum indicum, etc.

$4^{\circ}$ Hibernales ( $p l$. hibernales, hibernce), toutes celles qui fleurissent depuis le milieu de décembre environ jusqu'à la fin de février. Telles sont un grand nombre de Mousses, de Jungermanes, le Galanthus nivalis, l'Hellebor'us niger, etc.

C'est d'après la eonsidération de l'époque à laquelle les differentes plantes produisent leurs fleurs que Linnæus a établi son Calendrier de Flore. En effet, il y a un grand nombre de végétaux dont les fleurs paraissent toujours à la même époque de l'année, et d'une manière réglée. Ainsi, sous le climat de Paris, l'hellébore noir fleurit en janvier; le coudrier, le Daphne mezereum en février; l'amandier, le pêcher, l'abricotier, cn mars; les poiriers, les tulipes, les jacinthes, en avril; le lilas, les pommiers en mai, ete.

Non-seulement les fleurs se montrent à des époques différentes de l'anncé, dans les divers végétaux, mais il cn est encore un grand nombre qui s'ouvrent et se ferment à des heures déterminées de la journée; quelques-unes même ne s'épanouissent que pendant la nuit. De là on distingue les fleurs en dinurnes et en nocturnes. Ces dernières sont bien moins nombreuses que les premières. Ainsi la belle- 
de-nuit (Nyclago hortensis) n'ouvre ses fleurs que quand le soleil s'est eaehé derrière l'horizon.

Certaines fleurs même ont l'habitude de s'ouvrir et de se fermer à des heures assez fixes de la journée pour pouvoir annoneer d'après elles à quelle heure à peu près on se troure. Linnæus, si ingénieux à saisir tous les points de vue intéressans sous lesquels on pouvait eonsidérer les fleurs, s'est servi de ees époques bien eonnues de l'épanouissement de quelques espèees pour former un tableau auquel il a donné le nom d'Horloge de Flore. Les plantes, en effet, y sont rangées suivant l'heure à laquelle leurs fleurs s'épanouissent.

Les différens météores atmosphériques paraissent avoir une influenee marquée sur les fleurs de certains végétaux. Ainsi le Calendula pluvialis ferme sa fleur quand le eiel se eouvre de nuages, ou qu'un orage menace d'éelater. Le Sonchus sibiricus, au eontraire, ne s'ouvre et ne s'épanouit que quand le temps est brumeux et l'atmosphère chargée de nuages.

La lumière plus ou moins vive du soleil parait être une des eauses qui agissent le plus effieaeement sur l'épanouissement des fleurs. En elfet, son absenee détermine dans les fleurs, eomme dans les feuilles des plantes de la famille des Légumineuses, une sorte de sommeil. Par des expérienees extrêmement ingénieuses, mon ami M. Bory de Saint-Vineent est parvenu à faire fleurir eertaines espèces d'Oxalis, dont les fleurs ne s'étaient jamais épanouies naturellement, en les éelairant vivement pendant la nuit, et réunissant sur elles les rayons lumineux au moyen d'une lentille.

La durée des fleurs présente encore des différenees trèsnotables. Quelques-unes s'épanouissent le matin, et sont fanées avant la fin de la journée; on leur a donné le nom d'éphémères. Tels sont la plupart des Cistes, le Tradescentia virginica, 'quelques Cactus, cte. D'autres, au 
contraire, brillent du même éclat pendant plusieurs jours, et souvent même pendant plusieurs scmaines.

Enfin il est quelques fleurs dont la couleur varie aux différentes époques de leur développement. Ainsi l'Hortensia commence par avoir des fleurs vertes; petit à petit elles prenment une bellc couleur rosc, qui, avant qu'clles ne soient ḷ entièrement fanécs, devicmuent d'une teinte bleue, plus ou moins intense.

\section{CHAPITRE X.}

DES NECTAIRES.

Sous la dénomination générale de nectaures (nectaria); Linnæus a désigné non-seulement les corps glanduleux que l'on observe dans certaines fleur's, où ils secrètent une humeur micllcuse et nectarée, mais encore toutes les partics de la fleur qui, présentant des formes irrégulières et insolites, lui semblaient ne point appartenir aux organes floraux proprement dits, c'est-à-dire ni au pistil, ni aux étamines, ni aux cnveloppes florales.

On conçoit faeilement combien l'extension considérable donnée à ce moť a dû jeter de vague sur sa véritable signification, à tel point, qu'il est tout-à-fait impossiblc de donner une définition rigoureuse du mot nectaire, tel que Linnæus l'a entendu. Quelques exemples viendront à l'appui de notre assertion.

Toutes les fois qu'un des organcs constituans de la fleur offrait quelque irrégularité dans sa forme, dans son développement, ou quclque altération de sa physionomie habituelle, Linnæus lui donnait le nom de nectaire. On. pense bien qu'il a dû imposer de ce nom unc foulc d'organes très-différens les uns des autres.

Ainsi, dans l'ancolic, Linnæus décrit cinq nectaires en 
forme d'éperons recourbés et pendans entre les cinq sépales; dans les Delphinium il en existe deux qui se prolongent en pointe à leur partic postéricure, et sont contenus dans l'éperon que l'on observe à la base du sépale supéricur; dans les lıcllébores on en trouve un grand nombre qui sont tubuleux et comme à deux lèvres. Or ces prétendus nectaires des hellébores, des aucolies, et en général de tous les autres genres de la famille des Renonculacées, ne sont rien autre chose que les pétales.

Dans la capueine, le nectaire est un éperon qui part de la base du calice; dans les linaires, ce nectaire ou éperon est un prolongement de la base de la corolle. Il en est de même dans la violette, la balsamine, etc.

Linnæus a aussi donné le nom de nectaires à des amas de glandes placés dans différentes parties de la fleur. Aussi a-t-il confondu sous ce nom les disques comme dans les Crucifères, les Ombellifères, les Rosacées, etc. Dans le lis, le nectaire esí sous la forme d'un sillon glanduleux placé à la base interne des divisions du caliee; dans les iris, e'est un bouquet de poils glanduleux qui règne sur le milieu des divisions externes du ealiec.

Dans les Graminées, le neetaire se compose de deux petites écailles de forme très-variée, siluées d'un côté de la base de l'ovairc. Ces deux écailles ou paléoles forment la glumelle, organe qui n'efícctue aucune sécrétion. Daus les Orchidées, on a appelé nectaire la division inférieure et interne du calice, que d'autres botanistes, et Limmæus lui-même, ont désigné sous le nom de labelle.

Nous pourrions encore multiplier le nombre des exemples de genres où l'on a fait mention de nectaires. Mais ceux que nous avons cités suffisent pour faire voir combien ce mot est vague et peu défini dans la langue botanique, puisqu'on l'a appliqué tour à tour à des pétales, à des calices, à des étamines, à des pistils avortés et difformes, à des disques lıypogynes, périgynes et épigynes. 
Si l'on voulait eonserver cette expression de nectaire, nous pensons qu'il faudrait exclusivement la réserver pour les amas de glandes situées sur les différentes parties de la plante, et destinées à sécréter un liquide mielleux et neetaré, en ayant soin toutefois de ne pas confondre ee eorps avec les différentes espèees de disque, qui ne sont jamais des organes sécréteurs. Par ee moyen on ferait cesser le vague et la eonfusion que ce mot entraîne avec lui, et on le reudrait à sa véritable signifieation.

Nous avons dit, en commençant l'étude des organes floraux, que la fleur la plus complète n'était que la réunion de quatre verticilles de feuilles diversement modifiées. C'est un véritable bourgeon, mais qui, au lieu de donner naissance à un scion, a ses mérithalles tellement rapprochés les uns des autres, que les diverses parties qui composent ce bourgeon semblent naître d'un seul et même point qu'on a nommé réceptacle. Développons ici eette idée. Et d'abord nous croyons inutile de faire observer que le nombre des verticilles floraux varie suivant que la fleur est plus ou moins eomplète. Ainsi dans une fleur purement femelle, privée d'enveloppes florales, il n'y aura qu'un seul verticille; il y en aura deux dans une fleur hermaphrodite sans périanthe; trois dans celle à périanthe. simple, et quatre dans la fleur eomplète, c'est-à-dire celle qui, avec un périan the double, réunit des étamines et un ou plusieurs pistils. Chaeun de ces verticilles, avonsnous dit, est eomposé d'un nombre variable de feuilles diversement modifiées. Cette nature foliacée des parties constituantes de la fleur est d'abord très-facile à prouver pour le calice. En eflet les sépales ont eu général l'aspeet et la structure des véritables feuilles; ils sont généralement verts, pareourus de nervures saillantes dans lesquelles se trouvent des vaisseaux en spirale. Quand toutes 
les feuilles du ver tieille restent distinctes lesunes des autres, le caliee est dit poly-sépale: mais ces folioles peuvent se souder plus ou moins entre elles, et dès lors le ealice est dit monosépale ou gamosépale. La corolle est de même formée par un vertieille de feuilles plus intérieur que le caliee, et qui, pour cette raison, est déjà plus altéré dans sa nature; néanmoins il est encore extrèmement faeile de reeonnaître dåns les pétales d'un grand nombre de fleurs la même strueture que dans le caliee, avee quelques modifieations assez importantes. C'est ainsi par exemple que les trachées et les stomates qui existent dans le ealiee aussi bien que dans les autres feuilles proprement dites, manquent tout-à-fait daus la eorolle. Les folioles qui forment le vertieille eorollin peuvent rester distinetes les unes des autres ou se souder entre elles; de là les expressions de corolle polypétale et de corolle monopétale ou gamopétale. Les étamines forment le troisième verticille de la fleur. Leur analogie avee les pétales est très-grande, puisque l'on voit fréquemment les filets staminaux s'élargir en pétales, eomme par exemple dans toutes les fleurs qui doublent. Ainsi le filet d'une étamine peut done être eonsidéré eomme un pétale réduit à sa nervure médiane. Quant à l'anthère, c'est une feuille dont les bords se recourbent et se roulent vers la nervure médiane, et qui forment ainsi deux espèces de petits saes remplis d'un tissu cellulaire, dont les vésieules finissent par se séparer les unes des autres et forment le pollen. Le pistil peut également être considéré comme le résultat d'une ou de plusieurs feuilles verticillées. Quand il est uniloculaire et que les ovules qu'il renferme ne sont attaehés qu’à ux seul point de son intérieur, il est formé par úne scule feuille dont les bords convergent l'un vers l'autre, et se soudent pour constituer la cavité ovarienne. Quand au contraire l'ovaire est à plusieurs loges, ou mème quand il est à une seule loge, mais que les ovules sont attachés à 
plusieurs trophospermes pariétaux, dès lors il se compose d'autant de feuilles qu'il y a de loges ou de valves. Dans le premier cas, cclui de la plurilocularité, les bords des feuilles ont convergé vers l'axe de la fleur, et en se soudant latéralement entre elles par une partie de leur face externe, elles ont constitué les cloisons; dans le cas ou l'ovaire est uniloculaire, les feuilles ovariennes se sont soudées entre elles dans tout leur contour. Enfin, les ovules eux-mèmes doivent être considérés comme des espèces de petits bourgeons composés de plusieurs feuilles diversement modifiées.

Que l'on ne croie pas que la théorie que nous venons de présenter ici fort en abrégé, sur la naturc de la fleur et des parties qui la composent, soit une de ces idées spéculatives dont on embarrasse trop souvent l'étude des sciences. L'observation de la nature lui sert de base, et il n'est pas rare de voir ccrtaines fleurs, que l'on confond sous le nom de monstruosités, offrir d'une manière plus ou moins complèté les diverses parties de la fleur dans leur état normal et primitif, c'est-à-dire offrant l'aspect et la structure des véritables feuilles. Il n'est aucun botapiste qui n'ait été plusieurs fois à même d'observer un semblable phénomène. Pour n'en citer qu'unexemple trèsfrappant, nous dirons ici que nous avons eu en notre possẹsion une fleur de capucine (Tropceolum majus) que M. Du Petit-Thouars avait eu la bonté de nous communiquer, et dans laquelle le calice, la corolle, les étamines, le pistil et les ovules étaient sous la forme de feuilles, présentant la position naturelle et respective, des diverses parties constituantes de la fleur. Un phénomène semblable s'observe aussi dans plusieurs Crucifères, et entre autres dans le Turritis glabra.

Ainsi on peut donc dire que la fleur est un véritable bourgeon terminal, composé d'un nombre variable de yerticilles de feuilles diversement modifiées. 


\section{CHAPITRE XI.}

\section{DE LA FECONDATION.}

LA découverte de l'organe mâle et de l'organe femelle dans les végétaux a ouvert un nouveau champ à l'observation, en faisant étudier les phénomènes de l'aetion qu'ils exereent l'un sur l'autre. Ce n'est que depuis eette époque que l'on a bien connu le méeanisme de la féeondation. Cependant remarquons iei que les grandes vérités utiles à l'homme ont de tout temps été pressenties, en quelque sorte, par un instinet partieulier, par eeux mếme qui n'auraient pu en donner aueune explieation. Ainsi, quoique la déeouverte des sexes dans les végétaux ne'remonte point à plus de deux sièeles, cepcndant, de temps immémorial, les habitans de l'Arabie avaient remarqué que, pour que le datticr et le pistaehier pussent fruetifier, il était néeessaire qu'ils se trouvassent.rapprochés des individus sur lesquels ils n'avaient jamais vu des fruits. Aussi allaient-ils souvent ehcreher à de grandes distanees des rameaux de fleurs mâles pour les seeouer sur lesfleurs femelles, qui alors se convertissaient en fruits parfaits. Mais ils ignoraient entièrement la cause de ees phénomènes, n'ayant aueune idée de la présenec des deux sexes dans les végétaux.

Jusqu'en ees derniers temps on ne eomaissait pas plus le mécanisme de la féeonda tion dans les plantes que dans les animaux. Cependant on savait quel'organe femelle cst fécondé; que les ovules ou rudimens des graines renfermés dans l'intérieur de l'ovaire deviennent aptes à se développer et à reproduire plus tard des individus parfaitement semblables, toutes les fois que le pollen, renfermé dans les loges de l'étamine, a exereé son influence sur le stigmate. Mais on ignorait entièrement de quclle naturc 
cst l'influence que le pollen exerce sur le stigmate. Les recherches récentes de plusieurs observateurs, et en partieulier celles d'Amici et de M. Brongniart, ont jeté une vive lumière sur cette importante question; et fait voir que dans les plantes la fécondation paraît avoir le même mécanisme et la même cause que dans les animaux.

Iei, eomme dans ses autres ouvrages, nous avons lieu d'admirer la prévoyance de la nature et la perfection qu'elle sait domner aux instrumens qu'elle emploie. Les animaux, doués de la faculté de se mouvoir, pouvant se porter à volónté d'un lieu dans un autre, ont en général les organes de la génération séparés sur deux individus. Le mâle, à des époques déterminées, exeité par un sentiment intérieur, reeherehe sa femelle et s'en rapproche.

Les végétaux, au contraire, privés de eette faculté locomotriee, attachés irrévocablement au lieu qui les a vus naître, devant y croître et y mourir, ont en général les deux organes sexuels réunis, non-seulement sur le même individu, mais le plus souvent encore dans la même fleur. Aussi l'hermaphroditisme est-il très-commun dans les végétaux.

Cependant, il en est quelques-uns qui, au premier eoup d'œil, sembleraient ne pas se trouver dans des circonstances aussi favorables, et dans lesquels la fécondation paraîtrait avoir été abandonnée par la nature aux ehances du hasard. On voit que je veux parler des végétaux monoïques et diö̈ques. Ici, en effet, les deux organes sexuels sont éloignés l'un de l'autré, et souvent à des distances considérables. Mais admirons encorëici la prévoyance de la nature au lieu de l'accuser. Les animaux ayant la substanee fécondanteliquicle, l'organe mâle doit agir direetement sur l'organe femelle pour pouvoir le féconder. Si, dans les végétaux, ectte substance eût été de même nature que dans les animaux, nul doute que la fécondation n'eût éprouvé les plus grands obstacles dans les plantes monoïques et dioïques. Mais chez eux le pollen cst sous forme d'une 
poussière dont les molécules, légères et presque imperceptibles, sont transportécs, par l'air atmosphérique et les. vents, à des distanecs souvent inconeevables.

Remarquons cneore que le plus souvent, dans les plantes monoïques, les fleurs mâles sont situées vers la partie supérieurc du végétal, en sortc que lc pollcn, en s'échappant des loges de l'anthère, tombe naturellement et par son propre poids sur les flcurs femclles placécs au-dessous des premières.

Lcs fleurs hermaphrodites sont, sans contredit, celles dans lesquclles toutes les eireonstances aeecssoires sont les plus favorables à la féeondation. Les deux organes sexuels, cn effct, sc trouvent réunis dans la même fleur. Cettc fonetion commenee à l'instant où les loges de l'anthère s'ouvrent pour mettre le pollen cn liberté. Il est des plantes dans lesquelles la déhiseenee des anthères, et par eonséquent la féeondation, s'opèrc avant lc parfait épanouissement de la fleur. Mais, dans le plus grand nombre des végétaux, ee phénomène n'a lieu qu'après quc les cnveloppes florales se sont ouvertes et épanouies. Dans certaines fleurs hermaphrodites, la longucur ou la brièveté des étamincs, par rapport au pistil, semblerait d'abord un obstaele à la féeondation. Mais, eomme le remarque ingénieuscment Linnæus, quand les étamines sont plus longues que lc pistil, lcs flcurss sont cn général dressécs. Elles sont au contrairc renversées dans eelles où les étamines sont plus eourtes que le pistil. Nous n'avons bas besoin de fairc remarquer combien unc scmblable disposition est favorable à l'aete de la féeondation. Quand les étamines sont aussi longues que les, pistils, les fleurs sont indistinctement dressées ou pendantes.

Pour favoriser l'émission du pollcn et le mettre en contact avce le stigmate, les organes sexuels d'un grand nombre de plantes exéeutent des mouvemens très-scnsiblcs. A l'époque de la fécondation, les huit ou dix étamines 
qui eomposent les fleurs de la rue (Ruta graveolens) se redressent alteruativement vers le stigmate, y déposent une partie de leuripollen, et se déjettent ensuite en dehors.

Les étamines du Sparmannia africana, de l'épine-vinette, lor'squ'on les irrite avee la pointe d'une aiguille, se resserrent et se rapprochent les unes contre les autres.

Dans plusieurs genres de la famille des Urticées, dans la pariétaire, le mûrier à papier, ete., les étamines sont infléehies vers le centre de la fleur et au dessous du stigmate. A une certaine époque, elles se redressent avec élasticité, comme autant de ressorts, et laneent leur pollen. sur l'organe femelle.

Dans le genre Kalmia, les dix '-'mines sont situées horizontalement au fond de la fleur, et leurs anthères sont renfermées dans autant de petites fossettes qu'on aperȩoit à la base de la corolle. Pour opérer la féconda tion, ehacume des étamines se eourbe légèrement sur ellemême, afin de dégager son anthère de la petite fossette qui la eontient. Elle se redresse alors au-dessus du pistil, et verse sur lui son pollen.

Les organes femelles de eertaines plantes paraissent également doués de mouvemens qui dépendent d'une irritabilité plus développée pendant la fécondation.

Ainsi le stigmate de la tulipe et de plusieurs autres Liliacées se gonfle et paraît plus humide à cette époque.

Les deux lames qui forment le stigmate du Mimulus se rapproehent et se resserrent toutes les fois qu'une petite masse de pollen ou un eorps étranger queleonque vient à les toueher.

Il paraît même, d'après les observations de MM. de Lamarek et Bory Saint-Vineent, que plusieurs plantes développent àeette époque une ehaleur extrêmement manifeste; ainsi, dans l'Arum italicum et quelques autres plantes de la même famille, le spadice qui supporte les 
fleurs dégage une assez grande quantité de ealorique pour qu'elle soit apprćeiable à la main qui le touche.

Un grand nombre de plantes aquatiques, télles que les Nymphaca, les Villarsia, les Menyanthes, cte., ont d'abord les boutons de leurs fleurs caehés sous l'eau; peu à peu on les voit se rapprocher de sa surfaee, s'y montrer, s'y ćpanouir, et, quand la fćeondation s'est općrée, redescendre au-dessous de l'eau pour y mûrir leurs fruits.

Mais eependant la fécondation peut s'opérer dans les plantes entic̀rement submergées. Ainsi M. Ramond a trouvé dans le fond d'un lae des Pyrćnées le Ranunculus aquatilis reeouvert de plusieurs pieds d'eau, et portant cependant des fleurs et des fruits parfaitement mưrs. La fécondation s'était done općrće au milieu du liquide. Mon ami, M. Batard, auteur de la Flore de Maine-et-Loire, eut oeeasion de retrouver la même plante dans une circonstanee analogue. Il fit la euricuse remarque que chaque fleur, ainsi submergée, contenait entre ses membranes et avant son ćpanouissement une eertaine quantité d'air, et que c'ćtait par l'intermède de ee fluide que la féeondation avait lieu. L'air qu'il trouva ainsi renfermé dans les enveloppes florales eneore closes, provenait évidemment de l'expiration végétale dont nous avons précédemment ćtudić les phénomenes.

Cette observation, dont l'exaetitude a ćté plusieurs fois vérifiée depuis eette ćpoque, nous explique parfaitemènt le mode de fécondation des plantes submergées, quand clles sont pourvues d'enveloppes florales. Mais il devient impossible d'en faircl'applieation aux végétaux dćpourvus de ealice et de corolle. Tels sont le Ruppia, le Zostera, le Zanichellia, et d'autres eneore, dont la féeondation s'opère, bịen que leurs fleurs soient entièrement plongées dans l'eau.

Mais quel est le mode d'aetion du pollen sur le stigmate? L'opinion la plus généralement répandue jusqu'à présent parmi les botanistes, c'était que chaque grain de 
pollen réprésente une sorte de petite vésicule remplie d'un liquide dans lequel existe une foule de pétits grains que l'on regarde eomme la véritable substance propre à la féeondation. Aussitôt que ces grains de pollen s'échappent des anthères, ils se fixent sur le stigmate, dont la surfaee est en général inégale, visqueuse ou eouverte de poils. Là ils se renflent, se gonflent, s'ouvrent; la liqueur qu'ils eontiennent se répand sur le stigmate, et la fécondation a lieu.

Les observations eurieuses de M. Adolphe Brongniart sur la génération des végétaux ont jeté un jour tout nouveau sur eette question. Lorsque les grains de pollen sont mis en eontact a vec la surfaee du stigmate, ils projettent leur appendice tubuleux. Celui-ci, quand la surface du stigmate est nue, s'insinue plus ou moins profondément entre les utrieules stigmatiques. Les gramules polliniques ne tardent pas à se réunir vers l'extrémité libre de l'appendice, qui se renfle et devient légèrement opaque. Alors le grain de pollen s'affaisse et se flétrit. Peu de temps après, l'extrémité de l'appendiee s'ouvre, et les gramules polliniques sont mis à nu et en contact avee la matière mucilagineuse dont nous avous déjà parlé, et qui unit entre eux les utrieules stigmatiques. Ils s'y montrent sous l'aspeet de petits amas qu'on voit suecessivement pénétrer à de plus grandes profondeurs en se dirigeant vers le style. Quand les utrieules stigmatiques sont reeouverts d'un épiderme, l'appendice tubuleux s'applique à la surface de cet épiderme, se soude avec lui par son extrémité. L'un et l'autre venant à s'ouvirir, les granules polliniques se trouvent également mis en contact avec la matière intercellulaire du stigmate.

Les granules spermatiques, ajoute M. Brongniart, pénètrent done dans les intervalles intereellulaires du stigmate; mais là ees granules ne trouvent aucun vaisseau partieulier pour les transporter', ainsi que l'avaient prétendu quelques auteurs. Linck pensait que cette transmission 
se faisait à travers les parois des cellulcs. M. Brongniart, au contraire, dit qu'elle a licu par les cspaces interccllulaires. Dans le potiron, dit-il, le tissu utriculaire qui unit le stigmate et les ovules ne montre pas de globules dans ses intervalles avant la fćcondation; mais lorsque celle-ci s'est opérée, on suit avec la plus grande évidence dans ce tissu jaune la traînće brunâtre des granules spermatiques, et on les voit parvenir jusqu'aux ovules; les cellules n'en renferment jamais, ils se montrent toujours ct partout dans leurs intervalles. Ce transport paraît avoir lieu par suite de l'hygroscopéité des granules. Parvenus par cette voie jusqu'à l'ovule, les granules polliniques pénètrent par l'ouverture qui existe à ses deux membranes jusqu'à l'amande, soit directement par cette ou verture, soit, comme le pense M. Brongniart, par un tube membraneux et délié, qui sortant de cette amande, vient s'appliquer sur le placenta, et y puise lcs granules fécondans pour les porter dans l'intérieur de l'ovule. Ce tube aboutit intérieurement au point où doit se former l'embryon, c'est-à-dire à la petite vésicule que Malpighi a nommé sac de l'amnios. Cette vcsicule cst en quelquc sorte le moule où se figure l'embryon. On voit après l'imprégnation s'y développer des granules opaques, souvent vcrts, qui finissent par constituer l'cmbryon. Le col au moyen duquel cette vésicule était fixée au sac de l'amande se retrécit, se rompt et forme la radicule de l'embryon.

Tclle est la théorie de la gćnération des végétaux, ainsi qu'elle résulte des observations de Needham, de Smith, d'Amici, de Robert Brown, et particulièrement de M. Adolphe Brongniart. On voit qu'clle offre beaucoup d'analogie avec le mêmephćnomc̀ne observé chez les animaux; surtout si l'on admet la théorie des animalcules spcrmatiques.

Cctte explication paraît conforme à la naturc; dans le plus grand nombre des cas. Mais il cst d'autres circonstances dans lesquelles les phénomènes de la fécondation ne 
s'opèrent pas de la mème manière. Dans les plantes qui vivent eonstamment submergées, il est évident que les grains polliniques ne viennent pas se fixer'et se rompre sur le stigmate; et eependant la féeondation n'en a pas moins lieu. La surface du stigmate d'un grand nombre de plantes est extrêmement lisse, nullement visqueuse ; celle du châtaignier est'dure et eoriaee : le pollen ne peut y adhérer. Dans un grand nombre d'Orehidées et d'Apoeynées, le pollen, au lieu d'offrir une matière pulvérulente, composée d'une multitude innombrable de moléeules fines et légères, forme une masse entièrement solide. L'anthère s'ouvre; la masse pollinique ne ehange nullement de place, reste parfaitement entière, et la féeondation s'opère. Or, daus ee eas, le pollen n'a pas quitté l'intérieur de l'anthère pour aller sur le stigmate verser son fluide fécondant. Par la déhiseenee de l'anthère, il s'est trouvé simplement en eontact avee l'air atmosphérique, et eependant la plante a été féeondée.

C'est pour donner une explieation de ees faits que plusieurs auteurs ont pensé que, dans les végétaux, la féeondation pouvait, dans quelques eirconstanees, s'opérer sans contaet immédiat du pollen avec le stigmate, et seulement par suite de l'influenee d'une sorte d'émanation ou d'aura pollinaris. Mais eette question reste encore tout-à-fait indéeise.

Dans les plantes monoïques et diö̈ques, malgré la séparation, et souvent l'éloignement des deux sexes, la fécondation n'en a pas moins lieu.

L'air, pour les plantes dioïques, est le véhieule qui se eharge de transporter, souvent à de grandes distanees, le pollen ou l'aura pollinaris qui doit les féeonder. Les insectes et les papillons, en volant de fleur en fleur, servent aussi à la transmission du pollen.

Dans les plantes dioïques, les Palmiers, par exemple, on peut opérer artificiellement la fécondation. Il existait 
depuis long-temps au jardin botanique de Berlin un individu femelledu Chamarops humilis, qui tous les ans fleurissait, mais ne donnait pas de fruits. Gleditseh fit venir de Carlsruhe, des panieules de fleurs mâles, les secoua sur les fleurs femelles, qui donnèrent des fruit parfaits. Cette expérienee fut répétée plusieurs fois.

Ce mode de fécondation artificiclle est mis en pratique, depuis un temps immémorial, en Égypte et dans les autres parties de l'Afrique où le dattier est eultivé en abondance. A l'époque où les fleur's s'épanouissent, on monte au sommet des individus femelles, et on secoue au-dessus des grappes de fleurs, des régimes de fleur's mâles qui y répandent leur pollen. M. Delile rapporte que pendant la eampagne d'Égypte, eette pratique n'ayant pu être mise en usage à cause des hostilités continuelles entre les deux parties, la récolte des dattiers manqua entièrement.

Linnæus même prétend que non-seulement on peut, par ce procédé, féconder artificiellement une seule fleur d'une plante, mais qu'il est même possible de ne féconder qu'une scule loge d'un ovaire multiloculaire, en ne mettant le pollen en contact qu'avee une des divisions du stigmate. Mais eependant il a été prouvé que, bien que le pollen ne touchâl qu'un seul des lobes d'un stigmate, toutes les loges de l'ovaire étaient également féeondées.

L'expérience a encore prouvé que la fécondation, dans les plantes dioïques, peut avoir lieu à des distanees souvent fort considérables. Nous possédons un grand nombre d'exemples avérés, propres à démontrer ce fait. On cultivait déjà depuis long-temps, au Jardin des Plantes de $\mathrm{Pa-}$ ris, deux pieds de pistachiers femelles qui, chaque annce, se chargeaient de fleurs, mais ne produisaient jamais de fruits. Quel fut l'étonnement du célèbre Bernard de Jussieu, quand, une année, il vit ees deux arberes nouer et mûrir parfaitement leurs fruits! Dès lors il coujectura qu'il devait exister dans Paris, ou aux environs, quelque individu 
mâle portant des fleurs. Il fit des reeherches à eet égard, et apprit qu'à la même époque, à la pépinière des Chartreux, près le Luxembourg, un pied de pistaehier mâle avait fleuri. Dans ee eas, eomme daus les préeédens, le pollen, porté par le vent, est venu, par-dessus les édifiees d'une partie de Paris, féeonder les individus femelles.

Le Vallisneria spiralis, plante dioïque, que j'ai eu oceasion d'observer abondamment dans le eanal de Languedoe et les ruisseaux des environs de Beaueaire, offre un phénomène des plus admirables à l'époque de la féeondation. Cette plante est attaehée au fond de l'eau et entièrement submergée. Les individus mâles et femelles naissent pêle-mêle. Les fleurs femelles, portées sur des pédoneules longs d'environ deux ou trois pieds, et roulés en spirale ou tire-bouehon,, se présentent à la surface de l'eau pour s'épanouir. Les fleurs mâles, au eontraire, sont renfermées plusieurs ensemble dans une spathe membraneuse portée sur un pédoneule très-eourt. Lorsque le temps de la féeondation arrive, elles font effort contre eette spathe, la déehirent, se détachent de leur support et de la plante à laquelle elles appartenaient, et viennent à la surfaee de l'eau s'épanouir et féconder les fleur's femelles. Bientôt eelles-ei, par le retrait des spirales qui les supportent, redeseendent au-dessous de l'eau, où leurs fruits parviennent à une parfaite maturité.

Mais, quelle que soit la manière dont s'est opérée la féeondation, elle annonce toujours son influenee par des phénomènes visibles et apparens. La fleur, fraîehe jusque alors, et ornée souvent des eouleurs les plus vives, ne tarde point à perdre son riant eoloris et son éelat passager. La eorolle se fane; les pétales se dessèehent et tombent. Les étamines, ayant rempli les fonetions pour lesquelles la nature les avait eréés, éprouvent la même dégradation. Le pistil reste bientôt seul au centre de la fleur. Le stigmate 
et le style étant devenus inutiles à la plante, tombent également. L'ovaire seul persiste, puisque e'est dans son sein que la nature a déposé, pour y croître et s'y perfectionner, les rudimens des générations futures.

C'est l'ovaire qui, par son développement, doit former le fruit. Il n'est pas rare de voir le caliee persister avee cet organe, et l'aecompagner jusqu'à son entière maturité. Or il est à remarquer que eette cireonstanee a lieu prineipalement quand le ealice est monosépale : si l'ovaire est infère ou pariétal, le ealiee alors persiste néeessairement, puisqu'il lui cst intimement uni.

Dans l'Alkékenge (Physalis Alkekengi), le calice survit à la fécondation, se colore en rouge, et forme une coque vésieuleuse, dans laquelle lc fruit se trouve eontenu. Dans les narcisses, lcs pommiers, les poiriers, en un mot, dans toutes les plantes à ovaire infère ou pariétal, le caliee persistant forme la paroi la plus extérieure du fruit.

Peu de temps après que la féeondation a eu lieu, l'ovaire eommcnce à s'aeeroître; les ovulcs qu'il renferme, d'abord d'une substance aqueuse, et en quelque sorte inorganique, acquièrent peu à peu plus de eonsistance; la partic qui doit constituer la grainc parfaite, e'est-à-dire l'embryon, se développe suceessivement; tous ses organcs se prononcent, et bientôt l'ovaire a acquis les earaetères propres à eonstituer un fruit.

Nous terminons ici touí ce qui a rapport à la fleur proprement dite, considérée dans son ensemble et dans les différentes parties qui la composent. Avant de passer au fruit, il nous reste à faire connaître un organe accessoire de la fleur, qui manque quelquefois, mais qui, lorśqu'il existe, joue le plus grand rôle dans la coordination des plantes en familles naturelles. Cet organe est le disque. Nous nous oeeuperons cnsuite de l'insertion, c'est-à-dire de la position respeetive des diverses parties de la fleur, et principalement des organes sexuels. 
Le disque est un corps eharnu, de nature glanduleuse, ordinairemcnt jaunâtre, plus rarement vcrt, placé soit sous l'ovaire, soit sur son sommet, soit sur' la paroi internc du ealiec.

On distingue le disque en hypogyne, périgyne ct épigyne. $I^{\circ}$ Le disquc hypogyne portc le nom de podogyne lor'squ'il forme un corps clia'nu, distinct du réeeptaclc, et qui élèvc l'ovaire au-dessus du' fond de la fleur, eomme dans la rue, et les autres plantcs de la famille des Rutaeées; celui de pleurogyne, quand il naît sous l'ovaire ot qu'il se redressc sur une de ses parties latérales, "comme, par excmplc, dans la pervcnehe: On l'appelle 'épipode, lorsqu'il cst formé de plusieurs tubereules qui naissent sur le support de l'ovaire. Cette variété de disque se remarque surtout dans les plantes de la famille des Crueifères. $2{ }^{\circ}$ Le disque périgyne est formé par une substanee eharnue plus ou moins épaisse, épanehée sur là paroi interne du ealiee, comme dans le ecrisier, l'amandier, et dans certaines espèces de Diosma qui s'éloignc ainsi des autres espèees du mêmc genre.

$3^{\circ}$ Le disque épigyne cst eelui que l'on obscrve sur le sommet de l'ovaire quand ec dernier est infère, c'est-àdire quand il est soudé par tous les points de sa surface externe avec le tube du ealiee, conmme dans les Onbellifères,' les Rubiacées, ete.

\section{DE . L'insertion.}

L'inscrtion des étamincs sc distingue en absolue et en relalive. La premic̀re s'entend dc la position des étamines, abstraction faite du pistil ; e'est ainsi que l'on dit : étamines insérécs à la corolle, au calice, etc. La. sceonde fait connaître la posilion des étamines ou de la corolle monopétale staminifere, rclativement au pistil. Ainsi l'on dit dans 
ee sens : étamines insérées sous l'ovaire, autour de l'ovaire ou sur l'ovaire.

On distingue trois espéces d'insertion qui portent les noms d'hypogynique, périgynique et épigynique.

L'insertion hypogynique est celle dans laquelle les étamines ou la eorolle monopétale portant les étamines sont insérées sous l'ovaire. Par exemple, dans les Crueifères, les Labićes, ete.

L’insertion périgynique est eelle qui se fait au ealiee, comme par exemple dans les Rosacées. Enfin dans l'insertion épigynique, qui a licu tontes les fois que l'ovaire est infère, les étamines, ou la corolle monopétale staminif̣ère sont inșérées sur le sommet de l'ovaire. Les Ombellifèreses, les Rubiacćes, ete. , en offrent des exemples.

Ia position dị disque détermine en général l'inscrtion. Ainsi, toutes les fois qu'il y a un disque hypogyne, l'insertion est hypogynique; clle est périgyniqque, quand le disque est périgyne. Enfin eile est épigynique, tontes les fois qu'il y a un disque épigyne sur le sommet de l'ovaire,

\section{SECTION DEUXIEME.}

DU FRUIT, OU DES ORGANES DE LA FRUCTIFICATION PROPREEMENT DITS.

La fécondation s'est općrée, les enveloppes florales se sont fanées et détruites, les étamines soṇt tombées, le stigmate et le style ont abandonné l'ovaire qui seul a reçu, par l'influenee de eette fonetion, une vie nouvelle qu'il doit parcourir. Cette nouvelle époque du végétal eommence depris l'instant ou l'ovaire a été féeondé, et finit à celui de la dissémination des graines. On lui a domé le nom de fructification.

Le fruit n'est done que l'oraire féeondé et accru. Il se compose essentiellement de deux parties; savoir : le péricarpe et la graine. 


\section{CHAPITRE PREMIER.}

\section{DU PERICARPE.}

Le péricarpe est eette partie d'un fruit mûr et parfait, formés par les parois mêmes de l'ovaire féeondé, et eontenant dans son intérieur une ou plusieurs graines. C'est lui qui détermine la forme du fruit.

Le périearpe existe eonstamment ; mais quelquefois i] est si minee ou tellement uni avee la graine, qu'on le distingue avee peine dans le fruit mûr. Dans ee eas, plusieurs auteurs, pensant qu'il n'existait pas, ont dit que les graines étaient nues, comme dans les Labiées, les Ombellifêres, les Synantherées, ete. Mais il est prouvé aujourd'hui qu'il n'y a pas de graines nues, et que le péricarpe ne manque jamais.

Le périearpe offre ordinairement sur un des points de sa surfaee extérieure, le plus souvent vers sa partie la plus élevéc, les restes du style ou du stigmate, lesquels indiquent le sommet organique du péricarpe, et par eonséquent du fruit.

Le périearpe est toujours formé de trois parties; savoir : $x^{\circ} d^{\prime}$ une membrane extérieure, minee, sorte d'épiderme qui détermine sa forme et le recouvre extérieurement: on l'appelle épiccrpe; $2^{\circ}$ d'une autre nombrane intérienre qui revêt sa eavité séminifềre, elle a reẹu le nom d'endocarpe; $3^{\circ}$ entre ees deux membranes se trouve une partie parenehymateuse et eharnue qu'on appelle sarcocarpe ou mésocurpe. Ces trois parties, réunies et soudées intimement, eonstituent le périearpe.

Lorsque l'ovaire est infèr'e, e'est-à-dire toutes les fois qu'il est soudé avee le tube du ealiee, l'épicarpe est formé par le tube même du calice, dont le parenchyme se con- 
fond avec celui du sarcocarpe. Dans ee eas il est toujours faeile de recomnaitre l'origine de l'épicarpe, ear à sa partie supérieure il doit offrir, à une distanee variable du point d'origine du style et du stigmate, un rebord plus ou moins saillant, formé par les restes du limbe calyeinal, qui s'est détruit après la féeondation.

Le sarcocarpe ou mésocarpe est la partieparenehymateuse dans laquelle se trouvent réunis tous les vaisseaux du fruit. Il est extrèmernent développé dans les fruits charnus, tels que les pèches, les pommes, les melons, les potirons, ete. En effet toute la eliair de ees fruits est formée par le sarcocarpe.

L'endocarpe, ou membrane pariétale interne du fruit, est celle qui tapisse sa cavité séminifère. Presque toujours il est minee et membraneux. Mais il arrive quelquefois qu'il est épaissi extérieurement par une portion plus ou moins grande du sarcocarpe.' Quand cette partie du sarcocarpe devient dure et osseuse, elle enveloppe la graine, et eonstitue ee qu'on appelle mie noix ou noyau, quand il n'y a qu'une scule graine dans le fruit, et des nucules, quand il y en a plusieurs.

Lorsque le péricarpe est see et mince, il senible au premier abord que le sarcocarpe n'existe point. Nul doute que si l'on devait toujours entendre, par ee mot, une partic épaisse, eharnue et suceulcnte, il ne manquât fort souvent. Mais le caractère propre et distinetif du sarcocarpe est d'ètre le eorps vraiment vasculaire du péricarpe, c'est-à-dire d'ètre formé par les vaisseaux qui nourrissent le fruit tout entier; or, comme le péricarpe en contient toujours, le sarcocarpe existe eonstamment; mais quelquefois il est réduit à une très-peiite épaisseur, lorsque le fruit, étant parvenu à sa parfaite maturité, s'est déjà desséché. Cependant si l'on examine le péricarpe arce attention, on verra, entre l'épicarpe et l'endocarpe, des vaisseaux rompus qui servaicint à les unir l'un à l'autre, 
PÉTICINPE.

et qui sont les vestiges du sarcocarpe. Car, eomine eette partie est toujours abreuvée de sues aqueux avant la maturitédu fruit, le fluide qư'elle renferme s'étant évaporé, elle semble, au premier abord, avoir disparu et ne plus exister.

La eavité intérieure du périearpe, ou eelle qui renferme les graines, peut être simple; dans ee eas, le périearpe est dit uniloculaire (pericarpium uniloculare) ou à une seule loge; eomme par exemple dans le pavot (Papaver somniferum). D'autres fois il y a un nombre plus ou moins considérable de loges ou eavités partielles; de là les noms de biloculaire, triloculaire, quinquéloculaire, multiloculaire, domnés au périearpe, suivant qu’il présente deux, trois, cinq, ou un grand nombre de loges distinetes.

Les logęs d'un périearpe sont séparées les unes des autres par autant de lames vertieales qui prennent le nom de cloisons (dissepimenta).

Toutes les véritables eloisons n'ont qu'une seule manière de se former; l'endocarpe se prolonge dans l'intérieur de la eavité périearpienne, sous forme de deux proeessus lamellcux, adossés l'un à l'autre, et sont réunis ensemble par un prolongement ordinairement fort minee du sarcocarpe. Tel est le mode de formation de toutes les cloisons vraies. Celles qui ne sont pas formées de eette manière, doivent être eonsidérées eomme de fausses cloisons.

Il arrive quelquefois, dans certaines cloisons, que la partie parenehymateuse du sarcocarpe, qui unit les deux feuillets de l'endocarpe, se dessèche; alors ees deux lames se dessoudent et s'éeartent sensiblement l'une de l'autre, en sorte qu'elles paraissent au premier eoup d'ocil augmenter le nombre des loges du púricarpe. Mais on reconnaîtra facilement ectte déstuion, en observanl que les deux feuillets de l'endoccirpe offrent un de leurs eôtés parsemć de vaisseẩux rompus.

Outre leur mode d'origine ei de formation; un autro 
caractère distiuetif des cloisons vraies, e'est qu'elles alternent eonstamment avee les stigmates ou leurs divisions.

Certains fruits, au contraire, présentent de fausses cloisons dans leur eavité intérieure. Tels sont eeux de quelques Crucifères, de beancoup de Cucurbitacées, du pavot, ete. On distinguera les fulusses cloisons des vraies, $I^{\circ}$ en ee qu'elles ne sont pas formées par une duplieature de l'endocarpe proprement dit; $2^{\circ}$ parce que le plus souvent elles répondent à ehaque stigmate ou à chaeune de ses divisions, au lieu de leur être alternes, eomme les véritables cloisons.

Les eloisons sont distinguées eneore en complètes et en incomplètes. Les premières sont eelles qui s'étendent intérieurement depuis le haut de la eavité du péricarpe jusqu'à sa base, sans nulle interruption. Lies seeondes, au contraire, ne sout pas eontinues de la base au sommet, en sorte que les deux loges voisines eommuniquent entre elles. Le $D a$ tura stramonium nous offre un excmple de ces deux espèces de cloisons réunies dans le même fruit. Si on le coupe transversalement, il oflie quatre loges, et par conséquent quatre cloisons. Mais de ces cloisons, deux seulement sont complètes; les denx autres n'atteignent pas le sommet de la eavité intérieure du péricarpe; elles ne s'élèvent que jusqu'aux deux tiers de sa hauteur, et laisscnt communiquer ensemble, par leur partie supérieure, les deux loges qu'elles séparent inférieurement.

Pour arriver facilement à reeomaitre et à dénommer avee exaetitude les différentes parties qui eomposent le péricarpe, et les distinguer de eelles qui apparticunent à la graine, il est très-important d'étaßlir la juste limite entre ees deux organes. Toute graine devant recevoir sa nourriture du périearp̉e, il suit de là néeessairement qu'elle doit communiquer avec lui par quelqu'un des points de sa surface. Ce point a été nommé hile ou ombilic par les botanistes. Le lille doit être considéré eomme 
la limite précise entre le péricarpe et la graine; c'est-àdire que toutes les parties qui se trouvent en dehors et andessus du hile appartiemnent au péricarpe, et qu'au contraire on doit regarder eommc faisant partie de la graine, toutes celles qui sont situées au-dessons du fille.

Les graines sont attaehées dans l'intérieur dủ péricarpè; sur un corps eharnu partieulier, de grandeur et de formë variables, auquel on donne le nom de trophośperine (I): Dans le point intérieur du péricarpe; où est attäché le trophospermie, l'endocarpe est toujours pereé, parce que le sarcocarpe, étant la seule partie vasculairé du péricarpe, ét pouvant seul fournir lés mátériaux néeèssaires à la nutrition de la graine, il faut que l'endocarpe offre une ouverture, pour laisser passer les vaisseaux qui arrivent à eet organe.

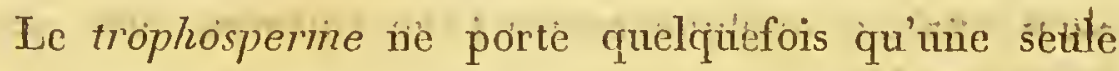
graine; d'autres fois il en porte un grand nombrè. Qtiarid sa surfacé oflre des pròlongenéniens mảnífesteś, dơnt chaeun soutient uné graine, on appelle eës prolongemens pódó-

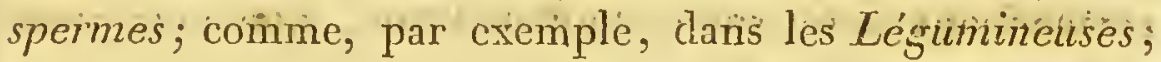
les Caryophyllées, les Portulacées, ete:

Le trophosperme, ou le podosperine, s'arrêtent ordinairement atitour du hile de la gräne. Lòrsquilils śe prolongent dì-delà de cê point, de mánière à recouvivir lâ grainè

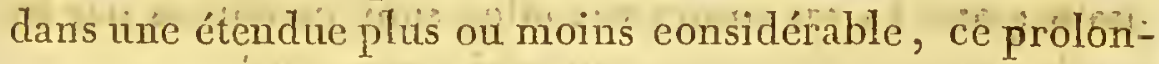
gement prend lic nom d'arille.

L'arille n'étant qu'une expansion du troplosperme, appartient, non point à la ğraine, eomme on le dit générálement, mais au périeàrpe.

- Examinons suecessivement les différentes parties internes du péricarpie; savoir : les cloisons, le trophospernié, et l'aillle.

(1) Placenita des auteurs. 


\section{$\S$ r. Des Cloisons.}

Nous avons déjà dit précédcmment qu'on a donné le nom de cloisons à des parties très-différentes les unes des autres; mais nous avons indiqué en même temps la manière dont les vraies eloisons sont formées. Toutes eelles donc qui ne présenteront point une semblable organisation, e'est-à-dire qui ne seront pas eonstituées par deux feuillets saillans de l'endocarpe, réunis par un prolongement du sarcocarpe, devront être considérées comme de fausses eloisons.

Les cloisons sont le plus souvent longitudinales, en sorte qu'elles s'étendent de la base vers le sommet de la eavité périearpienne.

Dans quelques eas très-rares, comme dans la casse (Cassia fistula), et quelques autres Légumineuses, elles sont transversales.

Les cloisons, eomme nous l'avons déjà dit, ont été distinguées encore en complètes et en incomplètes. Nous ne reviendrons point sur eelte distinetion, que nous avons suffisamment définie.

L'origine descloisons fausses est extrêmement variable. Tantôt, en effet, elles sont formées par uno saillie plus ou moins considérable dn trophosperme, eomme dans le pavot; tantôt, au contraire, elles sont produites par les bords rentrans des valves du péricarpe, etc.

\section{Du Trophosperme.}

Le trophosperme est eette partie du péricarpe à laquelle les graines sọnt attachées. Quelquefois il offre à sa surface un nombre plus ou moins grand de petits mamelons saillans, portant ehacun tme seule graine, et auxquels on donne le nom de podospermes.

Lorsqu'un péricarpe cst pluriloculaire, le trophosperme 
oceupe ordinairement son eentre, et alors on l'appelle central; daus ee eas, il est formé par la reneontre et la soudure des cloisons, et présente, dans l'angle rentrant de ehaque loge, une saillie plus ou moins considérable.

La forme du troplosperme est très-variée. Il est sphérique et presque globuleux: dans beaueoup de Primulacées, dans l'Anagallis arvensis, par exemple, ete.

Cylindrique, dans plusieurs Caryophyllées, tels que le Silene armeria, le Cerastium arvense, ete.

Trigone, dans le Polemonium cocrulcum.

Rayonnant (radiatum), eomme dans les Cucurbitacées, etc.

Suivant sa consistance, le trophosperme peut ètre :

Charnn; tel est eelui de la rne (Ruta graveolens), du Saxifraga granulata. Il est quelquefois coriace et dur, comme dans le pavot.

Subéreux, ou ayant la eonsistance du liége, eomme dans la stramoine (Datura stramonium), le tabac (Nicoliana tabacum), ete.

Suivant sa position, on ditcu'il est central ou axillaire, quand il oceupe le eentre ou l'axe du péricarpe. Par exemple, dans les Campanules, la digitale, ete.

Pariétal, attaché aux parois des loges du périearpe. Dans ee eas, il est appelé unilatéral, quand il est attaché d'un seul eôté du périearpe, eomme dans la plupart des Léguminenses et des Apocinées.

Bilatéral, attaché à deux des eôtés de la eavité intérieure du périearpe, eomme dans les Groseillers, ete.

Le podosperme offre aussi des formes très-variables; quelquefois il est grêle et filiforne, eomme dans la giroflée, le grosciller à maquereau, le frène, ete.

Unciforme, ou en forme de croehet, dans l'Acanthus mollis, ete.

D'autres fois, au contraire, il est plus épais et plus gros que la graine. 


\section{§. De l'A rille.}

L'arille, avons-nous dit, appartient essentiellement au péricarpe, puisqu'il n'est qu'un prolongement du trophosperine. C'est done à tort qu'un grand nombre de botanistes le eonsiderent eomme faisant partie de la graine, sur laquelle il est simplement appliqué sans y adhérer aueunemient, exeepté par le eontour du hile.

Peu de parties, dans leś végétáux, offrent autant de variétés dans leur forme et leur nature, que l'arille. Aussi, est-il très-difficile d'en donner une définition rigoureuse, et qui soit applieable à tous les cas.

Dans le muscadier (Myristica officinalis), l'arille forme une lame ehärnue, d'un rouge elair, découpée en lanières étroites et inégales : e'est eette partie qui est usitée en pharnaeie, et eonnue sous le nom de macis. Le Poly gala vulgaìis ä un arille trilobé, peu développé, formant une sorte de petite eouronie à la base de la graine. Daris le fusain ordinaire (Evonymus europceus), et le fusain à Jarges fenilles (Evonymus latifolins), l'arille, de couleur orangée, chveloppe et caehe la grainc de toutes partś ; dans le fusain à bois galeux (Evonymus verrucosus), il forme une cupule irrégulière, ouverte supérieurement.

D'après le petit nombre d'exemples que nous venous de eiter, on voit que eet organe est extrêmement variable, tant dans sa couleur que dans sa forme et sa eonsistanee. Mais son point d'origine étant le même dans tous les eas, il sera toujoirs faeile de le reeonnaitre, malgré les nombreuses formes sous lesquelles il peut se présenter.

Plusieurs parties ont été souvent prises pour des arilles. Ainsi, $\mathrm{r}^{\circ}$ la partie extérieure, manifestement charnue, du tégument propre de la graine, dans le jasmin, le Tabernomontana, ete.; $2^{\circ}$ l'endoearpe, eomme dans le café ( Coffcea arabica), les Rutacées, ete. 
Unc loi, jusqu'à présent rceonnue généralc, c'cst-àdire à laquelle il ne s'est point encore présenté d'exccption, c'est que l'arille ne se rencontre jamais dans des plantes dont la corolle cst monopétale. Lc Tabernomontana semblait cn quclque sorte contrcdirc cettc loi; mais, mieux examiné, son prétendu arille n'est quc la partie extćricurc du tćgument propre de sa graine, qui cst molle ct eharnue.

Tous venons d'ćtudier les parties constituiantes du péricarpc; savoir : les cloisons, les loges, lc trophosperme ct l'arillc; revenons maintcnant à d'autres considérations sur le péricarpe en général.

On distingue dans le péricarpe, commc dans l'ovảire, . I $^{\circ}$ sa base, ou lc point prar lequcl il est fixć au réccptacle ou au pédoncule; $2^{\circ}$ son. sommet, qui cst indiqué par la plaee qu'oecupait lc stylc ou lc stigmate sessile; $3^{\circ}$ enfin, son axe. Quelquefois cet axc est matćricl, et cxistc réellcment : on lui donne le nom de columelle. D'autres fois, au eontraire, il est fictif ct rationnel, c'cst-à-dire qu'il est représcnté par unc ligue imaginairc, dirigée de la basé vers lc sommet du péricarpe, qui passerait par son ećntrc.

La columelle formc une sorte dc petite colonne, sur laquellc s'appuient les différentes pièces du fruit, et qui persistc au centre du périearpe, quand colles-ei viennent à tomber : par exenplè, dans los Euphorbes, les Ombellifères, ctc.

- Les graines ćtant renfermécs dans le péricarpe, il faut, pour qu'à l'ćpoque de lcur maturitć elles puisscnt en sortir, que cclui-ci s'ouvre d'unc manière quclconquc. On donne le nom de délisscence à l'action par laçuclle un périearpe s'ouvre naturcllement. Ccpendant, il est dcs péricarpes qui ne s'ouvrent pas. On leur a donné le nom 
d'indéhiscens; tcls sont ceux des Synanthérées, des Labiées, des Graminćes, ete.

Parmi les péricarpes qui s'ouvrent naturellement à l'époque de la maturité, on distingue, $\mathrm{I}^{\circ}$ ecux qui sc rompent en pièces irrégulières, dont le nombre et la forme sont très-variables. On les appelle péricarpes ruptiles $2^{\circ}$ ceux qui ne s'ouvrent que par des trous pratiqués à leur partiesupéricure, comme dans les Antirrhinum; 30 eeux qui s'ouvrent à leur sommet par des dents d'abord rapprochées qui s'éeartent les uns des autres, telles sont beaucoup de Caryophyllées; $4^{\circ}$ enfin ceux qui sc partagent en un nombre déterminé de pièces distinctes ou panneaux qu'on appellc valves, sont les péricarpes vraiment déhiscens. Le nombre des valves d'un péricarpe est toujours annoneé par le nombre de sutures longitudinalcs, que l'on remarque sur sa surface extéricure. Les véritables valves sont toujours en nombre égal aux loges du périearpe. Ainsi, un fruit déhiscent, qui est quadriloculciire, sera également à quatre valves. Cependant il y a quelques exceptions. La eapsule de la violettc est à une seule loge et s'ouvre en trois valves.

Mais, dans quelques fruits, chacune des valves se partage en deux pièees, en sortc quc leur nombre parait double de eelui qui devrait naturellement exister.

Un péricarpe est appelć bivalve (pericarpium bivalve), quand il se partage de lui-même en deux valves égales et régulières, comme dans le lilas (Syringa vulgaris), lcs véroniques, ete.

Trivalve (pericarpium trivalve), celui qui s'ouvre en trois valves. Tels sont ecux de la tulipe, du lis, des violettes, ete.

Quadrivalse, ou à quatre valves (pericarpium quadrivalve), comme dans les épilóbes, la pomme épineuse.

Quinquésalve (pericarprum quinquevalve), cclui qui s'ouvre en cinq valves. 
Multivalve (pericarpium multivalve), quand il se partage en un nombre plus eonsidérable de valves ou segmens distincts.

La déhiscence valvaire peut se faire de différentes manières, relativement à la position respective des valves avec les cloisons. De là on a distingué trois espèces de délhiscences valvaires.

$\mathrm{x}^{\circ} \mathrm{Ou}$ bien eette déliscence se fait par le milieu des loges, c'est-à-dire entre les cloisons qui répondent alors à la partie moyenne des valves (valvis medio septiferis); onx l'appelle loculicide, comme dans la plupart des Éricinées.

$2^{\circ} \mathrm{D}$ 'autres fois la déhiscence a lieu vis-à-vis les cloisons, qu'elle partage le plus souvent en deux lames. On la nomme alors septicide, eomme, par exemple, dans les Serophularinées, les Rhodoracées, etc.

$3 \circ$ Enfin, elle a reçu le nom de déhiseence septifrage; quand la rupture a lieu vers la cloison, qui reste libre et entière au moment où les valves se séparent, eomme dans les Bignonia, le Calluna (Erica vulgaris).

Le plus souvent la déhiscence se fait par des sutures longitudinales; dans quelques eas cependant ees sutures sont transversales et les valves sont superposées. Cette espècè de fruit a reçu le nom de Pyxide; la jusquiame, le pourpier, le plantain, etc., en offrent des exemples. Lue périearpe, ou le fruit considéré dans son ensemble, est un des organes dont les formes sont les plus nombreuses et les plus variées. Ainsi, il est souvent sphéroüdal et arrondi, comme dans la pèehe, l'abricot, l'orange, etc.

Ové, comme celui d'un grand nombre de chênes, etc: Lenticulaire, c'est-à-dire approchant de la forme d'une lentille, comme dans un grand nombre d'Ombelliféres. Prismatique, c'est-à-dire ayant la forme d'un prisme à plusieurs faces, comme dans l'Oxalis.

Son sommet peut ètre aigu ou obtus; quelquefois le 
style persiste et forme sur lc fruit unc pointc plus ou moins remarquable. D'autres fois, c'cst le stigmatc qui acquiert un développement plus grand, comme dans la plupart des clématiles, et beaucoup d'anémoncs, où il forme des espèces d'appendices pluneux au sommet du fruit.

Le fruit peut être couronné par les dents du calice, Truand l'ovairc esı infèrc ou pariétal, comme dans la grenade ( Punica Granalum), la pomme, la poire, etc.

D'autres fois, il est surmonté par unc aigrctte (pappus), petitc touffe de poils soycux, qui doit être regardéc comme un véritable calice. C' cst ce quc l'on observe dans jresquc toutes les espèces de la nombreuse tribu des Synanthér 'ées. On tire de la forme et de la structure de l'aigr'etie de fort bons caractères génériques.

Ainsi, cette aigreıle peut ètre sessile (pappus sessilis), c'est-à-dirc immédiatement appliquée sur le sommet dc l'ovaire, sans le secours d'aucun corps intcrmédiaire, comme dans les genres Fieracium, Sonchus, Prenanthes, etc. (Voy. pl. 8, fig. I2.)

Daus d'autres genres, au contraire, cllc est portée sur une espèce de petit pivot ou support particulicr qu'on appelle stipes, et l'aigrette cst dite stipitée (pappus stipitatus), comme dans les genres Lacluca, Tragopogon, cte. (Voy.pl. 8, fig. 13.)

Les poits qui composent l'aigretle peurent être simples et non divisés; dans ec cas J'aigreite est dite simplement poilue (pappus pilosus), comme dans le Lacluca, lc Premanthes. (Toy. pl. 8, fig. 13.)

D'autres fois ils sont plumeux, c'cst-à-dirc ofliant sur lcurs partics latérales d'autres petits poils plus fins, plus déliés et plus courts, de manière à resscmbler aux barbes d'une plume. L'aigrette alors cst appelée plumeuse (pappus plumosus), comme dans les genies Jeontodon, Tragopogon, Picris, Cynara, etc. (Toy. pl. 8, fig. г a a.)

Dans les valérianes, l'aigrette, qui n’est manifestement 
que le limbe du caliee, est d'abord roulée en dedans de la fleur et se montre sous la forme d'un petit bourrelet circulaire à la partie supérieure de l'ovaire; mais quelque temps après la fécondatiou on voit ce calice se dérouler, s'allonger, et former une vérițable aigrette plurneuse.

Le pérịcarpe présente eneore assez souvent des espèees d'appendices membraneux en forme d'ailes, comme dans l'orme, les érables. (Voy. pl. 8, fig. 6.) D'après le nombre de ecs appendices, il est dit: diptère, triptère, télraptère, etc. Bcaucoup de genres de la famille des Sapindacées et des Acériuées offrent des exemples de cés différentes espèces de fruits.

D'autres fois il est couvert de poils longs et rudes, ressemblans à une sorte de fillasse, comme dans le Lontarus; ou même il est hérissé d'épines, comme le marronier d'Inde, la pomme épineuse (Datura stramonium), etc.

L'organisation du péricarpe et de la graine étant une des parties les plus difficiles de la botanique, afin de bien Ifaire eoucevoir les différens organes que nous venons de déicrire dans ce chapitre, nous allons faire l'analyse de quel1 ques fruits très-connus, et dénommer les différentes parties 'qui les composent; après quoi nous résumerons en peu de mots les objets que nous aurons suecessivement étudié.

Prenons le fruit du pécher (Amygdalus persica) pour exemple. (Voy. pl. 8, fig. 8.)

Le fruit étaut essenticllement composé de deux parties, ssayoir : du péricarpe et de la grainine, il s'agit d'abord de distinguer ces deux parties l'une de l'autre. Nous savons. que la graine est toujours eontenue dans l'intérieur du péricarpe; eherchons donc à la trouver au contre de cet. corgane. Si nous coupons une péche en deux, nous vcrious. son eentre occupé par une cavité ou loge, reufcrmant une scule graine, rarement denx. La graine une fois recounue, tout ce qui est en dehors d'elle appartient au péricarpe. Voyons à dénommer ses différentes parties. Nous trouyons 
d'abord, tout-à-fait à l'cxtéricur, unc pellicule mince; colorée, eouvertc d'un duvet très-court qu'on enlève facilcment : c'est l'épicarpe. La cavité intéricure du péricarpe est tapissćc par unc autre membranc lisse, intimement unie et eonfonduc avec la partic dure qui forme le noyau, c'est l'endocarpe. Toutc la partic épaisse, charnuc, parenehymatcuse, renferméc cntrc eettc dernière membrane et l'épicarpe, forme lc sarcocarpe. Mais à laquelle de 'ces trois partics appartient le noyau osscux qu'on trouve à l'intéricur? Est-ce, comme on l'a cru long-temps, un tégument propre de la grainc, un endocarpe épais et ligncux, ou bicn fait-il partic du sarcocarpe? Il nous scra très-facilc de résoudre ces qucstions. En cffet, examinons conımicnt s'est formće cette partie osscuse. Si nous prenons uncjeune pêche, long-temps avant l'époque de sa maturité, que nous la coupions en travers, nous n'éprouverons aucunc résistance; il n'y aura point encore de noyau solide. Or, à ecttc époque, les trois partics du péricarpe sont extrêmement distinctes les uncs des autres, et l'endocarpe est évidemment ici sous forme d'une simple membrane appliquée sur la face interne du sarcocarpe. Mais peu de temps après on voit la partic du sarcocarpe la plus voisine de ectte membrane intéricure devenir successivement plusblanche, plus scrrée, et passer graducllement par tous les degrés intermédiaires, avant d'acquérir la solidité osseuse qu'elle offre à l'époque de sa maturitć. Or, dans ce cas, quoique ecttc portion du sarcocarpe se soit intimement unic et confondue avec l'endocarpe, clle ne doit ccpendant ètre rapportéc en aucune manière à ce dernicr, mais bicn au sarcocarpe, puisque réellement ellc est formée par lui. Le noyau, ou la partic osseuse que l'on trouve au centre de la pêchic, est donc formé par l'endocarpe, auquel s'est jointc une portion ossifiéc du sarcocarpe. Ce que nous venons de dire de la pêche esı également applicable à l'abricot, la prune, la cerise, l'amande, etc., etc. 
PÉRICARPE.

Si nous prenons le fruit du pois ordinaire (Pisum sativum) (Voy. pl. 8, fig. 3), eonnu sous le nom de gousse, et que nous l'analysions, nous trouverons d'abord:

Que ee fruit est allongé et comprimé de manière à présenter deux bords tranehans, sur lesquels règnent deux sutures longitudinales; ee qui nous indique qu'il s'ouvrira à la parfaite maturité, en deux segmens ou valves; e'est done un périearpe bivalve. Si nous le eoupons longitudinalement, nous n'y verrons qu'une seule eavité intérieure, renfermant huit à dix graines, c'est-à-dire qu'il est uniloculaire, polysperme. Les graines sont toutes fixées, du côté de la suture supérieure, à une espèee de petit rebord épais, régnant tout le long de cette suture, et offrant un prolongement distinet pour ehaque graine. Tout ee qui est en dehors de la graine fait partie du péricarpe. Dénommons ces parties. 'Tout-à -fait à l'extérieur se trouve une membrane minee, très-adhérente à la partie sous-jacente; c'est l'épicarpe. La eavité intérieure est tapissée par une autre membrane, un peu moins intimement adhérente; c'est l'endocarpe. La partie eharnue, verte, vaseuleuse qui se trouve entre ees deux membranes, quoique peu épaisse, eonstitue le sarcocarpe. Le petit bourrelet longitudinal, qui descend le long de la suture, et auquel sont attaehées les graines, est le trophosperme. Chaque prolongement de ee eorps, partieulier à chaque graine, est un podosperme.

En résumé, nous voyons que le périearpe eșt eette partie du fruit qui forme les parois de la eavité simple ou multiple dans laquelle sont eontenues les graines; qu'il se eompose eonstamment de trois parties; savoir : $\mathrm{I}^{\circ}$ de l'épicarpe, ou membrane qui le reeouvre extérieurement; $2^{\circ}$ de l'endocarpe, ou membrane pariétale interne tapissant sa eavité intérieure; $3^{\circ}$ d'uṇe partie plus ou moins épaisse et charnue, quelquefois eependant mince et peu apparente, mais toujours vaseulaire, que 
que l'on nomme sarcocarpe ou mésocarpe : que souvent le péricarpe est partagé intéricurement par des cloisons en un nombre plus ou moins considérable de loges, nombre d'après lequel il est appelé biloculaire, quadriloculaire, multiloculaire, etc. Le point de la cavité péricarpienne, auquel sont attachées les graines, offre un renflement charnu plus ou moins développé, provenant du sareocarpe, qui a reçu le nom de trophosperme : on appelle, au contraire, podosperme chaque petit mamelon du trophosperme portant une sculc graine. Quand le trophosperme ou le podosperme recouvrent la graine de manière à l'embrasser dans une étenduc plus ou moins grande, ce prolongement particulier porte le nom d'arille.

Telles son toutes les partios qui entrent dans la composition du péricarpe. Etudions maintenant la graine.

\section{GH $\triangle$ PITRE II.}

DE LA GRAINE.

Nous venons de voir que le fruit est essentiellement for'mé de deux parties, le péricarpe et la graine.

La graine est ecte partic d'un fruit parfait, qui se trouve conteuuc dans la eavité intérieure du péricarpe, et qui renferme le corps qui doit reproduire un nouveau végétal. Il n’existe pas de graines nues propremeut dites, c'est-à-dire qui ne soicnt pas recouvertes par le péricarpe. Mais ce dernier est quelquefois si mince ou si adhérent à la graine, qu'on l'en distingue difficilement à l'époque de la maturité du fruit, parce qu’ils se sont soudés et confondus enscmble. Cependant ces deux parties ćtaient bicu distinetes dans l'ovaire après la fécondation. De là l'impéricuse nécessité d'étudier avec soin la stucture de l'ovaire, pour recommaitre celle que doit avoir le fruit. 
Ainsi, dans les Graminées, les Synanthérées; le péririearpe est très-minee et eollé intimement avec la graine dont il est très-diffieile de le distinguer. Il en est de mểme eneore dans beaueoup d'Ombellifères, ete.; tandis que si on les examine dans l'ovaire; cés deux parties sont fort distinetes l'une de l'autre.

Toute graine provient d'un ovule fécondé. Son cảraetère essentiel est de renfermer un eorps organisé, qui, mis dans des eireonstanees favorables, se développe et devient un être parfaitement semblable à eelui dont ìl a tiré son origine. Ge eorps est l'embryon. L'essence de la. graine eonsiste done daus l'embryon.

C'est à tort, selon nous, que l'on a domné le nom de graines aux eorpusenles reproduetifs des Fougères; des Mousses, des Champignons et de toutês les autres plantes agames. En effet, rien dans leur intérieur ne réssemble à un embryon. Il est vrai eependant qu'ils forment en se développant un végétal semblable en tout à eeluii dont ils proviennent. Mais il n'y a pas que l'embryon qui soit susceptible d'un pareil développement; 'les bourgeons des plantes vivaees, et surtout les bulbilles qui se développent sur différentes parties des végétaux, souvent mềne jusque i dans l'intérieur du périearpe, à la plaee des graines; peuvent également donner naissanee à un végétal eomplet. iOr personne n’a jamais été tenté, malgré eette grande : analogie de fonctions, de regarder les bulbilles et les bour: geons eomme de véritables graines: : les eorpuseules reproiductifs des agames, leur'étant parfaitement analogues, ne (doivent pas plus qu'eux porter le nom de graines.

La graine est formée de deux parties : $1^{\circ}$ de l'épispperme ou tégument propre; $2^{\circ}$ de l'amande, eontenue (dans l'épisperme (I).

(1) Telle est en effet la structure la plus générale de la graine fécondée è parvenue à sa maturité; mais cette structure s'éloigne beancoup de celle qui 
Nous étudierons séparément ces deux parties quand nous aurons parlé d'unè manière générale de la direction. et de la position des graines, relativement au péricarpe. Le point de la graine par lequel elle est fixée au péricàpe, se nomme l'ombilic ou le hile (hilus). Le hile est toujours marqué, sur le tégument propre, par un point ou espèce de cicatrice plus ou moins grande qui n'occupe jamais qu'une partie de sa surface, et au moyen. de laquelle les vaisseaux du trophosperme communiquaient avec ceux du tégument propre de la graine.

présente l'ovule avant l'imprégnation, et quc nous allons faire connaitre ici, d'après les observations curieuses qu'a publiées récemment notre célèbre ami M. Robert Brown. Avant la fécondation, l'ovule se compose de deux membranes et d'une amandc. La membrane extérieure on le testa présente quclquefois près du hile, d'autrefois dans un point plus ou moins éloigné; une petite ouverture ponctiforme, déjà apcrçe par quelques observateurs anciens, et à laquelle M. Turpin a donné le nom de micropyle. Cette ouverture n'a aucune communication directe avec les parois de l'ovaire. Selon M. R. Brown, elle indique la véritable base de l'ovule, et le point qui lui est opposć, son sommet, Les vaisseaux nourriciers da péricarpe qui arrivent à l'ovule par le hile, rampent dans l'ćpaisseur du testa jusque vers son sommet, où ils forment une sorte d'ćpanouissement, communiquant avec la membrane internc et qu'on nomme chalaze. Cette membrane interne a une direction opposée à celle du testa, c'est-it-dire qu'ellc s'insère par une base assez large au sommet de celui-ci, seul point par lequel ces deux membranes soient en communication l'une avec l'autre. Le sommet de la memhrane interne est aussi percé d'une petitc ouverture qui correspond exactement à celle de la base du testa. L'amande renfermée dans l'intérieur des deux tégumens dc l'ovulc, est un corps celluleux, ayant constamment la mĉme direction quc la membrane interne, c'est-à-dirc insérć à sa base, ou point opposć à son somunet perforé. Elle se compose de deux parties, l'une épaisse et celluleuse, c'est le chorion de Malpighi, l'autre interne, formant une sorte de petit sac celluleux, souvent rempli d'abord d'un fluide mucilan gineax, c'est l'amnios et sa liqueur. C'est dans ce sac intćrieur que l'enbryon commence à sc montrer. Sa radicule correspond toujours an somumet dc l'amande, c'est-à-dire à l'ouverture on base du tégument cxterne de l'ovule. L'endosperme qui accompagne souvent l'embryon, peut être formé soit par lc sac amniotique, soit par le chorion, l'amnios étant resorbé, soit par ces deux organes à la fois. 
Le centre du hile représente toujours la base de la graine. Son sommet est indiqué par lc point diamétralcment opposé au hilc.

Lorsqu'une graine est compriméé,, cellc de sés deux faces qui regarde l'axc du péricarpc portc lc nom dc face. proprement dite'; l'autre, qui cst tournéc du côté des parois du péricarpc, est appclée lc dos (dorsum). Le bord de la graine est représenté par lc point de jonction de la face et du dos.

Quand le hile est situć sur un des points du bord de la graine, elle est dite comprimée (semeñ compressum.). On dit, au contraire, qu'cllc est déprimée (semen depressum), quand le hile sc trouve sur sa facc ou son dos. Cette distinction est très-importantc à fairc.

La position des graines et surtout leur direction rclativement à l'axe du péricarpe cst importante à considércr, lorsque ces graines sont en nombrc déterminé. Elles fournissent alors d'excellcns caractères dans la coordination naturelle des plantes.

Ainsi toutc graine fixée par son cxtrémité mềme au fond du péricarpe ou d'une dc ses loges, quand il cst multiloculaire, et dont elle suit plus ou moins bicn la direction, est dite dressée (semen erectum), comme dans toutes les Synanthérées, ctc.

On l'appclle au contraire renversée. (semen inversum), quand clle est attachéc dc la mêmc manière au sommet de la loge du péricarpe; par exemple, dans lcs Dipsacées. Dans ces deux cas, le trophospcrme occupe la base ou le sommet de la loge.

Si, au contraire, le trophospcrme étant axillairc ou pariétal, la grainc dirige son sommct (ou la partie dia. métralement opposée à son point d'attachc) vers la partie supéricure de la $\log \mathrm{c}$, cllc est appcléc ascendante ( semen ascendens), comme daus la pomme, la poire, etc. (Voy. pl. 8, fig. 9.) 
On la dit, par opposition, suspenduie (s. appensum), quand son sommet regarde la base de la loge, comme dans les Jasminées, beaucoup d'A poeyniées, ètc.

On donne à la graine le nom de péritrope ( $s$. peritror puim ), quand son axe rationel, ou la ligne qui est censée passer par sa base et son sommet, est transversale relativement aux parois du périearpe.

\section{§. De l'Épisperme.}

L'épisperme; ou tégument propre de la graine, est presque toujours simple et uniqué antour de l'amandë: Cependant quelquefóis, eomme il présente une épaisseur assez notable, et qu'il est légèrement eharnu à son inté rieur, sa paroi interne se détaehe et s'isole, en sorte qu'il paraît eomposé de dcux tuniques, l'une extérieure, plùs épaisse, quelquefois dure et solide, à laquelle Goertner a domné le nom de testa; l'autre extérieure, plus minee, que l'on nomme tegmen. Cette disposition se remarque très-bien dans la graine du ricin (Ricinus communis); mais ees deux membranes ne sont pas plus distinetes l'une de l'autre que les trois parties qui composent le périearpe. 'Le liile est toujoúrs situé sur l'épisperme. Il offre un aspeet et une étendụe variables. Quelquefois il se présente sous la forme d'ü simple point, à peine visible. D'autres fois au eontraire il est très-large, eomme dans le marronicr d'Inde, par exemple; où sa "eoulcur blanchấtre le fait distinguer facilement du reste de l'épisperme, qui est d'un brun foneé.

Vers la partie eentrale du hile, quelquefois sur un de ses côtés, on voit une ouverture fort petite, à laquelle M. Turpin a donné le nom d'omphalode, et qui livre passage aux yaisseaux nourriciers qui, du trophosperme, s'introduisent dans le tissu de l'épisperme. Lorsque ce faisceau vasculaire se continue quelque temps avant de 
se ramifier, il forme une ligne saillante, à laquelle on a donné lc nom de vasiducte ou de raphé. Le point. intérieur où se termine le vasiduete porte le nom de chalaze ou d'ombilie interne. Le vasiduete est souvent peu apparent à l'extérieur : on ne le déeouvre alòs que par le seeours de la disseetion, eomme dans beaueoup d'Euphorbiaeées. D'autres fois il est très-saillant et visible, eomme dans les Orangers, où il s'allonge d'un bout à l'autre de l'épisperme.

Dans beaueoup de graines on trouve près du hile un organe perforé, souvent dirigé du eôté du stigmate, et que les botanistes désignent avee M. Turpin sous le nom de micropyle. Plusicurs auteurs pensent que e'est par eette ouverture que le fluide féeondant est apporté au jeune embryon.

M. Brown (voy. la note placée en tête de ce ehapitre, page 29I.) eonsidère eette ouverture eomme la base de la graine. La radieule de l'embryon lui eorrespond toujours exaetement.

On remarque quelquefois, plus ou moins loin du hile de quelques graines, une sorte de eorps renflé en forme de calotte, auquel Groethér a donné le nom d'embryotége, comme dans le dattier, l'asperge, la eomméline, ete. Pendant la germination, ec eorps se détaehe et livre passage à l'embryon.

L'épisperme est le plus souvent simplement appliqué sur l'amande, dont on le sépare avee faeilité. Mais il arrive quelquefois qu'il eontraete avee ellc üne adhérenee si intime, qu'on ne peut l'enlever çu'en le grattant.

L'épisperme n'offre jamais de loges ni de eloisons à son intérieur. Sa eavité est toujours simple. Cependant il peut, dans quelques eas rares, renfermer plusieurs embryons à la fois. Mais eette superfétation est une anomalie, une sorte de jeu de la nature, qui n'a rien de fixe ni de constant. 


\section{\2. De l'Amande.}

L'amande est toute la partie d'une graine mûre et parfaite, contenue dans la cavitć de l'épisperme. Elle n'a aucune espèce de communication vasculaire avec lui, à moins que ces deux organes nc soient soudés et confondus; car dans ce cas il devient difficile de déterminer s'il n'existe point quclquc communication vasculaire entre eux.

L'amande tout cntic̀re pcut être formće par l'embryon, comme dans le haricot, la lentille, la fêve de marais, etc.; c'est-à-dire qu'il remplit à lui scul toute la cavité intérieure de l'ćpisperme. (Voy. pl. 7, fig. 3, 7.)

D'autrcfois, outre l'embryon, l'amande renferme un autre corps accssoire, qu'on appelle endosperme (I), comme dans le ricin, le blé, etc. (Voy.pl. 7 , fig. 3, c.)

La structure de ces deux organes cst tellement différente, qu'il scra facile de les distinguer au premicr coup-d'oeil. L'embryon, en effet, est un être essentiellement organisé qui, par la germination, doit s'accroître et se développcr. L'endosperme, au contrairc, est une masse de tissu cellulaire, quelquefois durc et comme cornéc, d'autres fois charnuc et mollc qui, par la germination, sc fane et diminue ordinairement de volume, au lieu d'en acquérir. Ainsi donc la germination levera tous les doutes, pour déterminer la nature des deux corps reufermés dans l'ćpisperme, quand on n'y sera pas parvenu au moyen de l'analyse et de la dissection.

\section{§3. De l'Endosperme.}

L'endosperme est cctte partic de l'amande qui forme autour ou à côtć de l'embryon, un corps acccssoire, le-

(1) Périsperme de Jussieu; albunen de Gœrtner. 
quel n'a avec lui aucune eontinuité de vaisseaux ou de tissu. Le plus souvent il est formé de tissu cellulaire dans les mailles duquel se trouve renfermée de la fécule amylacée, ou un mucilage épais.

Cette substance sert de nourriture au jeune embryon. Avant la germination, elle est tout-à-fait insoluble dans l'eau ; mais à cette première époque de la vie végétale, elle change de nature, devient soluble, et sert en partie à la. nourriture et au développement de l'embryon.

Il est toujours assez facile de séparer l'endosperme de l'embryon, parce qu'il ne lui est aucunement adhérent.

Sa couleur est le plus souvent blanche ou blanchâtre; il est vert dans le gui (viscum album).

La substance qui le forme est en général très-variable; ainsi il est :

Sec é farineux dans un grand nombre de Graminées, le blé, l'avoine, l'orge, etc.;

Coriace et comme cartilagineux dans un grand nombre d'Ombellifères;

Oléagineux et charnu, c'est-à-dire épais et gras au toucher, eomme dans le ricin et beaucoup d'autres Euphorbiaeées;

Corné, tenaee, dur, élastique comme de la eorne, dans le cafée et beaucoup d'autres Rubiacées, la plupart des Palmiers, etc.;

Mince et membrancux, comme eelui d'un grand nombre de Labiées, ète.

La présence ou l'absence de l'endosperme est un trèsbon earactère générique; surtout dans les Monocotylédons. Cet organe doit donc jouer un grand rôle dans l'arrangement des familles naturelles des plantes.

L'endosperme peut exister dans une graine, quoique son embryon ait avorté, ou manque cntièrement.

Il est toujours unique, même dans les cas où il y a plusicurs embryons réunis dans la même graine. 


\section{§4. De l'Embryon.}

L'embryon est ce corps déjà organisé, existant dans une graine parfaitc aprc̀s la fćcondation, ct qui constitue le rudiment composé d'une nouvclle plante. C'est lui, en effet, qui, placé dans des circonstances favorables, va, par l'acte de la germination, devenir un végétal parfaitcment semblable en tout à celui dont il tire son origine.

Quand l'embryon existc scul dans la grainc, c'est-à-dirc qu'il est immédiatement recouvert par l'épisperme ou tégument propre, on l'appelle épispermique (embryo epispermicus), comme dans le haricot. (Voy. pl. 7, fig. 3, $4,5,6$.

$\mathrm{Si}$, au contraire, il est accompagné d'un endosperme, il prend le nom d'endospermique (embryo endospermicus), comme dans les Graminécs, lc ricin, etc. (Voyez pl. 6 , fig. 3, 4.)

L'cmbryon endospermique pcut offrir des positions différentes rclativemcnt à l'endosperme. Ainsi quelquefois il est simplement appliqué sur un point de sa surface, ct logé dans une petite fossette superficielle que celle-ci lui présente, comme dans lcs Graminées, ou bien il est roulé autour de l'cndospcrme qu'il enveloppe plus ou moins complétement. comme dans la bclle de nuit. Il a reçu dans ce cas lc nom d'extraire (embryo extrarius). (Voyez pl. 7 , fig. 8.)

D'autrcs fois il est totalement renfermé dans l'intérieur de l'endosperme qui l'enveloppe de toutes parts; il porte alors lc nom d'intraire (embryo intrarius), comme dans le ricin, ctc. (Voy. pl. 7, fig. 3, 4.)

L'embryon étant un végétal déjà formé, toutes lcs partics qu'il doit un jour développer y existent déjà, mais seulement à l'état rudimcntaire. C'est, comme nous l'avons dit, la véritable différence de l'embryon ct des corpuscules rcproductifs des plantes agames. 
L'embryon est cssentiellement formé de quatre parties'; savoir : ${ }^{\circ}$ du corps radiculaire ; $2^{\circ}$ du corps cotylédonaire; $3^{\circ}$ de la gemmule; $4^{\circ}$ de la tigelle.

I $^{\circ}$ Le corps radiculaire ou la radicule constitue une des extrémités de l'embryon. C'est lui qui, par la germination, doit donner naissanee à la raeine ou la former par son développement. (Voy.pl. 7, fig. $4, a, 8, a$. )

Dans l'embryon à l'état de repos, e'est-à-dire avant la germination, l'extrémité radieulaire est toujours simple et indivise. Lorsqu'elle se développe, elle pousse souvent plusieurs petits mamelons qui constituent autant de filets radieulaires, comme dans les Graminées.

Si, dans quelques eas, il est difficile avant la germination de reconnaître et de distinguer la radieule, cette distịnetion devient aisée lorsque l'embryon commenee à se développer. En effet, le corps radieulaire tend continuellement à se diriger vers le centre de la terre, quels quic soient les obstaeles qu'on lui oppose, et se ehange en raeine, tandis que les autres parties de l'embryon preninent une direetion eontraire.

Dans un certain nombre de végétaux le corps radiculaire lui-même s'allonge et se change en raeine par l'effet du développement que la germination lui fạit ąequérir. C'est ce que l'on observe dans un grand nombre de Dicotylédons; daus le cas où lạ radicule est extérieure et à nu, les végétaux prennent le nom d'exorhizes. Tels sont les“Labiées, les Crueifères, les Borraginées, les Synanthérées, etc., et la plupart des plantes dicotylédonées. (Voy. pl. 7, fig. 5, $6,7, a$.

Dans d'autres végétaux, au contraire, la radieule est rècouverte et eachée entièrement par une enveloppe partieulière qui se rompt à l'époque de la germination pour lui donner issue; ee eorps a reçu le nom de coléorhize; dans ee eas la radieule est intérieurè ou coléorhizée, et les plantes qui offrent cette disposition ont reȩu le nom de 
endorhizes. A eette division se rapporte la plus grande partic des vrais Monocotylédons, tels que les Palmiers, les Graminées, les Liliacées, etc. (Voy. pl. 7, fig. ro.)

Enfin dans quelques cas plus rares, la radicule est soudée et fait corps avec l'endosperme : on appelle synorhizes les plantes dans lesquelles on observe cette organisation. Tels sont les Pins, les Sapins, toutes les autres Conifères, les Cycadées, etc.

Toutes les plantes phanérogames eonnues viennent se ranger dans ces trois divisions. Aussi peut-on substituer avee avantage ees trois grandes classes à celles des Monocotylédons et des Dicotylédons, sujettes à d'assez nombreuses exceptions, comme nous le ferons voir tout à l'heure.

$2^{\circ}$ Le corps cotylédonaire peut être simple et parfaitement indivis; dans ee cas il est formé par un seul cotylédon, et l'embryon est appelé monocotylédoné (embryo monocotyledoneus), comme dans le riz, l'orge, l'avoine, le lis, le jonc, etc. (Voy. pl. 7, fig. 7, 8.) D'autres fois il est formé de deux corps réunis base à base, que l'on nomme Cotylédons, et l'embryon est dit alors dicotylédoné (embryo dicotyledoneus), eomme dans le ricin, la fêve, ete. (Voy.pl. 7, fig. 3, 5, 6.)

Toutes les plantes, dont l'embryon offre un seul eotylédon portent le nom de Monocotylédonées; toutes celles qui ont deux cotylédons sont appelées Dicotylédonées.

Les cotylédons, sont quelquefois au nombre de plus de deux dans le même embryon; ainsi il y en a trois dans le Cupressus pendula; quatre dans le Pinus inops et le $C_{e-}$ ratophyllum demersum; einq dans le Pinus laricio; six dans le cyprès chauve ( Taxodium distichum); huit dans le $P$ inzus strobus; enfin on en trouve quelquefois dix et même douze dans le Pinus pinea.

On voit done que le nombre des cotylédons n'est point 
le même dans tous les végétaux, et que la division en Monocotylédons et en Dicotylédons, rigoureusement observée, ne peut pas comprendre tous les végétaux connus; d'ailleurs il arrive assez souvent que les deux cotylédons se réunissent et se soudent, en sorte qu'au premier eoup d'œil jl est difficile de décider si un embryon est monocotylédoné ou dieotylédoné, eomme, par exemple, on l'observe dans le marronier d'Inde.

Ce sont ees motifs qui ont engagé mon père à prendre dans un autre organe que dans les eotylédons la base des divisions primordiales du règne végétal. La radicule nue ou eontenue dans une coléorhize, ou enfin soudée à l'endosperme, offrant des earaetères plus fixes, plus invariables, il s'en est servi pour former trois grandes elasses dans les plantes embryonées ou Phanérogames, qui sont :

$I^{\circ}$ Les Endorhizes, ou eelles dont l'extrémité radiculaire de l'embryon présente une coléorhize, sous laquelle sont un ou plusieur's tubercules radiculaires qui la déchirent, lors de la germination, et se changent en racines. Ce sont les véritables Monocotylédons.

$2^{\circ}$ Les Exorhizes, ou celles dont l'extrémité radieulaire de l'embryon est nue, et devient elle-même la racine de la nouvelle plante; tels sont la plupart des Dieotylédons.

$3^{\circ}$ Les Sxnormizes, ou plantes dans lesquelles l'extrémité radiculaire de l'embryon est intimement soudée à l'endosperme. Cette classe, moins nombreuse que les deux précédentes, renferme les Conifères et les Cycadées, qui s'éloignent des autres végétaux par des caractères si remarquables, et que le nombre de leurs cotylédons exclut égalemıent de la classe des Monoeotylédonés et des Dieotylédonés.

Les cotylédons paraissent être' destinés par la nature à favoriser le développement dc la jeune plante, en lui fournissant les premiers matériaux de sa untrition. En 
effet, les eolylédons sont presque eonstamment trèsépais et eharnus, dans les plantes qui n'ont pas d'endosperme, tandis qu'ils sont minees et comme foliaeés dans eelles où cet organe existe. C'est ee que l'on peut voir faeilement, en comparant l'épaisseur des eotylédons du haricot et du riein.

A l'époque de la germination, quelquefois les cotylédons restent eachés sous la tcrre, sans se montrer à l'extérieur; dans ce cas ils portent lc nom de eotylédons hypogés (cotyledones hypogei), eomme dans le marronier d'Inde.

D'autres fois ils sortent hors de terre, par l'allongement du collet qui les sépare de la radieule; on lcur donne alors le nom d'épigés (cotyled. epigei), comme dans le harieot et la plupart des Dieotylédonés. Quand les deux cotylédons sont épigés, et qu'ils s'élèvent au-dessus du sol, ils forment les deux feuilles séminales (folia seminalia). (Voy. pl. 7 , fig. $13, b, b_{\text {. }}$ )

$3^{\circ}$ De la gemmule. On donne le nom de gemmule (gemmula) au petit eorps, simple ou eomposé, qui naît entre les eotylédons, ou dans la eavité mĉme du eotylédon quand l'embryon n'en présente qu'un. On lui donnait autrefois le nom de plumule ( plunnula). Comme eet organe n'a le plus souvent aueune ressemblanee avee le eorps auquel on le eomparait, mais qu'au eontraire il forme toujours le premier bourgeon (gemma) de la jeume plante qui va se développer, le nom de gemmule est infuiment plus eonvenable, et mérite d'être préféré.

La gemmule est le rudiment de toutes les parties qui doivent se développer à l'air extérieur. Elie est fornée par plusieurs petites feuilles plissées diversement sur elles-mêmes, qui, en se développant par la germination, deviennent les feuilles primordiales (fol. primordialia). (Voy. pl. 7, fig. I 3, $d, d$.)

Quelquefois elle est libre et visible à l'extérieur, avant la germination; d'autrcs fois au eontraire elle ne devient 
apparente que lorsque celle-ei a eommencé ; enfin, dans quelques eas rares, elle est eaehée sous une sorte d'enveloppe analogue, en quelque façon, à eelle qui reeouvre la radieule des endorhizes, que l'on appelle coléoptile, et alors la gemmule est dite coléoptilée. Cette eoléoptile ne doit être le plus souvent eonsidérée que eomme un cotylédon minee, reeouvrant la gemmule à la manière d'un étui.

$4^{\circ}$ De la tigelle (cauliculus). Cet organe n'existe pas toujours d'une manière bien manifeste. Il se confond; d'une part, avec la base du eorps eotylédonaire, et de l'autre, avee la radieule, dont il est une sorte de prolongement. C'est par l'aeeroissement aequis par la tigelle, lors de la germination, que les eotylédons sont, dans quelques plantes, soulevés hors de terre et deviennent épigés.

Après avoir ainsi étudié suceessivement les quatre parties qui eomposent un embryon, savoir $x^{\circ}$ le corps radieulaire, $2^{\circ}$ le corps cotylédonaire, $3^{\circ}$ la gemmule, $4^{\circ}$ la tigelle, voyons quelles sont les différentes positions que l'embryon peut affeeter relativement à la graine qui le eontient, ou au périearpe lui-même.

Nous avons déjà vu que l'embryon pouvait être endospermique ou épispermique, suivant qu'il était aeeompagné d'un endosperme, ou qu'il formait à lui seul la masse de l'amande; que dans le eas où il était endospermique, il pouvait être intraire ou extraire, quand il était eontenu et renfermé dans l'intérieur de l'endosperme, ou simplement appliqué sur un des points de sa surface.

C'est par le moyen de ees deux extrémités de l'embryon que l'on peut déterminer sa direetion propre et sa direetion relative. L'extrémité radieulaire forme toujours la base de l'embryon. D'après eela on dit de l'embryon qu'il est : Homotrope (emb. homotropus), cuand il a la même 
direction que la graine, c'est-ì-dire que sa radicule répond au hile, comme cela s'observe dans beaucoup de Légumineuses, de Solanées et un grand nombre de Monocotylédons. L'embryon homotrope peut être plus ou moins courbé. Quand il est rectiligne, on lui donne le nom d'orthotrope ( $\mathrm{emb}$. orthotropus), comme dans les Rubiacées, les Synanthérées, les Ombilifères, etc.

On appelle embryon antitrope (embryo antitropus), celui dont la direction est opposée à celle de la graine, c'est-à-dire que son extrémité cotylédonaire correspond au hile. C'est ce que l'on peut observer dans les Thymélées, les Fluviales, le Mélampyrum, etc.

On donne le nom d'embryon amphitrope ( $\mathrm{emb}$. amphitropus ) à celui qui est tellement recourbé sur luimême, que ses deux extrémités se trouvent rapprochées et se dirigent vers le hile, comme on le voit dans les Caryophyllées, les Crucifères, plusieurs Atriplicées, etc.

Comme l'embryon monocotylédoné et l'embryon dicotylédoné diffèrent beaucoup l'un de l'autre, dans le nombre, dans la forme et l'arrangement des parties qui les composent, nous allons exposer isolément les caractères propres à chacun d'eux.

\section{§5. Embryon dicotylédoné.}

L'embryon dicotylédoné, ou celui dont le corps cotylédonaire présente deux lobes bien distincts, offre les caractères suivans: Sa radicule est cylindrique ou conique, nue, saillante; elle s'allonge lors de la germination, et deviẹt la véritable raciue de la plante. Ses deux cotylédons sont attachés à la même hauteur sur la tigelle; ils ont, dans beaucoup de cas, une épaisseur d'antant plus grande que l'endosperme est plus mince, ou qu'il n'existe point du tout. La gernmule est renfermée entre les deux cotylédons, qui la recouvrent et la cachent en grande partie. La tigelle est plus ou moins développée. 
Tcls sont les earactères communs aux embryons dieotylédonés en général. Cependant quelques-uns offrent des anomalies qui sembleraient d'abord les éloigner de cette classe; ainsi, quelquefois les deux cotylédons sont tellement unis et soudés ensemble, qu'ils semblent n’en plus former qu'un seul, comme dans le marronier d'Inde, et ordinairement le ehàtaignier. Mais on remarquera que cette soudure n'est qu'aceidentelle, car il arrive quelquefois qu'elle n'a pas lieu. C'est ce que l'on observe en effet pour le marronier d'Inde, et ce qui le fait rentrer dans l’organisation générale des embryons dieotylédonés. D'ailleurs on doit regarder comme véritablement dicotylédoné tout embryon dont la base du corps cotylédonaire est fendue entièrement ou partagée en deux, quoique lui-même paraisse simple et indivis à soil sommet.

\section{§2. De l'Embryon monocotylédoné.}

L'embryon monocotylédoné est eclui qui, avant la ger. mination, est parfaitement indivis, et ne présente aucune fente ni incision.

Si, dans le plus grand nombre des eas, il eșt assez facile de reconnaître dans l'embryon dicotylédoné les différentes parties qui lé eomposent, il n'en est pas toujours de même dans l'embryon monocotylédoné, où, fréquemment, toutes ees parties sont tellement unies et coufondues, qu'elles ne forment plus qu'une masse, dans laquelle la germination seule pent faire distinguer quelque chose. Aussi l'organisation de l'embryon des monocotylédonés est-elle bien moins parfaitement connue que celle des végétaux à deux cotylédons.

Dans l'embryon monocotylédoné, le corps radiculaire oceupe une des extrémités; il est plus ou moins arrondi, souvent très-peu saillant; formant comme une sorte de mamelon peu apparent. D'autres fois, au contraire, il est 
extrêmernent large et aplati, et forme la massc la plus considérable de l'embryon, comme dans la plupart des Graminćes. L'cmbryon est alors appclé macropode ( $\mathrm{emb}$. macropodus ). (Voy. pl. 7, fig. 8, 9.)

La radicule est renfermée dans une coléorhize qu'clle rompt à l'ćpoque de la germination. Cette radicule n'est pas toujours simple, comme dans les Dicotylédonés; clle est le plus souvent forméc de plusieurs filets radiculaires, qui percent quclquefois, chacun isolément, la coléorhize qui les renferme, comme cela s'obscrve principalement dans les Graminécs.

Le corps cotylédonaire est simple, et ne présente aucune incision ni fentc. Sa forme cst extrêmement variablc. Il est toujours latéral, relatirement à la masse totale de l'cmbryon. Le plus souvent, la gemmule est renfermée dans l'intéricur du cotylédon qui l'cnvcloppe de toutes parts, et lui forme muc espécc de coléoptile. (Voy. pl. 7 , fig. $9, b$, то , $b$.) Ellc sc compose de petites feuilles cmboîtécs les unies dans les autres. La plus extéricure forme ordinairement une espèce d'ćtui clos de toutes paris, cmbrassant et recouvrant les autres. M. Mirbcl lui a donnć le nom de piléole.

La tigelle n'existc pas lc plus souvent, ou cllc sc confond intimement avec le cotylédon ou la radiculc.

relle est l'organisation la plus ordinaire des cmbryons monocotylédonćs; mais, dans bcaucoup de circonstances on trouve des modifications propres à plusicurs végétaux. C'est ainsi, par excmple, que la famille des Graminces préscnte quclques particnlarités dans la structure de son embryon. En cflet, il cst composé, I d'un corps charnu, épais, discoüde en général, appliqué sur l'cndospcrme; ce corps a reçu le nom d'typoblaste (I) : cette partic ne

(1) G'est à ce corps que Gorrner donne le now de vitellius. La plupary 
prend aucun accroissement par la germination; elle peut être assimilée au corps radiculaire; $2^{\circ}$ du blaśe ou de lá partie de l'èmbíyon quii doit se développer. Il est appliquéé sur l'hyoblasle, et est formé de la tigelle, de la gemmule; renfermée dans le cotylédon, constituant une sorte de graine ou d'étui qui les enveloppe de toules parts. L'extrémité inférieure du blasle, par laquelle doivent sortir un ou plusieurs tubercules radicellaires, porte le nom de radiculode.

- Enfin on appelle épiblaste un aṕpéndice antéricur du blaste, qui le recouvre quelquiefois en partie, et qui semblè n'en être qu'un simple prolongement.

\section{CHAPITRE IIT.}

DE IA GERMINATION.

Ox donne le nom de germination à la série de phénomènes par lesquels passe une graine qui ; parvenue à son état de maturité, et mise dans des conditions favorables"; se gonfle, rompt ses enveloppes, et tend:à développer l'embryon qu'elle renferme dans son intérieur.

Pour qu'une graine germe, il faut le concours de cer-s taines circonstances dépendant de la graine elle-mèmę, ou qui lui sont accessoires et étrangères, mais qui n'exercent pas 'moins une influence incontestable sur les phénomènes de son dẹ́veloppement.

La graine doit être à son état de maturité : elle dọit avoir été fécondée et renfermer un, ẹbryon parfait dans toutes ses parties. Il faut de plus que la graine ne soit pas

des anteurs le regardent comme le cotylédon. Mais l'ánalogie se refuse à cèttc supposition.

Voyez le mémoire de mon pìre sur lés embryons endorhizes, inséré dans le $\mathrm{I} \mathrm{F}^{\mathrm{e}}$ volume des Ánnales du Musćum, année $\mathrm{I} 8 \times \mathrm{x}$. 
trop aneiennc; car ellc aurait perdu, par le temps, sa faculté germinative. Cepcndant il cst ccrtaines graines qui la eonservent pendant un nombre d'années considérablc : ce sont principalement eelles qui appartiennent à la famille des Légumineuses. Ainsi l'on est parvenu à faire germer des harieots conscrvés depuis soixante ans; on cite mêmẹ des graines de sensitive qui se sont parfaitement développées ccnt ans environ après avoir été récoltées. Mais il faut qu'clles aicnt été préservées du eontact de l'air, de la . Iumière et de l'humidité.

Les agens cxtćrieurs indispensables à la germination

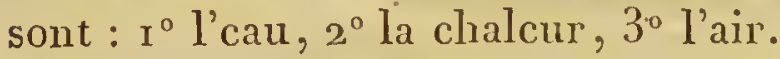

I L'eau, eommc nous l'avons déjà vu précédemment, est indispensable à la végétation ct aux phénomènes de la nutrition dans lcs végétaux. Ce n'est point sculement eomme substance alimcntaire qu'clle agit dans ce cas; mais e'est cneore par sa faeulté dissolvante et sa fluidité qu'ellc sert alors de menstruc et de vćlieule aux substances vraiment alibiles du végétal.

Ellc a, dans la germination, me manière d'agir parfaitemcnt analoguc. C'cst clle, cn cffet, qui, cn pénétrant dans la substanec de la grainc, ramollit ses enveloppes, fait gonfler l'embryon, détermine, dans la nature même de l'cndósperme ou des colylédons, des eliangemens qui les rendent souvent propres à fournir au jeunc végćtal les premicrs matériaux de sa nutrition. C'est elle encore qui se charge des substanees gazeuses ou solides qui doivent scrvir d'alimens à la jeune plantc qui commence à eroîtrc. Elle fournit aussi à son développernent par la décomposition qu'ellc éprouvc; ses élémens désunis se combinent avee lc carbone, et donnent naissancc aux différens principcs-immédiats des végétaux.

Cependant il uc faut pas que la quantité d'eau soit trop considérable; car alors les graincs éprouveraient unc sorte de macération qui détruirait leur faculté germinative, el 
s'opposerait à leur développement. Nous parlons ici des grainesqui appartiennent aux plantes terrestres; car celles des végétaux aquatiques germentétant plongées entièrement dans l'eau. Quelques-unes, néanmoins, quoiqu'en trèspetit nombre, montent à sa surface pour y germer à l'air, et ne pourraient sc développer si elles restaient submergées.

L'eau a donc évidemment déux modes d'action dans la germination : $\mathbf{l}^{\circ}$ elle ramollit l'enveloppe séminale et fávorise sa rupture; $2^{\circ}$ elle sert de dissolvant et de véhicule aux véritables alimens du jeune végétal.

$2^{\circ}$ La chaleur n'est pas moins nécessaire à la germination que l'eau. Son influence est, en effet, très-marquée sur tous les phénomènes de la végétation. Une graine mise dans un lieu dont la température est au-dessous de zéro, n'éprouve aucun mouvement de développement, réste inactive, comme engourdie, tandis qu'une chaleur douce et tempérée accélère singulièrement la germination, Mais cependant il ne faut pas que cette chaleur dépasse certaincs limites; sans quoi, loin de favoriser le développement des germes, elle les dessécherait et y détruirait le principe de la vie. Ainsi une chaleur de $45^{\circ}$ à $50^{\circ}$ s'oppose à la germination, tandis que celle qui ne s'élève pas au-dessus de $25^{\circ}$ à $30^{\circ}$, surtout si elle est jointe à une certaine humidité, accélère l'évolution des différentes parties de l'embryon.

L'air est aussi utile aux végétaux, pour germer et s'accroître, qu'il est indispensable aux animaux pour respirer et pour vivre. Une graine que l'on priverait totalcment du contact de ce finide n'acquerrait aucune espèce de développement. Cependant Homberg dit être parvenu à faire germer quelques graines dans le vide dè la machine pneumatique. Mais quoiqu'on ait, depuis lui, souvent répété cette expérience, on n'a jamais pu obtenir les mêmes résultats. L'on peut donc assurer que l'air est indispensabile 
à la gepmination. M. Théodore de Saussure, dont le témoignage est d'un si haut poids dans la partic expérimentale de la physiologic des végétaux, pense que les expériences de Homberg nc doivent nullement infirmer cette vérité, et que les conclusions qu'il en a tirées doivent être considérécs comme des résultats imparfaits ct peu cxacts.

Des graines enfoncécs trop profondément dans la terre, et sonstraites ainsi à l'action de l'air atmosphérique, sont souvent restécs pendant $m$ temps fort long. sans donner aucun signe de vic. Lorsque, par une causc quclconque, clles se sont trouvécs ramcnées plus près de la supcrficic de la terre, de manière à être en contact avec l'air ambiant, leur germination s'est effectuée.

L'air n'étant point un corps simple, mais étant au contrairc formé d'oxygène et d'azote, doit-il son action au mélange de ces deux gaz? Ou bicn est-ce l'un d'cux seulement qui détermine l'influcnce qu'il exerec sur les phénomènes de la germination?.

L'action de l'air sur les végétaux, à cette premic̀re époque de leur développement, présente les mèmes circonstances que pour la respiration dans les animaux. En effet, c'est l'oxigène de l'air qui agit principalement dans l'acte de la respiration pour donner au sang les qualités qui doivent le rendre propre au développement de tous les organes; c'est encore ect oxygène qui aide et favorise la germination des végétaux. Des graines placécs dans du gaz azote ou du 'gaz acide carbonique ne peuvent se développer, et ne tardent point à y périr. Nous savons qu'il cn serait de même des animaux que nous soumcttrions à de scmblaḅles influcnces. Mais ec n'est point à l'état de purcté et d'isolement que l'oxygène a une action aussi favorable à l'évolution des germes; car il l'accélère d'abord, mais bientôt la détruit par. l'activité trop puissante qu'il lui communique. Aussi les graines, les plantes et les animaux ne peuvent-ils ni se développer, ni respirer, ni vivre dans du 
gaz oxyģène pur. Il faut qu'une autre substance mélangéc avec lui tempèrc sa trop grande activité pour cqu'il devienne propre à la respiration et à la végétation. On a remarqué que son mélange avec l'luydrogène ou l'azote le rendait plus propre à remplir cette forction; el que les proportions les plus convenables de cc mélange étaicnt une partie d'oxygène pour trois parties d'azotc ou d'hydrogène.

L'oxygène, absorbé pendant la germination, se combine avec l'excès de earbone que contient lc jeune végétal, et forme de l'acide carbonique, qui est rejeté au dehors. C'est par cette combinaison nouvelle que, les principes de l'endosperme n'étant plus les mêmes, la fécule qui le compose, d'insoluble qu'elle était avant cette époquc, devient soluble, et souvcnt est en partie absorbće, pour servir de première nourriture à. l'embryon.

Certaines substances paraissent ayoir une influence bien manifestc pour aceélérer la germination des végétaux. C'est ce qui résulte des expériences de M. de Humboldt. Cet ilJustrc maturaliste, à qui presque toutes les branches des connaissances humaines doivent quelques-uns de leur's progrès, et.souvent la perfeetion où nous les voyons arrivées aujourd'hui, a démontré quc les graines du cresson alénois (Lepidium sativum) mises dans une dissolution de chlore, germaient en cinq ou six heures; tandis que dans l'eau purc ces mêmes graines avaient besoin de trente-six heures pour arriver au même résultat. Certaines grainès exotiques, qui jusqu'alors avaient résisté à tous les moyen's employés pour les faire germer, se sont parfaitement développées dans une dissolution de cette même substance. Il a dc plus fait remarquer que toutes les substances qui pouvaient céder faeilement une partie de leur oxygène à l'eau, tels. quc beaucouṕ d'oxydes métalliques, les aeides nitrique et sulfurique suffisamment étendus, hâiaient lc développement des graines, mais produisaient en même temps l'effet que nous avons signalé pour lc gaz oxygène 
pur, ćest-à-dire qu'ils épuisaient le jeunc cmbryon et ne tardaicnt pas à le faire périr.

La terre dans laqjuclle on place en général les graines, pour déterminer leur germination, n'est pas une condition indispensable de leur développement, puisque tous les jours nous voyons des graines germer très-bien et avee beancoup de rapidité sur des éponges fines, ou d'autres corps que l'on a soin d'imbiber d'eau. Mais eependant qu'on ne eroie pas que la terre soit tout-à-fait inutile à la végétation; la plaute y puise par ses racines des substances qu'elle sait s'assimiler, après les avoir converties en élémens nutritifs.

La lumière, loin de lsàter le développement des organes de l'embryon, le ralentit d'ume manière manifeste. En effet, il est constant que les graines germent beaueoup plus rapidcment à l'obscurité que lorsqu'elles sont exposécs à la lumière du soleil.

Toutes les graines n'emploient pas un espace de temps égal pour commenecr à germer. Il y a même à ect égard les diflérences les plus tranchées; ainsi il en est qui germent dans un temps très-conrt. Lc eresson alénois en deux jours; l'épinard, le navet, les haricots en trois jours; la laitue en quatre jours; los melons, les courges en cinq jours; la plupart des Graminćes en une semaine; l'hysopeau bout d'un mois. D'antres emploient un temps fort considérable avant de clonner aucun signe de développement; ce sont principalement eclles dont l'épisperme est très-dur, ou qui sont enviromnées d'un endocarpe ligneux, comme celles du phe cher, de l'amandier, qui ne germent qu'au bout d'un an; les graines du noisetier, du rosier, du cornouiller, et d'autres encore, ne se développent que deux amnées après avoir été mises en terre.

Après avoir passé rapidement èn revue les circonstanees aceessoires qui déterminent ou favorisent la germination, étudions les phénomènes génćraux de cette fonetion, après quoi nous donnerons quelques détails relatifs aux particu- 
larités qu'elle présente dans les plantes monocotylédonćes; et daus les dicotylédonées.

Le premier effet apparent de la germination est le gonflement de la graine, et le ramollissement des enveloppes qui la recouvrent. Ces enveloppes se rompent au bout d'un temps plus ou moins long, variable dans les différens végétaux. Cette rupture de l'épisperme se fait quelquéfois d'une manière tout-à-fait irrégulière, comme dans les haricots, les fèves : d'autres fois', au contraire, elle présente unc uniformité et une régularité qui se reproduisent de la même manière dans tous les individus de la même espèee. C'est ee que l'on observe principalement dans les graines pourvues d'un embryotége, sorte d'opercule qui se détache de l'épisperme pour livrer passage à l'embryon; eomme, par cxemple, dans l'éphémère de Virginie (Tradescantia virginica), la comméline (Commelina communis), le dattier (Phanix dactilifer $a$ ), et plusieurs autres Monoeotylédons.

L'embryon, dès le moment où il commence à se développer, prend le nom de plantule. On lui distingue deux extrémités, croissant constamment en sens inverse : l'une, formée par la gemmule, tend à se diriger vers la région de l'air et de la lumière; on l'appelle caudex ascendant. L'autre, au eontraire, s'enfoncant dans la terre, et suivant par conséquent une direetion tout-à-fait opposée à celle de la précédente, porte le nom de caudex descendant. Elle est formée par le corps radieulaire.

Dans le plus graind nombredes cas, e'est le eaudex descendant ou la radicule qui, la première, éprouve les effets dẹ la gcrmination. On voit eette extrémité devenir de plus en plus saillante, s'allonger et constituer la racine dans les exorhuzes. Dans les endonuzes, au contraire, la coléorhize, poussée par les tubercules radieellaires qu'elle renferme, s'allonge quelquefois, et se prête à tune distension assez considérable avant de se rompre; d'autres fois 
elle cède sur-lc-champ, et laisse sortir les tubercules radicellaires qu'clle recouvrait.

Pcudant ce temps la gemmulc ne reste pas incrte et statiommaire. D'abord cachée entre les cotylédons, elle se redresse, s'allonge, et cherche à se porter vers la superficic de la terre, quand clle y a ćté cnfouic. S'il y a unc coléoptile, clle s'allonge, se dilate; mais, plus rapide dans son accroisscment, la gemmule presse sur elle, la perec à sa partic supéricure et latéralc et sc montre à l'extérieur.

Quand le caudex ascendant commence à se développer au-dessous du point d'insertion des cotylédons, il les soulève, les porte hors de la terre. Ceux qui offrent ce phénomène sont alors appelés cotylédons épigés (1); ils sc développent, quelquefois mème s'amincissent, deviennent comme foliacés et portent alors le nom de feuilles séminales,

Si, au contraire, le caudex ascendant ne commence qu'au-dessus des cotylédons, ccux-ci restent cachés sous la terre, et, loin d'acquérir ancun accroissement, ils dimiment de volume, sc flétrissent et finissent par disparaître entièrement. On les nomme alors cotylédons hypogés (2).

Qnand unc fois la gemmule est parvenue à l'air libre, les folioles qui la composent se déroulent, se déploient, s'étalcnt, et aequièrent bicntòt tous les caractères des fenilles, dont elles ne tardent point à remplir les fonctions.

Mais quels sont les usages des parties aecessoiros de la graine; c'est-à-dire de l'épisperme et de l'cndosperme?

L'épisperme ou le tégument propre de la graine a pour usagc d'empêcher l'cau ou les autres matières daus les-

(I) Dérivé de $\varepsilon \pi \ell$, sur, au-dessus; et de $\not \eta$, terre, e'est-à-dire s'élerant au-dessus de la surface de la terre.

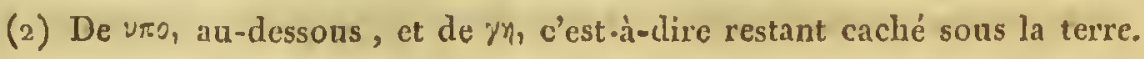


quelles une graine est saumise à la germination d'agir trop directement sur la subsianee mềme de l'enebryon; il remplit eu quelque sorte l'offiee d'un erible, à travers lequel ne peurent passer que des moléeules terreuses, fines: et très-divisécs. Duhamel, en effet, a remarqué que, les graines que l'on dépouille de leur tégument propre se développcnt rarement, ou donnent naissance à des végétaux grêles et mal eonformés.

L'endosperme, qui n'existe pạs toujours, n'est que le résidu de l'eau contenue dans la cavité de l'ovule où s'est développé l'embryou. Cette liqueur, que Malpighi a comparée à l'eau de l'amnios, quand elle n'a point été absorbée entièrement pendant la formation et l'aecroissement de l'embryon, prend peù à peu de la eonsistance, s'épaissit et fiṇit par former ume masse solide, dans laquelle l'embryon se trouve renfermé, ou sur la surface de, laquelle il cst simplement appliqué. Cette masse cst l'en-, dosperme. C'est pour eette raison que ee corps oflre touljours un aspect inorganique. Quelquefois tout le liquide, renfermé dans l'intéricur de l'ovule, et qui n'a point seryi à la nutrition de l'embryon, ne se solidific pas; une partie reste encore fluide. C'est ee que l'on observe très-bien dans le fruit du eocotier, par exemple, qui renferme dans l'intéricur de son noyau une quantité plus ou moins eonsidérable d'une sorte d'émulsion blanehâtre et douee, eonnue sous le nom de lait de coco.

L'origine et les premiers usages de l'endosperme nous, indiquent d'avance ceux que la nature lui a eonfiés lors de la germination. En effet, c'est lui qui fournit à la jeune plante sa première nourriture. Les ehangemens qu'il éprouve alors dans sa composition ehimique et la nature de ses élémens le rendent très-propre à eet usage.

Cependant l'endosperme, dans quelques végétaux, est tellement dur et compacte, qu'il lui faut un long espace de temps pour se ramollir et se résoudre en une substance 
plus ou moins fluide, qui puisse être absorbée par l'embryon. Mais ce phénomène a toujours licu.

Si l'on prive ou isole un embryon de l'endosperme qui l'accompagne, il ne se développcra aucuncment. Il est donc évident que cet organe est intimement lié à son accroisscment.

Les cotylédons, dans beaucoup de circonstances, paraissent remplir des fonctions analogues à celles de l'endosperme; aus`i est-ce pour cette raison que le célèbre physicien, Charles Bonnet, les appelait les mamelles végétales. Si l'on retranche les deux cotylédons d'un cmbryon, il se flétrira et ne donnera aucun signe de développement. Si l'on n'en enlève qu'un, il pourra cncore végéter, mais d'une manière faible et languissante, comme un être malade et mutilé. Mais un fait. des plus remarquables, c'est quc l'on peut impunément fendre et séparer en deux parties latéralcs un embryon dicotylédoné, celui du haricot, par excmple; si chaque partie contient un colylédon parfaitemcnt entier, cllc se développera aussi bien qu'un cmbryon tout enticr, ct domnera naissance à un végétal aussi fort ct aussi vigoureux.

Enfin, comme le prouvent les expériences de MM. Desfontaines, Thouin, Labillardière et Vastel, il suffit d'arroser les cotylédons pour voir tout l'embryon s'accroitre et dévclopper ses parties.

La grande différence de structure qui existe entre les embiryons monocotylédonés et les embryons pourvus de deux cotylćdons influe d'une manière notable sur le mode de germination qui leur est propre. Aussi croyons-nous niéccssaire d'en étudier séparément les phénomènes, afin de faire mieux connaitre le mécanisme de cctte fonction dans ces deux grandes classes. Nous commencerons par les embryons exorhizcs oụ dicotylédonés, parce que e'est en cux qu'il cst plus facile d'obscrver le développement successif des différens organes qui les composent. 


\section{$\S$ x. Germinalion des Embryons exorhizes ou 'dicoty- lédonés.}

Dans l'embryon dicotylédoné la radicule est, en général, conique et saillante. La tigelle est ordinairement cylindrique ; la gemmule est nue et caehée entre la base de deux cotylédons, qui sont placés face à face et immédiatement appliqués l'un contre l'autre (I).

Telle est la.disposition des parties constituantes de l'embryon avant la germination. Voyons les changemens qu'elles éprouvent quand cette fonction commence à s'exécuter. Pour mieux faire entendre ce que nous allons dire, prenons pour exemple le haricot, et suivons-le dans toutes les époques de son-aceroissement. Voyez planche 7 , figures I, 2, 3, 4, etc. Nous verrons d'abord toute la masse de la graine s'imprégner d'humidité, se gonfler; l'épisperme se déehirer d'une manière irrégulière. Bientôt la radicule, qui formait un petit mamelọn eonique, eommenee à s'allonger ; elle pénètre dans la terre, donne naissance à de petites ramifications. latérales extrêmement déliées. Peu de temps après, la gemmule, qui jusqu'alors était restée caehée entre les deux cotylédons, se redresse, se montre à l'extérieur. La tigelle s'allonge, soulève les eotylédons hors de terre, à mesure que la radieule s'y enfonce et s'y ramifie. Alors les deux cotylédons s'écartent; la gemmule est tout-à-fait libre et découverte; les petites folioles qui la conposent s'étalent, s'agrandissent, devicunent vertés et commencent déjà à puiser dans le sein de l'atmosphère une partie des fluides qui doivent être employés à l'aecroissement de la jeune plante.

(1) Dans quelques cas fort rares, les deux cotylédons, au lieu d'être imıédiatement appliqués face à face, sont manifestement écartés, et plus ou moins divergens. C'est ce que l'on observe par exemple dans les genres Monumia et Ruizia ou Boldea de la famille des Monimiées. 
Dès lor's la germination est terninée, et la sceonde époque de la vie du végétal eommenee.

Quand l'embryon est endospermique, c'est-à-dire lorsqu'il est aeeompagné d'un endospermé, les phénomènes se passent de la même mąnière, mais l'endosperme n'aequidrt aueun aeeroissement; on le voit au eontraire se ramollir et disparaître insensiblement.

Quelques végétaux dieotylédonés ont un mode partieulier dè germination. Ainsi, par exemple, on trouve fort souvent des embryons déjà germés dans l'intérieur de eertains fruits, parfaitement elos de toutes parts. C'est ee que l'on observe assez fréquemment dans les fruits du eitronnier, où il n'est pas rare de r'eneontrer plusicurs graines déjà en état de germination.

Le manglier (Rhizophora mangle), arbre qui liabite les maréeages et les rivages de la mer dans les régions équinoxiales, offre un genre partieulier de germination. qui n'est pas moins remarquable. Son cmbryon eommence à se développer, tandis que la graine est eneore contenue dans le périearpe. La radieule presse contre le périearpe, qu'elle use et finit par. pereer. Elle s'allonge à l'extérieur, quelquefois dé plus d'un pied. Alor's l'embryon se détaehe, en abandonnant le corps cotylédonaire dans la graine, il tombe, la radieule la première, s'enfonee dans la vase et eonitinue de s'y développer.

Dans le marronier d'Inde ou hippoeastane, dans le ehâtaignier, et quelques autres végétaux dicotylédonés, les deux cotylédons, qui sont très-gros et très-épais, sont le plus sotivent immédiatement soudés l'un avec l'autre. Voiei alors comment s'opère la germination : lá radieule, en s'enfonçant dans la terre, allonge la base des deux cotylédous et dégage ainsi la gemmule, qui ne tarde point à se montrer au-dessus de la terre; mais les denx eotylédons ne sont pas entrainés par la gemmule, ils restent hypogés. 


\section{\$2. Germination des Enbryons endorhizes ou mono-} cotylédonés.

Les embryons monocotylédonés éprouvent en général moins de changcmens, pcndant la germination, que eeux des plantes dicotylédonées, à cause de l'uniformité de leur strueture intérieure. En ellet, ils se présentent fort souvent sous l'apparence d'un corps charnu, dans lequel on distingue avec peine lès organes qui le eonstituent. Aussi est-on obligé de soumettre ì la gcrmination les embryons endorhizes dont on veut bien connaître la strueture.

C'est ordinairement, comme dans les dicotylédons, I'extrémité radiculaire qui se développe la première. Elle s'allonge et sa coléorhize se rompt pour laisser sortir le tubereule radicellaire qui se développe et s'enfonee dans la terre. Ordinairement plusieurs radicelles naissent des parties latérales et inférieures de la tigelle. Quand elles ont acquis un eertain développement, la radicule principale se détruit et disparaît. Aussi les plantes monocotylédonées n'offrent-elles jamais de raeine pivotante eomme les végétaux dicotylédons.

Le cotylédon, qui renferme la gemmule, s'accroît toujours plus ou moins avant d'être perforé par celle-ci. C'est le plus souvent par la partie latérale du cotylédon, presque jamais par son sommet, que sort la gemmule. En effet, elle est toujours plus rapprochée de l'un de ses côtés, et son sommet est constamment oblique. Lorsque la gemmule a perforé le cotylédon, celui-ci se change en une sorte de graine qui embrasse la gemmule à sa base. (Voycz pl. .7, fig. $14, b, c$.$) C'est à eette graine que l'on a donné le nom$ de coléoptile.

Mais il arrive assez souvent qqu'une partie du cotylédon restc engagéc, soit dans l'intérieur de l'endosperme, soit dans l'épisperme; en sorte qu'il n'y a que la partie la plus voisine de la radieule qui soit entraunée au dehors-par le développement de celle-ei. (Toy. pl. 7, fig. xo, c.) 


\section{CHAPITRE IV.}

CJASSIFICATION DES DIFFÉLENTES ESPËCES DE FRUITS.

Dans les deux ehapitres préeédens, 'nous avons étudié avee quelques détails les différens organes qui entrent dans la composition d'un fruit mûr et parfait. Nous avons fait voir qu'il était toujours composé de detix parties, le péricarpe et la graine.

Nous devons maintenant faire eonnaître les diverses modifieations que peut offrir le fruit; eonsidéré dans son ensemble, c'est-à-dirc dans la réunion des différentes parties qui le eonstituent.

On eonȩoit qu'il doit exister un grand nombre d'espèces de fruits, toutes plus ou moins distinetes les unes des autres, quand on considère les variétés de forme, de strueture, de consistanee, le nombre variable et la position respective des graines, ete., que présentent les fruits. Aussi leur elassification est-clle un des points les plus difficiles de la botanique. Malgré les effor ts et les travaux d'un grand nombre de botanistes eélèbres qui s'en sont spécialement oceupés, la elassification earpologique est eneore loin d'être parvenue à ee degré d'exactilude et de préeision anquel sont arrivées la plupart des autres branehes de la botanique. Quelques auteurs ont voulu réunir sous une dénomination eommune des espèees essentiellement différentes par leur forme et leur strueture; d'autres, au contraire, en multipliant à l'infini le nombre des divisions, et les établissant sur des earaetères trop minutieux ou trop peu eonstans, ont également uui aux progrès de cette partie de la earpologie. Aussi ne ferons-nous eomaître daus eet ouvrage que les espèees de fruits bien distinetes et bien caractérisées, que celles, en un mot, qui ont été eousa- 
crées par l'usage, ou adoptées par la plupart des botanistes.

Les fruits, eonsidérés en général, ont étédivisés de plusieurs manières, et ont reçu des noms partieuliers. Ainsi on appelle fruit simple eelui qui provient d'un pistil unique, renfermé dans une fleur; tel est eelui de la pêelıe, de la eerise, ete. On appelle, an eontraire, fruit multiple eclui qui provient de plusieurs pistils renfermés dans une même fleur; par exemple, la fraise, la framboise, eelui des renoneules, des elćmatites, ete.; enfin on doune le nom de fruit composé à eelui quï résulte d'un nombre plus ou moins eonsidérable de pistils rémis, et souvent soudés enscmble, mais provenant tous de fleurs distinctes, très-rapproehées les unes des antres, eomme eclui du mûrier.

Suivant la nature de leur périearpe, on a distingué les fruits en secs et en charnzus. Les premiers sont eeux dont le péricarpe est minee, ou formé d'une substance généralement peu fournie de sues; les seeonds, au eontraire, ont un péricarpe épais et suceulềnt, et leur sarcoearpe est surtont très-developpé; tels sont les melons, les pêehes, les abrieots, ete.

Les fruits peuvent resier parfaitement elos de toutes parts, ou s'ouvrir en un nombre plus ou moins grand de pièees nommées valves; de là la distinetion des fruits indéltiscens et des fruits déhiscens. Ces derniers, quand ils sont sees, portent également le nọm de fruits capsulaires.

Selon le nombre de graines qu'ils renferment, les fruits sont divisés en oligospermes et en polyspermes. Les fruits oligospermes sont ecux qui ne eontiennent qu'un nombre peu considérable de.graines, nombre qui est le plus souvent exaetement déterminé. De là les épithètes de monosperme, disperme, trisperme, tétrasperme, pentasperme, données au fruit, pour exprimer que le nombre de ses graines est un, deux, trois, quatre, einq, ete. Les fruits polyspermes sont tous eeux qui renferment un nombre 
eonsidérable de graines que l'on ne veut pas déterminer.

Il y a des fruits dans lesquels le périearpe a si peu d'épaisseur, et eontraete une telle adhérence avee la graine, qu'il se soude et se eonfond avee elle. Linnæus regardait ees fruits eomme des graines nues : on leur a donné le nom de pseudospermes. Tels sont ceux des Graminées, des Labiées, des Synanthérées, ete.

Il est très-important de bien eomnaître èt de pouvoir distinguer les différentes espèees de fruits. En effet, eet organe sert fort souvent de base à la disposition des plantes en familles naturelles; et les earaetères que l'on retire de son examen approfondi eonduisent en général aux résultats les plus heureux dans la classification mélhodique des végétaux.

Pour simplifier l'étude de la nomenclature des fruits, nous les diviserons en trois elasses; dans la première nous réunirons tous les fruits simples, e'est-à-dire tous ceux qui proviennent d'un seul pistil renfermé dans une fleur. Nous subdiviserons eelte élasse en deux seetions, dans l'unc desquelles seront plaeés les fruits sees, et dans la seeonde les fruits eharnus. La seeonde classe renfermera les fruits produits par la réunion de plusieurs pistils dans une même fleur, e'est-à-dire les fruits mulliples. Enfin dans la troisième elasse nous traiterons des fruits composés on de ceux qui sont formés par plusieurs fleurs d'abord distinctes qui se sont soudćes de ruamèrè à ne eonstituer par leur réunion qu'un mème fruit. 


\section{PREMIERE CLASSE.}

\section{DES FRUITS SIMPIYS.}

\section{SECTION PREMIÉRE.}

FRUITS SECS.

$\S \pi$. Fruits secs et indéhiscens.'

LEs fruits seces et indéhisccns sont ordinairement oligospcrmes, c'est-à-dire qu'ils renferment un très-petitnombre de graines. Leur péricarpe est en général assez mince, ou adhère avec le tégument propre de la graine; ce qui a porté les anciens à les considérç comme des graines nues ou dépourvues de péricarpes Ce sont là les véritables pseudospcrmcs. On distingue les espèees suivantes :] ॠथु

I $^{\circ}$ La cariopse (cariopsis, Rich.), fruit mouosperme, indéhiscent, dont le péricarpe, très-mince, est intimement confondu avec la graine, et nc peut en être distingué. Cettc espèce appartient à presque toute la famille des. Graminées, tels que le blé, l'orge, le riz, etc.

Sa forme est assez variable. Elle est ovoïde dans le blé (triticum), allongée ct plus étroite dans l'avoine (avena); irrégulièrement sphéroïdale dans le blé de Turquie (zea).

$2^{\circ}$ Liakène (akenium, Rich.), fruit monosperme; indélisccnt, dont le péricarpc est distinct du tégument propre de la graine; comme dans les Synanthérées, lc grand soleil (Helianthus aninuus), les chardons, ete.

Assez souvent l'akène est couronné par des soies, des paillettcs, qui constituent ee que nous avons désigné par le nom d'aigretle (pappus). (Voy. pl. 8, fig. x2 I3.) 
Quelquefois eette aigrette forme une simple petite couronue membraneuse, qui borde eiculairement la partie supérieure du fruit (pappus marginalis).

D'autres fois l'aigrelte est plumeuse ou soyeuse, selon la nature des poils qui la eomposent.

30 Le polakène (polakenium, Rich.). On appelle ainsi un fruit simple, qui, à sa parfaite maturité, se sépare en deux ou un plus grand nombre de loges, que l'on peut regarder elıacune comme étant un akène. De là les noms de diakène, triakène, pentakène, suivant le nombre de ces pièees. Exemple: les Ömbellifères, le panais, le persil, la eiguë, les Araliaećes, ete.

Dans les Ombellifères, c'est un diakène; dans la capucine, e'est un triakène; e'est un pentaliène ou polakène proprement dit, dans les Araliacées.

$4^{\circ}$ La samare' samara, Goertner ), fruit oligosperme, coriace, membraneux, très-comprimé, offrant une ou deux loges indéliseentes, souvent prolongées latéralenient en ailes ou appendiecs ćlargis. Par exemple, le fruit de l'orme (Ulmus campestris), des érables, ete. (Voyez pl. 8, fig. 6.)

- 5 Le gland (glans), fruit uniloeulaire, indéhiscent, monosperme (par l'avortement constant de plusieurs ovules ), provenant constamment d'un ovaire infère, pluriloculaire es polysperme, dont le péricarpe, uni intimement à la graine, présente toujours à son sommet les dents exeessivement petites du limbe du calice, et est renfermé en partie, rarement en totalité, dans une sorte d'involucre écailleux ou foliacé, nommé cupule. Par excmple, le fruit des chênes, du noisetier, ete. (Voy. pl. 8, fig. 7.)

La forme des glands esi en général très-variable. Il y elı a d'alongés, d'autres qui sont arrondis et comme sphériques; dans les uns, la cupulc est squamacéc ęt très-courte; dans d'autres, elle est fort développée et recouvre presque cutièrement le fruit. 
$6^{\circ}$ Le carcérule (carcerulus, Desvaux), fruit sẹe, pluriloeulaire, polysperme, indéhiseent; tel est eclui du tillcul.

$7^{\circ}$ On a appelé fruits gynobasiques ceux dont les loges sont tellement éeartées les unes des autres, qu'elles semblent eonstituer autant de fruits séparés, et que le style. paraît haître immédiatement du disque ou gynobase, par suite de la dépression eonsidérable que l'axe du fruit a éprouvée. Tel est le fruit des Labiées, des Boraginées, qui est formé de quatre akènes réunis à leur base sur un réeeptacle eommun; eelui des Simaroubées, etc.

\section{\$2. Fruits secs et déhiscens.}

Les fruits sees et déhiscens sont le plus souvent polyspermes; le nombre des valves et des loges qui les composent est très-variable. On les désigne, en général, par le nom de fruits capsulaires.

$1^{\circ}$ Le follicule ( folliculus), fruit géminé ou solitaire par avortement, ordinairement membraneux, uniloeulaire, univalve, s'ouvrant par une suture longitudinale, à laquelle s'attache intérieurement un trophosperme sutural, qui devient libre par la déhiseence du péricarpe. Rarement les graines sont attachées aux deux boids de la suture. Cette espèce de fruit est propre à la famille des $A$ poeynées, tels qu'au laurier rose (Nerium oleander), à l'Asclepias syriaca, au dompte-venin (Asclepias vincetoxicum), ete. (Voy.pl. 8, fig. II.)

$2^{\circ} \mathrm{L}$ a silique (siliqua), fruit sec, allongé, bivalve, dont les graines sont attachées à deux trophospermes suturaux. Ellc est ordivairement séparée en dcux loges par une fausse cloison parallc̀le aux valves, qui n'est qu'un prolongemont des trophospermes et qui persiste souvent après la chute des valves. Ce fruit appartient aux Crucifères; exemple: la giroflée, le choux, cic. (Voy. pl. 8, fig. I ${ }^{\text {re }}$ :) 
$3^{\circ}$ La silicule (silicula) diffère à peine de la précédente. On donne ce nom à une silique dont la hauteur n'est pas quatre fois plus eonsidérable que la largeur. La silieule ne contient quelquefois qu'une ou deux graines. Tels sont les fruits des Thlaspi, des Lepidium, des Isatis, etc. (Voyez pl. 8, fig. 2.)

Elle appartient également aux plantes Crucifères.

$4^{\circ} \mathrm{La}$ gousse, ou légume (legumen), est un fruit see, bivalve, dont les graines sont attaehées à un seul trophosperme, qui suit la direetion de l'une des sutures. Ce fruit appartient à toute la famille des Légumineuses, dont il forme le prineipal caractère. Par exemple, dans les pois, les fèves, les haricots, ete. (Toy. pl. 8, fig. 3.)

La gousse est naturellement uniloculaire; mais quelquefois elle est partagée en deux ou un plus grand nombre de loges par de fausses eloisons. Ainsi elle est biloculaire dans I'astragale.

Dans les easses, la gousse est séparée en un nombre considérable de loges par des diaphragmes ou fausses eloisons transversales. Ce caraetère appartient à tout le genre Cassia.

Quelquefois la gousse semble être formée de pièces artieulées; on dit alors qu'elle est lomentacée, eonme dans les genres Hippocrepis, Hedysarum, ete.

D'autres fois la gousse est enflée, vésieuleuse, à parois minees et demi-transparentes, comme dans les bagucnaudiers (Colutea).

Le nombre des graines que renferme la gousse varie beaucoup. Ainsi il y cn a une seule dans le Medicago lupulina, deux dans les véritables Ervum, ete.

Quelquefois la gousse est tout-à-fait indéhiseente, comme dans le Cassia fistula et d'autres espèees du mème genre; mais ees variétés sont rares et ne détruisent pas les caraetères propres à ectte espèce de fruit.

$5^{\circ}$ La pyxide (pyxidium, Erh.) est un fruit eapsulaire, 
see, ordinairement globulcux, s'ouvrant par une scissure transversale, en deux valves hémisphériques superposées. C'est ee que l'on observe dans le pourpier, le mouron, la jusquiamc, etc. Les auteurs la désigncnt communément par lc nom de boîte à savonnette (Capsula circumscissa, L.) (Voy. pl. ro, fig. S.)

$6^{\circ}$ L'élatérie (elaterium, Rich.), fruit souvcnt rclevé de côtes, se partageant naturellement à sa maturité en autant de coques distinctes s'ouvrant longitudinalement, qu'il présente de loges, comme daus les Euphorbiacées. Dc là les expressions de tricoque, mullicoque, domnées à ce fruit.

Ordinairement ees eoques sont réunies par une columelle centrale qui persiste après leur chute.

$7^{\circ} \mathrm{La}$ capsule (capsula); on donne ce nom général à tous les fruits sees et délhiscens qui ne peuvent être rapportés à aucume des espèces précédentes. On conçoit d'après cela que les capsules doivcnt être extrêmement variables.

Ainsi il y a des capsules qui s'ouvrent par des pores ou ouvcrtures pratiquées à lcur partic supérieure; telles sont celles des pavots, des Antirrhinum. D'autres fois ces pores sont situés vers la base de la capsulc. Plusjeurs ne sont déhiscentes que par leur sommet, fermé par des dents rapprochées, qui s'écartent lors de la parfaite maturité. C'csl ce que l'on remarque dans beaucoup de genres de la famille des Caryophyllées. (Voy. pl. 8, fig. 4.)

\section{SECTION II.}

TRUITS GHARIS.

Les fruits charnus sont indéhiscens. Leur péricarpe esł épais et pulpeux; ils renferment un nombre de graines variablc. Les espèces principalcs sont :

I ${ }^{\circ}$ La drupe (drupa) est un fruit charnu qui renferme un noyau dans son intéricur. Ce noyau est formé par l'en- 
doearpe endurci et ossifié, auquel s'est joint une partie plus ou moins épaisse du sareocarpe, eomme, par exemple, dans la pêehe, la prume, la ecrise, ete. (Voyez pl. 8, fig. 8.)

$2^{\circ}$ La noix (nux) ne diffèrc de la drupe que par l'épaisseur moins eonsidćrable de son sarcoearpe, qui porte alors le nom de brou (naucum). Tel est le fruit de l'amandier (Amygdalus contmunis), le fruit du noyer (Juglans regia), que l'on désigne même par le nom de noix proprement dite.

$3^{\circ}$ Le nuculaine (nuculaniunn, Rieh.) est un fruit eharnu provenant d'un ovaire libre, e'est-à-dire non eouronné par les lobes du ealiee adhérent, et renfermant dans son intćrieur plusieurs petits noyaux, qui portent alors le nom de micules (nuculce, Rich.). Tels sont les fruits du sureau, du Tierre, des Rhammées, du sapolilier' (Achras Sapota).

$4^{\circ}$ La mélonide (melonida, Rieh.) cst un fruit eharnu, provenant de plusieurs ovaires pariétaux réunis et soudés avce le tube du ealiec, qui, souvent très-épais et eharnu, se eonfond avee eux, eomme dans la poire, la pomme, la nèfle, le rosier, ete. (I). (Voy. pl.8, fig. 9.)

Dans la mélonide, la partie réellement eharnue du fruit n'est pas formée par le púriearpe lui-nuème; elle est due à un'épaississcment eonsidérable du ealiee; e'est ee que l'on peut voir facilement quand on suit a vee attention le développement de ce fruil.

(x) Cette espèce de fruit a jusqu'iei été fort inal définie par les autcurs?, puisqu'on la décrit eomme provenant d'un ovaire infère, multiloculaire, à loges distinctes. Mais nous avons déjà démontré préeédemment la grande différenee qui existe entre l'ovaire vraiment infère et l'ovairè simplement pariétal. L'inférité de l'ovaire en exelut toujonrs la pluralité dans la mêne fleur. Or, dans Ia plupart cles vraies Rosacées, il y a plusiears pistils, dont on peut suive graduellement les différens degrés d'adbérenee laténale avee Ia paroi interne dụ calice. Ainși, par exemple, dans le genre Rosa, lẹs piș- 
L'endoearpe quil revêt chaque loge d'une mélonide est cartilaginenx ou osscux; dans ce dernier cas il y a autant de nucules qu'il y a d'ovaires, comme dans la nèfle; ce qui fait qu'on a distingué ỉa nıélonide en deux variétés, savoir :

I $^{\circ}$ Mélonide à nueules, celle dont l'endocarpe est osseux, comme dans le Mespilus, lc Cralcegus.

$2^{\circ}$ Mélonide à pepius, celle dont l'endoearpe est simplement eartilagineux, eomme dans la poire, la pomme, ete.

La mélonide appartient exelusivement à la famille des Rosacées, dans laquuclle elle est associée à quelques autres espèces de fruits, qui n'en sont souvent que des variétés.

$5^{\circ}$ La balauste (balausta), fruit pluriloculaire, polysperme, provenant toujours d'un ovaire véritablement infère ct couronné par les dents du ealice, comme celui du grenadier et de toutes les véritables Myrtées.

$6^{\circ}$ La péponide (peponida, Rich.), fruit charnu, indéhiscent ou ruptile, à plusieurs loges éparses dans la pulpe, renfermant chacune une graine qui est tellement soudée avec la membrane pariétale interne de chaque loge, qu'on parvient difficilement à l'en séparer. Ce fruit se remarque dans le melon, le potiron et les autres Cucurbitacées, les Nymplićacées et les Hydrocharidées.

Il arrive quelquefois que le parenehyme charnu qui oecupe le centre de la péponide se rompt et se déchire par l'accroissement rapide du péricarpe. Dans ce cas la partie centrale est oceupée par une cavité irrégulière,

tils, qni sont au nombre de douze ou quinze, ne ticnnent aux parois $\mathrm{du}$ tube calicinial que par un petit pédieule de la basc de leur ovaire. Dans les genres Cracagus et Mespilus les ovaires sont soudés avec le calice par tout lcur côté externe. Dans les genres Pyrtis, Malus, etc., ces ovaircs sont nonseulement unis par lenr côtć extéricur avec le calice, mais sc sondent entre eux par tous les antres points. Cependant il arrive quelquefois dans certaincs poires que les ovaires restent distincls par leur côté internc, en sorte qươ trouve au centre da fruit une cavité plus ou moins grande, 
que l'on a, mais à tort, regardée eomme une véritable loge; c'est ee que l'on observe surtout dans le potiron (Pepo macrocarpus). Mais si l'on y fait quelque attention, on verra que eette prétendue loge n'est nullement tapissée par une membrane pariétale interne, e'est-à-dire un endoearpe; ee qqui démontre évidemment que eette cavité n'est qu'aecidentelle et ne constitue point une véritable loge. En effet, elle n'existe point dans toutes les espèees; et quand elle s'y montre, ee n’est que vers l'époque de léur maturité.

On peut voir dans la pastèque ou melon d'eau ( Cucurbita citrullus, L. ) la véritable organisation de la péponide. Dans eette espèee, la partie eentrale reste eonstamment pleine et eharnue à toutes les époques de son développement. Chaque graine est renfermée dans une loge particulière, avee les parois de laquelle elle ne eontraete d'autre adhérenee que par son point d'attaehe ou son hile. Il semble, dans ee eas, que la nature, qui, dans presque toutes les autres.espèees de eette famille altère, et modifie plus ou moins la véritable strueture de ee fruit, ait voulu, en quelque sorte, en ménager un qui pût faire eomnaître le type naturel et primitif des autres.

$7^{\circ}$ L'hespéridie (hesperidium, Desvaux), fruit eharnu, dont l'enveloppe est très-épaisse, divisé intérieurement en plusicurs loges par des eloisons membraneuses, qu'on peut séparer sans aueun déehirement, comme dans l'orge, le eitron, ete.

$8^{\circ}$ La baie ( bacca). Sous ee nom général on eomprend tous les fruits eharnus, dépourvus de noyau, qui ne font pas partic des espèces préeédentes. Tels sont, par exemple, les fruits du raisin, les groseilles, les tomates, ete. 


\section{DEUXIÈME CLASSE.}

DES FIUUITS MIUITIPLES (I).

Les fruits multiples sont eeux qui résultent de la réunion de plusieurs pistils renfermés dans une même fleur.

Le syncarpe (syncarpium, Rich.), fruit multiple, provenant de plusieurs ovaires appartenant à une même fleur, soudés et réunis ensemble, même avant la fécondation. Par exemple, eeux des Magnolia, des Anona, etc.

Le fruit du fraisier, du framboisier, est formé d'un nombre plus ou moins eonsidérable de véritables petites drupes, dont le sarcocarpe est très-minee, mais cependant très-manifeste dans la framboise, réunies sur un gynophore charnu, plus ou moins développé.

Plusieurs petits akènes réunis eonstituent le fruit des renoneules, ete.

(I) C'est à cette elasse qu'appartient réellement la mélonide, que nous n'avons laissée dans la précédente que pour zous conformer à l'usage généralement adopté. 


\section{TROISIÈME CLASSE.}

\section{D2S FRUITS AGREG}

Or donne ec nom à ccux qui sont formés d'un nombre plus ou moins considérable de petits fruits rapprochés, et souvent réunis et soudés cnscmble, proveuant tous de fleurs d'abord distinetes les unes des autres, mais qui ont fini par se réunir et se souder. Tels sont :

$1^{\circ}$ Le cônze ou strobile (conus, strobilus), fruit composé d'un grand nombre d'utricules membraneuses, cachıées dans l'aisselle de bractées très-dévcloppécs, sèches, et disposécs en forme de cône. Tel est le fruit des pins, des sapins, de l'aune, du boulcau, etc.

$2^{\circ}$ Le sorose. M. Mirbel dome ec nom à la réunion de plusicurs fruits soudés en un scul corps par l'intermédiaire de lcurs enveloppes florales, charnucs, très-dévcloppées et entregreffécs, de manic̀re à resscmbler à une baic mamcloméc. Tel est le fruit du mûrier, de l'ananas, ctc.

$3^{\circ}$ Le sycône. Sous ec nom M. Mirbel désigne le fruiı du figuicr, de l'Ambora et du Dorstenia. Il est formé par un involucre monophylle, charnu à son intéricur, ayant la forme aplatic, ou ovoüde et ferméc, et contenant un grand nombre de petites drupes, qui provicnnent d'autant de flcurs femelles.

Dans les vingt-cinq espèces de. fruits dont nons venons de domner les caractères abrégés se trouvent à peu près réunis tous les types auxçucls ou peut rapporter les nombreuses variétés que ect organe peut oflrir dans les végétaux. Ce tableau est loin d'être complet. Cette partic de la botani- 
que exige encore de longs et de pénibles travaux, une analyse soignée et serupuleuse, avant d'arriver à un état toutà-fait satisfaisant. Notre inlention n'a été ici que de présenter les espèees les mieux eonnues et les mieux déterminćes, afin de ne point jeter du vague ni de l'obscurité sur un sujet déjà si diffieile par lui-même.

Pour terminer tout ee qui a rapport aux organes de la fructifieation, il nous reste eneore à parler de la dissémination et des différens avantaģes que la médeeine, les arts et l'éeonomic domestique peurent retirer des fruits et des différentes parties qui les éomposent.

\section{CHAPITRE V.}

DE LA DISSÉMINATION.

Lorsqu'us fruit est parvenu à son dernier degré cle naturité, il s'ouvre; les différentes parties qui le eomposent se désunissent, et les graines qu'il renferme rompent bientôt les liens qui les retenaient eneore dans la eavité où elles se sont aeerues. On donnne le nom de dissémination à eette aetion par laquelle les graines sont naturellenıent dispersées à la surfaee de la terre, à l'époque de leur développement.

La dissémination naturelle des graines est, dans l'état. sauvage des végétaux, l'agent le ṕlus puissant de leur reproduetion. En effet, si les graines eontenues dans un fruic n'en sortaient point pour être dispersées sur la terre et s'y. développer, on verrait bientôt des espèees ne plus se reproduire, des raees entic̀res disparaitre; et, eomme tous: Jes végélaux ont une durée déterminée, il devrail néeessairement arliver une époque où tous auraient eessé de vivre, el où la végćtation aurait pour jamais disparu de la surface du globe. 
Le moment de la dissćmination marque le terme de la vie des plantes annuelles. En effet, pour qu'clle ai licu, il est nécessaire que le fruit soit parvenu à sa maturité, et qu'il se soit plus ou moins desséché. Or, ce phénomène n'arrive, dans les herbes amnuclles, qu'à l'époque où la végétation s'est entièrement arrêtée chez clles.'Dans les plantes ligneuses, la dissémination a toujours lien pendant la période du repos que ces végétaux éproụvent lorsque leur liber s'est épuisé à donnernaissance aux feuilles et aux organes de la fructification.

La fécondité des plantes, e'est-à-dire le nombre ćtonnant de germes ou de graines qu'clles produisent, n'est point une des eauses les moins puissantes de leur facile reproduction et de leur étomante multiplication. Rai a compté 32,00o graines sur un pied de pavol, et jusqu'à 360,000 sur un pied de tabac. Or, qu'on se figure la progression toujours croissaute de ce nombre, seulcment à la dixième génération de ees végétaux, et l'on conecvra avee peine que toute la surface de la terre n'en soit point recouverte.

Mais plusieurs eauses tendent à neutraliser en partie les effets de ectte surprenante fécondité, qui bientôt muirait, par son exeès même, à la reproduction des plantes. En effet, il s'en faut que toutes les graines soient mises par la nature dans des circonstances favorables pour se développer et croître. D'ailleurs un grand nombre d'animaux, et l'homme lui-méme, trouvant leur principale nourriture dans les fruits et les graines, en déiruisent une innombrable quantité.

Plusicurs circonstances favorisent la dissémination naturelle des graines. Les unes sont inhérentes au péricarpe, les autres dépendent des graines elles-mêmes.

Ainsi il y a des péricarpes qui s'ouvrent naturellement avec ume sorte d'élasticité, au moyen de laquelle les graines qu'ils renferment sont lancées à des distances plus ou moizs 
considérables. Les fruits du sablier, par exemple (Hura crepitans), du Dioncea muscipula, de la fraxinelle, de la Jalsamine, disjoignent leurs valves rapidement et par nnc sorte de ressort, en projetant leurs graincs à quelque distance. Le fruit de l'Ecballium elaterium, à l'époque de sa maturité, se détache du pédoncule qui le supportait, et, par la cicatrice de son point d'attache, lance ses graines avec une rapidité étounante.

Il y a un grand nombre de graincs qui sont minces et légères, et peuvent être facilement entraînées par les vents. D'autres sont pourvues d'appendices particuliers en forme d'ailes ou de couronnes, qui les rendent plus légères en augmentant par ce moyen leur surface. Ainsi les érables, les ormes, un grand nombre de Conifères ont leurs fruits garnis d'ailes membraneuses, qui scrvent à les faire transporter par les vents à des distances considérables.

La plupart des fruits de la vaste famille des Synanthérécs sont couromnés d'aigrettes, dont les soies fines et délicates, venant à s'écartcr par la dessiccation, leur scrvent. en quelque sorte de parachute pour les soutenir dans les airs. Il cn est de même des valériancs.

Les vents transportent quelquefois à des distances qui paraisscnt inconcevablcs les graines de certaines plantes. L'Erigeron canadense inondc et désole tous lcs champs de l'Europc. Limnæus pcnsait que cette plante ayait été transportée d'Amérique par les vents.

Les fleuves et les eaux de la mer servent aussi à l'émigration lointaine de certains végétaux. Ainsi l'on trouve quelquefois sur les côtes de la Norwège et de la Finlande' des fruits du Nouveau-Mondc apportés par les eaux.

L'homme et les différens animaux sont encore des moyens de dissémination pour les graines; les unes s'attachent à leurs vêtemens ou à leurs toisons, au moyen des crochets dont elles sont armées, telles que cclle des graterons, des aigremoines; les autres, leur servant de nourriture, sont 
transportểes daus les lieux qu'ils habitcnt, ct s'y développent lorsqu'clles y ont été abandomées et qu'elles sc trouvent dans des cireonstances favorables.

\section{Usages des Fruits et des Graines.}

C'est dans les fruits, et surtout dans les graines d'un grand nombre de végétaux, que sont contenues les substanecs alimentaires les plus riches en principes nutritifs, et souvent des médicamens doués de vertus très-énergiques. La famille des Graminées est sans contredit uuc de celles daus lesquelles l'homme trouve la nourriture la plus abondante, ct les animaux herbivores leur pâture la plus liabitucllc. Qui ne connaît, cn ef̊ct, l’usage général que toutcs les nations civilisées de l'Europe et des autres partics du monde font du pain? Or, cet aliment par cxecllence n'estil point fabriqué avec l'cudosperme farineux du Jlé, de l'orge et d'un grand nombre d'autres Graminécs? A ce seul ritre, ectte famille naturelle des plantes n'est-elle point pour l'homme une des plus intéressantes du r'ègne végétal?

Les péricarpes d'un grand nombre de fruits sont des alimens aussi aggréables qu'utiles. Tout le monde connait les usages ćconomiques auxquels on cmploie un grand nombre de fruits charnes, tels que les pêches, les pommes, les melons, les fraises, les groseilles, etc.

Le péricarpe charnu de l'olivicr (Otwea europeea), fourrait l'huile la plus pure et la plins cstiméc.

C'est avec le sue que l'on retire par expression des finits dela vigne, soumis à la fermentaion spiritncuse, que l'on fait le vin, cette boisson si utile à l'homme, quand il cu sait fairc un usage modéré. Plusieurs autres fruits, tcls que les pommes, les poires, les sorbes, ctc., fouruissent encore des liqueurs fermentées qui servent de boisson habituelle à des provinces cl à des nations entières.

Dans l'intérieur de pluṣieurs péricarpes de la famille des 
Légumineuses on trouve une substanee acidule ou douceâtre, quelquefois nauséabonde, qui jouit de propriétés laxatives, comme on l'observe dans la easse, le tamarin, lcs earoubes, les follicules du séné, ete.

Les dattes, les figues, les jujubes, les raisins sees sont des substanees alimentaires, remarquables par la grande quantité de principe sueré qu'clles renferment.

Les fruits du citronnier et de l'oranger contiennent de l'aeide eitrique presque à l'état de pureté.

Les petits nueulaines dé nerprum (Rhamnus catharticus) sont très-purgatifs.

Les graines ne sont pas moins riches en principes nutritifs quic les péricarpes. En effet, celles des plantes Céréales ou Graminées, d'un grand nombre deLégumineuses, etc., contịennent une quantité eonsidérable de féeule amylaeée, qui leur donne une qualité nutritive très-prononeée.

Los graines du lin, du cognassier, du psyllium, renferment aussi un prineipe mueilagineux très-abondant. Aussi sont-elles essentiellement émollientes.

Un grand nombre de graines se distinguent par un principe stimulant très-aromatique. Telles sont celles d'anis (Pimpinella cunisum), de fenouil (Anethum foniculum), de coriandre (Coriandrum salivum), de carvi (Carum carvi), qui ont reçu le nom de semences carmiñatives. D'autres au contraire sont appelées semenees froides, à eause de l'aetion émolliente et sédative qu'elles exercent sur l'éeonomie animale. Telles sont celles de la cálebasse(Cucurbita lagenaria), du coneombre (Cucumis sativus), du melon (Cucumis melo), de la citrouille (Cucurbila citrullus.)

Les semences carminatives appartiennent toutes à la famille des Ombellifères. C'est la famille des Cucurbitacées qui fournit les semences froides.

Qui ne connait l'usage habituel que font tous les peuples civilisés des graines torréfiées du café, du cacao, etc.?

On retire des graines de l'amandier, du noyer, du hêtre, 
du riein, du ehenevis, du pavot, du eolza, etc. 's une huile abondante qui jouit de propriétés modifiées dans ehaeun de ees végétaux par son mélange avee d'aut,res substanees.

Les graines du rocou (Bixa orellana) servent à teindre en rouge brun.

Nous ne finirions pas si nous voulions énumérer iei tous les avantages que l'homme peut retirer des fruits en général ou des parties qui les eomrosent. Mais un pareil travail nous éloignerait trop de rotre objet. Nous avons seulement voulu indiquer, quoique bien ineomplétement, les usages nombreux des fruits er des graines, soit dans l'économie domestique, soit dans la thérapeutique.

Ici se termine tout ee qui a rapport à la partie de la botanique que nous avons désignée par le nom d'Organographic. Nous y avons donné la deseription de tous les. organes des végétaux phanérogames, et des fonctions qu'ils remplissent. Nous allons maintenant faire connaître les diverses méthodes de elassifieation qui ont été proposées pour ranger et coordonner la quantité innombrable de plantes déjà eomnues et décrites par les différens auteurs. C'est à cette partie de la botanique que l'ou a domné le nom de taxonomie. 


\title{
DE LA TAXONOMIE,
}

\author{
DOU
}

\section{DES GLASSIFICATIONS BOTANIQUES}

\author{
EN GLNERAL。
}

Nous avons déjà vu que sous le nom de taxonomic on désigne eette partie de la botanique générale qui." a pour objet l'application des lois de la elassifieation au règne végćtal.

A l'époque où les seienees n'étaient eneore qu'à leur bereeau, e'est-à-dire quand un petit nombre de faits en composait tout le domaine, ceux qui se livraient à l'étude de ees seienees 'n'avaient besoin que de fort peu d'efforts, et seulement d'une mémoire assez heureuse, pour embrasser la eomnaissanee parfaite; et retenir les noms de tous les êtres à l'étude desquels ils s'étaient livrés: Aussi les preniers philosophes qui s'oceupèrent de la botanique parlent-ils des plantes sans adopter aucuin ordre, aueune méthode d'arrangement. Du temps de Théophraste, 'par exemple, qui le premier écrivit spécialement sur les végétaux, les fonctions des organes étaient méeonnues, les genres, les espèecs entièrement confondus, leurs caraetères distinetifs ignorćs; en un mot, quoiqu'on puisse'dire que ee philosophe ait eommeneć à éerire sur là botanique, on peut également assurer que cette seienee n'existait point eneore de son temps. Les earaetères des plantes ne reposaient que sur des eonnaissanees empiriques ou de simples 
traditions; car le nombre en était alor's si borné, qu'il était faeile de les eonnaître toutes individuellcment, sans qu'il fût néeessaire de lcs distinguer autrement que par un nom particulier à ehaeune d'elles, mais auquel ne se rattaelıait aueune idée de caraetère ou de eomparaison. Tel fut l'état de la botanique pendant un grand nombre de sièeles où, intimement unie à la médeeine, elle ne trouvait place que dans les ouvrages de ceux qui éerivaient sur l'art de guérir.

Mais quand, par des reeherehes mieux dirigées et des voyages lointains, le nombre des êtres dont s'oeeupe l'histoire naturelle devint plus grand, on sentit la nćeessité de mettre plus de prćeision dans le nom de ees différens objets, de les distinguer par quelques caraetc̀res, afin de pouvoir les reeonnaître. Bientôt la mémoire ne put retenir seule les noms d'un si grand nombre d'êtres, pour la plupart nouveaux et ineonnus jusqu'alors.

Ce fut dès eette époque que l'on eommença à sentir la néeessité de disposer les objets dans un ordre queleonque qui pût: en faeiliter la reeherehe, en domnant les moyens d'arriver plus promptement et avee plus de sûreté aux noms qui avaient ćtć donnés à ehaeun d'eux.

Mais ees arrangemens, d'abord purement empiriques, ne doivent point être regardés comme de véritables méthodes. En effet, ils n'étaient nullement fondés sur des connaissanees tirées des earaetères propres à chacun de ces êtres, et qui puissent servir à les distinguer les uns des autres, mais appuyés seulemcut sur quelques eireoustanees extérieures, et souvent étrangères à la nature même de l'objet. Ainsi l'ordre alphabétique suivant lequcl on rangea lcs végétaux ne pouvait avoir d'avantage que pour eeux qui les eonnaissaient déjà, mais qui voulaient se livrer à des reeherehes partieulic̀res sur quelques-uns d'entre eux. Il en est de mème de l'arrangement fondé sur les propriétés éeonomiques ou médieales des plantes, qui suppo- 
sent toujour's la eomnaissanee préalable des vertus de la plante dont on veut trouver le nom.

On pense bien que sur de semblables bases ne devaient s'élever que des elassifications aussi fautives qu'imparfaites, puisqu'elles reposaient, en général, sur des eonnaissanees étrangères à la nature et à l'organisation des végétaux. Elles ne pouvaient done en donner aueune idée satisfaisante.

L'expérience fit bientôt sentir la nécessité dè tirer de l'organisation mème des plantes et des parties qui les eomposent les caractères propres à les faire eonnaître et à les distinguer. Ce fut dès eette époque que la botanique devint réellement une seienee; ear ee fut alors que l'on eommenẹa à étudier l'organisation des végétaux pour pouvoir en tirer les earactères propres à les faire eonnaître et à les distinguer.

Dès lors les méthodes furent réellement eréées. Mais comme le nombre des organes des végétaux est assez eonsidérable, le nombre des méthodes fut égạlement trèsgrand, paree que ehaque auteur erut reeonnaître dans l'un d'eux les bases les plus solides d'une bonne elassifieation. Ainsi les uns fondèrent leur méthode sur la considération des raeines et de toutes les modifieations qu'elles peuvent offrir, les autres sur les tiges; eeux-ei sur les feuilles, tel que Sauvages, eeux-là sur l'infloreseence, ete.

Dans le seizième sièele, Gessner, né à Zurieh, fut le premier qui démontra que les earactères tirés de la fleur et du fruit étaient les plus ecrtains et les plus importans pour arriver à une bonne elassifieation des végétaux. Il fit de plus entrevoir qu'il existe dans les plantes des groupes eomposés de plusieurs̉ espèces réunies par des earaetères communs. Cette première idée de la réunion des végétaux en genres eut la plus grande influenee sur les progrès ultérieurs de la botanique.

Peu de temps après, Cæxalpin, né en I 5 r9, à Arezzo 
en Toscane, donna le modèle de la première méthode botanique. En effet, toutes les espèces y sont rangées d'après la considération des caractères que l'on peut tirer de la plupart des organes des végétaux, tcls que leur durée, la présence ou l'absence des fleurs, la position des graines, leur adhérence avee le calice, le nombre et la situation des cotylédons, ete. L'inventiou d'une semblable méthode, tout imparfaite qu'elle est, doit être considéréc comme le premier aperȩu d'une classification naturelle.

Cependant les découvertes nouvelles allaient toujours augmentant le nombre des végétaux connus, et chaque jour les ouvrages existans devenaient de plus en plus insuffisans. Plusieurs auteurs, parmi lesquels on doit eiter avec éloge les deux frères Bauhin, Rai, Magnol et Rivin, donnèrent successivement dans leurs écrits des preuves d'un mérite rare. Quelques-uns d'entre cux créćrent même des méthodes nouvelles, mais qui toutes furent éclipsées par celle que Joseph Pitton de Tournefort publia vers la fin du dix-septième siècle.

Ce botaniste célèbre, l'un de ceux dont les écrits ont fait le plus d'honneur à la France, était né à Aix en Provence, le 5 juin r656. Il fut professcur de botanique au jardin des plantes de Paris, sous le règne de Louis XIV, qui en I 700 lui donna une mission importante pour le Levant. Tournefort parcourut alors la Grìce, les bords de la mer Noire et les îles de l'Arehipel. Il revint à Paris, et publia la relation de son voyage, que l'ou peut citer comme un des modèles les plus parfaits en ce genre. Avant son départ, il avait déjà frit conmaître, dans son ouvrage intitulé Institutiones rei herbarice, sa nouvelle méthode, dans laquelle se trouvaient décrites dix mille cent quarante-six espèces rapportées ì six cent quatrevingt-dix-huit genres.

Le mérite de 'Tournefort n'est pas seulement d'avoir créé une méthode ingénieuse, dans laquelle se trouvent 
'décrites et rangées toutes les plantes connues jusqu'à lui ; mais son prineipal titre de gloire est d'avoir, le premier, distingué d'une manière plus précise et plus rigoureuse qu'on ne l'avait fait jusqu'alor's les genres, les espèces et les variétés qui peuvent s'y rapporter.

Avant lui, en effet, la science n'était encore que confusion et désordre; ehaque espèee n'était pas nettement distinguée de celles dont elle se rapproehait. Ce fut lui qui débrouilla ee ehaos, sépara les genres et les espèces par des phrases earaetéristiques, et, au moyen de son système ingénieux, rangea méthodiquement les plantes connues à cette époque.

Après Tournefort parurent encore un grand nombre de botanistes qui ont joui d'une certaine réputation. Quelques-uns d'entre eux proposèrent des méthodes nouvelles; mais aueune n'avait porté la moindre atteinte à celle de Tournefort. Cette gloire semblait réservée à l'immortel Linnæus. Son système, qu'il publia en 1734 , eut la vogue la plus surprenante, à cause de son extrême simplicité, et de la faeilité singulière qu'il offre pour parvenir à la connaissance du nom des végétaux.

Linnæus eut de plus la gloire de réformer, ou plutôt de créer la nomenclature et la synonymie botaniques, eneore si peu avancées par ses prédécesseurs. Tournefort lui en avait traeé la route, sens eependant en faire disparaitre tous les obstaeles. Chaque espèee, en effet, était eneore dénommée par une phrase caraetéristique, dans lacquelle on ne trouvait souvent pas les earactères propres à la distinguer. Or, ces phrases étant fort longues, il était trèsdiffieile d'en retenir' un grand nombrrs. Linnæus donna à chaque groupe ou genre un nom piropre ou générique, imitant en eela l'exemple de Tour.nefort ; mais de plus il désigna cliaque espèce de ces genres par un nom adjectif ou spécifique ajouté à la suite du zom générique. Par ee 
moyen ingénieux, il simplifia considérablement l'étude déjà fort étendue de la botanique.

Lesystème sexuel de Limnæus, séduisant par son extrême simplicité, exeita une révolution subite dans la seience, et fut aeeueilli partout avee un enthousiasme diffeile à déerire.

Quand le premier mouvement d'admiration qu'inspirc toujours une grande découverte fut un peu ealmé, on ne tarda point à s'apereevoir que ce système si ingénicux présentait eependant quelques ieonvéniens, et n'était point à l'abri de toute espèee de reproehe. En effet, fondé uniquement sur la eonsidération absolue d'un seul organe, il éloignait souvent des plantes que tous les autres earactères semblaient réunir trop étroitement pour que l'on pût jamais les isoler avee suecès; ear déjà l'on avait commencé à entrevoir que eertains genres de végétaux ont entre eux tant de points de contaet et de ressemblanee, que, réunis par l'ensemble général de leurs earaetères, ils paraissent en quelque sorte être tous membres d'une même famille. C'est ainsi, par exemple, qu'on avait déjà rapproché en tribus distinetes les Graminées, les Labiées, les Ombellifères, les Légumineuses, les Crueifères, ete., et plusieurs autrés groupes tout aussi naturels. Or, un grand défaut du système artifieiel de Linnæus était done de séparer ees plantes qui paraissaient devoir être pour toujours réunies. Ainsi les Graminées s'y trouvaient dispersées dans la première, la sceonde, la troisième, la sixième, la vingtunième et la vingt-truisième elasse de son système. Les Labiées étaient en partie dans la seconde elasse, et en partie dans la quatorzième. Il en était de même de la plupart des tribus naturelles cléjà reeomnues et conservées par un grand nombre de botanistes. Linnxus, obligé de suivre rigoureusement son système, s'était ainsi vu forcé de les séparer et de les disperser. 
Une nouvelle méthode qui, en eonservant les affinités déjà reeomnues de eertaines plantes, aurait offert l'ensemble de leurs earaetères distinetifs, eût done été préférable à ee système si ingénieux; mais qui péehait par un des points les plus essentiels.

Adanson avait donné la première esquisse de eette méthode. Bernard de Jussieu médita pendant quarante ans afin de trouver les earaetères les plus solides et les plus eonstans qui pussent lui servir de base. Il étudia avee un soin extrême l'affinité réeiproque des diverses espèees et des différens genres entre eux. Mais ee fut son neveu, Antoine Laurent de Jussieu, qui, rassemblant les riehes matériaux reeueillis par ses oneles, y joignant les nombreuses observations qu'il avait lui-même amassées, eréa réellement la méthode des familles naturelles, telle que nous l'exposerons bientôt. Ce fut dans son Genera plantarum, ouvrage marqué du sceau du génie, et l'un des plus beaux monumens des progrès de la botanique, qu'il posa les fondemens d'une méthode qui doit un jour être la seule suivie et adoptée par tous les bons esprits; car elle est, sans eontredit, de toutes les autres publicées jusqu'à ee jour, celle qui mérite la préférence.

En effet, elle n'a point pour base la eonsidération d'un seul organe; mais elle éludie l'ensemble des earaetères fournis par ehaeune des parties d'un végétal, et rapproehe les uns des autres tous eeux qui se touehent par le plus grand nombre de points de eontaet et de ressemblanee. C'est eette méthode qui, depuis près de quarante ans, a fait faire à la botanique de si rapides progrès et l'a placée au premier rang parmi les seienees naturelles.

Nous avons eru devoir entrer dans quelques détails sur les méthodes en général, avant de faire l'exposition particulière d'aneune d'elles. Il nous a semblé utile de jeter rapidement un eoup d'ocil sur les prineipales époques de la botanique, afin de faire mieux eonnaître l'impulsion et 
la face nouvelle que les trois classifications de Tournefort, de Linneus ct de Jussicu ont, chacune en particulier, donnécs à la botanique.

En terminant ces considérations généralcs, nous devous faire remarquer qu'il existc deux cspèces bien distinctes de classifications en histoirc naturellc. Dans l'une, en effet, on nc prend pour base que la considération d'un scul organe. Ainsi Tourncfort s'est servi de la corollc, Linnæus des étamines, pour établir lcurs principales divisions. On a donné le nom de systèmes à ces arrangcmens purcmeut artificicls. On conçoit qu'un système n'ayant uniquement pour but que de faịc arriver avec facilité au nom d'unc plante ne dome aucune idée de son organisation. Ainsi quand nous avons trouvé qu'une plante est de la première classe du système de Linneus ou de elcui de Tournefort, nous savons sculcment, dans le premicr cas, qu'cllc a une ćtaminc; dans le second cas, que sa corolle cst monopétale, régulière ct campaniforme; mais ces systèmes ne nous apprennent rien touchant les autres partics qui composent la plante dont ils nous ont sculement appris le nom. Dans la seconde espèce de classification, qui a reçu le nom de méthode proprement dite, comme les bases de chaque classe reposent sur la somme totale de tous les caractères tirés des différentes parties du végétal, lorsque l'on cst arrivé à l'une de ces classes, on connaît déjà les points les plus saillans de l'organisation de la plante dont on désire connaître le nom. Si, par exemple, au moyen de l'analyse nous sommes arrivés à savoir que telle plante est, je suppose, de la quatrième classe de M. de Jussieu, ectte connaissance nous apprendra que ectte plante est une Phanćrogame, que son embryon n'a qu'un scul cotylédon, qu'elle n'a qu'une scule cnveloppe florale, c'cst-à-dire qu'un calice monosépale adhérent arec un ovairc infère, que ses étamines sont insćrées sur l'ovairc, ctc., ctc. Onl voit combien l'étude de la méthode des familles naturclles domne 
des idées plus préeises et plus philosophiques sur la strueture ét l'organisation des différens végétaux. Elle mérite donc à juste titre la préférence sur toutes celles qui ont été inventées jusqu’à ee jour.

Il serait aussi long qu'inutile de faire iei l'exposition de toutes les méthodes qui ont été proposées par les différens botanistes pour grouper et coordonner cn elasses tous les végétaux connus. Le nombre de ees méthodes est d'ailleurs si considérable, que leur exposition ne peut être faite, môme d'une manière abrégéc, que dans un ouvrage spéeialement destiné à cet objet. Aussi nous contenteronsnous d'exposer ici seulement les trois classifications les plus importantes, qui sont cclles de Tournefort, de Linnæus et de Jussieu.

\section{DE LA MÉTHODE DE TOURNETORT.}

LE système de Tournefort, généralement eonnu sous le nom de méthode de Tournefort, est basé principalement sur la eonsidération des différentes formes de la eorolle. Un reproehe généralement fait à Tournefort est de n'avoir pas suivị l'exemple déjà donné par Rivin, et d'avoir eneore séparé les uns des autres les végétaux herbacés et les végetaux à tige ligneuse. Cet inconvénient est très-grànd, puisque souvent dans le même genre on troụve réunies eés deux modifications de la tige, et que même quelquefois, eomme nous l'avons prouvé préeédemment, eertaines cireonstances peuvenit agir assez directemcnt sur une même espèce pour la rendre tantôt ligneuse, tantôt herbacée. C'cst ee que nous avons fait remarquer pour' le ricin, la belle-de-nuit, ete. 
Cc système est composé de vingt-deux elasses, dont les caraetères sont tirés : $I^{\circ} \mathrm{dc}$ la consistance et dc la grandcur de la tige $; 2^{\circ}$ de la présencc ou de l'absenee de la corolle; $3^{\circ}$ de l'isolement de chaquc flcur ou de lcur réunion dans un involucre commun; ce qui eonstituc lcs flcurs composées; $4^{\circ}$ de l'intégritć de la corollc, ou de sa division en segmens isolćs, e'cst-à-dirc dc la eonsidération de la corolle monopétale ou polypétalc; $5^{\circ}$ de sa régularitć ou de son irrégularité.

$\mathrm{I}^{\circ}$ Sous le rapport de la consistanee ct de la duréc de leur tige, Tournefort divise les végétaux en hcrbcs et sousarbrisseaux, arbrisseaux ct arbrcs. Lcs hcrbcs et les sousarbrisscaux réunis sont renfermés dans les dix-sept premières elasscs; lcs cinq dernicres classes eontienncnt les arbrisscaux ct les arbres.

$2^{\circ}$ D'après la préscnce ou l'absence de la corollc, lcs hcrbes sont distinguécs en pétalées ct apétalćes. Les quatorze prcmières classes dcs hcrbes rcnferment toutes cclles qui sont pourvucs d'une corollc; les trois autrcs, cellcs qui en sont dépourvucs.

$3^{\circ}$ Les herbcs qui ont unc corollc ont leurs fleurs isolées et distinctcs ou réunies pour eonstituer des fleurs eomposées. Les onzcs prcmières classes renferment les herbes à flcurs simples; lcs trois suivantcs, cclles qui oflrent des fleurs composécs.

$4^{\circ}$ Parmi lcs plantcs herbacécs à fleurs simplcs, lcs unes ont une corolle monopétale; dans lcs autres, au contraire, elle est polypétale. Dans les quatrc prcmières classcs, Tourncfort a réuni les plantes à eorollc monopétale; dans les cinq qui suivent, eelles dont la corolle est polypétalc.

$5^{\circ}$ Mais cettc corolle monopétalc ou polypétale peut ètre régulière ou irrégulièrc; ce qui a servi à subdiviser cneorc chacune de ecs seetions.

Lcs plantes à tige ligneuse, avons-nous dit, sont renfcrmées dans les cinq dernières classes du système. Tour- 
nefort les a divisées d'après les mêmes considérations que les herbes. Ainsi elles sont apétalées ou pétalées; leur eorolle est monopétale ou polypétale, régulière ou irrégulière.

Il est important de faire remarquer que Tournefort appelait corolle les périanthes simples et eolorés, comme dans la tulipe, le lis, qui ont, selon lui, une corolle polypétale régulière.

Tels sont les prineipes qui ont dirigé Toumefort dans la formation des classes de son système, dont nous allons . présenter sommairement les caractères. 


\section{PREMIERE DIVISION.}

\section{HER18ES.}

\section{$§$ I. $\Lambda$ FLEURS SIMPLES.}

\section{$\int$ Première Classe.}

Campaniformes. Herbes à corolle monopétale réguličre, imitant une cloehe, eomme dans la eampanule, le liscron, cte., ou un grelot, eomme daus lemuguet, la bruyère, ete.

Corolle monopétale régulière. (Voy.pl. 5, fig. 3, 4.)

\section{Seconde Classe.}

Infundibulifonmes. Herbes à corolle monopétale rćgulic̀re, imitant la forme d'un entomnoir, comme le tabac, celle d'une coupe antique, c'est-ì-dire hypocratériforme: le lilas; ou d'unc rouc (cor. rotacée), eomme la bourrache. (Voy. pl. 5, fig. I, 2.)

\section{Troisième Classe.}

Personntes. Corolle monopétale irrégulière, imitant la forme d'un mufle de veau ou d'un masque antique, eomme celle des Antirrhinum, de la linaire, ete., ou ayant lc limbe plus ou moins ouvert, comme dans la digitalc, la scrophulairc; les plantes de eette elasse présentent toujours un ovairc simple au fond de leur ealiee. ( $V_{\text {oy }}$. pl. 5, fig. 7.)

\section{Quatrième Classe.}

Labiées. Corollc monopétalc irrégulière, dont le limbe est comme divisé en deux lè- 
suite. $\quad \begin{aligned} & \text { vres; plantes offrant un ovaire partagé en } \\ & \text { quatre lobes très-distincts }\end{aligned}$

Corolle monopétale irrégulière: des graines nues. Telles sont la sauge, le romarin, la bétoine, le thym, ete. ( $/$ oy. pl. 5, fig. 8.)

$\left\{\begin{array}{c}\text { Cinquième Classe. } \\ \text { Cruciformes. Corolle polypétale régu- } \\ \text { lière, composée de quatre pétales disposés } \\ \text { eñ eroix. Le fruit est une silique ou une si- } \\ \text { lieule. Ex. : la giroflée, le choux, le thlas- } \\ \text { pi, ete. (Voy.pl. } 5, \text { fig. } 9 \cdot) \\ \text { Sixième Classe. }\end{array}\right.$

Rosacées. Corolle polypétale régulière; eomposée de trois à dix pétales disposés en rose, comme dans le poirier, le pommier, le rosier sauvage, la fraise, la framboise, les

Corolle polypétale régulière.

\section{Septième Classe.}

Ombeliffìres. Corolle polypétale? régulière, eomposée de cinq pétales souvent inégaux, fleurs disposées en ombelle. Ex. : l'angélique, le panais, le fenouil, ete.

\section{Huitième Classe.}

CAryophyluérs. Corolle polypétale régulière, formée de èinq pétales longuement onguiculés, réunis dans un ealice monosépale; limbe étalé; par exemple, l'ocillet, la saponaire, l'A grostemma Githago, et en géral les Ceryophyllées. (Voypl. 5, fig. Io.) 
Suite.

Corolle polypétale régulière,

Corolle polypétale îrrégulière.

\section{Neuvième Classe.}

Liuracéts. Flcurs à corolle ]e plus souvent polypétale, composéc de six ou simplement dc trois pétales; quelquefois monopétale, à six divisions; le fruit est une capsule ou unc baic triloculairc. Ex. : lc lis, la tulipe, la jacinthe, ctc.

\section{Dixième Classe.}

Papintonacies, on Légumineuses. Corolle polypétale irrégulière, eomposée de einq pétales, l'un supéricur, nommé étcndard, deux latéraux, appelés les ailes, deux inférieurs, quelquefois rémis et soudés, eonstituant le carènc. Ex.: le pois, le harieot, la luzerne, etc. Le fruit cst toujours une gousse. (Voy. pl. 5, fig. I2.)

\section{Onzième Classe.}

Aromales. Cette classe renferme toutes les plantes herbacées dont la eorolle est polypétale irrégulicire et non papilionneéc; telles sont la violettc, la capucine, ete.

\section{\2. A FLEUnS compostes.}

\section{Douzième Classe.}

Composées.

Flosculeuses. Fleurs composécs de petites corolles monopétales régulières infundibuliformcs, à limbe découpé en cinq divisions. On dome à chacune de ees petites fleurs le nom de fleurons. Tcls sont les cliardons, les 
MÉTHOdE DE TOURNEFORT. artichauis, les centaurées, etc. (Voy. pl. 5, fig. 5.)

\section{Treiziène Classe.}

Srma-Froscticuses. Fleurs eomposées d'un grand nombre de petites eorolles monopétales irrégulières: clont le limbe est déjeté d'un Composées. eôté, et auxquelles on a donné le nom de denti-fleurons. Par exemple, la laitue, le salsifis, le pissenlit, etc. (Voy.pl.5, fig.6.)

\section{Quatorzième Classe.}

Radiées. Fleurs composées de fleurons nu centre et de demi-fleurons à la circonférence, comme dans le grand solẹil, la reine-marguerite, étc.

\section{PLANTES APÉTALES.}

\section{Quinzième Classe.}

Apt́tales. Plantes dont les fleurs n'ont point de véritable corolle, comme les Graminées, l'orge, le riz, l'avoine, le blé, ete. Dans quelques-unes, on trouve autour des organes sexuels un périanthe simple ou caliee, qui souvent subsiste après la floraison, Apétales. \{et s'aceroît avee le fruit, eomme dans les Rumex.

\section{Seiziène Classe.}

Aptetales sans fleurs. Plantes qui sont dépourvues d'organes sexuels et d'enveloppes florales proprement diles, mais qui ont des feuilles. Ce sont les Fougìres, tels que le polypodte, le cétćrach, l'osmonde, etc. 
Apélales, $\left\{\begin{array}{l}\text { Dix-septième Classe. } \\ \text { Apt́thles, sans flcurs ni fruits apparens, } \\ \text { comme les Champignons, les Mousses, les } \\ \text { Lichens, cte. }\end{array}\right.$

\section{DEUXIEME DIVISION.}

\section{AIRIRS.}

$\left\{\begin{array}{c}\text { Dix-Tuitième Classe: } \\ \text { Arbres ou arbrisscaux Apétales, c'est-à- } \\ \text { dire dont les fleur's sont dépourvues de co- }\end{array}\right.$ rolle. Ces arbres sont ou hermaplirodites, ou monoïques, comme le buis, beaucoup de Conifères, cte.; ou dioïques, comme le pistachicr, le lentisque.

\section{Dix-nenviome Classe.}

Anentacís. Arbres apétales, dont les fleurs sont disposées en chaton. Ils sont monoïques, comme le chène, le noyer, ete.; diö́ques, comme les saules, ete.

Monopétales, $\left\{\begin{array}{c}\text { Vingtiène Classe. } \\ \text { Arbres à eorolle monopétale régulière ou } \\ \text { irrégulière, tcls que le lilas, le sureau, le ca- } \\ \text { talpa, l'arbousicr, cte. }\end{array}\right.$ Vingt-zuniòme Classe.
réguliéres. $\left\{\begin{array}{c}\text { Arbres ou arbrisscaux à corolle polypétale } \\ \text { rosacée, comme le pommicr, le poirier, l'o- } \\ \text { ranger, le cerisicr, etc. }\end{array}\right.$ Polytépales $\left\{\begin{array}{c}\text { Vingt-deuxième Classe. } \\ \text { Arbres ou arbrisscaux dont la corolle est }\end{array}\right.$ irrégulières. papilionacéc, comme dans l'acacia, le faux ébénier, l'arlore de Judée, etc., etc. 
Telles sont les vingt-deux classes établies par Tournefort pour disposer tous lcs végétaux comnus. Quoiqu'au prcmicr abord, ec système paraisse simple ct d'une exécution facile, ccpendant il offre, dans plus d'un cas, des difficultés qu'il n'cst pas aisé de faire disparaître. Ein effct, la forme de la eorolle n'est pas toujours si bicn trañchée; que l'on puisse sur-le-champ décidcr à quellc classe elle appartien't réellement; car où est le point juste de séparation entre une eorolle hypoeratériforme, et unc corolle infundibuliforme; entre cette dernière et la eorolle campanulée?

Lc reproche le micux fondé que l'on puisse faire à ce système, c'est la séparation des plantes herbacées des ligneuses. En effet, les rapports les plus naturcls sont paŕ-là méconnus, et lics végétaux qui ont entrc eux la plus grande andlogie sont souvent éloignés et rejetés à de très-grandes distances lcs uns des antres, à cause de ccttc seule différencé.

Chaeune de ces classes a été divisée en un nombre plus ou moins considérablc de sections ou ordres, dont les caractèrcs ont été tirés des modifieations particulièreš que la formc de la corolle peut subir, de la consistance, de la composition et de l'origine du fruit, de la forme, de la disposition et de la composition des fcuillcs, etc., etc.

Enfin chacune de ces sections renferme un nombre plus ou moins considérable de genres, auxquels sont rapportćes toutes les espèces eomnues jusqu'à l'époquie où Toưrnefort éerivit. 


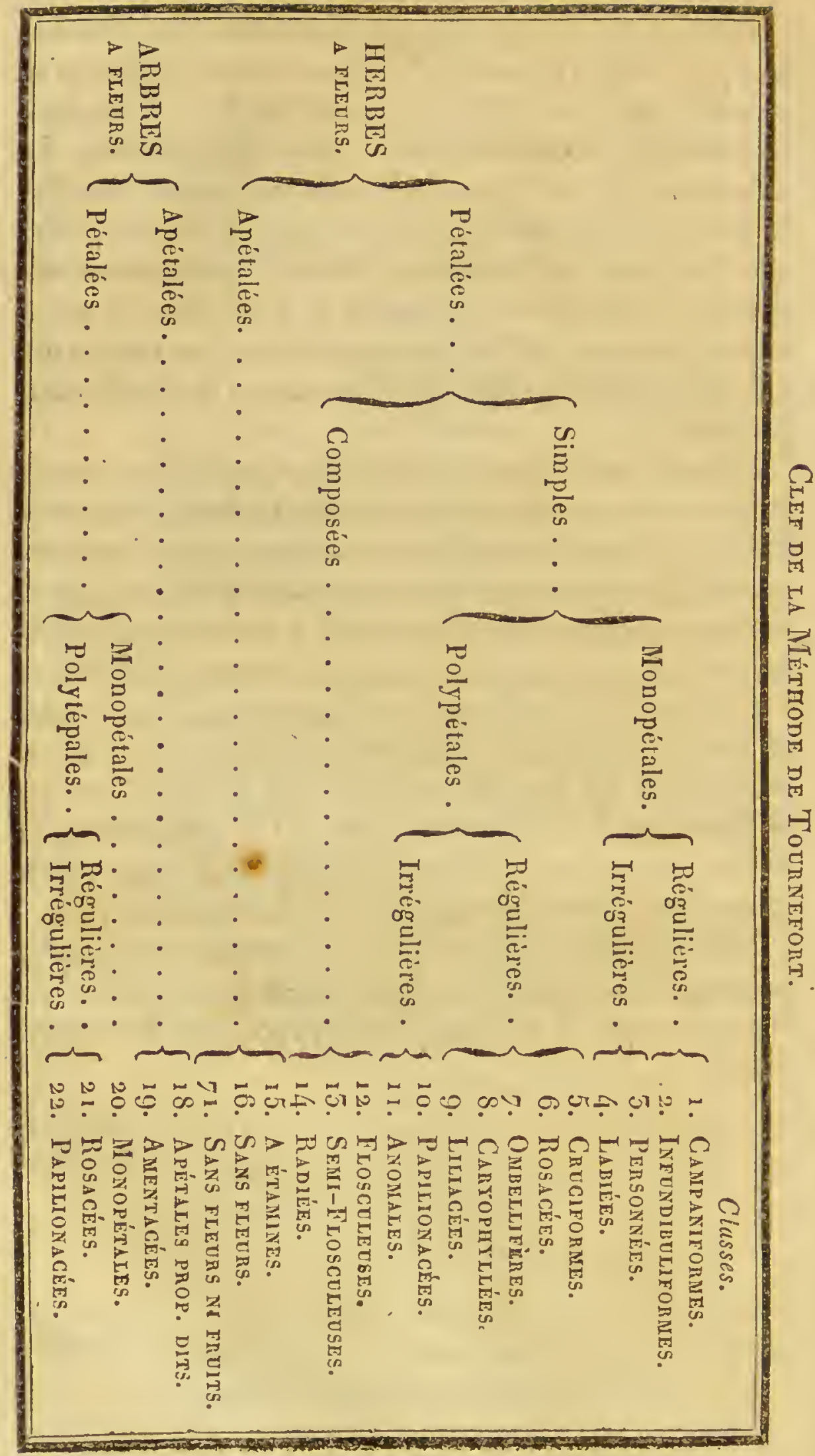




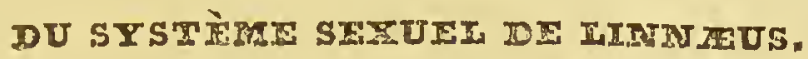

Les bases prineipales du système sexuel de Limnæus reposent presquc entièrement sur les différens earactères que l'on peut tirer des organes sexuels mâles, c'est-à-dire des étamines; de même quc celui de Tournefort est fondć sur les formes diverses que peut offrir la corolle: ee système est partagé en vingt-quatre classes.

Linnæus divise d'abord tous les végétaux connus en deux grandes seelions. Dans la première il range tous ceux qui ont des organcs sexuels, et par conséquent des fleurs apparentes. Ce sont les phanérogames ou phénogames. La seconde section eomprend les végétaux dans lesquels les organes sexuels sont eachés, ou plutôt qui en sont totalement dépourvus; on les nomme eryptogames. De là, deux prcmic̀res grandes sections dans le règne végétal :

$I^{\circ}$ Les phanćrogames.

$2^{\circ}$ Les cryptogames.

Mais, comme le nombre des végétaux de la premic̀re section est infiniment plus considérable quc cclui de la sceonde, les phanérogames ont été divisés en vingt-trois classes; les cryptogames au contraire ne forment que la vingt-quatrième ct dcrnière classe de ce système.

Parmi les plantes plianćrogames, les uncs ont des fleurs hermaphrodites, e'est-à-dire pourvues des deux scxes réunis; les autres sont unisexućcs.

Les vingt premic̀res classes du système sexuel renfcrment les végćtaux phanérogames à fleurs hermaphrodites ou monoclines; dans les trois suivantes sont placées les plantes diclines ou à fleurs unisexuées.

3० Phanérogames $\left\{\begin{array}{l}\text { monoelines. } \\ \text { dielines. }\end{array}\right.$ 
Les plantes monoclines ont les ćtamines libres et détachées du pistil; ou bien ees étamines sont soudées avec lui.

$$
4^{\circ} \text { Monoclines }\left\{\begin{array}{l}
\text { à étamines libres. } \\
\text { à étamines soudées au pistil. }
\end{array}\right.
$$

Les étamines dégagées de toute espèce de soudure avec le pistil peuvent être libres et distinctes les unes des autres; elles peuvent être réunies et soudées entre elles.

$5^{\circ}$ Etamines non $\{$ libres et distinctes. soudées au pistil. \{réunies entre elles.

Les étamines libres et distinctes sont égales ou inégales entre elles.

Celles qui sont libres et égales sont en nombre déterminé ou indéterminé.
60 Étamines libres ct égales en :
$\left\{\begin{array}{l}\text { nombre détcrminé. } \\ \text { nombre indéterminé. }\end{array}\right.$

C'est par des eonsidérations de cette nature que Limnæus est parvenu à former les bases de son système. On voit d'après cela qu'il est fondé, $I^{\circ}$ sur le nombre des étamines (les treize premières classes); $2^{\circ}$ sur leur proportion respective (quatorzième et quinzième); $3^{\circ}$ sur leur réunion par les filets (scizième, dix-scptième et dix-huitième); $4^{\circ}$ sur leur soudure par les anthères (dix-neuvième); $5^{\circ}$ sur leur soudure avec le pistil (vingtième); $6^{\circ}$ sur la séparation des sexes (vingt-unième, vingt-deuxième, vingt-troisième); $7^{\circ}$ enfin sur l'absenee des organes sexuels (la vingtquatrième et dernière).

Nous allons suceessivement étudier les earaetères de ces différentes classes, qui chaeune ont reçu des noms particuliers.

I Etamines en nombre déterminé et égales entre elles.

${ }^{\text {ro }}$ Classe. Monandris. Elle renferme toutes les plantes dont les fleurs n'ont qu'une seule étamine: l'Hippuris vulgaris, le Blitum, le Canna indica, ete. 
$2^{e}$ Classe. Diturnire. Deux étamines : le jasmin, le lilas, les véroniques, la sauge, le romarin, etc.

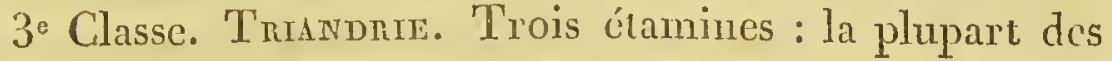
Graminées, les iris, etc.

4 C Classe. Tétraindrie. Quatre élamines : la garance, le caille-lait, les aspérules, les scabicuses, etc.

$5^{e}$ Classe. Pentandnie. Cinq étamines : les Borraginées, tclles que la bourrache, la pulmonaire; les Solanées, telles que la douce-amère, la belladone, la pomme de terre, l'allékenge, etc.; lcs Rubiacées exotiques, tels que les Cinchona, les Psychotria, etc.; les Ombellifères, tels que le panais, la cigüe, l'opoponax, la coriandre, etc.

6 e Classe. Hexandrie. Six étamines. Telles sont la plupart des Liliacées, le lis, la tulipe, la jacinthe; beaucoup d'Asparaginées, comme l'Asperge, le muguet, ctc.; le riz.

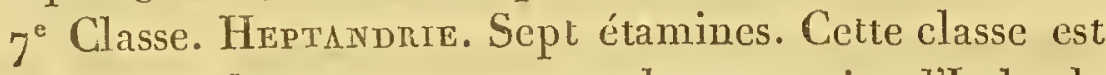
très-peu nombreuse: on y trouve le marronier d'Inde, le Saururus, etc.

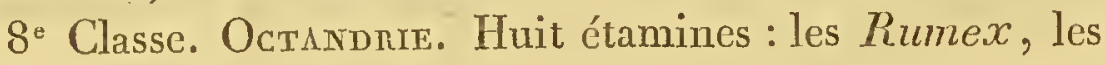
Polygonum, les bruyères.

$9^{e}$ Classe. Ennéandrie. Neuf étamines. $\Lambda$ cette classe se rapportent les différentes espèces de lauriers, de rhubarbes; le Butomus umbellatus, ,etc.

io Classe. Décandrie. Dix étamines. Nous trouvons ici presque toutes les Caryophyllées, telles que l'oillet, les Lychnis, les Silene, la rue, le Phytolacca decandra, etc.

$2^{\circ}$. Etamines en nombre non rigoureusement déterminé.

I ${ }^{e}$ Classe. Dodéchandir. De onze à vingt étamines. Exemples : l'A sarum europacum, le réséda, l'aigremoine, le Sempervivum tectorum, etc.

I $2^{{ }^{\circ}}$ Classe. Icosandrie. Plus de vingt étamines insérées sur le calice. Ici se rapportent toutes les vraies Rosacées; le prunier, l'amandier, le rosier, le fraisier, etc.; les myrthes, les grenadiers, etc. 
r $3^{c}$ Classe. Poryandure. De vingt à cent étamines, insérécs sous l'ovairc. Dans cettc classe sont réunies les véritables Renonculacées, telles que les anémones, les clématites, les renoncules, les hellébores, ctc.; la plupart des Papavéracćes, tels que le coquclicot, lc pavot, la chélidoinc, cte.

\section{$3^{\circ}$ Proporlion des élamines entre elles.}

I f $^{\mathrm{e}}$ Classc. Dinymanie. Quatrc étamincs, dont deux constamment plus petites, et deux plus longucs, toutcs insérées sur unc corolle monopétale irrégulièrc. Cette classe renferme les Labićes et les Personnćcs de Tournefort; telles sont le thym, la lavande, la bugle, la bétoine, les Anitrhimun, la digitalc, la scrophulaire, lc catalpa, ctc.

$15^{\mathrm{c}}$ Classe. Tétridoynamse. Six étamines, dont deux constamment plus petites que les quatre autres; corolle polypétale; fruit, une silicque ou une siliculc. Cette classe correspond parfaitcment aux Crucifères de Tourncfort.

\section{$4^{\circ}$ Soudure des étcimines par leur's filets.}

i $6^{\circ}$ Classc. Mona nelphue. Étamincs ell nombre variable, réunies el soudécs cnsemble cn un scul corps par leurs filcts. Excmple: la måuve, la guimauve, cte.

$17^{\circ}$ Classe. Diadeipme. Étamines en nombre variable, soudécs par leurs filets en deux corps distincts. Tels sont la fumeterre, le poljgala, et la plupart des Légumincuses, conme l'accacia, le cylise, la réģissse, le mélilot, ctc.

18 elasse. Polyadezphe. Étanines réunics par leurs filcts cn trois ou un plus grand nombre de faisccaux. Par excmple les IJpericum, l'oranger, les Mtelaleuca, ctc.

\section{5" Soudure des étamines par les antlieres.}

r $9^{\mathrm{C}}$ Classe. Srngtérisie. Cinc étamines réunies et soludécs par les anthères; flcurs ordinairement composées, 
rarement simples. Cette elasse renferme les Floseuleuses, les Semi-flosculeuses et les Radiées de Tournefort; elle con-tient aussi certaines autres plantes, telles que les Lobelia, les violettes, ete.

\section{$6^{\circ}$ Soudure du pistil et des étamines.}

$20^{\circ}$ Classe. Grinandrue. Étamines soudées en un seul corps avee le pistil; telles sont toutes les Orchidées, l'aristoloehe, ete.

\section{$7^{\circ}$ Fleurs unisexuées.}

2 I $^{\mathrm{e}}$ Classe. Monoecie. Fleurs mâles et fleurs femelles distineıs, mais réunies sur le même individu. Exemples : le chêne, le buís, le maïs, la sagyittaire, le ricin, elc.

$22^{\mathrm{C}}$ Classe. Droscre. Fleurs mâles et fleurs femelles existant sur deux individus séparés : la mercuriale, le dattier, le gui, les saules, le pistachier, etc.

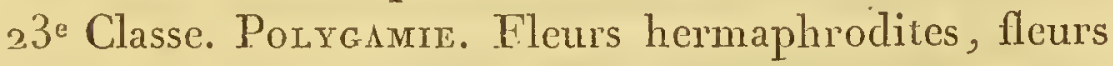
màles et fleurs femelles réunies sur un même individu ou sur des pieds différens. Par exemple : le frêne, la pariétaire, la croisette, le micoucoulier, ete.

\section{$S^{\circ}$ Fleurs invisibles.}

24 Classe. Criptoganie. Plantes dont les fleurs sont invisibles ou très-peu distinctes. Cette classe renferme les Fougères, telles que le polypode, l'osmonde, etc., les Mousses, les Lichens, les Prêles, les Algucs, les Champignons, elc., etc.

Nous venons d'exposer en peu de mots les caractères propres à chaeune des vingt-quatre classes établies par Linnæus dans le règne végétal. On voit que la marche de ce système est simple et facile à suivre. En eflet, il semble au premier abord qu'il ne faille que savoir compter le nombre c'es étamines d'une fleur, pour eonnaître à quelle 
classe elle appartient. Mais cependant nous ferons remarquer que, dans plusieurs cas, cetle détcrmination n'est point aussi aisćc qu'on le suppose d'abord, et que fort souvent on reste dans le doutc, surtout lorsque la plante présente quelquc anomalic insolite.

Oceupons-nous maintenant de fairc connaître les considérations d'après lesquelles ont été établis les ordres particulicrs à chaque classc.

Dans les treize premières elasses, dont les earactères sont tirés du nombre des étamines, ceux des ordres ont été puisés dans le nombre des styles ou des stigmates distinets. Ainsi une plante de la Pentandric, telle que le panais ou tout autre Ombellifère qui aura deux styles ou deux stigmates distinets sera du second ordre. Elle serait du troisième ordre, si clle en préscntait trois, ctc. Voyons les noms qui ont été domés à ees différens ordres.

I ${ }^{\text {er }}$ ordre. Monogynie, un seul style.

$2^{\mathrm{e}}$ ordre. Digyrie, deux styles.

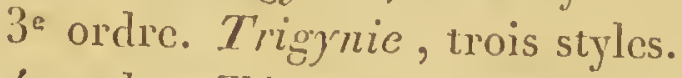

$4^{\mathrm{e}}$ ordre. T'étragynie, quatre styles.

$5^{\mathrm{e}}$ ordre. Pentagynic, eing styles.

$6 \mathrm{e}$ ordre. Hexagynie, six styles.

$7^{\mathrm{c}}$ oldre. Heptagynie, sept styles.

Se ordre. Décagynie, dix styles.

$9^{\text {e }}$ ordre. Polygynie, un grand nombre de styles.

Remarquons qu'il y a des classes dans lesquelles on n'observe point cette séric tout entic̀re d'ordres. Dans la Monandric, par exemple, on ne trouve que deux ordres: la Monogynic, comme daus l'Hippuris, et la Digynie, eomme dans le Blitum.

Dans la Tétrandric, il y a trois ordres, savoir : la Monosynie, la Dişrnie et la Tétragynie, Il cn a six dans la Pentandric, elc., etc.

Dans la quatorzième classe, ou la Didynamie, Linnecus a 
fondé les caractèrci des deux ordres qu'ily a établis d'après la strueture de l'ơaire. En effet, le fruit est tantôt formé de quatre petits àènes situés au fond du ealice, et qu'il regardait eomme quatre graines nues; tantòt, au eontraire, e'est une eapsule çui renferme un nombre plus ou moins considérable de gaines. Le premier de ees ordres porte lenom de Grmno:permie (graines nues); il eontient toutes les véritables Labiées, telles que le Marrube, les Plalomis, les Nepeta, le Sutellaria, ete.

Le seeond ordse, que l'on appelle Angiospermie (graines enveloppées), (t qui a pour earactère d'avoir , un fruik eapsulaire, réurit toutes les Personnées de Tournefort, telles que les Riinanthus, les Linaires, les Melampyrum, les Orobanches, ete.

La Tétradymmie, ou la quinzième elasse, offre également deux ordres, tirés de la forme du fruit, qui est une silique ou une silieule. De là on distingue la Tétradynamie en iliculeuse, ou eelle qui renferme les plantes dont le fruit est une silicule, telles que le pastel, le eochlćaria, le thlaspi, ete., et en siliqueuse, e'est-à-dire eelle dans lacuelle sont rangés les végétaux ayant une silique pour fruit, eomme la giroflée, le ehou, les cressons, ete.

Les seizièmıe, dix-septième et dix-huitic̀me elasses," c'est-à-dire la Monadelphie, la Diadelphie et la Polyadelphie, ont été établies, d'après la réunion des filets staminaux, en un, deux, ou un plus grand nombre de faisceaux distinets, abstraetion faite du nombre des étamines qui les composent. Linnæus a, dans ee.eas, employé les caraetères tirés du nombre des étamines pour former les ordres de ees trois classes. Ainsi on dit des plantes Monadelphes qu'elles sont triandres, tétrandres, pentandres, décandres, polyandres, suivant qu'elles renferment trois, quatre, einq, dix ou un grand nombre d'étamines soudées et réunies par leurs filets en un scul corps. Il en est 
de même dans la Diadclphie et la Poyadelphie, c'est-ìdire que le nom des ordres est le mime que celui des premières classes du système.

La Syngénésic, ou la dix-ncuvième classe du ssstème sexucl, est une de celles qui renfernent le plus grand nombre d'espèces. En efiet, les Synanhhérées forment à peu près la douzième partic de tous ler végétaux connus. Il étoit done très-important d'y multiplier les ordres, afin de faciliter la recherche des différertes espèces. C'est ce que Linnæus a tàché de faire en partajoant cette classc cn six ordres. Mais ici, comme le nomlre presque constant des ćtamines est cinq, ce nombre n’a pu offrir asścz de earactères pour devenir la base de ces dirisions; Linnxus l'a prise dans la structure mème de chazune des petites fleur's qui constituent les assemblages comus sous le nom de fleurs composées. En efiet, par suite d'avortemens constans, on troure avee les fleurs hemaphrodites des fleurs mâles et des fleurs femclles, souvent même des fleurs cutièrement ncutres. Linnæus, dont le gćrie poćtique se faisait remarquer dans tous les noms quil donnait aux différentes classes et aux différens ordres de son système, voyait dans ees réunions et ces mélanges de fleurs une sorte de polygamie. Aussi cst-ec le nom qu'il a donnć ì chaeun des six ordres de la Singénésie, cn icur ajoutant à chacun unc épithètc particulièrc. Voici leurs caractères: I er Ordec. Polygamie égale. Toutcs les fleurs sont hermaphrodites, et par conséquent toutes égalcment fécondcs, comme on le voit dans les chardons, les salsifis, ctc.

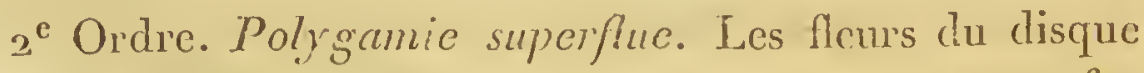
sont hermaphrodites; celles de la circonférence sont fcmelles; mais les unes et les autres doment de bonnes graincs. Par exemple, l'armoisc, l'absinthe.

$3^{*}$ Ordie. Polý samic frustranée. Les fleurs du disque sont hermaphrodites et fécondes; celles de la circonfé- 
rence sont neutre; ou femclles, mais stériles par l'imperfection de lcur sti’̧mate : elles sont donc tout-à-fait inutiles; daus l'orcl'e précédent elles étaicnt seulcment superflues. Fxemple: les centaurées, les Helianthus, ete.

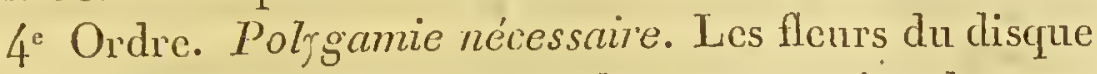
sont hermaphrodites, mais stériles par un vice de conformation du stignate; celles de la circonférence sont femelles, et fécondies par le pollen des premières : dans ce cas clles sont douc nécessaires pour la conservation de l'espèce, comme dans le souci, etc.

$5^{c}$ Ordre. Polygamie séparée. Toutes les fleurs sont hermaphrodites, rapprochées les umes des autres, mais cepcndaut contenues chacune dans un petit involucre particulier, eomme dans l'Echinops.

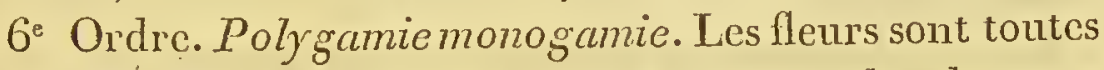
hermaphrodites; mais elles sont simples et isolées les uncs des autres, commc dans la violctte, les Lobelic, la balsamine, etc.

Ce dernier ordre, eomme il est facile de le voir, n’a aucune affinité avec les précédens. Il n'a de commun avec eux que la réunion des étamines par les anthères.

Dans la Gynandrie, ou la vingtic̀me classe du système scxucl, il y a quatre ordres qui sont tirés du nombre des étamines. Ainsi on dit: Gynandric-monandrie, comme dans l'Orchis, l'Ophrys;; Gynandric-diandrie, comme dans le Cypripedium; Gynandrie-hexandrie, comme dans l'aristoloche; Gynandric-polyandrie, les Arum.

La Monocie et la Dioccic préscntent en quelque sorte réunies toutes les modifications que nous avons remarquées dans les autres classcs. Ainsi la Monoccie renferme des plantes monandres, triandres, décandres, polyandres, monadelphes et gynandres. Cliacunc de ces variétés sert à à établir autant d'ordres distincts dans cette classe.

La Diocic en renferme cncore un plus grand nombre, dont les caractères, se rapportant à ceux de quelqu'une des 
classes préeédemment étal)lies, sont alors employés comme earactères d'ordres.

La vingt-troisième elasse ou la Polygamie, qui contient les plantes à fleurs hermaphrodites et à fleurs unisexuées mélangées, soit sur le même individu, soit sur deux ou trois individus distinets, a été pour ectte raison divisée en trois ordres: $\mathrm{I}^{\circ}$ la Woncecie, dans laquelle le même individu porte des flcurs monoelines at des fleurs diclines; $2^{\circ}$ la Diocie, dans laquelle on trouve sur un individu des fleurs liermaphrodites, et sur l'autre des fleur's unisexuées; $3^{\circ}$ enfin le Tricecie, dans laquelle l'espèee se compose de trois individus : un portant des fleurs hermaphrodites; un seeond des flcurs mâles, et le troisième des fleurs femelles.

La Cryptogamie, qui forme la vingt-quatrième et dernière elasse, est partagée en quatre ordres : $1^{\circ}$ les Foum gères; $2^{\circ}$ les Mousses; $3^{\circ}$ les Algues; $4^{\circ}$ les Champighons.

Après avoir fait comnaittre les bases du système sexuel, nous avons domué une esquisse des vingt-quatre elasses et des ordres nombreux qui s'y rapportent, tels qu'ils ont été établis par Linnæus. Lorsque l'on étudie ce systc̀me, on est frappé de son extrême simplicité et de la faeilité avee laquelle on arrive avee lui à la comnaissanee du nom d'une plante. Les elasses, en eflet, sont, pour la plupart, nettement tranchées et définics, surtout dans celles où les élamines sont en nombre déterminée. Non-seulement ce système contient toutes les plantes déjà comnues, mais il peut eneore comprendre toutes eelles que l'on pourrait découvrir; aussi a-t-il été uriversellement adopté à l'ćpoque où il a paru.

Mais il faut avouer eependant qu'il présente plus d'un ineonvénient grave. En effet, il n’est pas toujours aisé de déterminer si une plante appartient positirement à certaines classes. Ainsi, par exemple, la rue (Ru/a 
graveolens) a presque toutes ses fleurs munies de huit étamines; une seule au eentre de chacque assemblage de fleurs en présente dix. L'élève, dans ce eas, éprouverait quelque embarras, et serait tenté de plaeer eette plante dans la huitième elasse du système, e'est-à-dire dans l' $O c$ tandrie. Cependant Limneus la range dans la Décandrie, parce qu'il regarde la fleur à dix étaminies eomme étant la plus parfaite.

La Dodéeandrie n'est pas non plus caractérisée assez rigoureusement. On y plaee toutes les plantes qui ont de douze à vingt étamines. Mais l'aigremoine, que l'on y range, a souvent plus de vingt étamines.

Certaines Labiées ou Personnées qui appartiennent à la Didynamie ont leurs quatre étamines égales entre elles, et souvent l'irrégularité de la eorolle est à peine sensible.

Les ordres de la Syngénésie sont très-souvent d'une diffieulté rebutante pour pouvoir être reeonnus avee certitude. D'ailleurs le mélange des fleurs mâles, des fleurs femelles et des fleurs hermaphrodites en rejette plusieurs dans la Dioecie et la Polygamie.

Le sixième de ees ordres, la Polygamie monogamie; rapproche des Composées des plantes qui n'ont aucune "'alogie avee elles, telles que les violettes, la Lobelia, lis balsamines, ete.

La vingt-troisième elasse, c'est-à-dire la Polygamie; est un mélange eonfus de plantes qui appartiennent presque toutes aux différentes autres classes.

Si maintenant nous examinons les plantes rassemblées dans ehaeune de ees elasses, nous verrons que le plus souvent les affinités naturelles et reeonnues depuis si longtemps ont été entièrement rompues. Ainsi une des familles les plus naturelles, les Graminées, se trouve dispersée dans la Monandrie, la Diandrie, la Triandrie, l'Hexandric, la Monoeeie, la Dioceie et la Polygamie. Les Labićes sont en partie dans la Diandric, en partie dans la 
Didynamic. Il en est de même d'un grand nombre de familles tout aussi naturelles. Mais comme la classifieation établic par Linnæus est un système, e'est-à-dire un arrangement méthodique, mais purement artificiel, destiné sculcment à faire arriver avec facilité au nom d'une plante que l'on désire eomnaître, on ne saurait lui faire un reproche fondé d'avoir ainsi éloigné les unes des antres les plantes qui avaient entre elles beaucoup de rapports et d'affinité. Ce n'est done pas ec système qu'il faut étudicr lorsque l'on désire comnaîtré les rapports naturels des différens végétaux entre eux, tandis que parmi tous les systèmes artifieicls il méritc sans contredit la préférence pour arriver aisément au nom d'une plante.

Désirant faire disparaître de eet ingénicux système une partie des inconvénicns que nous avons signalés, et rendre son applieation plus facilc dans ecrtains points, feu mon père y a fait quelques modifieations importantes que nous allons faire connaître. C'était d'après le système de Linnæus modifié qu'étaient rangées les plantes du jardin de la faculté de médecine de Paris.

\section{SYSTÈME SEXUEL MODIFIÉ.}

Les dix premières elasses sont conservées saus aucun cliangement :

La $11^{\circ}$ elasse est la Polyandue, ainsi caractérisée: plus de dix élamines insérées sous le pistil simple ou multiple, c'est-à-dire dont l'insertion est hypogynique. Cette classe, qui remplaee la Dodécandrie, correspond parfaitement à la Polyandrie de Linnacus.

La $2_{2}{ }^{\circ}$ elasse est la Calxcandrie, ainsi earaetérisée: plus de dix étamines insérées sur le ealiec, l'ovaire étant libre ou pariétal; insertion périgynique. Cctte classe correspond en partic à la Lodécandrie, en partie à l'Ícosandrie. On y trouve toutes les viaies Rosacées.

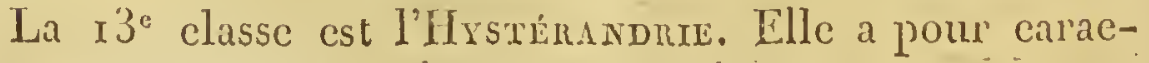


tère d'avoir plus de dix étamines insérées sur l'ovaire tout-à-fait infère, par conséquent à insertion épigynique. Cette classe correspond à une partie de l'Ieosandrie. Elle renferme les myrtcs, les Punica, Philadelphus, Psydium, etc.

Ces trois elasscs ainsi caractérisées sont beaucoup plus préeises, et eonservent mieux en même temps les rapports naturels, que celles primitivement adoptées par Linnæus, dont les caraetères, pris dans le nombre des étamines, pouvaient, dans beaucoup de circonstances, induire l'élève en erreur.

La $14^{e}$ elasse est la Didynamie, dont les ordres désignés par Linnæus sous les noms de Gymnospermie (graines nues) et d'Angiospermie (graines enveloppées), donnaient une idée fausse (puisqu'il n'existe pas de graines nues); ils ont été remplaeés par les suivans :

I Tomogynie (ovaire fendu et partagé). Ovaire profondément partagé en lobes distinets; style naissant d'uu enfoncement eentral de l'ovaire; fruit mur, tétrakène. Cet ordre renferme toutes les labiées.

$2^{\circ}$ Atomogynie (ovaire indivis). Fruit capsulaire, polysperme. Daus cette classe sont les Antirrhinécs, les Bignoniacées, ete.

r $9^{e}$ Classe. Sinnisuénie, remplaeant la Syngénésie, ainsi earactérisée : étamines réunies par les anthères seulement, de manière à former ure espèce de petit tube; ovaire moncsperme.

D'après ee caractèrc, on voit que cctte classe ne doit renfermer que les véritables plantes à fleurs dites eomposées, c'est-à-dire les Flosculeuses, les Sémiflosculeuses et les Radiées de Tournefort.

Les ordres de la Syngénésic de Linnzus étant tirés de caraetères trop minuticux, très-difficiles à reeonnaître, et souvent variables dans le même genre, ont ćtć changés en ceux qui suivent, très-facilcs à distingucr : 
I ${ }^{\text {er }}$ Ordre. Carduacées: eapitule composé de fleurons indifféremment hermaphrodites, mâles ou femelles; phoranthe garni de soies très-nombreuses, style offrant un léger renflement au-dessous du stigmate; connectif se continuant quelquefois au-dessus des anthères pour former un tube à cinq dents; tels sont les chardons, les centaurées, ete.

$2^{\mathrm{C}}$ Ordre. Corymbifères : capitule floseuleux ou radié; phoranthe nu ou garni de paillettes dont chacune accompagne une fleur. (Dans l'ordre précédent, elles étaient plusieurs à la base de chaque fleur.) Exemple : le tussilage, les Gnaphalium, les Erigeron, etc.

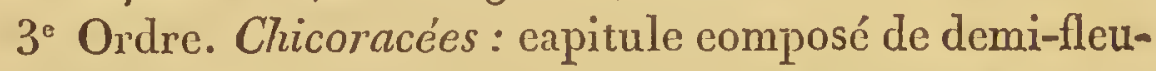
rons. Ex : la laitue, la chicorée, la scorzonère, etc.

$20^{\mathrm{e}}$ Classe. Symphysandrie. Cette elasse est formée du sixième ordre de la Syngénésie de Linnæus, la Polygamiemonogamic : elle a pour earaetères : des étamines soudées ensemble par leurs anthères, quelquefois même aussi par leurs filets, un ovaire polysperme, des fieurs simples; par exemple, les Lobéliacées, les Violettes.

La Gynandric, la Monoceic et la Dioecic sont conservées sans elangemens.

$24^{\circ}$ Classe. Anomaloegre. Fleurshermaphroditesoufleurs unisexuées sur le même ou sur des individus différens. Cette elasse correspond à la Polygamie de Linnæus.

${ }_{2} 5^{\mathrm{e}}$ Classe. Agamie. Végétaux dépourvus d'organes sexuels et se reproduisant au moyen de petits eorpuseules partieuliers, analogues aux bullbilles de eertaines plantes ct qu'on nomme sporules.

Tels sont les ehangemens que mon père a eru convenable de faire au système sexuel de Limnæus, afin d'en faire disparaître, autant que possible, les points qui pouvaient présenter des difficultés dans son cmploi. 


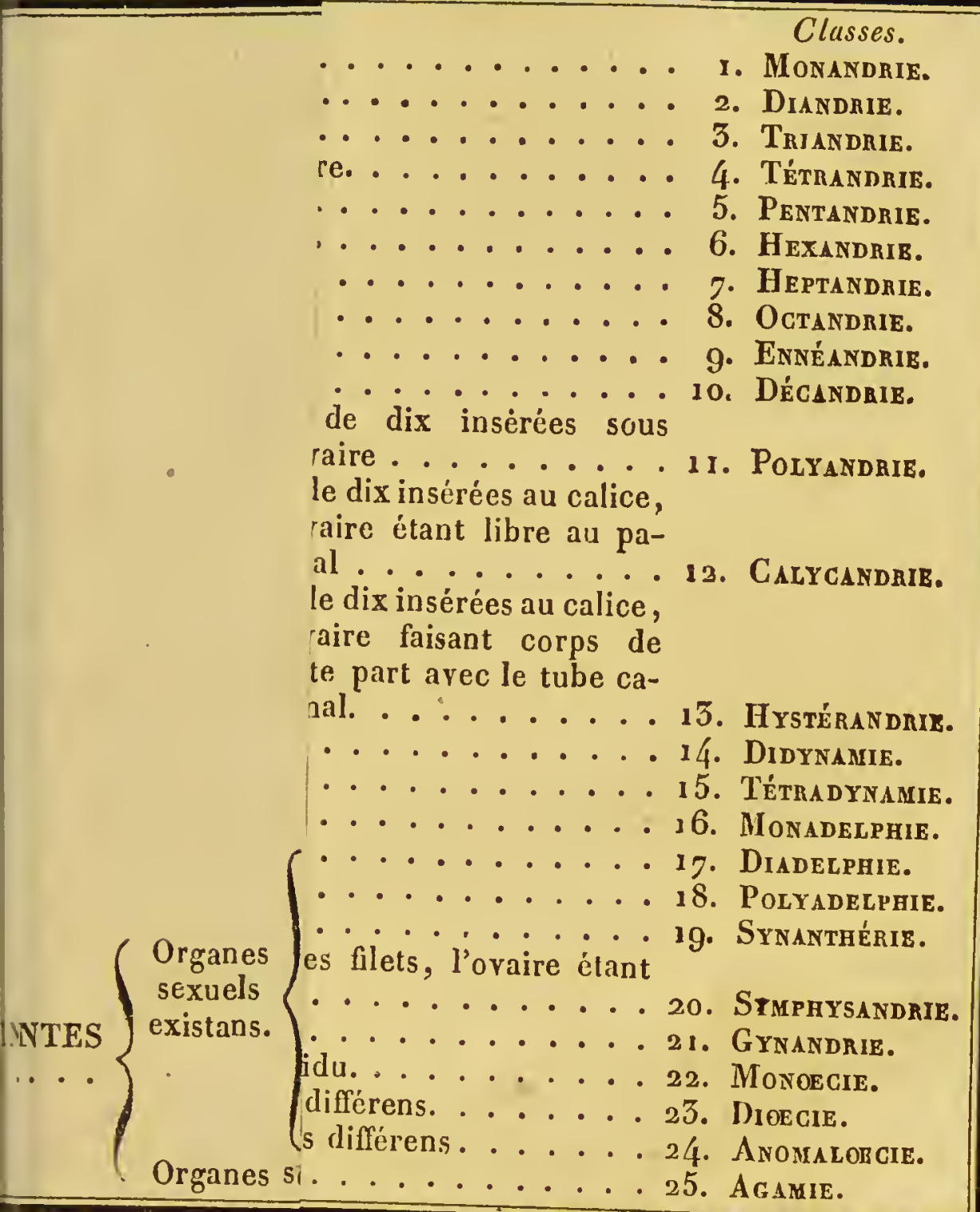





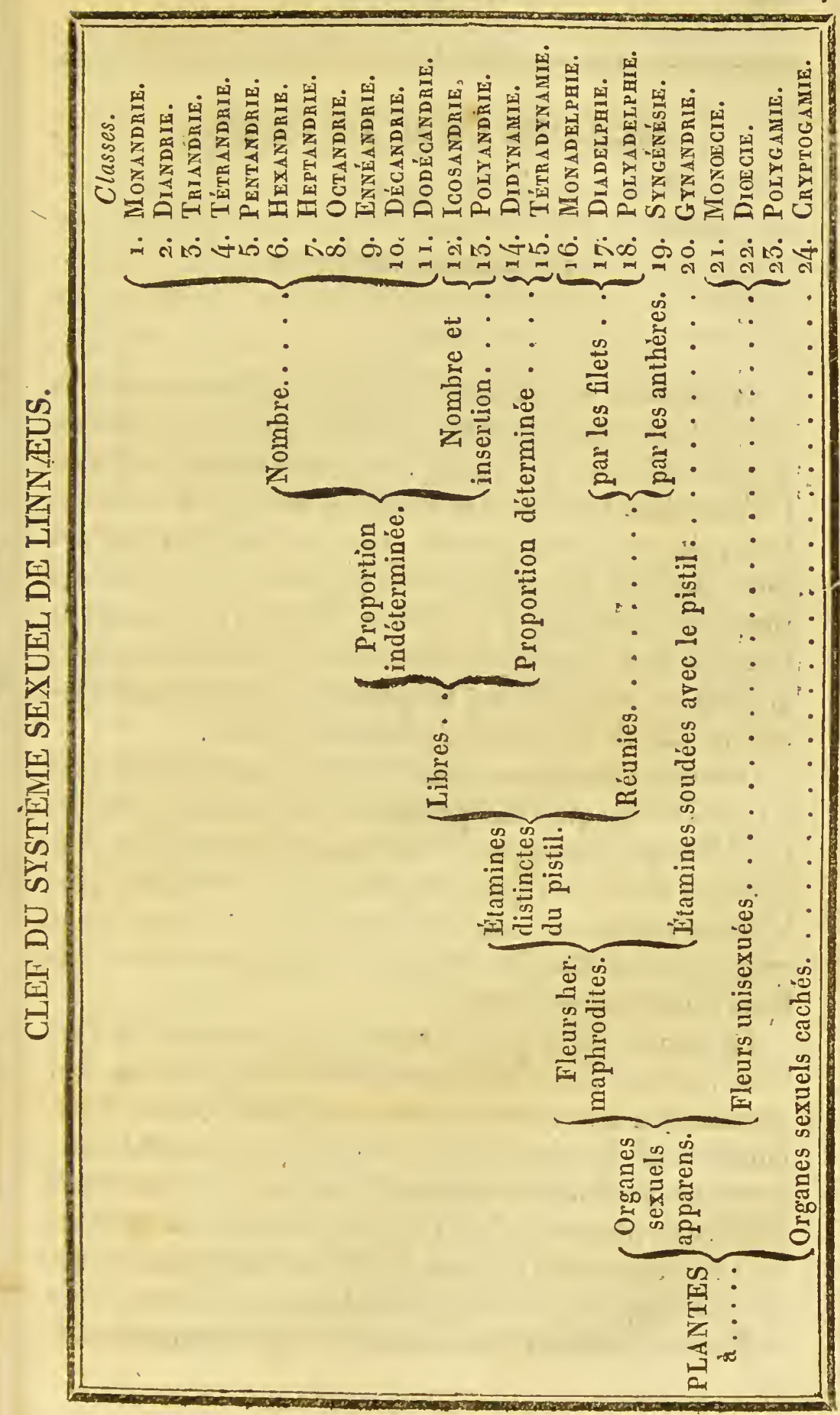




\title{
MÉTHODE DE M. DE JUSSIEU,
}

ou

\author{
DES FAMILLES NATUREL̈LES:
}

LA méthode des familles naturelles diffère essentiellement, dans sa marehe et ses caraetères, des deux systèmes de Tournefort et de Limnæus, dont nous venons de donner l'explieation. Dans eette méthode, en effet, les divisions ue sont point fóndées d'après la eonsidération d'un seul organe, mais les earaetères offerts par toutes les parties des végétaux concourent ì les former. Aussi les plantes qui se trouvent ainsi rapproehées sont-elles disposées de manière qu'elles ont a vee eelle qui les préeède ou les suit immédiatement plus de rapports qu'avee aueune autre.

Cette elassifieation est donc bien supérieure et préférable à toutes eelles qui l'ont préeédée, par les idées générales et philosophiques qu'elle nous donne sur toutes les produetions du règne végétal. En eflet, elle ne considère plus les êtres isolément, mais elle les réunit et les eoordonne en groupes ou familles, d'après le plus grand nombre de leurs caraetères communs.

La nature, en imprimant sur la physionomie de eertains végétaux un caraetère partieulier en rapport avee leur organisation intérieure, semble avoir voulu éelairer le botaniste dans la reeherehe des affinités qui existent entre toutes les produetions végétales. En effet, il y a un grand. nombre de plantes qui ont entre elles tant de ressemblanee dans la strueture et la eonformation de leurs parties, que de tout temps eette analogie a été aperçue, et que l'on a regardé ees différens végétaux comme appartcnant en quel- 
que sorte à une même famille. Ainsi les Graminées, les Labiées, les Crueifères, les Synanthérées ont toujours été réunics, quand on n'a pas saerifié les earaetères d'analogie et de ressemblanee aux bases d'un système artifieiel.

Lors donc que l'on s'occupa de réunir et de rassembler tous lcs végétaux en familles, e'est-à-dire cn groupes ou séries de genres se ressemblant par le plus grand nombre de earaetèrcs, on n'cut qu'à imiter la nature, qui avait en quelque sorte créé, comme pour servir de modèles, des types de familles cssentiellement naturelles. Ainsi les Légumineuses, les Crucifères, les Graminées, les Ombellifères, les Labiées, ete., vinrcut d'clles-mêmes se montrer au botaniste comme autant d'exemples dont il devait tâcher de se rapprocher.

Mais tous les végétaux n'ayant point, eomme ceux que nous yenons de nommer, des caractères cxtérieurs assez nets ni assez tranchés pour faire connaître à l'instant leur analogie avee certains autres, on eut recours à l'analyse, et l'on chercha dans tous leurs organes des modifieations qui pussent servir de caractères.

C'est dans le Genera plantarum de M. de Jussieu, véritable inventeur de la méthode des familles naturelles, qu'il faut étudier les prineipes de cette méthode, dont il est impossible de faire saisir l'esprit dans un exposé aussi sueeinct que celui que nous sommes forcés d'en donner.

Nous allons seulcment tầcher de faire connaître la manière dont les caractères ont été envisagés par cet auteur, et les principes sur lesquels reposent les bases de cette admirable classifieation.

Les caraetères doivent être considérés quant à.leur valeur, quant à lcur nombre, quant à leur affinité.

Sous le rapport de la valeur des caractères, on conçoit qu'ils doivcnt être d'autant plus fixes et plus importans, qu'ils sont tirés des organes les plus cssentiels des végétaux. Or, nous saỵons que ceux qui coneourent à la reproduc- 
tion joucnt le rôle le plus important dans la vic végétale; et que parmi cux cneore, l'embryon, qui est en quelque sortc le but eommun vers lequel sont dirigées toutes les fonetions de la plante, est eelui que son importance plaec au premier degré. C'est done dans l'embryon que M. de Jussicu a èherehé les premières bases de ses divisions. Les étamines et le pistil oecupent le seeond rang, et fournissent des caractères plus constans et plus préeieux que les enveloppes florales. Ces earactères ont d'autant plus de valeur qu'ils sont tirés, non du nombre et de la strueture de ees organes, qui sont fort sujets à varier, mais de leur position relative, qui est fixe. Ainsi, après l'embryon , la position relative des organes sexuels, ou leur insertion, fournit les caraetères les plus importans pour la coordination des végétaux. Enfin les tiges, les feuilles ct les raeines ne peuvent jamais être employées que eomme earactères aceessoires.

Quant à leur nombre, les earaetères se réunissent, se groupent et se eoordonnent; et de l'agrégation des caractères simples résultent les earactères généraux, qui servent à réunir sous une dénomination comnune un eertain nombre de végétaux.

Plusieurs earactères sont entre eux dans une dépendanee réciproque, et semblent inséparables les uns des autres. Ccux que l'on tire de la fleur ct du fruit sont prineipalement dains ee eas. C'est ainsi, par exemple, que l'ovaire infère néeessite eonstamment un ealiee monosćpale et une insertion épigynique. La corolle monopétale indique presquë eonstamment que les étamines sont insérées sur elle, et qu'elles sont en nombre déterminé, ete.

D'après la valeur et l'importance dont jouissent les différens earactères, il est facile de prévoir que les plus fixes, les plus constans, ont dù ètre employés pour les divisions fondamentales du règne végétal. Ainsi l'embryon a servi à former dans les végétaux les trois premic̀res grandes divisions. Les étamines et les cnveloppes florales ont ensuite 
été employées pour subdiviscr les trois premic̀res sections, établies d'après la considération dc l'cmbryon.

Cherchons maintenant à faire connậtrc par quels moycns on est parvenu à réunir les végétaux en familles ou groupes naturels. Et commençons d'abord par donncr une idéc des mots : espèce, variété, genre, ordre ct famille.

Les plantes disséminées sur la surface du globe forment les individus du règne végétal : quand on lcs cxamine avec attention, on ne tardc point à s'apcrcevoir qu'il cu existe un grand nombrc, s'offrant toujours à nos regards sous le même aspect, avec les mêmcs caractères extéricurs et intérieurs, et se reproduisant constamment sous la même forme. C'est à cette réunion d'êtres parfaitement scmblables, considérés abstractivemcnt, que l'on a donné lc nom. d'espèce. L'cspèce est donc l'cnscmblc d.cs individus qui se reproduisent constamment de la même manière. Unc graine provenue d'unc espècc quelconquc reproduit' toujours un individu qui lui est parfaitemcnt scmblable. Les caractères sur lesquels est fondée la distinction des différentes cspèces entre elles sont cn général tirés des organes de la végétation, c'est-à-dire des fetuilles, de la tigc et des racines. Les espèccs qui présentent quelques différences sous le rapport dc la couleur dc lcurs flcurs, du lietr qu'clles habitent, de leur hauteur plus ou moins considérable, constituent les variétés, qui sc distinguent des espèces proprement ditcs, en ce quc, dans l'état de nature, elles ne se rcproduisent point de graines avec tous lcurs caractères (I). Ainsi, par cxcmple, le lilas a habituellcment les flcurs d'une teintc violette tcndrc; mais quclqucfois scs fleurs sont blanchics, sans que pour ccla aucun des autres caractèrcs ait changé; le lilas blanc n'cst donc qu'une variété dc celui à flcurs violettcs. En efict, si l'on

(1) Nous arons cu soin de mettre dans l'ćtat de nature, car dans les plantes cultivées plụsieurs variétés se conservent de graine. 
sème des graines récoltées sur le lilas à fleur's blanehes, elles donneront naissanee à des individus dont ius fleurs seront indifféremunent violettes ou blanehes; ec qui prouvc que les variétés ne se eonservent pas toujours par le moyen des graines.

Le genre se eompose d'un nombre plus ou moins considérable d'espèces, réunies par des earactères communs tirćs des organes de la fruetifieation, mais toutes distinetes leș unes des autres par des caraetc̀res spécifiques, particuliers à chaeune d'elles, et fournis par les organes dc la végćtation. Ainsi le genrc Anagallis a pour caraclères une corollc monopétale rotacée, cinq ćtamines, et pour fruit unc pyxide, c'cst-à-dirc une capsulc globulcuse s'ouvrant circulairement par unc sortc d'op ereulc. Toutes lcs espèees de ec genre devront offrir ces différcns caractèrcs; mais elles se distingueront les unes des autres par la forme de lcur tige et de lcurs feuilles, cte. Il en est de même des autres genres.

En réunissant ensemble les genres de la même manièrc que les cspèces, c'est-à-dirc en rapproehant tous ceux qui ont des earactères communs ct analogues, on forme simplement des ordies proprement dits, si l'oul n'a égard qu'à un seul earaetère, tcl que lc nombre des stigmates ou de la forme du fruit, etc., et des familles ou ordres naturels, si l'on fait eoneourir à cette r'ćunion toutes les eonsidérations quc l'on peut tirer de la forme, de la ștrueture et de la disposition respeetive de tous les organes des végétaux quc l'on classe.

On doit donc entendre par ordre ou famille uaturelle de plantes, une sćrie ou réunion de genres plus ou moins nombreux, qui offrent tous les mèmcs caractères dans les olganes de la fructification.

Ainsi la famille des Crucifères a pour earaetères un embryon dieotylédoné, un fruit siliqueux ou silieuleux, or dinairement quatre pétales opposés deux à deux, des éta- 
mines en nombre déterminé, ete., ete. Tous les genres de eette famille devront offrir les mèmes earaetères, mais seulement avee quelques légères modifications, qui n’en altéreront point le type primitif, et qui serviront à établir les différenees des genres dont la réunion constitue cette famille.

C'est en suivant une marche semblable que l'on est parvenu à rassembler les végétaux en groupes ou familles naturelles. Mais, eomme ees familles sont en assez grand nombre, il a fallu les distribuer en différentes elasses plus ou móins nombreuses, en tâehant de eonserver entre elles la même analogie et la mème affinité. C'est à eette elassification des familles que l'on a donné le nom de Méthode de Jussieu, ou méthode des familles naturelles. Nous allons voir quels sont les earactères que cet auteur eélèbre a employés pour former ces différentes elasses.

Cette méthode a été divisée en quinze classes. Les premières divisions reposent sur les earaetères que l'on peut tirer de la présence ou de l'absenee de l'embryon : de là les Embryonés et les Inembryonés.

Les plantes embryonées sont distinguées, d'après le nombre de leurs cotylédons, I $^{\circ}$ en monoeotylédonées, $2^{\circ}$ en dieotylédonées. Tous les végétaux sont rangés dans ees trois grandes divisions primordiales :

Les Acotylédonés,

Les Monocotylédonés,

Les Dieotylédonés.

La seeonde considération, eelle qui sert vraiment à établir les elasses proprement dites, est fondée sur l'insertion relative des étamines ou de la eorolle monopétale staminifère. Or, nous avons vu qu'il existe trois espèces d'insertion:

I Insertion hypogynique, ou eclle daus laquelle l'ovaire étant entièrement libre, les étamines ou la corolle staminifère sont insérées au pourtour mêne de sa bise. 
2 Insertion périgynique, ou eelle dans laquelle l'ovaire étant librc ou pariétal, les étamines ou la eorolle monopétale staminifère s'insèrent au calice à une certainc distanec du pourtour de la base de l'ovaire.

$3^{\circ}$ Insertion épigynique, ou eelle dans laquelle l'ovaire est toujours infère, et où les étamines ou la eorolle staminifère sont insérées sur la partie supérieure de l'ovaire.

Ces trois sortes d'insertion servent à établir autant de classes.

Les Acotylédonés étant dépourvus d'embryons, et par conséquent de fleurs et de fruits, n'ont pu se prêter à cette division. Ils constituent la première classe.

Les Monoeotylédonés, pouvant offrir ces trois modes d'insertion, ont été partagés en trois classes : $x^{\circ}$ Monoeotylédonés à étamines hypogynes; $2^{\circ}$ Monoeotylédonés à étamines périgynes; $3^{\circ}$ Monocotylédonés à étamines épigynes.

Les Acotylédonés et les Monocotylédonés forment donc quatre elasses, savoir :

Acotylédonés ....................... I

Monocotylédonés $\left\{\begin{array}{l}\text { étamines liypogynes.... } 2^{\circ} \\ \text { étamines périgynes.... } 3^{\mathrm{e}} \\ \text { étamines épigynes.... } 4^{\mathrm{e}}\end{array}\right.$

Les Dicotylédonés étant beaueoup plus nombreux que Ies Acotýlédonés et les Monoeotylédonés réunis, on a dû. chereher à $\mathrm{y}$ multiplier le nombre des divisions. Sans abandonner l'insertion, elle n'est plus devenue qu'un caraetère seeondaire. Ainsi l'on a remarqué que ees plantes sont dépourvues de corolle ou apétales, ou qu'elles ont une corolle monopétale staminifère, ou bien que leur eorolle est polypétale. Cette distinetion a servi de base aux trois divisions que l'on a établics d'abord dans les Dicotylédonés, savoir :

I Dicotylédonés apétales; 
$2^{\circ}$ monopétales;

$3^{\circ}$ polypétales.

On s'est ensuite servi de l'insertion comme caractère secondaire pour subdiviser ces trois sections en classes. Ainsi les Apétales forment trois classes dans lesquellcs l'insertion est épigynique, périgynique et hypogynique.

Les Monopétales, dont la corolle porte toujours les étamines, constituent également trois classes, suivant que leur corolle staminifc̀re èst liypogynique, périgynique ou épigynique. Cette troisic̀me classe des Monopétales a été encore subdivisée, suivant que les étamines sont libres ou réunies par leurs anthèrcs, ce qui porte à quatrc le nombre des classes dans les corolles monopétales, savoir :

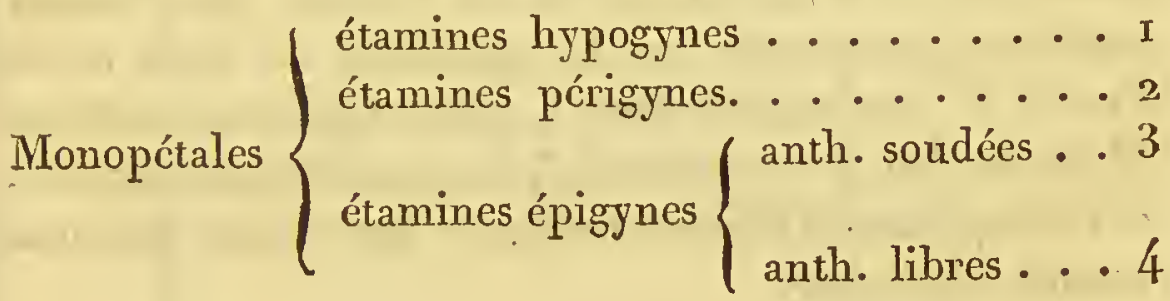

Ces quatre classes rćunies aux trois des Dicotylédonés apćtales, et aux quatre des Monocotylédonés et Acotylédonés forment déjà onze classes.

Les Polypétales ont égalcment été divisés en trois elasses', d'après leur mode d'insertion, qui cst épigynique, pćrigynique ou hypogynique.

Enfin, dans la quinzième et dernic̀re classe sont rangćes toutes les plantes dicotylćdonćes, dont les fleurs șont essentiellement unisexuécs, et séparées sur des individus distincts. On lcur a donné le nom de diclines irrégulières.

Telles sont les quinze classes quc M. de Jussieu a établies dans lc règne végétal, afin de pouvoir disposer méthodiquement les différentes famillcs de plantes, qu'il avait auparavant créées.

Chacune de ccs classes, en cffct, renferme un nombre plus ou moins considérable de familles naturelles toutes 
réunies par le earaetère eommun qui constitue la classe. Le nombre de ees familles n'est point définitivement arrêté, et ne peut pas l'être en effet. De nouvelles déeouyertes, des observations plus précises et plus exactes, en faisant eonnaître des objets nouveaux, ou en démóntrant les différenees qui existent entre des végétaux auparavant réunis et confondus, augmenteront eontinuellement le nombre des familles de plantes. Lorsqu'en i 789 , M. de Jussieu (I) publia son Genera plantarum, il déerivit roo familles; aujourd'hui nous allons donner les earaetères de plus de I 6o, et eneore ee nombre est-il suseeptible d'aug* mentation. M. de. Candolle a également publić une série de familles rangées dans un ordre partieulier, presque inverse de eelui adopté par M. de Jussieu. Sans vouloir nullement prononeer sur la supériorité de l'une ou de l'autre de' ees elassifications, nous exposerons eelle de M. de Jussieu, eomme étant la plus généralement adoptée, et eomme étant d'ailleurs conforme aux classes que nous venons d'indiquer.

(r) On avait reproché à M. dc Jussien dê n'avoir point donné de nom propre à chacune de ces quinze classes, comme Linnæus l'avait fait pour celles de son système. Ce célèbre botaniste a trop bien senti la justesse de cette obscrvation pour ne point y remédier. Il a donc donné à chacune dc ces classes un nom particulier. C'est dans une note qu'il a eu la honté de nons communiquer que nous avons puisć ces noms, que l'on trouvcra en tête de chaque classe dans la liste snivante. Le seul changement que nous nous soyons permis, c'est de leur donner une terminaison substantive. Ainsi nous avons dit Monohypogynic, an lieu de Monohypogynes; Peristaminie, au liea de Peristaminécs, etc., etc. 
MÉTHODE DE JUSSIEU.

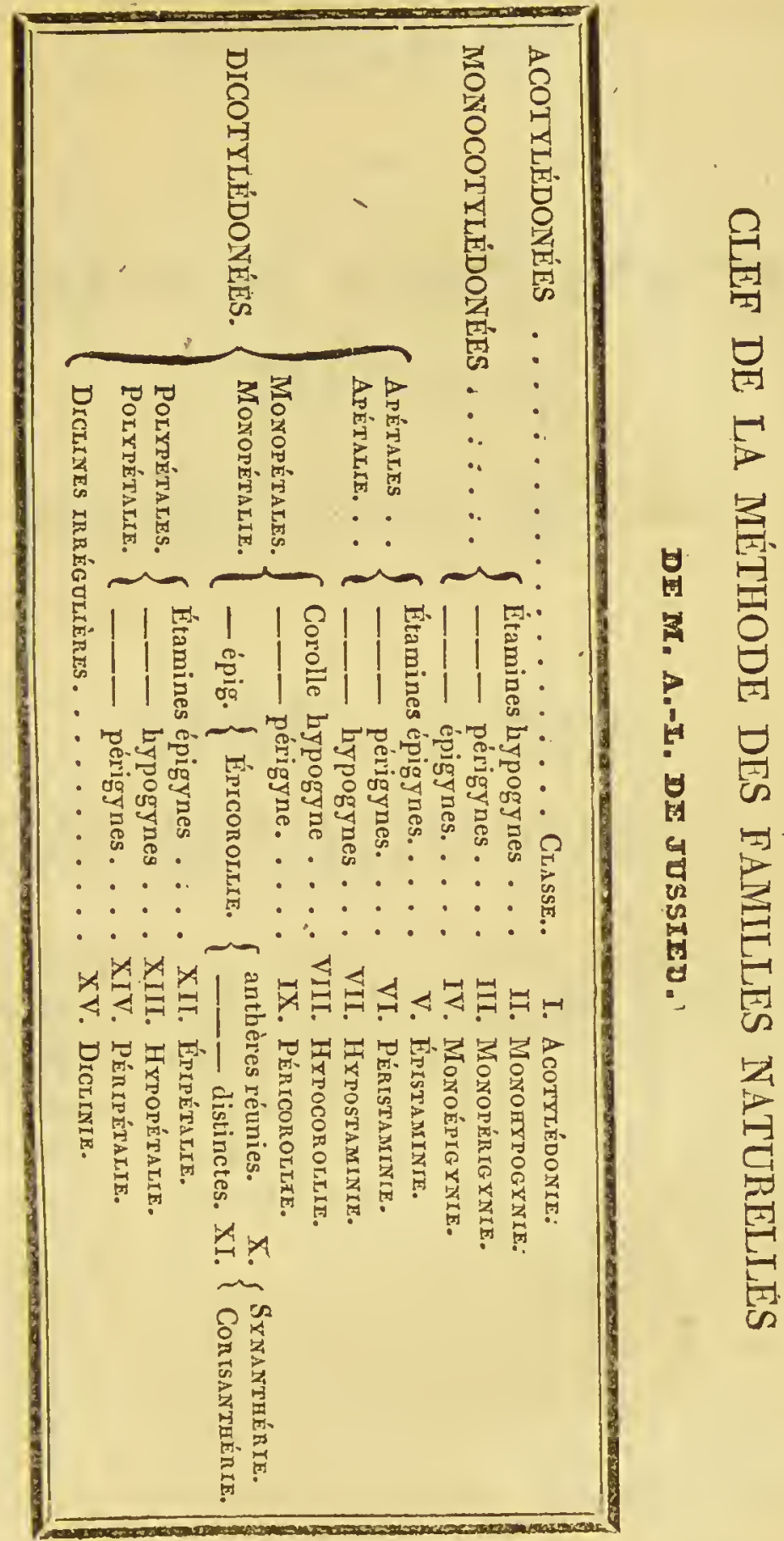





\section{TABLEAU}

DES

\section{FAMILLES DU RËGNE VÉGÉTAL,}

FATGÉES SELON IA MIÉTHODE

D'ANTOINE-LAURENT DE JUSSIEU.

PREMIERE DIVISION.

\section{PLANTES INEMBRYONEES (I).}

Cietre première division du règne végétal correspond à la Cryptogamie de Linnæus. Elle renferme tous les végétaux qui étant dépourvus de véritables organes de la génération, c'est-à-dire d'étamines et de pistil, ont reçu le nom de plantes agames, et se reproduisent au moyen de corpuscules analogues, dans leur structure et leur développement, aux bulbilles qu’on observe sur certains végétaux phanérogames. Linnæus nommait ces plantes cryptogames, parce qu'il croyait queleur fécondation avait lieu au moyen d'organes encore peu connus. M.de Candolle remarquant qu'un seul élément anatomique, le tissu cellulaire, entre dans leur composition, les appelle végétaux

(x) Nous donnerons ici quelques considérations générales sur l'organisation des plantes inembryonées, parce qu'elles présentent des particularités gane nous a'avons pa fairę connaître daus le cours de cẹt onvrage. 
cellulaires, par opposition au nom de végétaux vasculaires qu'il donne aux Plianćrogames. Nous eroyons devoir leur conserver le nom de plantes agames qui leur a été imposé par Neeker, paree qu'ainsi que nous lc prouverons bientôt, ils manquent réellement d'organes gćnérateurs, ou que, du moins, leurs organes de la reproduetion ont une structure entièrement différente de celle des mêmes parties dans les plantes phanérogames. Ainsi nous comprenons sous le nom de plantes agames toutes les plantes acotylćdonćes de M. de Jussieu, c'est-à-dire toutes celles qui ont étć rangćes par Linnæus dans la Cryptogamie ou dernic̀re 'classc de' son systc̀me.

Plusieurs auteurs les ont divisćes en deux classes, savoir: lcs cryptogames et lcs agames proprement ditcs. Au nombre des premières ils rangent les Salviniées, les Equisétacées, les Mousses, les Hépatiques, les Lycopodiacées et les Fougères, qu'ils regardent comme pourvues d'organes sexuels, mais très-petits et peu distinets. Dans la seeonde elasse se trouvent les plantcs véritablement agames, selon eux, telles que les Algues, Ics Lichens, et les Champignons, dans lcsquels on nc distingue rien qu'on puisse comparcr à dés étzmines ou à des pistils. Mais nous n'admettons point cette distinction. T'́ng ganisation de tous ces vćgétaux est trop manifestement différentc de celle des Phanérogames pour qu'on y retrouve les mêmcs organes. Nous pensons donc, comme Necker, que les plantes désignées par le nom de crýptogames sont cntièrement dépourvues d'organes sexuels; quc rien en ellcs ne peut-être raisonnablement assimilć à ees mêmes parties dans les phanérogames.

Plus d'une fois, dans le cours de eet ouvrage, nous avons montré l'extrême diflér cnce qui existc cutre toutes les parties de ecs végétaux et eelles des plantes phanérogamcs. Nous avons fait voir que les eorpuscules regardés par les auteur's comme des graines n’en sout point réelle- 
ment, puisqu'ils ne contienuent pas l'embryon. Ils donnent cependant naissance ì des ètres parfaitement semblaBles ì ceux dont ils se sont détachés. Mais, comme nous l'avous dit plusieurs fois, les bulbilles de certaines plantes vivaces, et un grand nombre de bourgeons produisent le mème pliénomènc, sans quc pour cette raison on puisse les assimiler aux véritables graines. D'ailleurs, comment s'opèrc cettc prétendue germination des plantes'agamcs? Peut-on la comparer à cellc des végétaux pourvus d'embryon? Un corpusculc reproductif d'une Fougère, d'un Champignon, etc., plaeé sur la terre, s'y développera, mais ee nc seront point, comme dans l'cúbryon d'une plante phanérogame, des parties déjà formées, seulement réduites en quelquc sorte à leur ćtat rudimentaire, qui acquierront successivement un plus grand développement; mais, au contrairc, des parties entièremcnt nonvelles seront produites. Ce ne sera point un aecroissement d'organes déjà cxistans, mais ln tissu mème de la sporule ou corpuseule reproductif, s'allongeant d'un côtépour s'cnfoneer dans la terre ct former une racine, lorsquc le végétal doit cn avoir une, formera de l'autre côté une tige en s'allongeant en sens inverse. Daus quelquc position qu'ume sporulc soit placée, le point en eontaet avcc la tcrrc s'allongera eonstamment pour former la raeine, et lc point opposédevicndra la tige. Ces deux organes n'cxistaicnt done point cncore avant ce dévcloppement; ils sc crécnt par l'influcnce de certaincs circonstances, qui paraissent comme fortuites ct étrangères à la nature mèmc du eorps qui les produit.

Si nous passons à l'examen des parties regardées comme les flcurs par les différens auteurs, nous verrons lin diversité la plus grande régner dans leurs opinions. Les uns, en elletet, appellent fleurs mâles ce que les autros décrivent cornme des fleurs femelles. Ainsi dans les Mousses, Linnæus regarde l'urne comme une fleur mále, Hedwig 
comme une fleur fenclle, Palisot-Beauvois comme unc fleur hermaphrodic.

Toutes les fois que ees végétaux présentent, eomme les Mousses, par exemple, deux sortes bien distinetes d'organes particuliers, regardés comme ceux de la fruetification, les auteurs n’ont dû être embarrassés que sur le clıoix qu'ils devaient en faire et la fonetion qu'ils devaicnt attribuer à chacun d'eux. Mais dans les Jongermanes, où l'on trouve quelquefois trois ou quatre sortes de fruetifications différentes entre elles par leur forme extéricure, comme il n'cxiste que deux espèces d'orģanes sexuels, les organes mâles et les organes femelles, on serai donc forcé ici d'en admettre quatı.e. Car si l'on a domné le nom d'organes sexuels à deux de ees parties, pourquoi le refuser aux deux autres, dont la st.rueture intéricure est la même, mais qui diffèrent seulesment par leurs formes extéricures ou leur disposition?

Dans les Fougères, au contraire, ou il n'existe évidemment qu'une scule espèce de fruetification entièrement formée par de petits grains, ordinairement renfermés dans des espéces de petites poches membraneuses, el que l'on a regardés comme des séminules, ou sont les étamines? où est le stigmate qui a recu l'influence de pollen? où sont les cordons pistillaires qui l'ont transmis aux ovules? Est-ce répondre à ectte question d'une manière satisfaisante pour la raison que de dire, comme Micheli et Hedwig, que les poils que l'on observe sur les jeunes feuilles sont les étamines; eomne Hill et Schmidel, que les fleurs mâles sont les ammeaux qui entourent les réecptacles où sont eontenus les séminules, ete., ete.?

Il faut en eonvenir, des opinions aussi diverses, et même toul-à-faii opposées sur le mème sujet, eonduisent ì une conséquenee qui nous paraît nécessaire : c'est que les prétendues fleurs des plantes agames, tantòt regardées corume renfermant des étamines, lantôt comme contenant des pistils, ne sont point réellement des fleurs. Cie sont des 
organes particuliers, des espèees de bourgeons, auxquels la nature a eonfié le soin de la reprodnetion de ees singuliers végétaux. Pourquoi, en effet, voudrions-nous restreindre dans les bornes étroites de nos eoneeptions la puissance de la nature? Ses moyens sont aussi variés que son pouvoir est grand. Et si elle a donné aux plantes agames une physionomie si différente de eelle des plantes phanérogames, des organes extérieurs qui n’ont souvent rien de comparable aux leurs, pourquoi ne lẹr auraitelle point aeeordé aussi un mode partieulier de reproduction, qui u’ait d'analogues avee eelui des végétaux phanérogames que les effets qu'il produit, e'est-à-dire la formation desorganes qui doivent servir à perpétuer l'espèee?

\section{PREMIERE CLASSE.}

ACOTYLÉDONIE.

\section{PREMIIEE FAMIL I.}

* hydrophytes. Hydrophrta. - Algee auct. Algarum pars. Juss.

Prineipe de l'organisation végétale, les plantes quì eomposent eette famille sont les plus simples que l'on connaisse. Quelques-unes se montrent à leur origine sous l'aspeet de petits globules ou vésicules isolées ou grouppées, qui, en se réunissant bout à bout ou en s'aggregeant diversement, forment desfilamens ou des tubes simples ou rameux, eontinus ou artieulés, des lames configurées de différentes manières, ou des espèees de réseaux. Les Hydrophytes, en effet, sont toutes ees plantes qui végètent 'dans les eaux douees ou salćes et les lieux inondés; leur tissu paraît, en général, homogène, composé de eellules de formes variées, et, selon Lamouroux et Bory de Saint-Vincent, de quelques vaisseaux constituant des fibres longitu- 
dinales. Leur's organes de la fi'uctification sont des sporanges déhiscens ou indéhiscens, renfermant de très-petites sporules. Ces organes sont diversement groupés; placés dans l'intéricur du tissu, rarcment à l'cxtéricur, sous la forme de tubereules. Ceux des hydrophytes tubulcuses sont tantôt réunis en globules, tantôt disposés cn lignes spirales. Les hydrophytes pprésentent toutes les nuances du vert ou du pourpre.

Cette famille reuferme les plantes généralement connues sous les noms d'Algues ou plantes marines. Elles se divisent en deux grandes tribus, que plasieurs auteurs ont eneore subdivisées, suivant qu'elles eroissent dans les eaux douceś, ou qu'elles babilent les eaux salées. Ces deux tribus sont les Conferves et les Thalassiophytes. Ces plantes ont été l'objet des travaux de plusienrs naturalistes modernes, parmi lesquels nous eiterons MM. Turner, Lyngbie, Lanouroux, Ronnemaison, Mertens, Agardh, et surtout Bory de SaintVineent. C'est aux onvrages de ees savans que nous renvoyons eeux qui voudraient avoir des détails plus circonstanciés sur la strueture et la elassifieation de ces végétaux.

La famille des IIydrophytes forme le lien et le passage entre les règnes animal et végétal. En effet, les Oseillaires et les Conjugées sont en quelque sorte des êtres mixtes qui ont tour à tour été rapportés aux animaux et aux vógétaux; les premières par les monvemens spontanés et variés qu'elles exécutent, les secondes par lenr mode de féeoudation et de développement, semblent avoir tous les caractères de l'animalité, tandis que par leur strueture, lenr forme, on ne pent les éloigner des Conferves, privées de toute espèee de mouvement, et appartenant eertainement an règne végétal. Il est done impossible de tronver de ligne de démareation bien tranchée entre les deux règnes animal et végétal. On a dit que eertaines $A \operatorname{lgg}_{\text {gnes }}$ étaient tour à tonr et suecessivement animaux et régétaux, c'est-à-dire qu'il y avait une véritable transmutation d'un règne dans un autre. Mais les observations récentes des observateurs les plus exaets ont prouré que eette transformation n'avait pas licu.

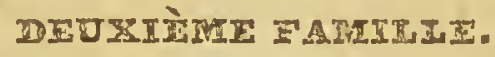 \\ * champrgnons. Filngi. Juss.}

Végétaux extrêmement variables dans leur forme, le ur' consistance, leur coulcur, etc. Ce sont des corps char un ou subéreux, ayant tantòt une forme comparable à celle 
d'un parasol, e'est ì dirc composés : I’ d'un chapeau ordinairement convexe porlant inféricurement des lames pcrpendiculaires, des tubes ou des ligncs anastomosćes; $2^{\circ}$ d'un pédiculc central ou latéral, au sommet duqucl on apcrçoit une membrane cireulaire, qui s'étend-jusqu'au pourtour du chapeau (colerette); tout le champignon est quclqucfois recouvert avaut son développement par une sorte dc bourse membraneuse complète ou incomplètc appelée volva. D'autres fois ee sont des masses globuleuses, ovoïdcs ou allongćes, des cspćces de coupcs, des filamens simplcs ou articulés, des troncs coralliformes, c'est-à-dire irrégulièremcnt ramifiés à la manière du eorail, et dont lcs couleurs sont extrêmement variables, offrant quelquefois les nuances les plus vives, mais leur tissu intérieur, qui sc composc' dc cellules irrégulièrcs, n'est jamais vert. Les sporules ou organcs reprodueteurs sont tantôt nues, tantôt renfermécs dans des espèccs dc petitcś capsules (thecce). Elles sont ou répandues à la surface du champignon, ou cnvcloppées daus un peridium ou coneeptaclc eharnu, ncmbraneux ou dur et ligneux.

Les Champignons sont, én général, des plantes parasites qui se développent, soit sur d'autres végétaux encore vivans, soit sur les corps organiques en état de décomposition putride, soit à la surface ou même dans l'intćrieur de la terre. Leur accroissement se fait quelquefois avec une rapidité extraordinaire, et leur durée est souvent très-fugitive, tandis que d'autrcs (Boletus igniaritus, ungrulatus, etc.), . végètent lentement et pendant plusienrs années consécutives. Un très-pctit nombre d'espèces croissent dans l'eau.

Les Champignons forment plasieurs groupes naturels qne quelques auteurs considèrent comme des familles distinctes. Ces groupes sont :

I Les Champrgaross proprement dits. Végétaux charnus, subéreux on ligneux, ayant les sporules placées dans des capsulcs dont la réunion constituc une membrane (hymenium) diversement replice et reeouvrant cn totalité ou en partie la surface du Champignon. Ex.: Agaricus, Boletus, MTerulius, Morchella, Clavaria, etc.

$2^{\circ}$ Les Ixcorerdacées sont formées d'un péridium ebarnu ou membraneux, d'abord clos, mais s'ouvrant ensuite et eontenant des sporules nucs, 
sans capsules et s'échappant du péridium oa réeeptacle sous formc de poussière. Ex.: Lycoperdum, Geastrun, Stemonitis, Desmodium, etc.

$3^{\circ}$ Lcs Hroxprúes, qui se préscntent sous la forme de tuhercules on coneeptacles, de formes très-variées, s'ouvrantpar une fente ou un pore, et contenant dans une sorte de pulpc gélatineuse de petites capsules (theca) pleines de sporules. Ex. : Hysterium, Splecria, Erysiphe, etc.

Nota. Il faut retrancher de ce groupe les Hypoxylécs Lichénoïdes, de de Candolle, qui, à l'exccption du genrc Hystérium, appartiennent aux Lichénées.

$4^{\circ}$ Lcs Mucḱdinérs. Ce sont des filamens ramenx et entreeroisés, portant des sporules dépourvues de eapsnles. Par cxemple, toutes les espèces de mucor, et les genres nombreux qu'on cn a formés.

$5^{\circ}$ Les UnédinéEs. Les sporules sont renfermées dans des capsules, on librcs, ou placćes sans ordre sur la surfacc d'une base filamenteuse ou pulvérulente. Ex. : Uredo, etc.

La famille dcs Champignons se distinguc des Algucs et des Lichénées par l'absence de toute espéce de fronde ou dc croùte, portant les organes de la fructification.

\section{THOISIG̀ME FAMILIE.}

* uichénévs. Lichenea. Horrm. - Algarum. Juss. et Mypoxylorum par's. DC.

Frondes ou thallus étendus sous la forme de membranes on eroîtes membraneuses de eonsistance variée, simples ou diversement lobécs, ou de tiges simples ou ramifiées, ou enfin simplement d'une sorte de poussière. Les sporules sont renfermées dans des eoneeptacles qu'on nomme généralement apothétions. Ceux-ci varient singulièrement dans leur forme, orbieulaire, allongée, linéaire, eonvexe, concave, ete.; leur couleur souvent brillante; leur position sur le thallus; ils sont de plus sessiles ou stipités, avee orı sans bord marginal, etc. C'est d'après ces diverses modifications qu'ont été établis les geures nombreux de cette famille, qui sont tous des démembremens de l'ancien genre Lielıen de Limneus.

Les Liøhens sont, en génćral, des plantes parasites, vivant sur. l'écorce des antres arbres, ou quelquefois sur la terre humidc, ou sur les roehes les plus 
stériles. Lear substance est, en général, assez sèche et comme cornée; elle se réduit par l'ébullition en une golée qu'on emploie comme aliment dans quelques espèces. - Les genres de cette famille sont exccssivement nombreux; et chacun des anteurs qui ont étadié cette famillc ont tous proposć une classification différente. Nous citerons comme exemples de cette famille les genres: Parnelia, Sticta, Usnea, Opegrapha, Stereocaulon, etc.

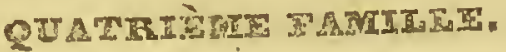

* mépatrques. Hepaticce. Iúss.

Ce sont desplantes intermédiaires'entre les Lichens et les Mousses, tantôt étendues en membranes simples ou lobées, pareourues par une nervure médiane que l'on a eonsidérée comme une tige, tantôtayant une forme dendroïde, e'està-dire composées d'une petite tige ramifiée portant des feuilles sessiles. Les organes générateurs sont fort variés, tantôt plaeés à la surface de la fronde, tantôt axillaires. Ce sont ou des globules, remplis d'un fluide visqueux et réunis dans une sorte de eapsule ou périanthe, tantòt des sporules dont la forme varie, et qui, réunies par des filamens roulés en spirale, sont contenues dans une eapsule qui s'ouvre soit parune fente, soit en quatre valves, et qui est aceompagnée d'une membrane, quti la reeouvre souvent en totalité avant son développement. Cette capsule est sessile ou portée sur un long filament ou pédieclle.

Les organes générateurs sont tellement variés dans cette famille, que dans le Blasia pusilla on en compte cinq formes différentes. C'est donc à tort que plusieurs auteurs ont cru trouver des flears mâles et des fleurs femelles dans les Hépatiques. Ils ont nommé ćtamines les globules remplis d'un fluide visqueux, et pistils les capsules remplies de sporules. Mais, quelle dénomination donner aux cinq organes différens que l'on remarque dans le Blasia?

Pour exemple de cettc famille, nous citerons les genres Marchantia, Riccia, Blasia, Jungermannia, etc. 


\section{CINQUIETE FAMTUx.}

* Mousses. MTusci. Juss.

Les Mousses sont de petites plantes qui aiment les lieux humides et ombragés; clles croissent à terre, sur le trone des arbres, ou les murs et les vicilles habitations; par leur port elles ressemblent à de petites plantes phanérogames cn miniature; leurs raeines sont très-fines ct touffucs, lcur tige simple ou rameuse; lcurs feuilles petites, de formes variées, mais commuuément étroites et subulées. Leurs sporules sont renfermées dans des 'espèces do capsules nommées urnes (theca) portécs sul une soie grèle et plus on moins longue, enveloppées d'abord dans unc sorte de bourse, qui se rompt circulairement par son milieu, ct dont la partie inférieure qui reste à la base de la soie se nomme la vaginule, tandis que la supérieure qui recouvrc le sommet de l'urne a reçu le nom'de eoiffe. L'urne 'elle-même préscnte intéricurement un axe central appelć columelle, ct s'onve au moyen d'un opercule circulaire. Lc contour de l'ouverture de l'urne se nomme péristome et se distinguc en interne et cxterne; il peut être garni de dents, de eils, bouclıé par une membrane ou toultà-fait nu. Indépendamment de ces organes on cn trouve cneore d'une autre sorte: ce sont des corps irrégulièrement ovoïdes et allongés portés sur un pédieule, très-eourt et accompagnés de filamens artieulés.

Les auteurs qui ont admis, dans les Mousses, l'existene de fleurs composées des mêmes organes que eelles des vćgćtaux phanćrogames, ont beaucoup varié sur les fonctions de ces organes et sux le nom qu'il convenait de leux donner. Ainsi Hedwig, dont les travanx ont jeté tant de lumière sur l'histoire des plantes de ectte famille, considère les Moasses comme pourvaes de fleurs mâles ct de flenrs femelles. Les corpuscules ovoïdes et vésieulenx, entremêlés de filamens articulés, sont pour lui des fleurs mâles dont chacune se compose d'un grain de pollen nu et pédicellć, Les urnes coustituent au 
contraire des flears femelles. Pour Palisot de Beauvois; l'urne est une fleur hermaphrodite, dont la columelle centrale est le pistil, et les granules qui l'environnent le pollen. Pour le mème autear, les fleurs nâlles d'Hedwig ne sont que de simples bourgeons ou des bulbilles d'ane nature particalière. Dillenins, au contraire, décrit l'urne comme une fleur mâle. Hill y voit une fleur hermaphrodite dont les séminules sernient les ovules et les eils du pćristome, les étamines, etc., etc.

Mais chacune de ces théories et un grand nombre d'autres, qu'il n'est pas de mon but defaire connaitre ici, se combattent muta ellemont et se détruisent en quclque sorte l'une par' l'autre. Il s'élève en effet une foule d'objections contre bacune d'elles. Quant à l'opinion d'Hedwig, sil'urne n'cst qu'an fruit provenai,t d'un ovaire fécondé, pourquoi le fruit est-il souvcnt déjà parvenu à son état de maturité, quand les prétendues ćtamines qui doivent les féconder cominencent à peine à paraítue? Comment s'opère la fécondation dans les espèces où l'on n'a point pu déeouvrir de fleurs mâles, etc.? etc.

Si l'nrne est une fleur hermaphrodite, que la columelle soit le pistil, et les séminales des grains de pollen, pourquoi, dans certains genres, cette columelle est-elle entièrement solide, et formće d'une substanee dure et parfaitement homogènc?

Si, comme le pense Hill, les dents da péristome en sont les ćtamines, où sont ees étamines dans les genres dont le péristome est nu? etc., etc.

Exemples : Sphagnum, Mniun, Hypnum, Buxbaunia, Tortula, etc. L'organisation des Mousses est tellement partieulière qu'il est impossible de les confondre avec les autres familles de plantes inembryonées.

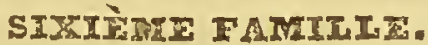

* uycopodiaées. Lycopodicace. Rreh.

Par leur part les Lyeopodiaeées ticnnent le milicu entre les Mousses et les Fougères. Elles sont pourvues d'une tige rameuse, souvent étalée et rampante, de feuilles trèsnombreuses et fort petites. Les organes de la fructification. offrent deux modifieations. Tantôt ce sont de très-petites eapsules globuleuses, trigones ou réniformes, uniloeulaires, contenant un grand nombre de sporules très-petites; tantôt ces eapsules sont un peu plus grosses, s'ouvrant en deux on trois valves et ne renfermant que trois ou quatre sporules plus volumineuses. Ces deux espèees de capsules, qui se trouvent quelquefois réunies sur le mème in- 
dividu, sont tantôt axillaires et solitaires, tantôt réunies ì l'aisselle de braetées et formant des épis simples ou digités.

Le genre Lycopodiun, qui forme le type de cette famille, avait été placé par Linné dans les Mousses, et par Jussieu au nombre des Fougères. Mais l'organisation et la position des organes reproducteurs distinguent faeilement les Lyeopodiacées de ees deux autres familles. Un grand nombre d'anteurs considèrent les capsales plus petites et remplies de granules très-nombrenx comme des fleurs mâles, et les plus grosses eomme des fleurs femelles. Mais elles ne nous paraissent être les unes et les autres que des réeeptaeles tout-ifait analogues à eeux que nous avons déjà observés dans les autres familles de plantes inembryonées.

Les genres qui composent eette famille sont les suivans : Lycopodium, Psilotum, Tmesipteris. Le professeur de Canclolle y réunit aussi le genre Isoëtes, quị nous paraît devoir rester parmi les Marsiléacées.

\section{SEPTIEAE TAMIEIE,}

\section{* fougieres. Filices. Juss.}

Plantes herbaeées et vivaees, devenant quelquefois arboreseentes dans les régions tropieales et s'élevant alors ì la manière des Palmicrs; leurs feuilles ou frondes sont tantôt simples, |tantôt plus ou moins profondément découpées, pinnatifides ou décomposées. Ces frondes offrent un earactère commun, eclui d'être roulées en erosse par leur extrémité, au moment où elles commeneent à se développer. Les organes de la fructifieation sont ordinairement situés à la faee inféricure des feuilles, le long des nervures ou à leur extrémité. Les sporules sont nues ou eontenues dans des espèces de petites eapsules. Ces eapsules, en se groupant, forment de petits amas qu'on nomme Sores. Ceux-ci sont en forme d'éeailles orbieulaires, réw niformes, sessiles ou stipitées, entourées quclquefois d'un anneau élastique, s'ourrant soit par leur eontour, soit par une fente longitudinale ou se déehirant irrégulièrement. Dans le genre Pteris, les sporules sont placées sous le bord replié des feuilles qui forme une ligne non inter- 
rompue. Dans les espèces d'Adianthum elles eonstituent de petites plaques saillantes et isolées, au moyen du bord replié des feuilles. Dans certains genres, elles sont isolées; dans d'autres, elles so groupent, forment des lignes plus ou moins allongées. Les sores commenecnt à se développer sous l'épiderme, qu'ils soulèvent de manière à en être recouverts. On nomme indusies les portions d'épiderme qui servent ainsi d'involucre aux sores. Dans quclques Fougères, telles que les Osmondes, les Ophioglosses, ete., les fructifications sont disposées eu grappes ou en épis.

Les genres de Fougères actuellement connus sont fort nombreux; ils forment cinq sections naturelles, savoir :

I0 Les Porypodracés. Capsules libres, se rompant d'une manière irrégulière, entourées d'un anneau élastique ćtroit et saillant, qui se termine en un pédicelle plus ou moins long. Ex. : Polypodium, Aspidium, Asplenizm; Pteris, ete.

$2^{a}$ Les Greicheñécs. Capsules dibres, sessiles, disposées régulièrement par groupes peu nombreux, entourées dans leur milieu d'an anneau élastique large et plat, s'ouirant par une fente transversale. Ex.: Ceratopteris, Gleichenia, Mlertensia, etc.

$3^{\circ}$ Les Osuundacées. Capsules libres, s'ourrant par une fente longitudinale en denx valves, anneau élastiqurc nul ou remplaeé par une calotte striée. Ex. : Anemia, Lygodium, Osmunda, etc.

40 Les Marattrées. Capsules, sessiles réunies et soudées, ct représentant ane capsule pluriloeulaire, point d'anneau élastique. Ex. : Dancea et MIarattia.

$5^{\circ}$ Les Opnrogrossíte. Capsules libres, en partie plongées dans la fronde, sans anneau élastique, s'ouvrant par une fente transversale. Ex.: Ophioglossum, Botrychium.

Les auteurs ont beaueoup varić sur la nature des organes reproducteurs dans les Fougères. Presque tous ont considéré les eapsules comme des organes femelles. Mais les uns commc Micheli et Hedwig ont regardé comme organes mâles les poils glanduleax qui se montrent quelquefois sur les jeunes feuilles; Ies antres, avee IIill, Schmidel, ont appclé étamines les anneaux des eonceptacles; quclqucs-uns enfin ont donné ce nom aux glandes miliaires et aux indusies. Mais ces diverses opinions peuvent toutes être facilement renversées, puisque toas les organes que l'on a considérés comme des étamines, ne sout nallement constans et manquent très-souvent. 


\section{FUITIEME TAMTILE,}

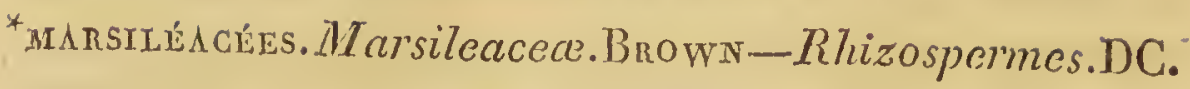

Ce sont de petites plantes aquatiques, fixées au fond de l'eau ou nageant à sa surface, avec ou sans tige apparentc. Les feuilles sont sétacées ou plus ou moins élargics. Les organes reproducteurs sont des espèces d'involucres eoriaces, tantôt d'une seule sorte, tantôt de deux sortes idifférentes. Ils sont épais, à une ou à plusicurs loges, séparées par des cloisons membraneuses, indéhiscens, ou s'ouvrant au moyen de valves. Ils renferment des corpuscules reproducteurs, qui tantôt sont tous organisés de la mềme manière et tantôt sont de deux espéces différentes : les uns plus gros que l'on considère comme des organes femelles; les autres plus petits comme des étamines. Ces involucres sont placés à la base des feuilles et quelquefois même adhérens à celles-ei. Quand les involucres sont de deux sortes sur la même plante, les uns sont membrancux, el contiennent unc grappe de corpuseules qu'on a regardés'comme des graines. Les autres, qui ont été décrits comme des organes mâles, contienucnt un grand nombre de granules sphérirques, attachés" par un long filament à une columelle centrale.

On a divisé cette famille en deux sections, savoir les Marsiléacées vraies; yui n'ont qu'ane seule espèce d'involucres, rcnfermant des 'granulcs de deux sortes et composées des genres|MLarsilea, Pilnlaria ct Isoetes, dernier genre quc quelques auteurs rapprochent des Lycopodiacécs, ct les Salvinices, dont les involucres sont de deux espèces différcntes et contiennent chacune des granules différemment organisés. $\Lambda$ cette seconde tribu appartiennent les genres Salvinia et Azolla.

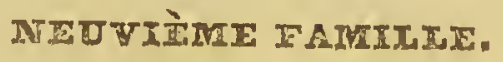

* esquisétacées. Equiselacece. DC.

Cette pelite famille ne comprend que le seul genre Equisetum, connu en français sous le nom de Presle. 
Toutes les espèces qui composent ee groupe sont des plantes herbacées, vivaccs. Leur's tiges, simples ou ramouses, sont en général erẹuscs, striées longitudinalement et offrant de distance en distance des noends, d'où naissent des gaines fendues en un grand nombre de languettes et semblant ètre des feuilles verticillées soudées entr'elles; quelquefois de ces nouds naissent des rameaux verticillés. Les fructifieations forment des épis terminaux. Ces épis se composent d'écailles épaisses et peltées, semblables à celles que l'on remarque dans lesfleurs mâles de plusieurs Conifères et entr'autres de l'if. A la face inférieure de ces écailles naissent des espèces de capsules disposéés sur une seule rangée et s'ouvrant par une fente longitudinale qui regarde du côté de l'axe. Ces capsules sont remplies de granules extrêmement petits, qui se eomposent d'une partie globuleuse, de la base de laquelle maissent quatre longs filamens articulés, renflés à leur partie supérieure, et roulés en spirale autour du corps globuleux qui est une veritable sporule.

Entrainé par l'analogie de forme qui existe entre les organes reproducteurs des Equisétacées et les étamines de quelques Conifères, Linné nommait ees organes des étamines, sans indiquer les organes qu’il regardait comme despistils. Hedwig, au contraire, considérait chaque granule comme une fleur hermaphrodite; la partie globuleuse était le pistil, et les filamens étaient quatre étamines dont le pollen était situé extérienrement. Mais ces filamens sont certainement analogues à ceux que l'on troupe dans les Jongermannes, les Marchantia, Targionia etc.

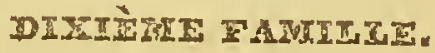

* characées. Chataceœ. Rich.

Plantes aquatiques et submergées, dont les tiges grèles, rameuses, verics et quelquefois translucides, portent de distance en distance des rameaux verticillés au nombre de huit à dix. Sur les rameaux des verticilles supérieurs ou trouve des espèces de sporanges ou de capsulcs au nom- 
bre de trois, quatre ou einq. Chacun d'cux est environné à sa base par deux ou trois bractées ou rameaux avortés que Linné considérait comme un ealice. Ils sont uniloculaires et contiennent des sporules nombreuses réunies en tune sculc masse, qu'on a regardéc comme une seule graine. Ces sporanges sont formés de deux tégumens, l'un externe; membrancux et transparcnt, très-mince, terminé supéricurement par cinq petites dents étalées en forme de rosace; l'interne est dur, sce, opaque, composé de cinq petites valves étroites contournées en spirale. Indépendamment de ces organes on observe encore sur les rameaux des espèces de tubercules rougeâtres sessiles et arrondis. La plupart des auteurs les décrivent comme des étamines. Ils sc composent d'unc membranc réticuléc, transparente, formant une sorte de vésicule remplic d'un fluide mucilagineux dans lequel on observe des filamens blânehâtres et articulés et d'autres filamens plus gros, fermés à l'une de lcurs extrémités, paraissant s'ouvirir à l'autre, et remplis d'un fluide rougeàtre. Ces tubereules, par les progrès de la végétation, s'affaissent, mais ne s'ouvrent pas.

Cette famille ne sc compose que du senl genre Chara. Il avait ćté établi par Vaillant en I 7 I 9 dans les Mlémoires de l'académic des seiences de Paris. Linné l'avait d'abord placé parmi les Cryptogames, tout prìs des Lichens; plus tard il changca d'opinion et lc rangea paruni les Phanérogames dans la monœeie monandrie. M. de Jussicu, dans son Genera, lc rénnit aux genres dont il forma sa famille des Nayades. Mais le professeur Liehard (in Michaux: flor. bor. am.) en fit le type d'unc famille distinctc sous le nom de Characées, famille qu'il plaẹa dans les Aeotylédoncs. Plus récemment M. Rob. Broun rapproche ee genre des Hydrocharidées, M. Léman des Onagraircs, et enfin MM. Martius, Walroth etBory de St-Vincent pensent qu'il a une très-grande analogie avec des Hydrophytes, et que c'cst dans eettc famille qu'il doit ètre placé. Mais si l'on compare la strueture des organes reproduten's des Characées avec celle des autres plantes acotylédones, on y trouvcra une trèsgrande analogie, particulièrement avec lcs Marsiléacécs, dont elles ne diffc̀rent que par lcurs sporanges plus petits, à cinq dents, à tégument double, et par les tubercules rougeâtres que l'on observe aussi sur leurs rameaux. 


\section{DEUXIEME DIVISION.}

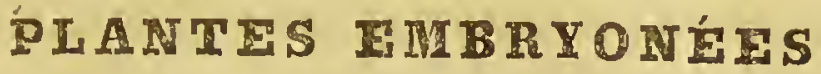

OU PHANÉROGAIIES.

Ce second grand 'embranchement du règne végétal se compose de toutes les plantes dont la strueture est plus compliquée, qui sont pourvues d'organes sexuels mâles et femelles, e'est-à-dire d'étamines et de pistils, et qui se reproduisent au moyen de véritables graines, ayant besoin d'être féeondées pour être aptes à donner naissanee à de nouveaux individus. D'après la structure de l'embryon, on les a divisées en deux groupes, les Monocoty. lédous et les Dicotylédons.

\section{Des puantes monogotylédones.}

C'est dans la strueture de l'embryon que réside le earactère essentiel des végétaux qui forment ee groupe. Cet embryon est monoeotylédon ( $V$. pag. 305 les earactères de l'embryon monoeotylédon). Mais indépendamment des caraetères tirés de l'embryon, il en a eneore d'autres empruntés aux organes de la végétation et de la floraison, et qui peuvent servir, au défaut des premiers, à reconnaître une plante monoeotylédone. Nous les indiquerons très-brièvement iei :

$I^{\circ}$ La strueture interne de la tige, qui se eompose d'une masse de tissu eellulaire, dans laquelle sont épars les faiseeaux vaseulaires. $(V$. pag. 74 , l'organisation de la tige des Monocotylédons, et pag. $9^{3}$, son mode de développement.) $2^{\circ}$ Les nervures des feuilles en général parallèles dans les Monocotylédons, tandis qu'elles sont irrégulièrement ramifiées dans les Dicotylédons. 
$3^{\circ}$ Le périanthe, eonstamment simple dans les plantes monocotylédones, e'est-à-dire qu'il n'y a qu'un caliee, quelquefois eoloré à la manière des pétales.

$4^{\circ}$ En général, dans les végétaux unilobés, les organes floraux sont au nombre de trois ou d'un multiple de trois, tandis que e'est le nombre einq qui domine dans les dieotylédons.

$5^{\circ}$ Mais e'est surtout le port, l'aspect gérééral qui est diffèrent dans ees deux grands embranehemens du règne végétal, et une fois que l'on a bien saisi les earaetères des prineipales familles des plantes monoeotylédones, eomme les Graminées, les Joneées, les Liliaeées, les Iridées, les Amomées, les Orehidées, les Palmiers, ete., on distingue ensuite très-faeilement, uniquement par le port, les plantes monoeotylédones des plantes dieotylédones.

Les Monoeotylédons se divisent en trois elasses suivant - que leur insertion est liypogyne, périgyne, ou épigyne.

\section{DEUXIEME CLASSE.}

MONOHXPOGYIE.

\section{OMZIENRE FAITIXIE.}

${ }^{7}$ nayades. Nayader. Juss.--Fluviales. Vent.-Polamophiles. Ricn.

Les Nayades, ainsi que l'indique leur nom my thologique, sont des plantes qui eroissent daus l'eau ou nagent à sa surfaee. Leurs feuilles sont alternès, souvent embrassantes à leur base; leurs fleurs, très-petitcs, sont unisexuées, monoïques ou plus rarement diö̈ques. Les fleurs màles consistent en une étamine nue ou aeeompagnée d'une éeaille, on enfin renfermée dans une spathe, qui eontient deux ou un plus grand nombre de fleurs. Les fleur's femelies se eomposent d'un pistil nu on renfermé dans ume spathe; elles sont tantôt solitaires, tantòt géminécs, ou culin 
xéunies en plus grand nombre ct quclquefois environnées de fleurs mâles daus unc enveloppc commune, de manière que leur réunion scmble rcprésenter une flcur hermaphrodite. L'ovairc est libre à unc seulc logc contenant un seul ovule pendant (dans lc genre nayas, il est latéral et presque basilaire). Le style cst généralement court, terminẹ par un stigmate tantôt simple, discoïde, planc ct membraneux (zanichellia); tantôt à deux ou trois divisions longues et linéaires. Le fruit est sec, monosperme, indéhiscent; la graine renferme sous son tégument propre un embryon le plus souvent recourbé sur lui-même, ayant sa radicule très-grossc et opposéc au hile.

Exemples : Nayas: Zostera, Ruppiä, Zanichellia et Potamogeton. - Les genres que nous venons de mentionner sout les seuls qui composeut la famille des Nayades, dont nous avons 'singulièrement modifié les caractères, ayaut donné de sa structure une explication différente de celle qui eu avait été donnée jusqu'à présent. On doit en exelure plusieurs genres qui y avaient été rapportés à tort: tels sont: Hippuris et Myriophyllum, qui forment la famille des Haloragées; Ceratophyllum, réuni aux Salicariées; Saururus et Aponogeton, formant la famille des Saururées; Callitriche, genre dicotylédone voisin des Euphorbiacées; Chara, genre acotylédone formant la famille des Characées.

La famille des Nayades est très-voisine des Aroïdées, dont elle se rapproche et par son port et par ses caractères: les Aroïdées en diffèrent surtout par leur ovule dressé et leur embryon coutenu dans un endosperme charnu.

\section{DOUZIİME FAMIILE.}

* aroünées. Aroüdece. Juss.

Plantes vivaccs à racine ordinairement tubéreuse; à feuilles souvent toutes radicalcs, ou alternes sur la tige; fleurs disposécs en spadices environnés cn général d'unc spathe de forme variable; unisexuées, monoïques, dépourvues d'envcloppes florales, ou hcrmaphrodites et entourées d'un calice à quatrc, cinq ou six divisions. Dans le premier cas, les pistils occupent en général la partie inférieure du spadicc, et doivent être considérés chacun 
eomme une fleur femelle, et les étamines comme autant de fleurs mâles; rarement les étamines et les pistils sont mélangés. Dans le seeond eas les fleurs, au lieu d'être eonsidérées eomme des fleurs hermaphodites, peuvent être déerites comme une réunion defleurs unisexuées; ainsi ehaque étamine et son éGaille eonstituent une fleur mâle ct le pistil eentral une fleur femelle. L'ovaire est en général à une seule loge contenant plusieurs graines attaehées à la paroi inférieure, oụ à trois loges; le stigmate est quelquefois sessile, plus rarement porté sur unstyle assez eourt. Le fruit est une baie ou plus rarement une capsule qui quelquefois est monosperme pas avortement. La graine se eompose, outre sou tégument propre, d'un endosperme eharnu dans lequel est placé un embryon cylindrique et dressé.

La fanille des Aroïdées se divise en trois tribus, savoir:

I $^{\text {re }}$ tibu. Les arö̈ó́es vrares; fleurs nues sans écailles; fruit eharnu. Arum, Arisarum, Caladium, Culcasia, Calla, Richardia.

$2^{\text {e }}$ tribu. Les orovtracírs; deurs entourées d'éeailles en forme de calice: Dracontium, Pothos, Carludovica, Orontium, Acortus.

3c tribu. Les rrstraées. Fruit sec et eapsulaire: Pistia, Ambrosinia.

Voisine des Nayades et des Typhacées, cette famille se distingue sartout. par son port, la disposition des fleuts, son embryou conteru dans un endosperme et plusieurs autres earactères.

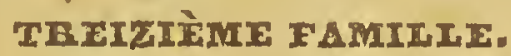

* typhnnées. Typhinece. - Typhoe. Juss. Pandanece. R. Brown.

Plantes aquatiques ou arborescentes et terrestres, à feuilles alternes engaînantes à leur base, à fleurs unisexuées, monoïques. Les fleurs mâles forment des chatons cylindriques or globuleux, composés d'ćtamines nombreuses, souvent réunies plusieurs ensemble par leurs filets, et cntremêlées de poils ou de petites éeailles, mais sans ordre et sans calicc propre. Les fleurs fernelles, disposées de la même manière, ont quel- 
quefois les écailles réunies au nombre de six autour du pistil et formant un calice de six sépales; ce pistil est sessile ou stipité, à une, plus rarement à deux loges, contenant chacune un ovule pendant. Le style, peu distinct du, sommet de l'ovaire, se termine par un stigmate élargi, comme membraneux et marqué d'un sillon longitudinal. La graine se compose d'un endosperme farineux contenant dans son centre un embryon cylindrique, dont la radicule est supérieure, c'est-à-dire a la même direction que la graine.

Cette petite famille ne se compose que des deux genres Typha et Sparganium. M. Robert Brown l'a réunie à la famille des Aroïdées, a vec laquelle elle a en effet plusieurs points de contact; mais néanmoins elle en diffère par plusieurs caractères, et entre autres par ses graines renversées et la structure de ses fleurs. Cependant ces denx familles mériteraient peut-être d'être réunies. Fant-il placer dans cette famille le genre Pandanus, qui ressemble tellement au genre Sparganium qu'il piraît en être en quelque sorte une espèce arborescente; ou faut-il, à l'exemple de Rob. Brown, en former une famille particalière sous le nom de Pandanées?

\section{QUATORRIÈME TAMIIIE.}

\section{SAURUbĖes. Saururece. Rich.}

Plantes qui croissent sur le bord des eaux ou nagent à sa surface. Leurs feuilles sont alternes simples pétiolées. Leurs fleurs sont hermaphrodites, dépourvues de périanthe, et ayant une simple écaille qui en tient lieu, et sur laquelle sont insérées les étamin es etles pistils. Les premières sont au nombre de sixà neuf, ayant lenrs filets subulés; et leur anthère à deux loges, qui s'ouvrent par un sillon longitudinal. Les pistils sont au nombre de trois à quatre au centre de chaque fleur. Ils sont à une seule loge contenant deux ou trois ovules dressés ou ascendans. Le style est marqué d'un sillon glanduleux sur le milieu de son còté interne, qui à son sommet s'élargit en stigmate. Le fruit se compose de petites capsules indéhiscentes contenant chacune une on deux 
graines. Celles-ci sous leur tégument propre contiennent un gros endosperme au sommet duquel est appliqué un très-petit embryon discoïde.

Cette famille se compose des genres Saururus et Aponogeton. Quant à l'Ouvirandra ou Hydrogeton, que l'on en a rapproché, il en diffère par la présence d'un calice et par son cmbryon sans endosperme. Ce dernier caractère, s'il est réel, ce que nous n'avons pas été à même de vćrifier, éloignerait ce genre des Saururées pour le rapprocher des Alismacécs.

\section{QUINZIÈME FAMILLE.}

cabombés. Cabombece. Rrar.

Petite famille uniquement composée des deux genres Cabomba et Hydropeltis, qui renferment des plantes herbacées vivaces croissant dans les eaux douces du nouveau continent. Leurs feuilles, qui nagent à la surface de l'eau, sont entières et peltées ou divisées en lobes plus ou moins fins. Les fleurs sont solitaires et longuement pédonculées. Leur calice est à six divisions profondes, ou à six sépales disposés sur deux rangées; les étamines varient de six à trente-six. Le nombre des pistils, réunis au centre de la fleur, est depuis deux ou trois jusqu'à dix-huit, c'està-dire en général moitié moindre que celui des étamines. Chaque pistil, qui est plus ou moins allongé, offre une seule loge contenant deux ovules pariétaux et pendans; le style est plus ou moins long, terminé par un stignate simple. Le fruit est indéhiscent, à une ou deux graines; celles-ci contiennent sous leur tégument propre un très-gros endosperme charnu ou farineux creusé à sa base d'une petite fossette dans laquelle repose un embryon presque discoïde, en forme de clou et parfaitement indivis.

Cette petitc famille a beaucoup de rapports avec les Saururées par son ovaire, son fruit et son embryon; mais dans cctte dernièrc famille les flemrs sont nues. Elle se rapproche anssi beaueoup par l'organisation de sa fleur des-Alisuacéces, lont ello diffère par son gros endosperunç ęt la forme de 
son embryon. M. de Candolle (Syst.nat. veget.) place les Cabombées parmi les Dicotylédones, et en forme une tribu des Podophyllées; mais cette manière de voir nous paraìt peu fondée, l'embryon des Cabombées étant bien certainément monocotylédon.

\section{SEIXIEMTE FAMIXIE.}

* cypéracíes. Cyperacece. Juss.

Végétaux herbacés croissant en général dans les lieux humides et sur le bord des eaux. Leur tige est un chaume cylindrique ou triangulaire, avec ou sans noeuds. Les feuilles sont engaînantes et leur gaîne est entière et non fendue, assez souvent garnie à son orifice d'un petit rebord membraneux nommé ligule. Les fleurs forment de petits epis ou épillets écailleux, composés d'un nombre variable de fleurs; chaque fleur se eompose d'une seule écaille, à l'aisselle de laquelle on trouve généralement trois étamines, un pistil formé d'un ovaire uniloculaire et monosperme, surmonté d'un style simple à sa base, portant en général trois stigmates filiformes et velus. Les étamines ont leur filet capillaire, leur anthère terminée en pointe à son sommet, bifide seulement à sa base. On trouve souvent en dehors de l'ovaire, des soies ou des éeailles en nombre variable, quelquefois même un utricule qui le reeouvre en totalité (Ex : Carex). Le fruit est un akène globuleux comprimé ou triangulaire. L'embryon est petit, placé vers la base d'un endosperme farineux, qui le recouvre par une lame très-mince.

Cette famille est très-naturelle et le nombre des genres qui la composent est très-considérable. Ises fleurs sont unisexuées ou hermaphrodites, et les étamines varient beaucoup en nombre. Les genres Scirpus, Crperis, Schocnus, Mariscus, Papyrus, etc, appartiennent à cettefamille. Elle a beaucoap d'analogie avec celle des Graminées, mais en diffère par quelques caractères, que nous exposerons à la suite de cette dernière famille. V. 'Graminéos.' 


\section{DOIX-SEPTIEME FAMIEIE.}

* graminées. Graminec. Juss.

Plantes herbaeées amnuelles ou vivaees, rarement soufrutescentes, d'un port tout partieulier et fort earaetéristique; ayant pour tige un chaume généralement fistuleux, offrant de distanee en distanee des nocuds pleins, d'où partent des feuilles alternes engainantes. Cette gaîne, qui peut être considérée eomme un pétiole élargi, est fendue dans toute sa longueur, et offre à son point de jonetion avee la feuille une sorte de petit collier mem. braneux ou formé de poils, qu'on nomme collure ou ligule. Les fleurs sont disposées en épis ou en panieules plus ou moins rameuses. Ces fleurs sont ou solitaires, ou. réunies plusieurs ensemble et formant de petits grouppes qu'on nomme épillets. A la base des épillets ou des fleurs solitaires, on trouve deux éeailles, l'une externe, l'autre interne formant la lépicène; rarement l'éeaille interne manque, et la léplec̀ne est univalve. Chaque fleur se compose de deux autres éeailles formant la glume, d'étamines généralement aunombre de trois, 'quelquefois moins, rarement plus; leurs filets sont capillaires, les anthères bifides à leurs deux extrémités; d'un pistil formé par un ovaire uniloeulaire, monosperme, marqué d'un sillon longitudinal sur l'un de ses eôtés, surmonté de deux styles que terminent deux stigmates poilus et glanduleux; plus rarement le style cst simple ou bifurqué à sa partic supérieure. En dehors de l'ovaire sur la faee opposée au sillon, on observe dans un grand nombre de genres deux petites paléoles de forme variée, qui constituent la glumelle ou nectaire. Le fruit est une cariopse, plus rarement un akène, nu, ou enveloppé dans les valves de la glume, qui se détache et tombe avec Iui. L'embryon a 
une forme disçö̀de et est appliqué sur la partie inférieure d'un endosperme farineux.

Cette famille est une des plus naturelles dı règne végćtal. Elle se conpose de tous ces végétanx connus sous les noms vulgaires de céréales ou de gramens; tels sont le blé, l'orge, l'avoine, le maïs, le panis, le riz, lc mìilet, etc. Les genres sont fort nombreux, et leurs caractc̀res fondés súr là forme variée des écailles, qui sont tantôt nues, tantôt portant à leur sommet ou sur leur dos unc arète ou une soie, quelquefois même plusieurs. Nous citerons comme exemples de genres de cette famille: les Triticum; Avena, Hordeum, Arundo, Poa, Sacharum, etc.

La famille des Graminées a la plus grande analogie avec celle des Cypéraeées par son port et plasieurs de ses caractères. Mais d'abord la gaîne des feuilles dans les Cypéracées est entière, et fendue dans les Graminées; dans ces dernières il y a deur éeailles pour chaque fleur, il n'y en a qu'une dans les Cypéracées; dans les Graminées il y a deux stigmates, et généralement trois dans les Cypéracées. L'embryon est plus compliqué dans les Graminées que dans les Cypéracées.

\section{TROISIEME GLASSE.}

MONOPERIGYNIE:

\section{DIX-HUIMIEMT FAMILIE.}

* palmirers. Palma. Juss.

Grande et belle famille, aussi remarquable par le port des végétaux quil la composent que par l'organisation intérieure de leurs diverses parties. Les Palmiers sont en général de grands arbres à tige simple, cylindrique, nue, et qu'on désigne sous le nom de stipe ou tige à colonne. A son sommet elle est courounée par un faisceau de feuilles très-grandes, pétiolées, persistantes, pinnées, ou déeomposées en 'un nombre plus ou moins eonsidérable de folioles de formes varićes. Les fleurs sont hermaphrodites ou plus souvent unisexuées, dioïques ou polygames, formant des chatons ou une vaste gratppenommée régime, et enveloppée avant son épanouissement dans une spathe eoriace et quelquefois ligneuse. Le périanthe est à six divi- 
sions, dont trois internes et trois externes, de manière à simuler un calice et une corolle. Les étamines sont au nombre de six, rarement de trois. Le pistil est simple, ou formé de la réunion de trois pistils distincts ou soudés; il offre une ou trois loges contenant chacune une seule graine; chaque pistil se termine par un style que surmonte un stigmate plus ou moins allongé. Le fruit est une drupe charnue ou fibreuse contenant un noyau osseux très-dur, à une ou à trois loges monospermes. La graine, outre son tégument propre, se compose d'un endosperme charnu ou cartilagineux, offrant quelquefois une cavité centralc ou la térale; l'embryon, très-petit, est cylindrique, placé horizontalement dans une petite fossette latérale de l'endosperme.

A l'exception dn Palmier éventail (Chamarops lumilis) tous les autres Palmiers sont exotiques. Ils habitent surtout dans les régions intertropicales du nouveau et de l'ancien continent. Ces arbres ne sont pas seulement remarquables par l'élégance de lears formes et la hautear prodigieuse à laquelle plasiears peuvent s'élever, mais ils sont ausi d'une très-grande importance par les services nombreux qu'ils rendent aux hahitans des contrées où ils croissent naturellement. Les fraits d'un grand nombre d'espèces, comme le Cocotier, le Dattier, le bourgeon terminal du choux palmiste, sont des alimens pour les habitans de l'Afrique septentrionale et de l'Inde. Plusieurs espèces fournissent unc fécule amilacée nommée Sagou; d'autres un principe astringent analogue au sangdragon; quelques-uns donnent une haile grasse, comme l' Ailais guineensis, qui fournit l'huile de Palme.

Le genres principaux de cette famille sont: Cocos, Phocnix, Chamarops, ALlais, Areca, Sagus, etc.

\section{DIX-NEUVIX̀ME FAMILIE.}

nestracées. Restiacece. R. Brown.

Plantes toutes exotiques, ayant le port des joncs ou de quelqucs Cypéracées, vivaces ou même soufrutescentes. Leurs feuilles sont étroites ou manquent quelquefois. Leurs chaumes sont nus ou couverts d'écailles engaînantes, à 
gaîne fendue d'un côté. Les fleurs, généralement unisexuées, sont réunies en épis ou en capitules, et souvent enviromnées de spathes. Leur calice, qui manque rarement, présente de deux à six divisions profondes. Les étamines varient d'une à six; quand il y en a moitié moins que de sépales au calice, elles sont opposées aux sépales internes (ce qui est le contraire dans la famille des Joncées): Les pistils sont libres ou soudés, à une seule loge contenant un ovule pendant; le style est simple, terminé par un stigmate subulé. Les fruits sont de petites capsules s'ouvrant longitudinalment d'un seul côtté, ou des espèces de noix indéhiscentes. La graine est renversée. L'endosperme est farineux, et l'embryon, qui est discoïde, est appliqué sur l'extrémité de l'endosperme opposée au hile.

Cette famille, qui se compose des genres Restio, Eriocaulon, Desvauxia, et d'un grand nombre de genres nouveaux originaires de la Nouvelle-Hollande, se distingue des Joncées par son embryon extraire et opposé au hile, par ses graines solitaires et pendantes, ses étamines opposées aux sépales interieurs, etc. Elle a anssi quelques rapports avec les Cypéracées, dont elle différe par ses gaineses fendues, la structure et la position de son embryon.

\section{YINGTIÈME FAMILLE.}

* Jon cées. Joncece. Delaharpe.-Juncorum pars auctor.

Plantes herbacées vivaces, rarement annuelles; ayant leur tige ou chaume cylindrique, nu ou feuillé, simple; leurśfeuilles, engaînantes à leur base, out leur gaîne tantôt entière, tantôt fendue dans toute sa longueur. Les fleurs sont hermaphrodites, terminales, disposées en panicule, ou en cime, renfermées avant leur épanouissement dans la gaîne de la dernière feuille, qui leur forme une sorte de spathe. Le calice est formé de six sépales glumacés, disposés sur deux rangs; les étamines, au nombre de six ou seulement de trois, sont insérées à la base des sépales internes; quand il n'y a que trois étamines, elles corres- 
pondent aux sépales extérieurs: L'ovaire est uniloculaire trisperme, ou triloeulaire polysperme plus ou moins triangulaire; lc style est simple, surmonté dc trois stigmates. Le fruit est une capsulc à une ou à trois loges ineomplc̀tes, contenant trois ou plusieurs graines, et s'ouvranten trois valves portant chacune une cloison sur le milicu de leur face interne. Les graines sont ascendantes; leur tégument est double; l'endosperme dur et farineux, contenant vers sa base un petit embryon arrondi.

Les genres qui composent aujoard'hui cette famille sont: Juncus; Luzula et Abama. M. de Jussieu (Genera Planturum) avait réuni dans sa famille des Joncs, un grand nombrc de genres fort différens entr'eux. Ces genres; mieux étudiés, sont devcnus les types d'un grand nombre de familles distinctes, sous les noms de Restiacées, Commélinées, Alismacées, Pontédériées, Colchicées.

Telle qu'elle a été limitée dans ces dcrniers temps par'M. dc La Harpc (Monograph. des 'oncées. Mém. soc. hist. nat., Paris, vol. 3), la famille des Joneées a quelques rapports avec les Cypéracées, dont clle diffère par sa fleur formée de six sépales et de six étamines; et avcc les Restiacées; mais celles-ci ont lear capsule à trois loges complètes, leurs graines pendantes, et lear embryon extrairc et opposé au hile.

\section{VINGT-UNIEME FAMITEE.}

\section{comménétes. Commelinece. R. Brown.}

-Petite famille formée desgenres Commelina et Tradescanlia, auparavant placés dans les Joncécs, et de quelques autres genres nouvcaux qui y ont été réunis. Les fleurs ont un calice à six divisions profondes, disposées sur deux rangs; les trois extérieures sont vertes et ealycinales, lcs trois intérieures sont colorées et pétaloïdes; les étamines, au nombre de six, rarement moins, sont libres. L'ovairc offre trois loges contenant chacunc un petit nombre d'ovules inserés à leur anglc internc, et est surmonté d'un stylc et d'un stigmate simplc. Le fruit est une capsule globuleuse, ou à trois angles comprimés, à trois loges, 
s'ouvrant en trois valves qui portent chacune une cloison sur le milien de leur face interne. Les graines sont rarement au-delà de deux dans chaque loge. L'embryon, en forme de toupie, est opposé au hile et placé dans une petite cavité d'un endosperme dur et charnu.

Les plantes qui composent cette famille sont herbacées; annuelles ou vivaces. Leur racine est formée de tabercules charnus; leurs feuilles alternes simples on engainantes; leurs flear's naes on enveloppées d'ane spathe foliacée.

Cette famille se distingue: $I^{0}$ des Joncées par son port, par son calice, dont les trois sépales intérieurs șont colorés, par la forme de son embryon; $2^{\circ}$ des Restiacées également par son calice, par la structure de sa capsule à loges polyspermes et ses graines axillaires et non pendantes.

\section{VINGT-DEUXIEME FAMILIE.}

rontédériacées. Pontederiacece.-Pontederece. Kunth.

Plantes vivant dans lể voisinage des eaux, portant des feuilles alternes, pétiolées, engaînantes à leur base, des fleurs solitaires, ou disposées en épis ou en ombelle et naissant de la gaîne des feuilles, qui est fendue. Le calice est monosépale tubuleux à six divisions plus ou moins profondes égales ou inégales; les étamines, au nombre de trois à six, sont insérées au tube ducalice; leurs filets sont égaux ou inégaux; l'ovaire est libre ou semi-infère, à trois loges polyspermes; le style et le stigmate sont simples. Le fruit est une capsule, quelquefois légèrement charnue, à trois, rarement à une seule loge, contenant une ou plusieurs graines, attachées à l'angle interne; cette capsule s'ouvre en trois valves septifères sur le milieu de leur face interne. Le hile est ponctiforme; l'endosperme, farineux, contient un embryon dressé, placé dans sa partie centralè et ayant la même direction que la graine.

Cette petite famille ne se compose que des deux genres Pontederia et Heteranthera. Elle a les rapports les plus grands d'une part avec les Commćlinées et d'autre parț avec les Liliacées. Elle diffère des premières par son 
embryon ayant la même direction que la graine, ce qui est le contraire dans Jes Commélinées; par sa graine, dont lc hile est ponctiforme, tandis qu'il en occapc tout un côté dans cellcs-ci; ellc cn diffère aussi par son calice tubuleux et les loges polyspermes de sa capsulc. Quant aux Liliacées, leors rapports nous paraissent encore plusintimes. Mais le port des Pontédériacées est différent; ce sont des plantes aquatiques à racine fibreuse; leur stigmate est simple. Néanmoins je ne suis pas éloigné de croire que les Pontédériacées pourzaient leur être réunies.

\section{VINGT-TROISIÈME FAMXXIE.}

${ }^{*}$ Alismacées. Alismacece.-Alismoüdes. Vent. Juncorum pars. Juss. Alismacea, Juncaginea, Butomea et Podostemea?? RICH.

Plantes herbacées annuelles ou vivaces, croissant pour le plus grand nombre dans les lieux humides et sur le bord des étangs ou des ruisseaux. Leurs feuilles sont pétiolées, engainantes à leur base. Leurs fleurs hermaphrodites, rarement unisexuées, sont disposées en épis, en panicule ou. en sertule. Leur calice qui manque dans le seul genre $L_{i}$ lcea, est formé de six sépales, dont les trois plus intérieurs sont généralement colorés et pétaloïdes. ILes étamines varient en nombre de six à trente. Les pistils sont réunis plusieurs ensemble dans chaque fleur, et restent distincts ou se soudent plus ou moins entr'eux. Leurovaire, qui est uniloculaire, contient un, deux ou un très-grand nombre d'ovules dressés, pendans ou fixés au côté interne. Les fruits sont de petits carpelles, secs, généralementindéhiscens. Leurs graines, ascendantes ou renversées, se composentd'un tégument propre, qui recouvre immédiatement un gros embryon droit ou recourbé en forme de fer à cheval.

- Nous réunissons ici en une seule les trois familles que mon père avait établies sous les noms d'Alismacées, de Juncaginées et de Butomées, mais que lui-même cependant n'était par éloigné de considérer comme trois sections naturelleș d'une mème famille. Il eșţ lẹ prẹmier quạ ait bien fait con- 
naître la structure de l'ovaire et de l'embryon dans ces trois groupes, qui deviennent ici des sections d'une même famülle. Aiusi nons diviserons les Alismacées en trois sections, savoir:

ro Les Juncagrnées, qui ont le calice uniforme, nal dans le genre Lilaca, une senle graine ou deux; dressées, et un embryon droil; tels sont les genres: Lilcea, Triglochin et Scheuchzeria.

$2^{0}$ Les AzrsuÉrs, qui ont le calice semipétaloïde, une ou deax graines sutarales, dressées ou ascendantes; un embryon droit ou recourbé en forme de fer à cheval: Sagittaria, Alisma, Damasonium.

3॰ Les витоми́es, dont le calice est semipétaloïde; les graines nombreuses, attachées à des veines qui adhèrent àl'intérieur de chaque loge, et l'embryon droit ou recourbé en forme de fer à cheval. Le mode d'adnexion des graines est fort singulier dans cette tribu, et se rencontre fort rarement. La famille des Flacourtianées dans les Dicotylédones en offre un second exemple: Les genres qui forment les Batomées sont: Butomus, Hydrocleis et Limnocharis.

La famille des Alismacées a beaucoùp de rapports avec les Nayades, surtout par son embryon dépourvu d'endosperme. Mais la graine des Nayades est renversée, et celle des Alismacées est dressée; la radicale est tournée vers le hile dans celles-ci, et lui est opposée dans celles-lì. Dailleurs la structure dcs fleurs offre aussi de très-grandes différences. Quant aux Joncées, dont les Alismacées faisaient primitivement partie, ces dernières en diffèrent surtout par leur embryon sans endosperme, tandis que les Joncées en ont constamment un.

Peat-être doit-on rapporter ici la famille des Podostémées indiquée par mon père, et qui ne diffère des Juncaginées que par la capsule polysperme.

\section{VINGT-QUATRIEME TAMIIIE.}
* corchicacées. Colchicacea. Dr Cand-Juncor'um par's. Juss.

Plantes herbacées, à racine fibrcusc ou hulbifère, à tige simple ou rameuse, portant des feuilles altcrnes et cngâ̂nantes. Les ,fleurs sont terminales, hermaphrodites ou unisexuécs; leur calice est coloré, à six divisions trèsprofondes, quelquefois tubulcux à sa base. Lcs étamines, au nombre de six, sont opposécs aux divisions du calice. Les ovaires sont au nombre de trois dans chaque flcur, tantôt libres, tantòt plus ou moins soudés de manière ì repré- 
senter un ovaire triloeulaire; chacun d'enx contient un grand nombre d'ovules attachés à leur angle interne. Le sommet de chaque ovaire porte un style, quelquefois trèslong, terminé par un stigmate glanduleux. Le fruit se compose de trois carpelles' distinets s'ouvrant par une fente longitudinale et intérieure; tantôt ees trois carpelles se soudent et forment une capsule à trois loges, mais qui finissent par se séparer de nouveau à l'époque de la maturité, et s'ouvrent ehaeun par une suture placée à leur angle interne. Les graines se composent d'un tégument membraneux ou rétieulé, surmonté quelquefois vers le hile d'un tubereule plus ou moins volumineux, d'un endosperme charnu, qui contient un embryon cylindriquéplacé vers le point opposé au hile.

Cette famillc tient en quelque sorte lc milien entre les Joncées, dont elle faisait autrefois partie, et les Liliacées. Elle sc distingue des premières par son calice coloré, ses capsules distinctes ou se séparant à la maturité. Cé dernier caractère, joint aux trois styles et au tćgument de la graine membraneux ct jamais crustacé, distinguent lcs Colchicées des Liliacées.

Les genres principaux de cette famille sont: Colchicum, Narthecium, $\mathrm{Te}$ ratrum, Merendera, Melanthium, Bulbocodium, etc.

\section{,VINGT-CINQUIËME FANIILE.}

${ }_{2}^{7}$ AsparaginéEs. Asparaginece.-Asparagorum par's. Juss. Smilacece. R. Brown.

Plantes herbacées vivaces ou frutescentes; à raeine fibreuse, à feuilles alternes, opposées ou vertieillées, quelquefois très-petites et sous'la forme d'éenilles. Fleurs quelquefois unisexuées, diversemeut disposées. Leur ealice, souvent coloré et pétaloïde, offre six ou huit divisions plus ou moins profondes, étalées ou dressées; des étamines en même nombre que les divisions ealycinales, à la base desquelles elles sont attachées ; leurs filets sont libres, rarement monadel phes. L'ovaire est librc, à troịs, rarement 
i une seule $\operatorname{loge}^{-}$, contenant chacune un ou plusieurs ovules insérés à leur angle intèrne; le style est tantôt simple, surmonté d'un stignate trilobé, ou bien il est triparti, et chaque division porte un stigmate. Le fruit est une capsule triloeulaire ou une baie globuleuse, quelquefois à une seule loge et à un seule graine par suite d'avortement. Les graines, outre leur tégument propre, se composent d'un endosperme charnu ou corné, contenant dans une cavité quelquefois assez grande, placée dans le voisinage de leur hile, un embryon très-petit.

La famille des Asparaginées, telle que nous venons d'en tracer les caractères, diffère de celle que M. de Jussieu avait établiè dans son Genera Plantarum. M. R. Browu, avec juste raison, a retiré de ce gronpe les genres à ovaire infère, dont il a fait une famille distinctesous le nom de Dioscorées. Le même botaniste réunit aux Asphodelées un grand nombre de genres des Asparaginées, ne laissant plus dans cette famille, qu'il nomme Smilacćes, que les genres dont le style est profondément trifide on qui portent trois ou quatre styles distincts,

Telle que nous l'avons caractérisée plus haut, la famille des Asparaginées forme deux sections ou tribus naturelles.

I $^{\text {re }}$ tribu. Asparaginées vraies: Stigmate simple ou trilobé: Dracana, Cordyline, Dianella, Asparagus, Callixcne, Lapageria, Convallaria, Polygonatum, Maianthemum, Ruscus, Smilax, etc.

$2^{\mathrm{e}}$ tribu. rarióes. Trois ou quatre stigmates distincts; Paris, Tril; lium, Medeola, etc:

\section{VINGT-SIXISHE FAMTILE.}

* IIIIAcéEs. Liliacece. - Lilia et Asphodeli. Juss. Hemerocallidec. Br.

Plantes à racine bulbifère ou fibreuse ; leurs feuilles, quelquefois toutes radicales, sont planes, ou cylindriques et creuses, ou épaisses et charnues. La tige ou hampé est en général nue, rarement elle porte des feuilles. Les fleurs sont tantôt solitaires et terminales, tantôt en épis simples, en grappes rameuses ou en sertules; elles sont quelquefois aceompagnées d'une spathe quiles enveloppait ayant leur 
épanouissement. Le calice est coloré et pétaloïde, formé de six sépales distincts ou unis par leur base et formant quelquefois un calice tubuleux. Ces six sépales sont disposés sur deux rangs, trois étant plus intérieurs et trois plus extérieurs. Les étamines sont au nombre de six, insérées à la base des sépales quand ceux-ci sont distincts, ou au haut du tube quand ils sont soudés. L'ovaire est à trois loges, et offre trois côtes saillantes; chacune d'elles contient un nombre variable d'ovules attachés à leur angle interne et disposés sur deux rangs. Le style est simple ou nul, terminé par un stigmate trilobé. Le fruit est une capsule à trois loges s'ouvrant en trois valves septifères sur le milieu de leur face interne. Leurs graines sont re- couvertes d'un tégument tantôt noir et crustacé, tantôt simplement membraneux. Leur endosperme est charnu, et contient un embryon cylindrique dont la radicule est tournée vers le hile; rarement cet embryon est contourné sur lui-même.

Nous réunissons ici en un seul groupe les deux familles établies par M. de Jussieu sous les noms de Liliacćes ct d'Asphodélées, et les Hémérocallidées de M. Brown. En effet ces deux premières familles offraient absolument la même organisation dans toutes leurs parties, et la seule différence qui cxistait entre elles consistait uniquement dans Icur mode de germination. Ainsi dans les Asplodẻles le cotylédon reste engagé dans l'intérieur de la graine par une de ses extrémités ct forme un prolongement filiforme qui éloigne la gemmule. Ce caractère joint à quelqucs différences dans le port, différences que l'habitade seule peut faire apprécier, sont les seuls signcs qui jistinguaient les Asphodèles dcs Liliacées : nous avons donc cru devoir les réunir.

Quant aux HÉMÉRocalridéres de Robert hrown, elles ne peuvent former une famille distincte, puisque leur seul caractère essentiel consisterait dans un calicc tubuleux à sa base. Ce groupe avait été ćtabli par le célèhre botaniste anglais pour les genres à ovaire libre de la famille des Narcissées de M. de Jussieu; tels sont Heinerocallis, Tubalgia, Blandfortia.

L'insertion présente quelques différences dans les genres qui composent les Lilineces. Ainsi, tandis que les étamines sont attaehées an calice dans un grand nombre dc genres, et en particulier dans la Jacinthe, le Lachenalia, l'Asphodèle, etc., et par conséquent périgynes, elles sont certaincment bypogyneş dans les lis, les auls, les aloës, le Tritoma, ctc. 


\section{VINGT-SEPTIEME FAMILIE.}

Bromélactes. Bromeliacea. Juss.

Les Broméliacées sont des plantes vivaces parasites. Leurs feuilles sont alternes, et en géuéral réunies en faiseeau à la base de la tige; elles sont allongées, étroites, souvent dentées et épineuses sur les bords. Dans un grand nombre d'espèees toute la plaute est eouverte d'une sorte de duvet ferrugineux; les fleurs forment des épis écailleux, des grappes rameuses ou des capitules, dans lesquels elles sont tellement rapproehées, qu'elles finissent par se souder ensemble. Dans un petit nombre d'espèces les fleurs sout terminales et solitaires. Leur calice est tubuleux, tantôt adhérent par sa partie inférieure avec le tube du ealice, tantôt entièrement libre. Le limbe présente six divisions plus ou moins profondes disposées sur deux rang̣s, dont les trois intérieures sont colorées et pétaloïdes. Les étamines sont en général au nombre de six, rarement en plus grand nombre. Liovaire est à trois loges, dans chaeune desquelles sont insérés un grand nombre d'ovules; le style se termine par un stigmate à trois divisions planes ou subulées. Le fruit est généralement une baie couronnée par les lobes du calice, à trois loges polyspermes. Quelquefois toutes les baies d'un niême épi se soudent ensemble et forment un fruit unique eomme dans l'ananas. Plus rarement le fruit est see et déhiscent. Les graines se composent d'un endosperme farineux, à la partie inféricure duquel est plaeé un embryon allongé et recourbé.

Nous divisons les genres de la famille des Broméliacées en deux tribas, savoir :

r. Tribn Thrarusrées. Ovaire libre: Tillandsia, Pilcrmia.

2. Tribu Broméracées. Ovaire infère: Neroplyyer, Guzmannia, Achnea, Brometia, Agave, Furcrea, etc. 
La famille des Broméliacées a de grands rapports avec la famillc des Narcissées, surtout par ses genres à ovairc infèrc, formant la tribu des Rromćliacées vraies; mais elle en differe par son calice dont les divisions sont disposées sur deux rangs, par ses fruits charnus et surtout par le port des végétaux qui la composent.

\section{QUATRIÈME CLASSE.}

MONOÉPIGYNIE.

\section{VINGT-HUITIEME FAITIXL.}

* prosconées. Dioscorece. R. Brown.

Les Dioscorées sont souvent des plantes sarmenteuses et grimpantes : leurs feuilles sont alternes ou quelquefois opposées, à nervures irrégulièrement ramifiées; leurs fleurs sont hermaphrodites ou uniscxuées; leur ovaire infère est adhérent avee un ealice dont le limbe est divisé en six lobes égaux. Les étamines, au nombre de six, sont libres ou rarement monadel phes, ayant leurs anthères introrses. L'ovaire est ì trois loges eontenant ehaeune, un, deux ou un plus grand nombre d'ovules qui sont tantôt aseendans, tantòt renversés. Le fruit est unc capsule minee et eomprimée ou une baic globuleuse, quelquefois allongée, couronnée par le limbe ealyeinal, et oflrant d'une à trois loges. Les graines contiennent un embryon placé vers le hile, dans l'intérieur d'un endosperme presque eorné.

Cette petite famille a été établie par Rohert Brown pour placer les genres de la famille des Asparaginées de Jussieu, dont l'ovaire est infère; tels sont Dioscorea, Tamnus, Rajania, Flugggea, etc.

\section{VINGT-INEUVIEME FAMILIE.}

* narcisstés. Narcissece.-Amaryllidece. R. Brown. Narcissor'um genera. Juss.

Plantes à raeine bulbifère ou fibreuse, à feuilles radi-eales; à lleurs solitaires, souvent très-grandes, ou disposćes 
en scrtules ou ombelles simples, enveloppécs avant leur épanouissement dans des spathes scaricuses. Le caliee est monosépale tubulcux, adhérent par sa base avee l'ovaire infère, à six divisions égales ou inégales; les étamines, au nombre de six, ont leurs filets libres, ou réunis au moyen d'une membrane; l'ovaire eist à trois loges polyspermes, le style simple et le stigmate trilobé. le fruit est une eapsule à trois loges et à trois valves sepifières; quelquefois e'est une baie qui par avortement ne eontient qu'une à trois graines. Celles-ei, qui offrent assez souvent une earoncule celluleuse, contiennent, dans un endosperme charnu, un embryon cylindrique et homotrope.

Robert Brown a partagé 1 a famille des Narcisses de M. de Jussieu en deux ordres naturels, les Hémérocallidées, où il a placé les genres à ovaire libre, et les Amaryllidées, qui sont les vćritables Narcissées à ovaire infère. Nous avons précédemment réuni les Hémérocallidćes aux Liliacćes. Les gcnres qui composent les vraies Narcissées sont: Narcissus, Anaryllis, Pancratium, Leucoium, Galanthus, etc. Le même botaniste anglais a aussi retiré des Narcisses de M. de Jussieu le genre Hypoxis, dont il a fait un groupe sous le le nom d'Iypoxidées, qui nous paraît pen différent des vraies Narcissćes. M. Kunth a également distrait de cette famille ic genre Pontederia, qui, avec l'Heteranthera, forme la famille des Pontédériacées, dont nous avons tracé précédemment les caractères.

\section{TRERTIEMS FANIIII. \\ * Iridées. Iridece. Juss.}

Famille très-naturelle, eomposée de végétaùx ordinairement herbacés, à raeinc tubéreuse et charnue, rarement fibreusc. Leur tige est cylindriquc ou eomprimée, portant des feuilles 'alternes planes, ensiformes. Leurs fleurs, qui sont souvent très-grandes, sont enveloppées avant leur épanouissement dans unc spathe membraneuse, mince ou scarieuse. Ces flcurs sont solitaires ou diversement groupées. Leur ealice est eoloré, tubuleux, à six divisions profondes, disposées sur deux rangées et sou- 
vent inégales. Les étamiues, constanment au nombre de trois, sont libres on monadelplies, opposćes aux divisions externes dil caliee. L'ovaire est à trois loges polyspermes. Le siyle est simple, terminé par trois stigmates simples, bifides ou découpés, el en lames minees el pétaloïdes. Le fruit est une eapsule à trois loges, s'onrrant cen trois valves septifẹres. Les graines se eomposent d'un tégument propre, et d'un embryon eylindrique homotrope plaeé clans un endosperme charnu ou eorné.

Cctte famille, composée d'un grant nomble de genrés, se divise en deux sections, suivant que ces genres ont les étamines lihres ou monadelphes. A la premicire appartiennent les genres Iris, Ixia, Gladiolus, Crocus, Antholy za, Watsonia, etc., etc. Dans la seconde on trouve les Sisyrinchinum, Galaxia, T'igridia, Viensseuxia, Ferraria, etc.

On distingue facilement les Iridées à lcur ovairc infère et à leurs étanines constamment au nombre de trois.

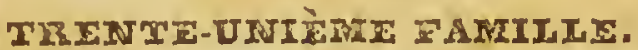

monomonacíes. Hamodoracee. R. Brown.

Les hémodoracées sout des plantes herbacées, vivaces, quelquefois sans tige, ayant les feuilles distiques simples, engainnantes à leur base; des fleurs disposées en corymbes on en épis. Leur calice est monosépale, à six divisions profondes, adhérent par sa base avec l'ovaire infère, exeepté dans le seul genre WVachendorfia. Les étamines imsérécs au ealiee sont au nombre de six on de trois; dans ee dernier eas elles sont opposées aux divisions intéricures. L'ovaire esi à trois loges, qui contiennent ehaeune une, deux ou "plusieurs ovules. Le style et le stigmate sont simples; le fruit est ume eapsule, quelquefois indéhiseente, on s'ouvrant soit par son sommet, soit par le moyen de valves. I cs graines contienuent un trèspelit embryon dans un endosperme assez dur.

Cette petite famille, par son port, sc rapproche beancoup des Iridées, mais elle en differe par ses tianines au nombre de șix, on, quancl il n'y en 
a que trois, par ses étamines opposées aux divisions intéricures du calice, et uon aur extérieures eomme dans les Iridées. Elle en différe eneore par son stigraatc constamment simple. Les gentes Dilatris, Lanaria, Heritiera, Wachendorfa, Hemodorum, Conostylis, Animozanthos et Phlebocarya composent cette fanille.

\section{TIR IRTE-DEURISHE TAMIUTE.}

Musucées. Musacere. Juss.

Plantes herbacées on viraces, dépourvues de tiges, quelquefois munies d'un stipe ou bulbe en forme de tige; feuilles longuement pétiolées, cmbrassantes à la base, très-entières; fleurs for t grandes, souvent peintes des couleurs les plus vives, réunics en grand nombre et renfermées dans des spathes. Leur calice est irrégulier, coloré, pétalö̈dc, adhércnt par sa base avec l'ovaire; son limbe est à six divisions, dont trois extérieures et trois interncs. (Dans le genre Musa, einy des divisions sont externcs, et, formant en quelque sortc unc lèvre supérieure, une seule est interne ct constitue la lèvre inférieure.) Les élamincs, a nombre de six, sont insérées à la partic intcrne des divisions ealycinales; les an thères sont linéaires introrses, à dcux loges, surmontées cn général par un appendice membraneux coloré, pétaloïde, qui est la tcrminaison du filet. L'ovaire infère cst à trois loges, contenant chacune un grand nombre d'ovules insérés à leur angle intcrnc. Dans le gearc Heliconia, il n'y a qu'un scul ovule maissant du fond de ehaque loge. Le style simple sc termine par un stigmate quelquefois concave, mais plus souvent à trois lobes ou trois lauièrcs. Lc fruit est ou unc eapsule à trois loges polyspermes, à trois valves portant l'une des eloisons sur le milieu dc leur face interne, ou un fruit charnu ct indéhiscent. Les graincs, quelquefois portécs sur un podosperıne et environnées de poils disposés circulaircment, se composent d'un té- 
gument quelquefois erustacé, d'un endosperme farineux contenant un cmbryon axile allongé et dressé.

Cette famille se compose des genres Mrusa, Héliconin, Strelizia et Urania. Intermédiaire entre les Narcissées et les Anomées, elle diffère des premières par son calice constamment irrégulier, et des secondes par ses étamines toujours au nombre de six.

\section{TRENTE-TBOISIEME FAMXIE.}

Aromés. Amomece. Ricr.-Canna. Juss. Scitaminece et Canna. R. Br. Drymyrthizées. Vent.

Les amomées sont des plantes vivaces, d'un port tout particulier, qui les rapproche un peu des orchidées; lcur racine est souvent tubéreuse et clarnue; leurs feuilles simples, terminées à leur base par une gaine entière ou fendue, et quelquefois munie d'une ligule. Les fleurs, rarement solitaires, sont aceompagnées de bractées assez larges, et forment en général des épis denses ou des panicules. Leur calice est double; l'cxtéricur, quelquefois tubulcux et plus court, est à trois divisions égales; l'intéricur a son limbe double; les trois divisions externes sont en géuéral égales : des trois internes, l'une est plus grande et dissemblable, et forme une sorte de labelle; les deux latérales sont plus petites et souvent même presque avortées. Il y a une seule ćtamine, dont le filet est souvent dilaté et comme pétaloïde; l'anthère est àdeux loges quelquefois séparées et distinctes. L'ovaire est à trois loges polyspermes; le style simple, terminé par un sligmate concave et en forme de coupe. A la base du style, sur le sommet de l'ováire, on trouve un petit tubercule bilobé, qui peut être considéré eomme deux ćtamines avortées. Le fruit est unc capsulc à trois loges, s'ouvrant en trois valves, portant chacume nue cloison sur le milieu de leur face interne. Les graines, quclqucfois aeeompaguces d'un arille, se composent d'un embryon cylindrique placé dans 
un endosperme farineux, et ayant sa radicule tournée vers le hile.

La description que nous venons de donner des caractères de la famille des Amomées est conforme à celle qui a été tracée par la plupart des auteurs; mais on pent en donıer une autre plus conforme aux affinités naturelles. Ainsi les Amomées, qui ont la plus grande affinité arcc les Musacées, peuvent être décrites comme ayant six étamines et ųn périanthe à six divisions comme ces dernières. L'une de ces étamines est fertile, les cinq autres sont stériles; deux sont représentées par lc tabercule bilobé qui existe à la basc du style, et les trois antres sont converties en appendices pćtaloïdes, et sont représentécs par les trois divisions les plus intérieures dn calice. Cette description de la fleur des Amomées est conforme à la nature, et de cette manière, cette famille se lie naturellement aux IIusacces, d'unc part, qui en sont en quelque sorte le type régulier, et aux Órchidées, d'une antre purt, dans lesquelles on observe des avortemens et des transformations analogues. M. Lestiboudois, professear de botanique à Lille, a le premier appelé l'attention des botanistes sur la structure de la fleur des Amomées; mais nous sommes loin de partager son opinion quand il pense que cette famille doit être réunie aux Musacées.

M. Brown a proposé dc séparer des Amomées quelques genres, tels que Canna; Maranta, Thalia, Phrynium et Myrosma, pour en former ane famille distincte sous le nom de Carwíes.

Indépendamment des genres cités précédemment, les Amomées comprennent encore l'Amomum, le Zingiber, l'Hellenia, le Costus, etc.

\section{TREDTE-QUATRIÈME FAMILIE.}

\section{* orchidées. Orchidec. Juss.}

Plantes vivàees, quelquefois parasites sur les autres végétaux, ayant une raeine composée de fibres simples et eylindriques, souvent aecompagnée d'un ou de deux tubereules eharnus, ovoïdes ou globuleux, entiers ou digités. Les feuilles sont toujours simples, alternes engaînantes; les fleurs, souvent très-grandes et d'une forme partieulière, sont solitaires, faseieulées, en épis ou en panicule. Leur ealice est à six divisions profondes, dont trois intérieures et trois externes. Celles-ei, assez souvent semblables entre elles, sont étalées, ou rapprochées les unes contre les autres à la partie supérieure de la fleur où 
elles forment une sorte de casque (calyx galeatus). Des trois divisions internes denx sont latérales, supéricures et semblables entre ellcs; l'une cst inférieure d'une figure toute particulière, et porte le nom de labelle ou lablier; il présente quelquefois à sa base un prolongement creux nommé éperon (labellum calcaralum). Du centre de la fleur s'élève sur le sommet de l'ovaire une sorte de columelle nommée gynostène, qui est formée par le style et les filets staminanx soudés, et qui porte à sa face antérieurc et supéricure une fossette glandulcuse qui est le stigmate, et ì son sommet une anthère à deux loges, s'ouvrant, soit par une suture longitudinale, soit par un opcreule qui en forme toute la partic supérieure. Le pollen contenu dans chaque loge de l'antbere est réuni en une masse qui a la même forme que la cavité qui la renferme. Au sommet du gynostème, sur les parties latérales de l'anthère, on trouve deux petits tubereules qui sont denx étamines avortées, ct qu'on nomme staminodes. Ces denx étamines sont, au contraire, développées dans le genre Cypripedium, tandis que celle du milieu avorte. Le fruit est une capsule à unc seule loge, contenant un très-grand nombre de graines très-petites attachées à trois trophospermes pariétaux, saillans et bifurqués du côté interne. Ces graines ont leur tégument extéricur formé d'un réseau léger, et se composent d'un endosperme, dans lequel est un très-petit embryon axile et homotrope.

Cette famille, qui peut êtrc regardéc comme une des plus naturelles du règne végétal, offre des particularités si remarquables dans l'organisation de sa fleur, qu'elle ue pent être confondue avec nulle autrc. La soudure des étamines, avec le filet et le stigmate, et surtout l'organisation du pollen réuni en masse (caractire qui ne s'observe que dans les $\Lambda$ selćpiadées et dans quelques mimeuses parmi les dicotylédons), sont les caractères distinctifs les plus saillans de cette famille. Les masses polliniques offrent dans leur composition trois modifications principales qui ont servi à étáblir trois tribus dans la famille des Orclidées. Tantòt elles sont formées de granules assez gros, cohérens entre 
eux par le moyen d'ane matière visquense, qui, lorsqu'on tend à les séparer; s'allonge sons forme de filament ćlastique; on donne à ces masses polliniques le nom de masses seetilcs; elles curactérisent la première tribu, on celles des Opurydírs qui contient eutre autres les genres Orchis, Ophrys, Satyrium, Serapias, Habenaria, ete. Tantòt les masses polliniques sont pulvérulentes, c'est-à-dire, formées d'ane matière comme pultacéc, ce qui s'observe dans la seconde tribu, ou celle des LrModories, qui eontient les genres Limodorun, Epipactis, etc. Enfin chaque masse polliniquc peut être formée de granules tellement eohérens et confondus értr'eux, qu'elle semble composce de cire; dans ce eas, qui s'observe dans la troisième triba ou celle des Epidendrées; on dit qu'elles sont solides. Exemples: Epidendrum, Angracum, Malaxis, Liparis, etc.

Les masses polliniques se prolongent quelquefois à leur partie inférieure en un appendiec filiforme nommé caudicule, qui souvent se termine par nne glande visqueuse de forme variée, et qu'on nomme rétinacle. Le nombre de ces masses polliniques rarie d'un à quatre pour chaque loge de l'anthère. Celle-ci est tantôt placée à la face antérieure et supérieure du gynostème, dont elle n'est pas distincte, comme dans la triba des Ophrydées, tantôt elle est placée dans une espèce de fossetze qui terminc le ğynostème à son sommet, et qu'on nomme clinandre, et clle s'ouvre et s'enlève comme une sorte d'opereule (anthera operculifornis) comme daus presque tous les genres des deux autres tribus, etc., etc.

\section{TIENTE-CINQUITHE FATILIE.}

\section{* hydrocharidées. HJ'drocharidece. Juss.}

Herbes aquatiques, ayant les feuilles caulinaires entières ou finement dentées, quelquefois étalées àla surface de l'eau. Lcs fleurs, renfermées dans des spathes, sont en général dioïques, très-rarement hermaphrodites. Les fleurs mâles, réunies ordinairement plusieurs ensemble, sont tantôt sessiles, tantôt pédicellées. Quant aux fleurs femelles ou aux fleurs hermaphrodites, elles sont toujours sessiles et renfermées dans une spathe uniflöre. Ice calice est toujours à six divisions, trois internes pétaloïdes et trois externes. Le nombre des étamines varie d'une à treize. L'ovaire est infère, quelquefois atténué à sa partic supérieure en un prolongement filiforne, qui s'élève au-clessus de la spathe et tient lieu de style. Les 
stigmates sont au nombre de trois à six, bifides ou bipartis, rarement simples. Le fruit est charnu intérieurement, offrant une cavité simple, ou divisée par des cloisons membraneuses en autant de loges qu'il y a de stigmates. Les graines, qui sont nombreuses et enveloppées d'une sorte de pulpe, sont dressées, ayant un tẹguunent propre men̉braneux très-mince, recouvrant immédiatement l'embryon qui est droit et eylindracé.

Parmi les genres qui composent cette famillc, nous citerons le Vallisneria, le Stratiotes, l'Hydrocharis, le Limnobiun, l'Otrelia, etc.

Cette famille est bien caractérisée par son ovaire infère, ses stigmates divisés, l'organisation intérieure de son fruit, qui est la même que celle des fruits de Cucurbitacées, et son cmbryon sans endosperme:

\section{TRENTE-SIXIEME FAMITLE.}

* Nymphéacées. Nympheacece. Salisb.

Grandes et belles plantes qui nagent à la surface des eaux, et dont la tige forme une souche souterraine rampante; leurs feuilles alternes entières sont cordiformes ou orbiculées, portées sur de très-longs pétioles. Leurs fleurs sont très-grandes, solitaires et portécs sur de très-longs pédoneules eylindricques. Le ealice est formé d'un nombre variable, et quelquefois très-grand de sépales disposés sur plusicurs rangs, de manière à représenter en quelque sorte un calice et une corolle polypétale. Les étamines sont très-nombreuses, insérćes sur plusicurs rangs au-dessous de l'ovaire, ou même sur sa paroi externe, qui se trouve ainsi recouverte par les étamines et par les sépales intérieurs qui ne sont probablement que des étamines transformées; ce que prouve la dilatation graduelle des filamens à mesure qu'on les observe plus extérieurement. I.es anthères sont introrses et à deux loges linéaires. L'ovaire est libre et sessile au fond de la fleur, divisé intérieurement en plusicurs loges par des eloisons 
membrancuses, sur les parois desquelles sont insérés de nombreux ovules pendans. Lc sommet de l'ovaire est couronné par autant de stigmates rayounans qu'il y a de loges à l'ovaire. La réunion de ces stigmates formc une sorte de disque qui couronne l'ovaire. Le fruit est indéhiscent et charnu-intéricurement, à plusicurs loges polyspermes. Les graines ont un tégument épais, quelquefois développé en forme de réscau, contenant un gros endospcrme farineux, qui porte à son sommct un embryon irrégulièrement globulcux ou napiforme dont la radicule cst touirnée vers le hile. Le cotylédon est mince, sous la forme d'une enveloppe particulière rccouvrant la.gẹmmule qui est bilobéc.

Cette famille, qui se compose des genres Nymphaca et Nuphar, est encore aujourd'hui un sujet de controverse parmi les hotanistes, puisque les uns la placent parmi les monocotylédons et les autres parmi les dicotylédons, auprès des Papakéracées; mais la structure de l'embryon et la germination sont certainement celles des autres monocotylédons. (Vojez dans le tome XII du Dictionnaire classique d'Histoire Naturelle l'article Nraphéacées, où nous discntons avec soin ces diverses opinions.) Nous terminons l'article cité iei par l'obscrvation suivante : doit-on laisser le genre Nelumbium dans la fanille des Nymphéacées, on doit-on en faire le type d'une famille distincte? Nous n'osons résoudre encore cette question. Le port est absolument le mêmc, et il pcut paraître fort étrange de séparer, comme ordres distincts, deux genres que quelques botanistes, en tête desquels se présente Linné, avaient crn devoir rénnir en un seul genre. Mais nous demanderons, d'un autre côté, si l'on peut admettre, dans la même famille, deux genres dont l'un a l'ovaire simple à plusienrs loges polyspermes, surmonté d'autant de stigmates qu'il y a de loges, et dont les ovules nombreux sont attachés à toute l'ćtendue des parois des cloisons, et dont l'antre, offrant au centre de sa fleur un très-grand réceptacle, ou torus en forme de cône renversé, présente un grand nombre de pistils distincts uniloculaires et monospérmes implảntés dans des alvcoles creusées à la facc supćrienre de ce réceptacle : deux genres, dont l'un cst muni d'ín très-gros endosperme charnu, qui manque cn totalité dans l'antre. Ces diffćrences nous paraissent tcllement importantes que nous ne sommes pas éloignés de les croire suffisantes pour ćtablit deux familles distinctes, mais qui doivent rester l'une près de l'aatre. 


\section{TRENTX-SEPTISME TAMITIE.}

balanophorézs. Balanophorece. Rien.

Petite famille composée de végétaux parasites d'un port partieulier, qui a quelque analogic avee celui, des clandestines et des orobanches, et qui, comme ces derniêres, vivent eonstamment implantés sur la raeine d'autres végétaux. Leur tige, dépourvue de feuilles, est ehargée d'éeailles ou nue. Les fleurs sont monoïques, formant des épis ovoïdes très-denses. Dans les fleurs mâles, le caliee est à trois divisions profoudes, égales et étalées; rarement une simple éeaille tieut lieu du ealice. Les étamines sont au nombre d'une à trois, rarement au-delà ;' elles sont soudées à la fois par leurs anthères et leurs filets; dans les fleurs femelles, l'ovaire est infére à une seule loge, contenant un scul ovule renversé. Le limbe du calice qui couronne l'ovaire, est entier ou formé de deux à quatre divisions inégales. Il y a un ou deux styles filiformes terminés par autant de stigmates simples. Le fruit est une earyopse globulcuse ombiliquée. La graine contient un très-petit embryon globuleux, placé dans une petite fossette superficielle d'un très-gros endosperme eharnu.

Les genres qui composent cette petite famille sont: Helosis, Langrdorffia, Cynomorium et Balanophora. Elle a des rapports avec les Aroïdées et les Hydrocharidées.

\section{DES PLANTES DICOTYLÉDONES.}

Ce sont toutes eelles dont l'embryon offre deux cotylédons; dans une seule famille, celle des conifères, on trouve souvent de trois à dix eotylédons verticillés.

L'organisation in térieure de la tige, dont toutes les parties sont disposées par conches eoneentriques; la disposition et la ramification des nervures; le nombre cinf ou 
un de ses multiples pour presque toutes les parties de la fleur; la présence très-fréquente d'un caliee et d'une corolle; et enfin, le port si différent de eelui des monocotyléfons, sont les'signes principaux qui distinguent les végétaux dicotylédons des plantes monoco tylédonées.

Les dicotylédons ont été d'abor'd divisés en apétales, monopétales, polypétales et díclines.

\title{
I. DICOTYLÉDONS APLTTAIES.
}

\section{CINQUTEME CLLSSE.}

\author{
EPISTAMINIE.
}

\section{TRENTE-HUITIEME FAMILIE.}

* aristolochiées. Aristolochice. Juss.

Famille composée des deux seuls genres aristoloche et azaret (I). Ce sont des plantes herbacées ou fruteseentes et volubiles, portant des feuilles alternes et entières, des fleurs axillaires. Leur calicc est régulier, à trois divisions valvaires, ou irrégulier, tubuleux, et formant une languette ou lèvre d’une figure très-variéc. Les étamines sont au nombre de dix ou de douze, insérées sur l'ovaire; elles sont tantôt libres et distinctes, tantôt soudées intimement avec le style et le stigmate, et formant ainsi une sorte de mamelon placé au sommet de l'ovaire. Sur ses parties latérales ce mamelon porte les six étamines qui sont biloculaires, et à son sommet il se termine par six petits lobes qui peuvent être considérés comme les stigmates. Le fruit est une capsule, ou une baie à trois ou six loges, contenant chaeune un très-grand nombre de graines

(I) MI. Robert Brown cite encore comme devant faire partie de cette fimille, qu'il pounuę Asarinfes, les genres Thowea ft Lragania. 
renfermant un très-petit embryon placé dans un endosperme cliarnu.

M. de Jussieu avait réani à cette famille le genre Cyrinus, qui est devenu le type d'tue famille distincte sous le nom de Cxтx́śrs.

\section{TRENTE-NEUVIUME TAMIIIE. \\ ${ }^{*}$ crtinées. Cytinece. R. Brown.}

Leurs fleurs sont unisexuées, monoïques ou dioïques. Le calice est adhérent, rarement libre (nepenthes); son limbe est à quatre ou einq divisions. Les étamines varient dehuit à seize, quelquefois même aul-delà; clles sont extrorses et monadelphes. L'ovaire est infère, excepté dans le nepenthes, à une ou quatre loges; les graines sont attachées à des trophospermes pariétaux. Le style est cylindrique, rarement nul, terminé par un stigmate dont les lobes sont égaux à eelui des trophospermes. Les graines ont un embryon cylindrique axile, placé au centre d'un endosperme eharnu.

Les genres qui eomposent cette petite famille sont : Cytinus, Rafflesia et Nepenthes. Les deax premiers sont parasites et dépourvus de fenilles. I.e troisième est remarquable par ses fenilles terminées à leur sommet par une outre qui se ferme au moyen d'un opercule mobile. Cetıe famille se distingue des Aristolochiées surtout par ses graines attachées ì des trophospermes pariétaux, par ses fleurs unisesuées et par le nombre quaternaire ou quinaire des différentes parties de la fleur.

\section{QUARANTIÈME FAMILLE.}

* santalactés. Santalacece. R. Brown.

Plantes herbacées ou frutescentes, ou arbres à feuilles alternes, rarement opposées, sans stipules, à fleurs petites, solitaires, ou disposées en épis ou en sertulc. Leur caliee est supère, à quatre on einq divisions valvaires. Les étamines, au nombre de quatre ì einq, sont opposées aux divisions calycinales et insérées à leur base. L'ovaire est 
infère, à uue seule loge, contenant um, deux ou. quatre ovules qui pendent au sommet d'un podosperme filiforme maissant et s'élevant du fond de la loge. Le style est simple, terminé par un stigmate lobé. Le fruit est indéhiscent, monosperme, quelquefois légèrement charnu. La graine offre un embryon axile dans un endosperme eharnu.

Celte famille, établie par Robert Brown, se compose des genres Thesium, Quinchamalium, Osyris, Fusanus, placés par IM. de Jussieu dans la famille des Eléagnées, et du genre Santalum qui faisait partie des Onagraires. Elle diffère surtout des Eléagnées par son ovaire infère et contenant plusieurs ovales pendans, tandis que celui-ci a l'ovaire libre contenant un seal ovule dressé. Elle a aussi des rapports avec la famille des Combrétacées. Mais celle-ci se distingue par ses ovnles pendans du sommet de la loge de l'ovaire, par ses graines sans endosperme et la corolle polypétale que l'on remarque dans quelques-ans de ses genres.

\title{
SIXIÈME CLASSE.
}

\author{
PÉRISTAMINIE.
}

\section{QUARANTE-UNIEYT FAMYLLE.}

* éléaginés. Eleagnce. A.Rich.-Eleagnotum gen.Juss.

Arbres ou arbrisseaux, à feuilles alternes ou opposées, sans stipules et entières. I,eurs fleurs sont diö̈ques ou hermaphrodites; les mâles sont quelquefois disposées en espèces de chatons. Le ealice est monosépale, tubuleux; son limbe est entier ou à deux ou quatre divisions. Les étamines, au nombre de trois à huit, sont introrses et presque sessiles sur la paroi interne du ealiee. Dans les fleurs femelles, le tube du caliec reeouvreimmédiatement l'ovaire, mais sans y adhérer. L'entrée du tube est quelquefois en partie bouchée par un disque diversement lobé. L'ovaire est libre, uniloculaire, contenant un seul ovule aseendant et pédicellé. Le style est eourt; le stigmate est simple, allongé, linguifor'me. Le fruit cst un alsène crus- 
taeé recouvert par le ealiee qui est devenu eharnu. La graine eontient, daus un endosperme très-mince, un embryon qui a la même direetion que eelle-ci.

La famille des Eléagnées, telle qu'elle avait été étahlie par M. de Jussieu, se composait de genres assez disparates. M. Robert Brown, le premier, a mieux circonserit les limites de cette famille en la réduisant aux seuls genres Elcagnus et Hippophae, anxquels nons avons ajouté les deux genres nouveaux, Shepherdia et Conuleun, qui tous ont l'ovaire libre et monosperme. Déjà M. de Jussieu avait retiré des Ėléagnées les genres Terminalia, Bucida, Painea, etc., pour en forner la famille des Terminaliées; mais M. Brown a fait, des genres primitiveıuent réunis dans les Eléagnées, trois familles, savoir : $I^{0}$ les Eléaguées vraies, telles que nous venons de les caractériser; $2^{\circ}$ les Santalacées, qui ont un ovaire infère, un ou plusieurs ovules pendans au sommet d'un podosperme basilaire; $3^{\circ}$ et les Combretacées, qui comprennent la plupart des genres des Terminaliées de M. de Jussieu, ct quelqques genres auparavant placés dans les Onagres.

\section{QUARANTE-OEUXIENTE FANITIE.}

\section{* thymétes. Thymelece. Juss.}

Arbrisseaux, rarement plantes herbacćcs, à feuilles alternes ou opposécs, très-entic̀res, ayant les fleurs terminales ou axillaires, en sertules, en épis, solitaires, ou rémies plusieurs cusemble à l'aisselle des feuillos. Le calice est généralement eoloré et pétaloïde, plus ou moins tubulcux, à quatrc on einq äivisions imbriquées avant leur épanouissenıent. Les étamines, en général au nombre de lunit, disposées sur deux rangs, ou de quatre, ou simplement de deux, sont insćrées et sessiles à la paroi interne du ealiee. L'ovaire est uniloculairc, et contient un seul ovule pendant. Le style est simple, terminé par un stigmate égal'ement simple. Le fruit est une sorte de noix légèrement eharnue extérieurement. L'embryon, qui est renversé eomme la graine, est contenu daus un endosperme eharnu et minee.

Les genres principaux de cette famille sunt: Daplene, Sicllera, Passcrina, Pimelen, Struthiola, fitc. 


\section{QUARANTE-TROISIEYKE TAMLEE:}

protéAcÉEs. Proteacec. Juss.

Les protéacées sont toutes des arbrisseaux ou des arbres exotiques, qui croissent en abondance au eap de BouneEspérance et à la Nouvelle-Hollande. Leurs feuilles sont alternes, quelquefois presque verticillées ou imbriquées. Leurs fleurs, généralement hermaphrodites et rarement unisexuées, sont tantôt groupées à l'aisselle des feuilles, tantôt réunies en une sorte de eône ou de chaton. Leur ealice se compose de quatre sépales linéaires, quelquefois soudés et formant un calice tubuleux à quatre divisions plus ou moins profondes et valvaires. Les étamines, au nombre de quatre, isont opposées aux sépales et presque sessiles au somnet de leur faee interne. L'ovaire est libre, à une loge eontenant un seul ovule attaché vers le milieu de sa hauteur. Le style se termine par un stigmate généralement simple. Les fruits sont des capsules de forme variée, uniloculaires et monospermes, s'ouvrant d'un seul eôté par une suture longitudinale, et dont la réunion eonstitue quelquefois une sorte de eône. La graine, qui est parfois ailée, se eompose d'un embryon droit dépourvu d'endosperme.

Les genres de cette famille sont nombreux. Nous citerons ici comme exemples les Protea, Petrophila, Branksia, Grevillea, Embothrium, Hakea, etc. Cette famille, à cause de la forme de son calice, de ses étamines sessiles au sommet des sépales, et surtont par son port, ne peut ĉtre confondue avec aucture autre.

\section{QUARANTE-QUATRIÈME FAMIIIE.}

\section{${ }^{*}$ LAurinées. Lautinea. Juss.}

Arbres ou arbrisseaux à feuilles alternes, rarement op= posées, entières ou lobécs, très-souvent coriaces, per- 
sistantes et ponetuces. Leurs fleurs, quelquefois unisexuées, sont disposées en panicules ou en eimes. Le calice est monosépale, à quatre ou six divisions profondes, imbriquées par leurs bords avant leurépanouissement. Les étamines sont au nombre de huit à douze, insérćes à la base du ealice; leurs filcts présentent à leur base deux appendices pédicellés, de forme variéc, et qui paraissent être des étamines avortées; les an thères sont terminales, s'ouvrant au moyen de deux ou quatre valvules qui s'enlèvent de la base au sommet. L'ovaire est libre, uniloculaire, eontenant un seul ovule pendant; le style est plus ou moins allongé, terminé par un stigmate simple. Le fruit est eharnu, accompagné à sa base par le calice qui forme une sorte de cupule. La graine contient sous son tégument propre un très-gros embryon renversé comme la graine, ayant des cotylédons extrêmement épais et eharnus.

Cette famille a pour type le laurier et quelques genres qui ont avee lui dn rapport, comme les Borbonia, Ocotea et Cassytha. Ce dernier est remarquable en ce qu'il est formé de plantes herbaeées, volubiles et sans feuilles. M. de Jussieu avait réuni aux Laurinées le Museadier; mais M. Robert Brown l'en a, à juste titre, retiré pour eu former une famille distiuete sous le nom de Myristicées. Ita famille des Laurinées est surtout caraetérisée par son port, ses étamines dont les anthères s'ouvrent au moyen de valvules. Le même carnetère s'observe eneore dans les Hamamélidées et les Berbérïdées; mais cette dernière famille appartient à la elasse des dicotylédones polypétales hypogynes.

\section{QUARANTE-CINQUIEัME FAMILIE.}

myristicíes. Myristicea. R. Br.

Arbres tous exotiques et eroissant sous les tropiques; ayantdes feuilles alternes, non ponctućes, entières; des fleurs dioïques, axillaires on terminales, diversement disposées. Leur calice monosépale est à trois divisions valvaires. Dans les fleurs niàles on trouve de trois à douze étamines monadelplies, dont les anthères rappro- 


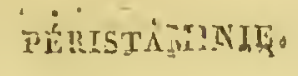

ehées et souvent soudées cnsemble, s'ouvrent parr un sillon longitudinal. Dans les fleurs femelles l'ovilure est libre, à une seule loge eonténant un senl ovule dressé. Le style est très-eourt, terminś par un stigmate lobé. Le fruit est une sorte de baic eapsulaire s'ouvrant en deux valves. La graine est reconverte par un arille eharnu, divisé en un grand nombre de lanières. L'endosperme est eharnu ou très-dur, marbré, contenant vers sa base un très-petit embryon dressé.

Cette famille a pour type le Muscadier. Elle est très-distincte des Laurinées par son calice à trois divisions; ses étamines monadelphes s'ourrant par un sillon longitudinal; sa graine dressée, arillée; son embryon très-petit; contenu dans un endosperme dur et marbré.

\section{QUARANTE-SIXIÈME FAMILIE.}

* poly goníes. Polggonea: Juss.

Plantes herbacées, rarement soufrutescentes, à feuilles alterues, engaîuantes à leur base, ou adhérentes à une gaîñe membraneuse et stipulaire, roulées en dessous sur leur nervure moyenne dans leur jeunesse. Fleurs quelquefois unisexuées, disposées en épis eylindriques ou en grappes terminales. Caliee monosépale, offrant de quatre à six segmens, quelquefois disposés sur deux rangs et imbriqués avant leur évolution. Etamines de rruatre à neuf, libres et à anthères s'ourrant longitudinalement. Ovaire libre, uuiloeulaire, offrant un seul ovule dressé; le fruit, assez souvent triaugulaire, est see et indéhiseént, quelquuefois reeouvert par le ealice qui persiste. La graine contient dans un cndosperme farineux, quelquefois très-mince, un einbryon renversé et souvent unilatéral.

Cctte famille sc compose des genres Polygonum, Rumex, Rheum, Coccoloba, ctc. Elle se distingue des Chénopodées par la gầue stipulaire de ses feuilles, par son opule dressé et șon embryon renversć. 


\section{QUARANTE-SXTMIBME FAMIILE.}

* chénopodées. Chenopodece. De Cardo-Atriplicea. Juss.

Plantes herbacées ou ligneuses, à feuilles altcrnes ou opposées, sans stipules. Leurs fleurs sont petites, quelquefois unisexuées, disposées soit en grappes rameuses, soit groupées à l'aisselle des feuilles. Leur calice monosépale, quelquefois tubuleux à sa base, est à trois, quatre ou einq lobes plins ou moins profonds, persistans. Les étamines varient d'une à cinq; eiles sont insérées soit à la base du calice, soit sous l'ovaire. Ces étamines sont opposées aux lobes du ealice. L'ovaire est libre, uniloculaire, monosperme, contenant un seul ovule dressé, et porté quelquefois sur un podosperme plus ou moins long et grêle. Le style, qui est rarement simple, est à deux, trois ou quatre divisions terminées chacune par un stigmate subulé. Le fruit est un akène ou une petite baie. Ia graine se compose sous son tégument propre d'un cmbryon cyliudrique grêle, recourbé sur un endosperme farineux ou roulé en spirale, et quelquefois sans endosperme.

Cette famille se compose des genres Chenopodium, Atriplex, Salsola, Beta, Salicornia, etc. Elle a, d'une part, beaucoup de rapports avee les Polygonées, qui en différent par la gaine stipulaire de leurs feuilles, par lenr embryon non recourbé et leur radienle supéricure. Elle a aussi, d'une autre part, beancoup d'analogie avec les Amaranthacées, dont eelles-ei ne diffèrent en réalité que par leur port et quelques autres caractères de peu d'importanee. Les Chénopodées nous offrent l'exemple de genres à insertion périgynique, comme les Beta, Blitum, Spinacia, et d'antres en plus grand nombre qui ont l'insertion hypogynique, tels que les Rivinia, Salsola, Camphorosma, Chenopodium, ete.

M. Robert Brown a proposé de séparer le gemre Phytolacca des Chénopodées pour en former le type d'une fanille distincte, sous le nom de Phytolaccées. Cette famille, qui nous parât peu distincte, diffêre par ses ovaireș réennis au nombre de dix̉ à douze, et soudés en un seul fruit. 


\section{SEPTIEME CLASSE.}

HYPOSTAMINIE.

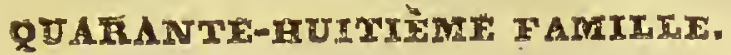

* amarañthacées. Amaranthacec. R. Br. - Amaranthacearum pars. Juss.

Les amaranthacées sont des plantes herbacées ou soufrutescentes, portant des feuilles alternes ou opposées, quelquefois munies de stipules scarieuses. Les fleurs sont petites, souvent hermaphrodites, quelquefois unisexuées, disposées en épis, en panicules ou en eapitules et munies d'éeailles qui les séparent. Le ealice est monosépale, souvent persistant, à quatre ou einq divisions très-profondes. Les étamines varient de trois à einq. Leurs filets sont tantôt libres et tantòt monadelphes, et formant quelquefois un tube membraneux, lobé à son sommet et portant les anthères à sa face interne. L'ovaire est libre, uniloculaire, reufermant un seul ovulc dressé et porté quelquefois sur un podosperme très-long, recourbé, au sommet duquel il est pendant. Le style est simple ou nul, terminé par deux ou trois stigmates. Le fruit, en général environné par le caliee, est un akènc ou une petite pyxide s'ouvrant par le moyen d'un opercule. L'embryon est cylindrique, allongé, recourbé autour d'un endosperme farinetix.

Cette famille, composée cntr'autres des genres Amaranthus, Colosia, Gomphrena, Achyranthes, etc., est teilcment rapprochée des Chénoporées, qu'il est extrèmement difficile de tracer la limite qui les sépare. En effet, l'insertion, qui est en général périgynique dans les Chénopodées, est aussi hypogynique dans plusicur's èorenres, comme nous l'avons dit prćcédenment; mais le port de ces deux familias est tout-i-fait différent; leṣ étamines sont 
souvent monadelphes dans les Amarantlacées, qui ont aussi quclquefois les feuilles opposćcs; mais quoiquc ces earactères distinctifs soient peu importans, cependant il est diffieile de réunir deux fanilles qui paraissent l'une et l'autre bien tranchées quand on ne considère quc leur port.

On a séparé des Amaranttacées ecrtains gcurcs à étamincs périgynes, snmme les Illecebrum, Paronychia, ete., qui, réunis à quelques autres tirés des Caryophyllées, forment uné samille distincte sous le nom de Paronychices.

\section{QUARANTIE-NIEUVIEME TANILIE.}

NúctagrnéEs. Nyctaginea. Juss.

Les Nyclaginées sont des plantes herbacécs, des arbustes, ou même des arbres dont les feuilles sont simples, le pliss souvent opposées, rfuclquefois alternes. Les fleurs sont axillaires ou terminales, souvent r'éunies plusieurs ensemble dans un involuere eommun ou ayant elacun une involuere propre et calyeiforme. Leur caliee est monosépale, coloré, souvent tubuleux, renflé à sa partie inférieure, qui souvent est plus épaisse, et persiste apuès la chute de la partic supériene. Le limbe est plus ou. moins divisé en lobes plissés. Les étamines varient de einq à dix et sont insérées au bord supérieur d'une sorte de disque hypogyne, souvent en forme de emprile. L'ovaire est à une seule loge eontenant un ovule dressé. Le style et le stigmate sont simples. Le fruit est, une eariopse recouverte par le disque et la partie inféricure du calice, qui sont crustacés et forment une sor'ie de périearpe accessoirc. Le véritable péricarpe est minee, adliérent avee le tégument propre de la graine. Celle-ei se eompose d'un embryon recourbé sur lui-même, ayant sa radicule repliće sur la faec d'un des eotylédons, et embrassart ainsi l'endosperme qui se trouve eentral.

Les genres Njetago, Allionia, Pisonia, Bocrrhaavia, ete, appartiennent ì ecttc famille. Quelques anteurs, partant des genres dont l'involucre est uniflore, comme dans le Nyctage ou bellc de, nuil, ont admis cet involucre comme un calice, et le calice comme une cr,rollc; mais l'analogie, et surtont 
les genres à involučre contenant plusieurs flears, prouvent que le périanthe est véritablement simple.

\section{HUITIEME GLASSE.}

HYPOCOROLLIE.

\section{CINQUANTIZSTE FAMITLE。}

* plantaginées. Plantaginece. Juss.

Petite famille de plantes uniquement eomposée des genres Plantain et Littorelle, et que l'on reeonnaît aux earaetères suivans: les fleurs sont hernaphrodites, unisexuées dans le seul genre Littorella, formant des épis simples, eylindriques, allongés ou globuleux; rarement les fleurs sont solitaires. Le ealiee est à quatre divisions profondes et persistantes, ou à quatre sépales inégaux, en forme d'éeailles, et dont deux plus extérieurs. La eorolle est monopétale, tubuleuse, à quatre divisions régulières, rarement entière à son sommet. Cette eorolle, dans le genre Plantain, donne attaehe à quatre étamines saillantes, qui, dans le Littorella, naissent du réeeptacle. L'ovaire eșt libre, à une, deux, ou très-rarement à quatre loges, eontetenant un ou plusieurs ovules. Le style est eapillaire, terminé par un stigmate simple subulé, rarement bifide à son sommet. Le fruit est une petite pyxide reeouverte par la eorolle qui persiste. Les graines se eomposent d'un tégument propre, qui reeouvre un endosperme charnu, au eentre duquel est un embryon eylindrique, axile et homotrope.

Les Plantaginées sont des plantes herbacées, rarement soufruteseentes, souvent privées de tige, et n'ayant que des pédoneules radieaux qui portent des épis de fleurs très-denses. Leurs feuilles sont soavent radieales, entières, dentées ou diversementincisées. Elles croissent en quelque sorte sous toutes les latitudes. Jussiea et la plupart des autres botanistes eonsidèrentles Plantaginées comme véritablement apétales. Pour eet illustre botaniste, l'organe que nọs 


\section{6}

avons décrit counme la corolle est le calice, et notre calice n'est qu'une réunion de braetées; mais il nous semble que la constanee et la régularité de ces deux organes doivent plutôt les faire considérer comme un périauthe. douhle, ainsi que l'a plus réeemment admis le eélèbre Robert Brown.

Les Plantaginées sont très-voisines des Plumbaginées, dont elles diffèrent surtout par leur style constamment simple, par leur ovaire à deux loges souvent polyspermes, tandis qu'il est eonstamment uniloeulaire et eontenant un ovule pendant du sommet d'un podosperme basilaire et dressé dans les Plumbaginées.

\section{CINQUANTE-UNIÈME TANILIE.}

\section{* plumbaginées. Plumbaginea. Juss.}

Famille naturelle des plantes dicotylédones, placée par les uns parmi les apétales, et par les autres dans les monopétales. Ce sont des végétaux herbacés ou soufrutescens, à feuilles alternes, quelquefois toutes réunics à la base de la tige, et engâinantes. Les fleurs sont disposécs en épis ou en grappes ramcuses et terminales. Lcur calice est monosépalc, tubuleux, plissé et persistant, ordinairement à cinc divisions; la corolle est tantòt monopétale, tantôt forméc de cinq pétalcs égaux, qui assez souvent sont légèrement soudés entr'eux par lenr hase. Les ćtamines généralement au nombre de cinq et opposées anx divisions de la corolle, sont épipétales, quand cclle-ci est polypétale, et immédiatcment hypogyncs, lorsque la corolle est monopétale (ce qui est le contraire de la disposition générale). L'ovairc est libre assez, souvent à cinc angles, à une scule loge contenant un ovule pcndant au sonmet d'un podospcrmc filiforme ct basilairc. Les styles, au nombre de trois à cinq, se tcrminent par autant de stigmates subulés. Le fruit est $m$ akène cnveloppé par le calice; la graine se compose, outre son tégument propre, d'un cndosperıc farinacé, au centre dnquel cst un embryon qui a la mime direction que la graine.

Cette petite famille se eompose des geures Plumbago, Statice, Limonium, Vogelia de Lamarck, Theta de Loureiro, Agialitis de Robert Brown. Elle 
UT'POCONOLIST.

diffère des Nyetaginées, qui sont monopérianthées, par son ovule porté sur un long podosperme, an sommer cluquel il est pendant; par plusieurs styles et plusieurs stigmates; par l'cmbryon droit et non reecur'bé sur lni-même, ete.

\section{CINQUANTE-DEUXIEMT FAMIIIS.}

* primulacées. Primulacece. Vent. - Lysimachice. Juss.

Les Primulaeées sont des plantes annuelles ou vivaees, à feuilles opposées ou vertieillées, très-rarement éparses. Leurs fleurs sont disposées en épis ou en grappes axillaires ou terminales; quelquefois elles sont solitaires ou diversement groupées. Le caliee, monosépale, est à einq ou à quatre divisions; la eorolle, monopétale, régulière, est tantòt tubuleuse à sa base, tantôt divisée très-profondément en einq lanières; les étamines, au nombre de einq, sont ou libres ou monadelphes, insérées au haut du tube de la eorolle, ou à la base de ses divisions; elles leur sont opposées, et leurs anthères introrses s'ouvrent ehaeune par un sillon longitudinal. L'ovaire est libre, à une seule loge eontemant un très-grand nombre d'ovules attaehés à un trophosperme eentral. Le style et le stigmate sont simples. Le fruit est une eapsule uniloeulaire et polysperme, s'ouvrant en trois ou einq valves, ou une pyxide opereulée. Les graines offrent un embryon eylindrique plaeé transversalement au hile dans un endosperme eharnu.

Les genres principaux qui composent cette famille sont: Prinula, Lysimachia, Hottonia, Anagallis, Cyclamen, Centunculus, etc. On y a aussi réani le Samolus, bien que son ovaire soit adhérent en grande partie avec le calice; mais, par tous ses autres caractères, il convient ì cette famille.

Les Primalacćes sont très-bien caractćrisées par leurs étamines opposées aux divisions de la corolle, leur capsule uniloeulaire, dont les graines sont attachćes à un trophosperme central, ct par leur embryon placé en travers devant le liile. Par ces différens caraclères; elles se rapprochent beaucoup des Myrsinées, qui n'en diffèrent que par leur frcit charm et lears graines enfoncées dans des espèes d'alvéoles clu trophosperme, qui est charnu et tress-gros. 


\section{CINQUANTI-TMOISIEME EAMTXIE.}

\section{* lentibularties. Lentibularia. Rrch.}

Petite famillc eomposée uniquement des deux gcnres Utricularia et Pinguicula, placés auparavant à la suite dcs Primulacées. Ce sont de petites licrbes vivant au milicu des eaux, ou dans les lieux humidcs et inondés. Leurs feuilles sont ou réunics en rosette à la base des tiges, ou divisées en segmens capillaires et souvent vésieuleux, dans les espèees qui nagent à la surface dcs eaux. Leur tige est toujours simple, portant une on plusicurs flenrs à leur extrémité. Leur ealice est monosépale pcrsistant, divisé eomme en deux lèvres; la corolle est monopétale, irrégulière, éperonnée, ćgalcment à deux lèvres. Les étamines, au nombre de dcux, sont incluses et insérécs touit-à-fait à la base de la corolle. L'ovaire est à une seule loge contenant un grand nombre d'ovules attaclıés à un trophosperme eentral. Le style est simple et très-eourt; le stigmate bilamellé. Le fruit est une capsule uniloeulaire, polysperme, s'ouvrant soit transversalement, soit par une fente longitudinale, qui partage son sommet en deux valves. Lesgraines offrent un embryon immédiatcment rceouvcrt par lc tégument propre.

Cette petite famille se distingue des Primulacées par sa corolle irrégnlière, ses deux étamines et son embryon sans endosperme; des Antirrhinées par son fruit à une seule loge, dont le trophosperne est central, et par son cubryon sams endosperme.

\section{CINQUANTE-QUATRIEME FAMILLE.}

* Globulariées. Globularia. DC.

Le genre Globularia, placé d'abord parmi les Primulaeées, eonstitue à lui seul eette pctite famille, dont voiei les principaux caractères : le calice est monosépale, tubulcux, 
persistant, à cinq divisions; la co rolle est monopétale, tubuleuse, irrégulière, à einq lanières étroites et inégales, disposées en deux lèvres ; les étamines, au nombre de quatre à cinq, sont alternes avec les divisions de la corolle. L'ovaire est unilocilaire, eontenant un seul ovule pendant. Le style est grêle et terminé par un stigmate à deux divisions tubuleusses et inégales; à la base de l'ovaire est un petit disque unilatéral. Le fruit est un akène reeouvert par le ealiee. L'embryon, presque cylindrique, axile, esi p̧lacé dans un endosperme eharnu.

I es Globulariées sont des plantes herbacées ou soufrutescentes, à feuilles toutes radicalcs ou alternes, à fleurs petites, violacćes, rćunies en capitule globuleux et accompagnćes de bractées. Slles diffèrent des Primulacées par leur corolle irrégulière, leurs étamines alterues, leur ovaire contenant un seul ovale renversé.

\section{CINQUANTE-CINQUIËRE FAMILIE.}

* orobanchées. Orobanchece. Vent.

Ce sont des végétaux tantôt parasites sur la raeine d'autres plantes, tantôt terrestres; leur tige est quelquefois dépourvue de feuilles, qui sont remplaeées par des éeailles. Les fleurs, accompagnées de bractées, sont terminales, tantôt solitaires, tantôt disposées en épis. Le ealice est monosépale tubuleux, ou divișć jusqu'à sa base en sépales distincts; la corolle est monopétale, irrégulière, souvent à deux lèvres; les étamines sont en général didynames; l'ovaire, appliqqué sur un disque hypogyne et annulaire, est à une seule loge qui eontient un très-grand nombre d'ovules altaehés ì deux trophospermes pariétaux et bifides par leur eôté libre. Le style se termine par un stigmate à deux lobes inégaux. Le fruit est une eapsule uniloculaire, s'ouvrant en deux valves qui portent ehaeune un trophosperme sur le milieu de leur face interne. Les graines, dont le tégument propre est double, offrent 
um endosperme eharnu qui porte un trés-petit embryon placé dans une fossette ereusée dans sa partie supéricure et latérale.

Les genres Orobanche, Phelippea, Lathreca, etc., forment cette famille, qui diffère des Scrophularinées par son ovaire uniloculairc, la position de son embryon et surtout le port des végétanx qui la composent.

\section{CINQUANTE-SIXIENEE FAMILIE.:}

\section{*scrophularin ées. Scrophularince. R. Brown:-Scrophu- lariae et Pediculares. Juss.}

Herbes ou arbustes à feuilles souvent opposées, quelquefois alternes, simples, à fleurs disposées en épis ou en grappes terminales. Leur calice est monosépale, persistant, à quatre ou cinq divisions inćgales; la corolle est monopétale, irrégulière, à deux lèvres et souvent personnée; les étamines, au nombre de deux à quatre, sont didynames. Tiovaire, appliqué sur un disque hypogyne, est ì deux loges polyspermes. Le style est simple, terminé par un stigmate bilobé. Le fruit est une eapsule biloculaire, dont le mode de déhiscence est très-variable. Tantôt elle s'ouvre par des trous pratiqués vers le sommet, tantôl par des plaques irrégulières, tantôt par deux ou quatre valves portant chacune la moitié de la cloison sur le milieu de leur face interne, ou opposées à la cloison qui reste entière. Les graines contiennent sous leur tégument propre, une amande composée d'un endosperme charnu, qui renferme un embryon droit cylindrique, ayant sa radicule tournée vers le hile ou opposée à ee point d'attaclie.

Nous avons suivi l'exemple de Robert Brown, qui rćunit en une seule les deux familles établies par M. dc Jussieu sous les noms de Scrophulaires et dc Pćdiculaires. La principalc difiérenec qui scrvait à distingucr ces deux familles, élait tirée du mode de déhiscence de la capsule, qui, dans lcs Scrophalaires, se fait par des trous ou des valves opposées à la cloison qui 
H XPOCOROLIIE.

reste jntacte; tandis que, dans les Pédiculaires, chaque valve porte sur le milieu de sa face interne la moitié de la cloison. Mais ces différences, qui paraissent fort tranchées, présentent les nuances nombreuses, et, par exemple, dans le genre Veronica, on les trouve presque toutes réunies. Cependant nous avons remarqué entre ces deux groupes une autre modification que nous n'avons pu observer sur tous les genres, mais qui nous a paru constante dans tous ceux dont nous arons pu analyser la graine, c'est que dans les Pédicnlaires de I. de Jussieu, l'cmbryon a toujours une direction. opposée à celle de la graine, c'est-ì-dire que ce sont ses cotylédons qui sont tonınés vers le hile, tandis que le contraire a lien dans les Scrophulaires.

$I^{0}$ Pédicelaires : Pedicularis, Rhinanthus, Melampyrum, Veronica, Euplirasia, Erinus, etc.

$2^{\circ}$ Scrophulaires : Antirrhinum, Linaria, Scrophularia, Digitalis, Gratiola, etc.

\section{CINQUANTE-SEPTIENE FAMIIIE.}

* solnnées. Solanece. Juss.

On trouve dans cette famille des plantes herbacées, des arbustes et même des arbrisseaux assez élevés, quelquefois munis d'aiguillons sur plusieurs de leurs parties, ayant des feuilles simples ou découpées, alternes, ou quelquefois geminées vers la partie supérieure des rameaux. Leurs fleurs, souvent très-grandes, sont ou extra-axillaires, ou formant des épis ou des grappes. Leur calice, monosépale et persistant, est à cinq; divisions peu profondes; leur corolle monopétale, régulière dans le plus grand nombre des cas, offre des formes très-variées, et cinq lobes plus ou moins profonds et plissés sur euxmêmes. Les étamines, en même nombre que les lobes de la corolle, ont leurs filets libres, rarement monadelphes par leur base. L'ovaire, assis sur un disque hypogyne, est ordinairement à deux, rarement à trois ou quatre loges polyspermes, dont les ovules sont attachés à l'angle intcrne. Le style est simple, terminé par un stigmate bilobé. Le fruit est ou une capsule à deux ou quatre loges polyspermes, s'ouvrant en deux. ou rquatre valres, ou une baie 
également à deux ou trois loges. Les graines, cquelquefois reuiformes et à épisperme chagrinć, ont un cmbryon plus ou moins reeourbé daus un endosperme charnu.

Les Solanées ont les rapports les plus intimes avec les Scrophularinécs. Elles en diffèrent en général par leurs feuilles eonstamment alternes, leur corolle régulière, leurs étamines en même nombre que les lobes de la corollc et surtout leur embryon reeourbé sur lui-même; ee dernicr caractère est nuême quelquefois le seul qui distingue réellement les Solanécs à corolle irrégulière de certaines Scrophularinées. Les genres des Solanées forment deux sections d'après la naturc de leurs fruits.

I. Fruit eapsulaire: Nicotiana, Verbascum, Hyoscianus, Datura, ete.'

II. Fruit charnn : Solanun, Atropa, Capsicun, Physalis, Lyciun ; etc.

\section{CINQUANTE-HUTTISME FAMILIE.}

\section{*Acarthacíes. Acanthacece. Juss.}

Les Acanthacées sont des herbes ou des arbrisseaux, à feuilles opposécs, à fleurs disposées en épis, ct accompagonées de bractées à leur base. Lcur calice cst monosépale, à quatre ou ciny divisions, régulières ou irrégulières. La córolle est monopétale, irrégulière, ordinairement bilabiée; les étamines sont au nombre de deux ou de quatre didynames. Liovaire est ì deux loges, qui conticnnent deux ou un plus grand nombre d'ovules; il est applicqué sur un disque hypogyne et annulairc. Le style est simple, terminé par un stigmate bilobé. Le fruit est unc capsule à deux loges, quelquefois monospermes, s'ouvrant avec élasticité en deux valves qui cmportent avec elles chaeune la moitié de la cloison. Ces graines sont en général portécs sur un podospcrıme filiforıne, et lcur cmbryon, placé immédiatement sous leur tégument propre, cst dépourvu. d'endosperme, ct a en général sa tradicule tournéc du côté du hilc.

Exemples : Justicia, Acanthus, Ruellia, Thunbergia, etc. Cette famille diffère des Scrophularinés par scs graines portées sur uu long podosperme; par son cubryon sans endosperme; etc. 


\section{CINQUANTE-INEUVIEMTE TAMILIE.}

* Jasminées. Jasminece. Juss. - Jasminées et Lilacées. Vent. Oléinées. Linck.

Cette famille se compose d'arbustes, d'arbrisseaux ou mème de très-grands arbres, à feuilles opposées, rarement alternes, simples ou pimnées. Les fleurs sont hermaphrodites, excepté daus le genre Frène, où elles sont polygames. Le calice est monosépale, turbiné dans sa partie inférieure; la eorolle est monopétale, souvent tubuleuse et irrégulière, à quatre ou cinq lobes, quelquefois assez profonds pour que la corolle paraisse polypétale (Ornus, Chionanthus); elle manque quelquefois entièrement. Les étamines sont au nombre de deux seulement. L'ovaire est à deux loges, contenant chacune deux ovules suspendus. Le style simple se termine par un stigmate bilobé. Le fruit est tantôt une capsule à uve ou deux loges, indéhiscentes ou s'ouvrant en deux valves, tantôt il est charnu et renferme un noyau osseux. Le tégument propre de la graine est mince ou charnu; l'endosperme est charnu ou dur; il contient un embryon ayant la même direction que la graine.

Les genres de cette famille, dont on avait fait trois familles distinctes, mais qui doivent rester réunies, ainsi que nous l'avons démontré (Mém. Soc. Hist. Nat., Paris, ton. II.), peuvent être divisés en deax sections de la manière suivante :

$x^{\circ}$ Fruit sec, Lrcacées: Lilas, Fontanesia, Fraximuts, Nyctanthes:

20 Fruit charnu, Jasßrsérs : Jasminum, Olea, Ligustrun, Philly. rea, etc.

\section{SOIXANTIEME FAMILLE.}

\section{* venbénacées, Verbenacece. Juss.}

Les Verbénaećes sont des arbres ou des arbrisseaux, rarement des plantes herbacécs, à feuilles ordinairement 
opposées, quelquefois composées. Les fleurs sont disposćes en épis ou en corymbes; plus rarement elles sont axillaires et solitaires. Leur caliee est monosépalé, persistant, tubuleux. La corolle est monopétale, tubuleuse, ordinairement irrégulière. Les étamines sont didynames, quelquefois au nombre de deux seulement; l'ovaire est à deux ou quatre lòges, eontenaut un ou deux ovules dressés. Le style se termine par un stigmate simple ou bifide. Le fruit est une baic ou une drupe, eontenant un noyau à deux ou quatre loges souvent monospermes. La graine se compose, outre son tégument propre, d'un endosperme minee et eharnu qui reeouvre un embryon droit.

Cetre famillc composée des genres Verbena, Vitex, Clerodendrum, Zapania, ctc. , se distingue des précédentes par son fruit charnu (execpté dans la Ferbena), et par ses graines, ordinairement solitaires dans chaque loge.

\section{SOIXANTE-UNIÈME FAMILIE.}

mroponinées. Myoporinea. R. Brown.

Arbustes généralement glabres, à feuilles simples, alternes ou opposées, à fleurs axillaires et sans bractées; leur caliee est persistant, à einq divisions profondes; leur eorolle monopétale est presque régulic̀re ou légèrement bilabiée; les étamines sont didynames ou quelquefois au nombre de einq, dont une reste parfois rudimentaire ; l'ovaire est libre, appliqué sur un disque hypogyne et ammulaire; il est à deux ou quatre loges, contenant chaeune un ou deux ovules pendans de leur sommet. Le style simple se termine par un stigmate également simple. Le fruit est une drupe eontenant un noyau à deux ou quatre loges renfermant chacune une ou deux graines, eomposées d'un embryon eylindrique, placé au eentre d'un endosperme assez dense.

Les Myoporinées, voisines des Verbénacées, dont elles diffèrent surtont par leurs graines pendantes et munies d'un endosperme épais, se composent des genres Myjoporum, Bontia, Pholidia, Stenochilus, Eremophila. 
HYPOCOROLLIE.

\section{SOIXANTE-DEUXILME FAMIIEE.}

* LAbiées. Labiata. Jüss.

Les Labiées forment une des familles les plus naturelles du règne végétal. Ce sont des plantes herbacées ou quelquefois des arbustes, dont la tige est carrée, les feuilles simples et:opposées, les fleurs groupées aux aisselles dẹs feuilles, et formant àinsi par leur réunion des épis ou des grappes rameuses. Leur calice est monosépale, tubuleux, à cinq dents inégales. La corolle, monopétale, tubuleuse et irrégulière, est partagée en deux lèvres, l'une supérieure et l'autre inférieure. Les étamines sont au nombre de quatre et didynames; quelquefois les deux plus courtes avortent. L'ovaire, appliqué sur un disque hypogyne, est profondément quadrilobé, très-déprimé. à son centre, d'où naît un style simple que summonte un stignate bifide; coupé en travers, l'ovaire offre quatre loges contenant chacune un ovule dressé. Le fruit se compose de quatre akènes monospermes, renfermés dans l'intérieur du calice qui persiste. La graine contient un embryon dressé au centre d'un endosperme charnu, quelquefois très-mince.

Les genres très-nombrenx de cette famille peuvent être divisés en deux sections suivant qu'ils ont deux ou quatre étamines didynames.

$\S$ I. Deux étamines : Salvia, Rosinarinus, Monarda, Lycopus, etc.

$\$$ II. Quatre étamines didynames : Betonica, Leonurus, Thymus, Ballota, Marrubiun, Phlomis, Satureia, etc.

\section{SOIXANTE-TROISIEMT FAMIXIE.}

* gorraginées. Borraginea. Juss.

Les Borraginées sont des herbes, des arbustes ou même quelquefois des arbres élevés, portant des feuilles altcrnes, souvent recouvertes, ainsi que les tiges, de poils très-rudes. Iseurs fleurs forment des épis unilatéraux, roulés en crosse à leur sommet, souyent réunis et formant 
une sorte de panieule. Leur caliee est monosépale, régugulier, persistant et à einq lobes; la eorolle est monopétale, régulière, à cinq lobes : elle offre dans un eertain nombre de genres, près de sa gorge, einq appendiees saillans, qui sont ereux dans leur intérieur et qui s'ouvrent extérieurement à leur base. Les einq étamines sont insérées au haut du tube du caliee, et alternent avee les appendiees dont nous venons de parler, quand eeux-ei existen t. L'ovaire, portésur un disque hypogyne, annulaire et sinueux, est profondément quadrilobé, à quatre loges monospermes, très-déprimé dans son eentre; le style naît de celte dépression et se termine par un stigmate à deux lobes. Le fruit se eompose de quatre earpelles monospermes, plus rarement ils so soudent et forment un fruit sec ou charnu, à deux ou quatre loges, quelquefois osseuses, ou uniloeulaire par avortement. Les graines ont leur embryon renversé dans un endosperme eharnu, trèsminee et qui mềne quelquefois n'existe pas.

La famille des Borraginées a des rapports avee les Labiées par la strueture de son pistil qui est la mème, et avee les Serophularinées. Mais on la distingue des prenières par sa tige eylindrique, ses feuilles alternes, sa corolle régnlière, ses étamines au nombre de einq, etc.; des secondes par la strueture de sou ovaire et de son fruit.

Nous eiterons iei comme exemples de genres de cette famille les suivans :

$\$ \mathrm{I}$. Genres sans appendices à la eorolle: Echium, Lichospermiun, Pulmonaria, Onosma, Cordia, ete.

\II. Genres munis d'appendices : Sympluyturn, Iycopsis, Anchusa, Borrago, Cynoglossum, ete.

Ventenat avait proposé de séparer des Borraginées le genre Cordia, à eause de son fruit simple et eharnu, et d'en former ane famille sons le nom de Síbestíniers. M. Rob. Brown (Prodr. fl. nov. Holl.) pense que les genres Hydrophyllum, Ellisia et Phacelia, qui ont un fruit eapsulaire, un gros endosperme cornć et des feuilles eomposées ou profondément lobées, forment une famille distinete qu'il nomme Hxnrophyxtés. Enfin le prof. Sehrader dans son exeellent mémoire sur les Borraginées, propose de les diviser en trois ordres distinets, savoir : les Borraginées, les Hydrophyllées et les Héliotropićes. Mais les différenees qui existent entre ces trois groupes, nous paraissent de trop peu d'importance pour justificr leur séparation comme familles distinctes. 


\section{SOIXANTE-QUATRISME FAHETE.}

* convolvulacées. Convolpulacece. Juss.

Plantes herbaeées ou soufruteseentes, souvent volubiles et grimpantes, ayant des feuilles alternes, simples, ou plus ou moins profondément lobées; des fleurs axillaires ou terminales; le ealice monosépale, persistant, à cinq divisions; la eorolle monopétale, régulière , également à einq lobes plissés; les cinq étamines insérées au-tube de la corolle. L'o vaire est simple et libre, porté sur un disque liypogyne; il offre de deux à quatre loges contenant un petit nombre d'ovules. Le style est simple ou double. Le fruit est une eapsule offrant d'une à quatre loges, eontenant ordinairement une ou deux graines, attachées vers la base des cloisons; elle s'ouvre en deux ou quatre valves, dont les bords sont appliqués sur les cloisons qui restent en place; plus rarement la eapsule reste close ou s'ouvre en deux valves superposées. L'embryon, dont les eotylédons sont planes et ehiffonnés, esi roulé sur lui-même, et plaeé au eentre d'un endosperme mou et comme mueilagineux.

Le caractère essentiel de cettc famille consiste dans sa capsule, dont les su= tures correspondent aux cloisons. Ce carnctèrc manquant dans quelques genres, auparavant réuais aux Convolvulacées, tels que Hydrolea, Nama, Sagonea et Diapensa, M. Rob. Brown a proposé d'en former une famille distincte sous le nom d'HYorouéacées. Les genres principaux des Conrolvulacées sont : Convolvulus, Iporncea, Cuscuta, Evolvulus, Cressa, etc.

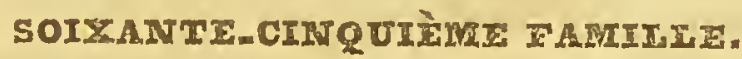

polémonicées. Polemoniacece. Juss.

Plantes herbacées ou ligneuses, quelquefois volubiles, munies de feuilles alterues ou opposées, sourent divisées 
et pinnatifides, et de fleurs axillaires ou terminales, formant des grappes rameuses. Chaque fleur se compose d'un calice monosépale, à eing lobes; d'une corolle monopétale, régulière, rarement irrégulière, à cinq divisions plus ou moins profondes; de cinq étamines insérées à la corolle; d'un ovaire appliqué sur un disque souvent étalé au fond de la flcur et lobé; cet ovaire offre trois loges contenant un ou plus souvent plusicurs ovules; le style est simple, terminé par un stigmate trifide. Le fruit est une capsule à trois Joges, s'ouvrant en trois valves septifères sur le milieu de leur face interne, ou portant sculement l'empreinte de la cloison, qui reste intacte au eentre de la capsule. Les graines offrent un embryon dressé all.centre d'un cndosperme charnu.

Cette famille tient en quelque sorte le milieu entre les Convolvulacées et les Bignoniacées. Elle diffère des premières par ses valves portant les cloisons sur le milieu de leur face interne et non eontiguës par leurs bords sur ees cloisons, et par son emluyon dressé; des secondes par sa corolle presque toujours réguliere, son ovaire à trois loges, ses valves portant les cloisons, ete. Les genres qui composent cette famille sont peu nombreux, tels sont: Polemonium, Plilox, Cantur, Bonplandia, et probablement Cobcea.

\section{SOIXARTE-SIXIEME FAMILIF.}

bIgnoniacḱrs. Bignoniaceœ. Juss. - Bignoniacece et Pedalinece. R. Brown.

Ce sont des arbres, des arbrisseaux ou phns rarement des plantes herbacées, dont la tige est souvent sarmenteuse et garnie de vrilles; lenrs feuilles, ordinairement opposées ou ternées, sont rarement aliemes, le plus souvent composées. Les fleurs, qui sont terminales ou axillaires, diversement groupées, ont un caliee monosépale, souvent persistant et à einq lobes; une corolle monopétale, plus ou moins irréćgulière et à cinq divisions; le plus souvent yuatre ćlamines didynames, accompagnées d'un 
filet stérile; qui est l'indiee d'tune einquième étamine avortée; dausquelques genres, les cinq étamines sont égales ou deux seulement sont fertiles. L'ovaire, porté sur un disque hypogyne, présente úne ou deux loges eontenant ordinairement plusieurs ovules; le style simple se termine par un stigmate bilamellé. Le fruit est une eapsule à une ou deux loges, s'ouvrant. en deux valves opposées à la eloison; rarement le fruit est eharnu, ou dur et indéhiseent. Les graines, souvent bordées d'une aile membrancuse dans tout leur eontour, renferment sous leur tégument propre un embryon dressé, dépourvu d'endosperme.

Les genres principaux de cette famille sont: Bignonia, Catalpa, Jacaranda, Tecoma, etc., dont les graines sont ailées; et le Sesamum, Martynia, Craniolaria, dont les graines sont sans ailes et qui constituent la tribu des Sésamées de M. Kunth. Quant aux genres Pedalium et Josephinia, dont M. Brown a fait une famille distincte sous le nom de Pedalinées, nous croyons qu'ils ont de trop nombreux rapports avec les genres qui forment la tribu des Sésamćes pour en être séparés.

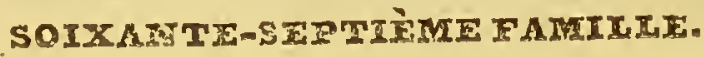

${ }^{*}$ gentianées. Gentianece. Juss.

Presque toutes les Gentianées sont des végétaux herbacés, rarement fruteseens, portant des feuilles opposées, entières, glabres; des fleurs solitaires, terminales ou axillaires ou réunies en ćpis simples. Lenr ealice monosépale, souvent persistant, est à einq divisions; la corolle, monopétale, est régulic̀re, ordinairement à einq lobes imbriqués avant leur développement. Les étamines, en même nombre que les divisions dela corolle, leur sont alternes. L'ovaire, quelquefois rétréei à sa base et eomme fusiforme, a une seulc loge eontenant un grand nombre d'ovules attaeliés à deux trophospermes pariétaux et suturaux, bifides:du eôté interne. Le style est simple ou profondément biparti; chacque division porte un stigmate, Le fruit est 
tume eapsule à une seule loge, contenant un très-grand nombre de graines; elle s'ouvre en deux valves, dont les bords sont plus ou moins rentrans pour s'unir aux trophospermes. Les graines sont en général fort petites, et leur embryon, qui est dressé, est renfermé dans l'axe d'un endosperme charnu.

Cette famille est bien caraetérisée par son port, ses feuilles opposées; entières, leur coulenr verte-glauque; elle a du rapport, d'une part, avee les Polémoniacées, dont elle diffère par ses feuilles opposées, ses ovaires à deux loges seulensent et le mode partieulier de déhiscenee de sa eapsule ; d'nne autre part, avec les Serophularinées; mais eelles-ci, par leur eorolle irrégulière, leurs quatre étamines didynames et la déhiseenee de leur fruit, s'en distinguent facilement. Nous eiterons parmi les genres de Gentianées les Gentiana, Erythraa, Chironia, Exacum, Villarsia, Menyanthes. Ces denx derniers sont remarquables par leurs feuilles alternes et ternées dans le Menyanthes.

\section{SOIXANTE-HUITIEME FAMILIE.}

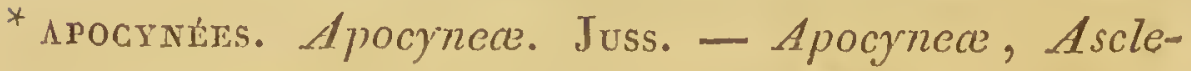
piadea Brown. - Strychnece. Juss.

Les Apoeynées présentent un aspeet très-varié. Ce sont des plantes herbaeées, des arbustes, ou même des arbres très-élevés, et en général laeteseens. Leurs fenilles sont simples et opposées, entières; leurs fleurs sont axillaires ou terminales, solitaires ou diversement réunies. Dans ehacune on trouve un ealiee monosépale, à einq divisions, tantôt étalé, tantôt tubulcux; une eorollc monopétale, régulière, d'une forme très-variée, offrant quelquefois cinq appendiees pétaloïdes, eoncaves, qui maissent de la gorge de la eorolle et se sondent en partie avee les étamines. Celles-ci, au nombre de einq, sont tantôt libres et distinetes, tantôt réunies par les filets et par les anthères et formant une sorte de tube qui reeouvre le pistil et se soude souvent à son sommet avee le stigmate. Les anthères sont ì deux loges, et le pollen qu'elles renferment est pulvérulent dans celles dont les étamines sont libres et 
en masses solides de même forme que l'intérieur de la loge dans celles où les étamines sont soudées; chaque masse pollinique est terminée à son sommet par une glande, qui se soude avec celle de la masse polliniqque à côté de laquelle elle est placée. Deux ovaires libres, appliqués sur un disque hypogyne, soudés ensemble par leur côté interne ou seulement par leur sommet, offrent chacun une $\operatorname{loge}$ qui renferme un grand nombre d'ovules placés à leur suture interne. Les deux styles se soudent quelquefois en un seul, et se terminent par un stigmate plus ou moins discoïde, quelquefois cylindrique et tronqué. Le fruit est un follicule simple ou double; plus rarement il est charnu et indéhiscent. Les graines, attachées à un trophosperme sutural, sont nues ou couronnées par une aigrette; elles contiennent dans un endosperme charnu ou corné un embryon droit.

Cette famille a été divisée en deux par M. Rob. Brown, savoir:

Io Les ÁpocynéEs vraies, qui ont la corolle dépourvue d'appendices et le pollen pulvérulent; tels sont les genres Apocynum, Vinca, Rauwolfia, Arduinia, Nerium, etc.

2。 Les AsclépLiadées, dont la corolle est nnnie d'appendices et le pollen en masses solides comme dans les Orchidées; tels sont les genres Asclepias, Hoya, Cynanchum, etc.

\section{SOIXANTE-NEUVIEME FAMXILE,}

sapotúes. Sapotea. Juss.

Arbrcs ou arbrisseaux tous exotiques et croissant pour laplupart sous les tropiques. Leurs feuilles sont alternes, très-entières, persistantes, coriaces; leurs fleurs hermaphrodites et axillaires. Elles ont un calice persistant et monosépale; une corolle monopétale, régulière, dont lcs lobes sont en nombre égal, double ou triple de ceux du calice. Les étamines sont en nombre défini; les unes sont fertiles, en même nombre que les lobes du calice, et op- 
posées aux pétales; les autres stériles, sont alternes avec les précédentes. L'ovaire est à plusieurs loges, eontenant chaeune un ovule dressé. Le style se termine cn général par un stigmate simple, quelquefois lobé. Le fruit est charnu, à une ou plusieurs losges monospermes, quelquefois osseuses. L'embryon est dressé, eontenu dans un endosperme eharnu qui manque rarement.

Les genres de cette famille sont: Achras, Mimusops, Syderoxylon, Imbricaria, Lucuma, etc. Elle a de grands rapports avec les Ébénacées, qui en diffèrent par leurs fleurs généralement unisexuées, leurs étamines dispo. sées sur deux rangs, leur style divisé et leurs graines pendantes.

\section{SOIXANTE-DIXIEME FAMILIE.}

mynsinées. Myr'sinea. R. Brown. - Ardisiaceß. Juss. Ophiosperma. Vent.

Les Myrsinées sont des arbres ou des arbustes, à feuilles alternes, très rarement opposées ou ternées, glabres, eoriaees, entières ou dentées, sans stipules; à fleurs disposées en grappes ou en espèees d'ombelles, ou enfin simplement grouppées à l'aisselle des feuilles ou au sommet des rameaux : ces fleurs sont hermaphrodites, rarement unisexućes; leur ealice généralement persistant est à quatre ou cinq divisions profondes; leur corolle est monopétale régulière, à quatre ou einq lobes, lcs étamines en même nombre que les lobes de la eorolle, quelquefois monadelphes, sont attachées à la base des lobes et leur sont opposées. Les filets sont eourts, les anthères sagittées. L'ovaire est libre, miloeulaire, eontenant un nombre variable d'ovules insérés à un trophosperme eentral, dans lequel ils sont quelquefois plus on moins profondément enfoneés. Le style est simple, terminé par un stigmate simple ou lobć. Le fruit est une sorte de drupe sèehe, ou une haie eontenant d'une à quatre graines. Celles-ei sont 
peltées; ayant leur hile eoncave; leur tégument simple recouvrant un endosperme charnu ou corné, dans lequel est placé un embryon eylindrique un peu recourbé et placé transversalement au hile.

Cette famille a de grands rapports avec les Sapotées et les Ébénacées, par son port et plusieurs de ses caractères; d'un autre côté, la structure de son ovaire, ses étamines opposées aux lobes dc la corolle, lui donnent quelqu'affinité avec les Primulacées. Lcs genres qui composent la famille des Myrsinées sont les suivans : Myrsine, Ardisia, Jacquinia, Samara, Wallenia et Agicera.

\section{SOIXANTE-ONZIEME FAMTLIE,}

ÉBÉNACÉEs. Ebenacere. Rich, - Guayacaneœ. Juss.

Cette famille se compose d'arbres ou d'arbustes non lacteseens, dont le bois très dur est souvent d'une teinte noire à son eentre. Leurs feuilles sont alternes, entières, souvent eoriaees et luisantes; les fleurs sont en général axillaires, rarement hermaphrodites; le plus souvent polygames; leur ealiee est monosépale à trois ou six divisions égales et persistantes; la eorolle est monopétale régulière, son limbe est à trois ou six divisions imbriquées, les étamines sont en nombre défini, tantôt insérées sur la eorolle, tantôt immédiatement hypogynes; elles sont en nombre double ou quadruple des divisions de la eorolle, très-rarement en nombre égal, et alors alternant avee elles; le plus souvent les étamines sont disposées sur deux rangs et ont leurs anthères linéaires laneéolées, a deux loges. L'ovaire est libre sessile, à plusieurs loges eontenant ehaeune un ou deux ovules pendans; le style est divisé, plus rarement simple, les stigmates sont simples ou bifides. Le fruit est une baie globuleuse, s'ouvrant quelquefois d'une manière presque régulière et contenant un petit nombre de graines comprimées. Leur tégument re- 
couvrc un endosperme cartilagineux, dans lequel est un embryon qui a la même direction que la graine.

Mon père a retiré de la famille des Guayacanées de M. de Jussieu an certain nombre de genres qui en sont fort différens, ct dont il a formé la farnille des Styracées. Telle qu'elle est limitée aujourd'hui par les botanistes modernes, la famille des Ebénacées se compose des genres Diospyros, Royena, $P_{a-}$ ralea, etc. Elle a des rapports avec les Sapotées; mais celles-ci ont leurs étamines en même nombre que les divisions de la corolle, auxqnelles elles sont opposées et en outre présentent plusieurs autres caractères distinctifs. Quant aux Styracées, nous indiquerons, à la suite de cette famille, les caractères qui les distinguent des Ebénacécs.

\section{NEUVIĖME CLASSE.}

PÉRICOROLIIE.

\section{SOIXANTE-DOUZIEME FAMIXIE}

* styrackes. Styracece. Rich, Symploceœ. Juss.

Cette petite famille renferme des arbres ou des arbrisseaux à feuilles alternes, sans stipules; à fleurs axillaires, quelquefois tcrminales. Leur ealice est libre ou adhérent avee l'ovaire infère; le limbe est entier ou divisé ; la corolle est monopétale, régulière. Les étamines, dont le nombre varie de six à seize, sont libres ou monadelphes par leur base. L'ovaire, eomme nous l'avons dit, est tantôt supère, tantôt infère, ordinairement à quatre loges, séparées par des cloisons membraneuses et trèsminees; chacune de ees loges eontient communément quatre ovules attachés à l'angle interne de la loge, et dont deux sont dressés et deux renversés. Le style est simple, terminé par un stigmate très-petit et simple. Le fruit est légèrement charnu; il contient d'une à quatre nucules osseuses et plus ou moins irrégulières. La graine est formée, outre son tégument propre, d'un endosperme charnu, qui contient un embryon cylindrique, ayant la même direction que la graine. 
Cette famille se compose des genres Halesia, Symplocos, Styrax, "Alstonia et Ciponima, qui faisnient autrefois partie de la famille des Ebénacées Mon père les en a retirés pour en former la nouvelle famille des Styracées, qui en diffère par son insertion périgynique, son ovaire dont les loges contiennent quatre ovules, dont deux dressés et deux renversés, et par son style simple.

\section{SOIXANTE-TBETZIEME FAMIIIE,}

* ÉrICrNíes. Ericinea. - Erica et Rhodora. Juss. Epa cridece. R. Br. Vacciniece. Desv.

Arbustes et arbrisseaux d'un port élégant, ayant en général des feuilles simples, alternes, rarement opposées, verticillées ou très-petites et en forme d'écailles imbriquées. Leur inflorescence est très-variable. Le caliee, monosépale, est tantôt libre, tantôt adhérent avee l'ovaire, qui alors est infère, à cinq divisions, quelquefois tellement profondes, qu'il paraît formé de sépales distincts. La corolle est monopétale, régulière, à quatre ou cinq lobes, quelquefois à quatre ou cinq pétales distincts. Les étamines, en génćral en nombre double des divisions de la corolle, ont leurs filets libres, rarement soudés entre eux à leur base. Les anthères sont introrses, à une ou deux loges, quelquefois terminées par deux appendices en forme de cornes à leur sommet où à leur base, et s'ouvrant en général par un trou vers leur sommet. Ces étamines sont généralement attachées à la corolle; mais quelquefois elles sont immédiatemeut hypogynes; l'ovaire est infère ou libre; dans ce dernier cas, il est sessile au fond de la fleur ou appliqué sur un disque hypogyne plus ou moins saillant et quelquefois sous la forme de lobes ou d'écailles. 11 offre de trois à cinq loges contenant chacune un assez grand nombre d'ovules attachés à leur angle iuterne. Le style est simple, terminé par un stigmate offrant autant de lobes qu'il y a de loges à l'ovaire. Le 
fruit est une baic ou plus souvent unc capsule, quelquefois couronnéc par lc limbe du calicc, et s'ouvrant en autant de valves qu'il y a de loges; tantôt ehaeune de ees valves entraîne avec elle une des cloisons sur lc milieu de sa faee internc (dćhiseenee loeulicide), tantôt la dćhisecnee a lieu cn faee de chaque eloison (déhisecnee septieide). Les graines se composent d'un endosperme charnu, au milieu duquel est un cmbryon axile, eylindrique, ayant la même dircetion que la graine.

Nous réunissons ici lcs Rhodoracées dc M. de Jussien; qui ne diffèrcnt des Ericinées que par leur capsule, dont les valves emportent les cloisons sur le milieu de leur face intcrne, tandis que dans les Ericinées, en génćral, la déhiscence a lieu en face des cloisons. Mais on observe l'un et l'autre de ces dcux modes dans plnsieurs genrcs des Ericinécs. Qunnt aux Epacridćes de M. Rob. Browis, il n'cxiste d'autre différence essentielle cntre ce groupe et les Ericinécs, que dans leurs anthères constamment uniloculaires et un port différent. Nous avons cra pouvoir les réunir et n'en former qu'une simple section. Nous diviserons de la manic̀re suivante la famille des Ericinées:

10 Vaccrmérs. Genres à ovaire infère. Facciminm, Escallonia, Gaylussaccia, etc.

$2^{\circ}$ Ericinérs. Ovaire librc, disque bypogyne, anthères biloculaires. Erica, Rhododendrum, Rhodora, Ledum, Clcthra, Arbutus, Andromeda, etc.

$3^{\circ}$ Eracridées. Ovaire libre : disquc sous forme de cinq écailles hypogynes, anthères uniloculaires. Epacris, Styphelia, Leucopogon, etc.

\section{SOIXANTE=QUATORZIÈIE FAMITXE.}

${ }^{*}$ gesnériacées. Gesncriaceœ. Rich.

Ce sont des plantes herbaeées, rarement soufrutcseentcs à leur base, portant des fcuillcs opposées ou alternes, des fleurs axillaires ou tcrminales. Le caliec cst monosépale, persistant, à cinq divisions, adhérant par sa base avec l'ovaire qui est généralcment infèrc. La eorolle est monopétalc, irrégulic̀re, à cinq lobes inégaux formant quclquefois comme dcux lèvres; on trouve deux ou quatre étamines insérées à la corolle. L'ovaire, comme nous 
l'avons dit, est infère ou libre; dans le premier cas, il est eouronné par un disque épigyne souvent lobé; dans le seeond eas, le disque est hypogyne et souvent latéral. Le style est très-simple, terminé par un stigmate simple et coneave dans son centre. L'ovaire présente une seule loge dans laquelle un nombre très-eonsidérable d'ovules sont attaehés à deux trophospermes pariétaux, ramifiés du côté de la loge. Le fruit est ou charnu ou see et formant une eapsule uniloeulaire s'ouvrant en deux valves.

On cite comme appartenant à cette famille les genres Gesneria, Gloxinia, Besleria, Columnea, Achimenes. Mais si l'on en excepte les deux premiers, qui ont l'ovaire infère, les trois autres, anxquels il fant rénnir le Ramondia, autrefois placé dans les Solanées, ne nous paraissent différer en rien des Orohanchées ; pent-être faudrait-il alors réduire les Gesnériées anx seuls genres à ovaire infère.

\section{SOIXANTE-QUINZIEMTE FAMIXIE.}

* enmpanulacées. Campanulacece. Juss.

Les Campanulaeées sont ordinairement des plantes herbacées ou soufrutescentes, remplies en général d'un sue blane et amer. Leurs feuilles sont alternes et entières, rarement opposées; leurs fleurs forment des épis, des thyrses ou sont rapproehées en capitules. Elles offrent un calice monosépale, à quatre, cinq ou huit divisions persistantes; une corolle monopétale régulière ou irrégulière, ayant son limbe partagé en autant de lobes qu'il y a de divisions au calice, quelquefois eomme bilabiée. Les étamines, au nombre de eing, sont alternes a vec les lobes de la eorolle. Leurs anthères sont libres ou rapproehées en forme de tube. L'ovaire est infère ou semi-infère, à deux ou plusieurs loges polyspermes. Le style est simple terminé par un stigmate lobé, quelquefois environiné de poils ou d'une sorte de godet eupuliforme. Le fruit est une capsule couronnéc par le limbe du ealiee, à deux ou à un plus grand nombre de loges, s'ouvrant soit par le 
moycn de trous qui sc forment vers la partie supérieure; soit par des valves incomplètes, et qui cntraînent avec elles une partie des cloisons sur le milicu de lcur face internc. Les graincs, très-petitcs ct fort nombreuses, renferment dans un endosperme charnu un embryon axile et dressé,

-Nous réunissons ici les familles des Campanulacées, des Lobéliacées, des Goodénoviées et des Stylidiées, qui ont entre elles des caractères communs trop intimes pour former antant de familles distinctes. Nous les considérons simplement comme des tribus d'un même ordre naturel.

10 Canraatulacérs. Corolle régulière, étamines distinctes, capsule à deux loges polyspermes. Ex. : Campanula, Phyteuna, Prismatocarpus, Jasione, etc.

$2^{\circ}$ Lobśtracḱts, Rrcr. Corolle irrégulièrc, étamines soudées par les anthìres, stigmate environné de poils. Ex. : Lobelia, Lysipomia, etc.

3० GoonÉNoviéEs, R. BRowr. Corolle irrégulièrc, étamines libres ou sondées par les anthères, stigınate environné d'une sorte de godet cupuliforme, eapsule biloculaire ou noix monospermc. Ex. : Goodenovia, Euthales, Lechenaultia, ete.

$4^{\circ}$ Struidićes, R. Brown. Corollc irrégulière; deux étamines, dont les filets sont soudés et confondus avec le style, et formant une sorte de colonne centrale; stigmate situé entre lesdeux anthères; capsule biloculaire, bivalve. Ex. : Stylidium, Leupenthookia, ete.

\section{DIXIËME CL $\Lambda$ SSE.}

ÉPICOROLLIE. - SYNANTHRRIE.

\section{SOIXANTE-SEIZIXNE FAMIILT.}

* synanthénxes. Synantherea. Rich.-Cichoracea, Corymbiferce et Cynarocephala. Juss. - Compositce. Auct.

Cette grande famille est une des mieux caractérisécs ct des micux limitées du règne végétal. Elle comprend des plantes herbacécs, des arbustes ou même des arbrisscaux plus ou moins élevés. Leurs feuilles sont communément alternes, rarcment opposécs. Leurs flcurs, généralcment petites, forment des capitules ou calathides, hemisphéri- 
ques, globulcux ou plus ou moins allongés. Chaque capitule se eompose : $I^{\circ}$ d'un réeeptaele eommun, épais et quelquefois eharnu, eonvexe ou eoncave, et qui a reẹ les noms de phoranthe ou de clinanthe; $2^{\circ}$ d'un involucre eommun qui environne le eapitule, et se eompose d'éeailles dont la forme, le nombre et la disposition varient suivant les genres; $3^{\circ}$ sur le réeeptaele on trouve fréquemment à la base de ehaque fleur de petites éeailles ou des poils plus ou moins nombreux. Les fleurs qui forment les eapitules sont: de deux sortes; les unes offrent une eorolle monopétale, r'égulière, infundibuliforme et en général à einq lobes réguliers; on les nomme des fleurons; les autres ont une eorolle irrégulière, déjetée latéralement en forme de languette; on les appelle des demi-fleurons. Tantôt les eapitules se eomposent uniquement de fleurons (Flosculeuses), tantôt uniquement de demi - fleurons (Semiflosculeuses), tantôt enfin leur eentre est oceupé par des fleurons, et leur eireonférenee par des demi-fleurons (Radiées). Chaque fleur offre l'organisation suivante : le ealiee, adhérent avee l'ovaire, a son limbe entier', membraneux, denté, formé d'éeailles ou de poils; la eorolle monopétale, régulière ou irrégulière; einq étamines à filets distinets, nıais dont les anthères sont réunies et forment un tube qui est traversé par un style simple, que termine un stigmate bifide. Le fruit est un akène nu à son sommet ou eouromné par un rebord membraneux, par des petites éeailles ou par une aigrette de poils simples ou plumeux, sessile ou stipitée. La graine est dressée, eontenant un embryon homotrope et sans endospcrme.

Cette famille, qui a été l'objet d'un grand nombre de traraux importans, pent se diviser en trois tribus principales de la manière suivantc :

Io Les Cynarocéphates, dont toutes les fleurs sont des fleurons, et qui ont leur réceptaclc garni de poils nombreux ou d'alvéoles, et dont le stylc est renflé et garni de poils an-dessous du stigmate; tels sont les genres Carthamus, Carduus, Cynara, Centaurea, Onopordon, etc. 
$2^{\circ}$ Les Cricoracírs, dont toutes les fleurs sont des demi-flearons; tels sont les genres Lactuca, Cichorium, Sonchus, Hieraciun, Prenanthes, ete.

$3^{\circ}$ Les Cor XMrifìres, dont les eapitules se eomposent en général de fleurons au eentre et de demi-fleurons à la eirconfércnee; ex. : Helianthus, Chrysanthemum, Anthemis, Matricaria, etc.

\section{SOIXANTE-DIX-SEPTIEME FAMIIIE,}

\section{calycénées. Calycerece. Rich. - Boopidece. Cassint.}

Ce sont des plantes herbacées, ressemblant assez par leur port aux Seabicuses. Leur tige porte des feuilles alternes, souvent découpées et pimnatifides. Les fleurs sont petites et forment des capitules globuleux, environnés d'un involucre eommun. Le réceptacle qui porte les fleurs est garni de squames foliacées qui se soudent quelquefois avec les fleurs, de manière à n'en êtrc pas distinctes. Le caliec est adhérent avec l'ovaire infère, ct les divisions de son limbe sont quelquefois roides ct épineuses. La corolle cstmonopétale, tubuleuse, infundibuliforme et régulière, au-dessous des cinq étamines sont cinq glandes nectarifères. Ces étamines sont soudécs à la fois par les filets ct les anthères, et formcnt un tube cylindrique, et chaque anthère s'ouvre par sa face interne. L'ovaire infêre est à unc seule loge, du sommet de laquelle pend un ovule renversé; le sommet de l'ovaire préscnte un disque épigyne, un style simple terminé par un stigmatc hémisphériquc. Dans le genre Acicarpha, toutes les fleurs sont soudées enscmble par leur's ovaires. Le fruit est un akène couronné par les deuts épineuses du calice. La graine offre sous son tégument propre un endosperme dans lequel est contenu un embryou renversé eomme la grainc.

Cette petite famille se eompose des genres Boopis, Calycera et Acicarpha. Elle tient le milieu entre les Synanthérées et les Dipsacées. Elle diffère des premières par son ovule renversé, ses étnmines soudées à la fois par les anthères et les filets et par son stigmate simplle; des Dipsacées par ses fevilles alternes et ses ćtanines sondées. 


\title{
ONZIEME CLASSE.
}

\author{
ÉPICOROLLIE.-CHORISANTHÉRIE.
}

\section{SOIXANTE-DIX-FIUITIEME FAMIILE.}

* dipsacées. Dipsacece. DC. - Dipsacearum gen. Juss.

La tige est herbacée, les feuilles opposées sans stipules; les fleurs réunies en capitules hémisphériques ou globuleux, accompagnés à leur base d'un involucrc de plusieurs folioles; le calice cst double, l'extéricur est monosépale, libre, entier ou divisé en lanièrcs étroites et sétacées. L'interne est adhérent avec l'ovaire, terminé par un limbe entier ou divisé. La corollc cst monopétale tubuleuse, à quatre ou cinq divisions inégales; les étamines, en même nombre que ces divisions, alternent avec elles. L'ovaire est infère, à une seule loge contenant un seul ovule pendaut; le'style et le stigmate sont simples. Le fruit est un akène eouronné par le limbe calycinal, et enveloppé dans le calice externe. La graine est pendante, et son embryon, qui a la même direetion, est plaeé dans un endosperme charnu assez mince.

Le professeur de Candolle a retiré de cetle famille, telle qu'elle avait été établie par M. de Jussieu, İe genre Valeriana et quelqnes autres analogues pour en former la famille des Valérianćes, qui diffère des vraies Dipsacées par ses fleurs non réunies en capitule; par son calice simple, son stigmate
lobé, etc.

Par leur port, et surtout leur inflorescence, les Dipsacées ont quelqu'analogie avec les Synanthérćes, mais elles en diffèrent par leur calice double, leurs anthères libres ct leur graine renversée. Les genres prineipaux de cette famille sont: Dipsacus, Scabiosa, Knantin. 


\section{SOIXANTE-DIX-NIUVIËME FAMILIE.}

\section{* valérianées. Valerianece. DC.}

Plantes herbaeées, à feuilles opposées, simples ou plus ou moins profondément ineisées; à fleurs sans calicule, ordinairement disposées en grappes ou panieules terminales. Leur ealiee est simple, adhérent avecl'ovaire infère, ayant son limbe denté ou roulé en dedans et formant un rebord entier. La eorolle est monopétalc, plus ou moins irrégulière, et quelquefois épcromnée à sa base, et à einq lobes.Les étamines varient d'une à einq, et sont alternes avee les lobes de la corolle. L'ovaire est à une seule loge (quelquefois on trouve deux autres cavités vides ou fausses loges, de manière que l'ovaire semble être triloeulaire). Cette loge contient un seul ovule pendant. Le style est simple, terminéle plus souvent par un stigmate trifide. Le fruit est un akène eouronné par les denıs dı ealiee ou par une aigrette plumeuse, formée par le déroulement du limbe. La graine eontient un embryon dépourvu d'endosperme.

Cette famille se compose des genres Valeriana, Centranthus, Fedia, $P_{a-}$ trinia, etc. Voy. la note placée à la suite des Dipsacées.

\section{QUATRE-VINGTIÈME FAMIXIE.}

* rubiacées. Rubiacea. Juss. - Operculariées.J.

On trouve dans ectte famille des plantes herbacées, des arbustes et des arbres d'me très-grande hauteur. Leurs feuilles sont ou opposées ou verticillées; dans le premier cas, elles offrent de chaque eôté une stipule, intrapétiolaire, qui souvent se soude avee les côtés des pétioles, ct forme une sorte de gaîne. Les fleur's sont axillaires ou terminales, quelquefois rémies en tête. Le ealice, adhićrent par sa base avec l'ovaire infère, a son limbe cnticr ou 
partagé en quatre ou einq lobes plus on moins profonds et persistans. La eorolle est monopétale régulièrc, épigyne à quatre ou cinq lobes. Lcs étamines sont en même nombre que les lobes de la eorolle et altcrnant avec eux. L'ovaire est infèrc, surmonté d'un style simplc ou bifidc. Cet ovaire présentc deux, quatre, cinq, ou un plus grand nombrc de loges, qui conticnnent chacune un ou plusieurs ovules dressés ou attachés à l'angle interne des loges. Le fruit est très-variable. Tantôt il se compose de deux petitcs coques monospermes et indéhisccntes, tantôt il est eharnu, et conticnt dcux noyaux monospermes; dans certains genres c'cst unc capsulc à dcux ou à un plus grand. nombrc de loges, s'ouvrant cn autant de valves; ou un fruit eharnu et indéhiseent. Toujours ec fruit estcouronné à son sommet par lc limbc ealycinal. Lcs graincs, quelquefois ailées ct mcmbrancuses sur lcur hord, contiennent dans un cndosperme dur ct corné, un embryon axilc et dressé, ou quclqucfois plaeé cn travers relativemcnt auhile.

Cette famille, extrêmement naturelle et très-facile à distingner, se divise en deux sections principalest: dans l'une on place tous les genres à feuilles verticillées, tels que Galium, Asperula, Rubia, Sherardia, Crucianella, etc,; dans l'autre les genres beaucoup plus nombreux, qui ont les feuilles opposées et les stipules intermédiaires, comme Cinchona, Coffea, Cephaelis, Psychotria, etc. Nous n'avons en Europe que des Rubiacées à feuilles verticillées.

Nous réunissons à cette famillc le grouppe des Orerculariées, qui ne diffère réellement pas des autres Rubiacćes.

\section{QUATRE-VINGT-UNIÈME FAMIXIE. \\ * Caprifoliacées. Caprifoliacea. Rich.}

Arbrisseaux ì fcuillcs opposées, raremcnt alternes, généralement simples, plus rarcnent imparipinnées, sans stipulcs; lcs fleurs sont axillaircs, solitaires, ou souvent gúminées et en partie souclécs ensemble par lcur calice; 
disposćes en cyme ou réunics en une sorte de capitule. Le calicc est toujours monosépale, adhérent par sa partie inférieure avec l'ovaire qui est infère; le limbe est à cinq dents persistantes; la corolle est monopétale, le plus souvent irrégulière; quelqucfois elle est formée de cinq pétales distincts. Les étamines sont au nombre de cinq, alternant avec les divisions de la corolle. L'ovaire offre d'une à cinq loges, contenant ehacune soit un sẻul ovule pendant, soit plusicurs ovules attachés à leur anglc interne. Le style est simple, terminé par un stigmate très petit et à peine lobé. Le fruit est quclquefois géminé, c'est-à-dire formé de la soudure de deux ovaires. Il est clarnu, à une ou plasicurs loges, quelquefois osseuses, et renfermant chacune une ou plusieurs graines. Celles-ci ont un tégument propre, quclquefois recouvert d'un noyau, un endosperme cliarnu, qui conticnt un embryon axile ayant la mème direction que la graine.

Cette famille peut être faeilement divisée en deux tribus naturelles, suivant que les loges de son ovaire sont monospermes ou suivant qu'elles sont polyspermes.

Io Hénéraḉes: loges de l'ovaire monospermes, Hedera, Cornus, Sambucus, Viburnum.

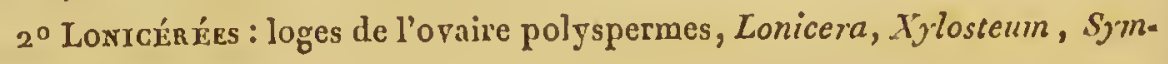
phoricarpos, ete.

Cette famille, voisine des Rubiacées, en diffère surtout par sn corolle irrégulière et l'absenee des stipules entre les feuilles.

\section{QUATRE-VINGT-DEUXIÈME FAMILLE.}

* ioranthées. Loranthece. Rich.

Les Loranthées sont pour la plupart des plantes vivaces et généralement parasites. Leur tige est ligneuse et ramifiéc; leurs feuilles simples et opposées, entières ou dentées, coriaces, persistantes, sans stipules. Les fleurs sont diversement disposćes, tantùt solitaires, tantôt en 
épis, en grappes ou en panicules axillaires ou terminales. Les fleurs, en général hermaphrodites, sont quelquefois diö̈ques. Le ealice est adhérent avee l'ovaire infère, son limbe est entier ou légèrement denté; ce caliee est aceompagné extérieurement de deux bractées, ou d'un second calice cupuliforme enveloppant quelquefois entièrement le véritable calice. La corolle se compose de quatre à huit pétales, insérés vers le sommet de l'ovaire; ces pétales, sont parfois soudés, et représentant une corolle monopétale. Les étamines sont en même nombre que les pétales; elles leur sont opposées, sessiles ou portées sur des filamens plus ou moins longs. L'ovaire est 'à une seul loge, qui contient un ovule renversé. Cet ovaire est couronné par un disque épigyne et annulaire. Le style est souvent long et grêle, quelquefois manquant complétement, le stigmate est souvent simple. Le fruit est généralement eharnu, contenant une seule graine renversée, adhérente, avec la pulpe du périearpe qui est épaisse et visqueuse. Cette graine renferme un endosperme charnu, dans lequel est placé un embryon cylindrique ayant la radieule tournée vers le hile.

Cette famille, dont les genres faisaient autrefois partie des Caprifoliacées, en diffère par sa corolle, le plus souvent polypétale, ses étamines opposées aux pétales; son ovaire uniloculaire et monosperme. Les genres principaux de cette famille sont: Loranthus, Viscum, Aucuba, etc.

\section{DOUZIÈME CLASSE.}

ÉPIPÉTALIE.

\section{QUATRE-VINGT-TROISIÈME FAMILIE.}

rhizophonées, Rhizophorea. R. Brown.

Ce sont des arbrcs tous exotiqucs, à feuilles opposées, simples, avee des stipules interpétiolaires comme dans 
les Rubiacées. Leur ealiee, adhérent avee l'ovaire, offre quatre ou einq divisions valvaires à son limbe, qui est persistant ; la corolle sc compose de quatre à einq pétales, les étamines varient de huit à quinze. L'ovaire, qui n'est quelquefois que semi-infère, offre constamment deux loges, qui eonticnnent chacune deux ou un grand nombre d'ovules pendans. Ie style est simple, et le stigmate biparti. Le fruit, qui est couronné à son sommet par le ealice, est uniloeulaire, monosperme et indéliscent. La graine qu'il renferme se eompose d'un gros embryon privé d'endosperme; cet cmbryon germe et se développe quelquefois dans l'intérieur du fruit, qu'il perfore à son sommet.

Les genres Rhizophora, Bruguiera et Carallia composent seuls cette famille, qui diffère des Caprifoliacées, parmi lesquelles ces genres étaient placés, par leur corolle polypétale, leur fruit coriace et lear embryon sans endosperme; et des Lorauthées par leur embryon sans endosperme.

\section{QUATRE-VINGT-QUATRIEME FAMILIE.}

\section{* ombeliffìres. Umbelliferce. Juss.}

L'une des familles les plus naturelles du règne végétal, les ombellifères sont des végétaux herbacés, dont la tige est souvent ereuse intérieurement. Les feuilles alternes, engainantes à leur base, généralement déeomposées en un très-grand nombre de segmens ou de folioles. Les fleurs, toujours fort petites, blanehes ou jaunes, sont disposées en ombelle; on trouve quelquefois à la base de l'ombelle de petites folioles dont la réunion eonstitue l'illvoluere, et les involucelles quand elles sont placées à la hase des ombellules. Chaque fleur se eompose d'un ealice adhérent avec l'ovaire infére, et dont le limbe est entier ou à peine denté, d'une eorolle formée de einq pétales plus ou moins étalés; de einct étamines épiggnes, alternes avee les pétales; d'un ovairc à deux loges, eontenant elracune un ovule renversé, et couronmé it son sommet 
' par un disque épigyne ct bilobé; de deux stylcs, terminćs chacun par un petit stigmate simplc. Lc fruit est un diakènc de forme trùs-variée, sc séparant à sa maturité en denx akc̀nes monospcrmes, réunis cntr'cux par une petite columelle filiformc. La graine est rcnversée, et contient dans un endosperme assez gros un très-petit embryon axile.

Les genres de cette famille sont extrềmement nombreux. Nous citerons entre autreslesDaucus, Conium, Carvi, Ammi, Scandix, Apium, Pastinaca, etc.

\section{QUATRE-VINGT-CINQUIEME FAMILIE:}

arditicéfs. Araliacece. Juss.

Les araliacćes constituent un grouppc à peine distinct (des ombcllifères. Ce sont des végétaux hcrbacés ou quel'quefois des arbres très-élevés. Lcurs flcurs, égalcment très-petitcs, sont disposécs en ombelles simples ou en omjbelles paniculées. Lcur calicc est également adhérent ct (denté; leur corolle, forméc de cinq à six pétales; leur covaire présente de deux à six loges monospermes, et est ssurmonté d'autant de stylcs, que terminent des stigmates ssimples. Lc fruit est tantôt charnu ct indéhiscent, tantôt ssec et sc séparant cn autant de coques monospermes qu'il y avait de loges à l'ovaire.

Cette famille est extrêmement voisine des Ombellifères, dont elle diffère E par le 'plas grand nombre de ses loges et de.ses styles ou par son fruit charnu. IEx. : Aralia, Panax, Gastonia, eitc.

\section{TREIZIEME CLASSE.}

HYPOPÉTALIE.

\section{QUATRE-VINGT-STXIEME FAMIIIE.}

* renongulacées. Ranunculacece. Juss.

Cette grandc famillc se compose de plantes herbacćes, Iportant des feuilles alterncs, embrassantes à leur basc, 
Ie plus souvent divisées cn un grand nombre de segmens; opposées dans le seul genre clématite; les fleurs varient beaueoup dans leur disposition, quelquefois elles sont aecompagnées d'un involuere formé de trois feuilles, éloigné des fleurs; ou rapproehć d'elles et ealyeiforme. Le caliee est polysépale, souvent eoloré et pétaloïde, rarement persistant; la eorolle est polypétàle, quelquefois nulle; les pétales sont quelquefois simples, avec une petite fossette ou une lame glanduleuse à leur base interne; plus souvent difformes ou irrégulièrement ercusés en cornet, et brusquement onguieulés à leur base. Les étamines génćralement en grand nombre, libres, à anthères eontinues aux filets; les pistils, quelquefois monospermes ag régés cn une sorte de capitule, ou polyspermes et réunis eirculairement et quelquefois plus ou moins intimement sondés. Le style est très-court, ordinairement latéral; le stigmate simple. Les fruits sont monospermes indéhiseens, en eapitule ou en épi ; ou hicn ee sont des eapsules agrégées, distinetes ou soudées, quelquefois solitaires, uniloculaires polyspermes, s'ouvrant par leur suture interne qui porte les graines; très-rarement e'est tine baie polysperme. Les graines ne sont pas arillées; l'embryon, très-petit, a la même direetion que la graine, et est renfermé dans la base d'un endosperme eharm on dur.

Les genres nombreux de cette famille peuvent se diviser en deux grandes sections, suivant que les ovaires sont monospermes ou polyspermes.

10 Ovaires monospermes.

A. Genres umnis d'un calice et d'une corolle.

Ranunculus, Ficaria, Cernocephalus, MYosurus, Adonis.

B. Genres sans corolle.

Anemone, Clcmatis, Thalictrum.

2) Ovaires polyspermes.

A. Genres sans corolle.

Paunia, Caltha.

13. Cenres mnnis diune corolie.

Trollins, Eranthis, Melleborse, Ligella, Garidella, Aquilegia, Delphinium, fcruitum, scica. 


\section{QUATRE-VINGT-SEPTIÈTE TAMTIJE.}

DiLLÉNincées. Dilleniacec. DC.

Arbres ou arbustes sarmenteux, ayant des feuilles alternes, très-rarement opposées, sans stipules, souvent embrassantes à leur base; des fleurs solitaires ou ęn grappes, quelquefois opposées aux feuilles. Leur calice est monosépale persistant, à cinq divisions profondes et imbriquées latéralement; leur corolle ordinairement de cinq pétales; leurs étamines, très-nombreuses, libres, disposées sur plusieurs rangs, sont libres, quelquefois unilatérales ou disposées en plusieurs faisceaux. Les carpelles varient de deux à douze, généralement·distincts, ils sont quelquefois soudés en un seul. Leur ovaire est uniloculaire, contenant deux ou plusieurs ovules attachés à la partie inférieure de leur angle interne et dressés. Les styles sont simples et terminés chacun par un stigmate également simple. Les fruits sont distincts ou soudés, charnus ou secs et déhiscens. Les graines ont un tégument crustacé, recouvrant un endosperme charnu, dans lequel est un embryon très-petit dressé, placé vers la base.

On compte dars cette famille les genres Tetracera, Davilla, Delima, Pachynema, Fivirandra, Dillenia, Hibbertia, etc. Elle se distingue des Magnoliacées et des Anonacées par le nombre quinaire des partics de sa fleur.

\section{QUATRE-VINGT-HUITIUME FAMILLE.}

MugNolincées. Magnoliacece. Juss.

Cette famille se compose de grands et beaux arbres ou d'arbrisseaux élégans, ornés de belles feuilles alternes, souvent coriaces ct persistantes, munies à leur base de stipules foliacées. Les fleurs, souveut très-grandes, et répandant une odeur suave, sont en général axillaires. Lc 
caliec se compose de trois à six sćpales eaducs; les pétales varient de trois à vingt-sept, disposés sur plusicurs rangées; les étamines, fort nombreuses et libres, sont dis. posćes sur plusieurs rangćes, et attachćes au réeeptacle qui porte les pétales. Les pistils sont nom]breux, tantôt réunis eireulairement et sur une seule rangée au centre de la fleur, tantôt formant un eapitule plus ou moins allongé. Ces pistils se composent, d'un ovaire uniloeulaire contenant un ou plusieur's ovules, d'un style à peine distinct ct d'un stigmate simple. Les fruits se composent de carpelles sees ou charrus, réunis circulairement ct sous forme d'étoile, ou disposés cn capitules, et quelquefois tous soudés entr'cux. Chaque earpelle est indéhiscent ou s'ouvre par une suture longitudinale, et la graine est quelquefois portée sur un trophosperme sutural et filiforme, qui pend en dehors, quand le fruit s'ouvre. Ces graines ont leur embryon dressé dans un endosperme eliarnu.

- La fumille des Magnoliacées se subdivise en denx tribns de la manière suivante :

Io ILricrérs : carpelles verticillés, rarement solitaires par avortement; feuilles marquées de points transparens, ex. : Illicium, Drings, Tasinannia.

$2^{\circ}$ Magnorrírs : earpelles disposés en eapitules; feuilles non ponetuées. Ex. : Magnolia, Michelia, Talauma, Lyriodendron, ete.

C̣ette famille est très-voisine des Anonaećes, dont elle diffère surtout par ses stipules et la strueture continue de son endosperme. Elle a aussi des rapports avee les Dilléniacées, qui en diffèrent par le nombre quinaire des parties de la fleur.

\section{QUATRE-VINGT-NEUVIEME FAMILLE.}

añonacén. Anonacea. Juss.

Les Anonacées sont des arbres ou des arbrisseaux ayaut les feuilles alternes, simples, dépourvues de stipules, earactère qui les distingue surtout des Magnoliacées. 'Leurs fleurs, ordinairement axillaires, sont quclquefois terminales. Leur ealice est persistant, à trois divisions pro- 
fondes; leur corolle formée de six pétales, disposés sur deux rangs; les étamines sont fort nombreuses, formant plusieurs rangées; leurs filets sont courts, et leurs anthères presque sessiles. Les carpelles, en général réunis en grand nombre au centre de la fleur, sont tantôt distincts, tantôt soudés entrị'eux; chacun d'eux offre une seule loge qui contient un ou plusieurs ovules attachés à leur suture interne, et formant souvent deux rangées longitudinales. Ces carpelles constituent soït autant de fruits distinets (rarement un seul par suite d'avortement); quelquefois ils se soudent tous entr'eux, et forment une sorte de cône eharnu et écailleux. Les graines ont leur tégument formé de deux lames; leur endosperme corné est profondément sillonné, contenant tun très-petit embryon placé vers le point d'attache de la graine.

Cette famille, daus laquelle on trouve les genres "Anona, Kadsura, Asimina, Uvaria, etc., est très-voisine des Magnoliacées, dont elle diffère surtout par l'absence des stipules, par les pétales, dont le nombre n'excède jamais six, et par l'endosperme, profondément et irrégulièrement sillonné.

\section{QUATRE-VINGT-DIXIEME FAMILLE.}

* Berbéridóes. Berberidece. Juss.

Herbes ou arbrisseaux à feuilles alternes, simples ou composées, accompagnées à leur base de stipules qui sọnt souvent persistantes et épineuses; leurs fleurs, généralement jaunes, sont disposées en épis ou en grappes. Ellès ont un calice de quatre à six sépales, rarement d'un nombre plus considérable ou moindre, accompagné extérieurement de plusieurs écailles; leurs pétales, en même nombre que les sépales, sont planes ou concavés et irrćguliers, mais constamment opposés aux sépales; ils sont souvent munis à leur base interne de petites glandes ou d'écailles glanduleuses; les étamines, en nombre égal aux pétales, leur sont opposées; les an- 
thères sessiles ou portées sur un filet plus ou moins long, sont à deux loges, qui ehacune s'ouvrent par une sorte de valve ou de panneau, ainsi que nous l'avons déjà observé dans la famille des Laurinées. L'ovaire est à une seule loge, qui renferme de deux à douze ovules dressés ou attaehés latéralement sur la paroi interne, et y formant une seule ou deux rangées. Le style, quelquefois latéral, est eourt, épais ou nul; le stigmate est généralement eoncave. Le fruit est sec ou eharnu, uniloculaire et indéliiscent. Les graines se eomposent d'un tégument propre, reecuvrant un endosperme charnu ou eorné, qui eontient un embryon axile et homotrope.

Cette famille, dont on a retiré plusieurs des genres qai y avaient été réunis par M. de Jussieu, se compose des suivans : Berberis, Mahonia, Nandinia, Leontice, Carlophyllun, Epinedium et Diphylleia. Elle est très-distincte de toutes les autres fomilles voisines par ses étamines opposées aux pétales et le mode de déhiscence de ses anthères.

\section{QUATRE-VINGT-ONZIÈME FAMILIE.}

Ménispenmées Menispermece. Juss.

Cette famille se compose d'arbustes sarmenteux et grimpans, dont les feuilles alternes sont généralement simples, rarement eomposées; les fleurs sont petites, unisexuées et le plus souvent diöques. Le caliee se compose de plusieurs sépales disposés par trois et formant plusieurs rangées; il en est de même de la eorolle, qui manque quelquefois; les étamines sont monadelphes ou libres, en même nombre que les pétales, ou en nombre double ou triple. Les pistils souvent en grand nombre, libres ou soudés par leur côtéinterue, sont à une seule loge contenant un ou plusieurs ovules. Les fruits sont des espèces de petites drupes monospermes, obliques et eomme reniformes, eomprimées. La graine qu'elles contiennent se eompose d'un embryon reeourbé sur lui-mème et généralement dépourvu d'endosperme. 
HYPOPETALIE.

Les Ménispermées, qui se composent entr'autres des genres Menisperinum, Cocculus, Cissampelos, Abuta, Lardizabala, etc., sont assex rapprochées des Anonacées; mais elles s'en distingueut par leur port, qui est tont-à-fait différent, par leurs étamines, généralement en nombre défini, et la structare de lears fruits.

\section{QUATRE-VINGT-DODZIZ̀ME FAMILIE. :}

\section{Ochnacées. Ochnacece. DC.}

Végétaux ligneux très-glabres dans toutes leurs parties, ayant des feuilles alterues munies de deux stipules à leur base, des fleurs pédoneulées, très-rarement solitaires ou plus souvent disposées en grappes rameuses; leurs pédoneules sont artieulés vers le milieu de leur longueur. Elles ont un ealiee à einq divisions profondes, imbriquées latéralement avant leur développement; une eorolle de einq à dix pétales étalés, imbriqués lors de la préfloraison. Les étamines varient de einq à dix et même au-delà ; ayant leurs filets libres, insérés ainsi que les pétales au-dessous d'un disque hypogyne très-saillant, sur lequel est implanté l'ovaire. Celui-ci est déprimé à son eentre, et paraît formé de plusieurs pistils distinets rangés autour d'un style eentral, qui semble naître immédiatement du disque. Le style est simple et porte à son sommet un nombre variable de lanières stigmatifères. Le fruit se eompose des loges de l'ovaire qui se sont séparées les unes des autres, et qui forment autant de earpelles drupaeés, portés sur le disque ou gynobase (I), qui a pris de l'aeeroissement. Ces earpelles, dont plusieurs avortent quelquefois, sont uni-loeulaires, monospermes et indéhiscens; ils paraissent en quelque sorte artieulés sur le gynobase dontils se séparent facilement. Leur graine renferme uń gros embryon dressé dépourvud'endosperme.

(1) Voyez, page 2,55, la deseription de l'ovaire gynol asique. 
A cette famille se rapportent les genres Ochna, Gomphia, Walkera, ILeesia, ete. Elle a beaneoup d'affinité avec la famille des Rutacées, et plus particulièrement avec la tribu des Simaroubées, dont elle diffère par ses feuilles simples et munies de stipules, par ses graines dressées et ses earpelles indéhiseens; d'un autre côté, les Ochnacées se rapprochent des Magnoliacées, et en particulier du genre Drymis.

\section{QUATRE-VINGT-TREIZIEME FAMILIE.}

${ }^{*}$ rutucírs. Rutacea. Adrien de Jussiev.-Zygophyllece et Diosmeæ. Brown. Simarubece. Ricr.

Grande famille composée d'arbres, d'arbustes ou de plantes herbaeées cu fruteseentes, ayant des feuilles opposées ou alternes, très-souvent marquées de points translucides, avee ou sans stipules, des fleurs en général hermaphrodites, très-rarement unisexuées; un calice de trois à cinq sépales soudés par la base, une eorolle de einq pétales, quelquefois soudés ensemble et formant une eorolle speudo-monopćtale, plus raremeut nulle; einq ou dix étamines, dont quelques-unes avortent quelquefois et offrent des formes varićes; l'ovaire se eompose de trois à cinq earpelles plus ou moins intimement soudés, et formant autant de côtes plus ou moins saillantes. Chaque loge conticnt souvent deux, plus rarement un, ou un assez grand nombre d'ovules, insérés à leur angle interne, et y formant deux rangées; les styles sont libres ou soudés. Ces carpelles sont en général appliqués sur un disque hypogyne plus ou moins saillant, et quelquefois ils forment par leur réunion un ovaire gynobasique, dont le style semble naitre d'une dépression très-profonde de sa partic eeutrale. Le fruit est tantôt simple, formant une eapsule, s'ouvrant en autant de valves septifères qu'il y a de loges, tantôt et plus souvent il se sépare en autant de coqucs ou de earpelles, le plus souvent monospermes indéhiseens, et quelquefois légèrement eharnus, ou secs et s'ouvrant en deux valves incomplètes. Les graines dont le tégument propre 
est souvent crustaeé, se eomposent d'un endosperme eharnu ou eorné, contenant un embryon à radicule supérieure, rarement tournée vers le hile qui est latéral; quelquefois l'embryon est dépourvu d'endosperme.

Nous avons adopté la famille des Ratacées, telle qu'elle a été limitée par notre ami M. Adrien de Jussieu, dans son excellent travail sur cette $\mathrm{fa}_{\text {- }}$ mille. Il y a réuni comme de simples tribas: les Zygophyllées de M. Brown et les Simaroubées établies par mon père, et l'a divisée en cinq tribus naturelles, qui sont :

I ${ }^{\circ}$ Les Zygophy'Luḱks: fleurs hermaphrodites, loges de l'ovaire contenant denx ou plusieurs ovules; endocarpe ne se séparant pas du sarcocarpe, endosperme cartilagineux, fenilles opposées. Ex.: Tribulus, Fagonia, Guaiacum, Zjgophyllum, etc.

$2^{\circ}$ Les Rutérs: fleurs hermaphrodites; deux on plnsieurs ovules dans chaque loge; endocarpe ne se séparant pas du sarcocarpe; endosperme charna, feuilles alternes. Ex. : Ruia, Peganum, etc.

3० Les'Dısmérs: fleurs hermaphrodites, deux ou plusieurs oviules; endocarpe sc séparant du sarcocarpe. Ex. : Dictamnus, Diosma, Boronia, Ticorea, Galipea, etc.

$4^{\circ}$ Les Simaroubérs : fleurs hermaphrodites ou nnisexuées; loges à un senl ovale; carpelles distincts, indćhiscens; embryon sans endosperme. Ex.: Simaruba, Quassia, Simaba, etc.

$5^{\circ}$ Les Zaxtroxy LÉks: fleurs unisexuées; loges contenant de deux ì quatre ovules; embryon placé au centre d'un endospcrme charnu. Ex. : Galvezia, Aylanthus; Brucea, Zanthoxylum, Toddalia, Ptelea, etc.

Cette famille a beaucoup d'affinité avec les Ochnacées, surtout la section des Simaroubées, qui offre comme ces dernières un ovaire gynohasique; mais elle en diffère par ses graines renversées, ses feuilles composées, sans stipules, etc.

\section{QUATRE-VINGT-QUATORZILME FAMEIIE,}

Pitcosponées. Pitlosporece. R. Brown.

Arbrisseaux quelquefois sarmenteux et volubiles, à feuilles simples et alternes, sans stipules; à fleurs solitaires, fasciculćes ou disposées en grappes terminales. Leur calice esı monosépale, à eing divisions profondes; la corolle se compose de cinq pétales égaux, réunis et soudés par leur Jjase, de manière à former une corolle monopétale tubi- 
leuse et régulière, ou étalće et eomme rotacée; les einq étamines sont dressées, hypogynes, de même que la eorolle; l'ovaire est libre, élevé sur une espèce de disque hypogyne; il présente une ou deux loges, séparées par des cloisons ineomplètes, qui souvent ne se joignent pas au centre de l'ovaire, et de là l'uniloeularité de cet organe. Les ovules sont nombreux, attaehés sur deux rangées longitudinales et distinetes vers le milieu de la cloison. Le style est quelquefois très-eourt, terminé par un petit stigmate bilobé. Le fruit est une eapsule à unc ou deux loges polyspermes, s'ouvrant en deux valves; ou un fruit charnu et indéhiseent. Les graines se eomposent d'un tégument propre un peu crustacé, d'un endosperme blane et eliarnu, et d'un embryon extrêmement petit, plaeé vers le hile, et ayant sa radieule tournée vers ee point.

Les genres qui composent cette famille étaient placés auparavant parmi les Rhamnées; mais leur insertion hypogynique les en éloigne de beaucoup. M. de Candolle place les Pittosporées eutre les' Polygalées et les Trankéniacées; mais il nous semble que cette famille doit être mise auprès des Rutacées, dont elle se rapproche singulièrement par une, foule de earactères. Voici les genres principaux de cette famille : Pittosponun, Billardiera, Bursaria, Senacia, etc.

\section{QUATRE-VINGT-QUINZIEYTE FAMILLE.}

* génaniacées. Geraniacece. Aug. Sit-Himare. Geraniacere, Oxalideœ, Tropoolee et Linacece. DC. Balscminea. A. Rich.

Plantes herbacées oú soufruteseentes à feuilles simples ou rarement eomposées, alternes, avec ou sans stipules à leur base. Les fleurs sont axillaires ou terminales. Leur caliee est formé de einq sépales souvent inégaux et soudés ensemble par leur base, quelquefois prolongé en éperon; la corolle se compose de cind pétales égaux on inégaux, libres ou légerement cohérens entr'cux par leur 
base; ces pétales sont en général tordus en spirale avant leur épanouissement. Les étamines sont au nombre de cinq à dix, rarement sept; elles sont libres, ou plus souvent monadelphes par la base de leurs filets; leurs anthìres sont à deux loges. Les carpelles sont au nombre de trois à cinq, plus on moins intinemcut unis entr'eux; ils offrent chacun une seule loge, contenant un, deux, ou un plus grand nombre d'ovules attachés à leur angle interne. Les styles, qui naissent du sommet de chaque ovaire, restent distincts, ou se soudent entr'eux, et se terminent chacun par un stigmate simplc. Le fruit se compose de trois à cinq coques, contenant une ou deux graines, restant indéhiscentes, ou s'ouvrant par leur côté interue; ou bien c'cst une capsule à cinq loges polyspermes, s'ouvrant en cinq valves, quelquefois avec élasticice. Les graines, dont le tégument propre est quelquefois charuu ou crustacé extérieurement, se composent d'un embryon droit, ou plus ou moins recourbé, immédiatement recouvert par le tégument propre, ou placé daus un endosperme charnu.

Nous avons adopté l'opinion de M. Auguste de Saint-Hilairc, qui, dans sa Flore du Brésil méridional, réunit en une seule les fumilles des Oxalidées, des Troprolées, des Linaeées et des Géranineées du professeur de Candolle. Ces diverses familles, ainsi que celle que nous avions établie sous le nom de Batsaniraties, ne forment en effet que des tribus d'ane même famille, qui doit conserver le nom de Géraniacées.

$\mathrm{I}^{\circ}$ OxaldDÉEs, feuilles ordinairement eomposées, sans stipules; fleurs axillaires; capsule à cinq loges polyspermes, styles distinets; embryon droit dans un end osperme charnu. Ex. : Oxalis.

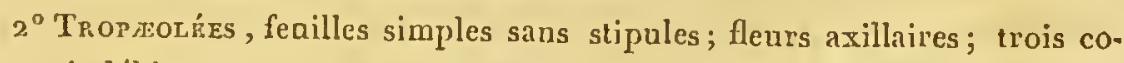
ques indébiscentes et monospermes; cmbryon sans endosperme: Tropocolum.

30 Thasamixées, feuilles simples, sans stipules; fleurs irrégulières; point de style; eapsule à einq loges polysperines, s'ouvrant avee élastieité; embryon sans endosperme: Bulsainina.

$4_{4}^{\circ}$ LrxacÉrs, fenilles simples sans stipules; fleurs terminales régulières; trois on cint styles distincts; capsule à cinq loges dispermes; endosperme mince, : Linum. 
$5^{\circ}$ GÉRantées, feuilles simples, wunies de stipules; flears opposées aux feuilles; styles soudés; coques indéhiscentes, et embryon généralement saus endosperme : Geranium, Erodium, Pelargonium, Monsonia.

\section{QUATRE-VINGT-SEIZIÈME FAMIIIE.}

* Malvacées. Malvacece. Kunth. Malvacearum. Juss.

Cette famille renferme à la fois des plantes herbacées, des arbustes et même des arbres à feuilles simples alticrnes, ou lobćes, munies de deux stipules à leur base. Les fleurs sont axillaires, solitaires, ou diversement groupécs, et formant des espèces d'ćpis. Le calicule esı souvent accompagné extérieuremeat d'un calicc formé de folioles variables en nombre, ct diversemcnt soudécs. Le calice est monosćpale, à trois ou cinq divisions, rapprochées en forme de valves avant leur épanouisscment. La corolle se compose généralement de einq pétalcs alternes avec les lobes du calicc, contournés en spirale avant leur déroulcment, souvent réunis cnsemble à leur base, au mojen des filets stamincux, de manièrc quc la corollc tombc d'unc pièce. Les étamines sont généralcmcut très-nombreuses, rarement en même nombre ou cn nombre double des pétales. Leurs filcts sont réunis, et forment un tube, ct leurs anthc̀res réniformes, et constamment uniloculaires. Le pistil sc composc de plusieurs earpelles, tantôt verticillés autour d'un axe central, et plus ou moins soudćs entr'eux, tantôt réunis cn unc sorte de capitule; ces carpclles sont uniloculaircs, contcnant un, deux ou um plus grand. nombre d'ovules attachés à leur angle interne. Les styles sont distinets, ou plus ou moins soudés, et portent chacun un stigmatc simple à lcur sommet. Lc fruit préscnte les mêmes modifications que les carpclles, e’cst-ìdirc que ceux·ci sont tantôt réunis circulairement autour d'un axe matériel, tantôt réunis cn tète, ou formant, par leur soudure, une capsulc pluriloculaire, qui s'ou- 
vie en autant de valves qu'il y a de loges mońospermes ou polyspermes; d'autres fois les earpelles s'ouvrent seulement par leur eòté interne. Les graines, dont le tégument propre est quelquefois chargé de poils cotonneux, se composent d'un embryon droit, généralement sans endosperme, ayant les eotylédons foliacés, repliés sur eux-mêmes.

La famille des Malvacées, telle qu'elle est aujourd'hui limitée par les botanistes, ne contient qu'une partie des genres qui y avaient d'abord été réanis par M. de Jussieu. Ventenat a d'abord séparé des Malvacéesle genre Sterculia, dont il a formé le type des Sterculiacćes. M. Rob. Brown considère les Malvacées, non comme une famille, mais comme une grandc trihu ou classe qui se compose des Malvacées de Jussieu, des Sterculiacées de Ventenat, des Chlénacées de Dupetit-Thouars, et des Tiliacées de Jussieu, et une nouvelle famille qu'il nomme Byttnćriacées. Notre savant ami, M. le prof. Kunth, ne place dans les Malvacées que les trois premières sections de Jnssieu, il adopte les Byttnériacées de M. Rob. Brown, et y rénnit les Sterculiacées dc Ventenat; enfin il forme une famille nouvelle sous le nom de Bombacées des genres Bombax, Cheirostemon, Pachira, Helicteres, Cavanillesia, Matisia, et Chorisia.

Ainsi définie, la famille des Malvacées se distingue surtout par ses pétales simples, ses anthères constamment uniloculaires et ses graines généralement sans endosperme. Parmi les genres qui la composent, nous citerons les snivans: Malope, Malva, Althoea, Lavatera, Hibiscus, Gossipium, Palava, Lagunea, etc.

\section{QUATRI- VINGT-DIX-SEPMIEME FAMTLIE.}

bonbacées. Bombacea. Kunth.

Ce sont des arbres ou des arbrisseaux, originaires des contrées intratropieales, ayant des feuilles alternes, simples ou digitées, munies à leur base de deux stipules persistantes. Le calice, quelquefois accompagné extérieurement de quelques braetées, est monosépalc, à cinq divisions imbriquées avant leur épanonissement, quelquefois entier; Ia corolle, qui manque quelquefois, se compose de cinq pétales réguliers. Les étarnines, au nombre de einq, dix, quinze ou davantage, sont monadelphes par leur base, et forment supérieurement cinq faisceaux, qui 
portent chacun une ou plusieurs anthères uniloculaires. L'ovaire est formé de cinq carpelles, tantôt distinets, tantôt soudés entr'eux, et terminés chacun parun style et un stigmate, qui quelquefois se soudent en un scul. Les fruits sont en général des capsules à einq loges polyspermes, s'ouvrant en einq valves, ou ils sont coriaces, eharnus intérieurement, et restent indéhiscens. Les graines, souvent environnées de poils ou de duvet, ont tantôt un endosperme eharnu, recouvrant un embryon, dont les eotylédons sont planes ou chiffonnés. L'endosperme manque quelquefois.

Cette famille, trés-voisine de la précédente, en diffère surtout par son calice entier ou dont les lobes ne sont pas appliqués en forme de valves avant leur épanouissement, par leurs filets disposés en cinq faisceaux et la structure de leur frnit.Les genres qui la composent sont: Bornbax, Helicteres, Matisia, Cavanilesia, Adansonia, etc.

\section{QUATRE-VINGT-DIX-ZIUITIÈME FAMIIIE.}

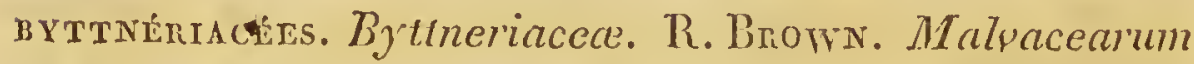
gen. et IIermannice. Juss. Sterculiacece. Vent.

Arbres ou arbrisseaux à feuilles alternes, simples, munies de deux stipules opposées; fleurs disposées en grappes plus ou moins rameuses axillaires or opposées aux feuilles. Le calice, nu ou aecompagné d'un calicule, est formé de cinq pétales plus ou moins soudés par leur base, et valvaires; la eorolle, de cinq pétales planes, roulés en spirale avant leur épanouissement, ou plus ou moins eoueaves et irréguliers; ees pétales mauquent quelquefois. Les étamines, en même nombre, ou double ou multiple des pétales, sont en général mouadelphes, et le tube qu'elles forment par leur réunion, préseńte souvent des appendiees pétaloïdes, placés entre les étamines anthérifères, et qui sont autant d'étamines avortécs. Les anthères sont constamment à deux loges. Les earpelles, au nombre de 
trois à cinq; sont plus ou moins complétement soudés. Chaque loge renferme deux ou trois ovules ascendans, ou un plus grand nombre, attachés à l'angle interne de chaque loge. Les styles restent libres, ou sont plus ou moins soudés entr'eux. Le fruit est en général une capsule globuleuse, accompagnée par le calice, à trois ou cinq loges, s'ouvrant en autant de valves, qui souvent portent la cloison sur le milien de leur face interne. Les graines offrent dans un endosperme charnu un embryon dressé.

Cette famille, qui se distingue surtout des Malvacées par ses anthères à deux loges, et ses graines en général inunies d'un endosperme charnu, a été partagée en six sections ou tribus naturelles, savoir :

$\mathrm{I}^{\circ}$ Les Strrcuriacézs, fleurs souvent unisexuées, calice na, pas de corolle; ovaire pédicellé, formé de cinq carpelles distincts; l'endosperme manque quelquefois. Ex. : Sterculia, Triphaca, Heritiera.

20 Les BytrnérréEs, les pétales sont irréguliers, concaves, souvent terminés à leur sommet par une sorte de ligule; les étamines sont monadelphes, l'ovaire est ì cinq loges, contenant en général deux ovules dressés : Theobroma, Abroma, Guazuna, Lyetneria, Ayenia, etc.

3o Les Lastopétatées, calice pétaloide; pétales très-petits en forme d'écailles, ou nuls; ovaire à trois ou cinq loges, contenant chacunc de deux à huit ovales : Seringia, Thomasia, Keraudrenia, etc.

$4^{\circ}$ Les Hermanirés, fleurs hermaphrodites, calice tuboleux, corolle de cinq pétales planes; roulés en spirale avant leur épanouissemcnt; cinq étamines monadelphes ou libres, opposées aux pétales, loges polyspermes : \#1elochia, Hermannia, Mahernia, etc.

5o Les Dombzxacḱzs, calice monosćpale, corolle de cinq pétales planes, étamines égales, nombreuses et monadelphes; ovaire à trois ou cinq loges; contenantdeux ou un plus grand nombre d'ovules: Ruizia, Dombeya, Pentapetes, etc.

$6^{\circ}$ Les Waltichíss, calice environné d'un involucre de trois à cing fojioles, pétales planes; étamines très-nombreuses, monadelphes inégales et formant une colonne analogue à celle des Malvacécs: Eriolcena, Wallichia, Grethea. 


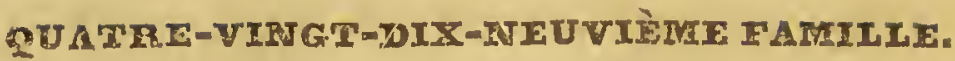

\section{chlénactes. Chlonacece. Dupetit-Thouars.}

Cette pelite famille se eompose d'arbrisseaux, tous originaires de l'île de Madagasear. Leurs feuilles sont alternes, munies de stipules, entières et eaduques. Les fleurs forment des grappes rameuses. Ces fleurs sont munies d'involueres persistans, qui eontiennent une ou denx fleurs. Leur ealice est petit, formé de trois sépales : les pétales varient de çinq à six; ils sont sessiles, ei quelquefois réunis par leur base. Les étamines, au nombre de dix, ou en nombre indéterminé, monadelphes par leurs filets, quelquefois eohérentes entr'elles par lcurs anthìres. L'ovaire est à trois loges, surmonté d'un style simple, et d'un stigmate trifide. Le fruit est une eapsule à trois, rarement à une seule loge par avortement, contenant ehaeune unc ou plusicurs graines, insérées à leur angle interne et pendantes. Ces graines eonticnnent uin embryon axile dans un endosperme eharnu ou eorné.

Les Chlénacées, cómposées des genres Sarcolana, Lepsolana, Schizolana et Rhodolana, ont été rapprochées des Malvacées par M. Dupetit-Thouars, à cause de leur calieule et de leurs étamines monadelphes, ete.; et par M. de Jussieu des Ébénacées, à cause de leurs pétales soudés et formant une sorte de eorolle monopétale, et de quelques autres earactères.

\section{CENTIEMT FAMITE.}

* tilincées. Tiliacece. Juss. Tiliacece et Elcoocarped. Juss.

Presque toutes les Tilincées sont des arbres ou des arbrisseaux, un petit nombre des plantes herbacées. Elles portent des feuilles altemes simples, accompaguées à leur base de deux stipules caduques. Leurs fleurs sont axillaires pédonculées, solitaires on diversement groujpées. 
Elles ont un calice simple, formé de quatre à cinq sépales, rapprochés en forme de valves avaut l'épanouissement de la fleur; une corolle d'un même nombre de pétales, qui manquent rarement, et soat souvent glanduleux à leur base. Les étamines sont en grand nombre, libres, et ont leurs anthères biloculaires; on trouve souvent en face de chaque pétale une glande pédicellée. L'ovaire présente de deux à dix loges, contenant chacune plusieurs ovules attachés sur deux rangs à leur angle interne. Le style est simple, terminé par un stigmate lobé. Le fruit est une capsule à plusieurs loges, contenant plusieurs graines et quelquefois indéhiscente, ou une drupe monosperme par avortement. Les graines contiennent un embryon droit ou un peu recourbé, dans un endosperne charnu.

Nous réunissons à cettc famille celle des Éléocarpées de M. de Jnssieu, qui n'en diffère que par deux caractères dc peu d'importance, savoir : des pétales frangés à leur sommet, et des anthères s'ouvrant seulement par deux pores. Nous en faisons une simple tribu des Tiliacées, que nous divisons en deux sections, savoir :

to Les Trliacées vraies, comprenant les genres Tilia, Sparmannia, Heliocarpus, Corchorus, Triumfetta, Apeiba, etc.

$2^{\circ}$ Les Elmocarrées, dans lesquelles sont les genres Elococarpus, Vallea, Decadia, etc.

Les Tiliacées ont de l'affinité avec les Malvacées, dont elles diffèrent par leurs étamines libres et leur embryon placéau centre d'un endosperme charnu; avec les Byttnériacées, dont elles se distinguent par leurs étamiues libres et nombreuses; leur style simple, etc.

\section{CENT UNIÈMT FAMILLE.}

ternstroenináés. Ternstromiacece. - Ternstromiaco et Theacece. Mirbel.

Arbres ou arbrisseaux, à feuilles alternes, sans stipules, souvent coriaces et persistantes; à fleurs quelquefois trèsgrandes, axillaires et terminales, ayant un calice formé de cinq sépales concaves inégaux, et imbriqués; une corolle composée de cinq pétales, quelquefois soudés à 
leur base et formant une corolle monopétale; des étamines nombreuses, souvent réunies par la base de leurs filets, et soudés avee la corolle. L'ovaire est libre, sessile, le plus généralement appliqué sur un disque hypogyne, divisé en deux à einq loges, eontenant ehaeune deux ou un plus grand nombre d'ovules pendans, à l'angle interne de chaque loge. Le nombre des styles est le même que celui des loges; ils se termincht chaeun par un stigmate simple. Le fruit offre de deux à cinq loges; il est tantôt eoriace indéhiseent, un peu eharnu intérieurement; d'autres fois il est see, eapsulaire, s'ouvrant en autant de valves. Les graines, souvent au nombre de deux seulement dans ehaque loge, ont leur embryon nu ou recouvert d'un eridosperme charnu souvent très-minee.

Nous arons eru devoir réunir les deux familles établies par M. le professeur Mirhel sous les noms de Théacées et de Ternstromiacées; ces deux familles en effet ne different pas sensiblement l'une de l'autre. Elles sont formées des genres T'ernstromia, Thea, Cancllia, Freziera, ete., qui avaient été placés dąns la famille des Aurantiées, dont ils diffèrent par leur ealico, la plaralité des styles, pár l'absence des points translucides, et par un endosperme, qui manque uéanmoins quelquefois. D'un autre côté, eette famille a quelques rapports avec celle des Ebénacées, placée parui les Monopétales. Mais en général, elle mérite d'être étudiée de noaveau, et nous attendons avec impatienee le travail que notre ami M. Cambessèdes prépare sur ce groupe et celui des Gattifères.

\section{CEAT DEUXEATE FAMTTLE.}

olacinées. Olacinece. Mrreel.

Cette petite famille formée aux dépens des aurantiacées, se eompose de végétaux ligneux, portant des feuilles simples, alternes, pétiolćes, sans stipules, des fleurs trèspetites et axillaires. Celles - ei se composent d'un caliee très-petit monosépale, persistant, entier ou denté, prenant souvent beaucoup d'aceroissement et devenan t charnu. La corolle est formée de trois à six pétales, coriaces, 
sessiles, val vaires, libres ou soudés par leur base. Ces pétales, qui portent quelquefois les étamines, sont réunis souvent deux à deux, et seulement séparés à leur sommet. Les étamines sont en général au nombre de dix, dont plusieurs avortent quelquefois, et existent sous la forme de filamens stériles. Ces étamines sout immédiatement hypogynes ou portées sur les pétales. L'ovaire est libre, à une seule loge, contenant en général trois ovules, qui sont pendans au sommet d'un podosperme central et dressé. Le style est simple, terminé par un stigmate très-petit et trilobé. Le fruit est drupaeé, iudéhiscent, souvent recouvert par le caliec devenu charnu, et contenant une seule graine. Celle-ci se compose d'un gros endosperme charnu, dans lequel est renfermé un petit embryon basilaire et homotrope.

Composée des genres Olax, Fissilia, etc., cette petite famille est très-distinctc dcs Aurantiacées par ses feuilles non ponctuées, ses étamines définies, son ovaire constamment uniloculaire et son embryon contenu dans un trèsgros endosperme.

Selon le célèbre Rob. Brown, le gcnre Olax serait apétale, c'est-à-dire que sa fleur aurait un involucre calyciforme et un calice formé de trois sépales; et à cause de la structure intérieure dc son ovaire, ce genre devrait ètre rapproché des Santalacées.

\section{CENT TROISTÈTE FAMILIE.}

\section{marcgraviaches, Mlarcgraviacece, Choisy.}

Arbrisseaux très-souvent sarmenteux et grimpans, parasites à la manière du lierre, ayant les feuilles alternes, simples, entières, eoriaces et persistautes; les fleurs généralement disposées en un épi court et en forme de eyme. Ces fleurs, longuement pédonculées, sont quelquefois obliques au sommet de leur pédoneule, qui porte assez généralement une braetće irrégulière, creuse et cuculliforme ou en cornet. Ces fleurs sont hermaphrodites, ayant un ealice de quatre à six ou sépt sépales, courts, imbri- 
qués, et généralement persistans; la corolle est monopétale, cntic̀re, s'cnlevant comme unc sorte de coiffe, ou formée de einq pétales sessiles. Les étamines, généralement en grand nombre (cinq seulement dans le Souyoubea) ont leurs filets libres. Liovaire est globulcux, surmonté d'un stigmate sessile et lobé cn étoile, qui est rarement porté sur un style; il présente une seule loge qui offre de quatre à douze trophospermes pariétaux, saillans en forme de deni-cloisons, divisés par leur bord libre en deux ou trois lames diversement contournées, et toutes couvertes d'ovules fort petits. Le fruit est globuleux, coriace, charnu intérieurement, indéhiseent, ou se vompant irrégulièrement en un eertain nombre de valves, dont la déhiscenee se fait de la base vers le sommet, et qui portent chacune un trophosperme sur le milieu de leur face interne. Les graines sont très-petites, et contiennent immédiatement sous leur tégument propre l'cmbryon, qui est homotrope.

Les genres qui eomposent cette famille sont Marcgravia, Antholoma, Noranthea et Souroubea. Ce groupe a des rapports avee les Guttifères; mais il en a aussi de'très-intimes avee les Bixinées et les Flacourtianées, qui ont également une corolle polypétale et des étamines indéfinies, un fruit uniloeulaire et des trophospermes pariétaux. Mais dans ees deux dernières familles les fenilles sont aecompagnées de stipules et l'embryon est recouvert par un endosperme:

\section{CENT QUATIIEMI FAMILIE.}

Guttifìnes. Guttiferce. Juss.

Cctte famille se compose d'arbres ou d'arbrisseaux; quelquefois parasites, et tous remplis de sues propres jaunes et résineux; leurs fcuilles, opposées, ou plus rarcment alternes, sont coriaecs et persistantes; lcurs deurs, disposécs en grappes axillaires ou en panicules terminales, sont hermaphrodites ou unisexućes ct polygames; leur calice est persistant, formé de deux à six sépales 
arrondis, souvent colorés; la corolle est composée de quatre à dix pétales, les étamines, très-nombreuses, rarement en nombre défini, libros; l'ovaire, simple, surmonté d'un style court, qui manque quelquefois et qui porte un stigmate pelté et radié ou à plusieurs lobes. Le fruit est tantôt capsulaire, tantôt charnu ou drupaeé ; s'ouvrant quelquefois en plusieurs valves, dont les bords, généralement rentrans, sont fixés à un plaeenta unique ou à plusieurs placentas épais. Les graines se composent d'un embryon homotrope sans endosperme.

Les Guttifères comprennent an assez grand nombre de genres, tous exotiques; tels sont les Clusia, Godoya, Mahurea, Garcinia, Calophyllum, etc. Elles diffèrent surtout des Hypéricinées par leurs étamines complétement li. bres, lear suc propre laiteax, l'absence des points translucides, etc,

\section{CENT CHTOUTEME FAMILIE.}

\section{* hypénicinées. Hypericinece. Juss.}

Plantes herbacées, arbustes ou même arbres souvent résineux et parsemés de glandes transparentes, ayant des feuilles opposées, très-rarement alternes, simples; les fleurs sont axillaires ou terminales, diversement grouppées. Leur caliee est à quatre ou cinq divisions très-profondes, un peu inégales; la corolle se compose de quatre à cinq pétales, roulés en spirale avant leur évolution. Les étamines sont très-nombreuses, réunies en plusieurs faisceaux par la base de leurs filets, quelquefois monadelphes ou libres. L'ovaire est libre, globuleux, surmonté de plusieurs styles, quelquefois réunis et soudés en un seul; il offre autant de loges polyspermes que de styles. Le fruit est une capsule, ou une baie à plusieurs loges polyspermes. Dans le premier cas, elle s'ouvre en autant de valves eontinues, par leurs bords avec les cloisons, qu'il y a des loges. Les graines, très-nombreuses et très-petites, contiennent un embryon homotrope sans endosperme. 
Cette famille, composée d'un petit nombre de genres, tels que Hypericun! Androscemum, Ascyrum, Vismia, etc., porte aussi le nonm de Millepertuis, parce que la plupart des espèces présentent dans l'épaisseur de leurs feuilles des glandes miliaires transparentes, qui vues entre l'œil et la lumière semhlent être autant de petits trous. Ce caractère, joint "i celui des étamines très-nombreuses, aux loges du fruit polyspermcs, distinguent parfaitement les Hypéricinées des autres familles voisines.

\section{CENT SIXIEME FAMITXE.}

* aurantiacées. Aurantiacece. Connéa. Airaníorum ge-
nera. Juss.

Arbres ou arbrisseaux très-glabres, quel quefois épineux, portant des feuilles alternes et artieulées, simples, ou plus souvent pinnées, munies de glandes vésieuleuses, remplies d'une huile volatile, transparente; des fleurs odorantes, généralement terminales. Leur ealiee est monosépale, persistant, à trois ou einq divisions plus ou moins profondes; leur eorolle, de trois à einq pétales sessiles, libres ou légèrement soudés entr'eux; les étamines, quelquefois cn même nombre que les pétales, ou doubles ou multiples de ee nombre, sont libres, ou diversement réunies entr'elles par leurs filets, et sont attachées audessous d'un disque hypogyne, sur lequel est appliqué l'ovaire. Celui-ei est globuleux, à plusieurs loges contenant un seul ovule suspendu, ou plusieurs ovules attachés à l'angle interne de la loge. Le style, quelquefois très-eourt et très-épais, est toujours simple, terminé par un stigmate diseoïde, simple on lobé. Le fruit est en général eharnu intérieurement séparé en plusieurs loges par des cloisons membraneuses très - minees, eontenant une ou plusicurs graines insérées à leur angle interne, et généralement pendantes. Extérieurement, le périearpe est épais et indéhiseent, rempli de vésieules pleines d'huile volatile. Les graines renferment un, quelquefois plusieurs embryons sans endosperme. 
HYPOPETALIE.

Los genres qui composent cette famille se distinguent surtout par leurs feuilles articulées, sonvent composées, munies de glandes vésiculcuses, qui existent aussi dans l'épaisseur de leurs pétales et de leur péricarpe, par leur style simple et leurs graines sans endosperme. Exemples : Eitrus, Limonia, Murraya, etc.

\section{CENT SEPTIEME FAMIMLE.}

* ampélidées. Ampelidece. Rich. Vites. Jurss.

Arbustes ou arbrisseaux volubiles, sarmenteux et munis de vrillcs opposées aux fcuilles. Celles-ci sont alternes, pétiolécs, simples ou digitées, munies à lcur base de deux slipules. Les fleurs sont disposées en grappes opposées aux feuilles. Le calice èst très-court, souvent entier et presque plane; la corolle, de cinq pétales, quelquefois cohércns entre cux par leur partie supérieure, et s'enlevant tous ensemble en forme de coiffe. Les étamines, au nombre de ciụ, sont dressécs, libres et opposées aux pétales; l'ovaire est appliqué sur un disque hypogyne annulaire et lobé dans son contour; il offre constamnent deux logcs, contenant chacune deux ovules dressés; le style, qui est épais et très-court, sc terminc par un stigmate à peine bilobé. Le fruit est unc baie globuleuse, contenant d'une à quatre graines dressées, ayant leur épisperme épais, leur endosperme corné, ct contenant vers sa basse un très-petit embryon dressé.

Cette petite famille, composée des genres Vitis, Cissus et Ainpelopsis, est très-distincte par ses fenilles munies de stipules, par ses vrilles opposées aúx fenilles, ses ćtamines opposćes anx pétales, et la structure de son fruit et de sa graine.

\section{CENT EUITIXYHE TAKIIEE.}

miprocraticécs. Hippocralicece. Juss. Hippoctaléacées. Kunth. DC.

Arbustes ou arbrisscaux généralement glabres ct sarinenteux, portau t des feuilles opposées, simples, coriaces, 
entières ou dentées; des fleurs petites, axillaires, fascieulées ou en eorymbes. Leur calice est persistant, à cinq divisions; leur corolle se eompose de einq pétales égaux; les étamines sont généralement au nombre de trois, rarement quatre ou einq, ayant leurs filets réunis par leur base, et formant un androphore tubuleux. L'ovaire est trigone, à trois loges contenant ehaeune quatre ovules attaehés à leur angle interne. Le stylc est simple, terminé par un ou trois stignates. Le fruit est tantôt eapsulaire à trois angles membraneux, tantôt eharnu; ehaque loge contient en général quatre graines. Celles-ei ont un embryon dressé, dépourvu d'endosperme.

Cette famille, composée des genres Ilippocratea, Anthodon, Raddisia, Salacia, etc. , est voisine des Acérinées et des Malpighiacées.

\section{CENT NEUVIÈME FAMTIL.}

* acérintes. Acerinece. DC.

Familleuniquement eomposée du genréérable (acer.L.) et offrant les earactères suivans : fleurs hermaphrodites, ou uniscxuées, ealiee à einq divisions, plus ou moins profondes, ou cntier; eorolle de cinc pétales, étamines en nombre double des pétales, insérées sur un disque hypogyne, qui oceupe tout le fond de la flcur; ovaire didyme et comprimé, à deux loges, eontenant ehaeune deux ovules, attaehés à l'angle intcrne; style simple, quelquefois tròs-court, terminé par deux stigmates subulés. Le fruit se eompose de deux samares, indéhiseentes, prolongées en ailes d'un eôté. Les graines offrent sous leur tégument propre un embryon roulé en spirale.

Les Acérinées sont des arbres à fcuilles opposées, simples ou pinnées; ct ì fleurs disposćes en grappes ou en cimes teriuinales. Lilles ticnnent en qucl que sorte le unilieu entre les Malpighiacćes, dont clles diffèrent surtout par leurs fruits membraneux, ailćs et senlement à denx loges, et les Hippocastanćes.

La famille des Acérinées, telle qu'elle avait étć établie par Jussieu, con- 
tenait plusienrs autres genres ; tels sont l' Zisculus, qni formc la famille des Hippocastanćes de M. de Candolle, et qui nous parait appartenir à celle des Malpighiacces, et l'Hippocratea; celle des Hippocraticées.

\section{[CENT DIXIEMIE FATRLI:}

\section{MILPIGHIAGÉEs. Míalpighiacece. Juss.}

Famille eomposée d'arbres, d'arbrisseaux ou d'arbustes, à feuilles opposées simples ou eomposées, souvent munies de poils en forme de navette (pili malpighiacei), aceompagnées souvent à̀ leur base, de deux stipules. Fleurs jaunes ou blanches formant des grappes, des eorymbes ou des sertules axillaires ou terminales. Les pédieelles qui supportent les fleurs sont souvent articulés et munis de deux petites braetées vers leur partic moyenne. Leur ealice est monosépale, souvent persistant, à quatrè ou cinq divisions profondes; leur corolle, qui manque quelquefois, se compose de einq pétales longuement ongui'culés. Les étamines, au nombre de dix, rarement moins, sont libres ou lég’èrement soudées par la base. Le pistil est tantôt simple, tantôt formé de trois carpelles, plus ou moins soudés entr'eux. Chaque carpelle ou chaque loge contient, soit un seul ovule suspendu à la partie supérieure de l'angle interne, soit deux ovules attachés à eet angle. Lcs styles, au nombre de trois, sont quelquefois soudés. Le fruit, qui est sec ou charnu, se compose de trois carpelles distincts, ou forme une capsule ou un nueulaine à trois, rarement à deux ou à une seule loge. La capsule est ordinairement relevée d'ailes membraneuses, très-saillantes, ou de pointes épineuses. Le nuculaine renferme tantôt trois nucules uniloeulaires, tantôt un noyau, à trois loges monospermes. Chaque graine se compose d'un tégument propre peu épais, recouvrant immédiatement un embryon un peu reeourbé.

Ctte famille, dans laquelle on trouve cntr'aatres les genres Malpighia, Eyrsonima, Irplage, Caudichaudia, Banisıeria, etc., est voisine des 
Acérinées et des Hypéricées. Elle se distingue des premiers par ses pétales longuement onguiculés, ses étamines monadelphes et les loges de son fruit tonjours monospermes; des secondes, par ses étamines définies, ses loges monospermes, ete. Nous réunissons ici le genre Zisculus, qui forme la famille des Hippocastanées de IM. de Candolle.

\section{CENT ONZIENE FAMIIUE.}

Ĺrythroxyuées. Erythroxylece. Kunth.

Arbres ou arbrisseaux à feuilles alternes ou opposécs, généralement glabres, munies de stipules axillaires. Les fleurs sont petites, pédiecllées, ayant un ealiee persistant à cinq divisions profondes; une corolle de einq pétales, sans onglet et munis intéricurement d'une petite éeaille. - Les étamines, au nombre de dix, sont monadelphes. L'ovaire est uniloculaire, contenant un seul ovule pendant, ou bien il est à trois loges, dont deux sont vides. De l'ovaire naissent trois styles, tantôt distincts, tantôt soudés presque jusqu'à leur sommet. Le fruit est une drupe monosperme, contenant une graine anguleuse, dont l'endosperme, dur et corné, contient un embryon axile et homotrope.

Cette petite famille ne se compose que dn genre Erythroxylum, placé jadis parmi les Malpighiacées et d'un genre nouvean établi par M. Knnth, sous le nom de Sethia. Elle diffère des Malpighiacces par ses pétales appendiculés, son fruit monosperme et son embryon, muni d'un endosperwe.

\section{CENT DOUXIIAE FAMTUE.}

Mémincérs. Meliacea. Juss. - DC. Cedrelea.R. Brown.

Arbres ou arbrisscaux ì feuilles alternes sans stipules, simples ou composées, ì fleurs tantôt solitaires et axillaires, tantôt diversement grouppées en épis ou en grappes, ayant un calice monosépale, à quatre ou einq divisions plus ou moins profondes; une corolle de quatre 
à einq pétales valvaires; des étamines généralement en nombre double des pétales, rarement en même nombre ou cnnombreplus eonsidérable. Ces étamines sont toujours monade]phes, et leurs filets forment un tube, qui porte les anthères tantôt à son sommet, tantôt à sa face interne. L'ovaire est porté sur un disque hypogyne et annulaire; il offre quatre à einq loges, eontenant généralement deux ovules eollatéraux et superposés. Le style est simple, terminé par un stigmate plus ou moins profondément divisé en quatre à einq lobes. Le fruit est tantôt see, capsulaire, s'ouvrant en quatre à einq valves septifères; tantôt il est eharnu et drupaeé, et parfois uniloeulaire par suite d'avortement. Les graines se eomposent d'un embryon, quelquefois enveloppé d'un endosperme minee ou eharnu, qui manque dans d'autres genres.

Les genres Ticorea et Cusparia, d'abord placés dans cette famille, ont été transportés par M. Robert Browin dans les Rutacées. Le même botaniste a formé des genres Cedrela et Sivictenia nnc famille distincte sous le nom de Cédrécress. Mais le professeur de Candolle en a simplement fait une tribu des Méliacées. Cette famille se divise en deux tribus naturelles; sayoir :

Io Mŕuracées vraies, loges da fruit contenant une ou deux graines sans ailes ni endosperme; embryon rcnversé; cotylédons planes et foliacés, on épais ct charnus. Ex. : Geruma,Huniria, Turrcea, Quivisia, Strigilia, Sandoricum, Melia, Trichilia, Guarea, etc.

$2^{\circ}$ CÉDréLées, loges du fruit polyspermes, graines généralement ailées, munies d'un endospermc charnu, embryon dressé, cotylédons foliacés. Ex. : Cedrela, Swictenia, etc.

Cette famille, voisine des Sapindacées et des Ampélidées, en diffère par ses étamines constamment monadelphes, et la structure de son fruit.

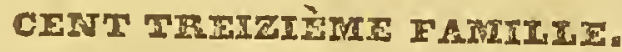

SAPIndACÉEs. Sapindacece. Juss.

Cette farnille se compose de grands arbres ou d'arbustes, quélquefois de plantes herbacées et volubiles, portant des feuilles alternes et généralement imparipinnćes, munies quelquefois de vrilles. Leur ealiee se com-. 
pose de quatre à cinq sép̧ales, libres, ou légèrement soudés par leur base. La corolle, qui manque quelquefois, est formée en général de quatre à cinq pétales, tantôt nus, tantôt glanduleux vers leur partie moyenne, où ils portent quelquefois une lame pétaloïde. Les étamines, en nombre double des pétales, sont libres et appliquées sur un disque hypogyue, plane, lobé, qui garnit tout le fond de la fleur. L'ovaire est à trois loges, contenant en général deux ovules superposés et attachés à l'angle interne de chaque loge. Le style, simple à sa base, est trifide à son sommet, qui se termine par trois stigmates. Le fruit est une capsule quelquefois vésiculeuse, à une, deux ou trois loges, contenant chacune une seule graine. Les graines se composent d'un gros embryon ayant sa radicule recourbée sur les cotylédons, et dépourvu d'endosperme.

Cette famille a été diviséc en trois trihus de la manière suivante:

I ${ }^{0}$ Pauririnéres, pétales appendiculés, disque formé de glandes distinetes, placées entre les pétales et les étamines; ovaire à trois loges monospermes; herbes où arbustes volubiles, munis de vrilles. Ex.: Cardiospermum, Urvillea, Serjania, Paullinia.

$2^{\circ}$ Sarrindées, pétales non appendiculés, mais glanduleux ou larbus, rarement nus; disque annulaire, ou quelquefois glandes soudées entre elles; ovaire à deux ou trois loges monospermes; arbres ou arbrisseaux non volubiles. Ex. : Sapindus, Talisia, Schmidelia, Euphoria, Thoninia, Cupania, ete.

$3^{\circ}$ Dodoréacées, pétales munis d'une écaille à leur base; ovaire à deux ou trois loges, contenant deux ovules; péricarpe vesiculeux ou ailé; cmbryon ayant ses cotylédons roulés en spirale. Ex. : Kolreuteria, Dodoncea, ete.

\section{CENT QUATORZIEME FAMITIE.}

* polygalées. Polygalece. Juss.

Nous trouvons dans cette fümille des plantes herbacées, ou des arbustes, ì feuilles alternes, simples et entières, à fleurs solitaires, axillaires ou cn épis. Chacune se 
HYPOPETALIE.

compose d'un calice de quatre à cinq sépales, imbriqués latéralement avant l'épanouissement de la fleur, et dont deux, quelquefois plus intérieurs, sont pétaloïdes et colorés; la corolle est formée de deux à cinq pétales, tantôt distincts, tantôt réunis ensemble par le moyen des filets staminaux, qui forment un tube fendı d'un côté. Les étamines, généralement au nombre de huit, sont monadelphes; leur androphore est divisé supérieurement en deux phalanges, portant chacune quatre anthères uniloculaires, et s'ouvrant en général par leur sommet. Plus rarement les étamines sont au nombre de deux à quatre et libres. L'ovaire est quelquefois accompagné à sa base par un disque hypogyne et unilatéral, ou formé de deux appendices latéraux et lamelleux; il offre deux, plus rarement une ou trois loges, contenant chacume un ou deux ovules. Le style est long, ordinairement recourbé, et portant un stigmate creux, bilobé ou unilatéral. Le fruit est une capsule ou une drupe. Dans le premier cas, il est à deux loges monospermes, et s'ouvre en deux valves septifères; dans le second cas, il est uniloculaire, monosperme et indéhiscent. Les graines sont pendantes, en général accompagnées d'une sorte de caroncule ou d'arille de forme variée. Leur embryon est tantôt placé dans un endosperme charnu, et tantôt dépourvu d'endosperme.

Le genre Polygala avait d'abord été placé par M. de Jussieu dans la famaille des Pédiculaires. Mon père, en faisant voir que sa corolle était véritahlement polypétale, a le premier indiqué la nécessité d'en former une Ifamille distincte, que M. de Jussieu a établie plus tard sous le nom de Poly! galées. Cette famille se rapproche par la forme générale de sa fleur des Légumineuses et des Fumariacées; mais par ses caractères elie doit être placée dans le voisinage des Droséracées et des Trémandrées de M. R. Brown. Outre le genre Polygala, on compte encore dans cette famillc les genres Salomonia, Comesperma, Badiera, Sonlamea, Kraneria, cte. 


\section{CENT QUINZIEME FAMIILE.}

tréMandréEs. Tremandrece. R. BRotwn.

Cettc petite famille, formće des deux seuls genres Tremandra ct Tetratheca, se compose d'arbustes ayant le port des Bruyères, tous originaires de la Nouvclle-Hollande, portant des feuilles alternes ou verticillées, sans stipules, simples ou dentées, et souvent garnies de poils glanduleux. Leurs fleurs sont axillaires et solitaires, ayant un calice de quatre à cinq sépales inégaux, rapprochés en forme de valves, avant l'épanouisscment de la fleur, et caducs. La corolle se compose de quatre à cinq pétales égaux, alternes avec les sépales, plus longs que les étamincs. Celles-ci, au nombre de huit à dix, sont placées par pairc en face de elraque pétale; leurs anthères, qui offrent deux ou quatre loges, s'ouvrent à leur sommet par un petit trou ou une sorte de túbe. L'ovaire est ovoïde, comprimé, à deux loges, contenant chacune deux à trois ovules pendans. Le style se termine par un ou deux stigmates, et le fruit est une capsule comprimée, biloculaire, s'ouvrant en deux valves septifères sur le milicu de leur face. Les graines, insérées au haut de la cloison, sont termincées par un appendice caronculiforme. L'embryon est dressé dans un endosperme charnu.

Cette famille a de nombrenx rapports avec les Polygalées, dont elle diffère par ses étamines libres, ses anthères ì deux ou quatre loges, sa corolle régulière, et avec les Droséracées, dont elle se distingue par ses anthères, les loges de son ovaire, qui ne contiennent que denx on trois ovules, etc.

\section{CENT SEIZIENTE TARILE.}

* Fumartacées. Fumariacece. DC.

Les Fumariacées sont toutes des plantes herbacées non lactescentes, ayant des feuilles alıerues et décomposées 
en un grand nombre de segmens étroits; des fleurs assez petites, généralement disposées en épis terminanx. Leur ealice se compose de deux sépales très-petits, opposés, planes et eaducs; la corolle est irrégulière, 'tubuleuse, formée de quatre pétales inégaux, quelquefois légèrement soudés entr'eux à leur base; le supérieur, qui est le plus grand, se termine à sa partie inférieure par un éperon court et recourbé. Les étamines, au nombre de six, sont diadelphes, c'est-à-dire formant deux androphores, qui portent chacun à leur sommet trois anthères, savoir : une mojenne à deux loges et deux latérales uniloculaires. L'ovaire est uniloculaire ct eontient quatre ou un grand nombre d'ovules attaehés à deux trophospermes longitudinaux, correspondant à ehaque.suture. Le style est court, surmonté d'un stigmate déprimé. Le fruit est tantôt un akène globuleux, monosperme par avortement, tantôt une capsule quelquefois vésiculeuse, polysperme, et s'ouvrant en deux valves. Les graines sont globuleuses, munies d'une caroucule, ct eontenant, dans un endosperme charnu, un embryon petit, un peu latéral, quelquefois recourbé et placé transversalement.

Cette famille, composée du genre Fumaria et des genres établis avec ses diverses espèces, comunc Corydalis, Diclytra, Cysticapnos, etc., se distingue des Papavéracées par l'ahsence da suc laiteux, la corolle irrégulière et les six étamines diadelphes.

\section{CENT DIX_SIPTIÈMEAMILIE.}

* papavénacées. Papaveracece.-Papaveracearum gen. J. Podophyllearum gen. DC.

Plantes herbacées ou plus rarement sous-arbrisseaux à feuilles altcrncs, simples, ou plus ou moins profondément découpées, remplies en général d'un suc laiteux blanc ou jaunâtre. Les fleur's sont solitaircs ou disposées en cymes ou en grappes rameuses. Le calice est formé de 
deux, très-rarement de trois sépales concaves et trèscadues. La eorolle, qui manque quelquefois, se eompose de quatre, très-rarement de six pétales, planes, chiffonnés et plissés avant leur épanouissement. Les étamines, en très-grand nombre, sont libres. L'ovaire est ovoïde ou globuleux, ou étroit et comme linéaire; à une seule loge, contenant un très-grand nombre d'ovules attachés à des trophospermes saillans, sous la forme de lames ou de fausses cloisons. Le style, très-court ou à peine distinet, se termine par autant de stigmates qu'il y a de trophospermes. Le fruit est une capsule ovoïde, eouronnée par le stigmate, indéhiscente, ou s'ouvrant par de simples pores au-dessous du stigmate; ou bien elle est allongée en forme de silique, s'ouvrant en deux valves, ou se rompant transversalement par des artieulations. Les graines, ordinairement fort petites, se eomposent d'un tégument propre, portant quelquefois une sorte de petite caroneule eharnue, d'un endosperme également eharnu, dans lequel est placé un très-petit embryon cylindrique.

M. de Jussieu avait réuni dans ses Papavéracées le genre Fumaria, qui, mieux étudié, est devenu le type d'une famille distinete. Les genres de Papavéraeées sont: Papaver, Argemone, Meconopsis, Sanguinaria, Bocco: nia, Romeria, Glaucium, Chelidonium, et Hypecoum.

Nous réunissons à cette famille le Podophyllum et le Jeffersonia, qui forment l'une des tribus de la famille des Podophyllées de M. de Candolle, famille dans laquelle ce professeur eélèbre réunit en outre des deux genres mentionnés iei, le Cabomba et l'Hydropeltis, qui forment une famille tout-àfait distincte et appartenant aux Monoeotylédons. Voy. Cabomhées.

\section{CENT DIX-HUITIEMTE FAMILIE.}

* crucifìres. Ctuciferco, Juss.

L'une des familles les plus grandes et des plus naturelles du règne végétal, eomposée de plantes herbaeées ou quelquefois soufrutescentes, croissant pour la plupart en Europe. Leurs feuilles sont alternes, simples, ou plus ou 
mnins profondément incisées; lcurs fleurs, disposées en épis ou grappes simples ou panieulées. Le calice est formé de quatre sépales caducs, et dont deux sont quelquefois bossus à leur base; la corolle se eompose de quatre pétales olguiculés, opposés en croix (de là le nom de Crucifères). Les étamines, au nombre de six, sont tétradynames, c'està-dire qu’il y en a quatre plus grandes rapprochées deux par deux et deux plus courtes et opposées. A la base des. étamines on trouve sur le réecpiacle dẹx ou quatre glandes, dont une entre chaque paire des grandes étamines et une plus grande sur laquelle cst imposée ehaque petite étamine. L'ovaire est plus ou moins allongé, à deux loges séparées par une fausse eloison. Chaque loge contient un ou plusieurs ovules attaehés au bord cxterue de la cloison membraneuse, qui n'est qu'un prolongement des deux trophospermes suturaux. Le style est eourt ou presque nul, et semble une continuation de la cloison; il se termine par un stigmate bilobé. Le fruit cst une silique ou une silicule, d'une forme variable, indéhiscente ou s'ouvrant en deux valves. Les graines sont attachées de chaque côté de la cloison. Leur cmbryou est immédiatement recouvert par le tégument propre; il cst plus ou moins reeourbé sur lui-mềme.

Les genres qui composent cette famille sont extrêmement nombreux. Linné les divisait en deux ordres, suivant que le fruit était une silicule ou une silique. Dans le premier on trouve entre autres les genres Lepidium, Thlaspi, 1satis, Myagrum, Cochlearia, Iberis, Lunaria, etc. Dans le second les genres Cheiranthus, Sisymbrium, Hesperis, Brassica, Liruca, Siuapis, etc.

\section{CENT DIX MEUVIEME FATIIEE.}

* cappatidóes. Capparidece.

Ce sont des plantes herbacées ou des végétaux ligneux qui portent des feuilles alternes, simples ou digitées, aecompagnées à leur base de deux stipules foliacées; leurs 
fleurs sont terminales, en forme d'épis ou de grappes, ou axillaires et solitaires; leur ealice se compose de quatrc sépales caducs, très-rarement soudćs ensemble par leur base; la corolle, formée de quatre à cinq pétales égaux ou inćgaux; les étamines sont tantòt en nombre défini, tantôt et plus souvent en nombre indéfini; l'ovaire est simple; souvent élevé sur un support plus ou moins allongé, qu'on nomme podogyne, à la base duquel sont insérés les étamines et les pétales; il offre une seule loge contenant plusieurs trophospermes saillans sous la forme de lames our fausses cloisons, portant un graud nombrc d'ovules. Le fruit est scc out charnu. Dans lc premicr cas, c'est une sorte de silique plus ou moins allongée, s'ouvrant cn deux valves comme dans la plupart des crucifères. Dans le second cas, c'est une baic uniloenlaire et polysperme, dont les graines sont ou pariétales, ou semblent éparses dans la pulpe qui remplit le fiuit. Ces graines sont en général réniformes composées d'un épisperme sec et comme crustacé, qui recouvre immédiatement un embryon un peu recourbé ct dépourvu d'endosperme.

Parmi les genres qui composent cette famille, nous citerons les snirans: Capparis, Cratcuva, Morisonia, Boscia, Cleome, etc. M. de Jussieu avait placć dans sa famille des Capparidées plusicurs genrcs qui sont devcnus les types de familles distinctes. Ainsi le Reseda forme la famille des RésÉdacÉEs; les Drosera, Pamassia, Aldrovanda ct Dioncea, les Droséracérs; Ie Marcgraviz et le Norantea, les Marcgraviacítes.

Les Capparidées ont les rapports les plus intimes avec les Crucifères, mais elles en diffèrent par leurs feuilles munies de stipules; leurs étamines nombreuses et la structure de leur frnit.

\section{CEMTT VINGTIEME FAMIILE. \\ * nésédać́es. Resedacea. DC.}

Plantes généralement herbacćes, rarcment soufrutescentes, à feuilles altcrnes, sans stipules, souvent munies de deux, glandes à leur base. Les fleurs forment des épis 
simples et terminaux. Le calice présentc de quatre à six divisions profondes et persistantes. La corolle se eompose d'un même nombrc de pétales alternes avec lcs sépales du calicc. Ces pétales sont, en général, eomposés de deux parties, l'une inférieure entière, l'autrc supéricure divisée cn un nombre plus on moins considérable de lanières; les étamines sont généralcment cn nombre indéterminé (de (quatorzc à vingt-six), leursfilamens sont libres, ct hypogynes; leurs anthères à deux loges, s'ouvrant chacunc par un sillon longitudinal. En dehors des étamines, e'cst-à-dire entrc les pétales et les filets on trouve une sortc de godet annulaire, glanduleux, plus élevé du côté supéricur, ct formant ainsi un disquc hypogyne d'unc nature partieulière. Le pistil, légèrement stipité à sa basc, paraît formé de la réunion intime de trois carpelles, ct se termine supérieurement par trois cornes portant chacune un stigmate à son sommet. Cct ovaire offre une seulc loge ouver te à son sommet, contenant un grand nombre d'ovules attachés à trois trophospcrmes pariétaux, qui offrent le caractère remarquable de nc point correspondre aux stigmates, mais d'alterner avcc cux. Le fruit, très-rarcment charnu, est ordinairemcnt une capsule, plus ou moins allongée, ouverte naturcllement à son sommet, qui se termine par trois angles, à une seule loge, ct dont les graines sont rangćes sur trois trophospermes pariétaux. Ces graines, très-souvent réniformes, sont composées d'un tégument assez épais, d'un endosper'mc charnu très-mince et d'un cmbryon reeourbé en forme de fer à cheval.

Ccttc famillc ne se compose que du genre Rescda et de l'Ochradcnus de M. Delile. Le genre Reseda avait été placć par M. de Jussieu dans la famille des Capparidées, et il faut convenir en effct qu'il a plusieurs points de contact avec cette famillc, et en particulier avec le genre Clcome. Mais M. Tristan (Ann. du Mus. d'Hist. nat., t. xvirr, p. 392) en a formé le type d'une famille distincte, adoptée par M. de Candolle, et placée par le premier de ces hotanistes, cntre les Passiflorées et les Cistées, mais nćanmoins plus près de ces derniìres. Dans ses Collectanea bolanicâ, t. XxI , M. J. Lindloy 
a donné une explication tout-à-fait diffćrente de la fleur du Résćda: Pour ce botaniste, le calice est un involncre commun, chaque pétale est une fleur stérile, et le nectaire ou disque est un calice propre qui environne une flenr hermaphrodite composée des étanines et du pistil; d'après cctte manière de voir, M. Lindley rapproche les Résédacées dcs Ephorbiacées, qui offrent une disposition à peu près analogue. Mais néanmoins nous croyons que cette famille ne saurait être éloignée des Capparidées et des Cistées.

\section{CENT VINGIT-UINIEME FAMILIE.}

flacountanées. Flacourtianex. Rich. - Bixinées. Kunth.

Arbrisseaux à feuilles alternes simples, entières souvent coriaees, persistantes et dépourvues de stipules; à fleurs pédonculées et axillaires, souvent unisexuées et dioïques, d'autres fois hermaphrodites. Leur calice est formé de trois à sept sépales distincts ou légèrement soudés par leur base; la corolle, qui manque quelquefois, se compose de einq ou sept pétales alternant avee les sépales. Les étamines, en nombre défini ou indéfni, ont leurs filets libres, leurs anthères à deux loges; ees étamines sont, ainsi que la corolle, insérées au pourtour d'un disque hypogyne et annulaire, qui manque rarement. L'ovaire est sessile ou stipité, gुlobuleux, à une seule loge dans tous les genres de la famille, exeepté dans le Flacourtia, qui en offre de six à neuf. Dans le premier cas, il renferme un assez grand nombre d'ovules attaeliés à des trophospermes pariétaux dont le nombre est le même que eelui des stigmates ou des lobes du stigmate. Le fruit est uniloeulaire, excepté dans le Flacourtia; il est indéhiscent ou déhiscent, et les valves portent ehaeune un trophosperme sur le milieu de leur face interne. En général, le tégument propre de la graine est charnu, et l'embryon, qui est homotrope, droit, est plaeé au centre d'un endosperme charnu.

Les genres qui composent cette famille ne nous paraissent point encore hien définitivement déterminés. Leur caractère essentiel consiste dans des placentas pariétaux, simples ou plus souvent étalés et ramifiés sous la 
forme de veines qui tapissent la paroi interne de l'ovaire, comme nous l'avous déjì fait remarquer dans la tribu des Butomées de la famille des Alismacées. Peut-être serait-il convenable de réunir aux Flacourtiacées la famille des Bixinées, établie par notre savant ami le professeur Kunth, et qui ne nous parait point en différer sensibleınent, Les genres principaux qui composent les Flacourtianées sont: Flacourtia, Roumea, Kiggellaria, Erythrospermum, etc. Cette famille a da rappport avee les Capparidées, dont elle diffère surtout par son embryon pourva d'un endosperme charnu, et par ses graines insérées sur le nilieu et non sur le bord des valves. Elle a aussi quelque affinité arec les Cistées et les Tiliacées.

\section{CENT VINGT-DEUXIEME FAMILIE.}

${ }^{*}$ cistéEs. Cislece. DC. - Cistorum genera. Juss.

Ce sont des plantes herbaeées annuelles ou vivaees, ou des arbustes ligneux, portant des feuilles souvent opposées entières, et parfois munies de denx stipules; des fleurs axillaires ou terminales, solitaires ou en épis, en grappes ou en sertules. Leur ealiee est à trois ou cinq divisions très-profondes, tantôt égales, tantôt inégạles et deux étant plus extérieures; leur eorolle à einq pétales ehiffonnés très-eadues, étalés en rose et sessiles; les étamines fort nombreuses et libres; l'ovaire globuleux, rarement uniloeulaire, plus souvent à cinq ou dix loges, contenant plusieurs ovules insérés au bord interne des cloisons. Dans l'ovaire uniloeulaire, les ovules s'attachent à des trophospermes pariétaux. Le style et le stigmate sont simples. Le fruit est une capsule globuleuse enveloppée dans le caliee, qui est persistant, offrant une, trois, cinq ou même dix loges, et s'ouvrant en trois, cinq ou dix valves, portant ehacune une des cloisons, ou un des trophospermes surle milieu de leur faee interne. Les graines, assez nombreuses dans ehaque loge, eontiennent un cmbryon plus ou moins reeourbé, ou roulé en spirale dans un endosperme charnu.

Cette petite famille ne se compose que des genres Cistus et IIeliantheınum, Telle qu'elle avaiț été établie par, M, dę Jussieq, dans son Genera 
plantarum, elle renfermait les genres Viola, Pipared, Piriqueta et Tachibota, qui forment aujourd'hni la famille des Violariées.

\section{CENT VINGT-THOISI音HE FAMILIE.}

\section{* muoséracées. Droseracece. DC.}

Plantes herbacées, annuelles ou vivaces, rarement soufrutescentes, ayant des feuilles alternes, souvent munies de poils glanduleux et pédicellés, et roulées en crosse avant leur développement. Leur caliee est monosépale, à cinq divisions profondes, ou à einq sépales distinets; leur corolle, de cinq pétales planes et réguliers; les étamines au nombre de cinq, quelquefois de dix, alternent avec les pétales, et sont libres; quelquefois on trouve en faee de chaque pétale des appendiees de forme variée. Ces étamines sont généralement périgynes et non hypogynes, comme on l'a dit jusqu'à présent. L'ovaire est à une scule $\operatorname{loge}$, rarement à deux ou trois; dans le premier cas, il eontient un grand nombre d'ovules attachés à trois ou cinq trophospermes pariétaux, simples ou bifides; dans le second cas, les eloisons paraissent formées par les trophospermes saillans en forme de larmes, et qui se reneontrent et s'unissent au eentre de l'ovaire. Les stigmates, généralement en même nombre que les trophospermes ou que les loges, sont sessiles et rayonnans. Le fruit est une capsule à une ou plusicurs loges, s'ouvrant sculement par sa moitié supéricure en trois, quatre ou cinq valves, portant sur le milieu de leur face interne un des trophospermes. Les graines, souventrecouvertes d'un tissu lâche, eonticnnent un embryon dressé, presque eylindrique, dans l'intérieur d'un endosperme minee qui manque quelquefois.

Les genres rapportés à cette famille par $M$. de Candolle sont Drosera, Aldrovanda, Romanzoffa, Byblis, Roridula, Drosophyllum, Dionae et Parnassia. Mais en traitant avec quelques détails des caxactères de cctte fawille; 
dans le tome 5 du Dietionnaire classique d'Histoire naturelle, article DroséRACÉES, p. 624, nous avons fait voir que l'on doit en retrancher, $I^{\circ}$ le Dioncea, qai a l'insertion réellement hypogyne et les graines toutes attachées au fond de la capsule, et qui se rapproche peut-être davantage des Hypéricées; $2^{\circ}$ le Romanzoffia, qui appartient aux Scrophulariées.

La famille des Droséracées diffère des, Violariées, dont elle se rapproche beaucoup, par son insertion périgyne, l'absence des stipules et la régularité constante de la flenr, etc.

\section{CENT VINGT-QUATRIIME FAMIUTE.}

\section{* violartíes. Violatiece. DG.}

Herbes ou arbustes à feuilles alternes, très-rarement opposées, munies de deux stipules persistantes. Les fleurs sont axillaires pédoneulées. Le ealice se eompose de einq sépales libres, ou légèrement soudés entr'eux à leur base, qui se prolonge quelquefois ain-dessous de leur point d'attaehe, et qui sont égaux ou inégaux. Lá corolle se eompose de cinq pétales inégaux, dont l'inférieur se prolonge à sa base en un éperon plus ou moins allongé; très-rarement la eorolle est formée de cinq pétales réguliers. Les étamines, au nombre de cinq, sont presque sessiles, rapprochées et contiguës latéralement entr'elles, à deux loges introrses; les deux qui sont placées vers le pétale inférieur, offrent assez sonvent un appendice en forme de corne recourbée, qui naît de leur partie dorsale et se prolonge dans l'éperon. L'ovaire est globulenx uniloculaire, contenant un grand nombre d'ovules attachés à trois trophospermes pariétaux. Le style est simple, un peu eoudé à sa base, renflé vers sa partie supérieure, qui se termine par un stigmate, un peu latéral, et offrant une petite fossette semi-circulaire. Le fruit est une capsule uniloculaire, s'ouvrant en trois valves, qui ehaeune portent un trophosperme sur le milieu de leur face interne. Les graines eontiennent un embryon dressé dans un endosperme charnu. 
Les Violariées; qui se composent des genres Viola, Ionidium, Hybanthus, Noisettia, Conhoria, Alsodeia, etc., se distinguent surtout des Cistées par leur corolle irrégulière, leurs cinq ćtamines, lcur stigmate renflé et concave, etc. Elles ont aussi des rapports avec les Polygalées, les Droséracées, etc.

\section{CENT VINGT-CINQUIEMI FANILIE.}

\section{* frankéniacées. Frankeniacece. Aug. Saint-Hilaire.}

Les Frankéniacées sont herbaeées ou fruteseentes, leurs feuilles sont alterıes ou verticillées, entières ou dentées en seie, avee des nervures latérales très-rapprochées, munies à leur base de deux stipules, qui manquent seulement dans le genre Frankenia. Les fleurs sont axillaires disposées en grappes simples ou composées, ou en pauicules. Ces fleurs sont hermaphrodites. Leur calice est formé de einq sépales, légèrement soudés à lcur base; la eorolle de cinq pétales, égaux ou inégaux. Dans le genre Sauvagesia, on observe de plus un verticille de filamens renflés en massue, et une corolle intérieure qui existe aussi dans le genre Luxemburgia. Les étamines sont au nombre de einq, de huit, ou indéfinies; elles sont libres, leurs anthères sont à deux loges extrorses, qui s'ouvrent par une fentelongitudinale ou un pore. L'ovaire est ovoïde allongé, ou trigone, souvent placé sur un disque liypogyne; il offre une seule loge, eontenant trois trophospermes pariétaux, portant cliacun un assez grand nombre d'ovules. Le style est grêle, terminé par un stigmate extrêmement petit. Le fruit est une capsule recouverte par le calice ou par la corolle intérieure, à une seule loge qui s'ouvre en trois valves, dont les bords, légèrement rentrans, forment trois cloisons incomplètes, portant les graines. Celles-ci, au centre d'un cndosperme charnu, contienuent un petit embryon axile, eylindrique et homotrope. 
Cette petite famille se compose des gentes Frankenia, Lavradia, Sauvágesia et Luxemburgia. Elle a les plus grands rapports avec les Cistées, les Violacées et les Droséracées; mais elle en diffère surtout par le mode de déhiscence de ses capsnles, dont les valves portent les graines sur leurs bords rentrans, tandis que les placentas sont placés sur le miliea de la face interne des valves dans les familles précédentes.

\section{CEIT VINGT-SIXIEME FAMILIE.}

* caryophyrlées. Cayyophyllea. Juss.

Les Caryophyllées sont herbacées, rarement soùfrutescentes à lcur basc; leurs tiges sont souvent noueuses et articulées; lcurs feuilles, opposćcs ou vcrticillées, sont simples; les fleurs, généralemcnt hcrmaphrodites, sont terminalcs ou axillaires. Lcur calice sc compose de quatre à cinq sépalcs distincts ou soudés entr'eux, et formant un tube cylindrique ou vésiculeux, simplcment denté à son sommet; la corolle, de cinq pétales, ordinairement onguiculés à leur base, manque trc̀s-rarcment; le nombrc des étamines, est cn général égal ou double des pétales; dans ce dcrnicr cas, cinq sont alternes avec les pétalcs, et cinq leur sont opposées ct se soudent inférieurement avec lcs onglets; toutes sont insérécs à un disque hypogyne qui supporte l'ovaire. Celui-ci présente dcpuis une jusqu’à cinq loges; il cst uniloculaire; les ovules, qui sont nombreux, sont attachés à un trophosperme ccntral; quand il est pluriloculairc, lcs ovules sont attachés à l'angle intcrne de chaque loge; les styles varicnt de deux à cinq, et se tcrmincnt chacun par un stigmate subulé. Le fruit est une capsule, très-rarement une baic, ayant d'une à cinq $\log c s$ polyspermes. Cettc capsulc s'ouvrc, soit par son sommet, au moycn de petites dents qui s'ćcartent les unes des autres, soit par des valves complètes. Les graines sont tantòt planes ct mcmbrancuscs, tantôt arrondies. Elles conticnncut un cmbryon recourbć on comme roulé aulour d'un endospermé farineux. 
Plusicurs genres, d'abord placés dans cette famille'; en ont été rctirés et réunis à quelques autres, tirés de la famillc dcs Amaranthacées, forment la nouvclle famille des Paronychiées, qui se distingue surtout par son inscrtion périgyniquc; tels sont les genres Polycarpon, Locffingia, Minuartia, Queria. Les genres Linum et Lechea, dont on a vait fait la famiile des Linacées; ont été réunis anx Géraniacées. Le Frankenia est devcnu lc type de la famille des Frankéniacées; Ic Sarothra a été reporté daus les Hypéricinées.

On pent diviser en deux tribus les genres de cette famille, savoir :

$x^{\circ}$ Les Drantrìes, qui ont un calice monosépale tubuleux; des pétales longuemcnt ouguiculés : Dianthus, Silene, Lychnis, Agrostemına, Cucubalus, etc.

$22^{\circ}$ Les Arsrivires, dont le calice est étalé ct les pćtales sans onglet : Arenaria, Alsine, Spergula, Cerastium, Mollugo, cte.

\title{
QUATORZILME C.LASSE.
}

\author{
PÉRTÉTALIE (I).
}

CENT VINGT-SEPTISRIF FAMIUIE.

* paronychiées. Paronychice. Aug. St.-Hil.

Plantes hicrbacées ou soufrutescentes, portant des feuilles opposées, souvent connées paṛ lcur base, avec ou sans stipules; des flcurs très-petites, axillaires ou terminales, nues ou accompagnécs de bractées scarieuses. Lcur calice, monosépale, souvent persistant, offre cinq divi-

(1) Indépendamment des familles dont nous avons tıacé les caractères, plusieurs autres appartiennent aussì à la mêmc classe. Mais commc leurs caractèrcs ne sont pas encore bien parfaitement déterminés, ou qu'clles ne se composent que d'un très-petit nombre de genres, nous avons cru devoir les négliger dans un livre de la naturc de cclui-ci. Telles sont, I" les Escar.coźkes (R. Brown), roisines des Saxifragées; $2^{\circ}$ les Stackuousícs (R. Broww), qui ne se composent que du genre Stachliousia; $3^{\circ}$ les Chadtrétrées (R. Browr); $4^{\circ}$ ct les Aquitarinérs (R. Brown), intermćliaires cntrc les Rhamnécs et les Térćbenthacées, et qui comprennent, les promières les genres Chailletia, Leucosia et Tapura, et les secondes les genres Aquilaria, Ophispermun et Grrinops. 
sions plus ou moins profondes; assez souvent il forme un tube à sa partie inférieure, qui est souvent épaissie par un bourrelet glanduleux; les pétales, au nombre de cinq, très-petits et squammiformes ou même nuls, sont insérés au haut du tube ealyeinal. Les étamines, également au nombre de einq, dont quelques-unes avortent parfois, sont alternes avee les pétales, et ont leurs anthères introrses. L'ovaire est libre, à une seule loge eontenant un seul ovule placéau sommet d'un podosperme basilaire quelquefois très-long, et dans ee eas l'ovule est renversé; d'autres fois plusieurs ovules sont attaehés à un trophosperme central très-eourt. Le stigmate est tantôt sessile et simple, tantôt il est bifide et porté sur un style assez eourt. Le fruit est une eapsule déhiscente au moyen de valves ou de fentes, ou bien elle reste elose. Les graines se composent, outre leur tégument propre, d'un embryon eylindrique appliqué sur un des eôtés ou roulé autour d'un endosperme farineux. La radicule est toujours tournée vers le hile.

Cette famille, établie par M. Ang. de St-Hilaire, se compose de genres retirés des Amaranlhacés, des Portnlacées et des Caryophyllées, dont ils s'éloignent surtout par leur insertion périgynique, tandis qu'elle est hypogynique dans les deux autres. Nous avons divisé les genres des Paronychiées en deux tribus, savoir:

I0 Les Sctératituieses, qui renferment les genres qui n'ont pas de bractées, dont les divisions calycinales ne sont pas scarieuses sur les hords; les feuilles sans stipules et connées. Ex. : Locfflingia, Minuartia, Queria, Sclerantlus, Mniarum et Larbrea.

$2^{\circ}$ Les Paronychí́es vraies, dont les genres ont leurs fleurs munies de bractées; leurs divisions calycinales scarieuses sur les hords, souvent charnues et creusées en gonttière, les feuilles accompagnées de stipules. Ex. : Gynnocarpus, Paronychia, Illecebrum, Anychia, Herniaria, Polycarpon, Hagea, etc. 


\section{CENT VINGT-HUITIRIE TAMIILE.}

* portulacées. Portulacece. Juss.

Plantes herbaeées, rarement frutescentes, ayant des feuilles opposées, quelquefois alternes, épaisses et eharnues, sans stipules; des fleurs généralement terminales. Leur ealice est en général formé de deux sépales plus ou moins soudés et souvent comme tubulé à la base; la eorolle se eompose de cinq pétales libres, ou légèrement soudés entr'eux, et formant une corolle monopétale; les étamines sont en même nombre que les pétales, insérées à leur base, et leur sont opposées; plus rarement elles sont plus nombreuses. L'ovaire est libre, ou presque semi-infère, à une seule loge eontenant un nombre variable d'ovules, naissant immédiatement du fond de la loge ou attaehés à un,trophosperme eentral. Le style est simple, terminé par trois ou einq stigmates filiformes. Le fruit est une eapsule uniloeulaire, contenant trois ou plusieurs graines, et s'ouvrant soit en trois valves, soit en deux valves superposées. Les graines, sous leur tégument propre, souvent erustacé, reeouvrent un embryon cylindrique qui est roulé sur un endosperme farineux.

Plasieurs genres, d'abord réunis à cette famille, en ont ćté retranchés. Ainsi le Tamarix forme la famille des Tamariscinées, qui diffère surtout par l'absence de l'endosperme; les genres Scleranthus, Gymnocarpus, et probablement le Telephium et le Corrigiola, ont été portés dans la nouvelle famille des Paronychiées, qui n'en diffèrent guère que par leurs étamines alternes et nour opposćes anx pétales; lenr stigmate simple ou bifide et non tri ou quinquéfide. Les genres qui restent parmi les Portulacées sont: Portulaca, Talinum, Montia, etc.

\section{CENT VINGT-NEUVIEME FAMITLE.}

* ficoüdÉEs. Ficoüdea. Juss.

Ce sont en général des plantes grasses eomme les Crassulacées, ayant leurs feuilles alternes ou opposées; leurs 
fleurs souvent très - grandes, axillaires ou terminales. Chaeune d'elles présente un ealice monosépale, souvent eampanulé et persistant, ayant son limbe quelquefois coloré, et à quatre ou cinq lobes; une eorolle polypétale, et dont les pétales sont quelquefois en nombre indéfini, d'autres fois soudés en une eorolle monopétale; plus rarement la corolle manque. Les étamines sont généralement assez nombreuses, libres et distinctes. L'ovaire est tantôt entièrement libre, tantột adhérent par sa base avee le calice; il offre de trois à einq loges, eontenant chacune plusieurs ovules attachés à un trophosperme qui naît de l'angle interne de ehaque loge; eet ovaire est surmonté de trois à einq styles, terminés ehacun par un stigmate simple. Le fruit est tantôt une bąie, tantôt une capsule environnée par le caliee, à trois ou einq loges polyspermes. Leurs graines offrent un embryon roulé autour d'un endosperme farineux.

Cette famille a de très-grands rapports avec les Portulacées, dont elle diffère par ses pétales et ses étamines, généralement en grand nombre, par sa pluralité de styles, et son ovaire à trois ou cinq loges, et non uniloculaire comme dans les Portulacées. Les genres principaux de la famille des Ficoïdées sont : Reaumuria, Mesambryanthemum, Nitraria, Tetragonia, etc. (Cette famille, qui, par son port, se rapproche des Cirassulacées, en diffère !par son ovaire simple.

\section{CENT TRENTIEME TAMILE.}

* saxifragées. Saxifragec. Juss. Cunoniacece. R. Brown.

Les Saxifragées sont des plantes herbaeées, rarement des arbustes ou des arbres, dont les feuilles sont alternes ou opposées, simples, et quelquefois eomposées, avee ou sans stipules. Leurs flcurs, tantôt solitaires, tantôt diversement groupées en épis, en grappes, ete., offrent un ealiee imonosépale, tubuleux inféricurement; où il se soude :avec l'ovaire, terminé supéricurement par trois ou cinq 
divisions; la corolle, qui manque très-rarement, est formée de quatre à cinq pétales, quelquefois soudés par leur base; les étamines sont en général en nombre double des pétales, quelquefois en nombre indéfini. L'ovaire est à deux, plus rarement à quatre ou cinq loges; il est tantôt tout-à-fait libre, tantôt semi-infère ou presque infère, terminé à son sommet par autant de styles qu'il y a de loges. Celles-ci contiennent ordinairement plusicurs, très-rarement un seul ovule. Ces ovules sont attachés à un trophosperme placé le long de la cloison. Le fruit, qui est rarement charnu, est en général une capsule, terminée supérieurement par deux cornes plus ou moins allongées, s'ouvrant en général en deux valves septifères. Les graines offrent, sous leur tégument propre, un endosperme charnu qui contient un embryon axile, homotrope, quelquefois un peu recourbé.

Cette famille, à laquelle nous réunissous les Cunoniacées de M. R. Brown; qui n'en diffèrent que par lenr tige ligneuse, se cormpose des genres Saxifraga, Heuchera, Tiarella, Cunonia, Weinnmannia, etc.

\section{CENT THENTE-UNIEME FAMIIIE.}

haMAMUÉLIDÉEs. Ilamamelidece. R. Brown.

Ce sont des arbustes à feuilles alternes, simples, munies souvent de deux stipuleš caduques. Les fleurs sont axillaires, ayant un calice composé de quatre sépales, quelquefois réunis en tube à leur partie inférieure, et soudés avec l'ovaire, qui est semi-infère; la corolle se compose de quatre pétales allongés, linéaires, valvaires, et un peu tordus avant l'épanouissement des fleurs; les étamines sont au nombre de quatre, alternes avec les pétales, ayant leurs anthères introrses, et à deux loges, s'ouvrant par une valvule qui est parfois commune aux deux luges, et qui occupe lcur face interne; devant chaque, pétale on trouve souvent une écaille de forme variée, et 
qui paraît tenir lieu d'une étamine avortée. L'ovaire est semi-infère, ou entièrement libre, à deux loges, contenant ehaeune un ovule suspendu; du sommet de l'ovaire naissent deux styles terminés ehaeun par un stigmate simple. Le fruit, enveloppé par le caliee, est see, à deux loges monospcrmes, s'ouvrant en général en deux valves septifères. Les graines se composent d'un embryon homotrope, recouvert par un endosperme eharnu.

Le genre Hamamelis, qui forme le type de cette famille, avait été placé par M. de Jussieuà la fin des Berbéridées; mais son insertion est hien réellement périgynique. M. Rób. Brown (in Abel Iter Chinens.) a proposé d'établir pour ce genre une famille particulière sous le nom d'Hamamélidécs. Il rapporte en outre à cette famille les genres Dicoryphe et Dahlia, et en rapproche le Fothergilla, qui cependant en diffère par plusieurs caractères. C'est auprès de cette nouvelle famille que l'illustre botaniste anglais pense qu'il faut placer sa famille des Bruniacées. Qaınt aux Hamamélidées elles-mêmes, elles nous paraissent avoir beancoup de rapports avec Ics Saxifragées.

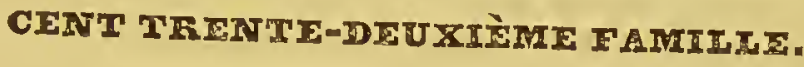

bruniacées. Bruniacece. R. Brown. Ad. Brong.

Les plantes qui forment eette famille sont des arbustes (qui, par leur port, ressemblent beaucoup aux Bruyères; cet aux Phylic a ou Bruyères du Cap. Tous sont originaires du cap de Bonne-Espérance; leursfeuilles sont très-pelites, rroides, entières, quelquefois imbriquées; les fleurs sont petites, disposćes en capitules, plus rarement cn paniccules. Le caliee est monosépalc à cinq divisions, adhérent cen général par sa base avec l'ovaire, qui est infère ou semiinfère (il est libre dans le scule genre Raspalia); les cinq divisions sont imbriquées, de même que la corolle, ivant leur épanouissement; lcs pétales sont au nombre de ing et alternes; les einq étamines sont alternes avce-les nétales, et lcurs filets adhèrent latéralement avee la base icc ehacun des pétales, ee qui a fait croire à quelques aucurs qu'ils étaient opposés aux pétales. L'ovaire est 
semi-infcre ou infère, ou cufin libre, à unc ou trois loges, contenant chacune un ou dcux ovules collatćraux et suspendus. Le style est simple ou bifide, ou les dcux styles sont distincts et terminés chacun par un très-petit stigmate. Le fruit cst sce, couronné par le calice, la corolle et les étamines, qui sont persistantes; indéhiscent, ou se séparant en deux coques généralcment monospermes, s'ouvrant par une fente longitudinale et intcrne. Les graines sont suspendues, contenant un très-petit embryon homotrope placć vers la base d'un endosperme elarnu.

Cetie petite fanille, indiquée par Rob. Brown ( in Abel Inter Chin.), a été adoptée par M. de Candolle (Prodr. syst., 2, p. 43). M. Adolple Brongniart en a fait l'objet d'un mémoire spécial, dans lequel il a mieux tracé et les caractères de la famille et ceux des genres qui la composent. Le genre Brunia, qui en forme le type, avait été placć par M. de Jussieu à côté du $P h y$ lica dans la famille des Rhamnées; mais il en diffère par plusieurs caractères, tels que ses étamines alternes et non opposées aux pétales; ses ovules souvent géminés et suspendus, et non solitaires et dressés, etc. M. Brown pense que les Bruniacées doivent être rapprochées des Hygrobiées et des Hamamélidées, tandis que M. de Candolle les place au voisinage des Rhamnées. Dans son travail sur cette famille, M. Brongniart énumère les genres suivans : Berzelia, Brunia, Raspalia, Staavia, Berardia, Linconia, Audoninia, Tittmannia et Tamnea.

\section{CENT TRENTE-TROISIEME PAMIIIE.}

* crassulacées. Crassulacec. DC. - Sempervivece. Juss.

Cette famille se compose de plantes herbacécs, ou d'arbustes dont les feuilles, les tiges et en général toutes les parties herbacćes, sont épaisses et charnucs. Ces feuilles sont alternes ou opposées. Lcurs fleurs, qui préscntent quclquefois des eoulcurs trc̀s-vives, offrent différens modes d'inflorescence. Lcur calice cst profondément divisé en un grand nombrc de segmens; leụr corolle se compose d'un nombre variablc, quclqucfois très - grand, de pétales réguliers, distincts ou soudés en une eorolle 
monopétale. La nombre des étanines est le même, ou plus rarement double des pétales, ou des lobes de la corolle monopétale. Au fond de la fleur on trouve consfamment plusieurs pistils distinets, et dont le nombre varie de trois à douze, et mème au-delà. Chaeun d'eux se eompose d'un ovaire plus ou moins allongé, à une seule loge, eontenant plusieurs ovules attaehés à un trophosperme sutural et interne. Le style et le stigmate sont simples. Les fruits sont des eapsules uniloeulaires, polyspermes, s'ouvrant par leur suture longitudinale et interne. Leurs graines offrent un embryon plus ou moins reeourbé, enveloppant. en quelque sorte un endosperme farineux.

Cette famille, composée de plantes grasses, a, par ses capsules polyspermes uniloculaires et s'ourrant par une seule suture longitudinale, du rapport avec les genres de la famille des Renonculacées, qủi offrent le même caractère. Mais elle se rapproche davantage des Saxifragées et des Ficoïdées, dont elle diffère surtout par ses pistils distincts au centre de la fleur. Les genres principaux sont : Tilloca, Buliardia, Crassula, Cotyledon, Bryophyllum, Sedum, Sempervivum.

\section{CENT TRENTE-QUATRYYME FAMIIIE,}

* nopalées. Nopalece. Vent. Cactus. Juss.

Cette famille se eompose uniquement du genre Cactus de Linnée, et des divisions qu'on y a établies. Ce sont des plantes vivaees, souvent arboreseentes, d'un port tout partieulier, qui n'a d'analogues que dans quelques Euphorbes. Leurs tiges sont ou eylindriques, rameuses, eannelées, anguleuses, ou eomposées de pièees artieulées, qui ont été eonsidérées eomme des feuilles. Les feuilles manquent presque eonstamment, et sont remplaeées par des épines réunies en faiseeaux. Les fleurs, qui sont quelquefois très-grandes et brillent du plus vif éelat, sont en général solitaires, et placées à l'aisselle d'un de ces faisceaux d'épines. Leur ealice est monosépale, adhérent avec 
l'ovaire infère, quelquefois écailleux extérjeurement, terminé à son sommet par un limbe, composé d'un grand nombre de lobes inégaux, qui sc confondent avec les pétales. Ceux-ci sont en géućral très-nombreux, ct disposés sur plusicurs rangs. Les étamines, également très-nombreuses, ont leurs filets grêles et capillaircs. L'ovaire est infère, à une seule loge, contenant un grand nombre d'ovulcs attachés à des trophospermes pariétaux, dont le nombre est très-variable et ordinairement en rapport avec celui des stigmates. Le style est simple, terminé par trois ou'un plus grand nombre de stigmates rayonnés. Le fruit est charnu, ombiliqué à son sommet. Ses graines ont un double tégument, et renferment un embryon droit ou recourbé, dépouryu d'endosperme.

M. de Jussieu avait réuni dans cette famille avec le genre Cactus, le genre Groseiller, dont M. de Candolle a formé sa famille des Ribésiées. Voy. cidessous les différenees qui existent entre ces deux familles.

\section{CENT TRENTE-CINQUITME FAMILIE,}

\section{* nibésrées. Ribesice. Ricri. Grossulariées. DC.}

Arbrisseaux buissonneux, quelquefois épineux, ayant des feuilles alternes, sans stipules; des fleurs axillaires, solitaires, géminées ou disposées en épis ou grajpes simples. Leur calice est monosépale, tubuleux, inférieurement où il adhère avec l'ovaire, ayant son limbe évasé et comme campaniforme, à cinq divisions étalées ou réfléchics; leur corolle est forméc de cinq pétales, quelquefois trèspetits; les étamines, en même nombre que les pétales et alternes avec enx, sont insérées vers le milicu du limbe calycinal. L'ovaire est infère, à une seule loge, contenant un grand nombre d'ovules attachćs sur plusicurs rangs à deux trophospermes pariétaux. Les deux styles sont plus ou moins soudés entr'eux, et se terminent chacun par un stigmate simple. Le fruit est une baie globuleuse, 
ombiliquée, polysperme, et ses graines se composent d'un gros embryon, immédiatement recouvert par le tégument propre.

Lc seul genre Ribcs, auquel on pourrait peut-être ajouter le genre Gronovia, auparavant placé dans les Cucurbitacées, compose cette famille. Elle est extrêmement voisine des Nopalées, dont elle diffère surtout par le port si différent des végétaux qui la composent, par leurs pétales et leurs étamines constamment au nombre de cinq, et non en nombre indéterminé comme dans les Cactus, par lears deux trophospermes et leurs deux styles. Dans un autre ouvrage (Botanique inédicale, p. 487) j'ai proposé de diviser les espèces nombreuses de ce genre en trois sections ou sous-genres, ayant pour type, l'ume le Ribes usa-crispa, l'autre le Ribes nignum, et la troisième le Ribes rubrum. J'ai appelé la première Grossularia, la seconde Ribes, et la troisième Botrycarpum.

\section{CENT TRTNTE-SIXIÈME FAMILIE,}

\section{* cucunbitacées. Cucurbitacece. Juss.}

Grandes plantes herbacées, souvent volubiles, couvertes de poils eourts el très-rudes. Leurs feuilles sont alternes pétiolées, plus ou moins lobées. Leurs vrilles, qui sont simples ou rameuses, naissent à eôté des pétioles. Les fleurs sont en général uniscxuées et monoïqués, trèsrarement hermaphrodites. Le calice est monosépale; dans les fleurs femelles, il offre un tube globuleux adhérent a vec l'ovaire infère; son limbe, qui est plus ou moins campanulé et à cinq lobes, est confondu et intimement soudé avec la corolle, et n'a de distinet que le sommet de ses lobes. La corolle est formée de cinq pétảles; réunis entre cux au moyen du limbe calyeinal, et représentant ainsi une corolle monopétale. Les étamines, au nombre de cinq; ont leurs filets monadelphes ou réunis en trois faisceaux, deux formés ehaeun de deux étamines; et le troisième, d'une seule étamine. Les anthères sont uniloeulaires linéaires, contournées sur elles-mêmes, en forme d' co placée horizontalemenit, et dont les branches seraient très-rap- 
proehées. Dans les fleur's femelles, le sommet de l'ovaire, qui est infère, est eouronné par un disque épigyne. Le style est épais, eourt, terminé par trois stigmates épais, et souvent bilobés. Cet ovaire est à une seule loge dans deux genres (Sicyos et Gronovia); il eontient un seul ovule pendant; mais en général il offre trois trophospermes pariétaux, triangulaires, très-épais, eontigus les uns aux autres par leurs eôtés, et remplissant ainsi toute la eavité de l'ovaire, et donnant attaehe aux ovules à leur point d'origine sur les parois de l'ovaire. Le fruit est charnu, ombiliqué à son sommet; e'est une péponide. Les graines, à la maturité du fruit, semblent éparses au milieu d'un tissu eellulaire, filamenteux ou eharnu. Le tégument propre est assez épais, et recouvre immédiatement un gros embryon homotrope dépourvu d'endosperme.

Les genres principaux de cette famille sont: Cucumis, Cucurbita, Pepo, Ecballium, Monordica, Bryonia, Gronovia, etc. Elle a des rapports assez grands avec la famille des Onagres, dont elle diffère très-bien par la structure de son périanthe, et surtout cclle de son fruil. Elle se rapproche également bcaucoup des Nopalées et des Ribésiées. Quant au genre Passifora, d'abord placć dans cette famillc, il est devenu le type d'un ordre distinct sous le nom de Passiflorécs.

\section{CENT TREMTE-SEPTIEME FAMILIE.}

\section{IoAsées. Loasece. Juss.}

Plantes herbaeées, rameuses, souvent couvertes de poils hispides, et dont la piqûre est brûlante eomme eelle des orties; leurs feuilles sont alternes ou opposées, entières ou diversement lobées; leurs fleurs, assez souvent jaunes et grandes, sont tantôt solitaires, tantôt diversement groupées. On y trouve un ealiee monosépale, tubuleux, libre ou adhérent avee l'ovaire infère, ayant son limbe à cinq divisions; une corolle de einq pétales réguliers, planes ou eoncaves; la gorge du calice est quelque. 
fois garnie de cinq appendices, ou d'un rebord déeoupé. Les étamines, généralement très-nombreuses, sont quelquefois en même nombre que les pétales. L'ovaire est libre ou infère, à une seule loge, offrant intérieurement trois trophospermes pariétaux, quelquefois saillans en forme de clọisons, et portant plusieurs ovules. Cet ovaire est surmonté de trois longs styles grêles, quelquefois réunis en un seul, et terminés chacun par un stigmate simple ou en forme de pinceau. Le fruit est une capsule, courommée par les lobes du calice, ou nue, s'ouvrant, par son sommet seulement, en trois valves qui portent un des trophospermes sur le milieu de leur face interne, excepté dans le genre Loasa, où les trophospermes correspondent aux sutures. Les graínes, quelquefois arillées, offrent un embryon homotrope dans un endosperme charnu.

Cette famille se compose des genres Loasa, Mentzelia, Klaprothia, anxquels M. Kunth a ajouté le Turnera et le Piriqueta. Elle a de grands rapports avec les Onagrairés et les Nopalées, mais en diffère par des caractères très-tranchés. Ainsi dans les Onagrairès l'ovaire est pluriloculaire; les étamines sont en nombre déterminé, etc. Dans les Nopalées, le fruit est charna et la graine sans endosperme.

\section{CLNT TRENTE-HUITIIME FAMITIE.}

passiflorées. Passiflorece. Juss.

Plantes herbacées, ou arbustes à tige sarmenteuse? munies de vrilles extra-axillaires, et de feuilles alternes simples ou lobées, et aecompagnées de deux stipules à leur base. Plus rarement, ce sont des arbres dépourvus de vrilles. Leurs fleurs sont en général grandes et solitaires; plus rarement elles forment une sorte de grappe. Ces fleurs sont hermaphrodites, ayant un calice monosépale, turbiné su longuement tubuleux, à cinq divisions plus ou moins profondes, quelquefois colorées; une eo- 
rolle de cinq pétales, insérés au haut du tube du calice; cinq étamines monadelphes par leur base, et formant un tube qui recoúvre le support de l'ovaire, et se soude avec lui; les anthères sont versatiles, à deux loges. En dehors des étamines, sont des appendices de forme très-variée, tantôt filamenteux, tantôt sous la forme d'écailles ou de glandes pédicellées, réunis crirculairement, et formant d'une à trois couronnes qui naissent à l'orifice et sur les parois du tube calycinal; quelquefois ces appendices, et même la corolle, manquent complétement. L'ovaire est libre, longuement stipité, ì une seule loge, offrant de trois à cinq trophospermes longitudinaux, qui parfois sont saillans en forme de fausses cloisons, et qui donnent attache à un grand nombre d'ovules; il est surmonté de trois ou quatre styles, terminés par autant de stigmates simples; rarement les stigmates sont sessiles. Le fruit est charnu intérieurement, contenant un très-grand nombre de graines; plus rarement il est sec, mais toujoures indéhiscent. Les graines ont un endosperme charnu, dans lcquel est un embryon homotrope et axile.

Selon M. dc Jussiea, Ics Passiflorées, de même que les Cucurbitacćes, n'auraient qu'un périanthe simple, et l'organe que nous avons décrit comme la corolle, et qui manque dans quelques genres, devrait être assimilé aux appendices nombrcux qui garnissent le tube da calice. Quelle que soit l'opinion que l'on adopte à cet ćgard, il n'en restc pas moins très-difficile de déterminer avec cxactitude la placc des Passiflorées dans la série des ordres naturels. Elles ne nous paraissent avoir que de bien faibles rapports avec les Cucurbitacćes, parmi lesquelles le genre Passiflore avait étć rangé. Mais cependant on pcut leur trouver quelque affinitééloignée avec certaines familles de plantes polypétales, et en particulier avec les Capparidées, et surtout avec les Loasées, dans le voisinage desqrelles elles nous paraissent devoir être rangées.

Les Passiflorées sc composcnt des genres Passiflora, Tacsonia, Murucuja, Malesherbia, Deidamia, Kolbia, ct probablement le Carica, placé aussi dans les Cucurbitacées. 


\section{CENT TRENTE-NEUVIME FAMILIE.}

* hygrobiées. Hy grobiere. Rich.Cercodiennes. Juss. Haloragere. R. Rrown.

Petite famille eomposée en ;énéral de plantes aquatiques, portant souvent des feuiles verticillées; des fleurs très-petites, axillaires, et quel[uefois unisexuées, ayant un caliee monosépale, adhérert avec l'ovaire infère, et terminé supérieurement par un limbe à trois ou quatre lobes; la corolle, qui manque quelquefois, se eompose de trois à quatre pétales alıernes avec les lobes du ealice. Les étamines sont en nombre igal ou double des pétales, auxquelles elles sont opposées Jans le premier cas. L'ovaire présente de trois à quatre loges, eontenant ehaeune un seul ovule renversé. Du sommet de l'ovaire naissent trois ou quatre stignates filiformes, glanduleux ou velus. Le fruit est une baie, ou une eapsule eouronnée par les lobes du calice, à plusieurs loges monospermes. Chaque graine, qui est renversée, contient dans un endosperme eharnu, un embryon eylindrique et homotrope.

Les genres qui composent cette famille avaient d'abord étć placés parmiles Onagraircs ou les Nayades. Ces genressont Myriophyllum, Haloragis, Cercodia, Proserpinaca, Trixis, etc. Elle differe surtout des Onagraires par son ovaire a loges monospermes, ses graines pendantes et son embryon pourvu d'un endosperme charnu.

\section{CENT QUARANTIEME FATIILIE.}

* onagrariées. Onagrarice. Juss.

Végétaux herbacés, rarement fruteseens, portant des feuilles simples, opposées ou éparses, et des fleurs terminales ou axillaires. Leur ealiee est adhérent avee l'ovaire infére; son limbe, à quatre ou einq lobes; la eorolle, forméc de quatre à cinq pétalcs, incombans latéralement 
et tordus en spirale, avat leur parfait épanouissement; cette corolle manque rrement. Les étamines sont en même nombre ou doublı, quelquefois moindre, des pétales; elles sont inséréesau tube du calice. L'ovaire, infère, offre de quatre à cinc loges, contenant un assez grand nombre d'ovules attaché à leur angle interne. Le style est simple, et le stigmate st tantôt simple, tantôt à quatre ou cinq lobes. Le fruit est une baie ou une capsule à quatre ou cinq loges, ne contenant souvent chacune qu'un petit nombre de graines, 3t s'ouvrant en autant de valves portant chacune une des cloisons sur le milieu de leur face interne. Les graines ofrent un tégument propre, en général formé de deux feuilets, et recouvrant immédiatement un embryon homotrope et dépourvu d'endosperme.

M. de Jussieu, dans sa famille des Onagres, avait d'abord placé un eertain nombre de genres qui en ont été suceessivement retirés. Ainsi le genre Mocanera nous parnît apparterir à la famille des Ternstræmiaeées; le Cercodea forme le type de la famille des Hygrobiées. Les genres Cacoucia, Combretum, rentrent dans les Combrétacées; le Santulum formc le type des Santalacées; les genres Mouriria et Petaloma nous paraissent appartenir aux Mélastomaeées, ct enfin les genres Loasa et Mentzelia eonstituent la famille des Loasées.

On trouve cntr'autres genres dans les Onagrariées les Epilobium, OErothera, Lopezia, Circosa, Jussicea, Fuschia, etc. Très-voisine des Myrtacées et des Mélastomacécs, la famille des Onagrariées se distingue des premières par ses feuillcs non ponetuées, ses étamines en nombre déterminé et son port; des Mélastomacées, par la straeture si différente de leurs feailles et de leurs anthères.

\section{CENT QUARANTE-UNIEME FAMIIIE.}

combrétucées. Combretacea. R: Brown. Elacagnorum et Terminaliarum gen. Juss.

Ce sont des arbres, des arbrisseaux ou des arbustes à feuilles opposées ou altermes, entières ou sans stipules; portant des fleurs hermaphrodites ou polygames, diversement disposées en épis axillaires ou terminaux; leur 
caliee est adhérent par sa base avee l'ovaire, qui est infère; son limbe, souvent tubulcux, est à quatre ou cinq divisions, et articulé avec le sommet de l'ovaire; la corolle manque dans plusieurs genres, ou se compose de quatre à cing pétales insérés entre les lobes du calice. Le nombre des étamines est en général double des divisions calycinales; cependant ee nombre n'est pas rigoureusement détcrminé. L'ovaire est à une seule loge, contenant de deux à quatre ovules pendans de son sommet. Le style est plus ou moins long, terminé par un stigmate simple. Le fruit est constamment uniloculaire, monosperme par avortement, et indéliscent. La graine, qui est pendante, se compose d'un épisperme qui reeouvre immédiatement l'embryon.

Les Combrétacées se composent de genıes, d'abord rapportés les uns aux Eléagnées, et les autres aux Onagraires; tels sont Bucida, Terminalia, Conocarpus, Quisqualis, Combretum, ete. Cette famille ne paraît pas, au premier conp d'œil, réunir des genres ayant entr'eux une très-grande affinité. En effet, les uns sont pourvas de pétales, et les autres en manquent; les uns ont les cotylédons planes, les autres les out roulés sur eux-mêmes. Mais le caractère vraiment distinctif de cette famille consiste dans son ovaire uniloculaire, contenant de deuxà quatre ovules pendans du sommet de la loge sans podosperme. Par ses genres apétales, cette famille tient aux Santalacées, qui s'en distinguent surtout par la présence d'un endosperme, et lenrs ovales dressés; par ses genres pétalés, elle se rapproche beaucoup des Onagres et des Myrtacées, entre lesquelles elle doit être placée.

\section{CENT QUARANTL-DSUXIEME FAMILLI,}

* myrtaé́es. Myrtacece. Juss.

Cette famille intéressante se compose d'arbres ou d'arbrisseaux d'un port élégant, dont les diverses parties sont pleines d'un suc résineux et odorant. Les feuilles sont opposées, cntièrcs, souvent persistantes, marquées de points translucides; les fleurs sont diversement disposées, soit à l'aisselle des feuilles, soit au sommet des rameaux. Leur 
calice est monosépale, adhérent par sa base avec l'ovaire infère, ayant son limbe à cinq, six ou seulement quatre divisions; la corolle, qui manque rarement, est formée d'autant de pétales qu'il y a de lobes au calice. Les étamines, génćralement très-nombrcuses, rarement en nombre déterminé, ont leurs filets libres ou diversement soudés, et leurs anthères terminales et généralement assez pelites. L'ovaire, infère, préscnte de deux à six loges, qui contiennent un nombre variable d'ovules attachés ì leur angle interne. Le stylc est généralement simple, et lc stigmate est lobć. Le fruit offre un grand nombre de modifications; il est tantôt sec, déliiscent en autant de valves qu'il y a de loges, tantôt indéliscent ou charnu. Les graines, généralernent dépourvues d'endosperme, offrent un embryon dont les cotylédons ne sont jamais ni convolutés, ni roulés en cornet l'un sur l'autre.

Le professeur de Candolle a divisé la famille des Myrtacées en cinq tribus naturelles, qui sont :

$I^{0}$ Les Chamílaucrés, fruit sec uniloculaire, graines basilaires, calice à cinq lobes; corolle de cinq pétales, manquant quelquefois; étamines libres ou polyadelphes. Les geures qui forment cette tribu sont tous originaires de la Nouvelle-Hollande : Calytrix, Chancelancium, Pileanthus, etc.

$2^{\circ}$ Les Leptosperućes, fruit sec déhiscent i plusieurs loges; graines attachées à l'angle interne, dépourvues d'arille et d'endosperme; feuilles opposées ou alternes. Arbrisseaux tous originaires de la Nouvelle-Hollande : Beaufortia, Calotannus, Tristania, Melaleuca, Eudesmia, Eucalyptus, Metrosyderos, Leptospermum, ete.

30 Les MyrtéEs, fruit eharnu, généralement à plusieurs loges ; graines sans arille ni endosperme; étamines libres; feuilles opposées. $\Lambda$ rbrisseaux presque tous originaires des Tropiques: Eugenia, Jambosa, Calyptranthes, Caryophyllus, Myrtus, Campornanesia, ete.

40 Les Barrongtowrées, fruit sec ou charnu, toujours indéhiseent à plasieurs loges; étamines monadelphes par la base; feuilles alternes non ponetuées. Arbres des régions équinoxiales de l'ancien et du nouvaeu continent: Dicalyx, Stravadium, Barringtonia, Gustavia.

$5^{n}$ Léçтhroées, fruit sec, s'ouvrant par un opercule (pyxide) ; étamines très-nombreuses, monadelphes; feuilles alternes non ponctuées. Grands arbres de l'Amérique équinoxiale : Leçtthis, Couratari, Couroupita, Bertholletia. 
La famille des Myrtacées, considérée dans son ensemble, forme une famille fort distinctc parmi les Dicotylédons à ovaire infère; elle a des rapports avec les Mélastomacées, qui en diffèrent par la disposition si remarquable et si constante des nervares de lenrs feuilles, et par le nombre et la structure dc lenrs étamines; avec les Onagres, qui s'en éloignent par leurs étamines en nombre déterminé; avec les Rosacées et les Combrétacćes, dont les feuilles alternes, les styles multiples dans la première, l'enbryon à lobes roulés dans la seconde de ces deux familles, forment les caractères distinctifs.

\section{CENT QUARANTEMROISIX̀ME FAMIIIE.}

mélastonacées. Melastomacex. Juss.

Les Mélastomacées sont de grands arbres, des arbrisseaux, des arbustes ou des plantes herbaeées, ayant des feuilles opposées, simples, munies généralement de trois à einq et même jusqu'à onze nervures longitudinales, d'où partent un très-grand nombre d'autres nervures transversales et parallèles, très-rapproehées. Les fleurs, quelquefois très-grandes, offrent en quelqque sorte tous les modes d'infloreseenee. Leur caliee est monosépale, plus ou moins adhérent avee l'ovaire, qui est infère ou semiinfère; son limbe est quelquefois entier, ou denté, ou enfin à quatre ou cinq divisions plus ou moins profondes; plus rarement il forme une sorte de eoiffe ou d'opereule; la eorolle se eompose de quatre à einq pétales; les étamines sont en nombre double des pétales. Leurs anthères présentent les formes les plus variées et les plus siugulières, et s'ouvrent à leur sommet par un trou ou pore eommun aux deux loges. L'ovaire est quelquefois libre, plus souvent adhérent avee le caliee; il offre de trois à huit loges eonteuant chaeune un très-grand nombre d'ovules. Le sommet de l'ovaire est souvent tapissé par un disque épigyne. Le style et le stigmate sont simples. Le fruit est tantôt scc et tantôt charnu, offrant le mềme nombre de loges que l'ovaire; il reste indéhiscent ou s'ouvre en autant de valycs septifères sur le milien de leur face interne. Les 
graines sont fréquemment reniformes; elles contiennent un embryon dressé ou légèrement reeuurbé, mais sans endosperme.

Cettc famille, qui vient d'être travaillée arcc soin par le professear de Candolle, dans le troisième volume de son Prodrome, est très-nombreusc en espèces, qui ont été groupées en un grand nombre de genres. Parmi ces genres on trouve les suivans: Melastoma, Rhexia, Miconia, Tristemma, Topoboca, etc. Elle est tellement distincte par la disposition des nervares de ses feuilles, qn'elle ne peut être confondue avec aucune autre de celles dont elle se rapproche, comme les Onagrcs, les Myrtacées et les Rosacées.

\section{CENT QUARANTE-QUATRIEME FAMILIE.}

\section{* salicaruées. Salicarice. Juss.}

Herbes ou arbustes à feuilles opposées ou alternes, portant des feuilles axillaires ou terminales; un ealice monosépale, tubuleux ou ureéolé, denté à son sommet; une eorolle de quatre à six pétales alternes avee les divisions du caliee et insérés à la partie supérieure de son tube; la eorolle manque dans quelques genres. Les étamines sont en nombre égal ou double des pétales, plus rarement en nombre indéfini. L'ovaire est libre, simple, à plusieurs loges, contenant ehaeune un assez grand nombre d'ovules. Le siyle est simple, terminé par un stigmate ordinairement eapitulé. Le fruit est une capsule recouverte par le caliee, qui est persistant, à une ou plusieurs loges, eontenant des graines attachées à leur angle interne. Ces graines se composent d'un embryon dépourvu d'endosperme.

Parmi les genres qui composent cctte famille, on peut citer les suivans: Lythrum, Cuphea, Ginoria, Lagocrtrocmia, Ammania, etc. Cette famille a de l'affinité avcc les Onagres, dont elle diffère par son ovaire libre; avec les Rosacées; mais celles-ci ont constamment des stipules et un grand nombre d'autres caractères qui les distinguent des Salicariées. 


\section{CENT QUARANTE-CINQUIEME FARILIE.}

* tamariscinées. Tamariscinece. Desvaux.

Arbustes ou arbrisseaux ayant des feuilles en général très-petites, squammiformes et engaînantes; des fleurs également petites, munies de bractées et disposées en épis simples, dont la réunion constitue quelquefois une panicule. Leur calice est à quatre ou cinq divisions profondes; rarement il forme un tube à sa partie inférieure; ses divisions sont imbriquées latéralement; la corolle se compose de quatre à cinq pétales persistans; les étamines, au nombre de cinq à dix, rarement de quatre, sont monadelphes par leur base. L'ovaire est triangulaire, quelquefois entouré à sa base d'un disque périgyne; le style simple ou triparti. Le fruit est une capsule triangulaire, à úne seule loge, contenant un assez grand nombre de graines attachées vers le milieu de la face interne des trois valves qui forment la capsule. L'embryon est dressé, dépourvu d'endosperme.

Cette petite famille se compose du genre Tamarix, que M. Desvaux, professeur de botanique à Angers, propose de diviser en deux genres, savoir : Tamarix et Myricaria. Ce genre Tamarix faisait d'abord partie de la famille des Portulacées, dont il diffère par son port et son embryon dépourva d'endosperme. Par ce dernier caractère, la famille des Tamariscinées a quelques rapports avec les Lythraires.

\section{CENT QUARANTE-SIXIỲME FAMIILE.}

* rosactes. Rosacede. Juss.

Grande famille composée de végétaux herbacés, d'arbustes ou d'arbres atteignant de très-grandes dimensions. Leurs feuilles sont alternes, simples ou composées, accompagnées à leur base de deux stipules persistantes, quelquefois soudées avec le pétiole. Les fleurs offrent différens modes d'inflorescence. Elles se composent d'un calice mo- 
nosépale, à quatre ou einq divisions, quelquefois aceompagné extéricurement d'une sorte d'involucre qui fait corps avee le ealiee, de manière que eelui-ci paraît à huit ou dix lobes. La corolle, qui manque rarement, est composée de quatre à einq pétales régulièrement étalés; les étamines sont généralement en grand nombre et distinetes. Le pistil présente plusieurs modifieations : tantôt il est formé d'un ou de plusieurs earpelles, entièrement libres et distincts, placés dans un ealíce tubuleux; tantôt ces carpelles adhèrent par leur côté extérieur aree le calice; tantòt ils sont soudés non-seulement avee lc ealiee, mais entr'eux; tantôt ils sont réunis en une sorte de eapitule sur un réceptacle ou gynophore. Chacun de ees carpelles est uniloculaire et contient un, deux ou un plus grand nombre d'ovules dont la position est très-variée. Le style est toujours plus ou moins latéral et le stigmate simple. Le fruit est extrêmement polymorphe; tantòt c'est une véritable drupe, tantôt une mélonide ou pomme, tantòt un ou plusieurs akènes, ou une ou plusicur's capsules déhiscentes, ou, enfin, une réunion de petits alènes ou de petites drupes formant un eapitule strr un gynophore quidevient eharnu. Les graines ont leur enubryou homotrope et déponurvin d'endosperme.

Cette grande famille a été divisée en tribus, dont quelques-unes ont été considérées pâr quelques auteurs comme des familles distinctes.

r re triba. Chrüsobatavérs (R. Brows), ovaire unique lilure, contenant deux ovules dressés; style filiforme, naissant presque de la base de l'ovaire; fleurs plas ou moins irrégulières; fruit drupacé. Ex. : Chrysobalanns, Parinarimm, Moquilea, etc.

${ }_{2}$ e tribu. Drupacies, D. C. , ovaire nnique libre, eontenant deux ovules collatéraux; style filiforme terminal; fleurs régulières; fruit drupacé. Ex.: Prudus, Amyscallus, Cerasus, etc.

3* tribu. Srrrícḱs (Rrch.), plusieurs ovaires libres ou légèrement soudés entr'eux par leur eóté interne; contenant deux ou quatre ovules collatéraux; style terminal; capsules distinetes uniloenlaires, on une seule capsule polysperme. Ex.: Spiraca, Kerria.

$4^{e}$ trilur. Fragarncés ( Rrem.), calice étalé, souvent muni d'un calicule 
extériear; plusiears carpelles monospermes incléhiscens; rćnnis quelquefois sur un gynophore charnu; style plus ou moins latćral. Ex. : Potentilla, Fragaria, Geum, Rubus, Dryas, Comarum, etc.

5e tribu. Sargursoruées ( J.), fleurs ordinairement polygames et quelquefois sans corolle; un ou deux carpelles, quelquefois adhérens avec le calice, terminés par un style et nn stignate en formede plume ou de pinceau. Ex. : Poterium, Clifforia, Alchemilla, etc.

$6^{e}$ tribu. Roséss (J.), calice tubuleux, urcéolé, contenant un nombre variable de carpelles monospermes attachés à la paroi interue du calice, qui dexient charnu et les recouvre. Ex. : Rosa.

$7^{e}$ tribu. Pomacíes (Rrce.), plusieurs carpelles uniloculaires, contcnant chacun deux ovules ascendans, rarement an grand nombre attaché au côté interne, soudés entr'eux et avec le calice, et formant un fruit charnn, connu sous le now de mélonide ou de pomme. Ex: Malus, Pyrus, Cracasus, Sorbus, Cydonia, etc.

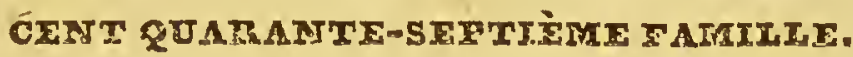

homalinées. Homalinea. R. Brown.

Les Homalinées sont des arbustes ou des arbrisseaux, tous originaires des eontrées chaudes du globe. Leurs fenilles sont alternes, pétiolées, simples, munics de stipules eaduques. Leurs fleurs sont hermaphrodites, disposées en épis, en grappes ou en panieulc. Leur ealice est monosépale, ayant soll tube eourt, eonique, adhérent avec l'ovaire; son limbe divisé en dix à trente lobes, dont les plus extérieurs sont plus grands et valvaires, et les intérieurs plus petits et en forme de pétales. La corolle manque; à la face interne et le plus souvent vers la base des sépales intérieurs sont situés des appendiees glanduleux et sessiles. Le nombre des étamines varic; il est quelquefois égal à eelui des lobes extérieurs du ealiee, et les étamines leur sont opposées; d'autres fois les étamines sont plus nombreuses et réunies par faisceaux. L'ovaire est généralement semi-infère, ì une seule loge eontenant un grand nombre d'ovules attachés à trois ou eing trophospermes pariétaux. Les styles, en mêtme nombre que 
les trophospermes, se terminent chacun par un stigmate simple. Le fruit est tantôt sec, tantôt charnu. Les graines ont leur embryon placé dans un endosperme eliarnu.

Famille encore peu connue, établie par M. R. Brown, dans son Mémoire sur les plantes du Congo, et adoptée par M. de Candolle (Prodr.syst., 2 , p. 53), qui y place les genres suivans: Homaliurn, Napimoga, Pineda, Blackwellia, Astranthus, Nisa, Myriantheia, Asteropeia et Aristotelia. Par la structure de son fruit, cette famille se rapproche des Flacourtianées et des Samydées, et par son insertion elle vient se placer près des Rosacées.

\section{CENT QUARARTE-HUITIÈME FAMIIIE.}

\section{samydées. Samydece. Vent.}

Arbrisseaux tous exotiques, et eroissant dans les régions les plus chaudes du globe, portant des feuilles alternes, distiques, simples, persistantes, le plus souvent marquées de points translueides, munies de deux stipulés à leur base. Les fleurs sont axillaires, solitaires ou groupées. Elles ont un calice formé de einq, plus rarement de trois à sept sépales, réunis ensemble à leur base, et formant quelquefois un tube plus ou moins allongé; le limbe offre des divisions plus ou moins profondes et colorées sur leur face interne. La corolle manque eonstamment. Les ćtamines sont en nombre égal, double, triple ou quadruple des divisions calyeinales, à la base desquelles elles sont insérées; elles sont monadelphes, et quelques-unes d'entre elles sont parfois stériles et réduites à leur filet, qui devient plane et velu. L'ovaire est libre, à une seule loge, eontenant un grand nombre d'ovules insérés à trois ou cinq trophospermes pariétaux. Le style est simple, terminé par un stigmate capitulé ou lobé. Le fruit est une eapsule uniloculaire, s'ouvrant en trois ou einq valves qui portent sur le milieu de leur face interne, les graines, enveloppées dans une pulpe plus ou moins abondante et eolorée. Ces graines offrent un endosperme charnu, dans lequel 
est un embryon très-petit, hétérotrope, e'est-à-dire ayant sa radieule opposée au hile ou point d'attaehe de la graine.

Cette famille se compose desgenres Sanyda, Anavringa et Casearia. Par la structure de son fruit elle se rapproche des Violettes et des Flacourtianées; mais son insertion, évidemment périgyne, la rejette du côté des Rosacées, dont plusieurs genres sont également apétales. Indépendamment des trois geures cités plus haut, on doit encore rapporter à cette famille le genre Piparea d'Aublet, rangé jusqu'à présent parmi les Violacées.

\section{CENT QUARANTE-NEUVIEME FAMITLE.}

\section{* Légumineuses. Léguminosce. Juss.}

Famille très-naturelle, et dans laquelle sont réunis des plantes herbaeées, des arbustes ou des arbrisseaux et des arbres souvent de dimensions eolossales. Leurs feuilles sont alternes, eomposées ou déeomposées, quelquefois simples, rarement les folioles avortent, et il ne reste que le pétiole, qui s'élargit et forme mne sorte de feuille simple. A la base de ehacune d'elles, sont deux stipules souvent persistantes. Les fleurs offrent une infloreseenee très-variée. Elles sont en général hermaphrodites. Leur ealiée est tantôt tubuleux, à cinq dents inégales, tantôt à einq divisions plus ou moins profondes et inégales; en dehors du caliee, on trouve une ou plusieurs bräetées, ou quelquefois un involueire ealyeiforme; la eorolle, qui manque quelquefois, se compose de einq pétales généralement inégaux, dont un supérieur, plus grand, qui enveloppe les autres et qu'on nomme étendard; deux latéraux appelés ailes; è deux inférieurs plus oü moins soudés ensemble, et formant la carènè; tantôt la eorolle est formée de einq pétales égaux. Les étamines sont généralement au nombre de dix, quelquefois plus nombreuses; le plus souvent leurs filets sont diadelphes, rarement monadelphes, ou entièrement libres, périgynes ou hypogynes. Liovaire est plus ou moins stipité à sa 
base; il est en général allongé, inéquilatéral, à une seule loge, contenant un ou plusieurs ovules attachés à la suture interne. Le style est un peu latéral, souvent recourbé et terminé par un stigmate simple. Le fruit est constamment une gousse. Les graines sont en général dépourvues d'endosperme.

Cette grande famille est composée d'un nombre très-considérable de genres qui peuvent être divisés en trois tribus naturelles, savoir :

$\mathrm{I}^{\circ}$ Les Parritoxacḱes, qui ont une corolle forniée de cinq pétales inégaux, formant la corolle irrégulière nommée papilionacée; dix ćtamines en général diadelphes. Ex.: Phaseolus, Faba, Lathyrus, Robinia, Glycine, Astragalus, Phaca, etc.

$2^{\circ}$ Les CAssréks, dont la corolle est en général formée de cinq pétales régaliers, les dix étamines en général libres. Ex.:Cassia', Bauhinia, Geoffrcca, ete.

$8^{\circ}$ Les MrMosÉes, renfermant les genres apétales, munis d'un involuere en forme de calice; des étamines très-nombreuses et libres. Ex. : Mimosa, Acacia, Inga, etc.

La famille des Légumineuses est très-voisine des Rosacées, et qnoiqu'au premier abord il paraisse très-aisé de les distinguer, cependant il faut convenir qu'il y a des genres qui servent en quelque sorte à étahlir le passage de l'un à l'autre.

\section{, CENT CINQUANTIÈME FAMILIE.}

* térébinthacées. Terebinthacec. Juss.

Arbres ou arbrisseaux souvent laiteux ou résineux; ayant des feuilles alternes, généralement composées, sans stipules; des fleurs hermaphrodites ou unisexuées, petites et généralement disposées en grappes; ehacune d'elles présente un calice de trois à einq sépales, quelquefois réunis ensemble à leur base, et soudés avec l'ovaire, qui est infère; une eorolle, qui manque quelquefois et se eompose d'un nombre de pétales, égal aux lobes du calice, et régulier. Les étamines sont en général en nombre égal, plus rarement double ou quadruple des pétales; dans le premier cas elles alternent avec les pétales. Le pistil se compose de trois à cinq carpelles, 
tantôt distincts, tantôt plus ou moins soudés entre eux, environmés à leur base d'un disque périgyne et annullaire; quelquefois quelques caspelles avortent, et il n'en reste qu'un, duquel naissent plusieurs styles. Chaque carčclle est à unc seule loge, eontenant tantôt un ovule porté au sommet d'un podosperme filiforme, qui naît du fond de la loge, tantôt un ovule renversé, tantôt deux ovules renversés ou eollatéraux. Les fruits sont secs ou drupacés, contenant généralement une seule graine. Cẻlle-ei renferme un embryon dépourvu d'endosperme.

Cette famille a été l'objet d'un excellent travail de la part de notre excellent ami le professeur Kunth. On peut diviser cette famille en sept tribus naturelles, savoir : 1o les Anacardiées on Cassuviérs, contenant les genres Anacardium, Mangifera, Pistacia, etc.; $2^{\circ}$ les Sumachisées, où l'on trouve les geures Rhus, Mauria, Duvaua, etc.; $3^{\circ}$ les Srondracíxs, qui comprennent les genres Spondias et Poupartia; $4^{\circ}$ les Burséracíes, oi sont réunis les genres Icica, Boswellia, Bursera, Canarium, etc.: 50 les Amyrinées, ex. : Anjris: 60 les Connañacées, ex: Connarus, Omphalobium, Cnestis, etc.; $7^{\circ}$ les Jugrandérs, ex. : Juglans, Carya, etc.

Cette famille a de très-grands rapports avec celle des Légumineuses, dont elle se distingue surtout par l'absence des stipules; elle offre aussi de l'affinité avec les Rhamnées, qui en diffèrent par leur ovaire constamment infère et leurs étamines opposées aux pétales.

\section{CERT CINQUANTE-UNIEME FAMILIE.}

* RHAMnÉEs. Rhamnece. R. Brown. - Rhamnorum pars. Juss.

Ce sont des arbres ou des arbustes à feuilles simples et alternes, très-rarement opposées, munies de deux très. petites stipules eaduques ou persistantes et épineuses. Les fleurs sont petites, hermaphrodites ou unisexuées, axillaires, solitaires ou réunies en sertute, en faiseeaux, ete. ; quelquefois formant des grappes ou des eapitules terminaux. Leur caliee est monosépale, plus ou moins tubuleux à sa partic inférieure, où il adhère avee l'ovaire qui est infère, ayant un limbe érasé, à quatre ou cinq lobes valvạires; 
la corolle se eompose de quatre à einq pétales onguieulés, très-petits, souvent roulés et coneaves; les étamines, en nême nombre que les pétales, sont placées en faee d'eux et en sont souvent em] rassées. L'ovaire est tantôt libre, tantôt semi-infère, ou eomplétement adhérent, à deux, trois ou quatre loges, eontenant ehaeune un seul ovule dressé; du sommet de l'ovaire, partent en général autant de styles qu'il y a de loges; la base du tube du ealiee, quand l'ovaire est libre, ou le sommet de ce dernier quand il est infère, présente un disque glanduleux, plus ou moins épais. I.e fruit est eharnu et indéhiseent ou sec et s'ouvrant en trois eoques. La graine est dressée, et eontient dans un endosperme charnu, quelquefois trèsminee, un embryon homotrope, ayant les eotylédons très-larges et très-minees.

La famille des Rhamnées, telle qu'elle avait été établie par le eélèbre auteur du Genera Plantarum, a vait été divisée en quatre seetions. M. Robert Brown le premier a proposé de former des deux premières sections une famille distinete, sous le nom de Célastrinées. Cette famille se distingue surtout par son ealice, dont les lobes sont imbriquées et non valvaires, par ses étamines alternes et non opposées aux pétales, et par son ovaire tou. jours libre, et dont les loges contiennent un ou deux ovules latéraux et superposés, par son fruit constamment see, et s'ouvrant au moyen de valves septifères snr le milieu de leur face interne.

M. Robert Brown a proposé de plus de faire une famille particulière ayant pour type le genre Brunia. Cette division de la famille a été adoptée par M. de Candolle dans le deuxième volume deson Prodromc, et par M. Brongniart fils, dans sa Dissertation sur la Famille des Rhamnées. Parmi les genres de Rhamnées, nous pouvons eiter iei les suivans: Rhamnus, Paliurus, Ceanothus, Colletia, ete.

\section{CENT CINQUANTE-DEUXIÈME TAMIXIE.}

* célastrinées. Celastrinea. R. Brown. Ad. Brong.Rhamnorum pars. Juss.

Cette famille est composée d'arbustes ou d'arbrisseaux à feuilles alternes, ou quelquefois opposées, à fleurs axil- 
laires disposées en cimes. Lè calice, légèrement tubuleux à sa base, offre un limbe à quatre ou cinq divisions ćtalées, imbriquées lors de leur préfleuraison. La corolle se compose de quatre à cinq pétales planes, légèrement charnus, sans onglet, insérés sous le disque. Les étamines alterues avec les pétales sont insérées, soit sur le bord du disque, soit sur sa face supérieurc. Le disque est périggne et pariétal, environnant l'ovaire. Celui-ci cst libre, à trois ou cuatre loges, contenant chacune un ou plusieurs ovules attachés par un podosperme filiforme à l'angle interue de chaque loge, et ascendant. Le fruit, qui est quelquefois une drupe sèche, est plus souvent une capsule à trois ou quatre loges s'ouvrant en trois ou quatre valves qui portent chacune une cloison sur le milieu de leur face interne. Les graines, quelquefois recouvertes d'un arille charnu, contiennent un endosperme charnu dans lequel est un embryon axile et homotrope.

Nous avons, en parlant des Rhamnées, indiqué les principales différences qui existent entre celte famille et celle des Célastrinées. M. de Candolle, dans son Prodrome, divise cette dernière famille en trois tribus, savoir : les Staphyléacées, les Evonymées et les Aquifoliacées. M. Adọ. Brongniart se range de la première opinion du célèbre professeur de Genève, qui, dans sa Théorie élémentaire, avait considéré les Aquifoliacées comme une famille distincte. En effet, ce groupe se distingue des vraies Célastrinées par sa corolle souvent monopétale, son insertion hypogyne, l'absence complète da disque; les loges de son ovaire contenant constamment un seul ovule pendant; son fruit charnu contenant de deux à six nucules osseux.

\section{CENT CINQUANTE-TROISTËME FAMIIIE.}

*aquifoliacées. Aquifoliacec. DC. - Ilicinece. Ad. Brong.

Arbrisseaux à feuilles alternes ou.opposées, coriaces, persistantcs, glabres, ̀̀ dents quelquefois épineuses, ayant leurs fleurs solitaires ou diversement groupées à l'aisselle des feuilles. Chacune d'elles offre un calice de 
quatre à six sépales pelits et imbriqués; une corolle d'un égal nombre de pétales alternes, soudés enscmble par leur base, et formant unc corolle monopétale, à divisions profondes et lypogynes. Les étamines, alternes avec les lobes de la corolle, sont insérćes à sa base; il n'y a pas de trace de disque; l'ovaire est libre, épais, tronqué, ayant de deux à six loges, qui contiennent chacune un seul ovule pendant du sommet de la $\operatorname{loge}$, et porté sur un podosperme cupuliforme; le stigmate est cn général sessile et lobé. Le fruit est constamment charnu, contenant de deux à six nucules indéhiscens, ligneux ou fibreux et monospermes. L'embryon est petit, homotrope et placé vers la base d'un endosperme cliarnu.

Cette famille, ainsi que nous l'avons démontrć en parlant des Célastrinées, est fort distinctc des vraies Rhamnćes et des Célastrinées, avec lesquelles ellc avait étć réunie. Ces différences sont mềme si grandes, que M. de Jussieu, et plus tard le professeur de Candolle, avaient eru pouvoir ranger les Aquifoliaeées parmi les Monopétales, auprès des Sapotacées et surtout des Ebénacces, dont elle ne diffère que par des caractères peu importans. Mais M. de Candolle a depuis abandonnć cette opinion, puisque, dans le second volnme de son Pródrome, il fait des Aquifoliacées une simple tribu des Célastrinées. Néanmoins la premic̀re opinion nous parât la plus vraie. Parmi les genres qui eomposent les Aquifoliaeées, nous trouvons les suivans: Ilex, Cassine, Myginda, etc.

\section{QUINZIÈME CLASSE.}

\section{DICLINIE. \\ 1 \\ CENT CINQUATTE-QUATRIF̈ME FAMILIE. \\ * euphonbiacées. Euphorbiacece. Juss.}

Les Euphorbiacées sont des herbes, des arbustes ou de très-grands arbres, qui croissent en général dans toutes les régions du globe. La plupart contiennent un suc laiteux et très-irritant; les feuilles, communément alternes, 
sont quelquefois opposées, accompagnées de stipules, qui manquent quclquefois. Lcs flcurs sont unisexućes; généralement petites, et offrent unc inflorescence trèsvariée. Lcur caliee cst monosépalc, à trois, quatre, cinq ou six divisions profondes, munies intérieurcment d'appendices écailleux et glandulcux. La corolle manquc dans le plus grand nombre des genres, ou se compose dc pétales tantôt distincts, tantôt rćunis en une corolle monopétale. Mais cette corolle nc paraît formée quc par des étamincs avortćcs et stériles. Dans les flcurs mâles, on compte un assez grand nombrc d'étamines; plus rarement ce nombrc est limité, ou mème chaquc étaminc peut être eonsidéréc comme une flcur (ainsi qu'on l'admet pour lc genre Euphorbe). Ces étamines sont libres ou monndelphes. Les fleurs femclles sc composent d'un ovaire libre, scssilc ou stipité, quelqucfois accompagné d'un disquc hypogync. L'ovaire est cn général à trois loges, contcnant chacunc un ou deux ovules suspendus. Du sommct de l'ovaire naissent trois stigmatcs généralcment sessiles et allongés. Le fruit cst sec ou légèrement charnu; il sc compose d'autant de coqucs contenant une ou dcux graincs, qu'il y avait de loges au fruit. Ces coqucs, qui sont osseuses intérieurcment, s'ouvicnt par leur angle internc en deux valves ct avec élasticité. Elles s'appuient par leur angle interne sur unc columellc centrale, qui souvent persiste après leur dispersion. Les graines, qui sont crustacées cxtćrieuremcnt, ct préscntent unc pctite caroncule charnuc, dans le voisinagc de lcur point d'attache, offrent un endosperme charnu, dans lequel est renfcrmé un embryon axile et homotrope.

On doit à M. Adrien de Jussieu une exeellente Monographie des gences de eette famille, qui $\mathrm{y}$ sont au nombie de quatre-vingt-six, eontenant environ unille quarante espèees. Parınices genres il nous suffira de eitcriciles suivans: Euphorbia, Mercurialis, Ricinus, Croton, Iutropha, Hura, Buxus, Acalypha, etc., etc. 
La famille des Euphorbiaećes est extrếmement distincte par la structure de son fruit. Elle a quelques rapports avec certaines Térébinthacées et Rhanonées.

\section{CENT CINQUANTE-CINQUIEME FAMILLE.}

\section{* urticées. Urticece. Kunth. - Urticece. Juss. ct Celtideæ. Rich.}

Plantcs herbacées, arbrisseaux, ou grands arbres quelquefois lactescens, à feuilles alternes, en général munies de stipules; ayant des fleurs uniscxuées, très-rarement hermaphrodites, solitaircs ou diversement groupées, et formant des chatons, ou réunies dans un involucre charnu, plane, étalé, ou pyriforme et clos. Dans les fleurs mâles, on trouve un calice formé de quatre à cinq sépales, distincts ou sondés, ct formant un tube, quatre à cinq étamincs alternes, ou très-rarement opposécs aux sépales. Les fleurs fcmelles ont un calice formé de deux à quatre sépales, ou une simple écaille à l'aissclle de laquellc elles sont placées. L'ovaire est libre, à unc seule loge, contenant un scul ovule pendant, et surmonté, soit de deux longs stigmates sessiles, soit d'un scul'stigmate, porté quelquefois sur un style plus ou moins long. Le fruit sc compose toujours d'un akène crustacé, enveloppé par le calice, qui quelqucfois devicnt charnu; d'autres fois, l'involucre, qui rcnfermait les fleurs femelles, prend de l'accroisscment, ainsi qu'on le remarque dans le figuier, le dorstenia, etc. La graine, outre son tégument propre, se compose d'un embryon en général rccourbé, souvent renfermé dans l'intérieur d'un endosperme plus ou moins mince.

A l'exemple de notre savant ami M. Ie professeur Kunth, nous ávons réuni anx Urtieées les genres Ulinus et Celtis, auparavant placés parmi les Amentaeces, et dont on avait fait la famille des Celtidées. En effet, ee dernier groupe ne diffère par aucun caraetère essentiel des autres Urticées. Cette 
famille, ainsi limitée, peut être divisée en trois tribus de la manière suivante :

Io Certró́gs (Rrch.), fleurs hermaphrodites; embryon sans endosperme. Ex. : Ulinus, Celtis.

$2{ }^{\circ}$ UrxicéEs vraies, fleurs unisexuées; fruits distincts; embryon renfermé dans un endosperme mince. Ex. : Urtica, Parictaria, Humulus, Cannabis, Morus.

3" Artocarpées ( D.C.), fleurs unisexuées; fruits réunis dans un involure charnu, plane ou pyriforme; embryon pourva d'un endosperme. Ex. :Dorstenia, Ficus, ete.

\section{CENT CINQUATTE-SIXIXIEE FAMIRTE.}

Monimúes. Monimice. Juss. Atherospermece. R. Brown.

Arbres ou arbrisscaux, à feuilles opposées, dépourvues de stipules, à fleurs unisexuées. Ces fleurs offrent un involuere globuleux ou ealyciforme, dont les divisions sont disposées sur deux rangées; dans le premier eas, cet involucre offre seulement quelques pelites dents à son' sommet, et dans les fleur's mâles il se rompt et s'ouvre en quatre lobes profonds et assez réguliers, dont toute la surface supérieure est ehargée d'étamines, à filamens eourts, et formant chacune une fleur màle. Dans le seeond eas (Ruizia), les étamines tapissent seulement la partie inférieure et tubuleuse de l'involucre; les filamens sont plus longs, et vers leur partie inférieure ils portent de chaque eôté un tubercule pédicellé, analogue à celui qu’on observe à la. même plaee dans les Laurinées. Les fleurs femelles se eomposent d'un involuere, absolument semblable à celui des fleurs mâles; dans les genres Monimia et Ruizia, on trouve au fond de eet involuere huit à dix pistils dressés, entièrement distincts les uns des autres et entremêlés de poils. Dans l'Ambora, ces pistils sont fort nombreux, entièrement plongés dans l'épaisseur des parois de l'involucre, n'ayant de libre et de visible que leur sommet, qui est un petit mamelon conoïde, et forme 
le véritable stigmatc. Chaeun de ees pistils est uniloculaire, et eontient un seul ovule pendant de son sommet. Dans les genres Ambora et Monimia, l'involucre est persistant, il prend même beaueoup d'aceroissement, et devient eharnu dans le premier de ees genres. Les fruits, qui dans l'A $A$ mbora sont contenus dans l'épaisseur même des parois de l'involuere, sont autant de petites drupes uniloculaires et monospermes. La graine se eompose d'un tégument propre, assez mince, recourrant un très-gros cndosperme eliarnu, dans la partic supéricure duquel est placé un embryon offrant la mème direction que la graine.

Cette fanille, établie par M. de Jusssieu, avait été divisée en deux familles distinetes par Robert Brown. Mais nous croyons que ces deux familles forment simplement deux tribus d'un même ordre naturel.

I'e tribu. Amnorírs, anthères s'onvrant par un sillon longitudinal; graines renversées: Ambora, Monimia, Ruizia.

$2^{e}$ tribu. Aтrúrosiermées, anthères s'ouvrant de la hase au sommet par le inoyen d'une valvule; graines dressées: Pavonia, Atherosperina, Citrosina.

Les Monimiées ont heaneoup de rapports avec les Urticées, auxquelles plusieurs des genres qui les composent étaient d'ahord réunis; mais elles en diffèrent surtout par leurs graines munies d'un très-gros endosperne, et par leur ovule pendant et non dressé. Le même caraetère les éloigne aussi des Laurinées, dont elles se rapprochent par la structure de lears étamines dans la tribu des Athérospermées.

\section{CENT CINQUANTE-SEPTILME FAMILIE.}

* salicinées. Salicince. Rich.

Famille qui se eompose des deux genres Saule et Peuplier. Ce sont de grands arbres à feuilles alternes, simples, munies de slipules eaduques. Leurs fleurs sont unisexuées, et disposées cn ehatons cylindriques ou ovoïdes. Les fleurs mâles se eomposent de deux à vingt étamines, plaećes à l'aisselle d'un écaille, ou sur sa face supérieure. Les fleurs femelles eonsistent en un pistil fusiforme, terminć par deux stigmates bipartis, situć à l'aisselle d'une 
écaille, et quelquefois aecompagnés à leur base d'un caliee en forme de cupule. Cel ovaire est ì unè ou deux loges contenant un assez grand nombre d'ovules dressés, attaehés au fond de la loge et à la base de deux trophospermes parićtaux. Le fruit est une petite capsule allongée, à une ou deux loges, eontenant plusieurs graines environnées de longs poils soyeux, et s'ouvrant en deux valves. L'embryon est dressé, homotrope, sans endosperme.

Formées aux dépens de la famille des Amentacées, les Salicinées constituent un groupe très-distinct par la structure de leur fruit.

\section{CENT CINQUANTE-HUITIEME FAMIILE.}

\section{mynucées. MYyricece. Rich. - Casuarinece. Minbel.}

Si l'on en exeepte le genre Casuarina, qui, par son port, ressemble à une presle (Equisetum) gigantesque, les Myricées sont des arbres ou des arbrisseaux à feuilles alternes ou éparses, avee ou sans stipules. Leurs fleur's sont eonstamment unisexuées et le plus souvent dioïques. Les fleurs mâles, disposées en ehatons, se composent d'une ou de plusicurs étamines souvent réunies ensemble sur un androphore rameux et placé à l'aisselle d'une braetée; les fleurs femelles, également en chatons; sont solitaires et sessiles à l'aisselle d'une braetée plus longue qu'elles. Chaque fleur se eompose d'un ovaire lentieulaire eontenant un seul ovule dressé ; le style, très-court, est surmonté de deux longs stigmates subulés et glanduleux. En dehors de l'ovaire on trouve deux, trois ou un plus grand nombre d'éeailles hypogynes et persistantes, se soudant quelquefois avee le fruit. Celui-ci est une sorte de petite noix monosperme et indéhiseente, quelquefois membraneuse et ailée sur ses bords. La graine qu'il renferme est dressée; son tégument reeouvre immédiatement un gros 
embryon ayant une direction entièrement opposéc à celle de la graine.

Formée de geures auparavant placés dans le groupe polymorphe des Amentacées, cette famille est voisine à la fois des Celtidées et des Bétulinées, mais elle diffère des premières par ses fleurs en chatons, et toujours unisexnées, par son ovule dressé; des secondes par son ovaire uniloculaire et son embryon sans endosperme.

\section{CENT CINQUANTE-NEUVIEME FAMIIIE.}

\section{* bétulintées. Betulinece. Rich.}

Arbres à feuilles simples, alternes, accompagnées à lcur base de deux stipules; fleurs unisexuécs, disposées cn chatons écaillcux. Dans les chatons mâles, chaque écaille, qui est quelquefois formée de plusicurs écailles soudées, porte deux ou trois fleurs nues, ou ayant un calice à trois ou quatre divisions profondes. Le nombre des étamines est très-variable dans chaque flcur. Les chatons femelles sont ovoïdes ou cylindriques, écaillcux; à la base interne de chaque écaille on trouve d'une à trois fleurs sessiles, nucs, préscntant un ovaire libre, comprimé, à deux loges, contenant chacune un seul oṿule attaché vers la partic supériéure de la cloison, et surmonté de deux longs stigmates allongés, cylindriques et glandulcux. Le fruit est un cône écailleux, dont les écailles ligncuses ou simplement cartilagineuses portent à leur base un ou deux petits akènes uniloculaires, monospermes par avortement et membraneux sur les bords. Leur graine se compose d'un gros embryon sans endosperme, ayant la radicule supéricure.

Les deux genres, Anne et Boulead, forment cette famille, qui diffèrc des Salicinées par son ovaire à deux loges monospermes, par ses fruits indéhiscens et ses graines dépourvues des longs poils qui recouvrent celles des Salicinées. Les Myricées ont aussi benucoup d'analogie avec les Bétulinées; mais leur ovaire toujours uniloculaire et leur ovule dressé sont les signes distinctifs qui existent entre cetre famille et cellc des Bétulinẹes. 
* cupulifitics. Cupuliferce. Rich. - Amentacecurum gen. Juss.

Ce sont des arbres à feuilles alternes, simples, mumies de deux stipules caduques à leur base. Leurs fleurs sont constamment unisexuées et presque toujours monoïques. Les mâles forment des chatons cylindriques et écailleux. Chaque fleur offre une écaille simple, trilobée ou calyciforme, sur la face supérieure de laquelle sont attachées de six à un grand nombre d'étamines, sans indice de pistil. Les fleurs femelles sont généralement axillaires, tantôt solitaires, tantôt groupées en capitules ou en chatons; dans tous les cas chacune d'elles est recouverte, en partie ou en totalité, par. une cupule écailleuse, et offre un ovaire infère, ayant son limbe peu saillant et formant um petit rebord irrégulièrement denté. Du sommet de.l'ovaire naît un style court qui se termine par deux ou trois stigmates subulés ou planes. Cet ovaire présente deux, trois ou un plus grand nombie de loges contenant chacune un ou deux ovules suspendus. Le fruit est constamment un gland, généralement uniloculaire, souvent monosperme par avortement, toujours accompagné d'une cupule; qui, quelquefois, recouvre le fruit en totalité à la manière.d'un péricarpe, comme'dans le châtaignier et le hếtre. La graine se compose d'un très-gros embryon dépourvu d'endosperme.

- Cette famille, composée de genres d'abord placés dans l'ancienne famille ḍes Amentaeées, comprendj les genres Quercus, Corylus, Carpinus, Cas: zanea et Fagus. Elle a quelques rapports avec les Conifères et les Bétulinées; mais les premières par leur port, la strueture de leurs fleurs femelles, lear embryon muni d'nn endosperme, les seeondes par leurs fleurs femelles disposées en eône; leur ovaire simplle, elc., s'en disstinguent suffisamment. 
Quant aux autres familles également formées aux dépens des Amentacées ; comme les Salicinées, les Myricées, leur ovaire libre est le caractère le plus saillant qui les éloigne des Cupulifères.

\section{CENT SOLXANTE-UNIEME FAMIXTE.}

* conifìres. Coniferce. J. Rrch.

Cette famille se compose de tous ces arbrisscaux et grands arbres, ayant de l'analogic avec le Pin et lc Sapin et que l'on désigne communément sous le nom d'arbres verts ct résineux. Leurs feuilles, coriaces et roides, persistent dans toutes les espèces, excepté dans le Mélèze et le Gingo; ces feuilles sont tantôt linéaircs, subulées, réunies au nombre de deux à cinq et accompagnées à leur base d'une petite gaine scarieuse; ou bien, elles sont en forme d'écailles imbriquées ou lancéolées, etc. Les fleurs sout constamment unisexuécs et en général disposées cn cònes ou chatons. Les fleurs mâles consistent essentiellement chacune dans une étamine tantôt nue, tantôt accompagnée d'une écaille à l'aisselle ou à la face inférieure de laquelle clle est placée; assez souvent plusieurs étamines s'entregicfient cusemble par leurs filets, et leurs antlières, qui sont uniloculaires, restent distinctes ou se soudent. L'inflorescence des flcurs femelles cst très-variable, quoique généralement elles forment des cônes ou clıatons écailleux; ainsi elles sont quelquefois solitaires, terminales ou axillaires, ou bien réunies dans un involucre charnu ou sec. Chacunc de ces fleurs préscnte un calice monosépale, adhérent avec l'ovaire, qui est en partie ou en totalitéinfère; son limbe, quelquefois tubuleux, est tantôt entier et tantôt à deux lobes divariqués, glanduleux sur leur face interue, et que l'on a généralement considérés comme deux stigmates. L'ovaire est à une seule loge et contient un seul ovule. A son sommet il présente communément une pe- 
tite eicatrice qui est le véritablc:stigmate. Tantôt ces fleurs femelles sont drcssées à l'aisselle des écailles ou dans l'involucre où ellcs sont placćes; tantôt elles sont renversées -et soudćes deux à deux, par un de leurs eôtés, à la faee interne et vers la base des éeailles qui forment lc eônc. Le fruit cst généralement un cônc ćeailleux ou bien un galbule, dont les écailles sont quelquefois charnues, se soudent ct représentcnt unc sorte de baie, comme dans les Genćvriers par cxemplc. Chaque fruit en particiulier, c'cst-à-dire chaquc pistil fécondé a un péricarpe souvent crustacć, quclquefois muni d'une aile membraneuse et marginale. Lc tégument propre de la graine est adbérent avec le péricarpe et recouvre une amande composée d'un endosperme charnu, contenant un embryon axile et cylindrique, dont la radicule est soudéc avcc l'endosperme et dont l'extrémité cotylédonairc se divise en deux, trois, quatre et jusqu'à dix cotylédons.

La famille des Conifères, sur laquelle mon père a pnblié un sỉ beau travail (Commentatio Botanica de Coniferis, in-fol., fig. Paris. I826), peut se diviser en trois ordres:

I TaxinéEs, fleur's femelles distinetes les unes des autres, attachées à une écaille ou dans une cupule. Fruit simple. Ex.: Podocarpus, Dacrydium, Taxus, Salisburia, Phyllocladus, Ephedra.

$2^{\circ}$ Cupressixérs, fleurs femelles dressées; réunies plusieurs ensemble à. l'aisselle d'écailles peu nombreuses, formant un galbule quelquefois charnu. Ex. Junipcrus, Thuya, Callitrix, Cupressns, Taxodium.

$3^{\circ}$ Abiesiríes. Ici se trouvent réunis tous les genres qui ont les fleurs femelles renversées, et pour fruit un véritable cône écaillenx. Ex. : Pinns Abics, Cunninghamia, Araucaria, etc.

\section{CENT SOIXARTE-DEUXIEYEE FANIXX.}

cycadées. Cycadece. Rich.

Lcs Cyeadćes, qui nc sc composcnt quc des deux genres Cycas et Zamič, sont des végétaux exotiques, ayant le. port des Palmicrs. Lcurs fcuilles, réunies au haut du 
stipe, sont pinnées et rouléés en crosse avant leur développement, eomme dans les Fougères. Les fleurs sont conm stamment dioïques. Les fleurs mâles constituent des chatons ou eônes, quelquefois très-grands, eomposés d'écailles spathulées, recouvertes à lcur faee inférieure d'un très-grand nombre d'étamines qui doivent être considérées chaeune comme une fleur mâle. L'infloresecnce des fleurs femelles n'est pas la même dans les deux genres Cycas et Zamia; daus le premier un long spadiee spathuliforme, aigu, denté sur ses côtés, porte à chaque dent une fleur femelle, enfoncée dans une petite fossette. Le Zamia a ses fleurs femielles également en eône, et ses écailles, qui sont épaisses et peltées, portent chacune à leur face inférieure deux fleurs femelles renversées. Ces fleurs se eomposent d'un calice globulcux, pereć d'une très-petite ouverture à son sommet, et appliqué sur l'ovaire avee lequel il est. en partie adhérent à sa base. Cet ovaire est uniloeulaire et contient un seul ovule; il se termine à son sommet par un stigmate en forme de mamelon. Le fruit est une sorte de noix forméc par le ealice, qui, quelquefois, est légèrement chamu. Le péricarpe est, en général, minee, crustacé et indéhiscent, adhércnt avee le tégument propre de la graine. L'amande se eompose d'un endosperme charnu, eontenant un cmbryou à deux cotylédons inégaux et quelquefois eohérens entr'eux, et dont la radicule est soudéc avec l'endosperme.

Pour peu qu'on compare la strueture des fleurs míles, et surtout des fleurs femclles des Cycadées avec celle des Conifères, on sera frappé de l'extrême resscmblance qui existe entre ces deux familles, et l'on devra adopter l'opinion de mon père, qui les place l'une à côté de l'autre. En effet, dans toutes les deux, les fleurs míles consistent chacune dans une seule anthère uniloculaire; les fleurs femelles se composent d'un périanthe monosépale, d'un ovaire semi-infère, à une seule loge et à un seul ovale. Le fruit et la graine offrent la même organisation : il est vrai que le port est tout-à-fait différent dans ces deux familles, puisque les Cycadées ressemblent entièrement anx Palmiers, et que la structure intérieure de lear tige 
est cclle des Monocotylćdons. Mais doit-on sacrilicr à ce caractère les anaIogies si importantcs qui existcnt clans l'organisation des fleurs̄ des Ciycadćcs et des Conifères? Doit-on placer parmi les Monocotylćdons unc famille dont l'embryon est évidemment à dcux cotylćdons? En admettant cette supposition, à côté de quelle lamillc monocotylédone placera-t-on lcs Cycadées? elles n'ont de rapport avec aucunc de cès familles; elles devront rester isolées; tandis que si l'on donne la préfércnce à la structure de l'embryon et à celle des fleurs, et qu'on 'place les Cycadécs parmi lcs Dicotylédons, il ne reste aucun doute sur la place qu'elles doivent occuper. Elles vieunent tout naturellement se classer à côté des Conifères. 


\section{HORLOGE DE FLORE,}

od

TABLEAU DE L'HEURE DE L'ÉPANOUISSEMENT DE CERTAINES FLEURS, $\Lambda$ UPSAL, PAR $60^{\circ}$ DE LATITUDE BOREALE.

\begin{tabular}{|c|c|c|c|}
\hline $\begin{array}{c}\text { HEUREs } \\
\text { du lever. } \\
\text { c'est-a-dire } \\
\text { de } \\
\text { L'épanouisse- } \\
\text { ment } \\
\text { des fleurs. }\end{array}$ & $\begin{array}{c}\text { NOMS } \\
\text { des } \\
\text { PIAATTES OBSER VÉES. }\end{array}$ & \multicolumn{2}{|c|}{$\begin{array}{c}\text { HEURES } \\
\text { du coucher, } \\
\text { c'est-à-dire } \\
\text { où } \\
\text { se ferment : } \\
\text { ces } \\
\text { mémes fleurs. }\end{array}$} \\
\hline 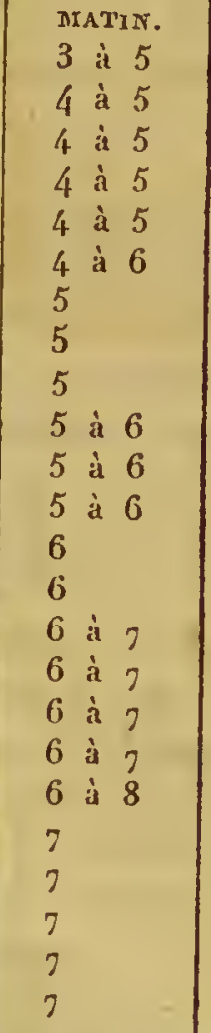 & 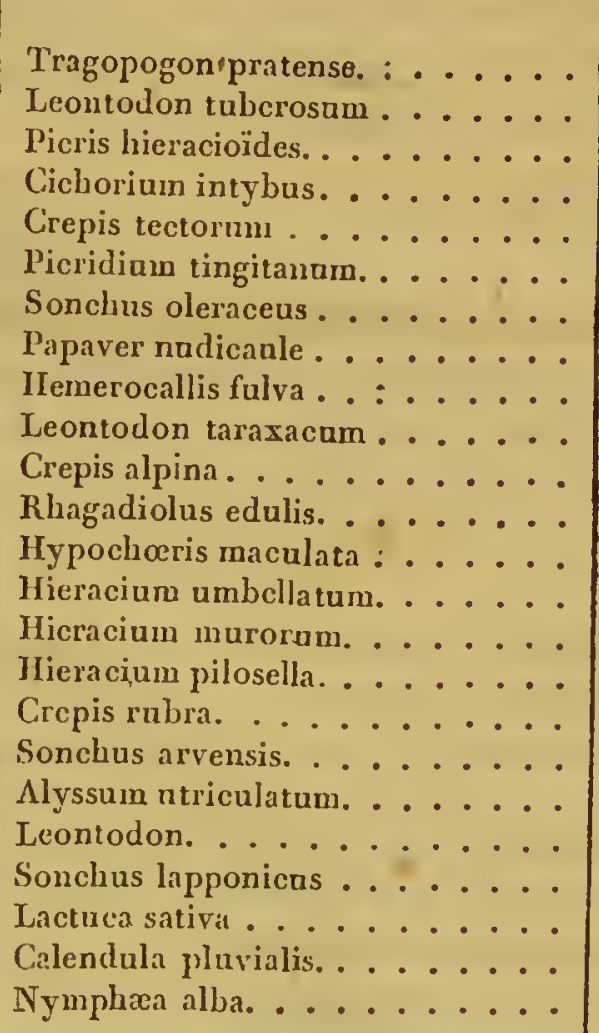 & 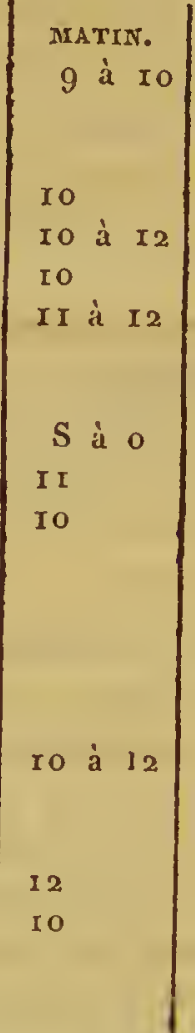 & 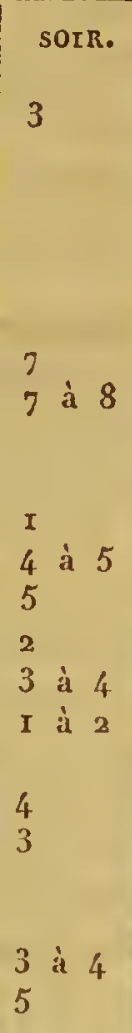 \\
\hline
\end{tabular}




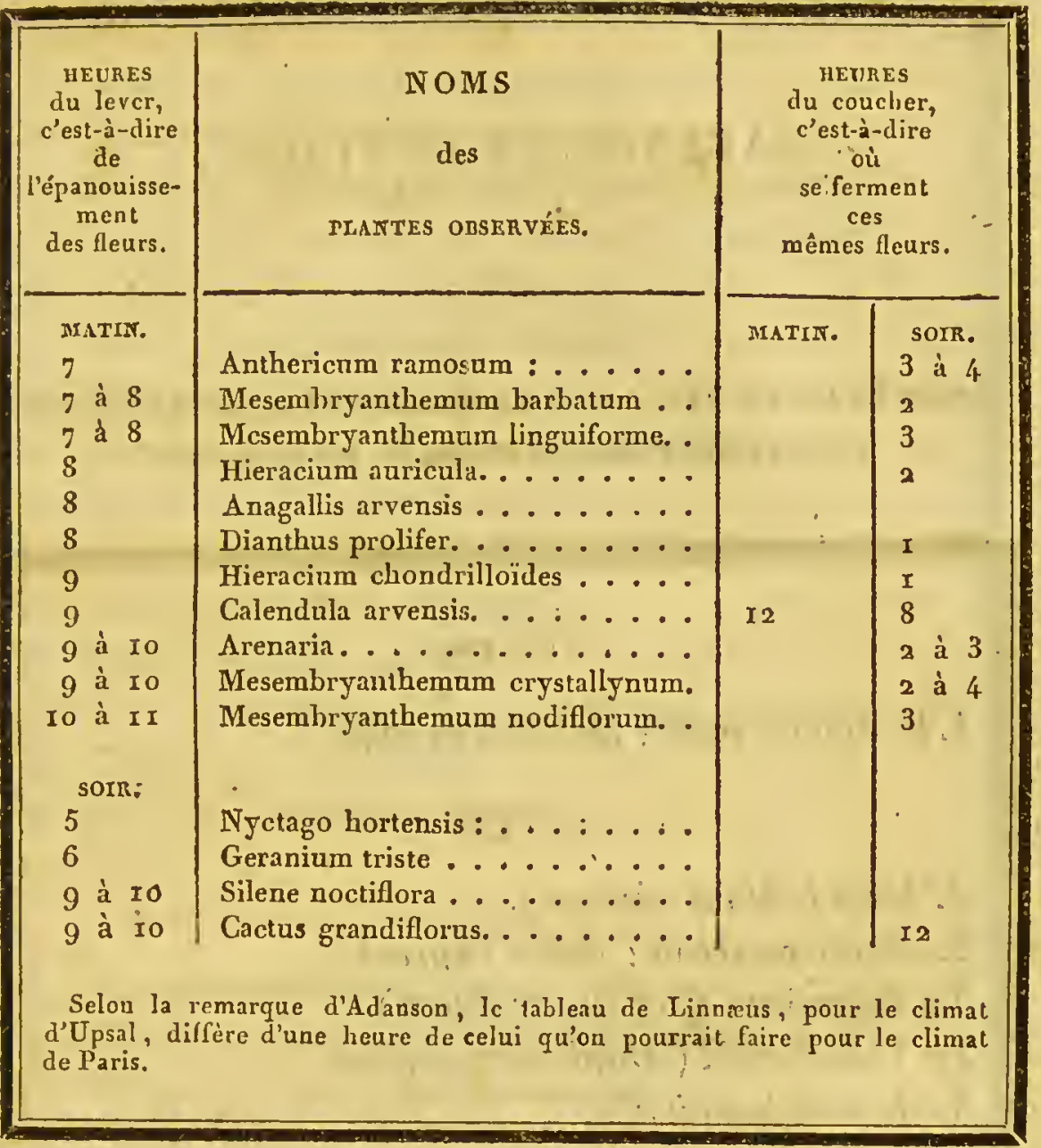




\title{
GALENDRIER DE FLORE,
}

\author{
ou
}

EPOQUES DE LA FLORAISON DE QUELQUES PLANTES SOUS LE CLIMÁT DEE PARIS, D’Ä́RES Ḿ. DE LAMARCK.

\section{JANVIER.}

L'Hellébore noir (Hellebor'us niger ).

\section{FÉVRIER:}

L'Aune (Alnus viscosa).

Le Saule marceau ( Salix caprcea).

Le Noisetier (Corylus avellana).

Le Bois-Gentil (Daphne mezereum).

Le Galanthus nivalis.

\section{MARS.}

Le Cornouiller mâle (Cornus mas).

L'Anémone hépatique (IIepatica triloba).

L'Androsace carnea.

La Soldanelle (Soldanella alpina).

Le Buis (Buxus sempervirens).

La Thuya (Thuya orientalis).

L'If ( Taxus baccata).

L'Arabis alpina.

La Renoncule ficaire (Ficaria Ranunculö̈des). L'Hellébore d'hiver (Helleborns hiemalis). 
L'Amandier (Amygdalus contmunis). Le Pècher (Amygdaluz persica). L'Abricotier (Armeniaca sativa). Le Groseiller à maquereau (Ribes Grossularia). Le Pétasite ( Tussilago Petasites). Le Pas-d'Ane (Tussilago Farfara). Le Ranuncultus auricomus.

La Giroflée jaune (Cheiranthus cheiri): La Primevère (Primula veris). La Fumeterre bulbeuse (Corydalis bulbosa). Le Narcissus pseudo-Narcissus.

L'Anemone Ranunculoïdes.

Le Safran printanier (Crocus vernus).

Le Saxifraga crassifolia.

L'Alaterne ( Rhamnus alaternus).

\section{AVRIL.}

Le Prunier épineux (Prunus spinosa). Le Rhodora de Canada (Rhodorc canadensis). La Tulipe précoce (Tulipa sulaveolens). Le Draba verna. Le Draba aizoüdes.:

Le Saxifraga granulata. Le Saxifraga tridactylites.

Le Cardamine pratènsis.

L'A sartum europceum.

Le Paris quadrifolia.

Le Pissenlit (Taraxacum Dens-Leonis).

La Jacinthe (Hyacinttius orientalis').

L'Ortie blanche (Lamium.album).

Le Prunier (Prunus domestica). La Sylvie (Anernone ńemorosa). 
L'Orobe printanier (Orobus vernus).

La petite Pervenche (Vinca minor). Le Frêne commun (Fraxinus excelsior). Le Charme ( Carpinus betulus).

L'Orme (Ulmus campestris).

L'Impériale (Fritillaria imperialis). Le Lierre terrestre (Glecoma hederacea). Le Junciss sylvaticus. La Luzula campestris. Le Cerastium arvense.

Les Érables.

Le Prunier mahaleb (Prunus mahaleb). Les Poiricr's.

Les Pommiers.

Le Lilas (Syringa vnlgaris).

Le Maronnier d'Inde (:Asculus Hippocastanum). Le Bois de Judée (Cercis Siliquastrum). Le Cerisier. (Cerasus communis). Le Faux Ébénier (Cilysus Laburnum). La Filipendule (Spircea Filipendula). La Pivoine (Pceonia officinalis). L'Erysimum alliaria.

La Coriandre (Coriandrum sativum). La Bugle ( Ajuga reptans). L'Aspérule odorante (Asperula odorata). La Bryone (Bryonia diö̈ca). Le Muguel (Convallaria maïalis). L'Épine-Vinette (Berberis vulgaris). La Bourrache (Borrago officinalis). Le Fraisicr ( Fragaria vesca). 
L'Argentine (Potentilla ar'gentea).

Le Chêne (Quercus Robur).

Les Iris, etc., et en général le plus grand nombre des plantes.

JUIN:

Les Sauges.

L'Alkékenge (Physalis Alkekengi).

Le Coquelicot (Papaver rhacas).

La Cardiaire (Leonurus cardiaca).

La Ciguë (Conium maculatum).

Le Tilleul (Tilia europcea).

La Vigne (Vitis vinifera).

Les Nigelles.

L'Heracleum sphondy lium.

Les Nénuphars.

La Prunélle (Prunella vuilgaris).

Le Lin (Linum usilatissimum).

Le Cresson de fontaine (Sisymbrium Nasturtium ).

Le Seigle (Secale cereale).

L'Avoine (Avena sativa.).

Le Froment (Triticum sativunn).

Les Digitales.

Le Pied-d'alouette (Delphinium consolida).

Les Hypericum.

Le Bleuet (Centaurea Cyanus).

L'Amorpha fruclicosa.

Le Melia Azedarach.

JUILLET.

L'Hysope ( Hyssopus officinalis.).

Les Menthes. 
L'Origan (Origanuin vulgare).

La Carotte (Daucus Carotta).

La Tanaisic (Tanacetum vulgare).

Les OEillets.

Le petite Centaurée (Erytluraca Centauriuin).

Le Monotropa Hypopitlys.

Les Laitues.

Plusicurs Inules.

La Salicaire (Lythrum Salicaria).

La Chicorée sauvage (Cichorium Intybus).

La Verge d'or (Solidago Virga auréa).

Le Catalpa (Bignonia Catalpa).

Le Cephalanthus.

Le Houblon (Humalus Lupulus).

Le Chanvre (Cannabis sativa), etc., ete.

AOUT.

La Scabiosa succisa.

Le Parnassia palustris.

La Gratiole (Gratiola officinalis).

La Balsamine des jardins (Balsamina hortensis).

L'Euphraise jaune (Euphrasia lutea).

Plusieurs Asters.

Le Laurier-Tin (Viburnum Tinus).

Les Coreopsis.

Les Rudbeckia.

Les Sylphium.

SEPTEM BRE.

Le Ruscus racemosus.

L'Aralia spinosa. 
Le Lierre (rictera ficlix).

Le Cyclamen (Cyclamen europaum).

$\mathrm{L}$ 'Amaryllis lutea.

Le Colchique (Colchicum antumnale).

Le Safran (Crocus sativus).

L'OEillet d'Inde ( Tagetes erecta).

OCTOBRE.

L'Aster grandiflorus.

Le Topinambour (Helianthus tuberosus).

L'Aster miser.

L'Anthemis grandiflora, etc.

Le Chrysanthemum indicum. 


\section{TABLE ANALYTIQUE}

\section{DES MATIÈRES.}

D

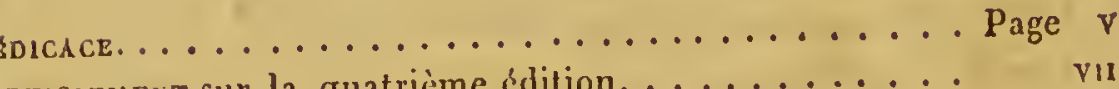

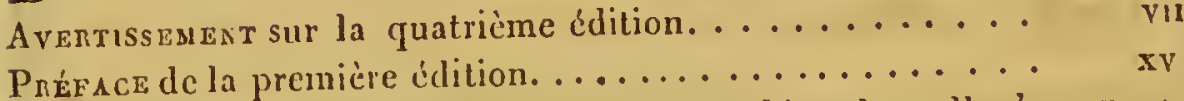

Inтroductior. Définition de la Botanique el objets dont cllc s'occupc, 1.

- Sa division en trois branches, la Botanique proprenient ditc, la Physique végétale, et la Botanique appliquée; Subdivisions de ces branclics, 2. - Ce qu'on cntend par un végćtal , 3. - Différence entre lcs végétaü ct les animaux, 4 .

Parties élémentaircs des végétaux ou Anatome végétate, 7. - Tissu cellulaire, 7. - Nouvelles obscrvations de MII. Dutrochet et Amici sur les ccllules, 3. - Formation ct développenent du tissu cellulairc ; Globuline dc M. Turpin; Chromule dc M. de Candollc, S.Tissu vasculairc ou tubulairc, ı. - Différentes cspèces de vaisscaux, 12. - Lcur's combinaisons, d'où résultent les fibres et le parencliyme qui, diversement combinés cux-mêmes, constituent les organcs, 14. - Distinction des vaisscaux cn vaisseaux sćvcux ou lymphatiques, $\mathrm{cr}$ vaisscaux propres ct cn vaisseaux aériens, 15. - Obscrvations de M. Amici sur les vaisseaux porcux, 16. - Systime de M. Dutrochet sur les pores des parois cellulaires, 17.-Desglandes, 19.-Diféerentes espèces de glandes, ibid. - Des poils, 20. - Dislinction des poils cu glandulifères, cxcréteurs ou lymphatiques, ibid.- Lcurs formes, 21. -Organes des végézaux; définition de cesorganes, savoir : de la racinc, de la tige, des fcuilles, ibid.;- des flcurs, du pistil, des étamines, 22 ; - de la corolle, du calice, du fruit, du péricarpe, des graincs, de l'ćpisperme, de l'amandc, 23. - L'amandc conticnt l'endosperme ct l'cubryon, qui cst formé lui-même du corps radiculairc, de la gemmule ct du corps cotylédonaire simple ou divisć, 24. - G:audc 
division des plantes en inembryonćes (cryptogames, agames, acotylćdones) et en embryonćes (phanérogames), fondéc sur l'absence ou la présence del'embryon,25.-Division des embryonées en monocotylédons et dicotylédons, suivant que le corps cotylédonaire est simplc ou divisé, 26.-Division des organes des végétaux en deux classes, suivant qu'ils servent à la nutrition ou à la reproduction, 27.

\section{Ire CLASSE. Organes de la nutrition ou de ta végétation.}

Chaprtre I'r. De la racine; définition et caractères de la racine, 3o. Il ne faut pas la confondre avec les tiges souterraines, 31 . - Diffćrentes parties sont susceptibles de produire des racines, ibid.-Analogie des racines et des tiges, 32. - Elle cst formée de trois parties, 33. - Division des racines suivant lcur durée, en annuelles, bisannuelles, vivaces et ligneuses, ibid. - Suivant leur structure, en pivotantes, fibrcuses, tubérifères et bulbifères, 34. - Suivant Iéur consistanice, 37. - Leur direction, ibid. - Leurs formes, ibid. Usages des racines, 3̊. - Leur division fondée sur leurs usages en médecine, 46 .

Crap. II. De la tige. Caractèrcs de la tige, 48. - Il ne faut pas la confondre avec la hampe et le pédonculc radical, ibid. - Cinq espc̀ces de tiges, le tronc, le stipe, le chaume, la souche, la tige proprement dite, 49.-Distinction des tiges suivant leur consistance, 5 r.Leurs formes, 53. - Leur composition, 55. - Leur direction, 56. Leur vestiture et leurs appendices, 57 . - Leur superficie, 'ibid. Leur pubescence, 58. - Leur armure, 60. - Structure anatomique des tiges, ibid.-Sect. Ire. Organisation de la tige dcs dicotylćdons, 6r.-Del'épiderme, ibid.-Observations microscopiques de M. Amici sur l'épiderme et les pores corticaux, 63. - Glandes Ienticulaires de Guettard, ou lenticelles de M. de Candolle, 65. - De l'enveloppe herbacće, ibid. - Des couches corticales, 67. - Du liber, ibid. De l'aubicr ou faux bois, 69. - Du bois proprement dit, ibid. - De l'étui médullaire, 71.-De la moellè, 72.- Sect. II. Organisation de. la tige des monocotylédons, 74 - - Sect. III. Organisation de la racine, 76. - Sect. IV. Considérations générales sur l'accroissement des végétaux, et en particulier sur le développement de la tige, $78 .-$ I Ier. Accroissement de la tige des arbres dicotylédonés, So. -A.Accroissement en diamètre, ibid.-Théorie de Duhamel, ibid:-Théorie de M. Du Petit-Thouars, 84. - Théoric de M. Mirbcl, $89 .-$ $B$. Accroissement en hautcur, 91: - $\int$ II. Accroissement de la tige des arbres monocotylédons, 93. - Opinion de l'autcur sur la nature de la' tige lignicuse des monocotylédons, 94. - Accroissement en largeur selon MM. Linck et Dutrocluct, 98. - Théorie de quelqucs 
procédés pour la multiplication artificielle des végétaux, expliqués par les lois de la physiologic végétale, 101.-Marcotte, ibid.-Bouture, 102. - Greffe, ibid. - \ler. Greffespar approche, 104.- $\int$ II. Greffes par scions, ibid. - $\int 111$. Greffes par gemmes ou boutons, 106. IIV. Grefres des parties ficrbacées des végétaux, ibid. - De la hauteur des arbres, 107. - De la grosseur des arbres, 108. - De la durće des arbres, 109. - Usage des tiges, ibid. - Leur division suivant leurs usages en mćdecine, 110.

Chras. III. Des bourgeons, III.-Des bourgeons proprement dits, ibid. - Leur division en nus et écailleux; 112. - Subdivision des seconds, suivant les parties qu'ils renferment, ibid. - Du turion, 114. -Di: bulbe, 117. - Des tubercules, ibid. - Des bulbilles , ibid. Úsages des bourgeons ct des bulbes, 119 .

Chap. IV. Des feuilles, 119. - Leurs différentes dispositions avant leur enticr développement, ilid. - Considérations sur la fcuille après ce développement; sa définition; son originc, 120. - Ses deux parties, lc pétiolc et le limbe, 121. - Faces du limbe. L'inférieurc présente les nervurcs dont Ies dispositions variables ont une grandc importance et peuvent ĉtre rapportécs à trois principalcs, 122 ct suiv. - Diverses nanières dont la feuille est unie à la tige, 124. - Division des feuilles en simples et composćes, 126 .

$\$ 1$. Des feuilles simples. Leur distinclion suivant leur point de départ. Leur disposition sur la tige ou les rameax, 129. - Leur direction relativement a la tige, 131 . - Leur circonseription ou figurc, ${ }_{32}$. - Les échancrures de leur base, 134. - Leur mode de terminaison à leur sommet, 135. - Lcur contour, ibid. - Lcurs incisions plus ou moins profondes, 136. - Les modifications de lcur bord, 138. Lcur expansion, 139. - Leur superficie, 140. - Leur pubescence, ibid. - Lenr consistance el leur tissu, ibid. - Lcur forme, 141.Lenr coToration, 142. - Lcur péliolation, 143. - Leur duréc, 144.

5 II. Des fcuilles composées, 144. - Leur division en composées proprement dites et décomposées, I 45. - Subdivision des unes, ibid.Et des autres, 147.

Structure, usages et fonctions des fenilles, $149 .-$ Opinions dc M. Dutrochet sur les mourcmens des feuilles, 153. - Défoliation ou chute des fenilles, i55. - Leurs usages économiques et médicaux, 156.

Crinp. V. Des stipules; leur définition , 158. - Importance des caractères qu'elles fournissent, ibid. - Variétés de leur conmexion, de leur consistance, de leur figure, de leup durée, 159.1 if 
Char. VI. Des villles, cirrhes ou mains, 160.

Crras. VII. Des épines et des aiguillons, 161 .

De la nutrition dans les végétaux, 163. -. De l'absorption ou succion, $164 .-$ De la marche de la sève, 169. - Observations nouvelles de M. Amici sur la circulation du suc dans Ics Chara et le Caulinia fragilis, 171.- Diverses hypothèscs relativement à la cause qui détermine la marchc de la sève, 173 . - De la transpiration, 176. -De l'expiration, 178. - De l'excrćtion, 179. - De la sève descendante, iso.

\section{CLASSE. Des organes de la reproduction ou de la} fructification.

Secrion Ire. Des organes de la floraison.

- Considérations générales sur la fleur, 184.

Chap. Ier. Du pédoncule et des bractćes, $189 .-$ Cé que sont les pédoncules, ibid. - Ce que sont les bractées, 19o. - Lcurs diversés dispositions, 191. - Unies, ellcs forment ou unc cupule, ou un involucre, qui prend, suivant les cas, le nom de calicule ou de spathe, ibid. - De ees parties dans les Graminées, 193.

Cr1s. II. De l'infloresscnce. Définition, 194. - Divers modes d'inflorescence. L'épi, 195. - La grappe, ibid. - Le thyrse, 196. - La panicule, ibid. - Le eorymbe. La cyme, ibid. - L'ombelle, ibid. Le sertule. Le vertieille. Le spadice. Le chaton', 197. - Le capitule, 198 .

Grap. III. Dc la préflemraison, 199.

Chap. IV. Des enveloppes florales en général, $20 \mathrm{r}$.

Chsp. V. Du calice. Définition et caraetc̀res, 204. - Distinction des caliccs en monosćpales ou gamosépales, et polysćpales, 205. LCaractères des premiers, ibid. - Leur distinction établie d'après leurs divisions plus ou moins profondes, 206. - D'après leur régularité ou irrégularité ct leurs formes diverses, $207 .-$ Nombre variable des sćpales dans les calices polysépales, 20g.

Chap. VI. De la corolle. Dćfinition et caractères, 210. - Division des corolles en monopétalcs et polypétales, 211. - Ce que c'est qu'un pétale, ibid. - Ses partics, ibid. - Différences des corolles monopétales et polypétales, 212. - Des partícs que présentent lcs preniè̀res, le tubb, le limbe et la gorge, ibid. - Corolle nonopétale r'gulic̀re, $2: 3$. - Irrógulière, 214 . Corolle poly pćlale, 217. 
Corolles polypétales régulic̀res qui peuvent étic cruciformes, rosacées, caryophyllées, 219. - Corolles pol ypćtales irrégulières qui sont papiiiunacécs ou anomales, 220. - Situation rclative des sćpales et des pétales, 221.

Chap. VII. Des organes sexuels. Histoire de leur découverte, 222. Des parties qui concourent à les former, ibid.

Cirsp. \{VIII. De l'ítanine ou organe sexuel male, 224. - Des parties qui la composent, ibid. - Nombre variable des étamines, 225. Leur granderrr relative, 226. - Leur situation rclativenıcnt aux divisions du calice et de la corollc, 227. - Leur dircction, ibid. Elles sorit libres ou récunies, 228 .

I. Du filct; ses différentes formes, 229.. - Lcs filets sont ou libres ou soudćs soit cn partic, soit en totalité, 230. - Lcur naturcet leur structurc organique, 231 .

$\int$ II. Dc l'anthèrc. Des parties qui eoncourcnt à la former, 23 I. - Dc ses loges ct de leur nombre, 232. - Points d'attache des anthèrcs, ibicl: - Lcurs formes, 233. - Lcs loges des anthères biloculaircs peuvent être soudécs, de différentes manières, 234. - Pcuvẹnt ctrc réunies par un connectif, ibid. - Mosles de déhiscence des anthìres, 235. - Les anthères peuvent être adherentes entre elles, ibid.

S III. Du pollen, 236. - Structure, uature de la superficie, et forma des grains polliniques, 237 . - Obscrvations de M. Guillcmin, ilid. -Olscrvations de M. Ad. Brongniart, 240. - Pollen en masse des Orchidées et des Asclépiadécs, 241 .

Cinsp. IX. Du pistil ou organc sexuel fermelle. Parties qui le composcnt, 242. - Du gynophore et du podogyne, ibid.

$\int$ I. Dc l'ovairc. Scs curactères, 243. - Son adhérence, ou noll-adhérence avee le calice; importance de ec carnctèe, 244. - Dvairc in. fere, ibid. - Ovaires parićtaux, ibid. - Ovaire gynobasique, 245 . - Cavités intérucures ou loges de l'ovaire variables en nombre , 246. - Des ovules qu'clles conticnnent, ilid.

i II. Du style, 247 . - Lc nombre des styles et leur pesition relativement a l'ovairc, 248. - Leurs formes, ibid.

\III. Du stigmäte. Délinition, 250. - Nombre des stigmates', ilid.Leur position sur le style, 251 . - Leur différence de sulsstance, illid. - Lcurs formes, ibid.

Nourclles considérations généralcs sur la flerır, 253. - De l'anthire, 254. 
Division des plantes suivant la saison de lcur foraison, ibid.

Influence des météorcs atmosphériques sur certaincs fleurs; 256 .

ChАे. X. Des neclaires, 257 .

De la fleur considćrée comme fornée de plusicurs verticilles de fleurs diversement moditiés, 259.

Crap. Xl. De Ia fécondation. Considérations générales sur son mécanisme et la manière dont la nature la favorise dans les divers vígétaux, 262. - Théorie de la fécondation selon M. Ad. Brongniart, 267 .

Du disque. Définition, 273. - Sa distinction én hypogyne, périgyne et épigyne, ibicl.

De l'insertion. Ce qu'on entend par ce mot, 273. - Distinctión des insertions en absolue et relative: ibid. - Trois sortes d'insertion relative, 274 .

SECT10s II. Du fruit ou des ớganes de la fructification proprement dits, 274 .

Cra p. Ier. Du péricarpe, 275. - Définition, ibid. - Des trois parties qui le forment, l'épicarpe, le sarcoearpe, l'cndocarpe, 275. - Des organes accessoires du péricalpe. De sa cavité intériéure simple ou offrant plusieurs loges, 277 .

\I. Des eloisons qui séparent ces loges, 280 .

§ II. Du trophosperme, 281 .

SIII. De l'arille, 282. - De la columelle, 283. - Déhiscence du péricarpe, ibid. - Péricarpes ruptiles ; 284. - Péricarpes déhiscenš ibid. - Nombre des valves, ibid. - La déhiscence valvaire peut être $1^{\circ}$ loculicide $; 2^{\circ}$ septieide $; 3^{\circ}$ septilrage, 235 . - Formes du péricarpe dans son ensemble, ibid. - Le fruit est quelquefois couronné d'une aigrette, 286. - Cette aigrette est sessile ou stipitée, ibicl. Elle est poilue ou plumeuse, $i \dot{b} i d$. - Ailes ou appendices membraneux du péricarpe, 287. - Exemples d'analyse de fruits propres a mieux faire connaltre l’organisation du péricarpe, ibid. - Analyse du fruit du pêcher, ibid. - Analyse du fruit du pois ordinaire, 289. - Résumé présentant toutes les parties qui constituent le péricarpe, ibid.

Crap. Il. Dé la graine, 29o. - Définition de la graine, ibiul - Il n'existe pas de graines nués, e'est-à-dire sans péricarpe, 291. - Nécessité d'ćtudier la structúre de l'ovaire pour connaître celle dù fruit, ibid. - L'essence et la perfection de la graine consistent dans lembryon, 291. - Dissemblance des véritables graines d'avec les corpus- 
cules reproduetifs des plantes agames, ibicl. - Note s!ır la ștructure de l'ovule antérieurement à l'impregnation, 29r. - La graine est formće de deux parties : 10 de l'épisperme ou tégument propre; $2^{\circ} \mathrm{de}$ l'aunande, ibid. - Du hilc ou point d'attaclie de la graine, 292. Sommet, face, bords de la graine, 293.-Graine comprimée, graine déprimée, ibial. - Position des graines, ibid. - Dressćes, ibid. Pienversées, ibid. - Ascendantes, suspenducs, péritropes, 293.

$\int$ Ier. De l'épisperme, 294. - Testa et teginen, ibid. - Hile, omphalode, vasiducte et chalaze, ibid. - Micropile, embryotége, ${ }^{29} 5$.

$\int 11$. De l'amande, 296.-Elle est formće par l'embryon scul ou uni à un cildosperme, ibid.

SIII. De l'endosperme, 296. - Sa couleur, sa substance, 297.

IV. Del'embryon, 298. - Embryon épispermique, ibid. - Embryon endospermique, ibid. - Embryon extraire et intraire, ibid.-L'embryon est formé de quatre parties : $1^{\circ}$ lc corps.radiculaire ou la radicule; $2^{\circ}$ le corps cotylédonaire; $3^{\circ}$ la geminule; $4^{\circ}$ la tigelle. La radicule peutêtre nue ou coléorhizée, c'est-à-dire renfermée dans une colćorhize, 299.-Lc corps cotylédonaire est à un seul, à dcux ou à un grand nombre de cotylédons, 3oo.-Embryon monocotylédoné, et dieotylédonć, ibid. - Nouvelle division des végétaux en endorhizes, exorhizes et synorhizes, 3n . - Ủsages des cotylédons, ibid. - Cotylédons hypogés et épigés, 302. - Feulles séminales, ibid. - De la gemmule ou plumule, ibid. - Fenilles primordiales, ibid. - De la coléoptile, 3o3. - De la tigelle, ibid. - Direction de l'embryon relativement au péricarpe, ibid. - Embryon homotrope, antitrope, ortlotrope et amphytrope, ibid.

SV. De l'embryon dicotylédoné, 304. - Caractères que présentent en général sa radicule, scs deux cotylćdons, sa gemmule, sa tigelle, ibid. - Ses anomalies, 305. - Soudure des deux cotylédons en un seul; le maronnier d'Incle, ibid.

$\int$ VI. De l'embryon monocotylédonć, 3c5. - Souvent on ne pcut bien reconnaitre ses différentes parties que par la germination, ibid. Corps radiculaire, embryon macropode, 3o6. - Radicule enfermée dans une colḱorhize; elle n'est pas toujours simple, ibid. - Corps eotylédonaire, ibid. - Il est simple, indivis, ibid. - Gemmulerenfer mée dans le cotylédon, composéc de petitcs feuilles emboitées les unes dans les autres, ibid.-Piléole, tigelle, se eonfond ordinairement avee le cotylédon ou la raaicule, ibid.-Structure del'embryon des Graminces, ibid. - De l'hy poblaste, 307. - Du blaste, ibid. De la radiculode, ibid. - De l'épiblaste, ibid.

Chap. III. De la germination, 307. - Définition de la germination, ibid. - Circonstances nécessaires à la gcrmination : lcs unes dépen- 
dent de la graine, les autres lui sont accessoircs ou étrangères, ibid. État où doit être la graine, ibirl. - Agens extéricurs indispensables à la germination, 303. - De l'eau, ibid. _ Elle sert de véhicule aux substances alimentaires du végétal, ibirl. - Sa trop grande quantité est nuisible aur graines, ibid. - Elle ramollit l'enveloppe séminale et favorise sa rupture, 3og. — De la chaleur, ibid. - Elle est aussi nécessaire que l'eau, mais ne doit pas passer certains degrés, ibid. Unc chaleur de 25 ì 30 legrés est la plus convenable, ibid. - De l'air, ibid. - Il est aussi utile aux végétaux pour germer et croître qu'aux animaux pour respirer et vivre, ilid._Expériences le Homberg, qui dit avoir vu germer des graines dans le vide de la machine pneunatique, per exactes, ibid. - Action de l'oxigène, 3ı. - Il aide et favorise la gerınination, ibid. - Pur, il l'aceélère d'abord, mais il ne tarde pas à l'arrêter par l'activité trop puissante quỉil lui communique, ibid. - Son action tempérée par sa réunion au gaz azote ou au gaz lyy drogène, ibid. - Proporlions les plus convenables de ce mélange, 3 I I. - L'oxigène, absorbé pendant la germination, se combine avec l'excès de carbone que contient le jeunc végétal, et forme de l'acide carbonique qui est rejeté an dehors; $i b i d$ : - Influence de cette combinaison sur l'enclosperme, ibid. - Expériences de M. de Humboldt avec le chlore, ibid. - Influence du sol et de la lumière sur la germination, 312. - Fhénomènes généraux de la germination, 312. - La radicule paraît la première, 313. - La gemmule parait peu de temps après, itid. - Usages de l'épisperme , 314. - Il sert à empêcher l'eau d'agir trop directement sur l'embryon, ilid. - Origine de l'endosperme, 315, - Usage de l'endosperme, qui fournit les prcmiers matériaux de sa nutrition au jcunic végétal, ibid.- Les cotylédons remplissent souventles mêmes usages que l'endospcrme, $3{ }_{1} 6$.

$\oint I^{e r}$. Germination des embryons exhorhizes ou dicotylédonés, 3 I 7 et suivans.

\II. Germination des embryons endorlizes ou monocotylédonés, 3 ig et suiv.

Chap. IV. Classification des différentcs espèces de fruits, 320. - Considćrés en général, les fr,uits sont disingués en simples, multiples et composés, 321. - Suivant la nature du péricarpe, les fruits sont secs ou charnus, ibid. - Les fruits secs sont déhiscens ou indéhiscens, ibid. - Selon le nombre des graincs qu'ils renferment, on distingue les fruits en oligospermes et en polyspermes, ibid. - Fruits pseudlospermes, 322 . 


\section{Première crasse. Des Fruits simples.}

Sretion première. Fruits secs.

$\$$ I cr. Fruits secs ct indéhiscens, 323.

$1_{0}$ La Caryopse, ibid.

2o L'Akèrae, ilid.-Akènc avec aigrette, ilid.

3o Le Polakène, -Diakène, - Tétrakène, etc. , 32 f.

$4 \circ$ La Samare, ibid.

5o Le Gland, ilid.

6० Le Carcérule, 325.

$\int$ II. Fruits sees et déhiscens, ibid.

10 Le Follicule, ibid.

20 La Silique, ibid.

$3^{\circ}$ La Silicule, $3_{2} 6$.

$4^{\circ}$ La Gousse. Gousse uni-biloculaire, lomentacée, ibid.

50 La Pyxide, ibid.

6o L'Élatérie, $32 \%$.

$7^{\circ}$ La Capsule, ibid.

Secrion II. Des fruits charnus, ibid.

$1^{\circ}$ La Drupe, ibid.

20 La Noix, $3_{2} 8$.

30 Le Nuculaine, ibid.

40 La Mélonide, ibid. - Note sur cette espèce de fruits, ibid. -

Mélonide à nucules, et Mélonide à noyaux, 329 .

50 La Balauste, ibid.

$6^{\circ} \mathrm{La}$ Péponide, ibid.

$7^{\circ}$ L'Hespéridic, 330.

8o La Baie, ibid.

\section{Deuxième classe. Des Fruils mulliples.}

Du Synearpe, 33 r.

Trolsième ciasse. Des Fruils agrégés ou composés.

1o Le Cóne ou Strobile, 332.

$2^{\circ}$ Le Sorose, ibid.

3० Le Sycone, ibid.

Cunp. V. De la Dissémination, 333. - Ce que l'on doit entendre par ce mot, ibid.-C'est le moyen le plus puissant de la reproduction des 
cspèces, ibid. - Circonstances q̨ui favorisent, 334. - Péricarpes qui

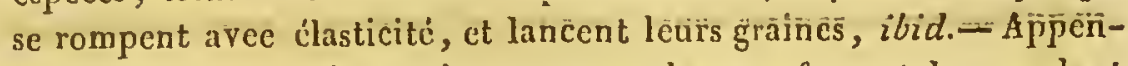
dices divers des graines qui augmentent leur surfaee et Ies rendent plus légères, 335. - Les vents, les flcuves, les eaux de la mer sont les agens de la dissémination, ibid. - Ùsages des fruits et des graincs, 335 et suir.

De la Taxonome, ou des Méthodes botaniques, 339. - Coup d'œil sur l'histoire de la Botanique, ibid.-Théophraste- ibid.-Gesner, 34r. - Les frères Bauhin, Rai,Magnol et Rivin,342.-Cæsalpin, ibid. - Tournefort, ibid. - Linnæus, 343. - Adarnson, Bernard de Jussieu, Antoine Laurent de Jussieu, $3 \not 5$.

Méthode de Tournefort, $3 q 6$ et suiv. - C̈lef de la méthode de Tournefort, 356 .

Système sexuel de Linnæus, 3.57 .

Système sexuel de Linnæus, modifié par M. Fiehard père, 364 .

Clef du système sexuel de Linnæus, 387 .

Clef du système sexuel modin̂é, ibid.

Méthode de M. de Jussieu, ou des familles naturelles, 388 .

Clef de la méthode de M. de Jussieu, 397.

Tableau des familles du règne végétal , 399 .

FIN DE IA TABLE ANAIYTIQUE. 


\title{
TABLE ALPHABBÉTIQUE
}

DES

\author{
FAMILLES DE PLANTES
}

DÉCRITES DANS CET OUVRAGE.

Acantiacées.

Acérinécs.

Algce.

Alismacées.

Alismoüles.

Amaranthacées.

Amaryllida.

Amentacearum genera.

Amomées.

Ampélidécs.

Anonacées.

Apocynées.

Aquifoliacées.

Araliacées.

Ardisiacea

Aristolochićcs.

Aroidécs.

Asclepiadea.

Asparaginées.

Asphodeli.

Atherosperina.

Atriplicea.

Aurantiacćcs.

Azarinea.

Balanophoréss.

Balsamina.

Berbćridées.

Bétulinées.

Bignoniacées.

Bixinées.

Bombacées.

Boopide.
Pag. 462 Borraginées.

510 Broméliacées.

403 Bruniacécs.

428 Bulomece,

lbid. Byttnériacécs.

453

434

563

$43 \mathrm{~S}$

509

490

470

555

487

472

445

412

470

430

$43 \mathrm{I}$

559

452

508

429

Cabomzées.

Calycérées.

Campanulacées.

Canna.

Capparidécs.

Caprifoliacécs.

Caryophyllées.

Casuarinea.

Cedrelece.

Célastrinécs.

Collidea

Cercodiennes.

Champignons.

Characées.

Chénopodées.

Chlénacées.

Chicoracea.

Cistées.

Colchicacées.

444 Combrétacées.

496 Cornmélinécs.

49. Composita.

562 Conifères.

468 Convolvulacées.

Corymbiferce.

499 Crassulacécs.

480 Crucifères.
465

433

533

428

500

420

480

477

438

519

483

527

56 r

512

554

558

541

404

413

452

502

478

523

429

542

426

4.78

563

467

478

534

518 
DES FAMILLES DE PIANTES.

Cucurbitacées.

Cunoniacea.

Cupulifères.

Cycadćes.

Cynarocephala.

Cyperacécs.

Cylinćcs.

Dizlénacíes.

Dioscorées.

Diosmece.

Dipsacées.

Droséracécs

Drymyrrhizées.

EBÉNAcÉEs.

Eléagnées.

Epacridece.

Equisćtacées.

Ericinées.

Erythroxylces.

Euphorbiacées.

Ficoỉdées.

Flacourtianées.

Fluviales.

Fougères.

Frankeniacées.

Fumariacées.

Gextiaxées.

Géraniacées.

Gesnériacées.

Globulariées.

Goodenoviae.

Graminées.

Grossularia.

Guayacanea.

Guttifcres.

Haloragea.

Hama mélidées.

Hemerocallidece.

Hćmodoracées.

Hépathiques.

Hermaninia.

Hippocastanea.

Hippocraticées.

Homalinćes.

Hydrocharidées.

Hydroléacées.

Hydrophytcs.

Hygrobićes.

Hypéricinées.

Hypoxylées. $j$
537 Ilicinea.

53 I Iridćes.

563

565

478

421

446

480

$43 !$

494

401

524

433

473

447

475

413

475

512 .

556

530

522

$410^{\circ}$

410

524
516

469

496

476

458

496

422

536

473

506

54

532

431

436

407

500

509

549

441

$45 \mathrm{I}$

403

$54 \mathrm{I}$

507

406

Joncées.

LA ВIÉES.

Lilacece.

Liliacécs.

Linacece.

Loasćes.

Mousses.

Myricécs.
J ASMUÉES.

Juncaginece.

Laurinćes.

Lćgumincuses.

Lcntibulariées.

Lichénées.

Lobeliacee

Loranthćcs.

Lycopodiacées.

Ly-simachice.

Magnoliacées.

Malpighiacées.

Malvacćes.

Marcgraviacées.

Marsiléacćes.

Mélastomacées.

Méliacées.

Mćnispermćes.

Monimiécs.

Musacées.

Myoporinées.

Myristicées.

Myrsinées.

Myrtacées.

555

435

463

425

428

465

449

55 r

458

406

463

43 I

496

538

477

484

409

457

489

511

$49^{8}$

505

412

545

512

$49^{2}$

559

408

437

464

56 I

450

472

543

434

416

535

454

442

493

504

463

486

$54 \mathrm{I}$

482

472

439

459

496 
588 TABLE ALPHABÉT. DES FAMTLLES DE PLANTES.

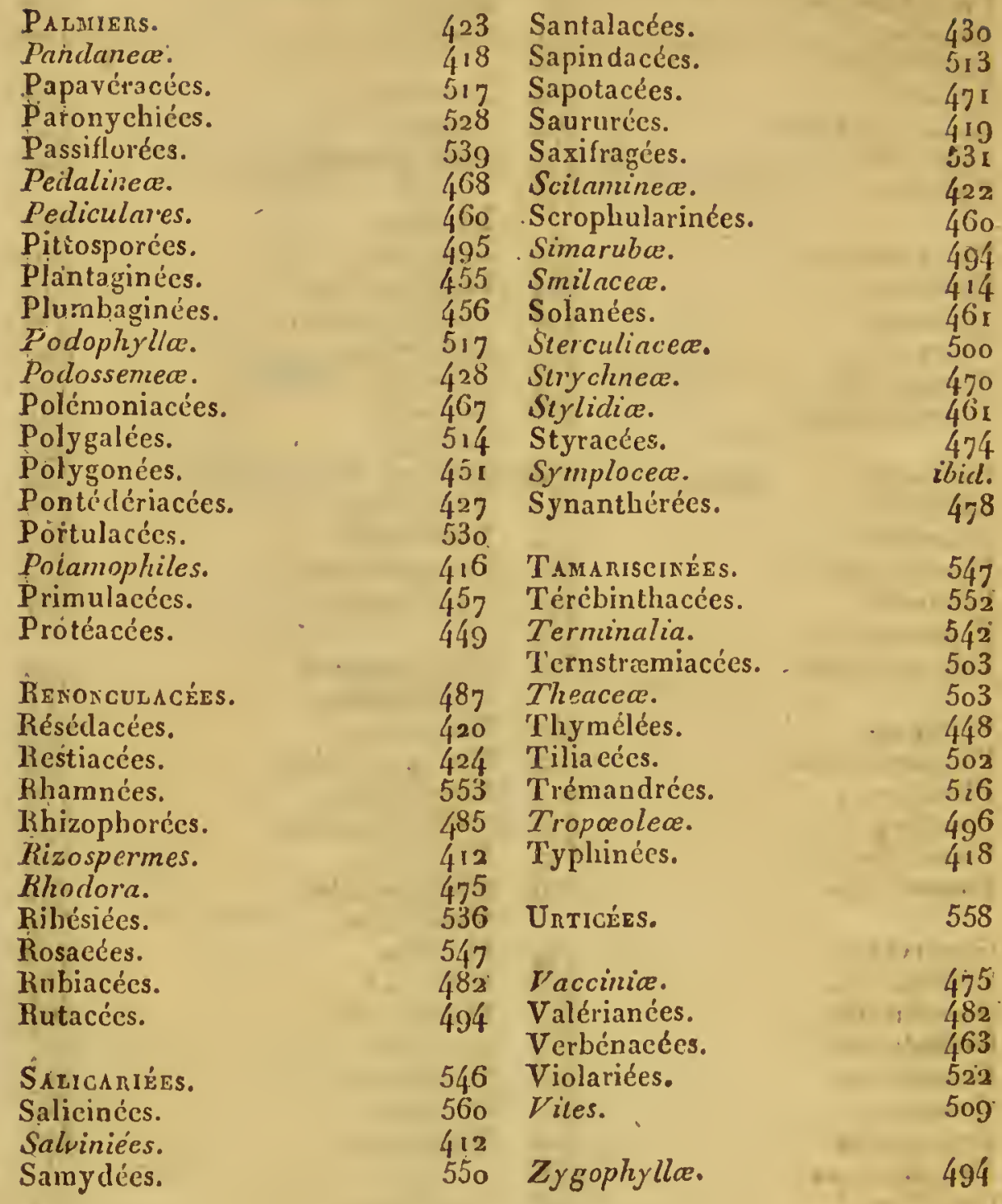



$I \%$,
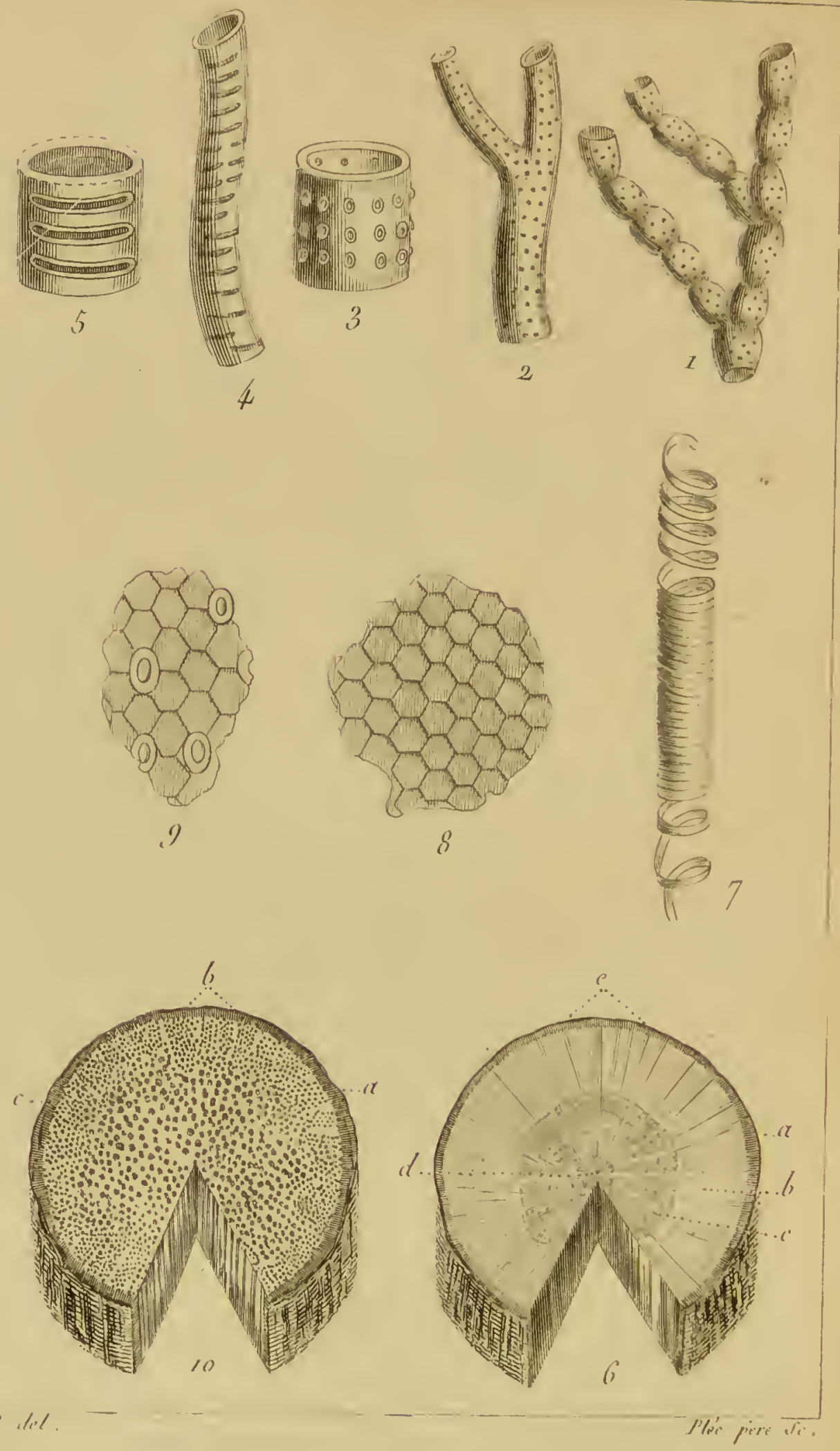

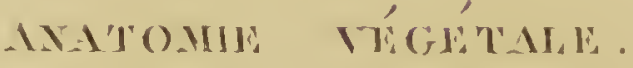


. 
II. 2

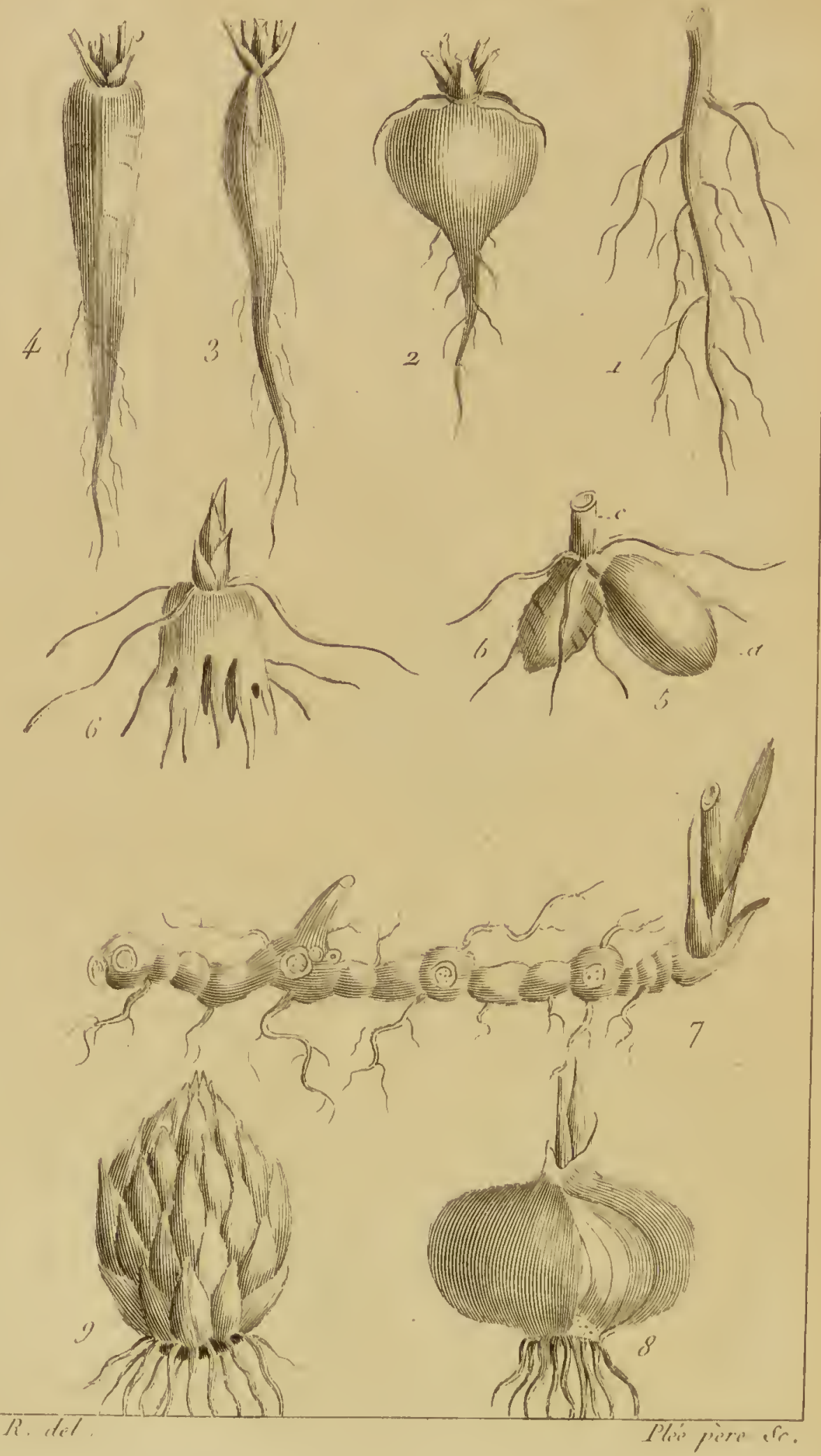

R.ICINHA, SOTCHL, BUTAISS. 


\section{EXPLICATION DES PLANCHES (1).}

\section{PLANCHE PREMLERE. ANatonie végétale.}

Fig. r. V exux moniliformes ou cn chapelet.

Fig. 2. Vaisseaux poreux:

Fig. 3. Portion de vaisseau poreux plus grossie.

Fig. 4. Vaisseau fendu ou fausse trachée.

-Fig. 5. Le même, plus grosșsi.

Fig. 6. Portion du tronc d'un arbre dicotylédoné, composé de couches concentriques : on voit en a l'écorce; en $b$ l'aubier ou faux bois ; en $\dot{c}$ le bois proprement dit; en $d$ le canal médullaire.

Fig. 7. Vaisseau en spirale ou trachée.

Fig. 8. Tissu cellulaire régulier.

Fig. 9. Portion d'épiderme pour faire voir les pores corticaux.

Fig. 10. Portion de stipe d'ụn arbre monocotylédoné, formé de faisceaux de fibres ligneuses, épars au milieu de la sübstance médullaire.

\section{PLANGHE DEUXIEME : RAGIṆES, SOUGHE, BuLbE.}

Fig. I. Racine rameuse, pivotante.

Fig. 2. Racine du radis. (Brasica Napus) Elle ëst. pivotante, simplé et napiforme.

Fig. 3. Racine de la ravc. (variété de l'espèce précédente) Elle est simple, charnue, fusiforme et pivotante.

Fig. 4. Rácine de lá carótte. (Dauctıs Carota, Ł.) Elle est simple, charnue, pivotante, conique.

Fig. 5. Racine tubérifère. (Orchis militaris, L.) Ellle cst didyme, à

(1) Nous avons emprunté plusieurs de nos figures aux Elémens de Physiologic de M. Mirbel et à l’İconographie do M. Turpin. Il était impcrssible de puiser à de meilleures sources. 
tubercules ovoïdes entiers : a est le tubercule qui doit pousser la nouvelle tige; $b$ est eclui qui a fourni la tige.

Fig. 6. hacine tubériforme (Orchis maculata, L.) à tubercules palnés. Fig. 7. Souclie, thizome, ou tige souterraine du sceau de Salomon. (Polygonatum vulgare, Desf.) On voit de distance en distance des empreintes circulaires qui proviennent des pousses des allnées précédentes. C'est à cette espèce de tige que l'on a donné les noms de racin e progressive, sigillée, succise, etc. C'est une véritable tige et non point une racine.

Fig. 8. Bulbe à tuniques de l'ogrion commun. (Allium Cepa, L.)

Fig. 9. Bulbe écailleux du lis. (Liliun candidum, L.) Il est composé d'écailles charnues, et imbriquées à la manière des tuiles d'un toit.

\section{PLANCHE TROISIÈME : Feullles simples.}

Figh. I. Foliole du rosicr. (Rosa centifolia, L.) Elle est ovale, obtuse, dentéc en scic ou serrée.

Fig. 2. Feuille elliptique, obtuse, cntière.

Fig. : 3. Feuille de la paquerette.(Bellis perennis, L.) Elle cst spathulée.

Fig. 4. Fenille du sceau de Notre-Dame ou Taminier. (Tamus communis, L.) Ellé est cordiforne, aiguë, cntière, basinerve.

Fig. 5. Feuille du nénuphar. (Nymphaca alba, L.) Elle est cordiforme, obtuse.

Fig. 6. Fcuille de l'asaret. (Asarum curopaum, L.) Elle est reniforme, obtuse et émarginée au sommet.

Fig. 7. Fénille sagittée ou en fer de flèche.

Fig. 8. Feuille hastée.

Fig. 9. Feuille de l'écuclle d'eau. (Iydrocotyle vulgaris, L.) Elle est orbiculaire, doublement crénelée et peltée.

Fig. Io. Feinilles supćricures du ehèvre-feuille. (Lonicera Caprifolium.) Elles sont cormées.

Fig. I1. Feuille du Buplevrum rotundifolium, L. Ellc est ovale, aiguë, perfoliée.

Fig. 12. Feuille de l'Irydrocolyle tripartita, Thunb. Elle est cuneiforme, quinqué-dentéc.

Fig. 13. Feuille du pissenlit. (Taraxacum dens leonis.) Elle est pinnatifidc et roncinée.

Fig. 14. Fcuille du séneçon vulgaire. (Senecio vulgaris, L.) Elle est pinnatifide, lyrée.

Fig, r 5. Feuille de la passiflore glauque. (Passiflora glauca, Jacq.) Elle est tripartite à lobes lancéolés', aigus, dentés en scie.

Fig. 16. Feuille de la passiflore bleue. (Passiflora carulea, Jacq.) Elle est quinqué-partice digitéc, à lobes lancéolés, sinueux. 
$\% 3$

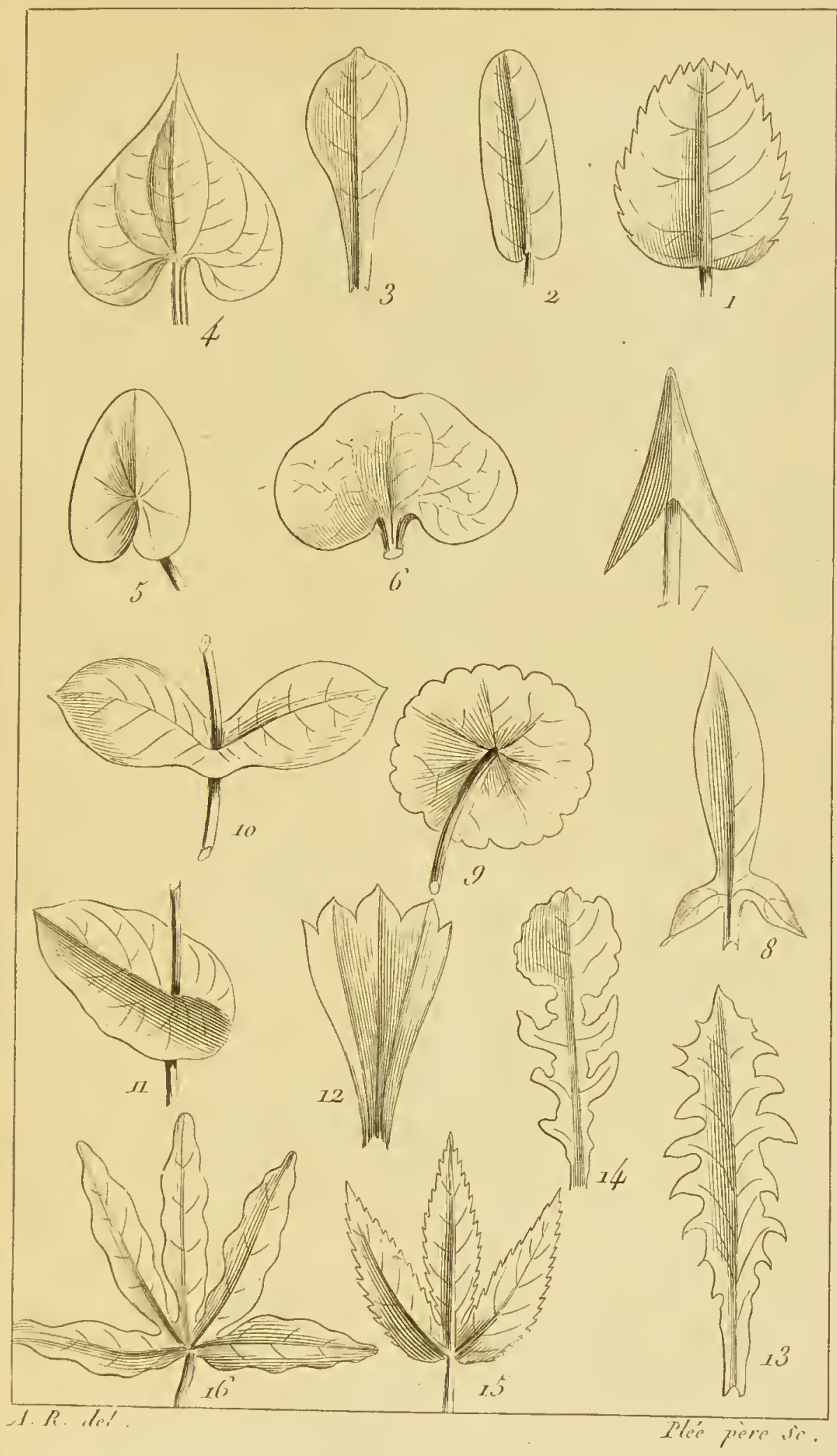

FHCTHALS SIMUTES. 

$\gamma$ 


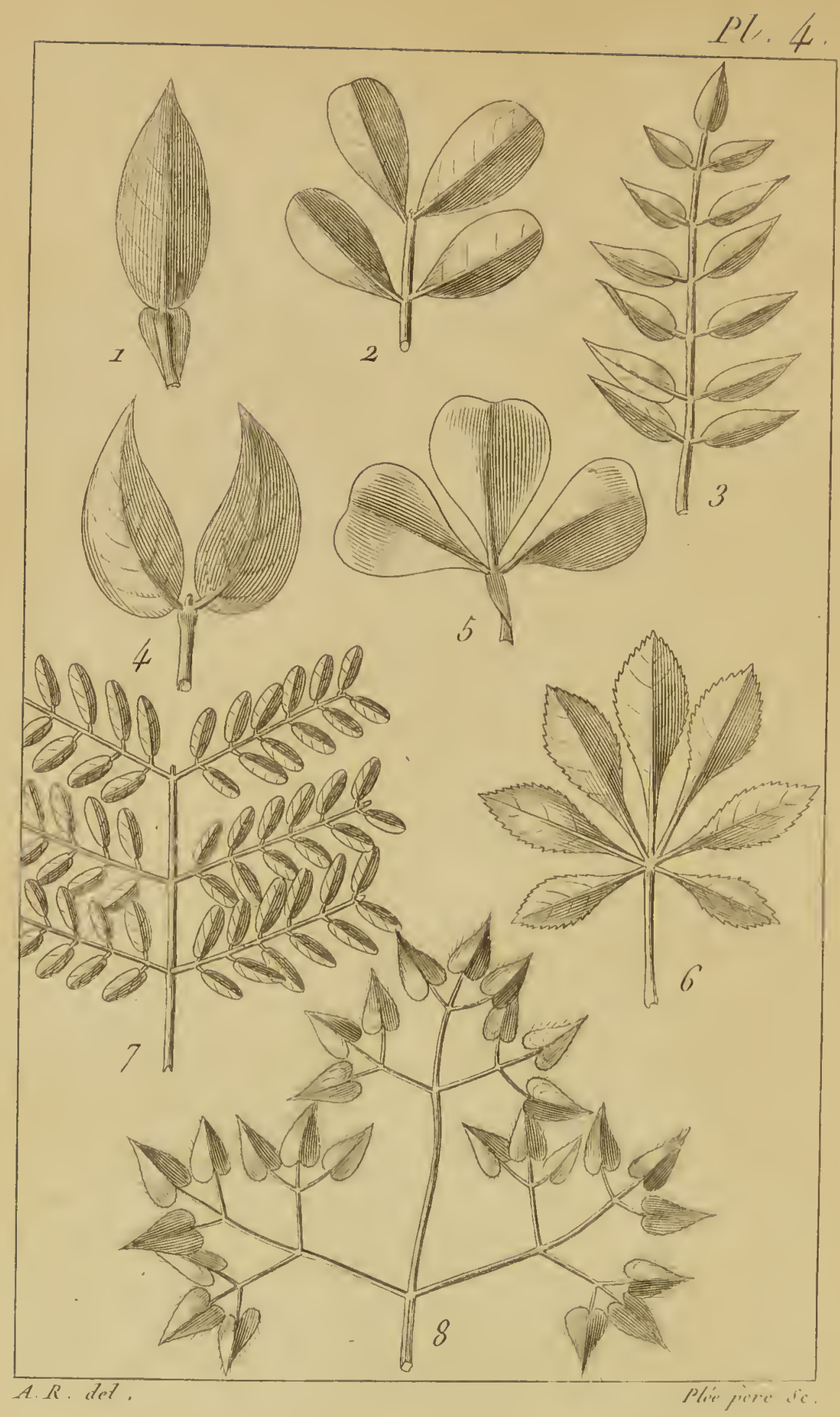

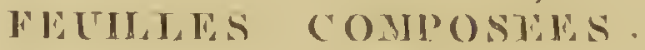



PL.

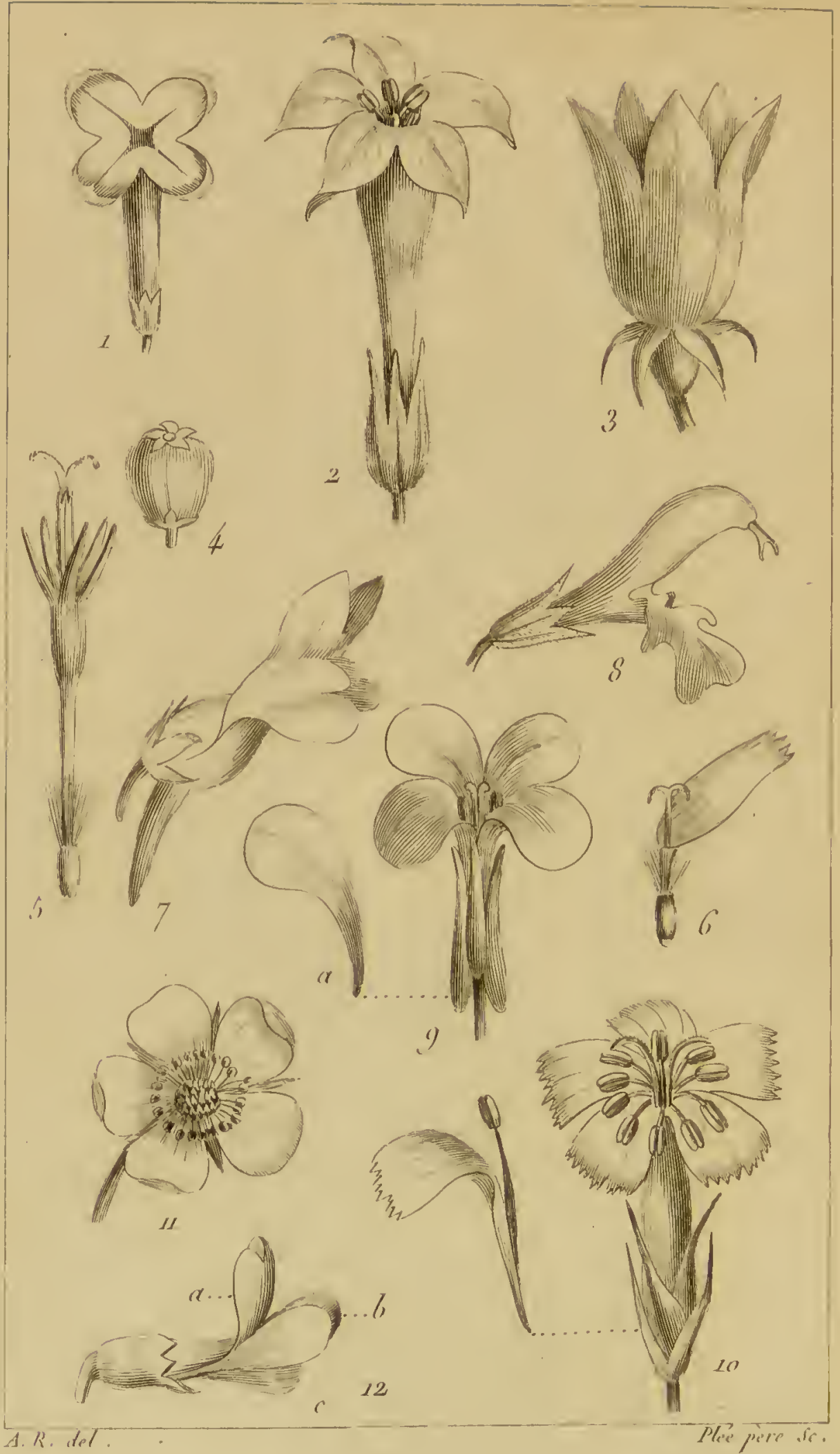

FTAURS. 
$P \% G$.

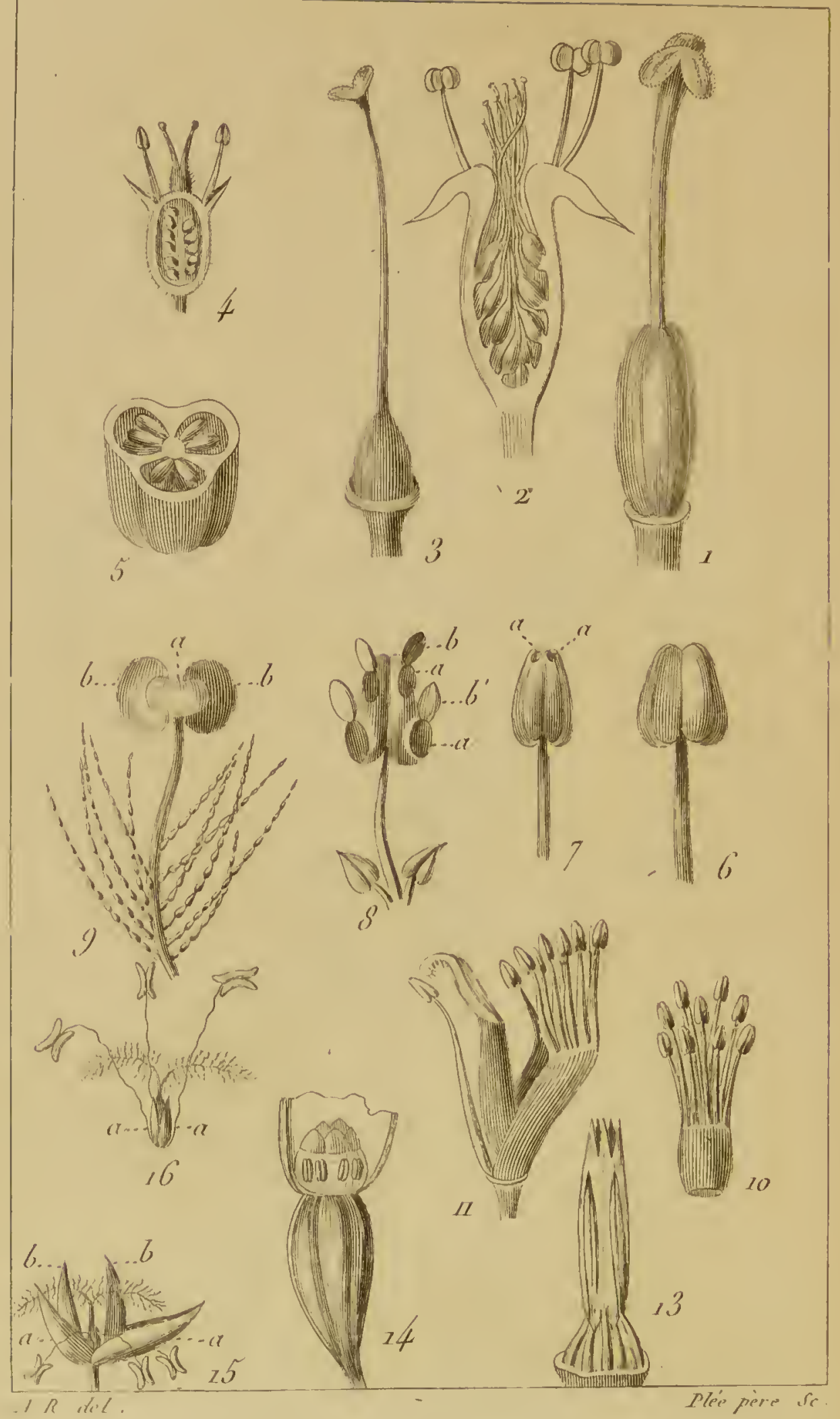




\section{PLANChE QUATRIÈme : Feurlies composées.}

Fig. I. Feuille de l'oranger. (Citjus Aurantium, L.) Elle est composée, unifolièe.

Fig. 2. Feuille paripennés ou pènée sans impaire, bijuguće.

Fig. 3. Feuille du frêne. (Fraxinus excelsior, L.) Elle est imparipennée, ou pennée avec impairé.

Fig. 4. Feuille unijuguée.

Fig. 5. Feuille digitée et trifoliée.

Fig. 6. Feuille du marronnier d'Inde. (Asculus hippocastanum, L.) Elle est cligitée, septem-foliolée ; à folioles obovales, aiguës, dentées.

Fig. 7. Feuille du Mimosa Julibrizin, L. Elle est décomposée, bipennée.

Fig. 8. Feuille décomposée, triıernée. (Epimedium alpinum, L.)

\section{PLANCHE Y. - FLEURS.}

1Fig. 1. Lilas. (Syringa vulgaris, L.) Corolle monopétale réguilière, hypocratériforme.

Fig. 2. Tabac. (Nicotiana Tabacum, L.) Corolle monopétale régulic̀re, infundibuliforme.

Fig. 3. Campanule. Corolle monopétale régulière, campanulée.

Fig: 4. Corolle monopétale régulière, en grelot.

Fig. 5. Fleuron d'un chardon.

Fig. 6. Demi-fleuron.

7ig. 7. Corolle monopétale irrégulière personnée de la linaire. (Antirrhinum Liriaria, L.)

8. Corolle monopétale irrégulière bilabiée.

ig. 9. Giroflée rouge. (Cheiranthus annuns.) Corolle polypétale régulière, cruciforme: $a$, un des pétalcs.

ig. 10. OEillet. (Dianthus caryophyllus.) Corolle polypétalerégulière, caryophyllée.

ig. 11. Fraisier. (Fragaria vesca, L.) Corolle polypétale réguliche, rosacée.

g. 12. Corolle poly pétale irrégulière, papilionacèe.

\section{PLANCHE VI. - ETAMINES Ë PISTILS.}

3. 1. Lis. (Liliume candiclum, L.) Ovaire libre a trois cótes, style clargi au sommet, et terminé par un stigmate trilobé. 
Fig. 2. Rosier des haies. (Rosa cunina, L.) Plusieursovaires parićtaux altachés au parois d'un calice monosépale urećolć.

Fig. 3. Gratiole. (Gratiola officinalis, L.) Ovaire libre, style trèslong, stigmate bilamellè.

Fig. 4. Groseiller épineux. (Ribes Grossularia, L, ) Ovaire infère surmonté d'un style biparti.

Fig. 5. Ovaire à trois loges, triloculaire.

Fig. 6. Étamine dont l'anthère est biloculaire, cordiforme.

Fig. 2. Anthère d'un Solanum s'ouvrant par un petit trou au sommet de ehaque loge.

Fig. 3, Laurier. (Laurus nobilis, L.) Anthère biloculaire, dont les loges s'ouvrent au moyen de petits panneaux : $a, a^{\prime}$ ouvertures des loges, $b, b^{\prime}$ panneaux.

Fig. 9. Éphémère de Virginic. (Tradescantia Virginica.) Étamines dont le filet est chargé de poils articulés; l'anthère est biloculaire didyme; les deux loges $\measuredangle 6$ sont éeartées l'une de l'autre par un connectif $a$.

Fig. 10. Dix étamines monadelphes.

Fig. 11. Dix étamines diadelphes.

Fig. 13. Cing étamines synanthères.

Fig. 14. Aristoloche clématite. (Aristolochia Clenatitis.) Ovaire infère, relevé de côtes; ćtamines soudées avee le style et le stigmate, c'est-à-dire gynandres.

Fig. 15. Graminée. Épillet uniflore : $a$ a les deux valves de la lépicène; $b \quad b$ celles de la glume embrassant le pistil et les trois étamines.

Fig. 16. Le même, dépouillé de la lépicène et de la glume : $a$ a Jes deux éeaillcs de la glumelle.

\section{PLANCHE VII. - Graines et Gehination.}

Fig. 1. Graine de harieot: $a$ hile, $b$ mieropile.

Fig. 2. La mĉ̀ne, dépouillée de son tégument propre on ćpisperme, $c^{2} e s t-a-d i r e$ embryon seul : $a$ radicule, $b$ b les déux cotylédons.

Fig. 3. La nuême, dont on a séparé un des colylédons : $a$ la radieule, $b$ li gemmule, $c$ le second cotylćdon.

Fig. 4. La mêmé, dont on a détaché les deux cotylédons: $a$ radieule, $b$ tigelle, $c c$ la gemmule.

Fig. 5. Haricot germant. On voit sortir la radicule.

Fig. 6. Ricin. (Ricinus communis, L.) a earoneule arilliforme.

Fig. 7 . La même, coupće longitudinalement : $a$ caroneule, $b$ cndosperme, $c$. cmbryon. 
11.7
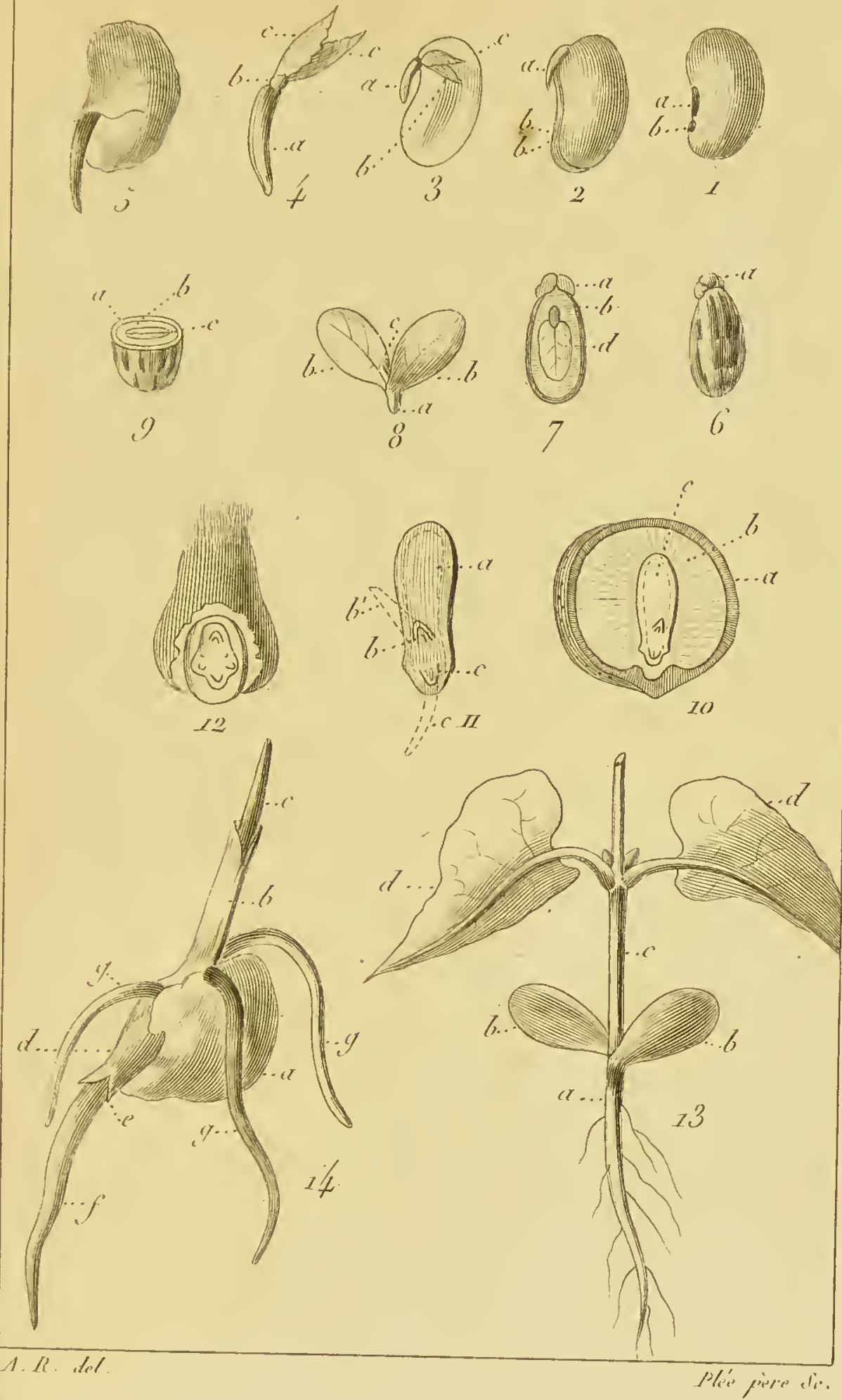

GIRANIA ef GJIRMIINITION. 



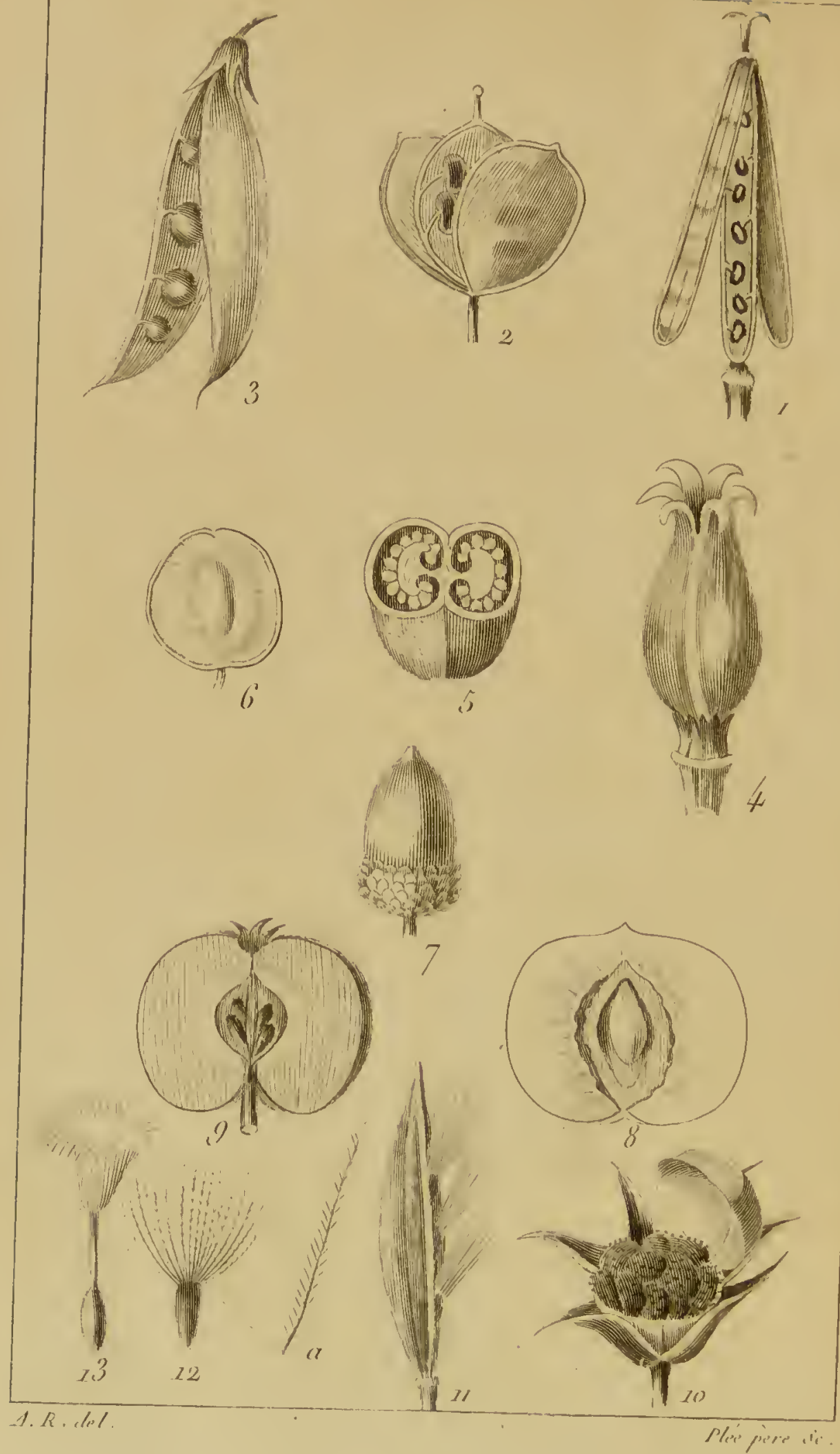
Fig. 8. Embryon séparé de l'intérieur de l'endosperine: a radicule, $b b$ cotylédons, $c$ gemmule.

Fig. 9. La même graine, coupće transversalement.

Fig. 10. Graine de balisier (Canna indica, L.), coupée longitudinalement : $a$ son épisperme, $b$ son endosperme, $c$ son embryon, qui est monocotylédon.

Fig. 11. L'embryon monocotylédon de la graine préeédente séparé : $a$ le eutylédon, $b$ la gemmule renfermée dans le cotylédon , qui en s'allongeant percera le cotylédon latéralement, et deviendra $b^{\prime}$; $c$ la radicule, renfermée dans une coléorhize; qu'elle doit percer en c'pour s'enfoncer dans la terre.

Fig. 12. Le blé (Triticum sativum) dont on a mis à nu l'embryon qui est monocotylédoné.

Fig. 13. Haricot déjà germé : $a$ la radicule, $b b$ les deux cotylédons, qui sont devenus les feuilles séminales, $c$ la tige, $d d$ les follioles de la gemmule, formant les deux feuilles primordiales.

Fig. 14. Graine de maïs ( $Z$ ea Mais, L.) germant: $a$ le corps de la graine formć par l'endosperme farineux, $b$ le cotylédon qui s'est allongé, et contenait dans son intérieur la gemmule qui l'a percé à sa partie supérieure et latérale $(c) ; d$ la coléorhize qui renfermait la radicule principale, $e$ point où la radicule $f$ a peré la coléorhize, sgg radicelles.

\section{PLANCHE VIII. - FRUITS.}

Fig. 1. Silique.

Fig. 2. Silicule.

Fig. 3. Goussc ou Iégume du pois. Fig. 4. Capsule d'un Lyclunis s'ourrant par des dents à sa partie su-
périeure.

Fig. 5. Capsulè biloculaire polysperme.

Fig. 6. Samare.

Fig. 7. Gland d'un chêne.

Fig. 8. Drupe du pêcher.

Fig. 9. Mélonide ou pomme.

Fig. 1o. Pyxide ou eapsule en boite à savonette.

Fig. x I. Follicule.

Fig. 12. Alène couronné d'une aigrette sessile plumeuse : $a$ un des poils détachés.

Fig. 13. Akc̀ue couronné par une aigrette stipitée, poillws.

FIN. 







$$
\text { - }
$$

$\cdot$

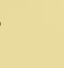

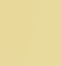
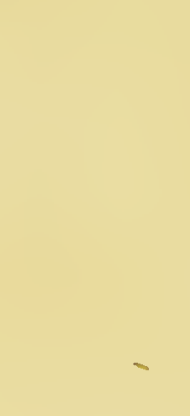


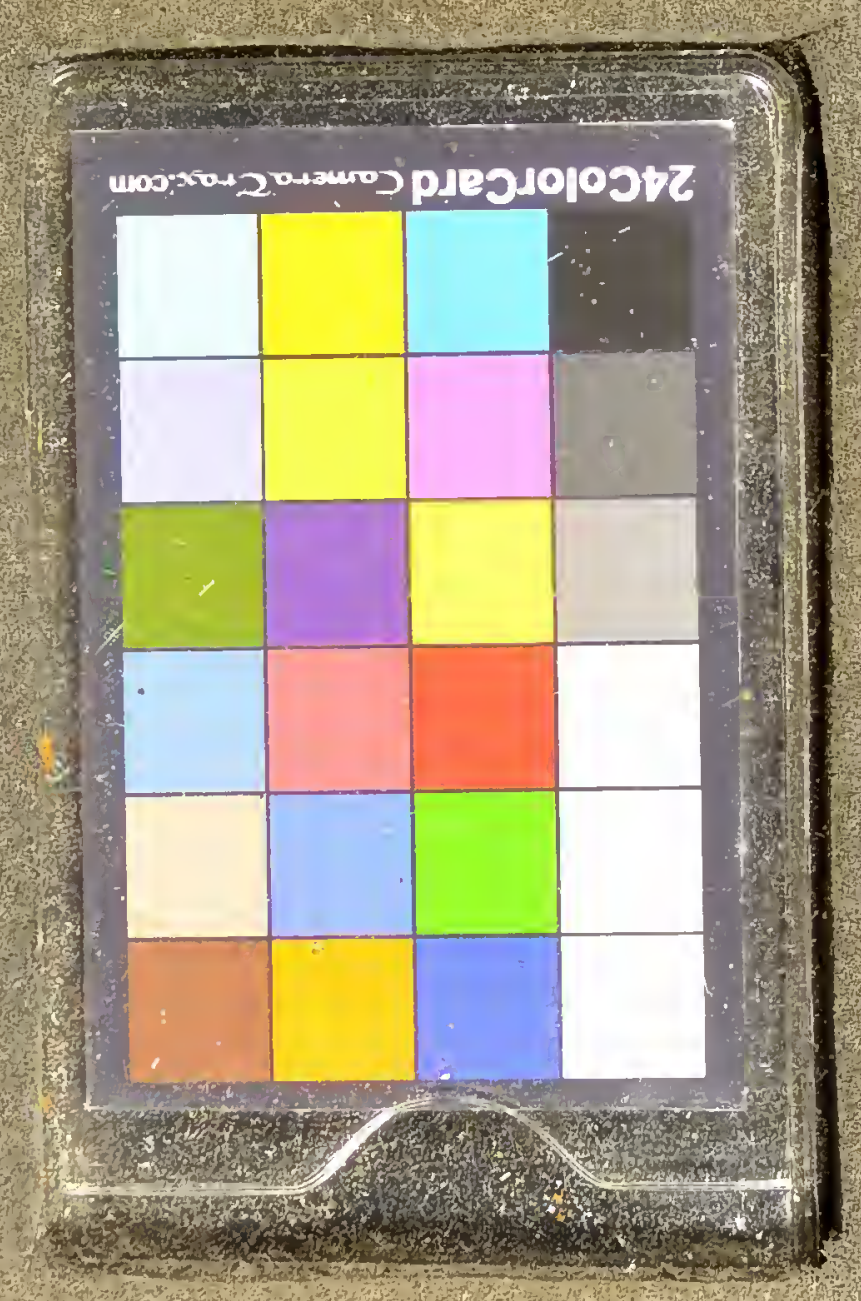

